\title{
ESTABLISHMENT AND \\ MAINTENANCE OF A \\ COAL SAMPLE BANK AND \\ DATA BASE
}

Final Report

April 8, 1988 to

September 28, 1993

by

Alan Davis

David C. Glick

Coal and Organic Petrology Laboratories

The Pennsylvania State University

University Park, PA 16802

Published November, 1993

PREPARED FOR THE

UNITED STATES DEPARTMENT OF ENERGY under Contract No. DE-AC22-87PC79997

U.S. DOE Patent Clearance not required prior to the publication of this document 


\section{TABLE OF CONTENTS}

DISTRIBUTION LIST . . . . . . . . . . . . . . . . . . . . . v v

LIST OF TABLES ...................................... vij

LIST OF FIGURES . . . . . . . . . . . . . . . . . . . . . . . ix

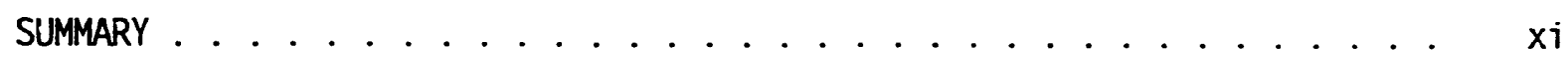

STATEMENT OF OBJECTIVES . . . . . . . . . . . . . . . . . . . 1

TASK I. COAL SAMPLE BANK OPERATION . . . . . . . . . . . . . . . . . 1

A. BACKGROUND .......................................... 1

B. STORAGE OF COAL SAMPLES ...................... . . . 3

Anaiysis of Head Space Oxygen in Bulk Storage Containers . . . . . . . . 3

Initial Studies of Foil Laminate Bags . . . . . . . . . . . . . . . . . 12

Adoption of Foil Laminate Bags and Steel Drums for DECS Samples . . . . 21

Monitoring of Sample Condition . . . . . . . . . . . . . . . 23

C. COLLECTION OF SAMPLES, SAMPLE HANDLING, AND PROCESSING . . . . . . . 51

Collection Methods . . . . . . . . . . . . . . . . . . . . . 51

Sample Processing and Packaging ............... . . 53

Sample Container Inventory . . . . . . . . . . . . . . . . . 53

Samples and Sample Types Collected . . . . . . . . . . . . . 55

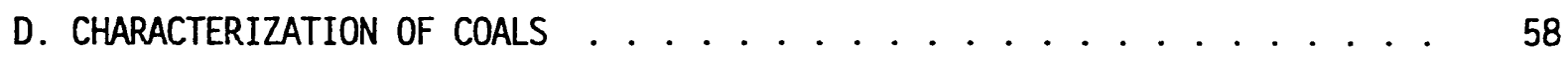

E. DISTRIBUTION OF COAL SAMPLES TO USERS . . . . . . . . . . . . . 60

TASK II. COAL DATA BASE ............................. 63

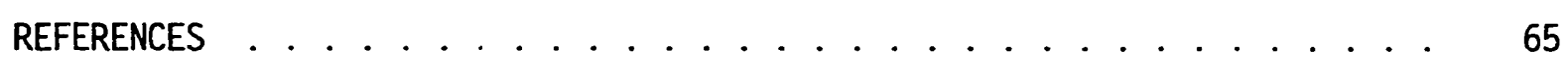

APPENDIX A. Monitoring of DOE Sample Bank Coals to Evaluate

Sample Equivalence and Sample Integrity . . . . . . . . . . 67

APPENDIX B. Coal Data Base Printouts of DECS Samples . . . . . . . . . . . 147

APPENDIX C. List of Agencies Supplied with Coal Samples and/or Data . . . 259 


\section{DISTRIBUTION LIST}

A. Michael J. Baird

U.S. Department of Energy

Pittsburgh Energy Technology Center

P.0. Box 10940, MS 922-H

Pittsburgh, PA 15236

B. Joann C. Zysk

Contract Administrator

U.S. Department of Energy

Pittsburgh Energy Technology Center

P.0. Box 10940, MS 921-118

Pittsburgh, PA 15236

C. Paul Scott

FE-14, B-125 GTN

U.S. Department of Energy

Washington, DC 20545

D. Office of Patent Counsel

Chicago Operations Office

U.S. Department of Energy

9800 South Cass Avenue

Argonne, IL 60439

E. U.S. Department of Energy

Office of Technology Transfer

Pittsburgh Energy Technology Center

P.0. Box 10940. MS 58-M217

Pittsburgh, PA 15236

H. Joanne Wastek

U.S. Department of Energy

Pittsburgh Energy Technology Center

P.0. Box 10940, MS 922-H

Pittsburgh, PA 15236

I. Malvina Farcasiu. Project Manager

U.S. Department of Energy

Pittsburgh Energy Technology Center

P.0. Box 10940, MS 922-H

Pittsburgh, PA 15236 


\section{LIST OF TABLES}

$\underline{\text { Table }}$

Page

1 PSOC sample weights collected and remaining . . . . . . . . . 2

2. Headspace oxygen content of polyethylene drums sampled to evaluate the extent of coal deterioration: Fal1, 1988

3 Comparison of head space oxygen in drums and buckets of PSOC samples after three years in storage . . . . . . . . . . 5

4 Influence of container type on coal characteristics after storage. PSOC-1536 (mvb, Lower Kittanning seam), initialiy sealed in argon . . . . . . . . . . . . . . . . . .

5 Liquefaction data from DECS-12 (hvAb, Pittsburgh seam) after storage ... . . . . . . . . . . . . . . 20

6 Particle size distributions of some minus 20 mesh Argonne National Laboratory Premium Coal Samples . . . . . . . . . . . . 20

7 Moisture, ash, and sulfur levels of DECS samples in foil laminate bags monitored through time ... . . . . . . . . . 25

8 ASTM repeatibility and reproducibility values ......... 26

9 DECS samples collected under the contract . . . . . . . . . . 56

10 Equivalence of moisture, ash, and sulfur levels in splits of DECS Samples .................. . . 59

11 Samples and data distributed $4 / 09 / 88$ to $9 / 08 / 93$. . . . . . . . . 61

12 Numbers of requests for samples and data by agency classification . 62 


\section{LIST OF FIGURES}

Figure

Page

1 Changes in drum oxygen content with time (polarographic oxygen analyzer) 6

2 Changes in container argon content with time (gas chromatograph) . . 9

3 Changes in container oxygen content with time (gas chromatograph) $\quad 10$

4 Changes in container nitrogen content with time (gas chromatograph) 11

5 Changes in Gieseler fluid range with time (mvb Lower Kittanning seam sample) .................... . . . 15

6 Changes in sulfate sulfur (as percent of total sulfur) with time (mvb Lower Kittanning seam samp?e) . . . . . . . . . . . . . 15

7 Changes in calorific value, carbon, and alkali extraction with time (mvb Lower Kittanning seam sample) . . . . . . . . . . . . . 16

8 Changes in fluorescence alteration with storage time (mvb Lower Kittanning seam sample) . . . . . . . . . . . . . . 18

9 Changes in Gieseler fluid range with time (hvAb Pittsburgh seam sample) 19

10 Gieseler fluidity for Argonne Premium Coal Sampies stored in foil laminate bags and original ampoules ............. 22

11 DECS-1 (Upper Wilcox Group, unnamed seam) condition over time . . . 27

12 DECS-2 (Illinois No. 6 seam) condition over time . . . . . . . 28

13 DECS-3 (Coal Basin M seam) condition over time . . . . . . . . 29

14 DECS-4 (Blue seam) condition over time . . . . . . . . . . 30

15 DECS-5 (Hiawatha seam) condition over time . . . . . . . . . 31

16 DECS-6 (Blind Canyon seam) condition over time .......... 32

17 DECS-7 (Adaville No. 1 seam) condition over time .......... 33

18 DECS-8 (Smith-Roland, "Wyodak" seam) condition over time . . . . . . 34

19 DECS-9 (Dietz seam) condition over time ............ 35

20 DECS-10 (Rosebud seam) condition over time . . . . . . . . . 36 
21 DECS-11 (Beulah seam) condition over time . . . . . . . . 37

22 DECS-12 (Pittsburgh seam) condition over time . . . . . . . . 38

23 DECS-13 (SEwell seam) condition over time . . . . . . . . . 39

24 DECS-14 (Upper Kittanning seam) condition over time . . . . . . . 40

25 DECS-15 (Lower Sunnyside seam) condition over time . . . . . . . . . 41

26 DECS-16 (Blind Canyon seam) condition over time . . . . . . . 42

27 DECS-17 (BTind Canyon seam, refrigerated sample) condition over time 43

28 DECS-18 Kentucky No. 9 seam) condition over time . . . . . . . . . 44

29 DECS-19 (Pocahontas No. 3 seam) condition over time . . . . . . . 45

30 DECS-20 (Elkhorn No. 3 seam) condition over time . . . . . . . . 46

31 Gieseler fluid range of bituminous PSOC and DECS samples after storage in drums and foil laminate bags: Linear regression on all appropriate samples ............... .

32 Sulfate sulfur levels of PSOC and DECS samples after storage in drums and foil laminate bags: Linear regression on all samples

33 Heating value of PSOC and DECS samples after storage in drums and foil laminate bags: Linear regression on all samples

34 Apparatus for inert gas purging of samples in steel drums . . . . 52

35 Typical processing scheme for a DECS sample . . . . . . . . . . 54 


\section{SUMMARY}

This DOE contract continued support for the DOE Coal Sample Bank and Data Base at Penn State. Its objective was the maintenance of a set of 50 (1ater modified to 54) high-quality coal samples and related compositional data for the use of DOE contractors and grantees. Fresh samples have been collected to counteract problems arising from deterioration of older sampies during storage. The five-year contract, a three-month no-cost extension, and a three-month unfunded period are covered in this report.

At the beginning of the contract, a new type of container was evaluated for long-term storage of coal samples ranging in quantity from a few grams to over 10 kg (22 lbs). Gieseler fluid behavior, oxidation of pyrite to sulfates, loss of heating value, and other properties were monitored over time. Based on preliminary results. these foil and polyethylene laminate bags were believed to be a significant improvement over the drums, buckets and cans previously used. Use of the bags was therefore instituted with DECS-1. the first sample collected under the contract. Monitoring of the condition of the DECS samples in foil bags and the older (PSOC-series) samples still supported by the contract has verified that the bags preserve the coal samples better than the other containers. All DECS samples. including the bulk reserves, have been stored under conditions where they are not subject to freezing or large temperature changes. Some splits of each DECS sample. and al1 of DECS-17, have been stored under refrigeration since 1991.

Twenty-two DECS samples were collected, ranging in rank from lignite to anthracite and representing the five most productive coal provinces of the U.S. Samples from the other two U.S. coal provinces (Pacific and Alaskan) are available from among the PSOC samples still supported by the contract. Over 6500 containers of DECS samples were created under the contract and 750 containers of 34 PSOC samples are supported by the contract. A new computer program used to track the sample container inventory was finalized.

Representative splits of the samples were distributed to DOE contractors. grantees, and other coal researchers. The standard package was approximately $300 \mathrm{~g}$ (2/3 lb.) of coal at minus 20 mesh. but other quantities and mesh sizes were also available. One sample (DECS-17) was specially processed into $30 \mathrm{~g}$ ( $1 \mathrm{oz}$ ) bags of minus $0.250 \mathrm{~mm}$ ( -60 mesh) coal for use in dispersed catalyst liquefaction research. Distribution has included 932 samples (one or more containers of one sample sent to one recipient at one time) supported by this contract. plus 379 bags of DECS- 17 .

Each sample was characterized by proximate and ultimate analysis. petrographic (vitrinite reflectance and maceral) analysis, physical and thermoplastic testing. and inorganic element analysis. The resulting data, and geologic data on each sample, were entered in a data base which can be used to produce a formatted five-page or one-page printout for each sample. An interactively operated data base can be searched, sorted or summarized to produce tables of selected data or to identify samples meeting a requestor's criteria. During the period covered. 2.313 printouts, and 204 special data reports resulting in distribution of data on 34.086 sanples, were provided on request. 


\section{STATEMENT OF OBJECTIVES}

The objective of this project was the establishment. on a limited scale, of a coal sample bank and related data base activities at Penn State. including:

(1) establishment of a sample bank of no more than fifty U.S. coal samples of proven value to process research personnel:

(2) management of the sample bank to provide high quality coal samples and related compositiona? data:

(3) provision of professional coal expertise for assistance in determining suitable sample specifications and in identifying specific samples to best fit the needs of an individual requestor's coal research;

(4) distribution of samples and scientific data in response to requests from the coal research community: and

(5) replenishment of depleted samples as required to assure adequate supply for future sample requests.

\section{TASK I: COAL SAMPLE BANK OPERATION}

\section{TASK IA. BACKGROUND}

Since 1967. Penn State has been involved with the maintenance of a set of coal samples from across the country. suitable for many aspects of coal researcin. Initially intended for use by various Penn State departments, its value was soon recognized by other research agencies. Provision of samples and data to other researchers has been supported by U.S. Department of the Interior's Office of Coal Research (1966-1975), U.S. Energy Research and Development Agency (19751980). and U.S. Department of Energy (1980-1993). Samples collected through the end of the previous contract had been identified by sequentially assigned numbers from PSOC (Penn State / Office of Coal Research) -1 through PSCC-1535.

These years encompassed a continuing program directed towards improvement in consistent sample collection. processing, distribution, analytical techniques. data computerization and container inventory operation. Thus. facilities for all of these aspects of the project were already in place when the contract began in Apri1. 1988.

Distribution of samples and data continued uninterrupted from the previous contract. Table 1 shows the samples supported at the onset of the contract and the amounts of samples remaining at the conclusion of the reporting period. 
Table 1. FSOC sample weights collected and reinaining

\begin{tabular}{|c|c|c|c|}
\hline \multirow{2}{*}{$\begin{array}{l}\text { PSOC } \\
\text { Number }\end{array}$} & \multicolumn{3}{|c|}{ Approximate Sample Weights in Kilograms } \\
\hline & Collected & Remaining $4 / 88$ & Remaining $8 / 93$ \\
\hline $\begin{array}{l}1107 \\
1442 \\
1443 \\
1444 \\
1445 \\
1446 \\
1447 \\
1448 \\
1451 \\
1461 \\
1468 \\
1469 \\
1470 \\
1471 \\
1474 \\
1475 \\
1482 \\
1485 \\
1486 \\
1487 \\
1488 \\
1489 \\
1490 \\
1491 \\
1492 \\
1493 \\
1494 \\
495 \\
1496 \\
1497 \\
1498 \\
1499 \\
1500 \\
1501 \\
1502 \\
1503 \\
1504 \\
1506 \\
1507 \\
1508 \\
1515 \\
1516 \\
1517 \\
1518 \\
1519 \\
1520 \\
1521 \\
1522 \\
1523 \\
1524 \\
1532 \\
1533 \\
1534 \\
1535\end{array}$ & $\begin{array}{l}907 \\
260 \\
260 \\
326 \\
239 \\
321 \\
327 \\
316 \\
256 \\
365 \\
359 \\
320 \\
279 \\
302 \\
340 \\
419 \\
293 \\
300 \\
329 \\
385 \\
338 \\
335 \\
231 \\
252 \\
284 \\
332 \\
241 \\
259 \\
267 \\
245 \\
332 \\
406 \\
452 \\
286 \\
251 \\
339 \\
350 \\
267 \\
246 \\
586 \\
359 \\
327 \\
275 \\
252 \\
277 \\
339 \\
334 \\
264 \\
320 \\
323 \\
213 \\
161 \\
212 \\
194\end{array}$ & \begin{tabular}{|l|}
882 \\
214 \\
194 \\
323 \\
228 \\
319 \\
326 \\
305 \\
192 \\
363 \\
332 \\
304 \\
273 \\
287 \\
336 \\
390 \\
290 \\
293 \\
322 \\
383 \\
335 \\
328 \\
229 \\
249 \\
266 \\
283 \\
213 \\
252 \\
264 \\
243 \\
330 \\
403 \\
449 \\
283 \\
244 \\
319 \\
347 \\
248 \\
198 \\
579 \\
354 \\
300 \\
141 \\
244 \\
269 \\
305 \\
332 \\
261 \\
317 \\
315 \\
202 \\
154 \\
204 \\
183 \\
\end{tabular} & 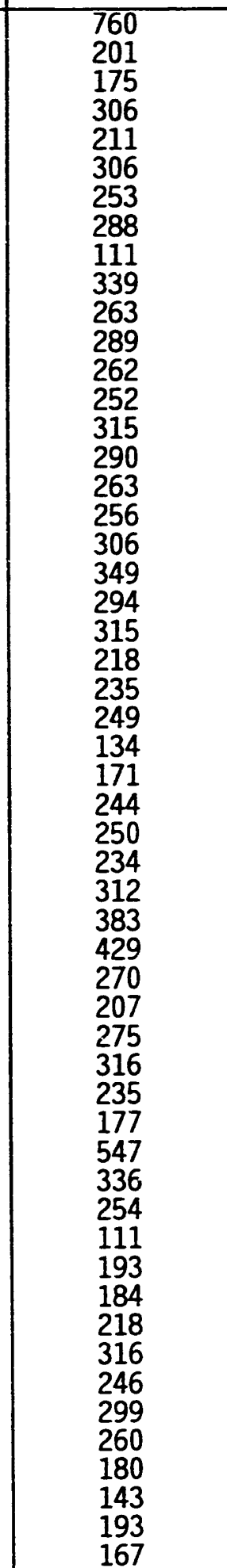 \\
\hline
\end{tabular}


TASK IB. STORAGE OF COAL SAMPLES

For the samples collected during the previous contract and supported by the subject contract, the processing procedure generally produced two $100 \mathrm{~kg}$ (220 $\mathrm{lb}$ ) drums of minus $25 \mathrm{~mm}\left(-1^{\prime \prime}\right)$ coat, one $50 \mathrm{~kg}(110 \mathrm{lb})$ drum of minus $6 \mathrm{~mm}\left(-1 / 4^{\mathrm{n}}\right)$ coal, and three $12 \mathrm{~kg}(26 \mathrm{lb})$ buckets of minus $6 \mathrm{~mm}\left(-1 / 4^{\mathrm{N}}\right)$ coal to be stored for future use and to meet requests for larger amounts of samples. The fourth bucket created was used to produce minus $0.85 \mathrm{~mm}(-20$ mesh) coal, stored in number 2 size steel cans of at least $225 \mathrm{~g}(1 / 2 \mathrm{lb})$ net weight, for distribution and analysis.

Past experience of both DOE and Penn State had led to the belief that preservation of a sample's initial characteristics over long-term storage could be improved. Argon gas was already being used to displace air inside sample containers before sealing. but samples showed varying levels of change nonetheless.

In order to quantify these changes, the contract provided for a program to monitor the condition of all supported samples through time. Thie plan was to use a polarographic gaseous oxygen analyzer to test the atmosphere inside twc drums of each sample (the instrument's probe pierces the drum lid, withdraws a sample, and the hole is sealed: the drum need not be opened). For each sample, one of the drums of minus $25 \mathrm{~mm}\left(-1^{\prime \prime}\right)$ coal was to be tested every six months without the drum being opened. The drum of minus $\epsilon \mathrm{mm}\left(-1 / 4^{\prime \prime}\right)$ coal was to be tested every year, and would then be opened in order to split out $2.3 \mathrm{~kg}(5 \mathrm{lb})$ of coal for chemical evaluation of the coal's condition. Moisture. ash. and total sulfur would indicate how well the subsample matched the subsample originally analyzed and other subsamples used for monitoring. Other analyses would ind:cate the extent of change fram the coal's original condition. Gieseler fluid properties (maximum fluidity aiid flutid temperature range) are among the most sensitive indicators of deterioration during storage for bituminous coals: decreases in these parameters indicate deterioration. Another sensitive indicator. particularly in coals with abundant iron sulfide minerals, is the oxidation of sulfides to sulfates, which can be measured by the increase in sulfate sulfur level. Decreases in calorific value are less severe but also indicate deterioration. Alkali extraction was also to be performed in order to evaluate its application for such monitoring.

\section{Analysis of Head Space Oxygen in Bulk Storage Containers}

\section{Ingold Polarographic Oxygen Analyzer}

The head space oxygen contents of primary (113 liter. 30 gal.) coal storage containers as well as selected secondary (19 liter. 5 gal.) containers were measured. Tables 2 and 3 show the results of this effort. including comments on the physical appearance of the coals observed when selected containers were opened to obtain representative splits to document sample integrity. 
Table 2. Headspace oxygen content of polyethylene drums sampled to evaluate the extent of coal deterioration: Fall, 1988

\begin{tabular}{|c|c|c|c|c|}
\hline $\begin{array}{l}\text { PSOC } \\
\text { Number }\end{array}$ & Seam Name & Rank & $\begin{array}{l}\% \text { Head } \\
\text { Oxygen }\end{array}$ & Comments* \\
\hline $\begin{array}{l}1107 \\
1442 \\
1443 \\
1444 \\
1445 \\
1446 \\
1447 \\
1448 \\
1451 \\
1461 \\
1468 \\
1469 \\
1470 \\
1471 \\
1474 \\
1475 \\
1482 \\
1485 \\
1486 \\
1487 \\
1488 \\
1489 \\
1490 \\
1491 \\
1492 \\
1493 \\
1494 \\
1495 \\
1496 \\
1497 \\
1498 \\
1499 \\
1500 \\
1501 \\
1502 \\
1503 \\
1504 \\
1506 \\
1507 \\
1508 \\
1515 \\
1516 \\
1517 \\
1518 \\
1519 \\
1520 \\
1521 \\
1522 \\
1523 \\
1524 \\
1532 \\
1533 \\
1534 \\
1535\end{array}$ & $\begin{array}{l}\text { King Cannel } \\
\text { Darco } \\
\text { unnamed } \\
\text { unnamed } \\
\text { Blue \#1 } \\
\text { New Mexico \#8 } \\
\text { Red } \\
\text { York Canyon } \\
\text { Pittsburgh } \\
\text { Mammoth } \\
\text { Buck Mountain } \\
\text { Mary Lee } \\
\text { Pratt } \\
\text { Pee Wee } \\
\text { Splashdam } \\
\text { Elkhorn \#3 } \\
\text { Hagel } \\
\text { Rosebud } \\
\text { Big Dirty } \\
\text { Adaville \#1 } \\
\text { Dietz } \\
\text { Pust } \\
\text { Brazi1 Block } \\
\text { Indiana \#6 } \\
\text { I11inois \#5 } \\
\text { Illinois \#6 } \\
\text { Kentucky \#9 } \\
\text { Bevier-Wheeler } \\
\text { Tebo } \\
\text { I11inois \#2 } \\
\text { Wadge } \\
\text { Colorado B } \\
\text { Basin B } \\
\text { Juanita C } \\
\text { Hiawatha } \\
\text { Blind Canyon } \\
\text { U. Sunnyside } \\
\text { Banner } \\
\text { Beulah } \\
\text { Pocahontas \#3 } \\
\text { Pa. Anthracite } \\
\text { L. Kittanning } \\
\text { L. Kittanning } \\
\text { M. Kittanning } \\
\text { Pittsburgh } \\
\text { Smith-Roland } \\
\text { L. Hartshorne } \\
\text { Sewel1 } \\
\text { \#5 Block } \\
\text { U. Kittanning } \\
\text { \#4 Usibelli } \\
\text { \#3 Usibelli } \\
\text { Waterfal1 } \\
\text { Deadfal1 Syn. \#4 }\end{array}$ & 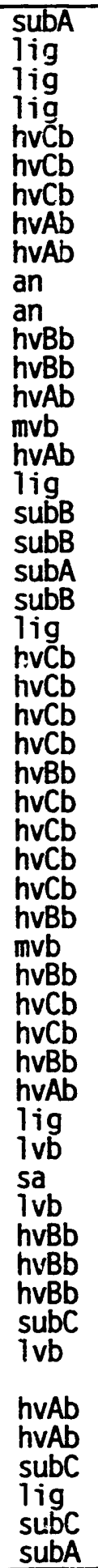 & $\begin{array}{r}r \\
3.2 \\
3.8 \\
1.4 \\
5.7 \\
12.3 \\
13.0 \\
18.7 \\
13.7 \\
19.7 \\
17.8 \\
15.0 \\
8.6 \\
15.7 \\
16.3 \\
9.5 \\
1.6 \\
8.1 \\
4.3 \\
13.4 \\
3.4 \\
10.6 \\
16.4 \\
15.0 \\
16.1 \\
3.0 \\
13.7 \\
15.8 \\
12.8 \\
11.9 \\
15.6 \\
17.1 \\
19.7 \\
16.4 \\
6.2 \\
16.9 \\
18.7 \\
19.4 \\
6.7 \\
20.7 \\
19.8 \\
20.5 \\
16.4 \\
18.5 \\
18.3 \\
78.8 \\
18.8 \\
19.1 \\
20.3 \\
15.7 \\
17.2 \\
13.6 \\
20.8 \\
\end{array}$ & $\begin{array}{l}\text { moist, trace } Y \text { sulfate } \\
\text { moist, trace } Y . \text { W sulfate } \\
\text { moist } \\
\text { moist. trace } Y \text { sulfate } \\
\text { trace } Y \text { sulfate } \\
\text { moist } \\
\text { moist, trace } Y . W \text { sulfate } \\
\text { moist } \\
\text { moist } \\
\text { moist, trace } Y \text { sulfate } \\
\text { iron stain } \\
\text { trace } W \text { sulfate } \\
\text { W sulfate } \\
\text { moist }\end{array}$ \\
\hline
\end{tabular}

*KEY: $Y=$ yellow $W=$ white moist $=$ visible condensation inside drum 
Table 3. Comparison of head space oxygen in drums and buckets ci PSOC samples after three years in storage

\begin{tabular}{|c|l|c|c|c|}
\hline $\begin{array}{c}\text { PSOC } \\
\text { Number }\end{array}$ & Se $\approx \pi$ Name & Rank & $\begin{array}{c}\% \text { Head Oxygen } \\
\text { in Drum }\end{array}$ & $\begin{array}{c}\text { \% Hoad Oxygen } \\
\text { in bucket }\end{array}$ \\
\hline 1446 & New Mexico \#8 & hvCb & 12.3 & 20.6 \\
1470 & Pratt & hvBb & 8.6 & 17.9 \\
1488 & Dietz & subB & 3.4 & 18.0 \\
\hline
\end{tabular}

Procedure

An Ingold 507 Head Space Oxygen Analyzer with a hard package sampling arm was used to measure the percentage of oxygen in the atmosphere within the containers. The hard package sampling arm aliows a needle to puncture the container lid and about $3 \mathrm{cc}$ of gas is drawn through the needle into a cuvette. A membrane in the cuvette allows gaseous oxygen to pass into an electrolyte gel where it is reduced to hydroxyl. The sensor generates a signal proportional to the hydroxyl concentration; the signal is displayed on a digital panel meter as percent oxygen. Calibration is achieved by sampling normal air and adjusting the signal to read 20.9 percent $0_{2}$. The time required from intake of the sample until a stable reading is achieved is about 30 seconds. Accuracy is reported by the manufacturer to be plus or minus $0.25 \%$. Increased accuracy at low oxygen levels may be achieved by calibration agiinst a gas standard; this method was not employed.

Results

At the onset of the gas and coal sampling program a problem with the 19 liter ( $5 \mathrm{gal}$.) buckets was noted. The screw-top 1 ids on about 20 percent of the containers had loosened. Head space oxygen analysis on the other 80 percent of the buckets revealed that many of them had also undergone leakage. Table 3 provides evidence of the susceptibility of these containers to atmospheric ?eakage. Because of the obvious inadequacy of the screw-top polyethylene buckets, further documentation of the gas conteric of these containers was not undertaken.

The oxygen contents of three primary containers were periodically measured over 36 days. Two drumis of subbituminous coal (PSOC-1488) and one drum of anthracite (PSOC-1468) were opened and then resealed after flushing with argon. In order to achieve a still higher argon content. vacuum pumping followed by' more argon introduction was then performed. Figure 1 shows how the oxygen contents of these drums changed during storage. 


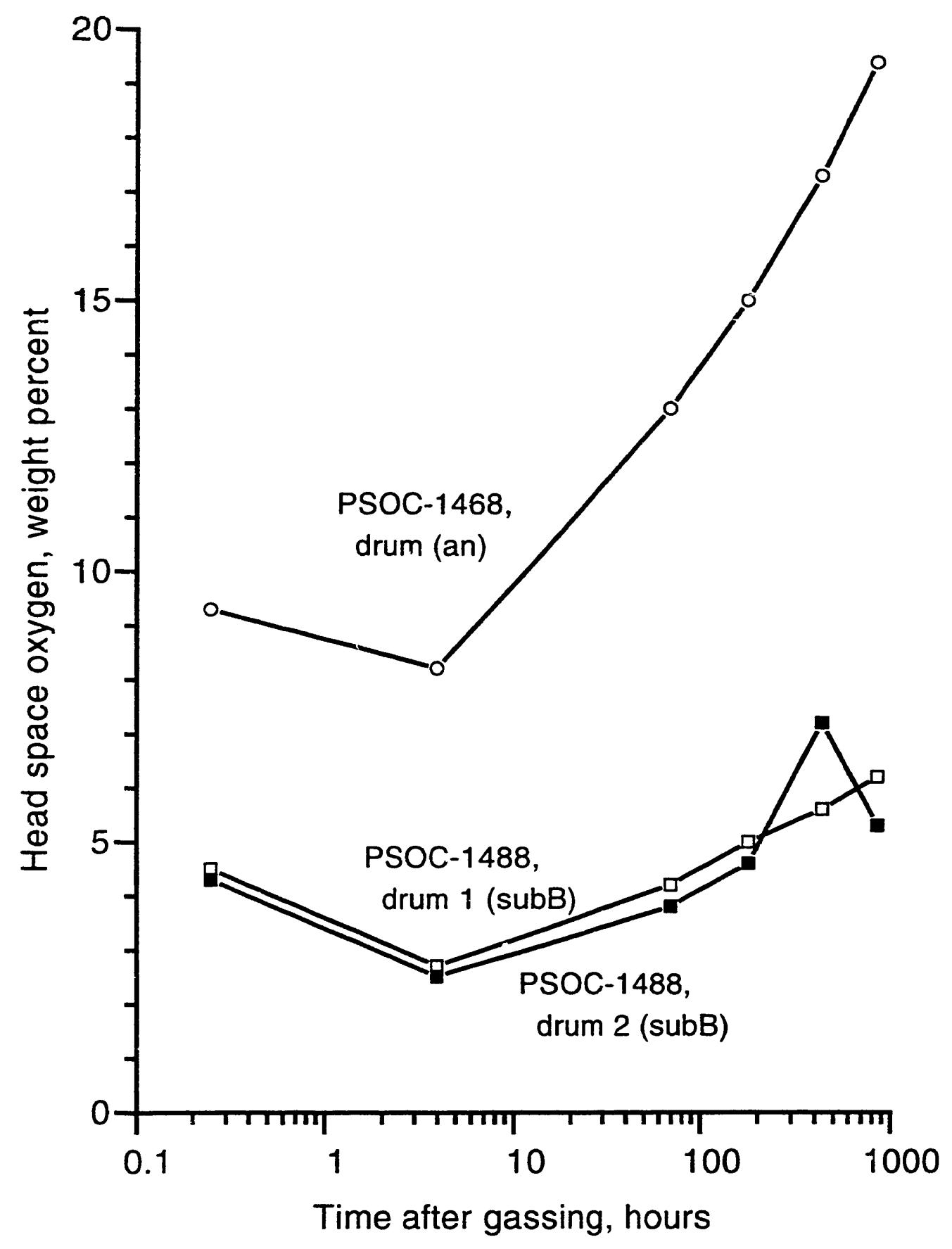

Figure 1. Changes in drum oxygen content with time (polarographic oxygen analyzer) 


\section{Gas Chromatograph}

Three bituminous coal samples were selected from the Penn State Coal Sample Bank for container atmosphere analysis by gas chromatography. This study was undertaken entirely with support from non-DOE sources. The samples were chosen for their relative freshness and similarity in rank, storage time, composition, mesh size, and weight in the storage containers. Two (F $30 C-1529$ and 1531) were samples from the Pittsburgh seam and one (PSOC-1530) was from the Sewickley seam. All three had been collected over a period of two days in July, 1987. One 113 liter (30 gal.) polyethylene drum and one 19 liter ( 5 gal.) polyethyle..e bucket of each sample were used. No DOE samples met the requirements for similarity as well as the chosen samples, and no single sample was available in similar mesh size and quantity in three drums and three buckets.

\section{Procedure}

The gas chromatograph was calibrated fur analysis of oxygen. nitrogen and argori. The results are expressed in mole percent of each gas. Before the coal sample containers were gassed, the argon from the argon tank to be used was analyzed. It was found to contain no measurable oxygen (the detection limit of the instrument was approximately 0.1 percent oxygen).

The six containers were moved to the gas chromatography lab and were fitted with gas sampling ports fabricated from tire valves and resealing membrane caps. After being opened for this procedure, argon gas was introduced into the six different containers by the following alternative methods:

for 113 liter (30-gal.) drums:

1) The lid was sealed, and the container was pumped with a small vacuum pump for approximately 2 minutes, then argon was introduced until a slight positive pressure was reached.

2) Same, except with large vacuum pump.

3) The lid was placed loosely over the container to allow the introduction of a stainless steel wand, through which argon was introduced to the bottom of the container. The wand was gradually withdrawn while the argon flowed for approximately 4 minutes.

for 19 liter (five-gal.) buckets:

1) The lid was sealed. and the container pumped with a small vacuum pump for approximately 30 seconds. until the bucket began to deform. Argon was introduced until a slight positive pressure was reached.

2) Same, except that the pumping and argon introduction were repeated after 3 hours.

3) A wand was used as in \#3, under drums, above. 
Gas samples were withdrawn through the membrane sampling ports with a $10-\mathrm{ml}$ syringe and analyzed immediately for nitrogen, oxygen and argon with a Varian model 3700 gas chromatograph.

Results

Results of the analyses on gas samples taken approximately 30 seconds. 4 hours, 24 hours, 4 days, and 75 days after gassing are plotted in Figures 2,3 and 4 using logarithmic scales for time. The three figures show levels of argon. oxygen and nitrogen respectively. The plotted argon levels at 0.01 hinurs (30 seconds) show the varying efficiency of the argon introduction methods. The initial argon content of the two vacuum-pumied drums was lower than that of the other four containers, but this would have been increased by longer pumping times.

The gas compositions after four hours probably reflect both diffusion into the interstices and pores of the coal samples and leakage of the containers. In the three containers treated once with a vacuum pump, gas samples were withdrawn approximately $10 \mathrm{~cm}$ from the argon introduction ports, and there was little time for diffusion before the first gas sampling. In comparison, the other three containers (treated with a wand or pumped twice) would have undergone more diffusion before the initial sampling. Their rate of argon level decrease at the sampling port over the first four hours would have been influenced less by diffusion and more by leakage.

Further drops in argon levels ( 1 day, 4 days and 75 days) are assumed to be related primarily to container leakage. The bucket treated with the wand, which appeared to leak the least over the first 4 hours, apparently developed a leak before 24 hours. By 75 days, the argon levels in al1 6 containers had dropped below 2 mole percent.

Figure 3 shows that measured oxygen levels in all containers rose between the 30-second and 4-hour samples. This is likely to have been caused at least in part by movement of gases within the containers. 0xygen would have migrated out of pores in the coal into the free gas, where it was measured. In four of the six containers, oxygen levels subsequently declined, which is believed to reflect adsorption of oxygen onto the sample and oxidation of the coal in an atmosphere of limited oxygen. Since the argon levels show that all the containers leak, the oxygen levels are assumed to reflect an equilibrium between oxygen leakage and uptake rates. The four containers with final oxygen contents of less than $5 \%$ show oxygen uptake by the coal to have occurred faster than the oxygen leakage. In the two buckets with final oxygen levels of approximately $20 \%$, the leakage rate was high enough that the gas composition was nearly that of air. Both final oxygen contents over 20 mole percent are assumed to be in error and should be 18.9 mole percent, the level of oxygen in atmospheric air.

The nitrogen levels (Fig. 4) reflect the leakage of air into the containers. with the coal depleting the atmosphere of oxygen in the four moderately tight containers, thereby enriching it in nitrogen. 


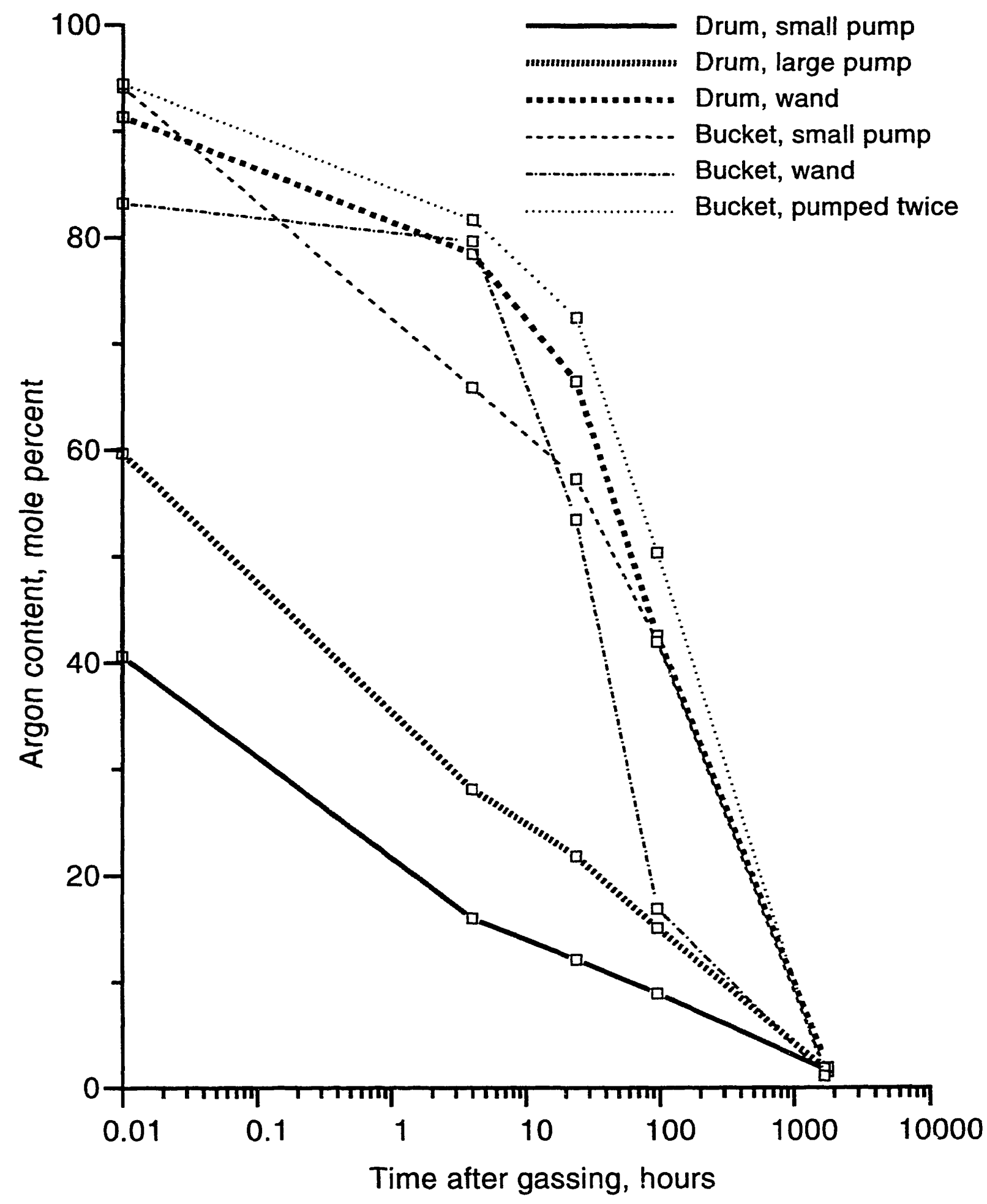

Figure 2. Changes in container argon content with time (gas chromatograph) 


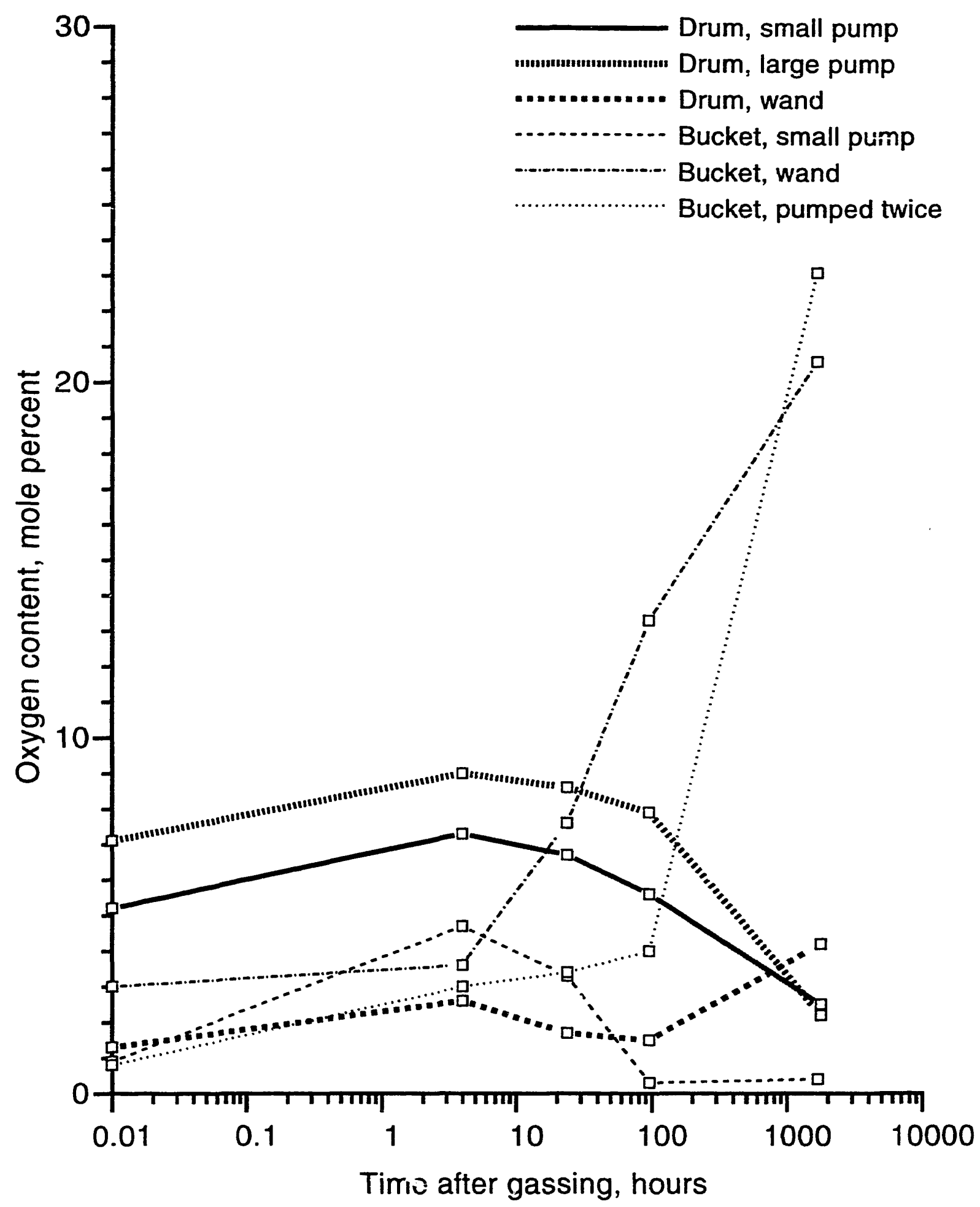

Figure 3. Changes in container oxygen content with time (gas chromatograph) 


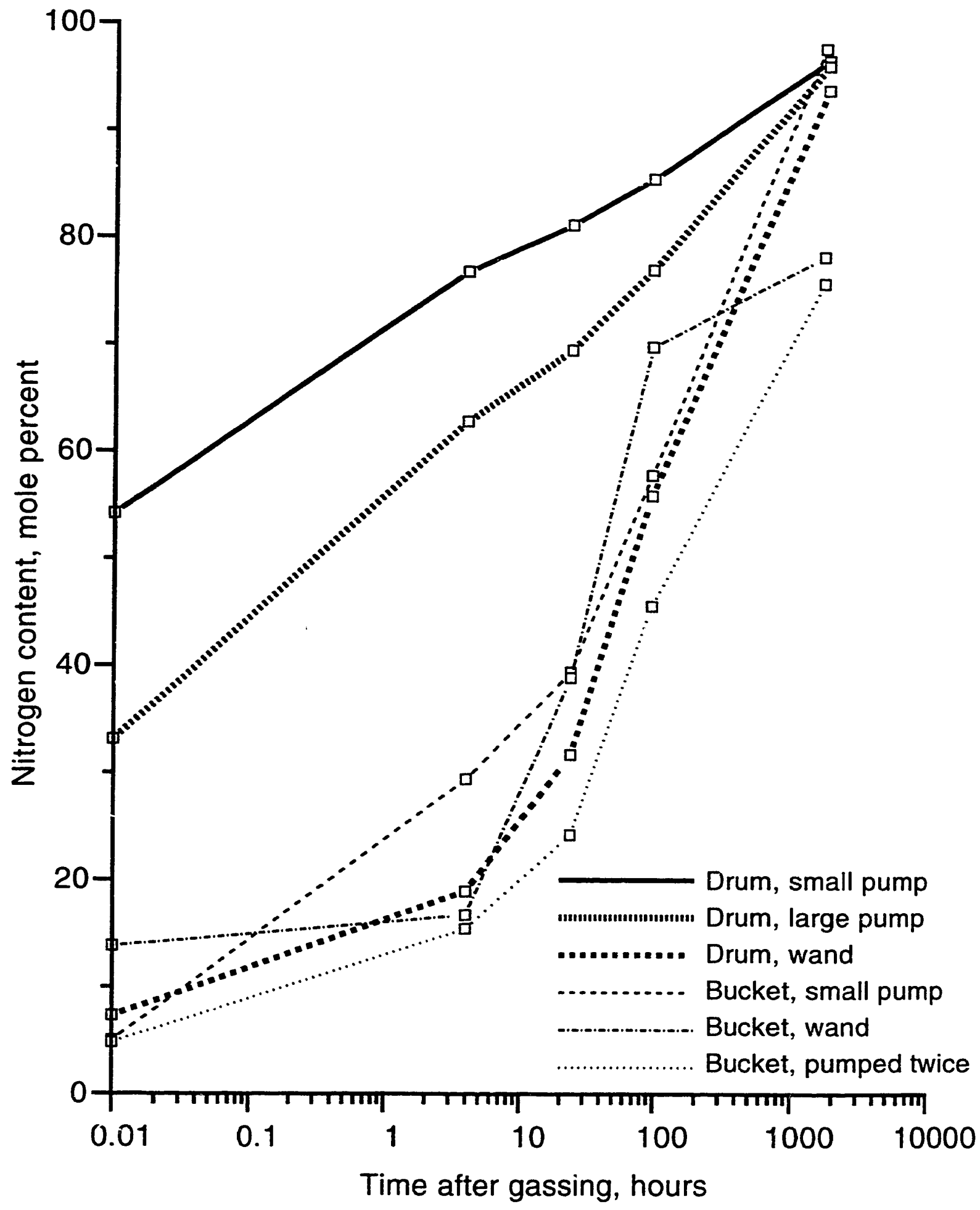

Figure 4. Changes in container nitrogen content with time (gas chromatograph) 
Conclusions

Data from two studies using different instruments indicated that atmospheric oxygen was leaking into the primary and secondary bulk storage containers of PSOC samples. The methods and containers tested cannot maintain an inert atmosphere over the long term. The actual percentage of oxygen in the containers is thought to be influenced by several factors:

1) Flushing with argon by any method only displaces part (50 to $80 \%$ ) of the atmospheric air present in the container after it is filled with coal. Because the atmosphere may not be homogeneous, and because leakage may be a factor before it becomes homogeneous by diffusion. this fraction is difficult to determine by sampling and analysis.

2) The amount of air displaced depends upon the argon introduction method used and its duration. Methods which allow time for diffusion of air to an exit before argon introduction ends appear to be most effective.

3) After sealing the containers, oxygen in the container is consumed by the coal: the amount and rate of consumption are thought to be a function of coal rank.

4) Leakage of air into the container may replace some or all of the oxygen consumed by the coal.

5) Full containers generally exhibit lower head space oxygen contents.

6) The lid seals of the 19-7iter (five-gal.) screw-top containers are unreliable.

7) The rate of leakage and the rate of oxygen consumption are likely to show disparate responses caused by seasonal temperature variation.

After the first year of polarographic and gas chromatographic research, it became apparent that the containers used did not exclude oxygen successfully for long-term storage. DOE agreed that the oxygen analyses had served their purpose and that they could be discontinued. The contractor's efforts were then concentrated on researching better types of containers for long-term storage.

\section{Initial Studies of Foil Laminate Bags}

Foil laminate bags had been shown to successfully preserve moisture in subbituminous coal samples for short-term storage (Sharp, 1986). Several types of laminate bags are available; military specification Mil B-131, the type used by Sharp. was also recommended by the distributor for its durability. A polyethylene inner layer allows heat-sealing and shields the contents from the middle aluminum foil layer which provides a barrier to gas transport and light. An outer spun-bonded polyethylene fiber layer resists tears and abrasions. Sealing may be accomplished by manual flushing with inert gas and a manually operated sealer, or an automated vacuum/inert gas sealer as used in the food industry. 
Some of these bags and an appropriate manual heat-sealer were acquired and a program was planned to test their ability to preserve pyritic sulfur. Gieseler fluidity, calorific value, and an inert atmosphere for a bituminous coal sample. The work was performed with support from the Penn State Cooperative Program in Coal Research.

Medium volatile bituminous Lower Kittanning seam coal (PSOC-1536)

A run-of-mine sample of the medium volatile bituminous (mean-max. $R_{0} 1.27 \%$ ) Lower Kittanning seam was collected as PSOC-1536 and promptly processed.

Gieseler fluidity. FSI, alkali extraction and preparation of petrographic pellets were accomplished within 32 hours after extraction of the coal from the mine face. A split was submitted for initial chemical analysis, and others were sealed for other studies.

Splits of the sample were packaged in several container types for the study: $-6 \mathrm{~mm}\left(-1 / 4^{\prime \prime}\right)$ coal in polyethylene buckets and foil laminate bags within buckets: $-0.85 \mathrm{~mm}$ ( -20 mesh) coal in steel cans, foil laminate bags and an open container exposed to t'ie atmosphere. After 1, 4, 12, 22, and 27 months containers were opened and samples re-analyzed.

All re-analysis results for the various coniainer types are shown in Table 4. Figure 5 shows that foil laminate bags maintained fluid range better than steel cans and much better than buckets. After storage for 12 months, the sample stored in the bucket had lost its thermoplastic character. However. the fluid range of the bagged $-0.85 \mathrm{~mm}(-20$ mesh) coal had dropped by only 5 percent (within the range of experimental error). Maximum fluidity and free-swelling index showed similar trends.

The initial analysis for the sample showed 5.88 percent total sulfur and 0.06 percent sulfate sulfur. Figure 6 shows that foil laminate bags avoided production of sulfates better than cans and much better than buckets. As with fluid range, the bucket sample was deteriorated even more than the sample exposed to the atmosphere. The bucket sample had much higher moisture than the exposed sample. perhaps caused by leakage, trapping of moist air, and subsequent condensation. Surface moisture would have accelerated the sulfide to sulfate reaction.

Figure 7 shows the change over time of the alkali extraction test, carbon content, and calorific value of the mvb sample over time of storage in foil bags as opposed to a sample left exposed to the laboratory atmosphere. All of these results show that the foil bags tended to preserve the initial condition of the coal. compared to the desarioration which occurs when the coal is exposed.

The foil laminate bags performed best at maintaining maximum Gieseler fluidity. fluid range and low sulfate sulfur (expressed as $\%$ of total sulfur to minimize effects of variability in the sulfate sulfur analysis). Coal at minus 6 $\mathrm{mm}\left(-1 / 4^{\prime \prime}\right)$ in foil laminate bags within caulked buckets maintained maximum fluidity and low sulfate sulfur better than coal at minus $0.85 \mathrm{~mm}(-20$ mesh) in 
Table 4. Influence of container type on coal characteristics after storage, PSOC-1536 (mvb, Lower Kittanning seam), initially sealed in argon

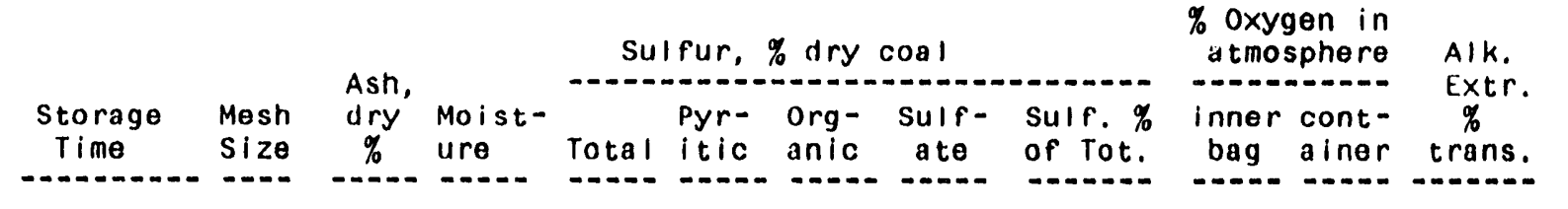

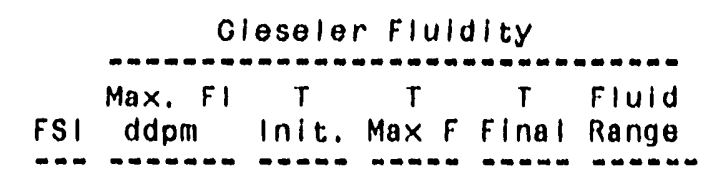

Foil Iaminate bags, -20 mesh coal

$\begin{array}{llllllll}\text { original } & & 21.37 & 1.34 & 5.88 & 4.54 & 1.28 & 0.06 \\ 4 \text { weeks } & 20 M & 20.50 & 1.43 & 5.31 & 4.45 & 0.79 & 0.06 \\ 16 \text { weeks } & 20 M & 21.40 & 1.38 & 5.47 & 4.98 & 0.43 & 0.06 \\ 52 \text { weeks } & 20 M & 1.22 & 1.29 & 5.69 & 4.35 & 1.31 & 0.04 \\ 93 \text { weeks } & 20 M & 1.46 & 1.08 & 5.35 & 4.63 & 0.68 & 0.03 \\ 116 \text { weeks } & 20 M & .9 .55 & 1.27 & 5.30 & 3.71 & 1.55 & 0.04\end{array}$

1.0
1.1
1.1
0.7
0.6
0.8

$\begin{array}{rr}- & 100.0 \\ 0.29 & 100.0 \\ 0.32 & 97.9 \\ & 96.2 \\ 0.47 & 98.9 \\ & 97.1\end{array}$

9.0
8.0
8.0
8.0

Steel can with inner polyethylene bag, -20 mesh coal

$\begin{array}{llllllll}\text { original } & & 21.37 & 1.34 & 5.88 & 4.54 & 1.28 & 0.06 \\ 4 \text { weeks } & 20 M & 21.20 & 1.03 & 5.23 & 4.36 & 0.80 & 0.07 \\ 16 \text { weeks } & 20 M & 21.20 & 1.42 & 5.37 & 4.98 & 0.34 & 0.05 \\ 52 \text { weeks } & 20 M & 21.35 & 1.63 & 5.71 & 4.23 & 1.19 & 0.20\end{array}$

$$
\begin{aligned}
& 1.0 \\
& 1.3 \\
& 0.9 \\
& 3.5
\end{aligned}
$$

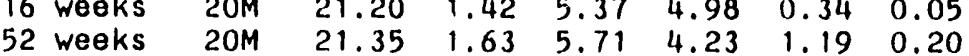

$\begin{array}{rrr} & - & 100.0 \\ 1.33 & 1.20 & 99.2 \\ 1.48 & 97.4 \\ & 0.60 & 94.9\end{array}$

$$
9.0
$$

9.0

9.0
8.5

Polyethylene bucket, $-1 / 4$ " coal

$\begin{array}{llllllll}\text { original } & 21.37 & 1.34 & 5.88 & 4.54 & 1.28 & 0.06 & 1.0\end{array}$

$\begin{array}{llllllll}4 \text { weeks } 1 / 4^{\prime \prime} & 20.40 & 2.22 & 5.27 & 4.24 & 0.85 & 0.17\end{array}$

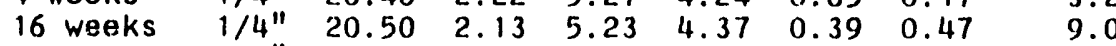

52 weeks $1 / 4$ " $15.61 \quad 5.21$ ? $4.87 \quad 2.44 \quad 1.26 \quad 1.18 \quad 24.2$

$\begin{array}{rr}17.80 & 100.0 \\ 16.20 & 100.0 \\ 13.30 & 97.4\end{array}$

8.0

Polyethylene bucket with caulked Iid, $-1 / 4^{\prime \prime}$ coal

$\begin{array}{lllllll}\text { original } & 21.37 & 1.34 & 5.88 & 4.54 & 1.28 & 0.06\end{array}$

2.8

$0.35 \quad 100.0$
07.5

$9.0 \quad 1224$

$\begin{array}{rr}9.0 & 560 \\ 7.5 & 1\end{array}$

$\begin{array}{ll}7.5 & 1 \\ 6.0 & 0\end{array}$

$\begin{array}{rrr}1224 & 410 & 472 \\ 968 & 417 & 472\end{array}$

$\begin{array}{rrr}950 & 415 & 474 \\ 1937 & 410 & 470\end{array}$

$472 \quad 505 \quad 95$

$931 \quad 410 \quad 467 \quad 505 \quad 95$

Foil laminate bags within polyethylene bucket with caulked Iid, $-1 / 4 "$ coal

origina 1

$\begin{array}{llllllll}21.37 & 1.34 & 5.88 & 4.54 & 1.28 & 0.06\end{array}$

$\begin{array}{lllllll}4 \text { weeks } 1 / 4 " & 21.30 & 1.30 & 5.50 & 4.64 & 0.82 & 0.04\end{array}$

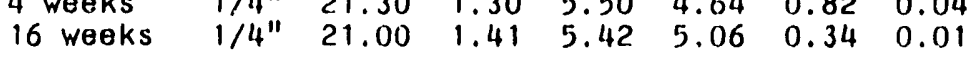

1.0

0.7

$\begin{array}{rrr}0.28 & 20.70 \quad 100.0 \\ 0.70 & 99.9\end{array}$

$\begin{array}{ll}9.0 & 12.24 \\ 8.5 & 1173\end{array}$

$0.1720 .30 \quad 97.8$

8.51169

$\begin{array}{lllr}410 & 472 & 510 & 100 \\ 409 & 1172 & 508 & 99 \\ 417 & 474 & 508 & 91\end{array}$

Exposed to atmosphere, -20 mesh coal

$\begin{array}{llllllll}\text { original } & & 21.37 & 1.34 & 5.88 & 4.54 & 1.28 & 0.06 \\ 4 \text { weeks } & 20 M & 22.60 & 0.54 & 5.34 & 4.25 & 1.06 & 0.03 \\ 16 \text { weeks } & 20 M & 20.30 & 0.94 & 4.98 & 4.37 & 0.42 & 0.18 \\ 52 \text { weeks } & 20 M & 21.89 & 0.66 & 5.39 & 4.18 & 1.43 & 0.39 \\ 116-9 \text { wks } & 20 M & 20.60 & 1.34 & 5.34 & 2.30 & 1.95 & 1.09\end{array}$

1.0

0.6

3.6

$116-9$ wks $20 M$

$\begin{array}{llllll}20.60 & 1.34 & 5.34 & 2.30 & 1.95 & 1.09\end{array}$

20.4

$\begin{array}{rr}(20.9) & 100.0 \\ (20.9) & 99.6 \\ (20.9) & 96.8 \\ (20.9) & 94.2 \\ & 93.0\end{array}$

$\begin{array}{rrrrrr}9.0 & 1224 & 410 & 172 & 510 & 100 \\ 8.5 & 568 & 414 & 472 & 504 & 89 \\ 8.0 & 155 & 426 & 472 & 505 & 80 \\ 7.0 & 2 & 447 & 1166 & 189 & 42\end{array}$




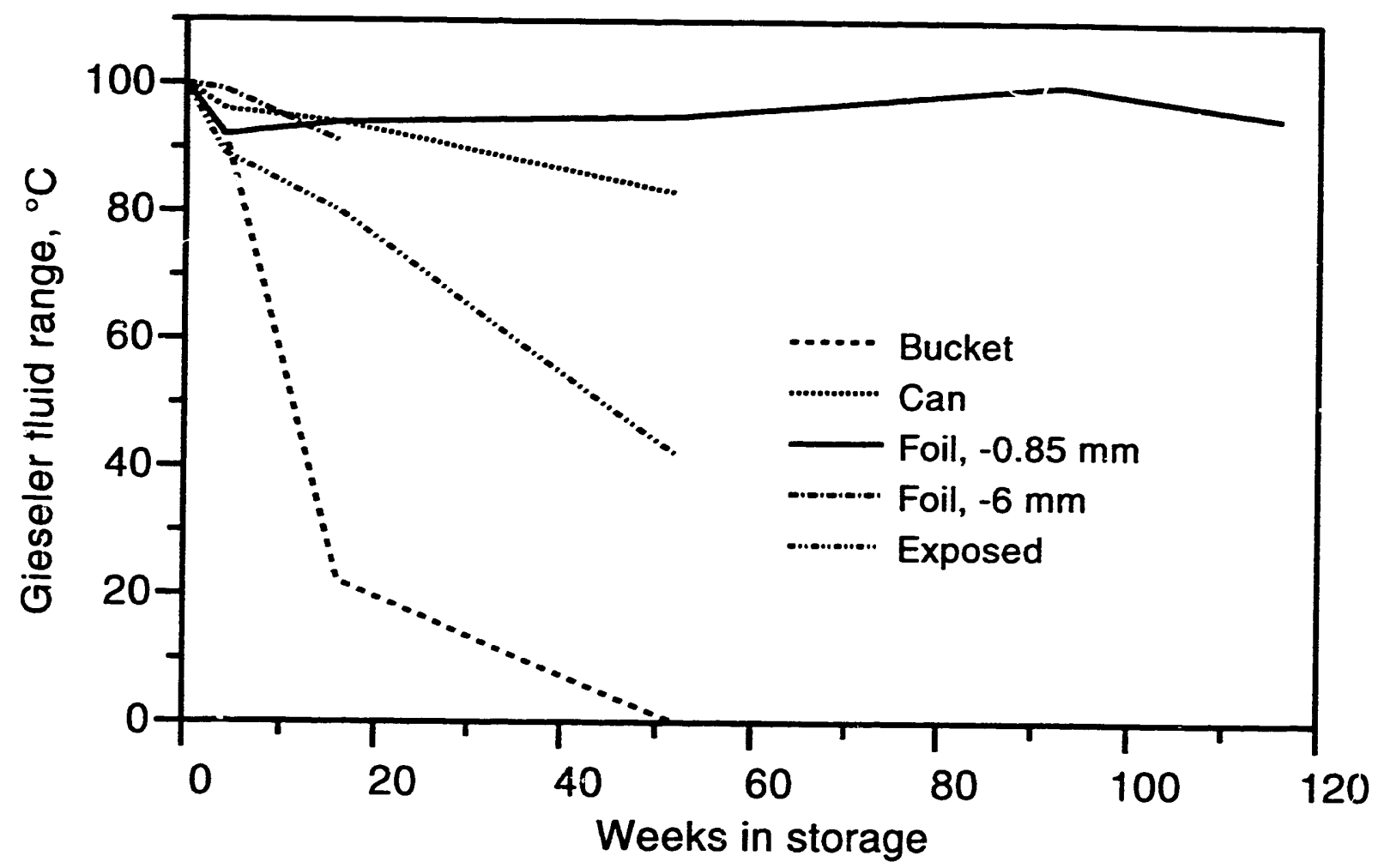

Figure 5. Changes in Gieseler fluid range with time (PSOC-1536, mvb Lower Kittanning seam)

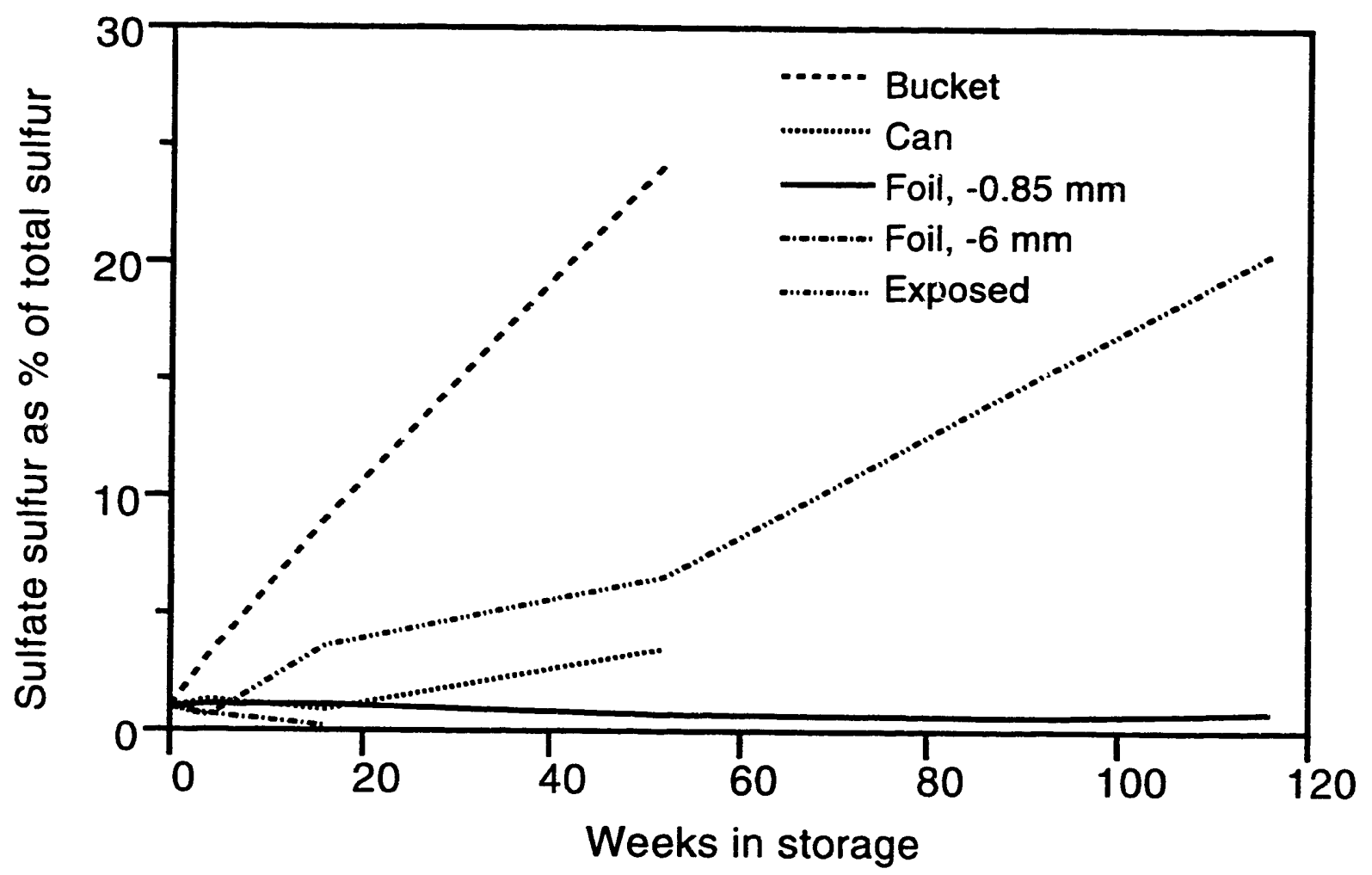

Figure 6. Changes in sulfate sulfur (as percent of total sulfur) with time (PSOC-1536, mvb Lower Kittanning seam) 

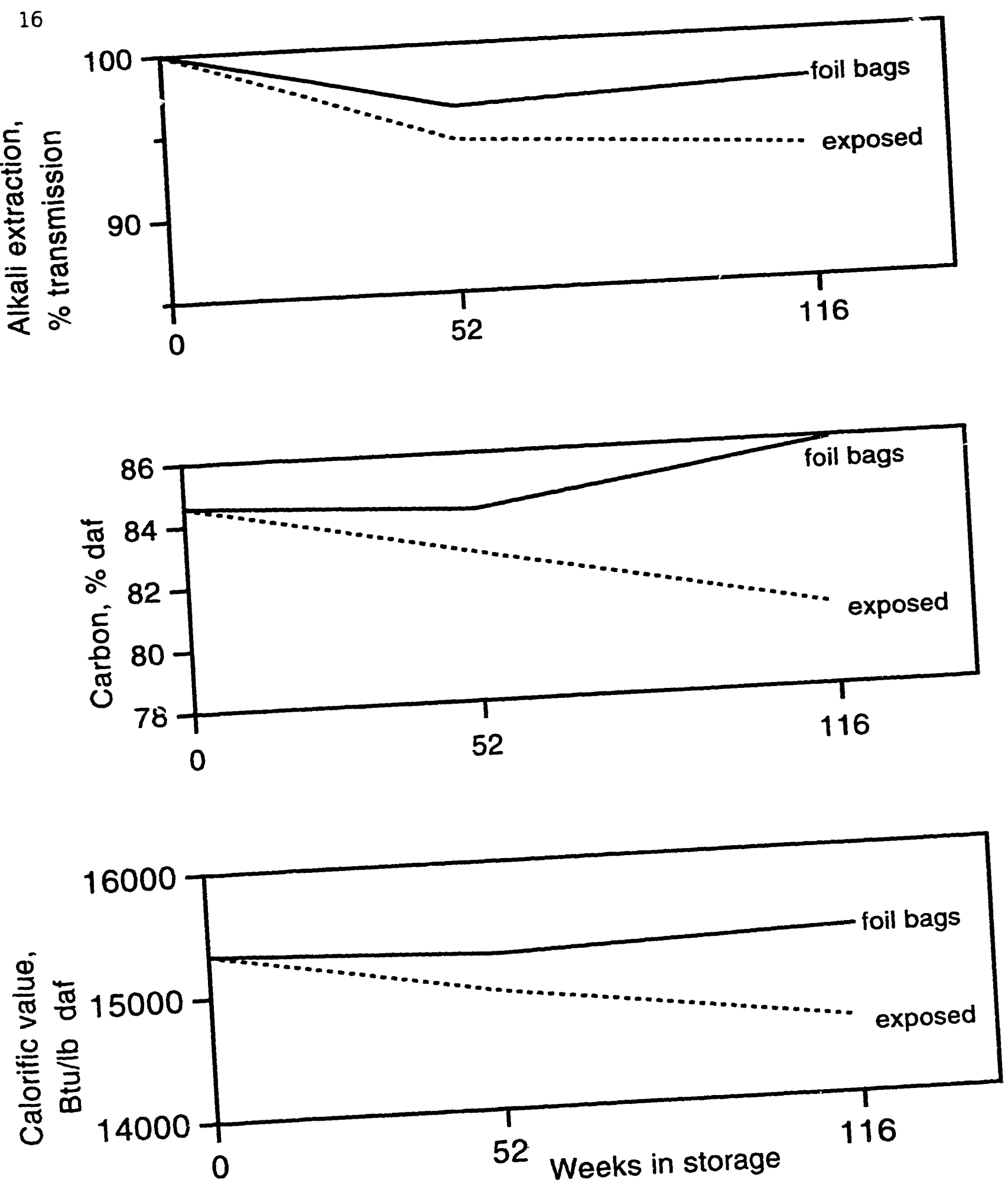

Figure 7. Changes in calorific value, carbon, and alkali extraction with time (PSOC-1536, mvb Lower Kittanning seam sample) 
foil laminate bags stored without enclosing buckets. Because the atmosphere in the buckets approached air in its oxyger, content. it is assumed that the mesh size and not the enclosing bucket caused the difference in preservation. The four oxygen levels within foil bags were equivalent when the range of error of the oxygen ana'yzer at low oxygen levels is considered.

The next best container was the steel can with an inner polyethylene bag containing minus $0.85 \mathrm{~mm}(-20$ mesh) coal. Values for sulfate sulfur as percent of total sulfur, oxygen, and maximum Gieseler fluidity were not as good as those from the foil bags. The steel can, not the polyethylene bag, provides the barrier to oxycjen.

The poly'ethylene buckets and the open container ("exposed") performed poorly at maintaining low sulfate sulfur and high Gieseler fluidity. The polyethylene bucket with caulked lid performed poorly even though the oxygen level was low.

ther tests were performed on selected samples from the various container types. Figure 8 is a plot of the change in fluorescence intensity over time of irradiation and shows a negative alteration for telocollinite for this medium volatile coal. Moreover, there appears to be little difference in alteration for the different storage samples with the exception of the subsample stored for 16 weeks in a bucket, without the protection of a foil bag. For this sample, which al so showed the greatest change in fluid properties, tr. initial alteration is again negative, but after about 8 minutes of alteration there is a slight increase in intensity. This increase may be the first indication of weathering effects in storage, although the pattern is inconclusive.

FTIR spectra were obtained on the fresh coal and that from foil bags and polyethylene buckets after 4 and 16 weeks: no definite changes in functionalgroup composition could be detected, either in the original spectra or the first derivatives.

Conclusions

The foil laminate bags performed best to maintain maximum Gieseler fluidity. fluid range. low sulfate sulfur, and other initial properties of the coal.

High volatile A bituminous Pittsburgh seam coal (DECS-12)

A sample with greater initial fluidity than PSOC-1536 might provide a more sensitive indication of deterioration in storage. When the hvAb Pittsburgh seam sample (DECS-12) was collected in July. 1990, it was designated for detailed monitoring in addition to the normal annual monitoring. Figure 9 shows how Gieseler fluid range decreased for a sample stored exposed to air. and was maintained near its initial value for samples stored in foil laminate bags.

The sample was also tested for liquefaction performance while fresh and again after 6 months in storage in foil bags and exposed to air. The conditions of hydrogenation were selected not to optimize conversion, but to emphasize minor 


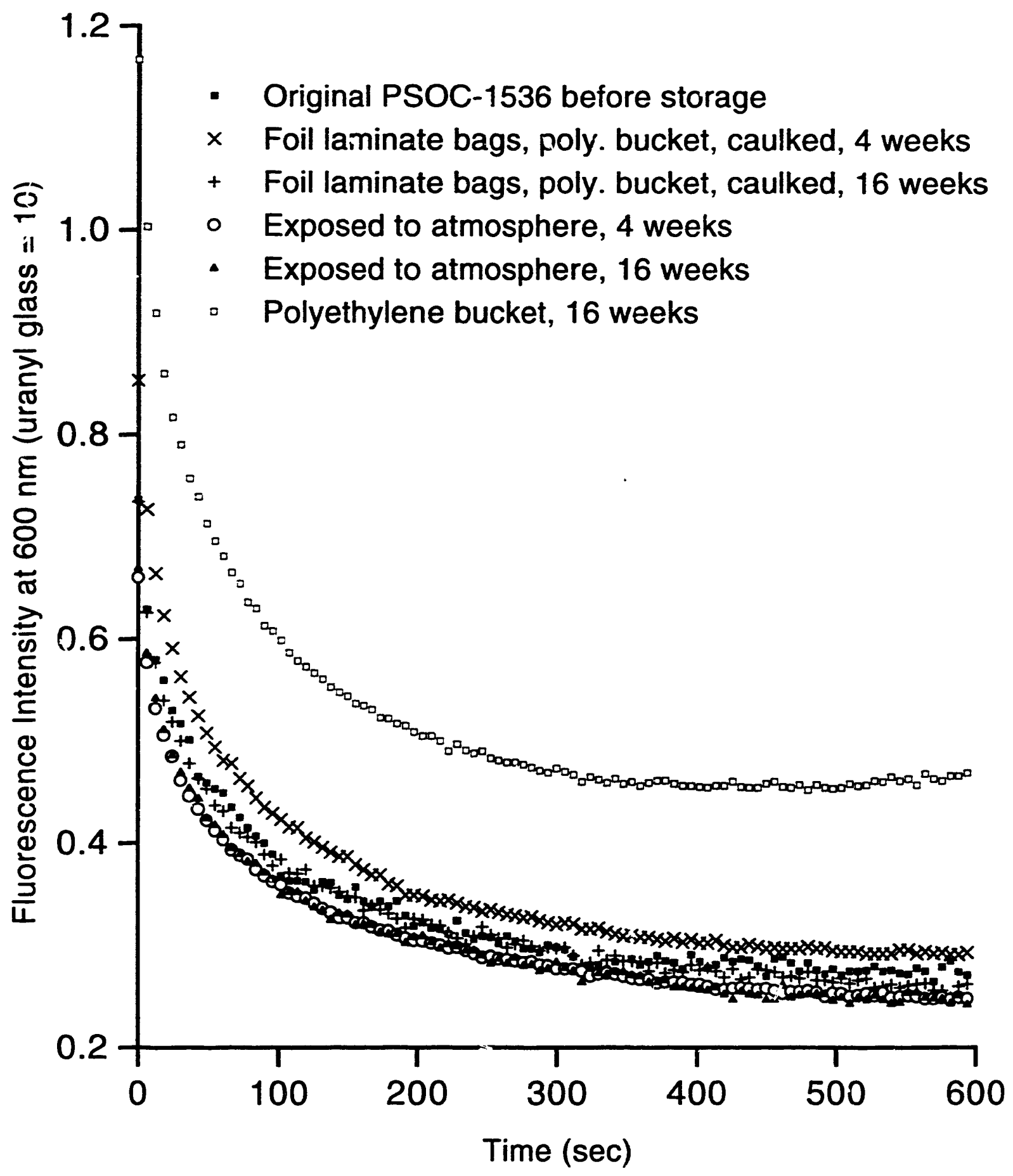

Figure 8. Fluorescence alteration of PSOC-1536 (mvb): Effect of storage 


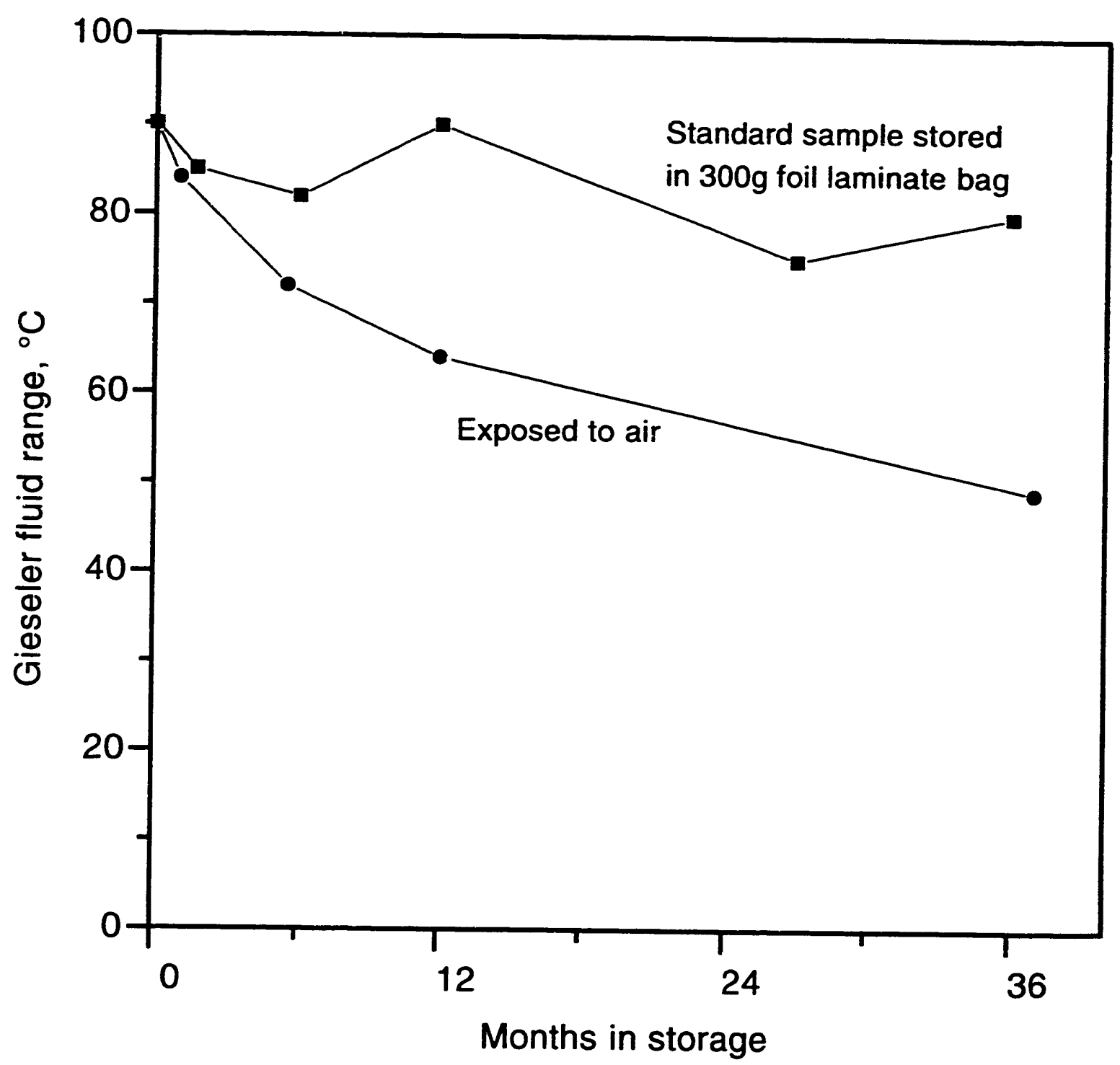

Figure 9. Changes in Gieseler fluid range with time (hvAb Pittsburgh seam sample) 
compositional differences in the products derived from the two stored samples. Liquefaction runs were conducted at $350^{\circ} \mathrm{C}$ for 30 minutes, 1000 psig $\mathrm{H}_{2}, 2: 1$ tetralin: coal ratio. Table 5 shows that whereas the liquefaction response of the sample stored in the foil bag had not deteriorated in 6 months. there was a smali but significant decrease in conversion of the exposed coal.

Table 5. Liquefaction data from DECS-12 (hvAb, Pittsburgh seam) after storage

\begin{tabular}{|c|c|c|c|}
\hline & Initial & $\begin{array}{c}\text { Exposed } \\
5.5 \text { months }\end{array}$ & $\begin{array}{l}\text { Foil Bag } \\
6 \text { months }\end{array}$ \\
\hline$\%$ Conversion & $43.1 \quad 0.6$ & 40.5 & 45.3 \\
\hline \% Asphaltenes & $\begin{array}{ll}41.2 & 0.7\end{array}$ & 42.5 & 41.7 \\
\hline$\% 0 i 1+$ Gas & 1.90 .9 & -2.0 & 3.6 \\
\hline
\end{tabular}

Conclusions

The foil laminate bags performed well to maintain Gieseler fluid range and liquefaction behavior of the high volatile A bituminous coal.

\section{Study Employing Argonne Premium Coals}

At DOE's request, a further study of Gieseler fluid properties and alkali extraction tests was undertaken on six bituminous coal samples from the Argonne National Laboratory's (ANL) Premium Coal Sample Bank. ANL supplies coal samples in flame-sealed, nitrogen-filled glass ampoules (Vorres, 1990). The Gieseler fluidity test had not been undertaken at the time of collection for these coals. Therefore, the initial fluid range was assumed. using typical values reported for samples in the immediate geographic vicinity of the subject coals, and results reported by ANL after the sample had been stored. Evaluation of possible deterioration during storage was complicated by the fine size distribution of the nominal minus $0.85 \mathrm{~mm}$ ( -20 mesh) samples provided by ANL (Table 6 ): size distributions finer than the $-0.425 \mathrm{~mm}$ ( -40 mesh) specified for the Gieseler test are known to increase the measured fluidity. It is possible that the true loss in fluid range might have bsen greater than that plotted.

The procedures and interim results of this study were reported by Glick. Mitchell and Davis (1991). Ten ampoules each of the six bituminous coals available from ANL were obtained. Four of each were placed in a nitrogen-filled glovebox where they were opened and the contents resealed in foil laminate bags. of the six left unopened. two were used for initial analyses of Gieseler fluidity and alkali extraction. After eight months and again after two years, analyses comparing the coal resealed in foil laminate bags with that in the undisturbed ampoules were performed. 
Table 6. Particle size distributions of some minus 20 mesh Argonne National Laboratory Premium Coal Samples

\begin{tabular}{|c|c|c|}
\hline Mesh sizes & $\begin{array}{l}\text { ANL-701 } \\
\text { Lewiston-Stockton }\end{array}$ & $\begin{array}{c}\text { ANL-101 } \\
\text { Upper Freeport }\end{array}$ \\
\hline$+0.600 \mathrm{~mm}(+30$ mesh $)$ & $1.0 \%$ & $0.2 \%$ \\
\hline$-0.600+0.425 \mathrm{~mm}(-30+40$ mesh $)$ & $6.1 \%$ & $0.5 \%$ \\
\hline$-0.425+0.250 \mathrm{~mm}(-40+60$ mesh $)$ & $30.3 \%$ & $3.2 \%$ \\
\hline$-0.250+0.150 \mathrm{~mm}(-60+100$ mesh $)$ & $62.6 \%$ & $11.5 \%$ \\
\hline$-0.150 \mathrm{~mm}$ ( -100 mesh) & & $84.6 \%$ \\
\hline
\end{tabular}

Figure 10 shows that the samples repackaged in foil laminate bags and stored for two years compared well to those stored in their original ampoules. After two years, maximum fluidities of these pairs of samples matched within experimental error for all except ANL-701. Fluid ranges matched within experimental error for all except ANL-501 and -701. The variation in results for ANL-701 is probably caused by problems with the Gieseler plastometer. Dashed lines on the graphs for ANL-301, -401 and -601 indicate that the 8-month test could not be successfully completed on the amount of coal allocated because multiple repeat runs were required to meet the ASTM precision requirement.

Conclusions

Favorable comparison to Argonne National Laboratory's glass ampoules supports the use of foil laminate bags for long-term storage of samples in the DOE Coal Sample Bank.

\section{Adoption of Foil Laminate Bags and Steel Drums for DECS Samples}

The studies described above indicated that foil laminate bags are greatly superior to the polyethylene bulk storage containers, and somewhat superior to the steel cans, used previously for PSOC samples. Another study verified the preservation ability and showed the bags to be comparable to glass ampoules when sealed under the same circumstances. The availability of the bags in a variety of sizes also removed the problem of differences in preservation efficiency between bulk storage containers and smaller distribution containers (cans). The bags are available in a wide variety of sizes, and could therefore replace both 19 liter buckets and $560 \mathrm{ml}$ cans. Samples for bulk storage would be split into lots no larger than $12 \mathrm{~kg}$ (26 lbs) so that the entire amount of each sample would be stored in only one type of container. Based on these considerations, DOE agreed that future samples should be stored in foil 1 aminate bags.

However, these bags are not strong enough to survive rough handling when containing large blocks of coal, so they are not suitable for field collection. 


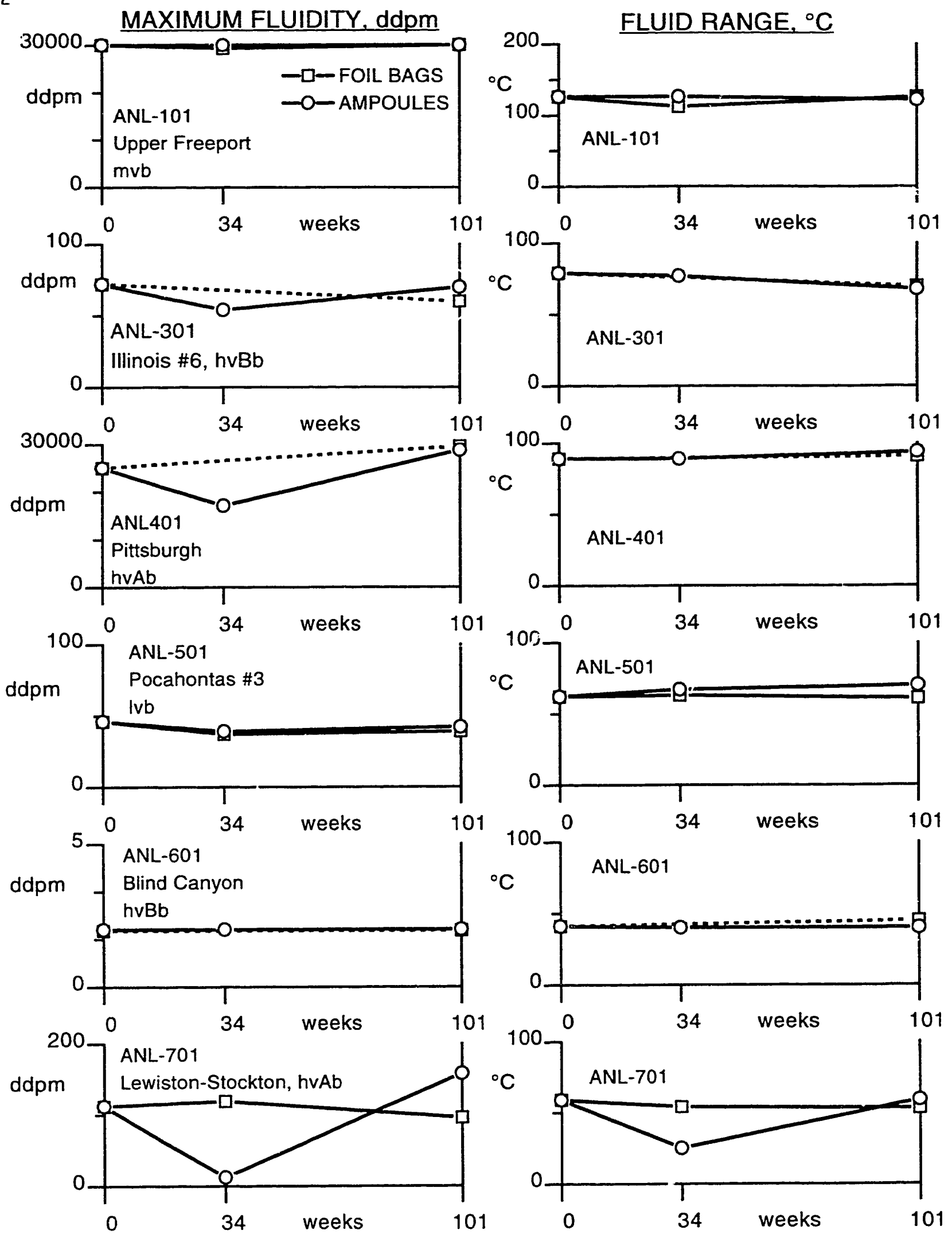

Figure 10. Gieseler fluidity for Argonne Premium Coal Samples stored in foil laminate bags and original ampoules 
Possibilities for field containers included the previously used polyethylene drums (in which the argon could be renewed daily before shipping. and which could be shipped by express service to minimize leakage) and 189 or 113 liter (55 or 30 gal.) steel drums with heavy duty closure bands and food-grade gaskets (Harvey and Kruse, 1988). Following some testing of the latter, they were adopted for use as described under Task IC (page 51).

\section{Monitoring of Sample Condition}

Appendix A shows the results of the analyses (including gaseous oxygen levels). performed in five annual programs over the five years of the contract. Monitoring of PSOC samples was performed on coal stored at minus $6 \mathrm{~mm}\left(-1 / 4^{\prime \prime}\right)$ in polyethylene drums in the Penn State Coal Sample Bank building (with no temperature control). Monitoring of DECS samples was performed on ccal stored at minus $0.85 \mathrm{~mm}$ ( $-20 \mathrm{mesh}$ ) in foil laminate bags (nominally $300 \mathrm{~g}, 2 / 3 \mathrm{lb}$ ), the same bags distributed routinely to users. A11 DECS samples have been stored at controlled temperature, either at room temperature or under refrigeration.

For the PSOC-series samples collected under the previous contract (DE-AC2284PC-70760, November 6, 1984 to April 5, 1988), the first column for each sample in Appendix A shows the initial analysis, performed as soon as possible after collection and processing. In some cases a second sample was also analyzed for verification of some results: these analyses are shown in the second column. At the beginning of the subject contract, a can of each coal at minus $0.85 \mathrm{~mm}(-20$ mesh) which was a possible candidate for monitoring by Gieseler fluidity and/or alkali extraction was analyzed. The results were used to indicate the best possible condition of these samples at that time in their storage history (because the cans were the best containers for sample preservation then in use). and are shown in the next column.

In five successive programs, corresponding roughty to the five years of the contract, the drum of minus $6 \mathrm{~mm}(-1 / 4 \mathrm{inch})$ coal of each sample was opened and approximately $2.3 \mathrm{~kg}$ (5 lbs) was removed by a representative subsampling procedure using either a riffle splitter or a rotary turning-tube divider. Each drum was then resealed, purged with argon gas, and returned to storage. The subsamples were stage-ground to appropriate mesh sizes and subjected to analysis. Moisture, ash, total sulfur, forms of sulfur, and calorific value analyses were performed on all subsamples. Geiseler fluidity testing was performed on those samples in the bituminous rank range which had previously demonstrated thermoplastic behavior. Alkali extraction testing was initially performed on samples in the bituminous rank range, and was continued onty for those samples (generally hvBb rank or higher) for which the results seemed to indicate that its use was appropriate (above approximately $80 \%$ transmittance). For PSOC samples, alkali extraction results were reported for a $10 \mathrm{~mm} 1$ ight path and were not corrected to the $17 \mathrm{~mm}$ 1ight path now adopted under ASTM D5263 as a standard condition (ASTM, 1993). The analytical resuits from these five programs are shown in the final columns for each sample in Appendix A. For samples which were superseded by newly collected samples during the contract. monitoring of the old sample was discontinued and monitoring of the new (DECS) sample was initiated. 
The format of entries in Appendix A for DECS samples is similar to that for PSOC samples. There was not a major gap in coverage between collection and initial monitoring, and all DECS samples were stored in the same container type. so there is no equivalent to the cans of PSOC samples analyzed to determine tic best possible condition for initial monitoring. For DECS samples, alkali extraction results were mathematically corrected to a $17 \mathrm{~mm}$ light path. Samples DECS-21 and DECS-22 were collected in the last year of the contract and were not monitored before the end of the contract.

Ash yield and total sulfur analyses show the degree to which the subsamples are representative of the total sample and the degree to which they match the initially analyzed subsample. Moisture analyses also show this, but could be influenced by storage conditiuns (temperature and humidity) as opposed to processing and subsampling conditions. Moisture, ash and sulfur levels are shown for DECS samples in Table 7. Variability between these subsamples is similar to that observed between subsamples analyzed for equivalence when the samples were first packaged.

Table 8 shows repeatability values (ASTM, 1993) applicable to the results presented in the various tables, figures and appendices. The results of duplicate analyses are expected to agree within the repeatability values shown if they are performed in the same laboratory by the same operator on the same equipment, and within the reproducibility values shown if performed in different laboratories. Samples in the monitoring studies were sent to the same laboratory, but over a period of years, so in some cases different operators would have been involved. Thus the expected tolerances for these tests may lie between the repeatability and reproducibility values shown. The random variation in ash between splits will also increase the variation in results.

Examination of monitoring results from PSOC samples (Appendix A) shows the problems associated with the bulk storage containers. Of the 29 PSOC samples which initially exhibited fluid properties with the Gieseler plastometer, only 7 maintained any fluidity through the monitoring project, and only one maintained more than 5 percent of its initial maximum fluidity. Each of the 7 did maintain at least half their fluid temperature range. Fluid temperature range is also less subject to variation between instrumental runs, and has therefore been used in this report as a more stable indicator of fluid behavior.

A11 samples except those lowest in total sulfur (PSOC-1447, 1468, 1498. 1499. 1501, 1502, 1503, 1504, 1508, and the four Alaskan samples, 1532-1535) showed significant increase in sulfate sulfur, indicating oxidation of sulfides. The Interior Province samples (PSOC-1490 through -1497) fared worst, with some increasing from below $0.05 \%$ initial sulfate sulfur to over $2.5 \%$, as much as half their total sulfur.

Monitoring results of samples DECS-1 through DECS-20 (Appendix A) are graphed in Figures 11 - 30. Each of these samples was a replacement for a PSOC sample with similar properties, usually collected from the same mine. These comparable PSOC samples were also monitored through time, and their conditions 
Table 7. Moisture, ash and suliur levels of DECS samples in foil laminate bags monitored through time

\begin{tabular}{|c|c|c|c|c|c|c|c|}
\hline \multirow{2}{*}{$\begin{array}{c}\text { DECS } \\
\text { sample }\end{array}$} & \multicolumn{5}{|c|}{ Monitoring Analyses: Moisture, percent coai } & \multicolumn{2}{|c|}{$\begin{array}{c}\text { Equivalence } \\
\text { Analyses }\end{array}$} \\
\hline & Year 1 & Year 2 & Year 3 & mean & st. dev. & mean & st. dev. \\
\hline 1 & 28.20 & 29.00 & 29.30 & 28.83 & 0.57 & 29.49 & 0.52 \\
\hline 2 & 10.30 & 10.10 & 9.80 & 10.07 & 0.25 & 10.18 & 0.16 \\
\hline 3 & 0.87 & 0.82 & 0.72 & 0.80 & 0.08 & 0.99 & 0.08 \\
\hline 4 & 15.00 & 14.80 & $\cdots$ & 14.90 & 0.14 & 15.05 & 0.13 \\
\hline 5 & 7.30 & 7.10 & $\cdots$ & 7.20 & 0.14 & 7.29 & 0.18 \\
\hline 6 & 4.35 & 4.06 & 4.45 & 4.29 & 0.20 & 4.51 & 0.13 \\
\hline 7 & 16.80 & 17.00 & $\cdots$ & 16.90 & 0.14 & 17.02 & 0.25 \\
\hline 8 & 28.30 & 28.60 & $\cdots$ & 28.45 & 0.21 & 28.22 & 0.31 \\
\hline 9 & 23.80 & 24.80 & $\cdots$ & 24.30 & 0.71 & 24.44 & 0.17 \\
\hline 10 & 21.10 & 22.40 & . & 21.75 & 0.92 & 21.88 & 0.87 \\
\hline 11 & 32.50 & 32.90 & $\cdots$ & 32.70 & 0.28 & 32.79 & 0.56 \\
\hline 12 & 2.47 & 2.99 & 1.98 & 2.48 & 0.51 & 2.35 & 0.03 \\
\hline 13 & 1.80 & 1.50 & - & 1.65 & 0.21 & 1.73 & 0.18 \\
\hline 14 & 1.70 & 1.40 & - & 1.55 & 0.21 & 1.68 & 0.17 \\
\hline 15 & 2.90 & 2.60 & $\cdots$ & 2.75 & 0.21 & 3.03 & 0.18 \\
\hline 16 & 4.90 & 5.00 & $\cdots$ & 4.95 & 0.07 & 5.13 & 0.12 \\
\hline 17 & 4.00 & 3.60 & $\cdots$ & 3.80 & 0.28 & 3.93 & 0.11 \\
\hline 18 & 7.30 & . & - & 7.30 & - & 7.47 & 0.39 \\
\hline 19 & 0.80 & $\cdots$ & $\cdots$ & 0.80 & -. & 0.96 & 0.06 \\
\hline 20 & 2.70 & $\cdots$ & $\cdots$ & 2.70 & - & 2.92 & 0.09 \\
\hline \multicolumn{8}{|c|}{ Ash, percent dry coal } \\
\hline 1 & 16.20 & 15.20 & 16.40 & 15.93 & 0.64 & 16.08 & 0.42 \\
\hline 2 & 16.10 & 16.20 & 15.90 & 16.07 & 0.15 & 16.08 & 0.16 \\
\hline 3 & 5.24 & 5.26 & 5.52 & 5.34 & 0.16 & 5.27 & 0.16 \\
\hline 4 & 6.20 & 6.20 & $\cdots$ & 6.20 & 0 & 6.27 & 0.09 \\
\hline 5 & 8.70 & 8.70 & $\cdots$ & 8.70 & 0 & 8.68 & 0.22 \\
\hline 6 & 5.96 & 5.92 & 6.00 & 5.96 & 0.04 & 5.92 & 0.13 \\
\hline 7 & 4.30 & 4.30 & $\cdots$ & 4.30 & 0 & 4.22 & 0.04 \\
\hline 8 & 12.80 & 13.10 & - & 12.95 & 0.21 & 13.31 & 0.41 \\
\hline 9 & 6.70 & 6.50 & $\cdots$ & 6.60 & 0.14 & 6.33 & 0.14 \\
\hline 10 & 13.00 & 13.10 & $\cdots$ & 13.05 & 0.07 & 12.67 & 0.34 \\
\hline 11 & 10.40 & 9.50 & . & 9.95 & 0.64 & 9.63 & 0.28 \\
\hline 12 & 11.05 & 10.35 & 9.52 & 10.31 & 0.77 & 10.12 & 0.32 \\
\hline 13 & 4.30 & 4.60 & $\cdots$ & 4.45 & 0.21 & 4.70 & 0.31 \\
\hline 14 & 10.30 & 10.60 & - & 10.45 & 0.21 & 10.76 & 0.32 \\
\hline 15 & 10.10 & 9.80 & $\cdots$ & 9.95 & 0.21 & 9.09 & 1.61 \\
\hline 16 & 15.20 & 15.70 & $\cdots$ & 15.45 & 0.35 & 15.74 & 1.82 \\
\hline 17 & 6.50 & 6.50 & $\cdots$ & 6.50 & 0 & 6.69 & 0.09 \\
\hline 18 & 11.30 & $\cdots$ & $\cdots$ & 11.30 & - & 11.85 & 0.68 \\
\hline 19 & 5.50 & . & .. & 5.50 & . & 5.92 & 0.79 \\
\hline 20 & 5.20 & .. & $\cdots$ & 5.20 & .. & 5.17 & 0.20 \\
\hline
\end{tabular}


Table 7. (continued)

\begin{tabular}{|c|c|c|c|c|c|c|c|}
\hline \multirow{2}{*}{$\begin{array}{l}\text { DECS } \\
\text { sample }\end{array}$} & \multicolumn{5}{|c|}{ Monitoring Analyses: Sulfur, percent dry coal } & \multicolumn{2}{|c|}{$\begin{array}{c}\text { Equivalence } \\
\text { Analyses }\end{array}$} \\
\hline & Year 1 & Year 2 & Year 3 & mean & st. dev. & mean & st. dev. \\
\hline 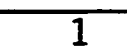 & 1.03 & 0.97 & 1.07 & 1.02 & 0.05 & 1.06 & 0.05 \\
\hline 2 & 4.33 & 4.54 & 4.50 & 4.46 & 0.11 & 4.41 & 0.08 \\
\hline 3 & 0.65 & 0.67 & 0.64 & 0.65 & 0.02 & 0.66 & 0.03 \\
\hline 4 & 0.57 & 0.54 & $\ldots$ & 0.55 & 0.02 & 0.57 & 0.09 \\
\hline 5 & 0.63 & 0.67 & $\ldots$ & 0.65 & 0.03 & 0.64 & 0.05 \\
\hline 6 & 0.43 & 0.35 & 0.43 & 0.40 & 0.05 & 0.43 & 0.02 \\
\hline 7 & 0.99 & 1.04 & . & 1.02 & 0.04 & 1.02 & 0.03 \\
\hline 8 & 0.77 & 0.81 & $\ldots$ & 0.79 & 0.03 & 0.80 & 0.04 \\
\hline 9 & 0.42 & 0.44 & $\ldots$ & 0.43 & 0.01 & 0.45 & 0.03 \\
\hline 10 & 1.14 & 1.33 & $\ldots$ & 1.24 & 0.13 & 1.09 & 0.10 \\
\hline 11 & 0.74 & 0.74 & $\cdots$ & 0.74 & 0 & 0.76 & 0.02 \\
\hline 12 & 1.18 & 1.16 & 1.17 & 1.17 & 0.01 & 1.16 & 0.03 \\
\hline 13 & 0.62 & 0.65 & $\ldots$ & 0.64 & 0.02 & 0.64 & 0.03 \\
\hline 14 & 1.71 & 1.80 & $\ldots$ & 1.76 & 0.06 & 1.76 & 0.04 \\
\hline 15 & 1.75 & 1.62 & $\ldots$ & 1.69 & 0.09 & 1.35 & 0.36 \\
\hline 16 & 0.48 & 0.46 & $\ldots$ & 0.47 & 0.01 & 0.47 & 0.01 \\
\hline 17 & 0.44 & 0.43 & .. & 0.44 & 0.01 & 0.42 & 0.02 \\
\hline 18 & 3.91 & $\ldots$ & $\ldots$ & 3.91 & . & 4.00 & 0.13 \\
\hline 19 & 0.78 & .. & $\ldots$ & 0.78 & $\ldots$ & 0.76 & 0.02 \\
\hline 20 & 0.96 & $\cdots$ & .. & 0.96 & -. & 0.98 & 0.01 \\
\hline
\end{tabular}

Table 8. ASTM Repeatability and reproducibility values

\begin{tabular}{|c|c|c|}
\hline ASTM Procedure & Repeatabi7ity & Reproducibility \\
\hline $\begin{array}{l}\text { Moisture, D3173-87 } \\
\text { coals }<5 \% \text { moisture } \\
\text { coals }>5 \% \text { moisture }\end{array}$ & $\begin{array}{l}0.2 \\
0.3\end{array}$ & $\begin{array}{l}0.3 \\
0.5\end{array}$ \\
\hline $\begin{array}{l}\text { Ash, D3174-89 } \\
\text { no carbonates present } \\
\text { carbonates present } \\
\text { coals with more than } 12 \% \text { ash. } \\
\text { containing carbonates and pyrite }\end{array}$ & $\begin{array}{l}0.2 \\
0.3 \\
0.5\end{array}$ & $\begin{array}{l}0.3 \\
0.5 \\
1.0\end{array}$ \\
\hline Total Sulfur. D4239-93 method C (infrared) & $0.03+0.04 \dot{x}$ & $0.05+0.06 \bar{x}$ \\
\hline BTU/1b (dry basis), D2015.93 & 50 & 100 \\
\hline $\begin{array}{c}\text { Forms of Sulfur. D2492-90 } \\
\text { Sulfate } \\
\text { Pyritic }\end{array}$ & $\begin{array}{c}0.02 \\
0.08+0.09 \bar{x}\end{array}$ & $\begin{array}{c}0.04 \\
0.15+0.27 \dot{x}\end{array}$ \\
\hline $\begin{array}{c}\text { Gieseler Plastometer, D2639-90 } \\
\text { Temperature points, }{ }^{\circ} \mathrm{C} \\
\text { Fluidity, ddpm }\end{array}$ & $\begin{array}{l}5 \\
0.1 \bar{x}\end{array}$ & $\begin{array}{l}\text { not specified } \\
\text { not specified }\end{array}$ \\
\hline
\end{tabular}



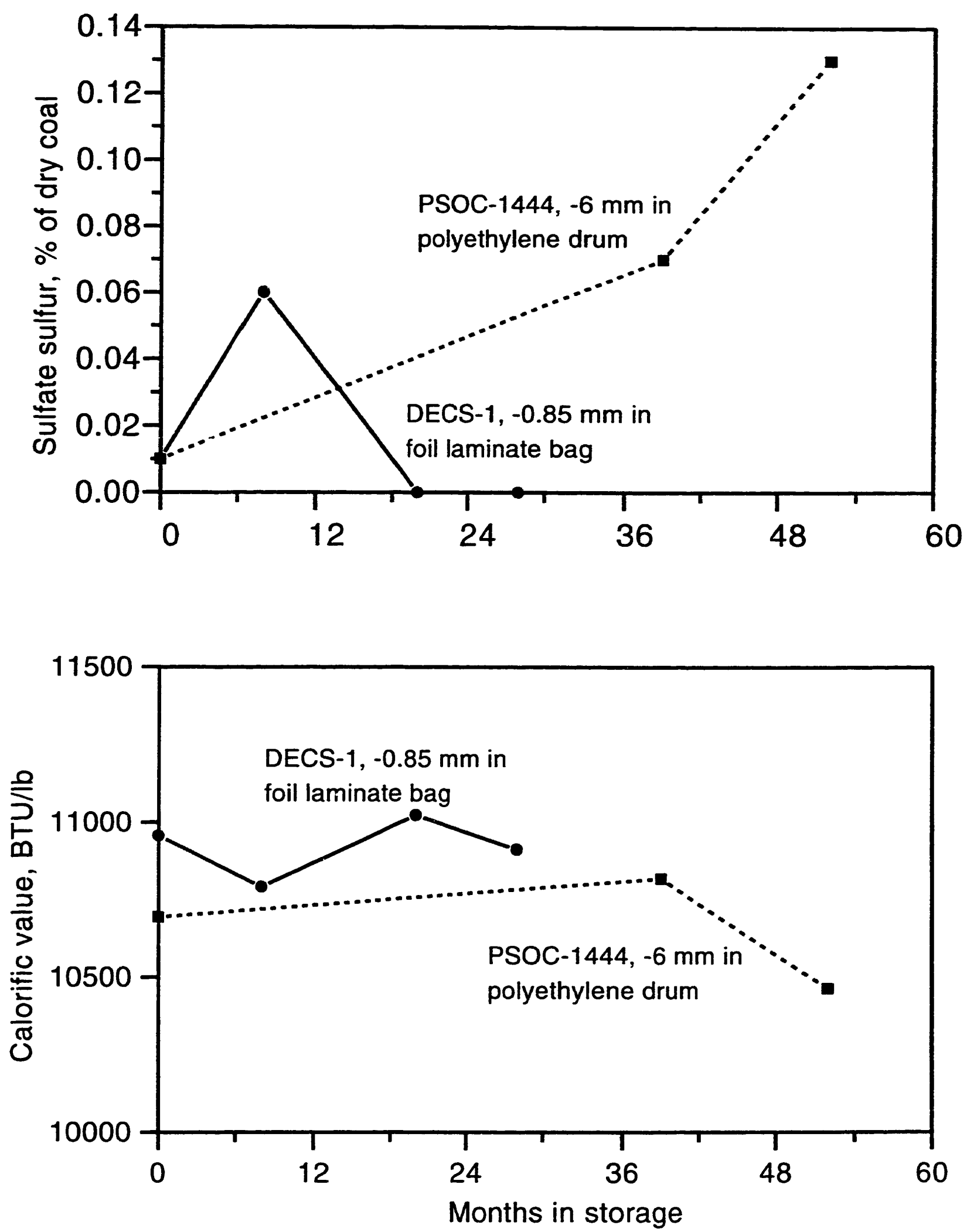

Figure 11. DECS-1 (Upper Wilcox Group, unnamed seam) con ition over time, showing PSOC-1444 for iparison 

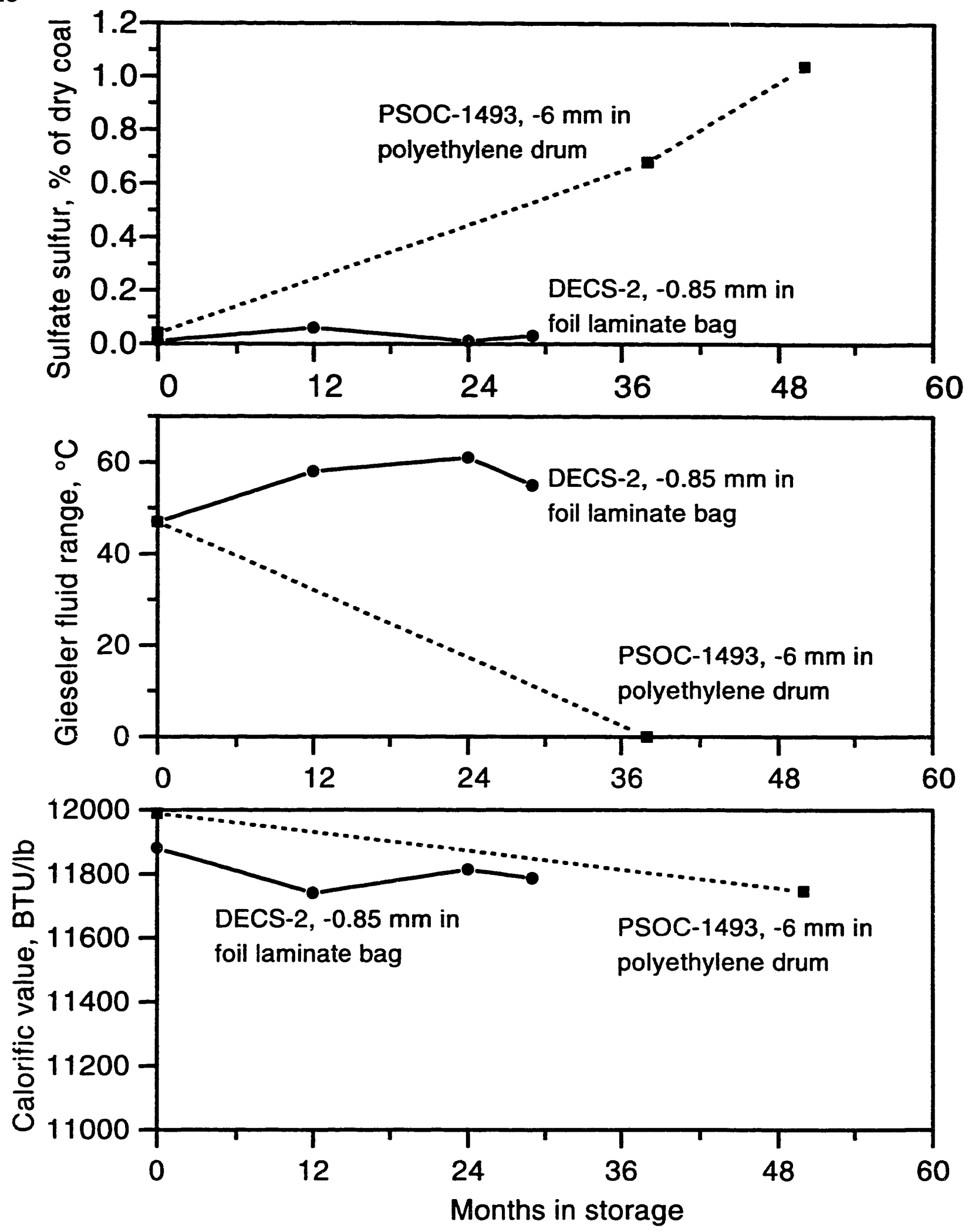

Figure 12. DECS-2 (Illinois No. 6 seam) condition over time, showing PSOC-1493 for comparison 

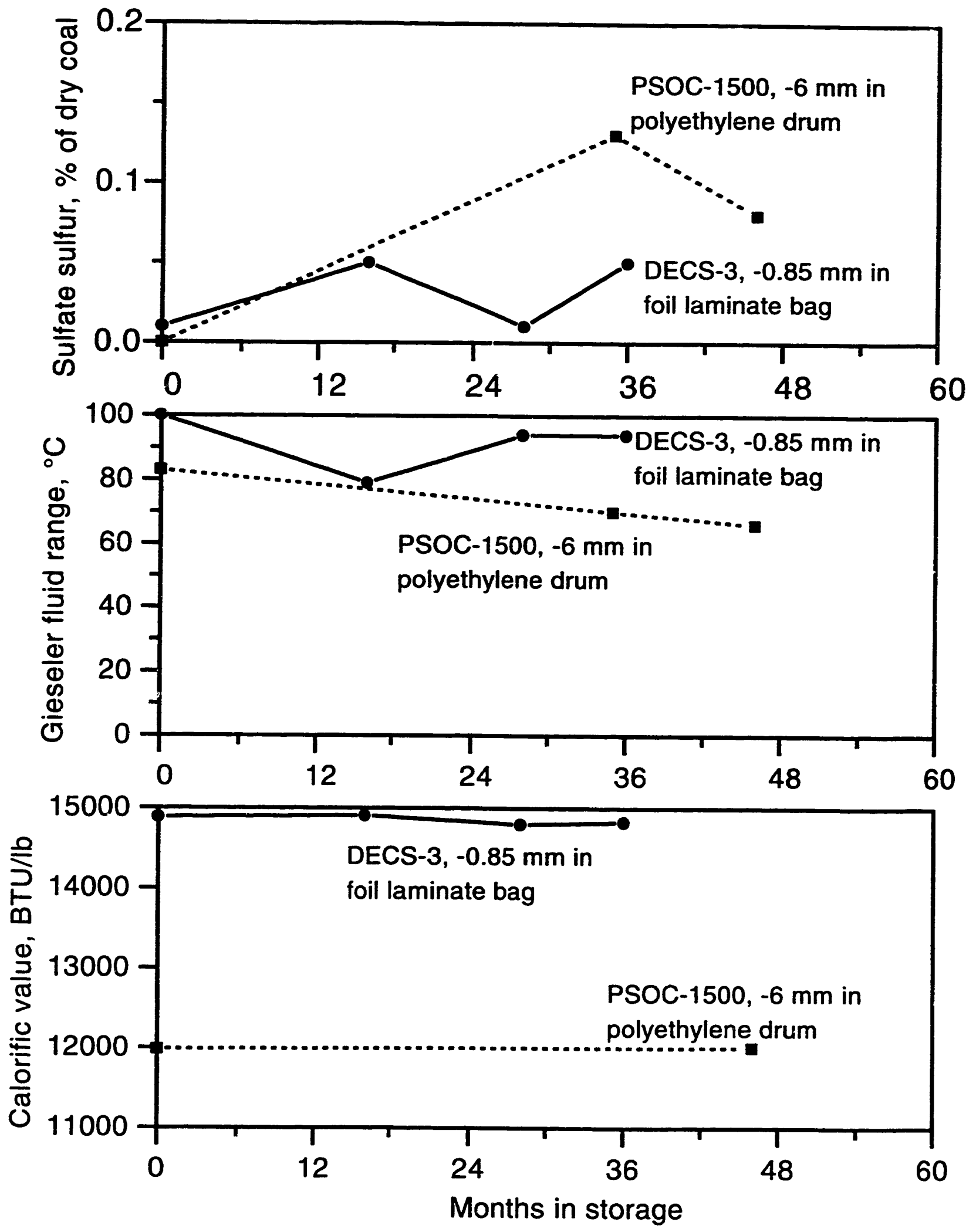

Figure 13. DECS-3 (Coal Basin M seam) condition over time, showing PSOC-1500 for comparison 

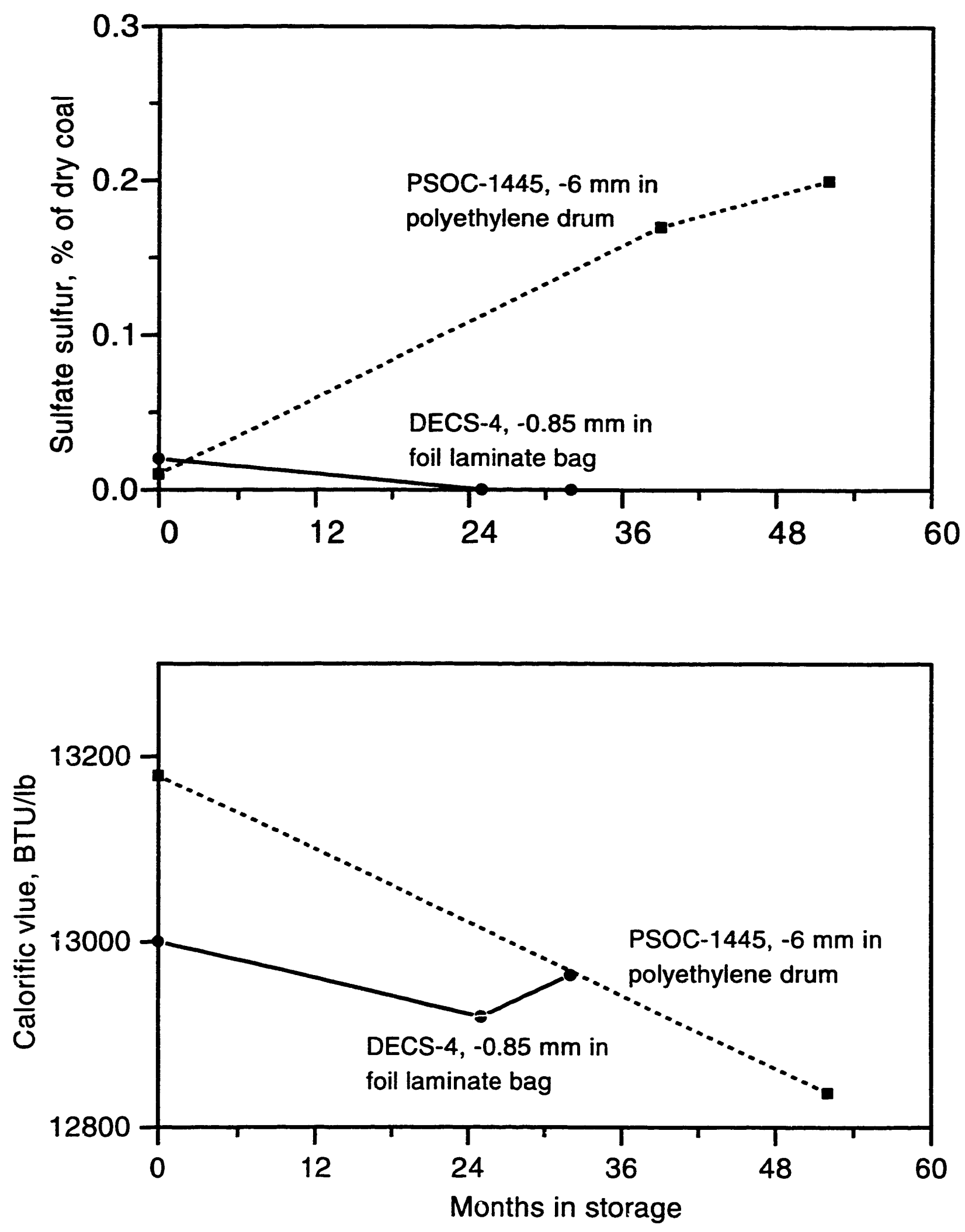

Figure 14. DECS-4 (Blue seam) condition over time, showing PSOC-1445 for comparison 

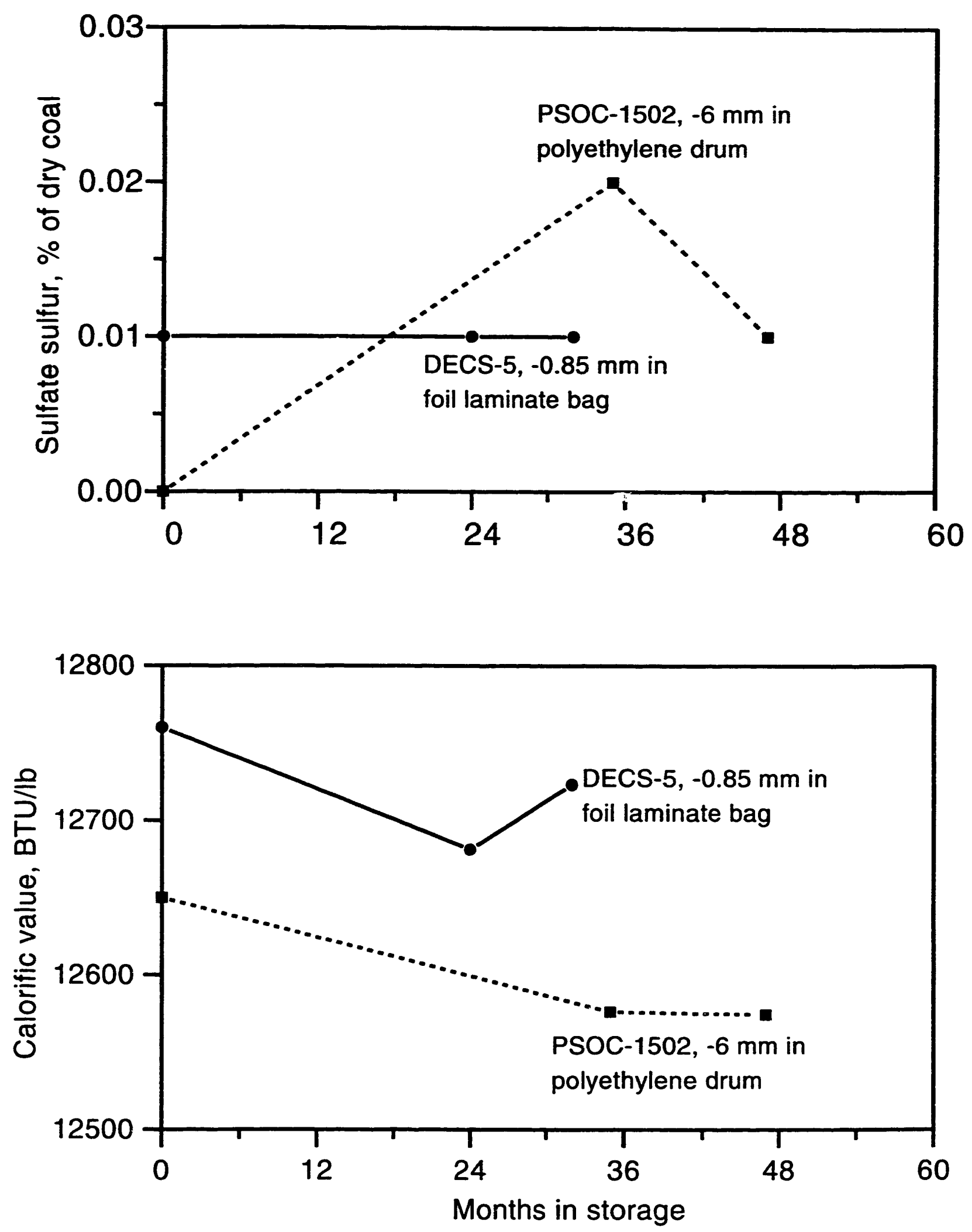

Figure 15. DECS-5 (Hiawatha seam) condition over time, showing PSOC-1502 for comparison 

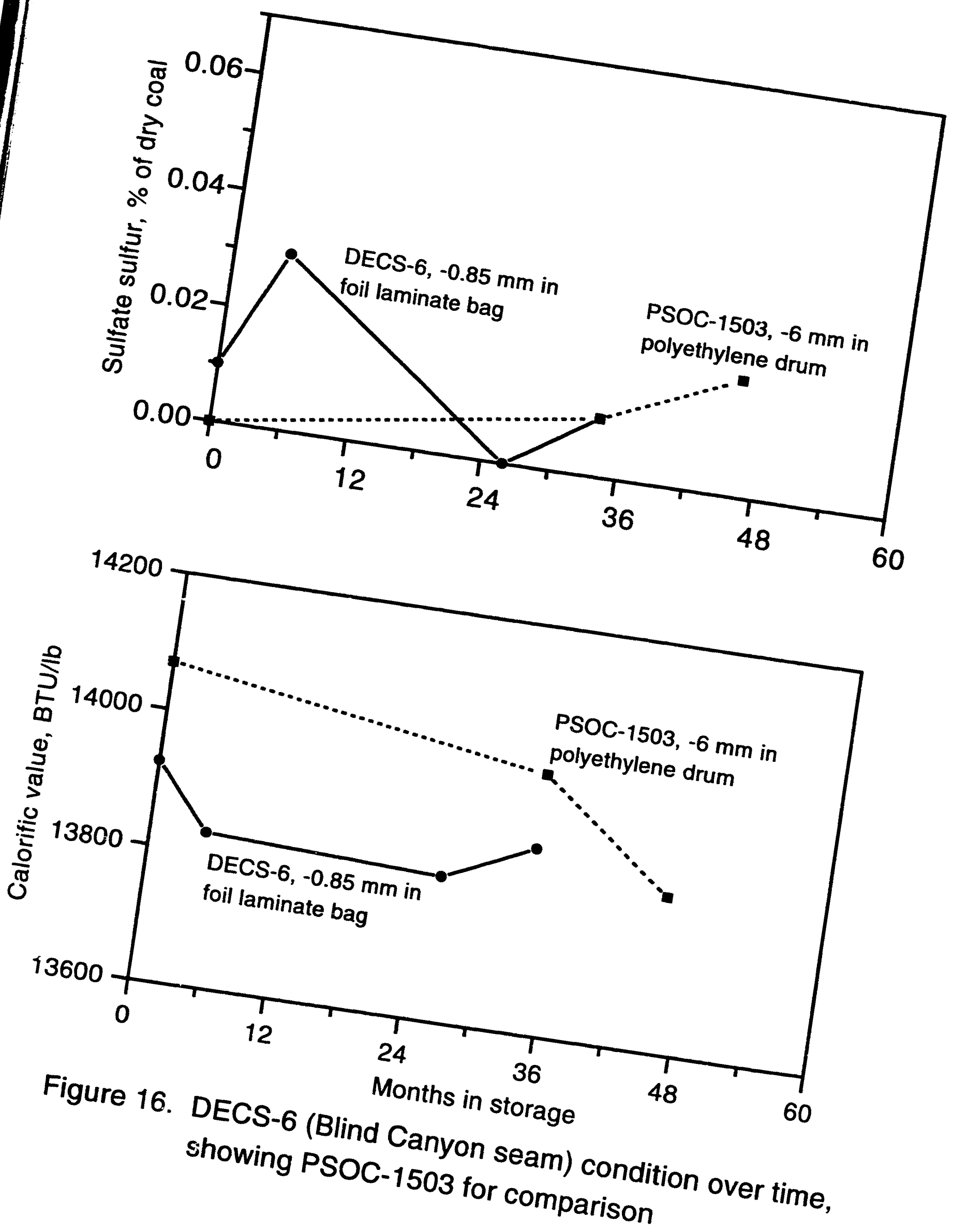

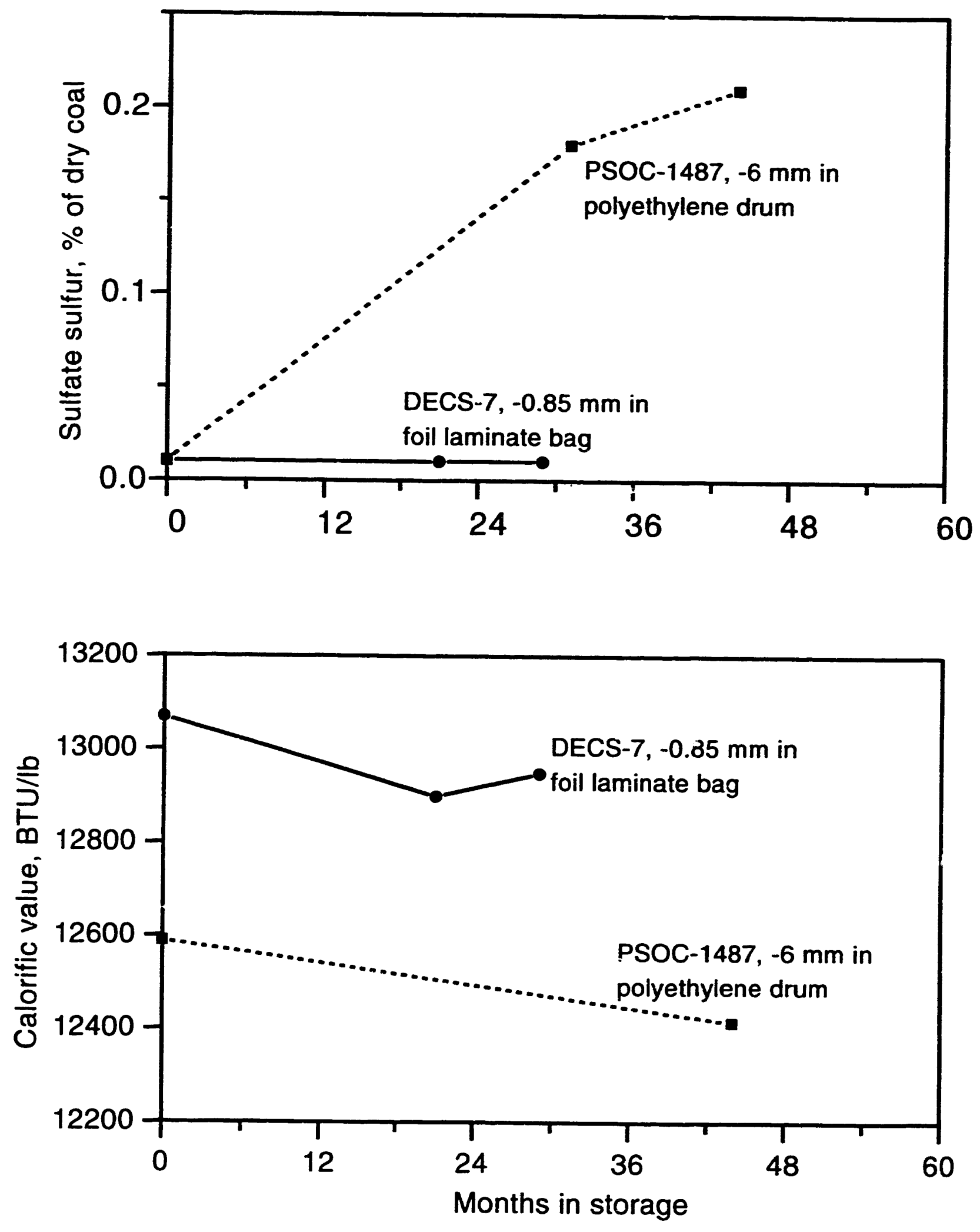

Figure 17. DECS-7 (Adaville No. 1 seam) condition over time, showing PSOC-1487 for comparison 

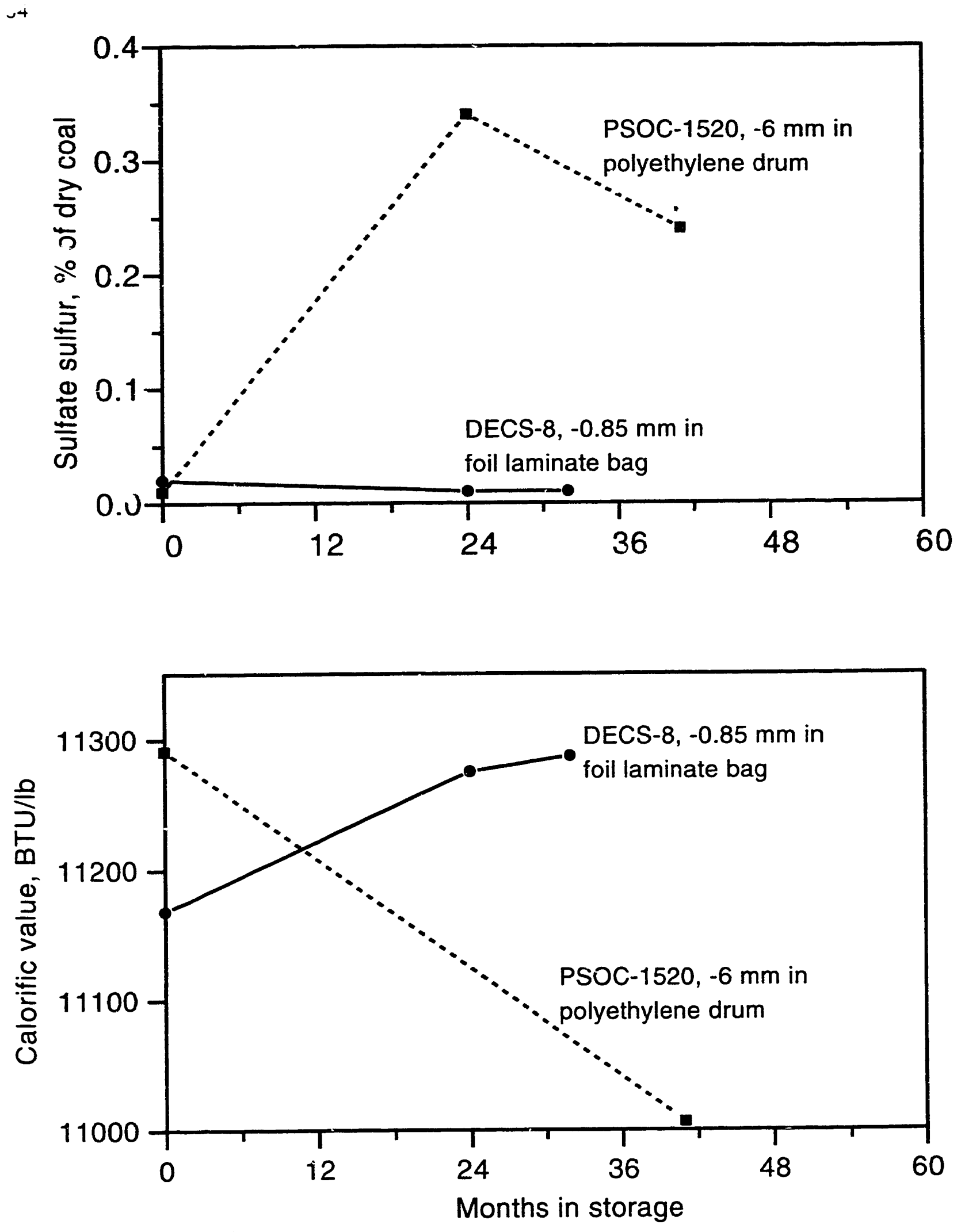

Figure 18. DECS-8 (Smith-Roland, "Wyodak" seam) condition over time, showing PSOC-1520 for comparison 

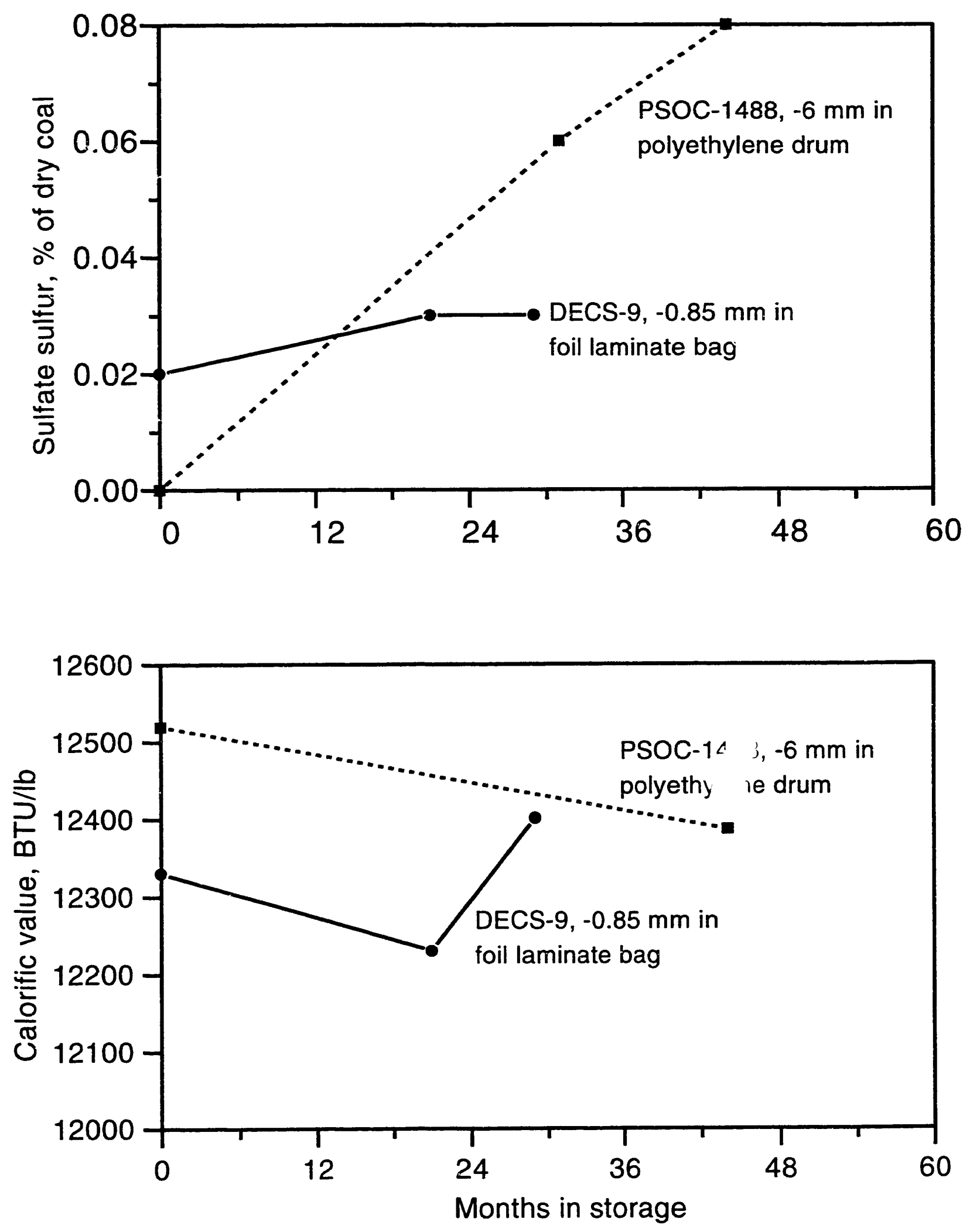

Figure 19. DECS-9 (Dietz seam) condition over time, showing PSOC-1488 for comparison 

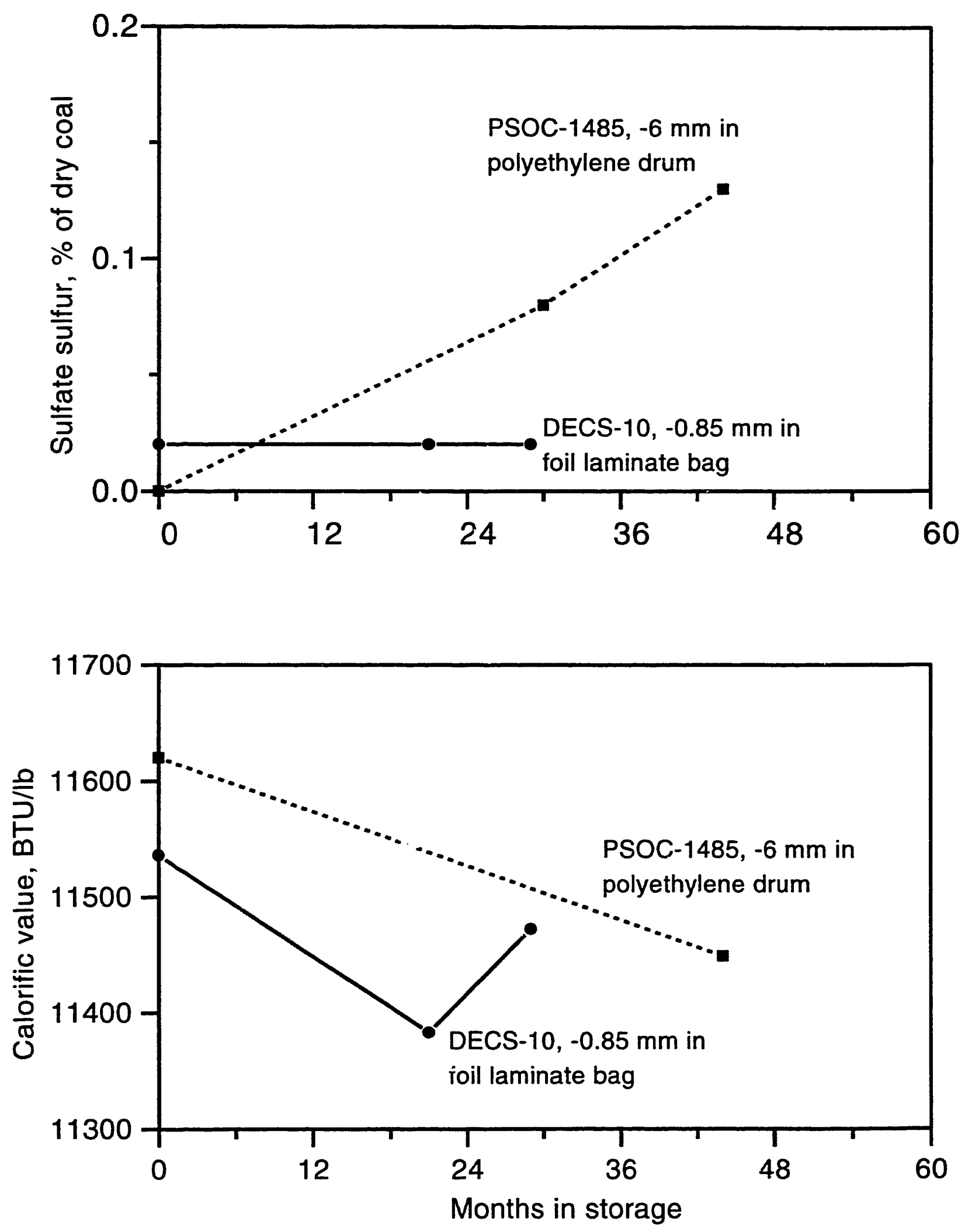

Figure 20. DECS-10 (Rosebud seam) condition over time, showing PSOC-1485 for comparison 

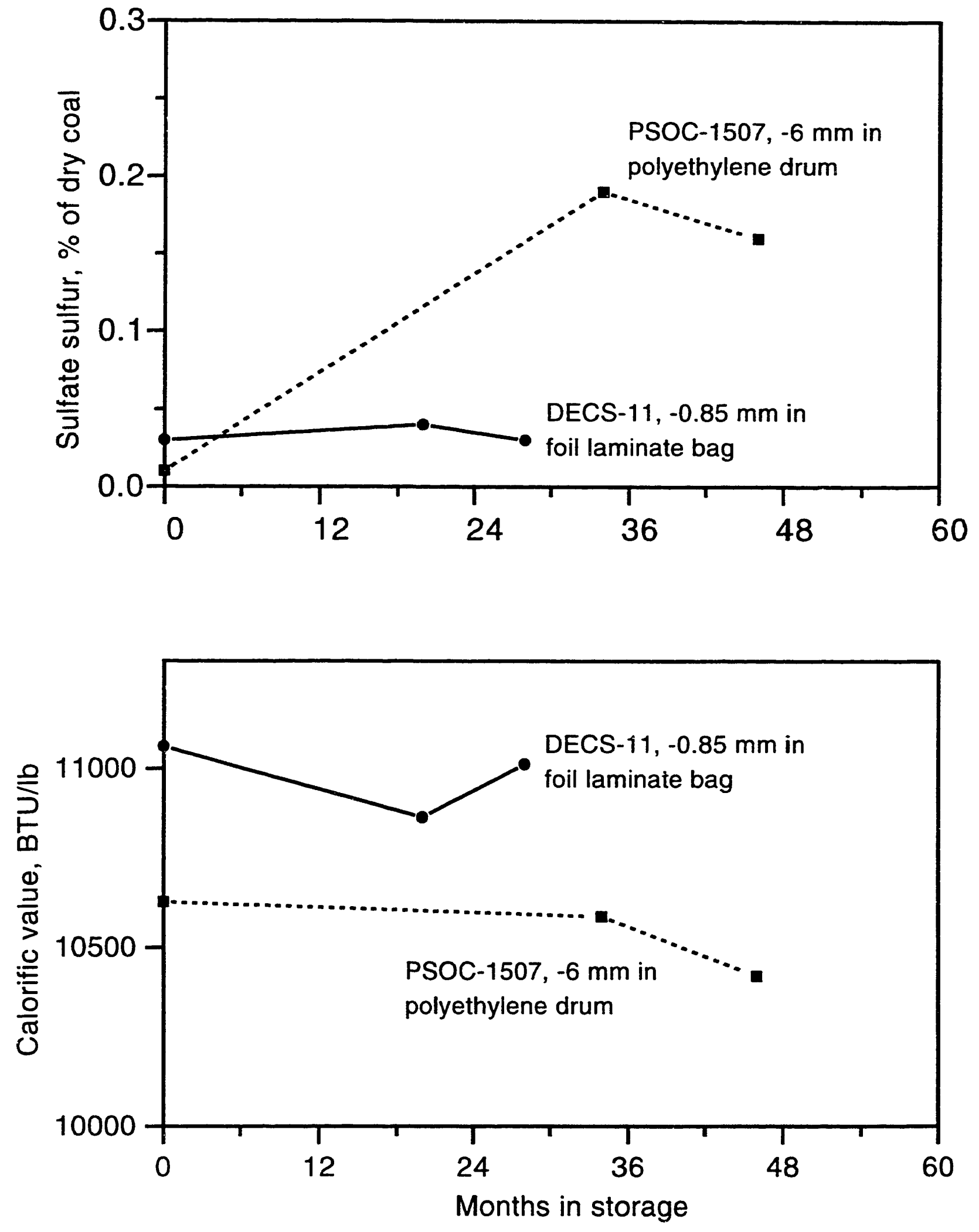

Figure 21. DECS-11 (Beulah seam) condition over time, showing PSOC-1507 for comparison 

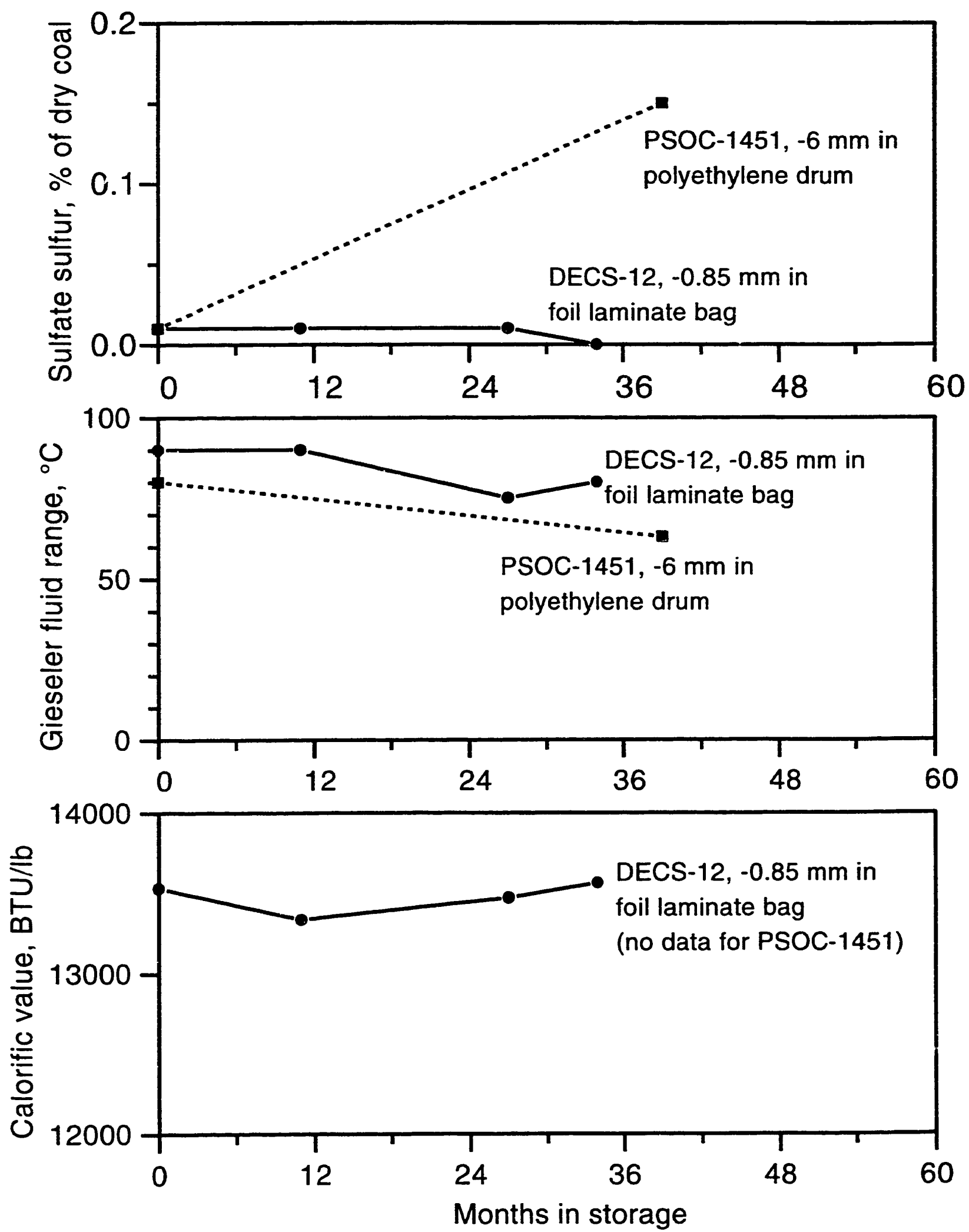

Figure 22. DECS-12 (Pittsburgh seam) condition over time, showing PSOC-1451 for comparison 

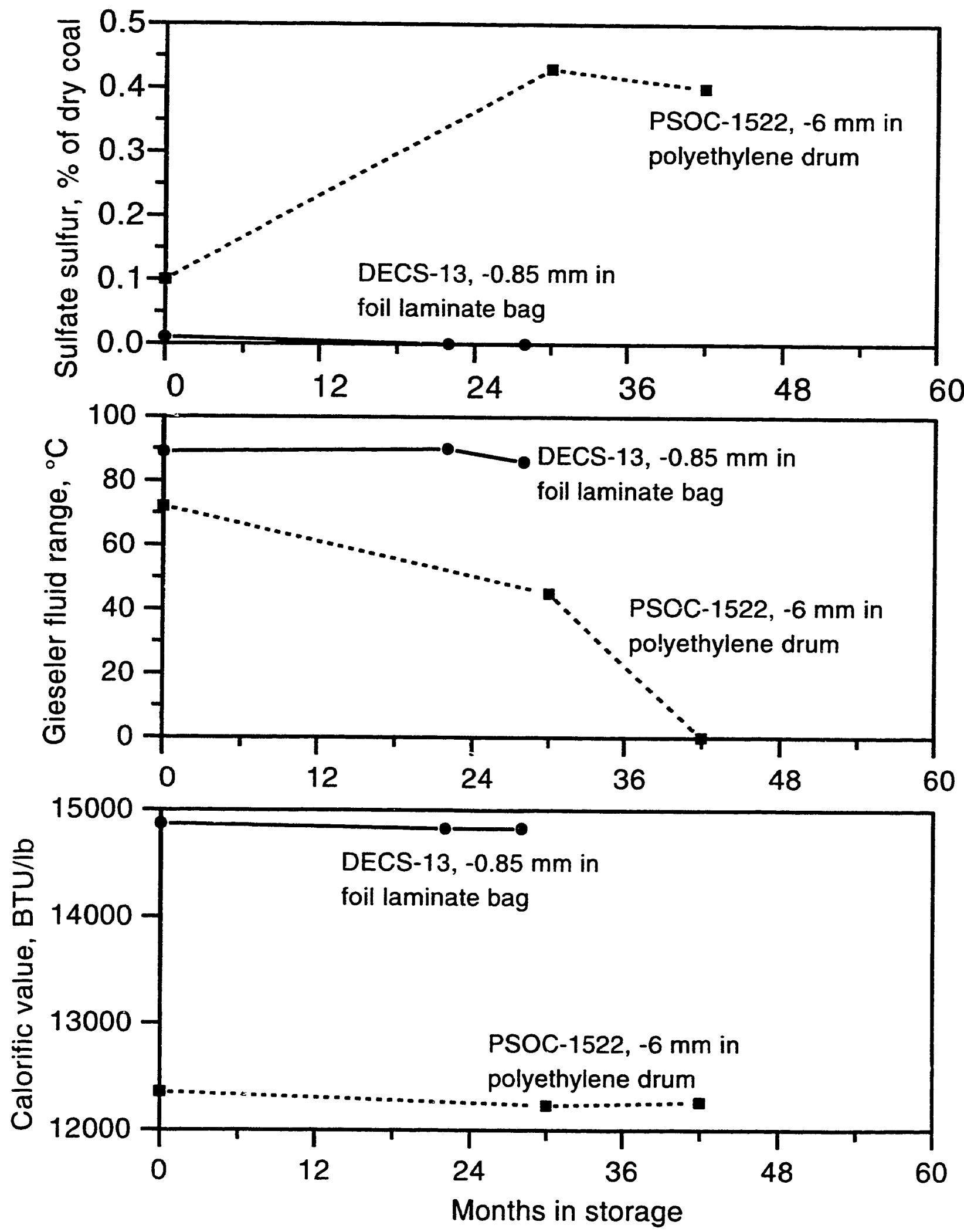

Figure 23. DECS-13 (Sewell seam) condition over time, showing PSOC-1522 for comparison 

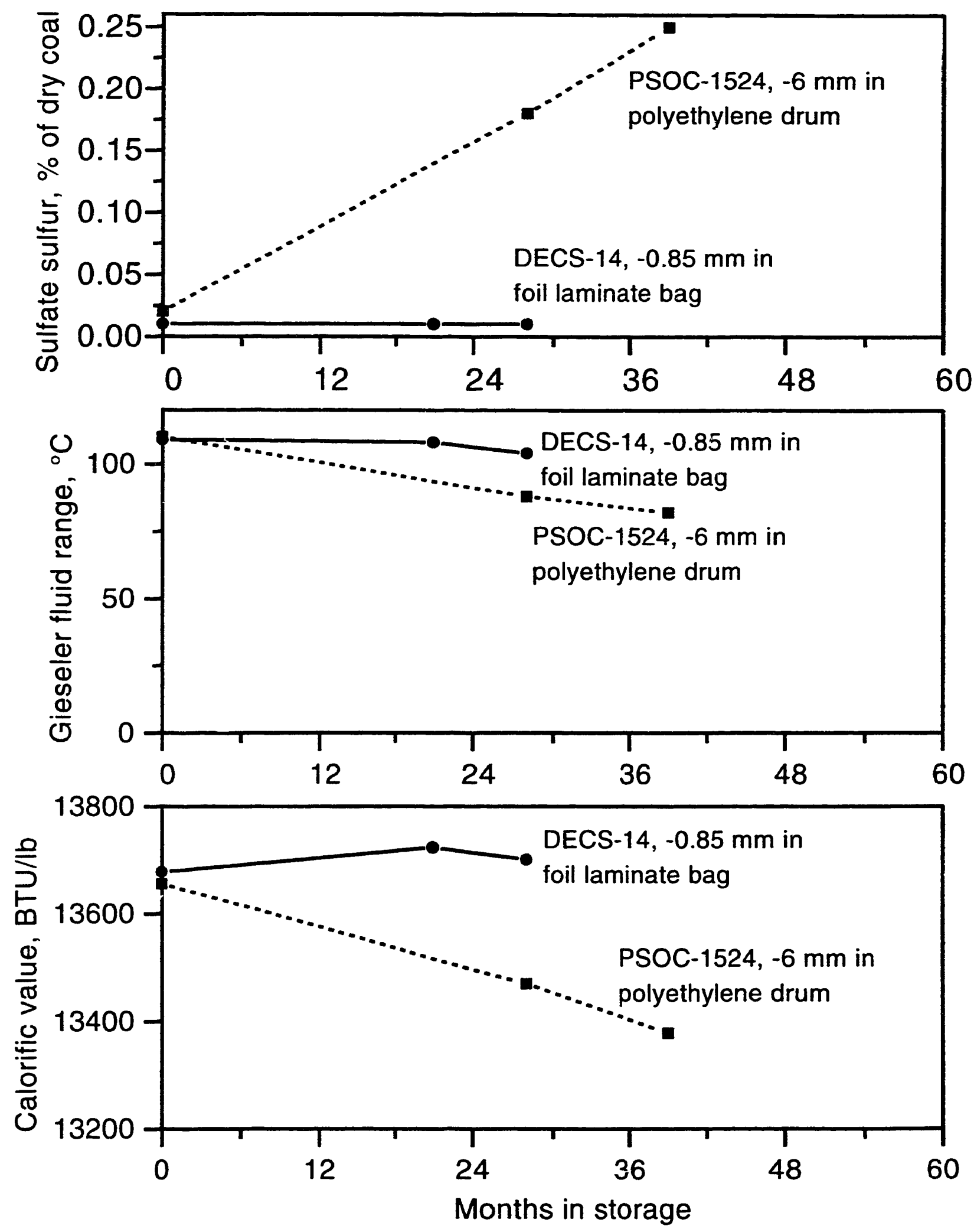

Figure 24. DECS-14 (Upper Kittanning seam) condition over time, showing PSOC-1524 for comparison 

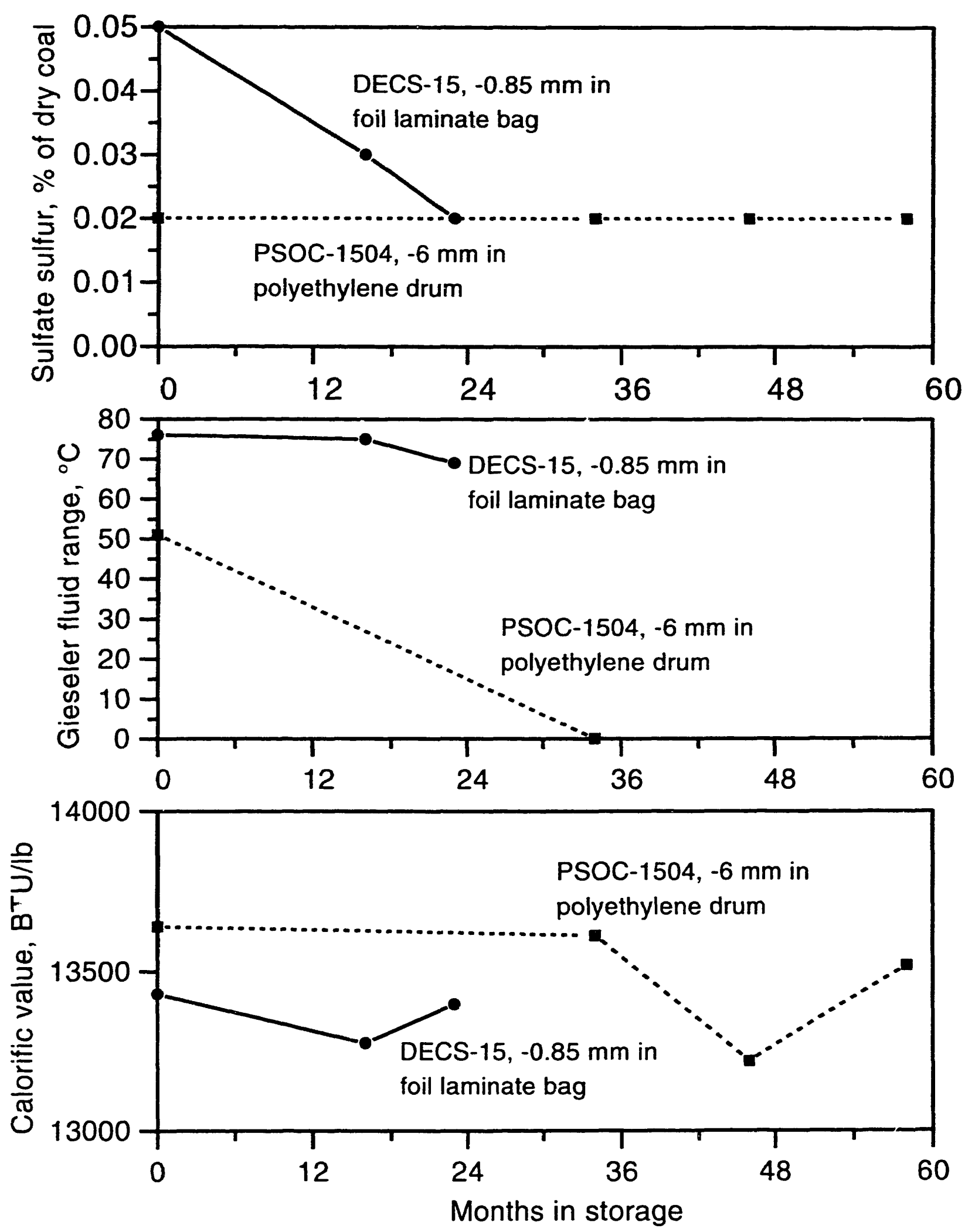

Figure 25. DECS-15 (Lower Sunnyside seam) condition over time, showing PSOC-1504 for comparison 


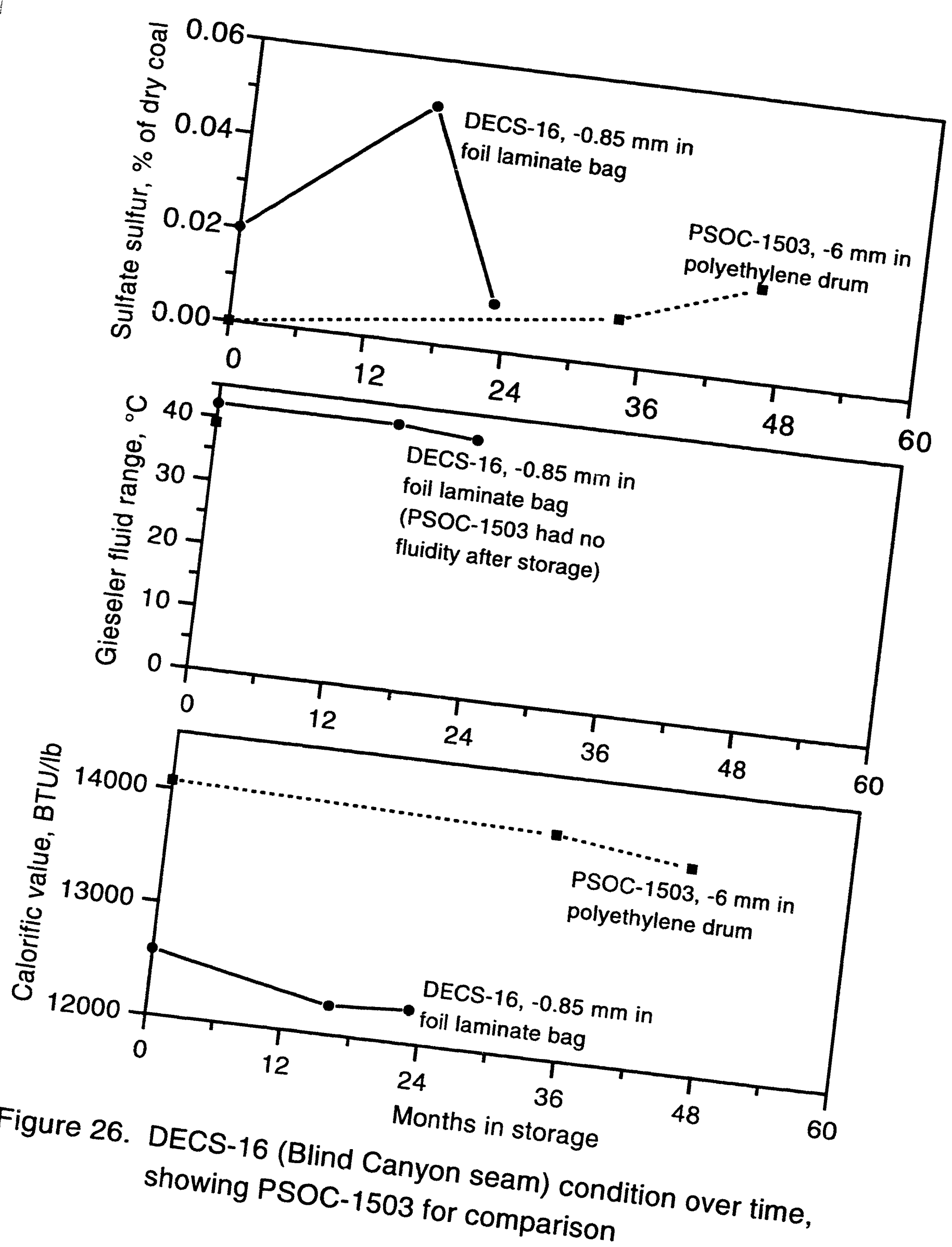



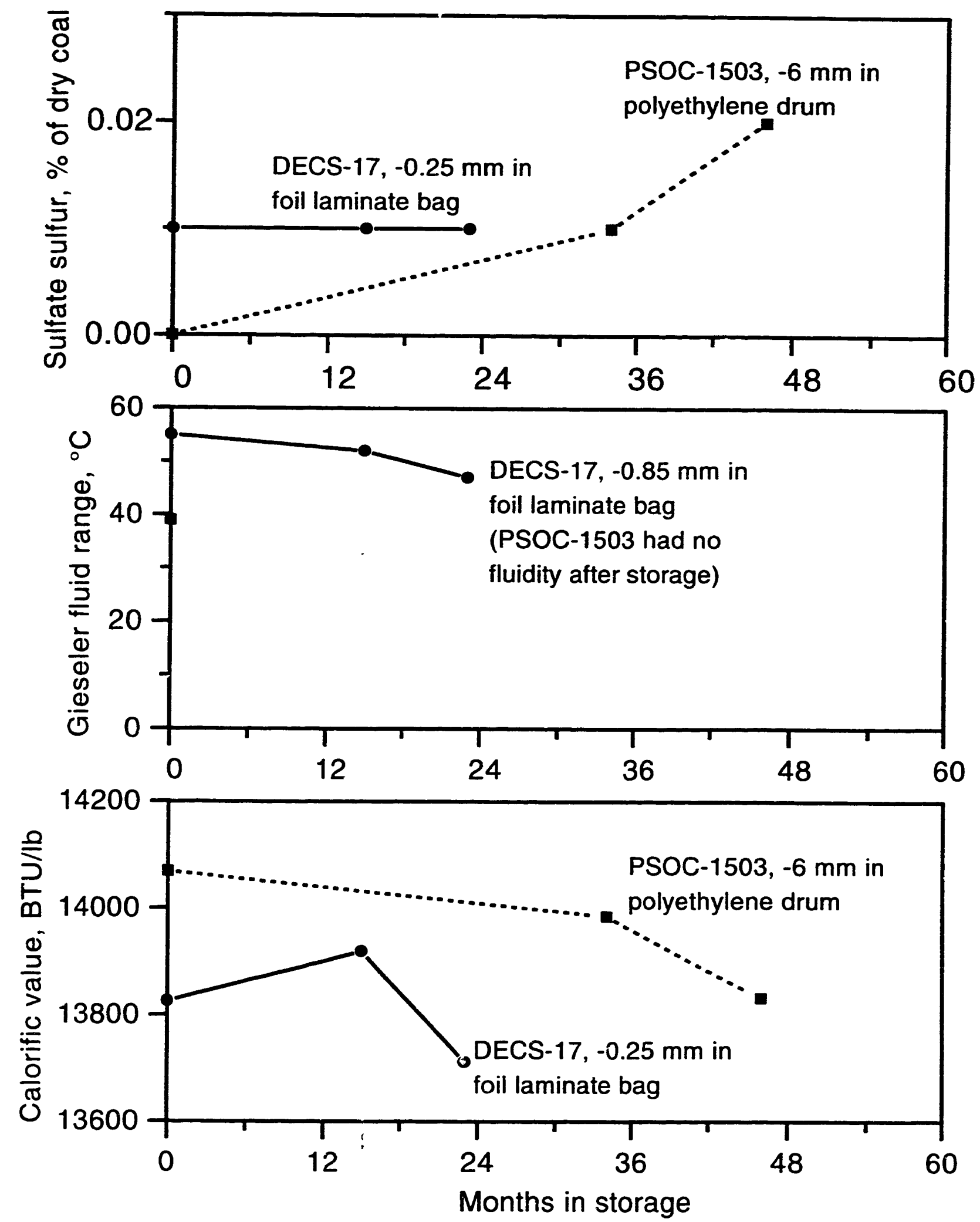

Figure 27. DECS-17 (Blind Canyon seam, refrigerated sample) condition over time, showing PSOC-1503 for comparison. Anaylses on samples stored at $-0.25 \mathrm{~mm}$ ( -60 mesh) except Gieseler fluidity on $-0.85 \mathrm{~mm}$ (-20 mesh) sample. 

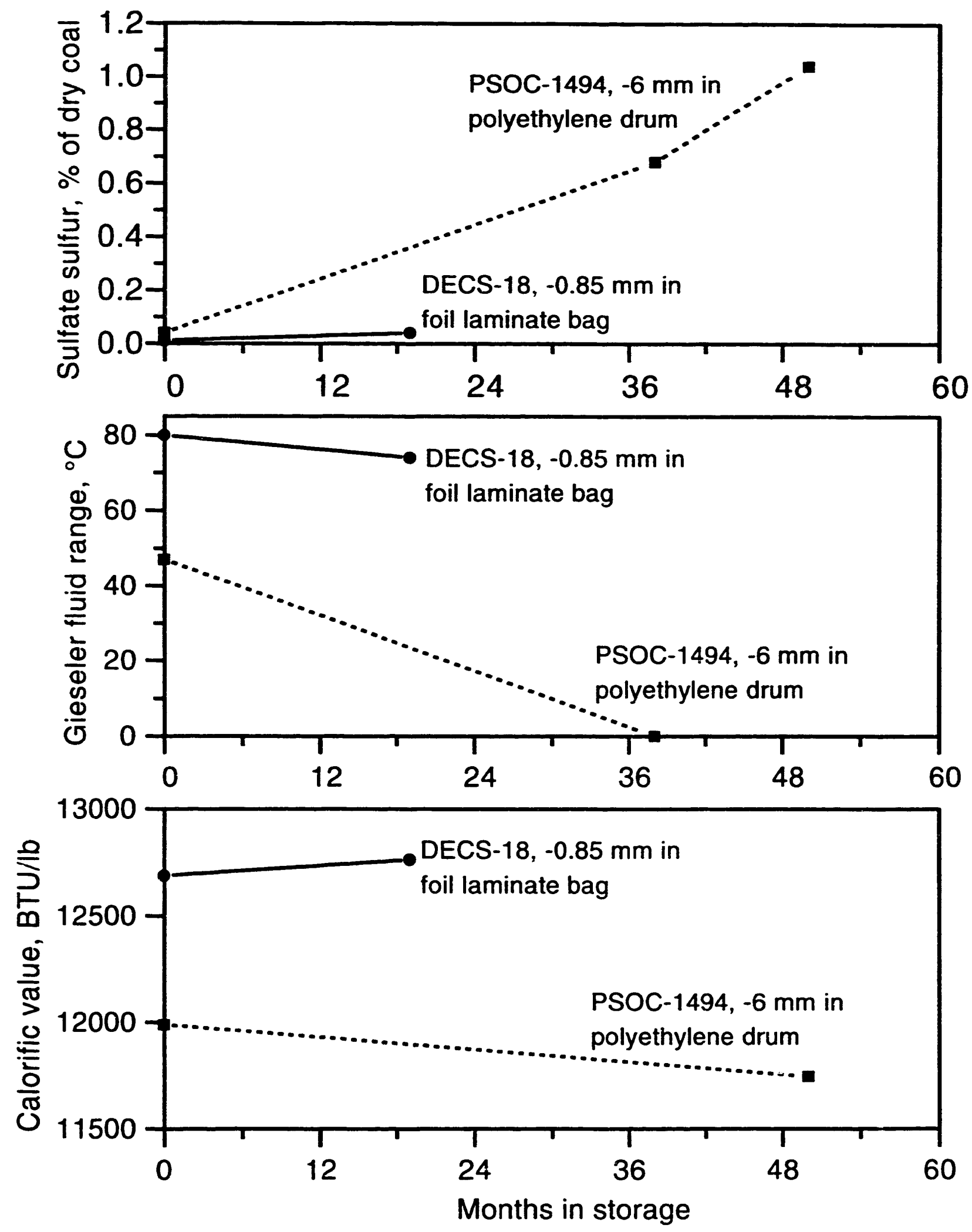

Figure 28. DECS-18 (Kentucky No. 9 seam) condition over time, showing PSOC-1494 for comparison 


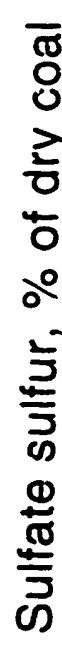

0.04

$0^{60} \longrightarrow$ DECS-19, $-0.85 \mathrm{~mm}$ in

DECS-19, $-0.85 \mathrm{~mm}$ in

0.00

r........

foil laminate bag

진

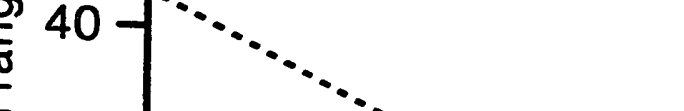

윽

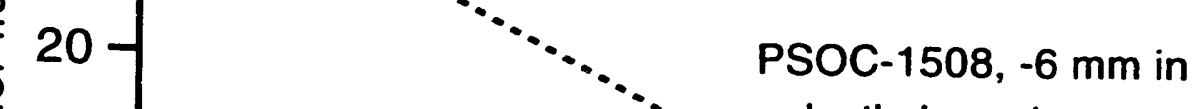

$\Phi$

$\stackrel{\mathscr{d}}{\mathscr{0}}$
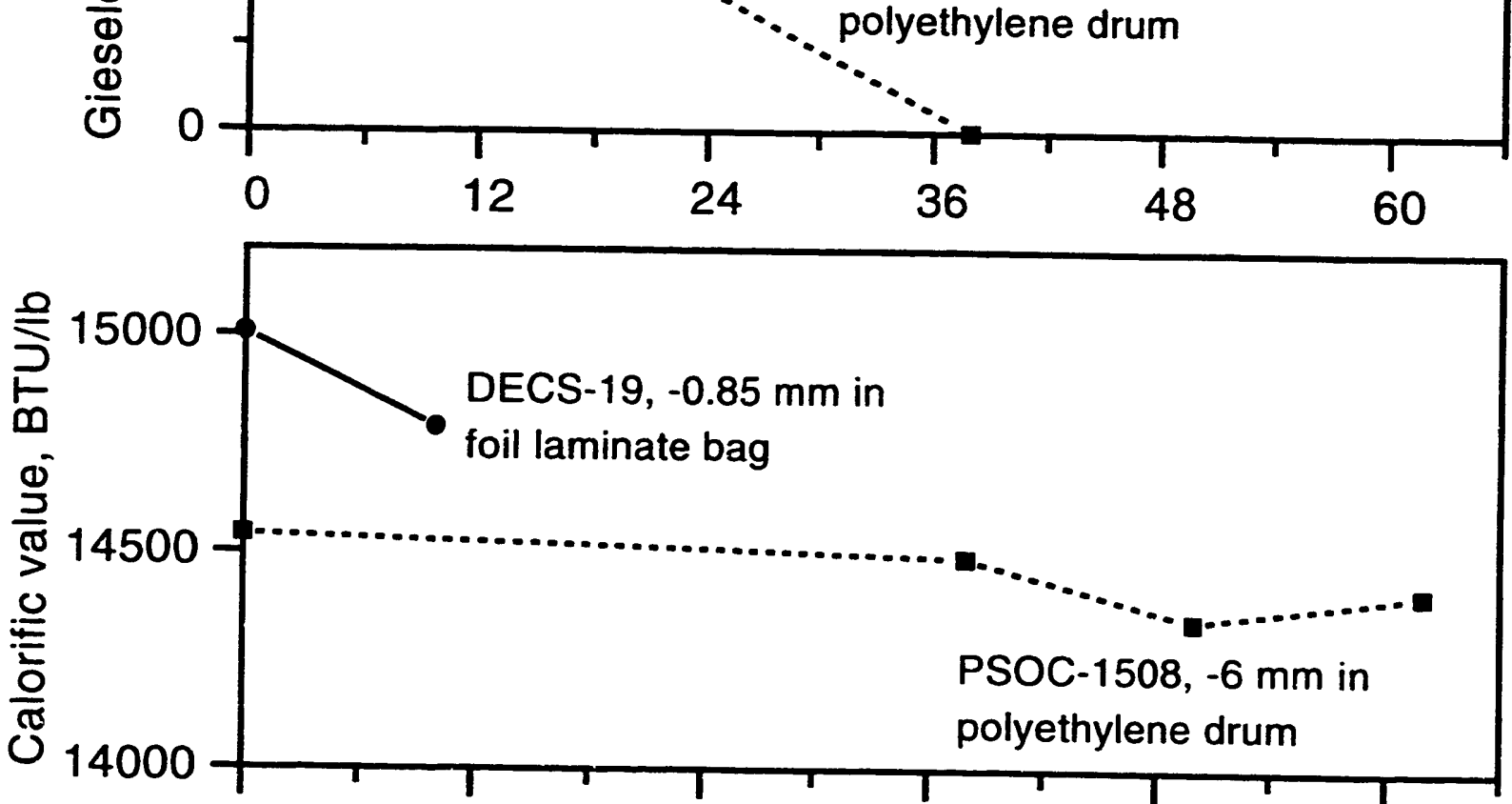

36

48

60 polyethylene drum

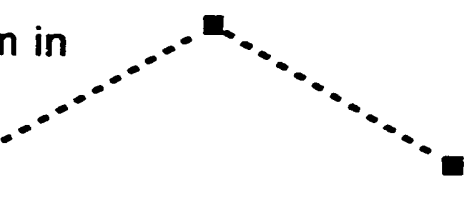



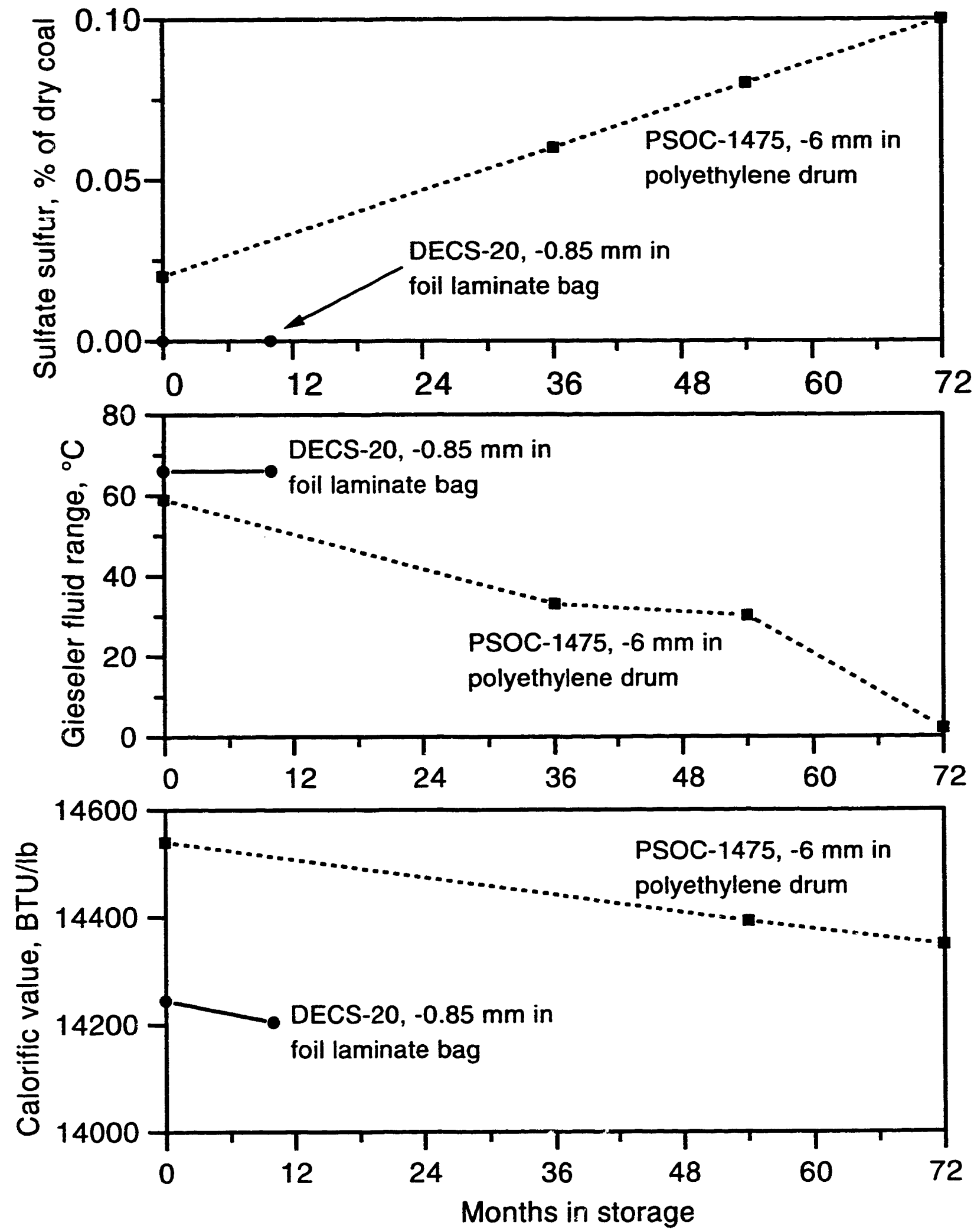

Figure 30. DECS-20 (Elkhorn No. 3 seam) condition over time, showing PSOC-1475 for comparison 
are shown for comparison. Sulfate sulfur and calorific value graphs are shown for all samples, and Gieseler fluid range is shown for those bituminous samples which maintained fluid behavior after storage. In each case the initial values of the DECS and PSOC sample showr may differ: it is the trends or slopes of the lines and not their vertical position which show the difference in storage behavior. Some random variations in readings are, iesent, and seem large where scales are expanded, so fluctuations must be interpreted cautiously. In general, DECS samples stored in foil laminate bags maintained their initial properties through time. whereas the bulk reserves of PSOC samp? es stored in polyethylene drums deteriorated.

DECS-2 (Figure 12), the IIlinois No. 6 seam, shows such a difference between DECS and PSOC storage for a high-sulfur Interior province sample.

Although the two samples were collected from different mines, they are similar in their total sulfur and initial sulfate sulfur. fluid range, and calorific value. DECS-18, the other Interior province sample. shows similar trends.

Eastern samples (DECS-12. -13, -14, -19, and -20) show similar trends. although sulfate sulfur is not as high. DECS-19 has decreased in calorific value more than PSOC-1508. but only one data point is available thus far.

Even several western samples with low total sulfur (DECS-4, -7, -8, -9, - 10. and $\cdot 11$ ) showed a significant increase in sulfate sulfur when stored in drums. but not when stored in foil laminate bags. Utah samples (DECS-5, -6, -15, -16, -17) had low total sulfur and maintained low sulfate sulfur: slight differences are exaggerated by the scale of the graphs.

The abilities of the various container types to preserve sample characteristics through time are summarized in Figures 31,32 and 33 using data from Appendix A. These are 1 inear regression plots comparing initial properties of coal to properties after storage in drums, cans, and laminate bags for various time periods.

Gieseler fluid range (Figure 31) decreased significantly for samples stored in polyethylene druilis. The graph shows a decrease for drums stored for 14-35 months, a slight increase for drums stored 36-51 months, and a greater decrease for drums stored more than 51 months. The inconsistency is believed to be an artifact of the particular samples measured in the different time periods; it was not observed with an interim data set using some of the same samples. The true trend seems to be a continuous decrease in fluid range with time. DECS samples stored in foil laminate bags show only a slight decrease in fluid range. less than that for cans and much less than that for drums of PSOC samples.

Production of sulfate sulfur from sulfides (Figure 32 ) was high for PSOC samples stored in either drums or cans, averaging as much as ten-fold for drums stored more than 68 months. DECS samples stored in foil bags maintained their initial sulfate sulfur levels much better. showing a very slight apparent decrease with time. This was probably an artifact caused by a few high initial sulfate sulfur data points. 

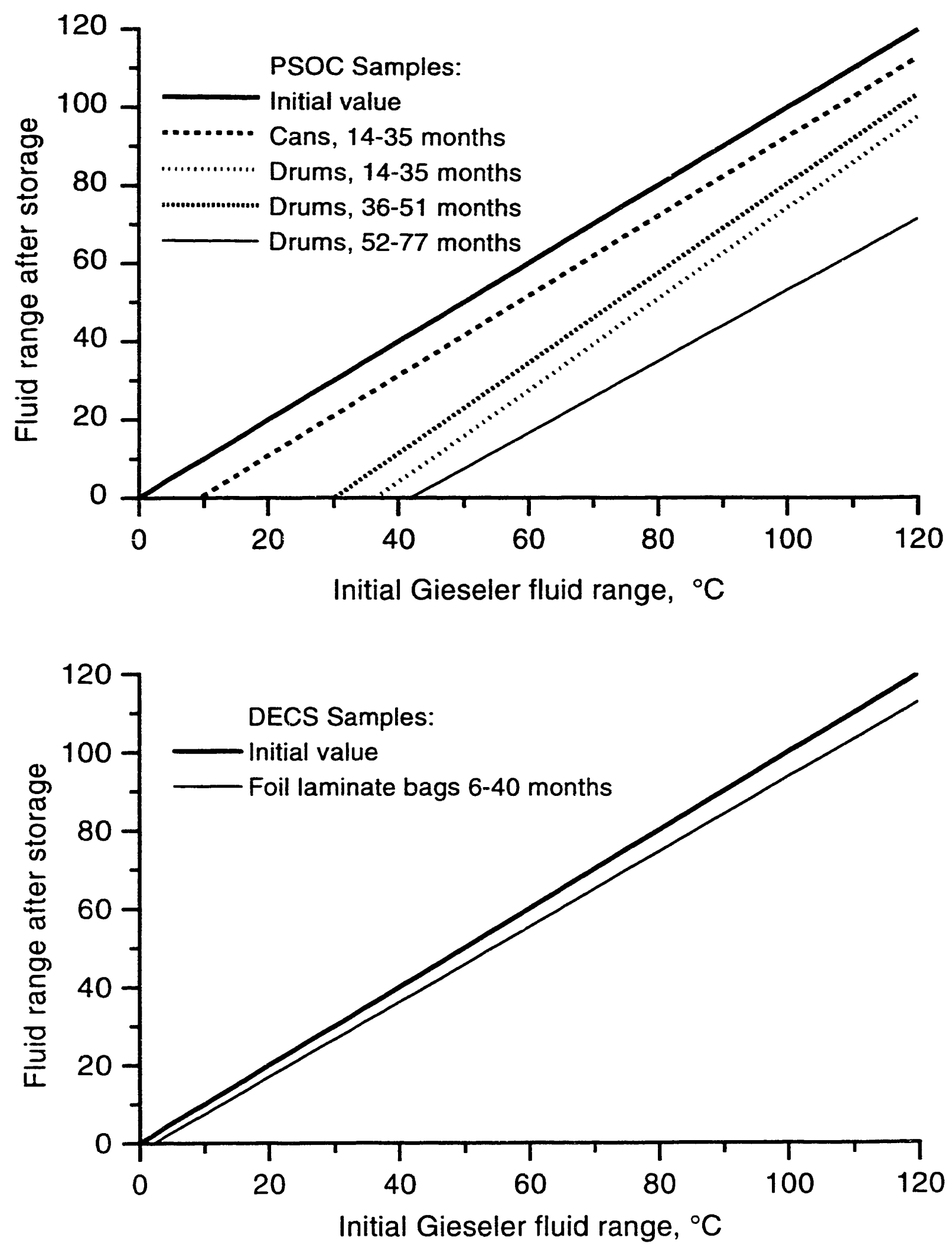

Figure 31. Gieseler fluid range of bituminous PSOC and DECS samples after storage in drums and foil laminate bags: Linear regression on all appropriate samples 

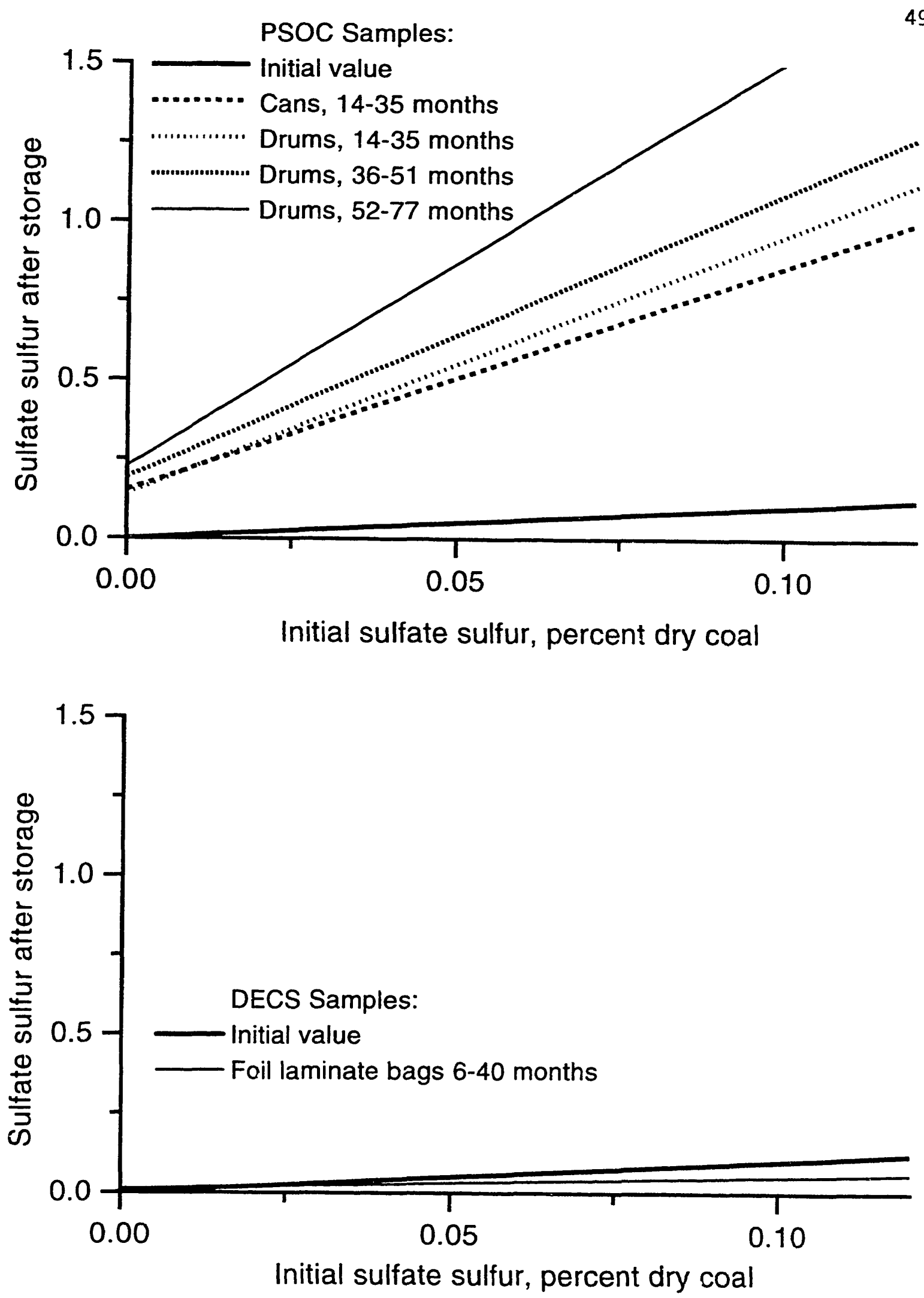

Figure 32. Sulfate sulfur levels of PSOC and DECS samples after storage in drums and foil laminate bags: Linear regression on all samples 

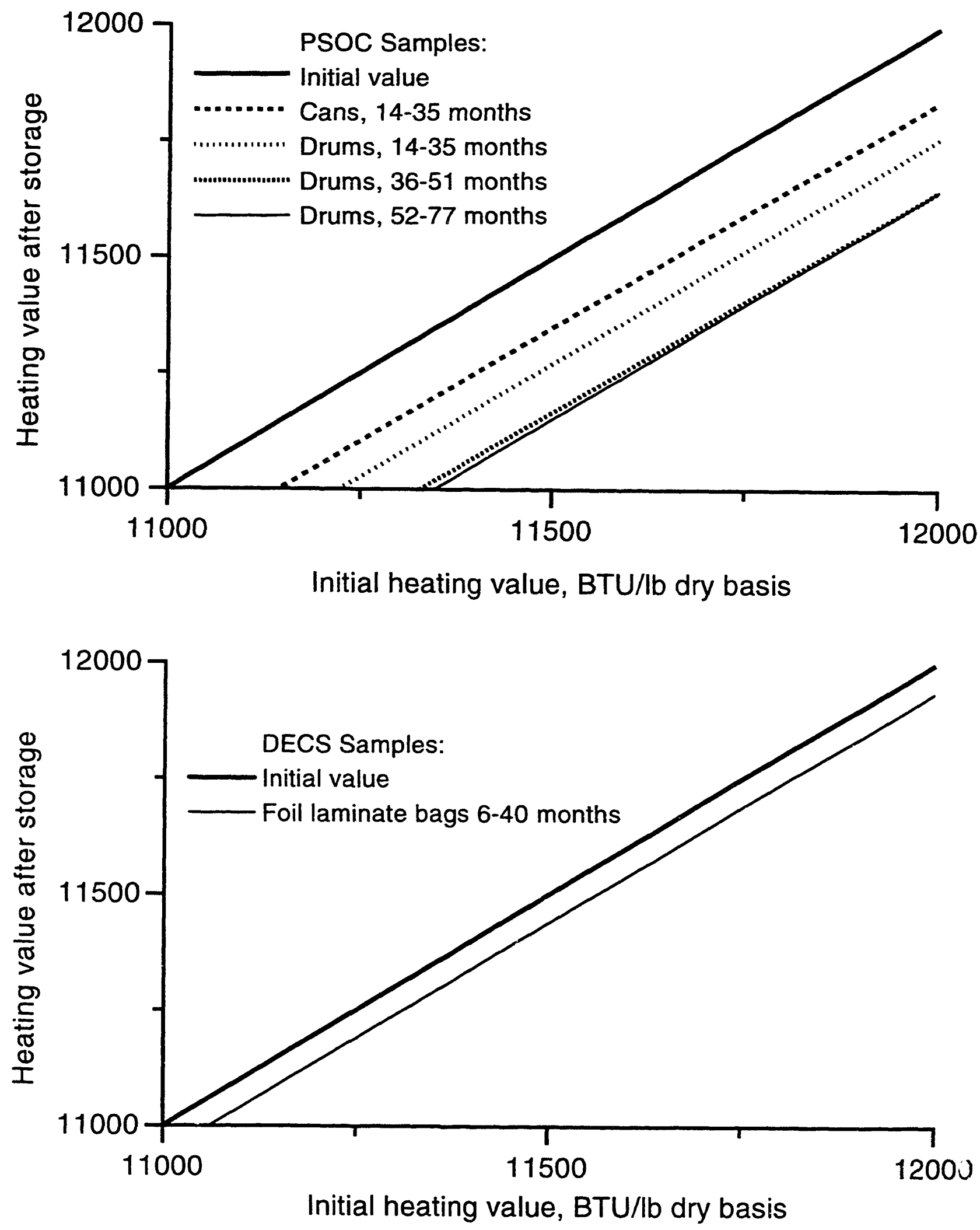

Figure 33. Heating value of PSOC and DECS samples after storage in drums and foil laminate bags: Linear regression on all samples 
Reduction in calorific value during storage was slight even for PSOC samples stored in drums: a graph showing the full range of values from 8000 to 16000 BTU/1b shows the regression lines for the various container types to be indistinguishable. When the scales are greatly expanded as in Figure 33. the expected trend of decrease over time can be seen. DECS samples lcst about 60 $\mathrm{BTU} / 1 \mathrm{~b}$ while in storage for one to three years, about a third as much as PSOC samples stored in drums for the same time.

\section{TASK IC. COLLECTION OF SAMPLES, SAMPLE HANDLING, AND PROCESSING}

The contract called for the collection of twenty-four samples, to be selected based on depletion rates of the existing PSOC samples. However. the monitoring studies described above showed that deterioration needed to be considered as wel1. Therefore, arrangements were made to recollect those samples identified as being most important to DOE liquefaction projects as soon as possible. These included an Eastern Texas seam. the I1linois No. 6 seam, the Blind Canyon seam (Utah), the Smith-Roland or "Wyodak" seam (Wyoming), and the Pittsburgh seam (Pennsylvania).

\section{Collection Methods}

\section{Steel Drums for Field Collection}

As soon as DOE had agreed to the use of foil laminate bags for storage and steel drums with high-density gaskets for field collection, the first sampling trip was organized to test these methods. Two of the most needed samples, the Texas lignite and the Illinois No. 6 seam, were selected. Gaskets were fabricated from Tygon tubing. and laboratory tests showed the gaskets and hardware to be gas-tight. The samples were collected, sealed in the drums, and purged with argon. In the field. Teaks were experienced in both the gaskets and the brass hardware affixed to the drum to introduce the argon. Argon was used to purge the drums as needed (daily) during transportation of the samples to Penn State to prevent introduction of air.

Prior to the next trip, the proper high-density gaskets were received. The hardware arrangement on the drum lids was changed to allow fewer connections and a lower profile of the hardware above the lid (Figure 34). This involved machining of valve bodies so that the valve body outside the drum lid and the 0 ring sealing fixture inside the lid hold the sheet metal of the drum lid securely between them. Two such assemblies (inlet and outlet ports) are fitted to holes in the drum lid. The inlet port has a stainless steel tube extending through the coal to the bottom of the drum for argon introduction. The outlet port provides a valved hole for the air to escape at the opposite end of the container from the argon introduction tube. During purging, argon flows from the tube at the bottom of the drum up through the coal sample. displacing the lighter air and expelling it at the outlet port. The proper gaskets and the hardware modifications have successfully allowed 20 subsequent samples ( 40 drums) to be collected and transported without major leakage problems. 
INLET PORT

OUTLET PORT

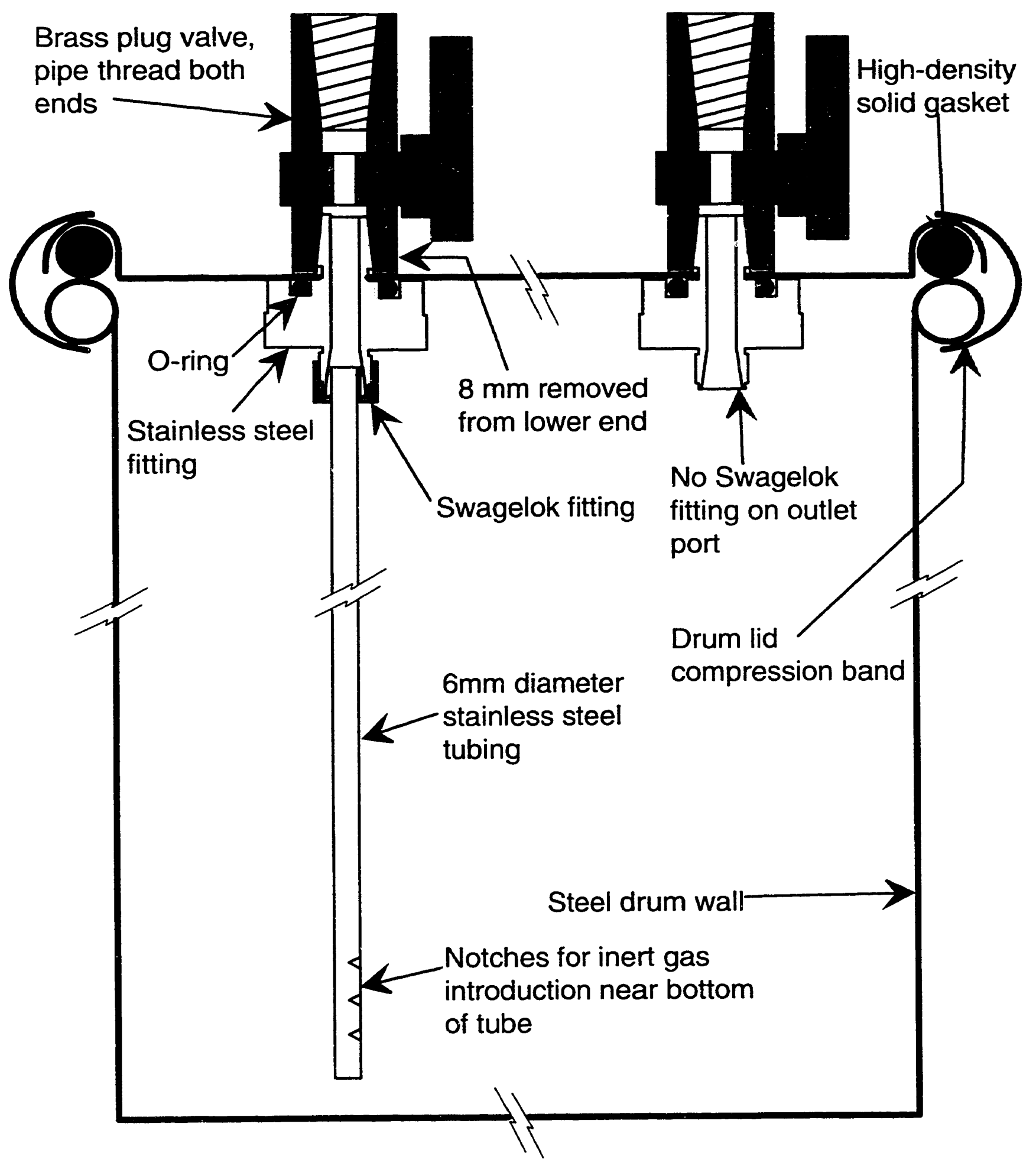

Figure 34. Apparatus for inert gas purging of samples in steel drums 
After agreement with DOE. the minimum sample size was reduced from $270 \mathrm{~kg}$ $(600 \mathrm{lb})$ to $180 \mathrm{~kg}(400 \mathrm{lb})$.

\section{Sample Processing and Packaging}

PSOC samples collected under previous contracts had been processed to provide approximately 32 steel cans $(300 \mathrm{~g}, 2 / 3 \mathrm{lb}$.) of coal at minus $0.85 \mathrm{~mm}(-20$ mesh) for distribution to users. These cans preserved the coal well, but when the initial batch of cans was depleted, the roal in bulk storage designated to replenish the cans had deteriorated. There was also some risk that production of these small batches of cans over many years might introduce variations from differences in processing methods. Therefore a new processing scheme was devised which would create 256 containers for distribution (approximately half the amount collected). This requires much more work initially, but less effort subsequently and no concern over differences between batches of containers. The general scheme, which requires some adjustment for individual samples based on total amount collected, is shown in Figure 35. These adjustments vary the fraction of coal crushed to minus $0.85 \mathrm{~mm}(-20$ mesh) in order to obtain the constant quantity (90 kg, 200 lbs) needed for the final splits.

The coal destined for the 256 bags is riffled into 8 splits, each of which is crushed to minus $0.85 \mathrm{~mm}$ ( $\cdot 20$ mesh). Each split is then riffled into four, producing $32 \mathrm{splits}$ of no more than $3 \mathrm{~kg}(6.6 \mathrm{lb})$. Each of these is divided into 8 final subsamples of nominally $300 \mathrm{~g}(2 / 3 \mathrm{lb})$ each using an 8 -tube rotary divider. Each subsample is placed in a polyethylene bag; the bag's top is folded over once and it is placed in a labelled and numbered foil laminate bag. The inner unsealed bag prevents the sealing area of the foil bag, and the sealer apparatus, from being contaminated by coal particles. The bags are then placed in the chamber of a sealer which is programmed to evacuate the chamber (including the bag). introduce argon to near atmospheric pressure, and heat-seal the top of the foil bag. As the bags are removed from the sealer their weights are recorded. Five bags are removed from the run; one is the principal analytical sample and the other four are analyzed to evaluate equivalence to the principal sample. The first 36 bags of each sample are kept in the laboratory where they are readily accessible for distribution: the remainder are stored for later use.

\section{Sample Container Inventory}

Data (such as bag number, weight, mesh size, storage location, etc.) for every bag of every sample are entered in a computerized inventory program running on Penn State's research mainframe computer. This program was written under the previous contract to replace an older program on a minicomputer, and has been further improved under the subject contract. Data may be entered for individual containers or for groups of containers which differ only in their weights (the 256 finai bags of each sample). The new program performs routine listings of containers for a specified sample about 100 times faster than the old one. When an order for samples is received. the inventory is queried and the locations. weights and particle sizes of the desired samples are shown. The list can be 


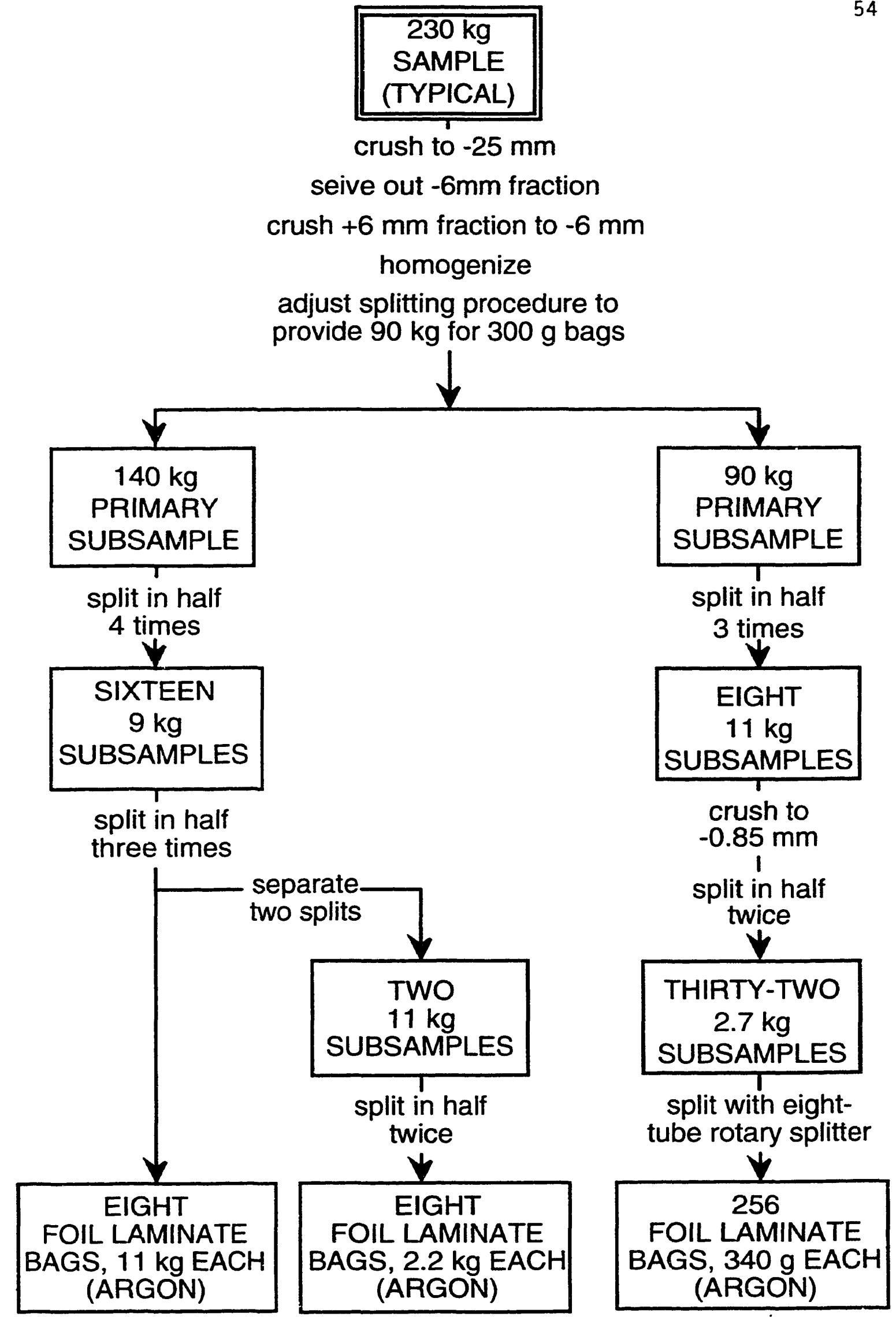

Figure 35. Typical crushing and splitting steps for a DECS sample 
printed out and used as a checklist while retrieving containers. The program is then used to remove the entry for each of those containers from the inventory 1ist: it prompts the user to enter information about the recipient of a container, and that information along with the original data on the container is transferred to a separate file of transactions.

The program stores its files in the format of a commercial data management package. so both the main file and the transactions file can be examined and manipulated with a wide range of commands if the inventory program does not provide the functions required. This is also the format of the Coal Data Base, so although the inventory and the Data Base are normally completely separate. they can be merged if needed for specific tasks.

\section{Samples and Sample Types Collected}

The existing PSOC samples had been chosen to provide a range of ranks, chemical compositions, and geographic and geologic sources and to represent the output of major mining operations across the country. Samples to be recollected in the DECS series were chosen to meet current research needs, particularly for liquefaction. The specific mines were chosen based on their current exposure of coal of the desired composition. The same mines from which Argonne National Laboratory Premium Coal Samples had been sampled were chosen when feasible.

The 22 DECS samples collected under the contract are listed in Table 9. The goal for each sample is to collect a sample which represents the entire thickness of the seam. Whenever feasible this is achieved by collection of a channel sample of the full seam. For particularly thick seams, lack of access to the entire seam. coupled with the quantity per unit thickness required to achieve a representative sample, make a channel sample inappropriate. In these cases a run-of-mine sample is usually collected. For mines where only a part of the vertical extent of the seam is normally worked, a channel sample of that section (working-section channel sample) is taken. The ability to collect fresh coal is also considered when deciding on the location and type of sample to be collected. Samples collected as other than whole-seam channels are listed below.

DECS-5. Hiawatha seam. Utah: Only the bottom 80 percent of the seam was being mined: the upper $56 \mathrm{~cm}$ (22 in) was inaccessible in the mine roof. Our working section channel sample coincided with the normal extent of mining.

DECS -6, Blind Canyon seam, Utah: The lowest $15 \mathrm{~cm}$ (6 in) of coal was not mined at the sample site and was not included in our working-section channel sample.

DECS-8, Smith-Roland ("Wyodak") seam, Wyoming: The vertical pit wall over 30 meters (100 feet) high was not safely accessible. A run-of-mine sample was collected using the automatic sampler at the preparation plant; amounts taken while top and bottom sections were being mined were gauged to provide a representative sample of the whole seam. 
Table 9. DECS samples collected under the contract

\begin{tabular}{|c|c|c|c|c|c|c|c|}
\hline $\begin{array}{l}\text { Sample } \\
\text { Number }\end{array}$ & $\begin{array}{l}\text { Replaces } \\
\text { PSOC. }\end{array}$ & Seam name & $\begin{array}{c}\text { Sample } \\
\text { Type }\end{array}$ & County & State & Rank & Year \\
\hline DECS-1 & 1444 & unnamed & Chan-SEAM & Freestone & $\mathrm{TX}$ & subc & 1989 \\
\hline DECS-2 & 1493 & Illinois \#6 & Chan-SEAM & Randolph & IL & $h v C b$ & 1989 \\
\hline DECS-3 & 1500 & Coal Basin M & Chan-SEAM & Pitkin & $\mathrm{CO}$ & $\mathrm{mvb}$ & 1990 \\
\hline DECS - 4 & 1445 & Blue & Chan-SEAM & Mckinley & NM & $h v C b$ & 1990 \\
\hline DECS - 5 & 1502 & Hiawatha & Chan-Work & Sevier & UT & $h v C b$ & 1990 \\
\hline DECS- 6 & 1503 & Blind Canyon & Chan-Work & Emery & UT & hvAb & 1990 \\
\hline DECS - 7 & 1487 & Adaville \#1 & Chan-SEAM & Lincoln & WY & $\mathrm{hvCb}$ & 1990 \\
\hline DECS - 8 & 1520 & Smith-Roland & Run-of-mine & Campbe11 & $W Y$ & subc & 1990 \\
\hline DECS -9 & 1488 & Dietz & DrII-SEAM & Bighorn & MT & subB & 1990 \\
\hline DECS - 10 & 1485 & Rosebud & Chan-Work & Rosebud & MT & subB & 1990 \\
\hline DECS-11 & 1507 & Beulah & Chan-SEAM & Mercer & ND & lig & 1990 \\
\hline DECS - 12 & 1451 & Pittsburgh & Chan-SEAM & Greene & PA & hvAb & 1990 \\
\hline DECS-13 & 1522 & Sewell & Chan-SEAM & Greenbrier & $W V$ & $\mathrm{mvb}$ & 1990 \\
\hline DECS-14 & 1524 & Upper Kitt. & Chan-Work & Barbour & WV & hvAb & 1990 \\
\hline DECS-15 & 1504 & L. Sunnyside & Chan-SEAM & Carbon & UT & hvAb & 1991 \\
\hline DECS-16 & 1503 & Blind Canyon & Chan-Work & Emery & UT & hvAb & 1991 \\
\hline DECS-17 & 1503 & Blind Canyon & Chan-Sect & Emery & UT & hvAb & 1991 \\
\hline DECS - 18 & 1494 & Kentucky \#9 & Chan-SEAM & Union & KY & $h v B b$ & 1991 \\
\hline DECS-19 & 1508 & Pocahontas \#3 & Chan-SEAM & Buchanan & VA & lvb & 1991 \\
\hline DECS-20 & 1475 & Elkhorn \#3 & Chan-SEAM & Pike & KY & hvAb & 1991 \\
\hline DECS-21 & 1461 & Lykens V. \#2 & Chan-SEAM & Columbia & PA & an & 1992 \\
\hline DECS-22 & 1107 & Upper Kitt. & Chan-Lith & Armstrong & PA & hvAb & 1993 \\
\hline
\end{tabular}

DECS-9. Dietz seam. Montana: Only half of the 26 meter (84 foot) seam was freshly exposed at a time in the mining operation. The mine operators use an air rotary drill rig equipped with an automatic cyclonic sampler to take samples ahead of the mine face for quality prediction. They have studied their sampling process and report that the air drilling process does not significantly affect moisture content. The rig deposits about $450 \mathrm{~kg}$ (1000 lbs) of drill chips in a cone beside the hole. Opposite quarters of a cone from a freshly drilled hole were taken as our sample.

DECS-10. Rosebud seam. Montana: At least $15 \mathrm{~cm}$ (6 in) of material below the floor of the mine was a mixture of pyrite and coal and is not normally mined. Also. the top 1 meter ( $3 \mathrm{ft}$ ) of coal at the freshly exposed sample site was inaccessible from our ladder. These sections were excluded from our working section sample, which thus comprised approximately 84 percent of the seam. 
DECS-14. Upper Kittanning seam. West Virginia: Neither the roof nor the floor of the coal seam were exposed in this underground mine. Our workingsection channel was extended down to reach the bottom of the coal. but not up to reach the top.

DECS-16. BTind Canyon seam. Utah: Collected from the same mine as DECS-6. and the same situation prevailed. At least $25 \mathrm{~cm}$ (10 in) of coal below the mine floor was not included in the working-section channel sample.

DECS-17. Blind Canyon seam, Utah: This sample was required by DOE to be lower in ash than DECS-16, so a lithotype channel sample excluding two high-ash zones was taken (see discussion below).

DECS-22. Upper Kittanning seam (canne1), Pennsylvania: The upper $203 \mathrm{~cm}$ $\left(80^{\prime \prime}\right)$ of the $280 \mathrm{~cm}\left(110^{\prime \prime}\right)$ seam thickness at the sample site was a cannel coal. To provide an unmixed cannel coal sample. the cannel coal section was taken as a lithotype channel sample. The lower section of banded coal was taken as another lithotype channel sample. PSOC-1560, which is not part of the DOE Sample Bank.

Special Sample for DOE Dispersed Catalyst Research

During the contract, a goal of DOE/PETC was to use a single coal for testing the comparative behaviors of dispersed catalysts. The initial criteria for selecting the coal were:

1) reasonably large reserves being actively mined.

2) high volatile bituminous rank.

3) high vitrinite content.

4) low pyritic sulfur.

5) low iron (to avoid the catalytic activity found in high pyrite coals such as the Illinois No. 6), and

6) low ash.

The Blind Canyon seam, Utah, was selected by DOE as the best candidate among several considered. Although sample DECS- 6 has already been collected from this seam. it was not used because the proposed sample was to be stored entirely under refrigeration after processing to preserve its properties, and a larger amount of sample and special restrictions on distribution were to be used to keep the sample available for research over an extended time period.

A trip was made to Utah and $275 \mathrm{~kg}$ (600 lb) each of samples DECS-16. Blind Canyon seam, and DECS-15, Lower Sunnyside seam (as a possible alternate), were collected. The samples were returned to Penn State and promptly processed. analyzed, sealed, and stored under refrigeration. As expected, analyses showed DECS-16 to be more suitable than DECS-15. However, the ash yield of DECS-16 was higher than had been expected based on previous samples. In an effort to provide the best possible sample for the long-term catalyst research projects, another trip to Utah was undertaken and $275 \mathrm{~kg}(600 \mathrm{lb})$ of sample DECS-17. B1 ind Canyon seam. was collected. To reduce the amount of ash, two high-ash zones in the seam were excluded from the sample. This sample was also promptly treated and was accepted by DOE as the sample for the dispersed catalyst 1iquefaction research. 
Reduction in Number of Samples Required

The collection effort planned under the contract had been intended to visit each region of the country only once to save travel time and costs. Unexpected costs were associated with the two additional trips to Utah, processing three larger than normal samples, and providing refrigerated storage for the special sample. As a result. DOE agreed in 1991 to reduce the number of samples required under the contract from 24 to 22.

\section{TASK ID. CHARACTERIZATION OF COALS}

All samples collected were subjected to characterization using the following routine compositional analyses and tests:

1. Proximate analysis

2. Ultimate analysis

3. Sulfur forms analysis

4. Calorific value determination

5. Maceral analysis

6. Vitrinite reflectance analysis

7. Ash fusion determination

8. Free swelling index determination

9. Hardgrove grindability index determination

10. Major inorganic elements analysis

11. Equilibrium moisture determination

12. Gieseler plasticity (for bituminous samples)

13. Carbon dioxide content

14. Chlorine content

The results of these analyses are shown in the individual Coal Data Base printouts included as Appendix B.

The alkali-extraction test (Lowenhaupt and Gray, 1980) for weathering of bituminous coals was applied to all samples in the appropriate range of ranks. For DECS samples, measurements from a $10 \mathrm{~mm}$ cuvette were mathematically corrected to a $17 \mathrm{~mm}$ light path per ASTM standard D-5263 (ASTM, 1993). Results are included in Appendix A.

Four additional subsamples of each DECS sample were submitted for ash. moisture. and total sulfur tests in order to evaluate the degree of variability among the subsamples distributed to researchers. Results of these tests for equivalence (Table 10) generally show low variability among subsamples. The table shows mean and standard deviation (S.D.) of each set of five analyses: however, because the means are not of similar magnitude, it is difficult to evaluate the variability using the standard deviations. The coefficient of variation (C.V.) is the standard deviation divided by the mean; coefficients of variation can be compared directly with each other to evaluate the variability within each set of analyses. 
Table 10. Equivalence of moisture, ash and sulfur of splits of DECS samples

\begin{tabular}{|c|c|c|c|c|c|c|c|c|}
\hline \multirow{2}{*}{$\begin{array}{l}\text { DECS } \\
\text { sample }\end{array}$} & \multicolumn{5}{|c|}{ Equivalence Analyses: Moisture, percent } & \multicolumn{3}{|c|}{ Statistics } \\
\hline & Principal & 1 & 2 & 3 & 4 & Mean & S.D. & C.V. \\
\hline 1 & 30.00 & 29.57 & 29.56 & 29.71 & 28.61 & 29.49 & 0.52 & 0.018 \\
\hline 2 & 10.43 & 10.22 & 10.10 & 10.03 & 10.11 & 10.18 & 0.16 & 0.015 \\
\hline 3 & 1.10 & 0.90 & 1.05 & 0.92 & 0.99 & 0.99 & 0.08 & 0.085 \\
\hline 4 & 15.13 & 15.08 & 15.21 & 14.90 & 14.93 & 15.05 & 0.13 & 0.009 \\
\hline 5 & 7.54 & 7.27 & 7.38 & 7.09 & 7.18 & 7.29 & 0.18 & 0.024 \\
\hline 6 & 4.73 & 4.48 & 4.51 & 4.38 & 4.45 & 4.51 & 0.13 & 0.029 \\
\hline 7 & 17.34 & 16.90 & 17.19 & 16.69 & 16.99 & 17.02 & 0.25 & 0.015 \\
\hline 8 & 28.42 & 28.29 & 28.53 & 27.74 & 28.11 & 28.22 & 0.31 & 0.011 \\
\hline 9 & 24.68 & 24.46 & 24.32 & 24.25 & 24.51 & 24.44 & 0.17 & 0.007 \\
\hline 10 & 21.58 & 23.40 & 21.21 & 21.47 & 21.72 & 21.88 & 0.87 & 0.040 \\
\hline 11 & 33.38 & 31.95 & 33.23 & 32.65 & 32.74 & 32.79 & 0.56 & 0.017 \\
\hline 12 & 2.40 & 2.36 & 2.37 & 2.32 & 2.32 & 2.35 & 0.03 & 0.015 \\
\hline 13 & 1.45 & 1.79 & 1.81 & 1.67 & 1.93 & 1.73 & 0.18 & 0.105 \\
\hline 14 & 1.46 & 1.61 & 1.92 & 1.71 & 1.68 & 1.68 & 0.17 & 0.100 \\
\hline 15 & 2.71 & 3.07 & 3.13 & 3.10 & 3.12 & 3.03 & 0.18 & 0.059 \\
\hline 16 & 5.20 & 4.95 & 5.26 & 5.09 & 5.15 & 5.13 & 0.12 & 0.023 \\
\hline 17 & 3.74 & 4.01 & 3.92 & 3.99 & 3.99 & 3.93 & 0.11 & 0.028 \\
\hline 18 & 6.81 & 7.51 & 7.51 & 7.67 & 7.85 & 7.47 & 0.39 & 0.053 \\
\hline 19 & 1.01 & 1.02 & 0.93 & 0.87 & 0.99 & 0.96 & 0.06 & 0.065 \\
\hline 20 & 2.79 & 2.90 & 2.98 & 2.90 & 3.01 & 2.92 & 0.09 & 0.029 \\
\hline 21 & 3.99 & 4.10 & 4.09 & 3.97 & 3.96 & 4.02 & 0.07 & 0.017 \\
\hline 22 & 0.91 & 0.93 & 0.95 & 1.01 & 0.96 & 0.95 & 0.04 & 0.040 \\
\hline \multicolumn{9}{|c|}{ Ash. percent dry coal } \\
\hline 1 & 15.81 & 16.51 & 16.55 & 15.91 & 15.63 & 16.08 & 0.42 & 0.026 \\
\hline 2 & 16.16 & 16.31 & 16.02 & 15.89 & 16.02 & 16.08 & 0.16 & 0.010 \\
\hline 3 & 5.37 & 5.01 & 5.44 & 5.27 & 5.26 & 5.27 & 0.16 & 0.031 \\
\hline 4 & 6.23 & 6.41 & 6.33 & 6.19 & 6.20 & 6.27 & 0.09 & 0.015 \\
\hline 5 & 8.54 & 8.47 & 8.98 & 8.55 & 8.85 & 8.68 & 0.22 & C. 026 \\
\hline 6 & 5.84 & 6.13 & 5.85 & 5.83 & 5.94 & 5.92 & 0.13 & 0.021 \\
\hline 7 & 4.17 & 4.24 & 4.22 & 4.27 & 4.22 & 4.22 & 0.04 & 0.009 \\
\hline 8 & 13.83 & 13.48 & 12.70 & 13.30 & 13.25 & 13.31 & 0.41 & 0.031 \\
\hline 9 & 6.37 & 6.41 & 6.50 & 6.16 & 6.21 & 6.33 & 0.14 & 0.022 \\
\hline 10 & 12.56 & 12.24 & 12.76 & 13.17 & 12.62 & 12.67 & 0.34 & 0.027 \\
\hline 11 & 9.56 & 10.12 & 9.52 & 9.49 & 9.46 & 9.63 & 0.28 & 0.029 \\
\hline 12 & 10.25 & 10.36 & 9.57 & 10.13 & 10.29 & 10.12 & 0.32 & 0.031 \\
\hline 13 & 4.22 & 4.54 & 4.95 & 4.90 & 4.89 & 4.70 & 0.31 & 0.067 \\
\hline 14 & 10.52 & 11.27 & 10.51 & 10.62 & 10.88 & 10.76 & 0.32 & 0.030 \\
\hline 15 & 10.13 & 10.19 & 10.46 & 7.37 & 7.29 & 9.09 & 1.61 & 0.177 \\
\hline 16 & 13.89 & 15.19 & 16.83 & 14.46 & 18.32 & 15.74 & 1.82 & 0.115 \\
\hline 17 & 6.57 & 6.68 & 6.81 & 6.65 & 6.75 & 6.69 & 0.09 & 0.014 \\
\hline 18 & 12.25 & 11.31 & 11.04 & 11.97 & 12.70 & 11.85 & 0.68 & 0.057 \\
\hline 19 & 4.60 & 6.39 & 5.76 & 6.49 & 6.34 & 5.92 & 0.79 & 0.133 \\
\hline 20 & 5.47 & 5.10 & 4.93 & 5.23 & 5.13 & 5.17 & 0.20 & 0.038 \\
\hline 21 & 11.15 & 11.34 & 11.15 & 10.97 & 11.18 & 11.16 & 0.13 & 0.012 \\
\hline 22 & 23.27 & 23.20 & 23.17 & 22.91 & 22.93 & 23.10 & 0.16 & 0.007 \\
\hline
\end{tabular}


Table 10. (continued)

\begin{tabular}{||c|cccccc|ccc|}
\hline DECS & \multicolumn{2}{c}{ Equivalence Analyses: Total sulfur. percent dry coal } & \multicolumn{3}{|c|}{ Statistics } \\
\cline { 2 - 10 } sample & Principal & 1 & 2 & 3 & 4 & Mean & S.D. & C.V. \\
\hline 1 & 0.99 & 1.05 & 1.12 & 1.10 & 1.05 & 1.06 & 0.05 & 0.048 \\
2 & 4.51 & 4.32 & 4.34 & 4.47 & 4.41 & 4.41 & 0.08 & 0.018 \\
3 & 0.65 & 0.65 & 0.63 & 0.70 & 0.68 & 0.66 & 0.03 & 0.042 \\
4 & 0.46 & 0.57 & 0.71 & 0.56 & 0.54 & 0.57 & 0.09 & 0.159 \\
5 & 0.56 & 0.63 & 0.70 & 0.64 & 0.66 & 0.64 & 0.05 & 0.080 \\
6 & 0.40 & 0.44 & 0.44 & 0.44 & 0.43 & 0.43 & 0.02 & 0.040 \\
7 & 0.98 & 1.00 & 1.06 & 1.03 & 1.05 & 1.02 & 0.03 & 0.033 \\
8 & 0.73 & 0.81 & 0.81 & 0.82 & 0.82 & 0.80 & 0.04 & 0.048 \\
9 & 0.41 & 0.48 & 0.48 & 0.44 & 0.45 & 0.45 & 0.03 & 0.065 \\
10 & 1.16 & 0.91 & 1.10 & 1.11 & 1.16 & 1.09 & 0.10 & 0.095 \\
11 & 0.74 & 0.76 & 0.78 & 0.74 & 0.76 & 0.76 & 0.02 & 0.022 \\
12 & 1.12 & 1.12 & 1.19 & 1.18 & 1.17 & 1.16 & 0.03 & 0.029 \\
13 & 0.62 & 0.60 & 0.67 & 0.65 & 0.65 & 0.64 & 0.03 & 0.043 \\
14 & 1.80 & 1.70 & 1.76 & 1.79 & 1.74 & 1.76 & 0.04 & 0.023 \\
15 & 1.67 & 1.56 & 1.59 & 0.96 & 0.95 & 1.35 & 0.36 & 0.267 \\
16 & 0.47 & 0.48 & 0.49 & 0.46 & 0.46 & 0.47 & 0.01 & 0.028 \\
17 & 0.44 & 0.44 & 0.42 & 0.41 & 0.39 & 0.42 & 0.02 & 0.051 \\
18 & 4.21 & 3.95 & 3.87 & 4.00 & 3.98 & 4.00 & 0.13 & 0.032 \\
19 & 0.74 & 0.78 & 0.77 & 0.75 & 0.76 & 0.76 & 0.02 & 0.021 \\
20 & 0.97 & 1.00 & 0.98 & 0.99 & 0.98 & 0.98 & 0.01 & 0.012 \\
21 & 0.50 & 0.52 & 0.54 & 0.53 & 0.51 & 0.52 & 0.02 & 0.030 \\
22 & 1.71 & 1.71 & 1.66 & 1.58 & 1.70 & 1.67 & 0.06 & 0.033 \\
\hline
\end{tabular}

TASK IE. DISTRIBUTION OF COAL SAMPLES TO USERS

Distribution of samples took place under the conditions of the contract. Up to $1 \mathrm{~kg}$ ( 2 lbs) each of up to 15 samples per year per requestor were distributed to DOE contractors and grantees at no charge to the recipient. Requests for more than $4.5 \mathrm{~kg}$ (10 lbs) of a single PSOC coal were referred to the DOE Project Manager for approval. It was agreed with DOE that requests for more than $2.3 \mathrm{~kg}$ ( 5 lbs) of a single DECS coal would be referred to the Project Manager for approval.

A record of distribution of samples and data is shown in Table 11. For record-keeping under the contract, and this Table, a "sample" is any amount of one coal sample provided to one requestor at one time. Thus one $10 \mathrm{~kg}$ bucket of PSOC-1451 sent out is one "sample." but one $300 \mathrm{~g}$ bag each of DECS-1 and -2 sent ever! year for four years is 8 "samples." The exception is special sample DECS17. for which each $30 \mathrm{~g}$ bag is counted individually. The bottom section of Table 10 shows the record of DECS-17 distributed. Over 1300 supported samples (379 bags of DECS-17 and 932 other COE-supported samples) were distributed during the 
Tab?e 11. Samples and data distributed $4 / 09 / 88$ to $9 / 28 / 93$

DOE samples

\begin{tabular}{|c|c|c|c|c|c|c|c|c|c|c|}
\hline & (ex & 1. DECS & $-17)$ & Othe & $r$ PSOC $s$ & imples & & & pecia & data req. \\
\hline Quarter & & to & to & & to & to & Total & Print & & imited info \\
\hline ending & Total & DOE ctr & others & Total & DOE ctr & others & spls & outs & \# & on \# spls \\
\hline $7 / 88$ & 21 & 0 & 21 & $\frac{49}{49}$ & 0 & $\frac{\pi}{49}$ & 70 & $\overline{144}$ & 5 & 3043 \\
\hline $10 / 88$ & 91 & 46 & 45 & 32 & 2 & 30 & 123 & 95 & 7 & 513 \\
\hline $1 / 89$ & 29 & 11 & 18 & 63 & 44 & 19 & 92 & 162 & 10 & 1631 \\
\hline $4 / 89$ & 46 & 20 & 26 & 93 & 43 & 50 & 139 & 88 & 7 & 2584 \\
\hline $7 / 89$ & 56 & 41 & 15 & 16 & 6 & 10 & 72 & 114 & 8 & 1259 \\
\hline $10 / 89$ & 55 & 32 & 23 & 58 & 15 & 43 & 113 & 125 & 10 & 1372 \\
\hline $1 / 90$ & 95 & 73 & 22 & 15 & 0 & 15 & 110 & 51 & 6 & 385 \\
\hline $4 / 90$ & 36 & 7 & 29 & 15 & 0 & 15 & 51 & 251 & 17 & 2989 \\
\hline $7 / 90$ & 14 & 6 & 8 & 11 & 3 & 8 & 25 & 42 & 7 & 2083 \\
\hline $10 / 90$ & 70 & 29 & 41 & 19 & 4 & 15 & 89 & 57 & 13 & 2138 \\
\hline $1 / 91$ & 44 & 19 & 25 & 23 & 15 & 8 & 67 & 65 & 10 & 4479 \\
\hline $4 / 91$ & 67 & 29 & 38 & 37 & 4 & 33 & 104 & 237 & 6 & 569 \\
\hline 7/91 & 63 & 17 & 46 & 22 & 7 & 15 & 85 & 121 & 8 & 285 \\
\hline subtotal & & & & & & & 1140 & & & \\
\hline $10 / 91$ & 25 & 8 & 17 & 16 & 0 & 16 & 41 & 28 & 7 & 553 \\
\hline $1 / 92$ & 44 & 7 & 37 & 31 & 0 & 31 & 75 & 113 & 6 & 312 \\
\hline $4 / 92$ & 41 & 30 & 11 & 15 & 5 & 10 & 56 & 121 & 14 & 2044 \\
\hline $7 / 92$ & 38 & 32 & 6 & 12 & 3 & 9 & 50 & 92 & 16 & 2331 \\
\hline $10 / 92$ & 30 & 12 & 18 & 50 & 2 & 48 & 80 & 188 & 15 & 1089 \\
\hline $1 / 93$ & 11 & 9 & 2 & 20 & 3 & 17 & 31 & 41 & 7 & 298 \\
\hline $4 / 93$ & 25 & 22 & 3 & 14 & 0 & 14 & 39 & 57 & 13 & 1130 \\
\hline TOTALS & 901 & 450 & $\overline{451}$ & $\overline{611}$ & $\overline{156}$ & $\overline{455}$ & $1 \overline{512}$ & $\overline{2192}$ & $\overline{192}$ & 31087 \\
\hline $5 \mathrm{yr}$ ctrct & & & & & & & & & & \\
\hline $5 / 30 / 93$ & 12 & 11 & 1 & 6 & 4 & 2 & 18 & 60 & 5 & 2436 \\
\hline $9 / 08 / 93$ & 19 & 10 & 9 & 17 & 1 & 16 & 36 & 61 & 7 & 563 \\
\hline TOTALS & $\overline{932}$ & $\overline{471}$ & $\overline{461}$ & $\overline{634}$ & $\overline{161}$ & $\overline{473}$ & $1 \overline{566}$ & $\overline{2313}$ & $\overline{204}$ & $\overline{34086}$ \\
\hline
\end{tabular}

overall

\begin{tabular}{|c|c|c|c|c|}
\hline & \multicolumn{2}{|c|}{ DECS-17 } & \multirow{3}{*}{$\begin{array}{l}\text { PSOC } \\
\text { spls } \\
1140\end{array}$} & \multirow{2}{*}{$\begin{array}{l}\text { PSOC Spls } \\
+ \text { DECS-17 } \\
\text { bags } \\
\end{array}$} \\
\hline $\begin{array}{l}\text { Quarter } \\
\text { ending }\end{array}$ & requests & $\begin{array}{c}30 \mathrm{~g} \\
\text { bags }\end{array}$ & & \\
\hline previous & & & & 1140 \\
\hline $10 / 91$ & 9 & 90 & 41 & 131 \\
\hline $1 / 92$ & 6 & 65 & 75 & 140 \\
\hline $4 / 92$ & 5 & $48^{\star}$ & 56 & 104 \\
\hline $7 / 92$ & 4 & 56 & 50 & 106 \\
\hline $10 / 92$ & 6 & 80 & 80 & 160 \\
\hline $1 / 93$ & 0 & 0 & 31 & 31 \\
\hline $4 / 93$ & 0 & 0 & 39 & 39 \\
\hline TOTALS & 30 & $\overline{339}$ & $\overline{1512}$ & $\overline{185 i}$ \\
\hline $6 / 30 / 93$ & 1 & 16 & 18 & 34 \\
\hline $9 / 08 / 93$ & 2 & 24 & 36 & 60 \\
\hline TOTALS & 33 & $\overline{379}$ & $\overline{1566}$ & $\overline{1945}$ \\
\hline
\end{tabular}

*incl. 8 300-gram bags 
contract and following interim period. Over 600 other samples were also distributed for a total of over 1900 samples.

Requests for samples were received from a wide variety of agencies, classified as university, corporate, or government and as domestic or foreign. Appendix $C$ lists the agencies receiving samples and/or data during the contract and the following interim period. Numbers in parentheses enumerate separate requests from the same agency. In total. 724 requests were received; most were for more than one sample or data printout. Table 12 summarizes the numbers of requests received from the various classes of agencies.

Table 12. Numbers of requests for samples and data by agency classification

\begin{tabular}{|l|c|c|c|}
\hline \hline Requesting Agency & Domestic & Foreign & Totals \\
\hline University & 433 & 25 & 458 \\
Corporate & 177 & 2 & 179 \\
Government & 76 & 11 & 87 \\
\hline Totals & 686 & 38 & 724 \\
\hline
\end{tabular}

A brochure describing the DOE Coal Sample Bank and Data Base. 1isting supported samples and conditions for distribution of samples to DOE contractors and grantees, was printed in Apri1, 1988 and distributed. After two years of zollection of DECS samples, a new version including information on 20 DECS amples was printed in July, 1992 and widely distributed. This version included rot only the list of 54 supported samples but also their ash. sulfur, calorific value, carbon, and hydrogen analyses.

A booklet describing the DOE Coal Sample Bank and Data Base as well as the larger Penn State Coal Sample Bank and Data Base was also written. The first edition, printed in Apri1. 1988, consisted of 83 pages and included a complete list of over 1400 samples by state. Three hundred copies were printed and distributed. When they had run out, a second edition was composed, adding a sample number -. state cross reference, and a discussion and list of DECS samples. The second edition condensed the booklet into 61 pages to reduce printing and postage costs. Four hundred copies were printed in October, 1990 , and distributed over the next two years. Another 50 unbound copies of that booklet, with a separate up-to-date list of DECS samples, have been distributed in response to more recent requests. 


\section{TASK II: COAL DATA BASE}

Data pertaining to the 22 DECS samples were entered into the Coal Data Base maintained on the Penn State research mainframe computer. The Data Base software accepts raw data as reported by the analyst and also recalculates the data to other bases of expression which are of value to researchers. Both the raw data and calculated data are checked for accuracy and consistency.

The Data Base may be interactively queried to identify samples matching requestor's criteria. When a list of samples has been produced, a table of data may be formatted and printed, or the list of samples may be used to specify that a one-page or five-page ciata printout be produced for each sample. Subsets of the Data Base or formatted tables may also be transmitted in electronic form. Appendix $B$ includes a five-page printout for each of the 22 DECS samples collected and analyzed under the contract.

During the contract, the Data Base has been improved, new data fields have been added, and ancillary calculation programs have been written. The writing of an entirely new program for creation of standard data printouts is well under way. This was originally instigated in order to make the format of data printouts more easily modified as new types of analyses are incorporated in the Data Base, to create a vertical (portrait) format printout making use of modern printers. to improve readability, and to allow for new data fields (including those expressed in metric system units). It has recently become a necessity because the operating system under which the current printout program operates will be unavailable after April, 1994. 


\section{REFERENCES}

ASTM, 1993, Annual Book of ASTM Standards, Gaseous Fuels; Coal and Coke, V. 05.05. ASTM, Philadelphia, 526 p.

Glick, David C., Gareth D. Mitchell and Alan Davis, 1991. Monitoring the Oxidation of Coals in Storage, Am. Chem. Soc. Div. Fuel Chem. Preprints, v. 36. rio. 3, pp. 861-868.

Harvey, Richard D. and Carl W. Kruse, 1988. The Illinois Basin Coal Sample Program: Status and Sample Characterization, J. Coal Qual., v. 7, no. 4, pp 109-113.

Lowenhaupt, D.E., and R.J. Gray, 1980. The Alkali-Extraction Test as a Reliable Method of Detecting Oxidized Metallurgical Coal. Int. J. Coal Geol., v. 1. pp 63-73.

Sharp. P.A., 1986, Storage of 8 Mesh Sub-bituminous Coa1, J. Coal Qual., v.5 no. 4. pp 131-132.

Vorres, Karl. 1990. The Argonne Premium Coal Sample Program, Energy \& Fuels, v.4, pp. 420-426. 


\author{
APPENDIX A \\ Monitoring of DOE Sample Bank Coals \\ to Evaluate Sample Equivalence and
}

Sample Integrity

Five annual programs completed August, 1993 


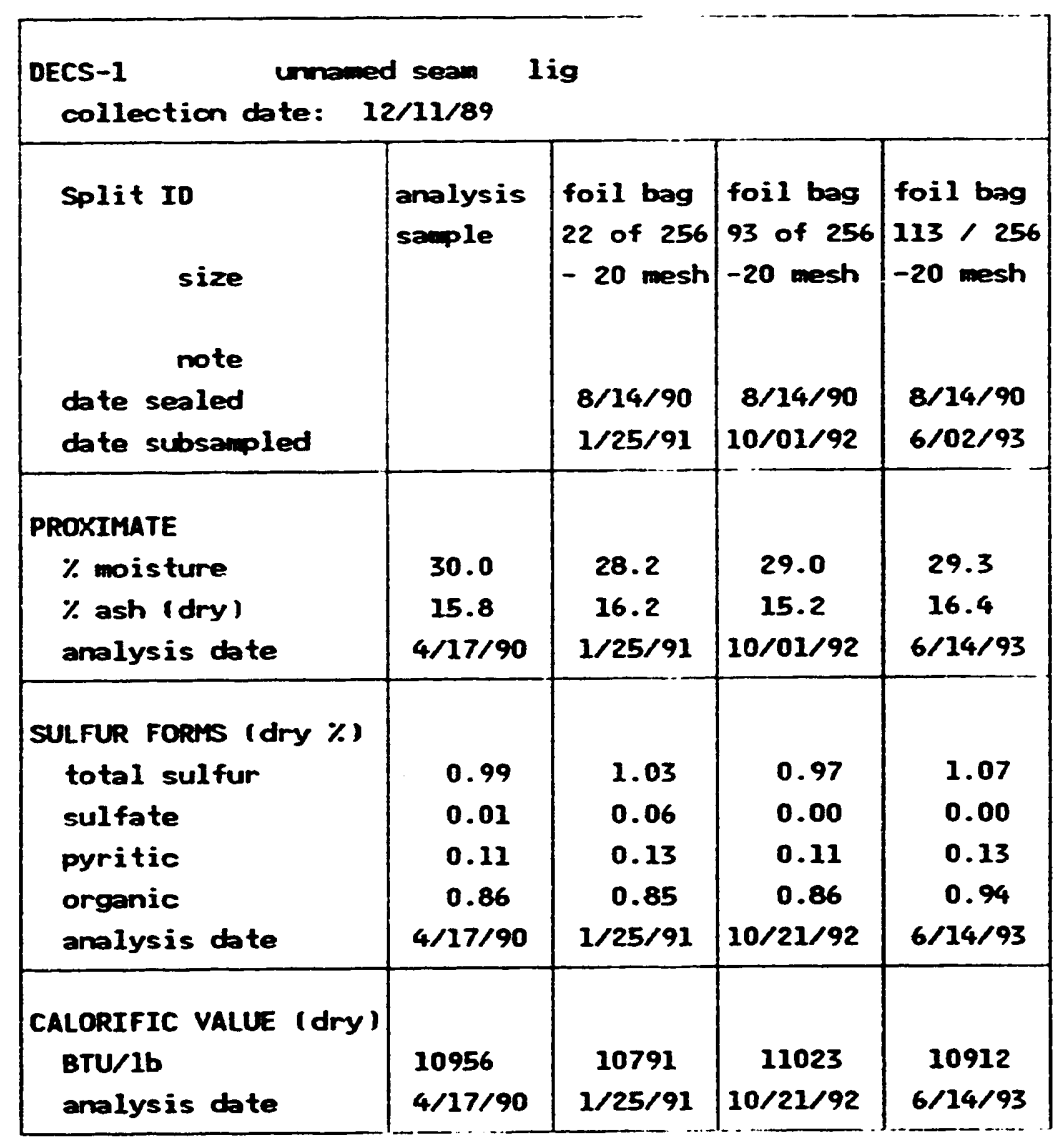




\begin{tabular}{|c|c|c|c|c|}
\hline $\begin{array}{l}\text { DECS-2 Illino } \\
\text { collection date: }\end{array}$ & $\begin{array}{l}\text { is } \# 6 \\
2 / 13 / 89\end{array}$ & Cb, Ro mas & $x=0.524$ & \\
\hline $\begin{array}{l}\text { Split ID } \\
\text { size } \\
\text { note } \\
\text { date sealed } \\
\text { date subsampled }\end{array}$ & $\begin{array}{l}\text { analys is } \\
\text { sample }\end{array}$ & $\begin{array}{l}\text { foil bag } \\
21 \text { of } 256 \\
-20 \text { mesh } \\
8 / 06 / 90 \\
1 / 25 / 91\end{array}$ & \begin{tabular}{|c|} 
foil bag \\
$100 / 256$ \\
-20 mesh \\
\\
$8 / 06 / 90$ \\
$10 / 20 / 92$
\end{tabular} & $\begin{array}{l}\text { foil bag } \\
109 / 256 \\
-20 \text { mesh } \\
\\
8 / 06 / 90 \\
6 / 02 / 93\end{array}$ \\
\hline $\begin{array}{l}\text { PROXIMATE } \\
\% \text { moisture } \\
\% \text { ash (dry) } \\
\text { analysis date }\end{array}$ & $\begin{array}{c}10.4 \\
16.2 \\
2 / 28 / 90\end{array}$ & $\begin{array}{c}10.3 \\
16.1 \\
1 / 25 / 91\end{array}$ & $\begin{array}{c}10.1 \\
16.2 \\
11 / 27 / 92\end{array}$ & $\begin{array}{c}9.8 \\
15.9 \\
6 / 14 / 93\end{array}$ \\
\hline $\begin{array}{l}\text { GIESELER FLUIDITY } \\
\text { max. ddpm } \\
\text { fluid range } \\
\text { analysis date }\end{array}$ & $\begin{array}{c}8 \\
47 \\
2 / 08 / 90\end{array}$ & $\begin{array}{c}9 \\
58 \\
2 / 11 / 91\end{array}$ & $\begin{array}{l}10 \\
61 \\
2 / 02 / 93\end{array}$ & $\begin{array}{l}7 \\
55 \\
7 / 19 / 93\end{array}$ \\
\hline $\begin{array}{l}\text { SULFUR FORMS (dry \%) } \\
\text { total sulfur } \\
\text { sulfate } \\
\text { pyritic } \\
\text { organic } \\
\text { analysis date }\end{array}$ & $\begin{array}{c}4.51 \\
0.01 \\
2.27 \\
2.24 \\
2 / 28 / 90\end{array}$ & $\begin{array}{c}4.33 \\
0.06 \\
2.27 \\
2.00 \\
1 / 25 / 91\end{array}$ & $\begin{array}{c}4.54 \\
0.01 \\
2.31 \\
2.22 \\
11 / 27 / 92\end{array}$ & $\begin{array}{c}4.50 \\
0.03 \\
2.06 \\
2.41 \\
6 / 14 / 93\end{array}$ \\
\hline $\begin{array}{l}\text { CALORIFIC VALUE }(d r y) \\
\text { BTU/lb } \\
\text { analysis date }\end{array}$ & $\begin{array}{c}11880 \\
2 / 28 / 90\end{array}$ & $\begin{array}{c}11740 \\
1 / 25 / 91\end{array}$ & $\begin{array}{c}11814 \\
11 / 27 / 92\end{array}$ & $\begin{array}{c}11787 \\
6 / 14 / 93\end{array}$ \\
\hline
\end{tabular}




\begin{tabular}{|c|c|c|c|c|}
\hline $\begin{array}{l}\text { DECS-3 Coal B } \\
\text { collection date: }\end{array}$ & $\begin{array}{l}\operatorname{asin} M \\
5 / 31 / 90\end{array}$ & mub, Ro max & $x=1.28$ & \\
\hline $\begin{array}{l}\text { Split ID } \\
\text { size } \\
\text { note } \\
\text { date sealed } \\
\text { date subsampled }\end{array}$ & $\begin{array}{l}\text { analysis } \\
\text { sample }\end{array}$ & \begin{tabular}{|c|} 
foil bag \\
5 of 256 \\
-20 mesh \\
$8 / 24 / 91$ \\
$1 / 25 / 91$
\end{tabular} & $\begin{array}{c}\text { foil bag } \\
13 \text { of } 256 \\
-20 \text { mesh } \\
\\
8 / 24 / 91 \\
10 / 01 / 92\end{array}$ & $\begin{array}{l}\text { foil bag } \\
31 \text { of } 256 \\
-20 \text { mesh } \\
8 / 24 / 91 \\
6 / 03 / 93\end{array}$ \\
\hline $\begin{array}{l}\text { PROXIMATE } \\
\% \text { moisture } \\
\% \text { ash (dry) } \\
\text { analysis date }\end{array}$ & $\begin{array}{c}1.10 \\
5.37 \\
8 / 23 / 90\end{array}$ & $\begin{array}{c}0.87 \\
5.24 \\
1 / 25 / 91\end{array}$ & $\begin{array}{c}0.82 \\
5.26 \\
11 / 27 / 92\end{array}$ & $\begin{array}{l}0.72 \\
5.52 \\
6 / 14 / 93\end{array}$ \\
\hline $\begin{array}{l}\text { GIESELER FLUIDITY } \\
\text { max. ddpm } \\
\text { fluid range } \\
\text { analysis tate }\end{array}$ & $\begin{array}{r}9774 \\
100 \\
7 / 11 / 90\end{array}$ & $\begin{array}{c}17(a) \\
79 \\
11 / 06 / 5 i\end{array}$ & $\begin{array}{c}383(b) \\
94 \\
11 / 27 / 92\end{array}$ & $\begin{array}{l}234 \\
94 \\
7 / 20 / 93\end{array}$ \\
\hline $\begin{array}{l}\text { SULFUR FORMS (dry \%) } \\
\text { total sulfur } \\
\text { sulfate } \\
\text { pyritic } \\
\text { organic } \\
\text { analysis date }\end{array}$ & $\begin{array}{c}0.65 \\
0.01 \\
0.10 \\
0.54 \\
8 / 23 / 90\end{array}$ & $\begin{array}{c}0.65 \\
0.05 \\
0.08 \\
0.52 \\
1 / 25 / 91\end{array}$ & $\begin{array}{c}0.67 \\
0.01 \\
0.05 \\
0.61 \\
11 / 27 / 92\end{array}$ & $\begin{array}{c}0.64 \\
0.05 \\
0.05 \\
0.55 \\
7 / 20 / 93\end{array}$ \\
\hline $\begin{array}{l}\text { CALORIYIC VALUE (dry) } \\
\text { BTIJ/lb } \\
\text { analysis date }\end{array}$ & $\begin{array}{c}14889 \\
8 / 23 / 90\end{array}$ & $\begin{array}{c}14907 \\
1 / 25 / 91\end{array}$ & $\begin{array}{c}14797 \\
11 / 27 / 92\end{array}$ & $\begin{array}{c}14828 \\
6 / 14 / 92\end{array}$ \\
\hline $\begin{array}{l}\text { ALKALI EXTRACTION* } \\
\text { \% transmission } \\
\text { analysis date }\end{array}$ & $\begin{array}{c}96.3 \\
7 / 11 / 90\end{array}$ & $\begin{array}{c}98.5 \\
10 / 02 / 91\end{array}$ & $\begin{array}{c}99.3 \\
11 / 27 / 92\end{array}$ & $\begin{array}{c}98.1 \\
7 / 13 / 93\end{array}$ \\
\hline
\end{tabular}

* calculated to 17 mm light path

(a) erratic behavior curing testing ( 3 runs performed)

(b) erratic behavior during testing (6 runs performed) 


\begin{tabular}{|c|c|c|c|}
\hline $\begin{array}{c}\text { DECS-4 Blue } \\
\text { collection date: }\end{array}$ & $6 / 04 / 90$ & Cb, Ro & $x=0.51$ \\
\hline $\begin{array}{l}\text { Split ID } \\
\text { size } \\
\text { note } \\
\text { date sealed } \\
\text { date subsampled }\end{array}$ & $\begin{array}{l}\text { analys is } \\
\text { sample }\end{array}$ & $\begin{array}{l}\text { foil bag } \\
6 \text { of } 256 \\
-20 \text { mesh } \\
\\
9 / 27 / 90 \\
11 / 27 / 92\end{array}$ & $\begin{array}{l}\text { foil bag } \\
16 \text { of } 256 \\
-20 \text { mesh } \\
9 / 27 / 90 \\
6 / 03 / 93\end{array}$ \\
\hline $\begin{array}{l}\text { PROXIMATE } \\
\% \text { moisture } \\
\% \text { ash (dry) } \\
\text { analysis date }\end{array}$ & $\begin{array}{c}15.1 \\
6.2 \\
10 / 29 / 90\end{array}$ & $\begin{array}{c}15.0 \\
6.2 \\
11 / 27 / 92\end{array}$ & $\begin{array}{c}14.8 \\
6.2 \\
6 / 14 / 93\end{array}$ \\
\hline $\begin{array}{l}\text { GIESELER FLUIDITY } \\
\text { max. ddpm } \\
\text { fluid range } \\
\text { amalysis date }\end{array}$ & $\begin{array}{c}0 \\
0 \\
2 / 12 / 91\end{array}$ & $\begin{array}{c}0 \\
0 \\
2 / 02 / 93\end{array}$ & $\begin{array}{c}0 \\
0 \\
7 / 21 / 93\end{array}$ \\
\hline $\begin{array}{l}\text { SULFUR FORAs (dry \%) } \\
\text { total sulfur } \\
\text { sulfate } \\
\text { pyritis } \\
\text { organic } \\
\text { analysis date }\end{array}$ & $\begin{array}{c}0.46 \\
0.02 \\
0.04 \\
0.41 \\
10 / 29 / 90\end{array}$ & $\begin{array}{c}0.57 \\
0.00 \\
0.04 \\
0.52 \\
11 / 27 / 92\end{array}$ & $\begin{array}{c}0.54 \\
0.00 \\
0.04 \\
0.50 \\
6 / 14 / 93\end{array}$ \\
\hline $\begin{array}{l}\text { CALORIFIC VALUE (dry) } \\
\text { BTU/lb } \\
\text { analysis date }\end{array}$ & $\begin{array}{c}13000 \\
10 / 29 / 90\end{array}$ & $\begin{array}{c}12919 \\
11 / 27 / 92\end{array}$ & $\begin{array}{c}12964 \\
6 / 14 / 93\end{array}$ \\
\hline
\end{tabular}




\begin{tabular}{|c|c|c|c|}
\hline \multirow{2}{*}{\multicolumn{2}{|c|}{$\begin{array}{l}\text { DECS-5 Hiawatha } \\
\text { collection date: } 6 / 06 / 90\end{array}$}} & \multirow{2}{*}{\multicolumn{2}{|c|}{ mucb, Ro $\max =0.59$}} \\
\hline & & & \\
\hline $\begin{array}{l}\text { Split ID } \\
\text { size } \\
\text { note } \\
\text { dote sealed } \\
\text { date subsampled }\end{array}$ & $\begin{array}{l}\text { analys is } \\
\text { sample }\end{array}$ & $\begin{array}{l}\text { foil bag } \\
11 \text { of } 256 \\
-20 \text { mesh } \\
9 / 07 / 90 \\
10 / 02 / 92\end{array}$ & $\begin{array}{l}\text { foil bag } \\
32 \text { of } 256 \\
-20 \text { mesh } \\
9 / 07 / 90 \\
6 / 03 / 93\end{array}$ \\
\hline $\begin{array}{l}\text { PROXIMATE } \\
\% \text { moisture } \\
\% \text { ash (dry) } \\
\text { analysis date }\end{array}$ & $\begin{array}{c}7.5 \\
8.5 \\
10 / 29 / 90\end{array}$ & $\begin{array}{c}7.3 \\
8.7 \\
10 / 02 / 92\end{array}$ & $\begin{array}{c}7.1 \\
8.7 \\
6 / 14 / 93\end{array}$ \\
\hline $\begin{array}{l}\text { SULFUR FORMS (dry \%) } \\
\text { total sulfur } \\
\text { sulfate } \\
\text { pyritic } \\
\text { organic } \\
\text { analysis date }\end{array}$ & $\begin{array}{c}0.56 \\
0.01 \\
0.27 \\
0.28 \\
10 / 29 / 90\end{array}$ & $\begin{array}{c}0.63 \\
0.01 \\
0.26 \\
0.35 \\
10 / 02 / 9 ?\end{array}$ & $\begin{array}{c}0.67 \\
0.01 \\
0.24 \\
0.42 \\
6 / 14 / 93\end{array}$ \\
\hline $\begin{array}{l}\text { CALORIFIC VALUE (dry) } \\
\text { BTU/lb } \\
\text { analysis date }\end{array}$ & $\begin{array}{c}12760 \\
10 / 29 / 90\end{array}$ & $\begin{array}{c}12681 \\
10 / 02 / 92\end{array}$ & $\begin{array}{c}12723 \\
6 / 14 / 93\end{array}$ \\
\hline
\end{tabular}




\begin{tabular}{|c|c|c|c|c|}
\hline $\begin{array}{l}\text { DECS-6 BIind } \\
\text { collection date: }\end{array}$ & $\begin{array}{l}\text { Camyon } \\
6 / 07 / 90\end{array}$ & $\checkmark A b, R o$ mas & $x=0.66$ & \\
\hline $\begin{array}{l}\text { Split ID } \\
\text { size } \\
\text { note } \\
\text { date sealed } \\
\text { date subsampled }\end{array}$ & $\begin{array}{l}\text { analys is } \\
\text { sample }\end{array}$ & $\begin{array}{l}\text { foil bag } \\
12 \text { of } 256 \\
-20 \text { esh } \\
7 / 19 / 90 \\
1 / 25 / 91\end{array}$ & $\begin{array}{l}\text { foil bag } \\
107 / 256 \\
-20 \text { mesh } \\
\\
7 / 19 / 90 \\
10 / 01 / 92\end{array}$ & $\begin{array}{l}\text { foil bag } \\
125 / 256 \\
-20 \text { mesh } \\
\\
7 / 19 / 90 \\
6 / 03 / 93\end{array}$ \\
\hline $\begin{array}{l}\text { PROXIMATE } \\
\% \text { moisture } \\
\% \text { ash (dry) } \\
\text { analysis date }\end{array}$ & $\begin{array}{c}4.73 \\
5.84 \\
8 / 23 / 90\end{array}$ & $\begin{array}{c}4.35 \\
5.96 \\
1 / 25 / 91\end{array}$ & $\begin{array}{c}4.06 \\
5.92 \\
10 / 21 / 92\end{array}$ & $\begin{array}{c}4.45 \\
6.00 \\
6 / 23 / 93\end{array}$ \\
\hline $\begin{array}{l}\text { GIESELER FLUIDITY } \\
\text { max. ddpm } \\
\text { fluid range } \\
\text { analysis date }\end{array}$ & $\begin{array}{c}3 \\
38 \\
2 / 14 / 91\end{array}$ & $\begin{array}{c}0 \\
0 \\
11 / 04 / 91\end{array}$ & $\begin{array}{l}\text { discon- } \\
\text { timued }\end{array}$ & \\
\hline $\begin{array}{l}\text { SUlfuR forats (dry \%) } \\
\text { total sulfur } \\
\text { sulfate } \\
\text { pyritic } \\
\text { organic } \\
\text { analysis date }\end{array}$ & $\begin{array}{c}0.40 \\
0.01 \\
0.02 \\
0.37 \\
8 / 23 / 90\end{array}$ & $\begin{array}{c}0.43 \\
0.03 \\
0.02 \\
0.38 \\
1 / 25 / 91\end{array}$ & $\begin{array}{c}0.35 \\
0.00 \\
0.02 \\
0.33 \\
10 / 21 / 92\end{array}$ & $\begin{array}{c}0.43 \\
0.01 \\
0.02 \\
0.40 \\
6 / 23 / 93\end{array}$ \\
\hline $\begin{array}{l}\text { CALORIFIC VALUE (dry) } \\
\text { BTU/Ib } \\
\text { analysis date }\end{array}$ & $\begin{array}{c}13923 \\
8 / 23 / 90\end{array}$ & $\begin{array}{c}13828 \\
1 / 25 / 91\end{array}$ & $\begin{array}{c}13814 \\
10 / 21 / 92\end{array}$ & $\begin{array}{c}13874 \\
6 / 23 / 93\end{array}$ \\
\hline $\begin{array}{l}\text { ALKALI EXTRACTIONE } \\
\% \text { transmission } \\
\text { analysis date }\end{array}$ & & $\begin{array}{c}83.3 \\
11 / 04 / 91\end{array}$ & $\begin{array}{c}95.3 \\
10 / 01 / 92\end{array}$ & $\begin{array}{c}90.7 \\
7 / 13 / 93\end{array}$ \\
\hline
\end{tabular}

* calculated to $17 \mathrm{~mm}$ light path 


\begin{tabular}{|c|c|c|c|}
\hline \multicolumn{4}{|c|}{$\begin{array}{l}\text { DECS-7 Adaville No. } 1 \text { macb, Ro max }=0.45 \\
\text { collection date: } 6 / 08 / 90\end{array}$} \\
\hline $\begin{array}{l}\text { Split IO } \\
\text { size } \\
\text { note } \\
\text { date sealed } \\
\text { date subsamled }\end{array}$ & $\begin{array}{l}\text { analysis } \\
\text { sample }\end{array}$ & $\begin{array}{l}\text { foil bag } \\
21 \text { of } 256 \\
-20 \text { mesh } \\
12 / 10 / 90 \\
10 / 15 / 92\end{array}$ & $\begin{array}{l}\text { foil bag } \\
26 \text { of } 256 \\
-20 \text { mesh } \\
\\
12 / 10 / 90 \\
6 / 03 / 93\end{array}$ \\
\hline $\begin{array}{l}\text { PROXIMATE } \\
\% \text { mois ture } \\
\% \text { ash (dry) } \\
\text { aralysis date }\end{array}$ & $\begin{array}{c}17.3 \\
4.2 \\
1 / 16 / 91\end{array}$ & $\begin{array}{c}16.8 \\
4.3 \\
10 / 15 / 92\end{array}$ & $\begin{array}{c}17.0 \\
4.3 \\
6 / 15 / 93\end{array}$ \\
\hline $\begin{array}{l}\text { SULFUR FOPis (dry \%) } \\
\text { total sulfur } \\
\text { sulfate } \\
\text { Pyritic } \\
\text { organic } \\
\text { analysis date }\end{array}$ & $\begin{array}{r}0.98 \\
0.01 \\
0.10 \\
0.87 \\
1 / 16 / 91\end{array}$ & $\begin{array}{c}0.99 \\
0.01 \\
0.08 \\
0.90 \\
10 / 15 / 92\end{array}$ & $\begin{array}{c}1.04 \\
0.01 \\
0.08 \\
0.94 \\
6 / 15 / 93\end{array}$ \\
\hline $\begin{array}{l}\text { CALORIFIC VALUE (dry) } \\
\text { BTU/lb } \\
\text { analysis date }\end{array}$ & $\begin{array}{c}13068 \\
1 / 16 / 91\end{array}$ & $\begin{array}{c}12898 \\
10 / 15 / 92\end{array}$ & $\begin{array}{l}12947 \\
6 / 15 / 93\end{array}$ \\
\hline
\end{tabular}




\begin{tabular}{|c|c|c|c|}
\hline $\begin{array}{l}\text { DECS-8 Smith-Ro } \\
\text { collection date: }\end{array}$ & $\begin{array}{l}\text { land } \\
6 / 11 / 90\end{array}$ & subc, Ro a) & $x=0.37$ \\
\hline $\begin{array}{l}\text { Split ID } \\
\text { size } \\
\text { note } \\
\text { date sealed } \\
\text { date subsampled }\end{array}$ & $\begin{array}{l}\text { analys is } \\
\text { sample }\end{array}$ & $\begin{array}{l}\text { foil bag } \\
27 \text { of } 256 \\
-20 \text { mesh } \\
10 / 22 / 90 \\
10 / 21 / 92\end{array}$ & $\begin{array}{l}\text { foil bag } \\
127 / 256 \\
-20 \text { mesh } \\
\\
10 / 22 / 90 \\
6 / 03 / 93\end{array}$ \\
\hline $\begin{array}{l}\text { PROXTMATE } \\
\% \text { moisture } \\
\% \text { ash (dry) } \\
\text { analysis date }\end{array}$ & $\begin{array}{c}28.4 \\
13.8 \\
10 / 30 / 90\end{array}$ & $\begin{array}{c}28.3 \\
12.8 \\
10 / 21 / 92\end{array}$ & $\begin{array}{l}28.6 \\
13.1 \\
6 / 15 / 93\end{array}$ \\
\hline $\begin{array}{l}\text { SULfuR Fords (dry \%) } \\
\text { total sulfur } \\
\text { sulfate } \\
\text { pyritic } \\
\text { organic } \\
\text { analysis date }\end{array}$ & $\begin{array}{c}0.73 \\
0.02 \\
0.28 \\
0.43 \\
10 / 30 / 90\end{array}$ & $\begin{array}{c}0.77 \\
0.01 \\
0.20 \\
0.56 \\
10 / 21 / 92\end{array}$ & $\begin{array}{c}0.81 \\
0 . \Gamma 1 \\
0.22 \\
0.58 \\
6 / 15 / 93\end{array}$ \\
\hline $\begin{array}{l}\text { CALORIFIC VALUE (dry) } \\
\text { BTU/lb } \\
\text { analysis date }\end{array}$ & $\begin{array}{c}11168 \\
10 / 30 / 90\end{array}$ & $\begin{array}{c}11275 \\
10 / 21 / 92\end{array}$ & $\begin{array}{c}11287 \\
6 / 15 / 93\end{array}$ \\
\hline
\end{tabular}




\begin{tabular}{|c|c|c|c|}
\hline $\begin{array}{l}\text { DECS-9 Dietz } \\
\text { collection date: }\end{array}$ & & UbB, Ro ma & $x=0.38$ \\
\hline $\begin{array}{l}\text { Split ID } \\
\text { size } \\
\text { note } \\
\text { date sealed } \\
\text { date subsampled }\end{array}$ & $\begin{array}{l}\text { analys is } \\
\text { sample }\end{array}$ & $\begin{array}{l}\text { foil bag } \\
82 \text { of } 256 \\
-20 \text { mesh } \\
\\
1 / 10 / 91 \\
10 / 21 / 92\end{array}$ & $\begin{array}{l}\text { foil bag } \\
91 \text { of } 256 \\
-20 \text { mesh } \\
1 / 10 / 91 \\
6 / 03 / 93\end{array}$ \\
\hline $\begin{array}{l}\text { PROXIMATE } \\
\% \text { moisture } \\
\% \text { ash (dry) } \\
\text { analysis date }\end{array}$ & $\begin{array}{c}24.7 \\
6.4 \\
1 / 18 / 91\end{array}$ & $\begin{array}{c}23.8 \\
6.7 \\
10 / 21 / 92\end{array}$ & $\begin{array}{c}24.8 \\
6.5 \\
6 / 15 / 93\end{array}$ \\
\hline $\begin{array}{l}\text { SULFUR FORMS (dry \%) } \\
\text { total sulfur } \\
\text { sulfate } \\
\text { pyritic } \\
\text { organic } \\
\text { analysis date }\end{array}$ & $\begin{array}{c}0.41 \\
0.02 \\
0.08 \\
0.31 \\
1 / 18 / 91\end{array}$ & $\begin{array}{c}0.42 \\
0.03 \\
0.06 \\
0.33 \\
10 / 21 / 92\end{array}$ & $\begin{array}{c}0.44 \\
0.03 \\
0.05 \\
0.35 \\
6 / 15 / 93\end{array}$ \\
\hline $\begin{array}{l}\text { CALORIFIC VALUE (dry) } \\
\text { BTU/lb } \\
\text { analysis date }\end{array}$ & $\begin{array}{c}12330 \\
1 / 18 / 91\end{array}$ & $\begin{array}{c}12230 \\
10 / 21 / 92\end{array}$ & $\begin{array}{c}12401 \\
6 / 15 / 93\end{array}$ \\
\hline
\end{tabular}




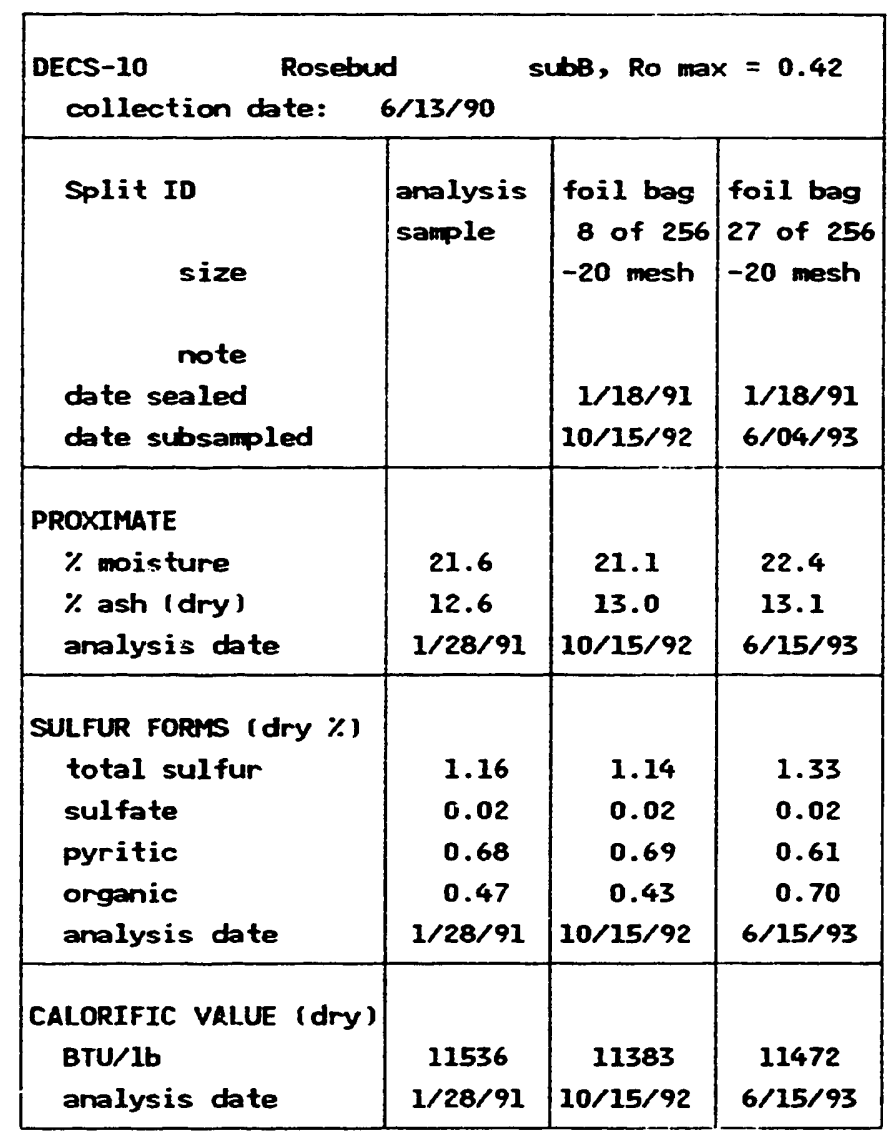




\begin{tabular}{|c|c|c|c|}
\hline $\begin{array}{l}\text { DECS-11 Beulah } \\
\text { collection date: }\end{array}$ & $6 / 15 / 90$ & ligA, Ro ma: & $x=0.35$ \\
\hline $\begin{array}{l}\text { Split ID } \\
\text { size } \\
\text { note } \\
\text { date sealed } \\
\text { date subsampled }\end{array}$ & $\begin{array}{l}\text { analysis } \\
\text { sample }\end{array}$ & $\begin{array}{c}\text { foil bag } \\
27 \text { of } 256 \\
-20 \text { mesh } \\
\\
1 / 25 / 91 \\
10 / 21 / 92\end{array}$ & $\begin{array}{l}\text { foil bag } \\
119 / 256 \\
-20 \text { mesh } \\
\\
1 / 25 / 91 \\
6 / 04 / 93\end{array}$ \\
\hline $\begin{array}{l}\text { PROXIMATE } \\
\% \text { moisture } \\
\% \text { ash (dry) } \\
\text { analysis date }\end{array}$ & $\begin{array}{c}33.4 \\
9.6 \\
2 / 06 / 91\end{array}$ & $\begin{array}{c}32.5 \\
10.4 \\
10 / 21 / 92\end{array}$ & $\begin{array}{c}32.9 \\
9.5 \\
6 / 15 / 93\end{array}$ \\
\hline $\begin{array}{l}\text { SULFUR FORAts (dry \%) } \\
\text { total sulfur } \\
\text { sulfate } \\
\text { pyritic } \\
\text { organic } \\
\text { analysis date }\end{array}$ & $\begin{array}{c}0.74 \\
0.03 \\
0.31 \\
0.39 \\
2 / 06 / 91\end{array}$ & $\begin{array}{c}0.74 \\
0.04 \\
0.24 \\
0.46 \\
10 / 21 / 92\end{array}$ & $\begin{array}{c}0.74 \\
0.03 \\
0.22 \\
0.49 \\
6 / 15 / 93\end{array}$ \\
\hline $\begin{array}{l}\text { CALORIFIC VALUE (dry) } \\
\text { BTU/1b } \\
\text { analysis date }\end{array}$ & $\begin{array}{c}11062 \\
2 / 06 / 91\end{array}$ & $\begin{array}{c}10863 \\
10 / 21 / 92\end{array}$ & $\begin{array}{c}11012 \\
6 / 15 / 93\end{array}$ \\
\hline
\end{tabular}




\begin{tabular}{|c|c|c|c|c|}
\hline \multirow{2}{*}{\multicolumn{2}{|c|}{$\begin{array}{l}\text { DECS-12 Pittsburgh } \\
\text { collection date: } \quad 7 / 25 / 90\end{array}$}} & \multirow{2}{*}{\multicolumn{2}{|c|}{ hvAb, Ro $\max =0.871$}} & \\
\hline & & & & \\
\hline $\begin{array}{l}\text { Split ID } \\
\text { size } \\
\text { note } \\
\text { date sealed } \\
\text { date subsampled }\end{array}$ & $\begin{array}{l}\text { analys is } \\
\text { sample }\end{array}$ & $\begin{array}{l}\text { foil bag } \\
86 \text { of } 256 \\
-20 \text { mesh } \\
7 / 30 / 90 \\
7 / 31 / 91\end{array}$ & $\begin{array}{l}\text { foil bag } \\
117 / 256 \\
-20 \text { mesh } \\
\\
7 / 30 / 90 \\
10 / 23 / 92\end{array}$ & $\begin{array}{l}\text { foil bag } \\
120 / 256 \\
-20 \text { mesh } \\
7 / 30 / 90 \\
6 / 04 / 93\end{array}$ \\
\hline $\begin{array}{l}\text { PROXIMATE } \\
\% \text { moisture } \\
\% \text { ash (dry) } \\
\text { analysis date }\end{array}$ & $\begin{array}{c}2.40 \\
10.25 \\
8 / 23 / 90\end{array}$ & $\begin{array}{c}2.47 \\
11.05 \\
7 / 31 / 91\end{array}$ & $\begin{array}{c}2.99 \\
10.35 \\
11 / 27 / 92\end{array}$ & $\begin{array}{c}1.98 \\
9.52 \\
6 / 15 / 93\end{array}$ \\
\hline $\begin{array}{l}\text { GIESELER FLUIDITY } \\
\text { max. ddpm } \\
\text { fluid range } \\
\text { analysis date }\end{array}$ & $\begin{array}{c}20002 \\
90 \\
7 / 27 / 90\end{array}$ & $\begin{array}{c}6458 \\
90 \\
7 / 24 / 91\end{array}$ & $\begin{array}{c}4309 \\
75 \\
10 / 27 / 92\end{array}$ & $\begin{array}{l}2173 \\
80 \\
7 / 21 / 93\end{array}$ \\
\hline $\begin{array}{l}\text { SULFUR FORMS (dry \%) } \\
\text { total sulfur } \\
\text { sulfate } \\
\text { Pyritic } \\
\text { organic } \\
\text { analysis date }\end{array}$ & $\begin{array}{c}1.12 \\
0.01 \\
0.38 \\
0.73 \\
8 / 23 / 90\end{array}$ & $\begin{array}{r}1.18 \\
0.01 \\
0.29 \\
0.88 \\
7 / 31 / 01\end{array}$ & $\begin{array}{c}1.16 \\
0.01 \\
0.17 \\
0.98 \\
11 / 27 / 92\end{array}$ & $\begin{array}{c}1.17 \\
0.00 \\
0.35 \\
0.82 \\
6 / 15 / 93\end{array}$ \\
\hline $\begin{array}{l}\text { CALORIFIC VALUE (dry) } \\
\text { BTU/lb } \\
\text { analysis date }\end{array}$ & $\begin{array}{c}13532 \\
8 / 23 / 90\end{array}$ & $\begin{array}{c}13337 \\
7 / 31 / 91\end{array}$ & $\begin{array}{c}13471 \\
11 / 27 / 92\end{array}$ & $\begin{array}{l}13563 \\
6 / 15 / 93\end{array}$ \\
\hline $\begin{array}{l}\text { ALKALI EXTRACTION* } \\
\% \text { transmission } \\
\text { analysis date }\end{array}$ & $\begin{array}{c}91.9 \\
7 / 27 / 90\end{array}$ & $\begin{array}{c}95.0 \\
7 / 24 / 91\end{array}$ & $\begin{array}{c}98.1 \\
10 / 23 / 92\end{array}$ & $\begin{array}{c}94.3 \\
8 / 18 / 93\end{array}$ \\
\hline
\end{tabular}

* calculated to $17 \mathrm{~mm}$ light path 


\begin{tabular}{|c|c|c|c|}
\hline \multicolumn{2}{|c|}{$\begin{array}{l}\text { DECS-13 Sewell } \\
\text { collection date: } 12 / 13 / 90\end{array}$} & \multicolumn{2}{|c|}{ mub, Ro $\max =1.35$} \\
\hline $\begin{array}{l}\text { Split ID } \\
\text { size } \\
\text { mote } \\
\text { date sealed } \\
\text { dote subsampled }\end{array}$ & $\begin{array}{l}\text { analysis } \\
\text { sample }\end{array}$ & $\begin{array}{c}\text { foil bag } \\
7 \text { of } 256 \\
-20 \text { mesh } \\
\\
2 / 05 / 91 \\
12 / 07 / 92\end{array}$ & $\begin{array}{l}\text { foil bag } \\
23 \text { of } 256 \\
-20 \text { mesh } \\
2 / 05 / 91 \\
6 / 04 / 93\end{array}$ \\
\hline $\begin{array}{l}\text { PROXIMATE } \\
\% \text { moisture } \\
\% \text { ash (dry) } \\
\text { analysis date }\end{array}$ & $\begin{array}{c}1.5 \\
4.2 \\
2 / 18 / 91\end{array}$ & $\begin{array}{c}1.8 \\
4.3 \\
12 / 07 / 92\end{array}$ & $\begin{array}{c}1.5 \\
4.6 \\
6 / 15 / 93\end{array}$ \\
\hline $\begin{array}{l}\text { GIESELER FLUIDITY } \\
\text { max. ddpm } \\
\text { fluid range } \\
\text { analysis date }\end{array}$ & $\begin{array}{l}909 \\
89 \\
2 / 18 / 91\end{array}$ & $\begin{array}{l}1294 \\
90 \\
2 / 03 / 93\end{array}$ & $\begin{array}{l}915 \\
86 \\
7 / 23 / 93\end{array}$ \\
\hline $\begin{array}{l}\text { SULFUR FORIS (dry \%) } \\
\text { total sulfur } \\
\text { sulfate } \\
\text { pyritic } \\
\text { organic } \\
\text { analysis date }\end{array}$ & $\begin{array}{c}0.62 \\
0.01 \\
0.07 \\
0.54 \\
2 / 15 / 91\end{array}$ & $\begin{array}{c}0.62 \\
0.00 \\
0.07 \\
0.55 \\
12 / 07 / 92\end{array}$ & $\begin{array}{c}0.65 \\
0.00 \\
0.08 \\
0.55 \\
6 / 15 / 93\end{array}$ \\
\hline $\begin{array}{l}\text { CALORIFIC VALUE (dry) } \\
\text { BTU/lb } \\
\text { analysis date }\end{array}$ & $\begin{array}{c}14871 \\
2 / 15 / 91\end{array}$ & $\begin{array}{c}14827 \\
12 / 07 / 92\end{array}$ & $\begin{array}{c}14830 \\
6 / 15 / 93\end{array}$ \\
\hline $\begin{array}{l}\text { ALKALI EXTRACTION* } \\
\% \text { transmission } \\
\text { analysis date }\end{array}$ & $\begin{array}{c}96.0 \\
3 / 05 / 91\end{array}$ & $\begin{array}{c}95.0 \\
10 / 29 / 92\end{array}$ & $\begin{array}{c}95 .: \\
8 / 18 / 93\end{array}$ \\
\hline
\end{tabular}

* calculated to $17 \mathrm{~mm}$ light path 


\begin{tabular}{|c|c|c|c|}
\hline \multicolumn{4}{|c|}{$\begin{array}{l}\text { DECS-14 Upper Kittaming } \\
\text { collection date: } 12 / 14 / 90\end{array}$} \\
\hline $\begin{array}{l}\text { Split ID } \\
\text { size } \\
\text { note } \\
\text { date sealed } \\
\text { date subsampled }\end{array}$ & $\begin{array}{l}\text { analys is } \\
\text { sample }\end{array}$ & $\begin{array}{c}\text { foil bag } \\
6 \text { of } 256 \\
-20 \text { mesh } \\
2 / 08 / 91 \\
10 / 14 / 92\end{array}$ & $\begin{array}{l}\text { foil bag } \\
20 \text { of } 256 \\
-20 \text { mesh } \\
2 / 08 / 91 \\
6 / 04 / 93\end{array}$ \\
\hline $\begin{array}{l}\text { PROXIMATE } \\
\% \text { moisture } \\
\% \text { ash (dry) } \\
\text { analysis date }\end{array}$ & $\begin{array}{c}1.5 \\
10.5 \\
2 / 15 / 91\end{array}$ & $\begin{array}{c}1.7 \\
10.3 \\
11 / 27 / 92\end{array}$ & $\begin{array}{c}1.4 \\
10.6 \\
6 / 15 / 93\end{array}$ \\
\hline $\begin{array}{l}\text { GIESELER FLUIDITY } \\
\text { max. ddom } \\
\text { fluid range } \\
\text { analysis date }\end{array}$ & $\begin{array}{c}29930 \\
109 \\
2 / 29 / 91\end{array}$ & $\begin{array}{c}27625 \\
108 \\
2 / 04 / 93\end{array}$ & $\begin{array}{r}23619 \\
104 \\
7 / 23 / 93\end{array}$ \\
\hline $\begin{array}{l}\text { SULFUR FORIS (dry \%) } \\
\text { total sulfur } \\
\text { sulfate } \\
\text { Pyritic } \\
\text { organic } \\
\text { analysis date }\end{array}$ & $\begin{array}{r}1.80 \\
0.01 \\
0.94 \\
0.85 \\
2 / 15 / 91\end{array}$ & $\begin{array}{c}1.71 \\
0.01 \\
0.03 \\
1.62 \\
11 / 27 / 92\end{array}$ & $\begin{array}{c}1.80 \\
0.61 \\
1.02 \\
0.78 \\
6 / 15 / 93\end{array}$ \\
\hline $\begin{array}{l}\text { CALORIFIC VALUE (dry) } \\
\text { BTU/lb } \\
\text { analysis date }\end{array}$ & $\begin{array}{c}13678 \\
2 / 15 / 91\end{array}$ & $\begin{array}{c}13723 \\
11 / 27 / 92\end{array}$ & $\begin{array}{c}13701 \\
6 / 15 / 93\end{array}$ \\
\hline $\begin{array}{l}\text { ALKALI EXTRACTION* } \\
\text { \% transmission } \\
\text { analysis date }\end{array}$ & $\begin{array}{c}91.0 \\
3 / 05 / 91\end{array}$ & $\begin{array}{c}99.5 \\
10 / 14 / 92\end{array}$ & $\begin{array}{c}94.1 \\
8 / 18 / 93\end{array}$ \\
\hline
\end{tabular}

* calculated to $17 \mathrm{~mm}$ light path 


\begin{tabular}{|c|c|c|c|}
\hline \multicolumn{4}{|c|}{$\begin{array}{l}\text { DECS-15 Lower Sunnyside } \\
\text { collection date: 5/06/91 }\end{array}$} \\
\hline $\begin{array}{l}\text { Split I0 } \\
\text { size } \\
\text { note } \\
\text { date sealed } \\
\text { date subsampled }\end{array}$ & $\begin{array}{l}\text { analys is } \\
\text { sample }\end{array}$ & $\begin{array}{l}\text { foil bag } \\
3 \text { of } 256 \\
-20 \text { mesh } \\
\\
10 / 15 / 92 \\
10 / 26 / 92\end{array}$ & $\begin{array}{c}\text { foil bag } \\
6 \text { of } 256 \\
-20 \text { mesh } \\
\\
10 / 15 / 92 \\
6 / 04 / 93\end{array}$ \\
\hline $\begin{array}{l}\text { PROXIMATE } \\
\% \text { moisture } \\
\% \text { ash (dry ) } \\
\text { analysis date }\end{array}$ & $\begin{array}{c}2.9 \\
10.0 \\
7 / 05 / 91\end{array}$ & $\begin{array}{c}2.9 \\
10.1 \\
11 / 27 / 92\end{array}$ & $\begin{array}{c}2.6 \\
9.8 \\
6 / 15 / 93\end{array}$ \\
\hline $\begin{array}{l}\text { GIESELER FLUIDITY } \\
\text { max. ddpm } \\
\text { fluid range } \\
\text { analysis date }\end{array}$ & $\begin{array}{l}959 \\
76 \\
6 / 13 / 91\end{array}$ & $\begin{array}{l}258 \\
75 \\
2 / 05 / 93\end{array}$ & $\begin{array}{c}170 \\
69 \\
7 / 26 / 93\end{array}$ \\
\hline $\begin{array}{l}\text { SULFUR FORtS (dry \%) } \\
\text { total sulfur } \\
\text { sulfate } \\
\text { pyritic } \\
\text { organic } \\
\text { analysis date }\end{array}$ & $\begin{array}{c}1.65 \\
0.05 \\
0.61 \\
0.99 \\
7 / 05 / 91\end{array}$ & $\begin{array}{c}1.75 \\
0.03 \\
0.94 \\
0.78 \\
11 / 27 / 92\end{array}$ & $\begin{array}{c}1.62 \\
0.02 \\
0.69 \\
0.91 \\
6 / 15 / 93\end{array}$ \\
\hline $\begin{array}{l}\text { CALORIFIC VALUE (dry) } \\
\text { BTU/lb } \\
\text { analysis date }\end{array}$ & $\begin{array}{c}13429 \\
7 / 05 / 91\end{array}$ & $\begin{array}{c}13275 \\
11 / 27 / 92\end{array}$ & $\begin{array}{c}13396 \\
6 / 15 / 93\end{array}$ \\
\hline $\begin{array}{l}\text { ALKALI EXTRACTION* } \\
\% \text { transmission } \\
\text { analysis date }\end{array}$ & $\begin{array}{c}95.5 \\
7 / 09 / 91\end{array}$ & $\begin{array}{c}91.8 \\
10 / 26 / 92\end{array}$ & $\begin{array}{c}91.0 \\
8 / 18 / 93\end{array}$ \\
\hline
\end{tabular}

* calculated to $17 \mathrm{~mm}$ light path 


\begin{tabular}{|c|c|c|c|}
\hline $\begin{array}{l}\text { DECS-16 Blind } \\
\text { collection date: }\end{array}$ & $\begin{array}{l}\text { Canyon } \\
5 / 07 / 91\end{array}$ & INAb, Ro ma & $x=0.64$ \\
\hline $\begin{array}{l}\text { Split ID } \\
\text { size } \\
\text { note } \\
\text { date sealed } \\
\text { date subsampled }\end{array}$ & $\begin{array}{l}\text { analys is } \\
\text { sample }\end{array}$ & $\begin{array}{c}\text { foil bag } \\
7 \text { of } 256 \\
-20 \text { mesh } \\
\\
8 / 25 / 92 \\
10 / 31 / 92\end{array}$ & $\begin{array}{l}\text { foil bag } \\
36 \text { of } 256 \\
-20 \text { mesh } \\
8 / 25 / 92 \\
6 / 04 / 93\end{array}$ \\
\hline $\begin{array}{l}\text { PROXIMATE } \\
\% \text { moisture } \\
\% \text { ash (dry) } \\
\text { amalysis date }\end{array}$ & $\begin{array}{c}5.4 \\
13.9 \\
7 / 05 / 91\end{array}$ & $\begin{array}{c}4.9 \\
15.2 \\
11 / 27 / 92\end{array}$ & $\begin{array}{c}5.0 \\
15.7 \\
6 / 15 / 93\end{array}$ \\
\hline $\begin{array}{l}\text { GIESELER FLUIDITY } \\
\text { max. ddpm } \\
\text { fluid range } \\
\text { analysis date }\end{array}$ & $\begin{array}{c}6 \\
42 \\
6 / 14 / 91\end{array}$ & $\begin{array}{c}3 \\
42 \\
2 / 08 / 93\end{array}$ & $\begin{array}{l}2 \\
41 \\
7 / 26 / 93\end{array}$ \\
\hline $\begin{array}{l}\text { SULFUR FORis (dry \%) } \\
\text { total sulfur } \\
\text { sulfate } \\
\text { pyritic } \\
\text { organic } \\
\text { analysis date }\end{array}$ & $\begin{array}{c}0.47 \\
0.02 \\
0.06 \\
0.38 \\
7 / 05 / 91\end{array}$ & $\begin{array}{c}0.48 \\
0.05 \\
0.07 \\
0.37 \\
11 / 27 / 92\end{array}$ & $\begin{array}{c}0.46 \\
0.01 \\
0.06 \\
0.39 \\
6 / 15 / 93\end{array}$ \\
\hline $\begin{array}{l}\text { CALORIFIC VALUE (dry) } \\
\text { BTU/Ib } \\
\text { analysis date }\end{array}$ & $\begin{array}{c}12580 \\
7 / 05 / 91\end{array}$ & $\begin{array}{c}12253 \\
11 / 27 / 92\end{array}$ & $\begin{array}{c}12297 \\
6 / 15 / 93\end{array}$ \\
\hline $\begin{array}{l}\text { ALKALI EXTRACTION* } \\
\% \text { transmission } \\
\text { analysis date }\end{array}$ & & $\begin{array}{c}79.9 \\
10 / 31 / 92\end{array}$ & $\begin{array}{c}83.4 \\
8 / 18 / 93\end{array}$ \\
\hline
\end{tabular}

* calculated to 17min light path 


\begin{tabular}{|c|c|c|c|}
\hline \multicolumn{4}{|c|}{$\begin{array}{l}\text { DECS-17 Blind Canyon, Refrig. InAb, Ro } \max =0.59 \\
\text { ( standard }-60 \text { mesh package for distribution ) } \\
\text { collection date: } 8 / 05 / 91\end{array}$} \\
\hline $\begin{array}{l}\text { Split ID } \\
\text { size } \\
\text { note } \\
\text { date sealed } \\
\text { date subsampled }\end{array}$ & $\begin{array}{l}\text { analys is } \\
\text { sample }\end{array}$ & $\begin{array}{l}\text { foil bag } \\
60 \text { of } 512 \\
-60 \text { mesh } \\
12 / 04 / 91 \\
4 / 16 / 93\end{array}$ & $\begin{array}{l}\text { foil bag } \\
61 \text { of } 512 \\
-60 \text { mesh } \\
12 / 04 / 91 \\
6 / 07 / 93\end{array}$ \\
\hline $\begin{array}{l}\text { PROXImaTE } \\
\% \text { moisture } \\
\% \text { ash (dry) } \\
\text { analysis date }\end{array}$ & $\begin{array}{c}3.7 \\
6.6 \\
8 / 27 / 91\end{array}$ & $\begin{array}{c}4.0 \\
6.5 \\
4 / 16 / 93\end{array}$ & $\begin{array}{c}3.6 \\
6.5 \\
6 / 18 / 93\end{array}$ \\
\hline $\begin{array}{l}\text { GIESELER FLUIDITY } \\
\text { max. ddpm } \\
\text { fluid range } \\
\text { analysis date }\end{array}$ & $\begin{array}{l}5 \\
55 \\
8 / 16 / 91\end{array}$ & $\begin{array}{l}\text { not done } \\
\text { on }-60 \\
\text { mesh }\end{array}$ & \\
\hline $\begin{array}{l}\text { SULFUR FORAS (dry \%) } \\
\text { total sulfur } \\
\text { sulfate } \\
\text { pyritic } \\
\text { organic } \\
\text { analysis date }\end{array}$ & $\begin{array}{c}0.44 \\
0.01 \\
0.02 \\
0.41 \\
8 / 27 / 91\end{array}$ & $\begin{array}{c}0.44 \\
0.01 \\
0.03 \\
0.39 \\
4 / 16 / 93\end{array}$ & $\begin{array}{c}0.43 \\
0.01 \\
0.04 \\
0.38 \\
6 / 18 / 93\end{array}$ \\
\hline $\begin{array}{l}\text { CALORIFIC VALUE (dry) } \\
\text { BTU/Ib } \\
\text { analysis date }\end{array}$ & $\begin{array}{c}13826 \\
8 / 27 / 91\end{array}$ & $\begin{array}{c}13919 \\
4 / 16 / 93\end{array}$ & $\begin{array}{c}13712 \\
6 / 18 / 93\end{array}$ \\
\hline $\begin{array}{l}\text { ALKALI EXTRACTION* } \\
\% \text { transmission } \\
\text { analysis date }\end{array}$ & $\begin{array}{c}86.0 \\
8 / 16 / 91\end{array}$ & $\begin{array}{c}93.8 \\
4 / 06 / 93\end{array}$ & $\begin{array}{c}93.1 \\
8 / 19 / 93\end{array}$ \\
\hline
\end{tabular}

* calculated to 17m light path 


\begin{tabular}{|c|c|c|c|}
\hline \multicolumn{4}{|c|}{$\begin{array}{l}\text { DECS-17 Blind Camyon, Refrig. Iwab, Ro max }=0.59 \\
\text { collection date: } 8 / 05 / 91\end{array}$} \\
\hline $\begin{array}{l}\text { Split I0 } \\
\text { size } \\
\text { note } \\
\text { date sealed } \\
\text { date subsampled }\end{array}$ & $\begin{array}{l}\text { analys is } \\
\text { sample }\end{array}$ & $\left|\begin{array}{l}\text { foil bag } \\
204 / 256 \\
-20 \text { mesh } \\
12 / 04 / 91 \\
10 / 28 / 92\end{array}\right|$ & $\begin{array}{l}\text { foil bag } \\
205 / 256 \\
-20 \text { wesh } \\
\\
12 / 04 / 91 \\
6 / 07 / 93\end{array}$ \\
\hline $\begin{array}{l}\text { PROXIMATE } \\
\% \text { misture } \\
\% \text { ash (dry) } \\
\text { analysis date }\end{array}$ & $\begin{array}{c}3.7 \\
6.6 \\
8 / 27 / 91\end{array}$ & $\begin{array}{c}3.9 \\
6.6 \\
4 / 16 / 93\end{array}$ & $\begin{array}{c}3.8 \\
6.7 \\
6 / 15 / 93\end{array}$ \\
\hline $\begin{array}{l}\text { GIESELER FLUIDITY } \\
\text { max. ddpm } \\
\text { fluid range } \\
\text { analys is date }\end{array}$ & $\begin{array}{c}5 \\
55 \\
8 / 16 / 91\end{array}$ & $\begin{array}{c}4 \\
52 \\
10 / 28 / 92\end{array}$ & $\begin{array}{l}2 \\
47 \\
7 / 27 / 93\end{array}$ \\
\hline $\begin{array}{l}\text { SULFUR FORIS (dry \%) } \\
\text { total sul fur } \\
\text { sulfate } \\
\text { pyritic } \\
\text { organic } \\
\text { analysis date }\end{array}$ & $\begin{array}{c}0.44 \\
0.01 \\
0.02 \\
0.41 \\
8 / 27 / 91\end{array}$ & $\begin{array}{c}0.43 \\
0.01 \\
0.03 \\
0.39 \\
4 / 16 / 93\end{array}$ & $\begin{array}{c}0.42 \\
0.01 \\
0.05 \\
0.36 \\
6 / 15 / 93\end{array}$ \\
\hline $\begin{array}{l}\text { CALORIFIC VALUE (dry) } \\
\text { BTU/lb } \\
\text { analysis date }\end{array}$ & $\begin{array}{c}13826 \\
8 / 27 / 91\end{array}$ & $\begin{array}{c}13869 \\
4 / 16 / 93\end{array}$ & $\begin{array}{l}13904 \\
6 / 15 / 93\end{array}$ \\
\hline $\begin{array}{l}\text { ALKALI EXTRACTION* } \\
\% \text { transmission } \\
\text { analysis date }\end{array}$ & $\begin{array}{c}86.0 \\
8 / 16 / 91\end{array}$ & $\begin{array}{c}90.0 \\
4 / 07 / 93\end{array}$ & $\begin{array}{c}94.1 \\
8 / 19 / 93\end{array}$ \\
\hline
\end{tabular}

* Calculated to 17min light path 


\begin{tabular}{|c|c|c|c|}
\hline \multicolumn{4}{|c|}{$\begin{array}{l}\text { DECS-17 Blind Canyon, Unref. TwAb, Ro max }=0.59 \\
\text { collection date: 8/05/91 }\end{array}$} \\
\hline $\begin{array}{l}\text { Split ID } \\
\text { size } \\
\text { note } \\
\text { date sealed } \\
\text { date subsampled }\end{array}$ & $\begin{array}{l}\text { analys is } \\
\text { sample }\end{array}$ & $\begin{array}{l}\text { foil bag } \\
7 \text { of } 16 \\
-20 \text { mesh } \\
8 / 15 / 91 \\
10 / 28 / 92\end{array}$ & $\begin{array}{l}\text { foil bag } \\
15 \text { of } 16 \\
-20 \text { mesh } \\
8 / 15 / 91 \\
6 / 07 / 93\end{array}$ \\
\hline $\begin{array}{l}\text { PROXIMATE } \\
\% \text { moisture } \\
\% \text { ash (dry ) } \\
\text { analysis date }\end{array}$ & $\begin{array}{c}3.7 \\
6.6 \\
8 / 27 / 91\end{array}$ & $\begin{array}{c}3.9 \\
6.4 \\
11 / 27 / 92\end{array}$ & $\begin{array}{c}3.7 \\
6.5 \\
6 / 18 / 93\end{array}$ \\
\hline $\begin{array}{l}\text { GIESELER FLUIDITY } \\
\text { max. ddpm } \\
\text { fluid range } \\
\text { analysis date }\end{array}$ & $\begin{array}{l}5 \\
55 \\
8 / 16 / 91\end{array}$ & $\begin{array}{c}5 \\
50 \\
10 / 28 / 92\end{array}$ & $\begin{array}{l}2 \\
36 \\
7 / 28 / 93\end{array}$ \\
\hline $\begin{array}{l}\text { SULFUR FORIS (dry \%) } \\
\text { total sulfur } \\
\text { sulfate } \\
\text { pyritic } \\
\text { organic } \\
\text { analysis date }\end{array}$ & $\begin{array}{c}0.44 \\
0.01 \\
0.02 \\
0.41 \\
8 / 27 / 91\end{array}$ & $\begin{array}{c}0.44 \\
0.01 \\
0.03 \\
0.40 \\
11 / 27 / 92\end{array}$ & $\begin{array}{c}0.43 \\
0.00 \\
0.05 \\
0.38 \\
6 / 18 / 93\end{array}$ \\
\hline $\begin{array}{l}\text { CALORIFIC VALUE (dry) } \\
\text { BTU/lb } \\
\text { analysis date }\end{array}$ & $\begin{array}{c}13826 \\
8 / 27 / 91\end{array}$ & $\begin{array}{c}13858 \\
11 / 27 / 92\end{array}$ & $\begin{array}{l}13836 \\
6 / 18 / 93\end{array}$ \\
\hline $\begin{array}{l}\text { ALKALI EXTRACTIONA } \\
\% \text { transmission } \\
\text { analysis date }\end{array}$ & $\begin{array}{l}86.0 \\
8 / 16 / 91\end{array}$ & $\begin{array}{l}88.9 \\
3 / 25 / 93\end{array}$ & $\begin{array}{c}92.1 \\
8 / 19 / 93\end{array}$ \\
\hline
\end{tabular}

* calculated to 17 might path 


\begin{tabular}{|c|c|c|c|}
\hline \multicolumn{2}{|c|}{$\begin{array}{cc}\text { OECS-18 } & \text { Kentucky } \$ 9 \\
\text { collection date: } 10 / 10 / 91\end{array}$} & \multicolumn{2}{|c|}{ Indb, Ro $\max =0.56$} \\
\hline $\begin{array}{l}\text { Split ID } \\
\text { size } \\
\text { note } \\
\text { date sealed } \\
\text { date subsampled }\end{array}$ & $\begin{array}{l}\text { analys is } \\
\text { sample }\end{array}$ & $\mid \begin{array}{c}\text { foil bag } \\
3 \text { of } 256 \\
-20 \text { mesh } \\
\\
10 / 09 / 92 \\
6 / 07 / 93\end{array}$ & \\
\hline $\begin{array}{l}\text { PROXIMATE } \\
\% \text { mois ture } \\
\% \text { ash (dry ) } \\
\text { analysis date }\end{array}$ & $\begin{array}{c}6.8 \\
12.3 \\
11 / 22 / 91\end{array}$ & $\begin{array}{c}7.3 \\
11.3 \\
6 / 18 / 93\end{array}$ & \\
\hline $\begin{array}{l}\text { GIESELER FLUIDITY } \\
\text { max. ddpm } \\
\text { fluid range } \\
\text { analys is dete }\end{array}$ & $\begin{array}{l}527 \\
80 \\
2 / 11 / 93\end{array}$ & $\begin{array}{l}409 \\
74 \\
7 / 29 / 93\end{array}$ & \\
\hline $\begin{array}{l}\text { SUlfuR FOAis (dry \%) } \\
\text { total sulfur } \\
\text { sulfate } \\
\text { pyritic } \\
\text { organic } \\
\text { analysis date }\end{array}$ & $\begin{array}{c}4.21 \\
0.01 \\
2.37 \\
1.83 \\
11 / 22 / 91\end{array}$ & $\begin{array}{r}3.91 \\
0.04 \\
1.78 \\
2.10 \\
6 / 18 / 93\end{array}$ & \\
\hline $\begin{array}{l}\text { CALORIfIC VALUE (dry) } \\
\text { BTU/Ib } \\
\text { analysis date }\end{array}$ & $\begin{array}{c}12687 \\
11 / 22 / 91\end{array}$ & $\begin{array}{c}12763 \\
6 / 18 / 93\end{array}$ & \\
\hline $\begin{array}{l}\text { ALKALI EXTRACTION* } \\
\% \text { transmission } \\
\text { analysis date }\end{array}$ & $\begin{array}{c}92.5 \\
3 / 26 / 93\end{array}$ & $\begin{array}{c}97.5 \\
8 / 19 / 93\end{array}$ & \\
\hline
\end{tabular}

* calculated to 17 min light path 


\begin{tabular}{|c|c|c|c|}
\hline $\begin{array}{l}\text { DECS-19 Pocahon } \\
\text { collection date: } 1\end{array}$ & $\begin{array}{l}\operatorname{tas} \$ 3 \\
2 / 17 / 91\end{array}$ & Ivb, Ro mas & $=1.71$ \\
\hline $\begin{array}{l}\text { Split ID } \\
\text { size } \\
\text { note } \\
\text { date sealed } \\
\text { date subsampled }\end{array}$ & $\begin{array}{l}\text { analysis } \\
\text { sample }\end{array}$ & $\begin{array}{l}\text { foil bag } \\
10 \text { of } 256 \\
-20 \text { mesh } \\
7 / 14 / 92 \\
6 / 07 / 93\end{array}$ & \\
\hline $\begin{array}{l}\text { Proximute } \\
\% \text { moisture } \\
\% \text { ash (dry) } \\
\text { analysis date }\end{array}$ & $\begin{array}{c}1.0 \\
4.6 \\
8 / 21 / 92\end{array}$ & $\begin{array}{c}0.8 \\
5.5 \\
6 / 18 / 93\end{array}$ & \\
\hline $\begin{array}{l}\text { GIESELER FLUIDITY } \\
\text { max. ddpm } \\
\text { fluid range } \\
\text { onalys is date }\end{array}$ & $\begin{array}{l}17 \\
50 \\
1 / 22 / 93\end{array}$ & $\begin{array}{l}9 \\
52 \\
7 / 30 / 93\end{array}$ & \\
\hline $\begin{array}{l}\text { SUlfuR Foris (dry \%) } \\
\text { total sulfur } \\
\text { sulfate } \\
\text { pyritic } \\
\text { organic } \\
\text { analysis date }\end{array}$ & $\begin{array}{c}0.74 \\
0.00 \\
0.21 \\
0.52 \\
8 / 21 / 92\end{array}$ & $\begin{array}{c}0.78 \\
0.00 \\
0.24 \\
0.53 \\
6 / 18 / 93\end{array}$ & \\
\hline $\begin{array}{l}\text { CALORIFIC VALUE (dry) } \\
\text { BTU/Ib } \\
\text { analysis date }\end{array}$ & $\begin{array}{c}15006 \\
8 / 21 / 92\end{array}$ & $\begin{array}{c}14790 \\
6 / 18 / 93\end{array}$ & \\
\hline $\begin{array}{l}\text { ALKALI EXTRACTION* } \\
\% \text { transmission } \\
\text { amalysis date }\end{array}$ & $\begin{array}{c}86.9 \\
7 / 29 / 92\end{array}$ & $\begin{array}{c}89.0 \\
8 / 19 / 93\end{array}$ & \\
\hline
\end{tabular}

* calculated to 17min 1ight path 


\begin{tabular}{|c|c|c|c|}
\hline $\begin{array}{l}\text { DECS-20 ElkJ } \\
\text { collection date: } 1\end{array}$ & $\begin{array}{l}\text { horn } \# 3 \\
2 / 18 / 91\end{array}$ & NAb, Ro mas & $=0.87$ \\
\hline $\begin{array}{l}\text { Split Io } \\
\text { size } \\
\text { note } \\
\text { date sealed } \\
\text { date subsampled }\end{array}$ & $\begin{array}{l}\text { analysis } \\
\text { sample }\end{array}$ & $\begin{array}{l}\text { foil bag } \\
16 \text { of } 256 \\
-20 \text { mesh } \\
7 / 15 / 92 \\
6 / 07 / 93\end{array}$ & \\
\hline $\begin{array}{l}\text { Proximute } \\
\% \text { moisture } \\
\% \text { ash (dry) } \\
\text { analysis date }\end{array}$ & $\begin{array}{c}2.8 \\
5.5 \\
8 / 21 / 92\end{array}$ & $\begin{array}{c}2.7 \\
5.2 \\
6 / 18 / 93\end{array}$ & \\
\hline $\begin{array}{l}\text { GIESELER FLUIDITY } \\
\text { max. ddpo } \\
\text { fluid range } \\
\text { analysis date }\end{array}$ & $\begin{array}{l}1612 \\
66 \\
1 / 19 / 93\end{array}$ & $\begin{array}{l}1149 \\
66 \\
7 / 30 / 93\end{array}$ & \\
\hline $\begin{array}{l}\text { SURFUR Foris (dry } \% \text { ) } \\
\text { total sulfur } \\
\text { sulfate } \\
\text { pyritic } \\
\text { organic } \\
\text { analysis date }\end{array}$ & $\begin{array}{c}0.97 \\
0.00 \\
0.21 \\
0.75 \\
8 / 21 / 92\end{array}$ & $\begin{array}{c}0.96 \\
0.00 \\
0.17 \\
0.78 \\
6 / 18 / 93\end{array}$ & \\
\hline $\begin{array}{l}\text { CALORIFIC VALUE (dry) } \\
\text { BTU/1b } \\
\text { analysis dote }\end{array}$ & $\begin{array}{c}14244 \\
8 / 21 / 92\end{array}$ & $\begin{array}{c}14204 \\
6 / 18 / 93\end{array}$ & \\
\hline $\begin{array}{l}\text { ALKALI EXTRACTIOA* } \\
\% \text { transmission } \\
\text { analysis dote }\end{array}$ & $\begin{array}{c}94.2 \\
3 / 30 / 93\end{array}$ & $\begin{array}{c}91.6 \\
8 / 19 / 93\end{array}$ & \\
\hline
\end{tabular}

* calculated to 17m light path 


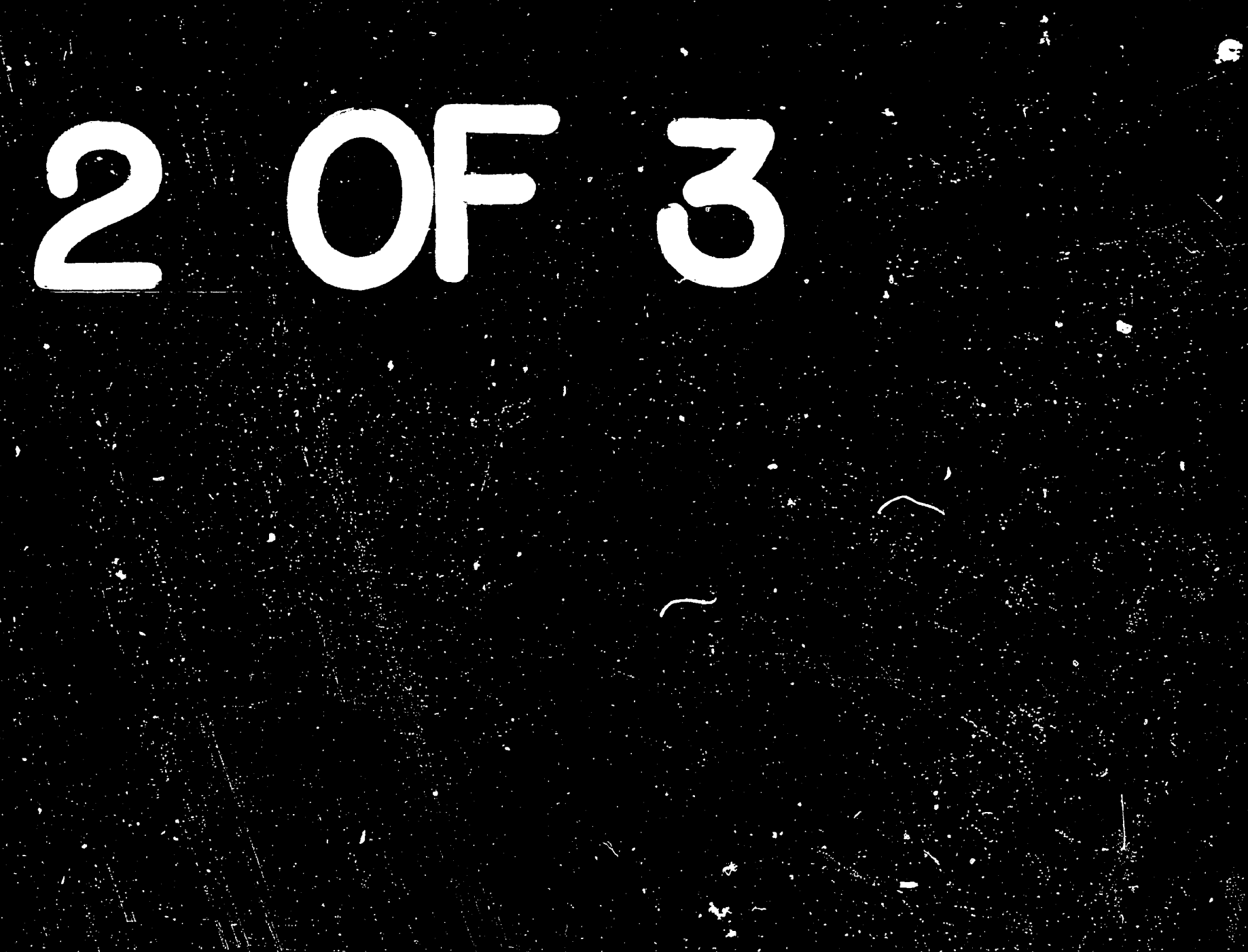

-

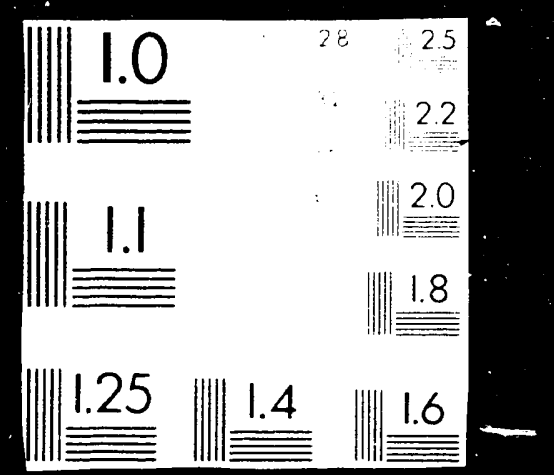

4

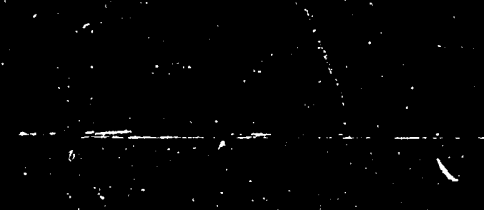




\begin{tabular}{|c|c|c|c|}
\hline $\begin{array}{l}\text { DECS-21 Lykens Val } \\
\text { collection date: } 1\end{array}$ & $\begin{array}{l}\text { ley \#2 } \\
3 / 07 / 92\end{array}$ & an, Ro max & $=5.19$ \\
\hline $\begin{array}{l}\text { Split ID } \\
\text { size } \\
\text { note } \\
\text { date sealed } \\
\text { date subsampled }\end{array}$ & $\begin{array}{l}\text { analys is } \\
\text { sample }\end{array}$ & $\begin{array}{l}\text { foil bag } \\
\text { of } 256 \\
-20 \text { mesh }\end{array}$ & \\
\hline $\begin{array}{l}\text { PROXIMATE } \\
\% \text { moisture } \\
\% \text { ash (dry) } \\
\text { analysis date }\end{array}$ & $\begin{array}{c}3.99 \\
10.71 \\
5 / 03 / 93\end{array}$ & & \\
\hline $\begin{array}{l}\text { SULFUR FORHS (dry \%) } \\
\text { total sulfur } \\
\text { sulfate } \\
\text { Pyritic } \\
\text { organic } \\
\text { analysis date }\end{array}$ & $\begin{array}{c}0.50 \\
0.00 \\
0.06 \\
0.43 \\
5 / 03 / 93\end{array}$ & & \\
\hline $\begin{array}{l}\text { CALORIFIC VALUE (dry) } \\
\text { BTU/lb } \\
\text { analysis date }\end{array}$ & $\begin{array}{c}13284 \\
5 / 03 / 93\end{array}$ & & \\
\hline
\end{tabular}




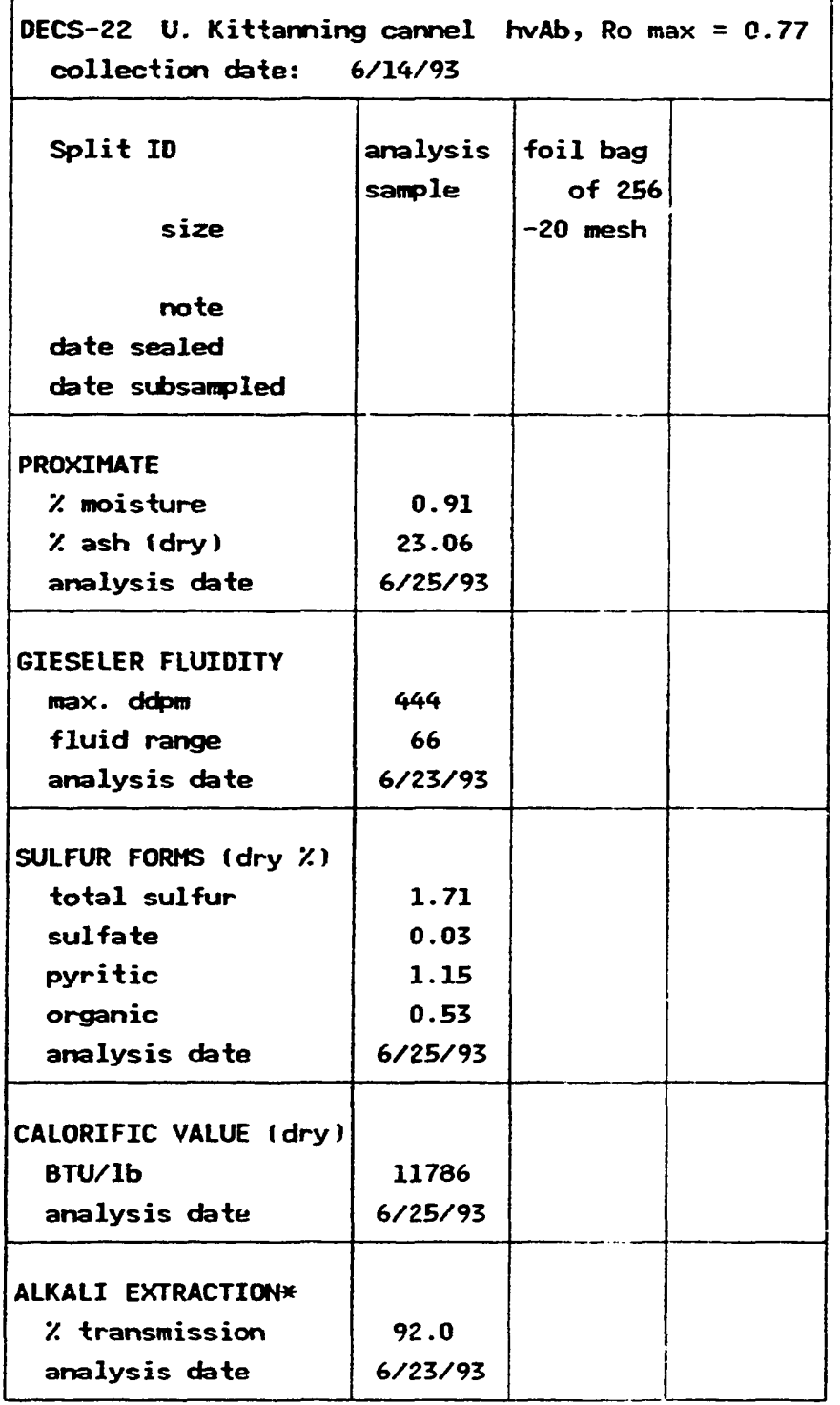

* calculated to $17 \mathrm{~mm}$ light path 


\begin{tabular}{|c|c|c|c|c|c|c|c|}
\hline \multirow{2}{*}{$\begin{array}{l}\text { PSOC-1107 } \\
\text { collection date: } \\
\text { Split ID } \\
\text { size } \\
\% \text { oxygen } \\
\text { note } \\
\text { date sealed } \\
\text { date oxygen/subspl }\end{array}$} & $\begin{array}{l}\text { KING CANNEL } \\
08 / 08 / 78\end{array}$ & \multicolumn{3}{|c|}{ subA, Ro $\max =0.55$} & \multirow[b]{2}{*}{$\begin{array}{l}30 \text { gal. } \\
1 \text { of } 1 \\
-1 / 4 " \\
11 / 14 / 91\end{array}$} & \multirow[b]{2}{*}{$\begin{array}{l}30 \text { gal. } \\
1 \text { of } 1 \\
-1 / 4 " \\
10 / 22 / 92\end{array}$} & \multirow[b]{2}{*}{$\begin{array}{l}30 \mathrm{gal} \\
2 \text { of } 2 \\
-1 / 4 " \\
\text { c } \\
5 / 10 / 93\end{array}$} \\
\hline & $\begin{array}{l}\text { analysis } \\
\text { sample } 1\end{array}$ & $\begin{array}{l}\# 2 \text { can } \\
15 \text { of } 23 \\
-20 \text { mesh } \\
\\
a \\
06 / 12 / 84\end{array}$ & $\begin{array}{l}30 \text { gal. } \\
1 \text { of } 1 \\
-1 / 4 " \\
-- \\
a\end{array}$ & $\begin{array}{l}30 \mathrm{gal} \\
1 \text { of } 1 \\
-1 / 4 " \\
18.6 \\
9 / 18 / 89\end{array}$ & & & \\
\hline $\begin{array}{l}\text { PROXIMATE } \\
\% \text { moisture } \\
\% \text { ash (dry) } \\
\text { analysis date }\end{array}$ & $\begin{array}{c}7.7 \\
19.2 \\
11 / 04 / 83\end{array}$ & $\begin{array}{c}14.2 \\
19.4 \\
06 / 13 / 88\end{array}$ & $\begin{array}{l}11.2 \\
19.2 \\
3 / 07 / 89\end{array}$ & $\begin{array}{c}12.1 \\
19.2 \\
10 / 16 / 89\end{array}$ & $\begin{array}{c}11.2 \\
20.2 \\
11 / 22 / 91\end{array}$ & $\begin{array}{c}15.8 \\
20.0 \\
11 / 27 / 92\end{array}$ & $\begin{array}{c}12.5 \\
19.5 \\
5 / 27 / 93\end{array}$ \\
\hline $\begin{array}{l}\text { SULFUR FORMS (dry \%) } \\
\text { total sulfur } \\
\text { sulfate } \\
\text { Pyritic } \\
\text { organic } \\
\text { analysis date }\end{array}$ & $\begin{array}{r}2.16 \\
0.92 \\
0.43 \\
0.81 \\
11 / 04 / 83\end{array}$ & & $\begin{array}{c}1.71 \\
0.80 \\
0.45 \\
0.46 \\
3 / 07 / 89\end{array}$ & $\begin{array}{c}1.85 \\
0.84 \\
0.42 \\
0.59 \\
10 / 16 / 89\end{array}$ & $\begin{array}{c}2.08 \\
0.93 \\
0.56 \\
0.59 \\
11 / 22 / 91\end{array}$ & \begin{tabular}{r|}
$(b)$ \\
1.96 \\
0.01 \\
0.23 \\
1.72 \\
$11 / 27 / 92$
\end{tabular} & $\begin{array}{c}1.96 \\
0.94 \\
0.50 \\
0.52 \\
5 / 27 / 93\end{array}$ \\
\hline $\begin{array}{l}\text { CALORIFIC VALUE (dry) } \\
\text { BTU/lb } \\
\text { analysis date }\end{array}$ & $\begin{array}{c}10012 \\
11 / 04 / 83\end{array}$ & & $\begin{array}{c}9736 \\
z \cdot n 7 / 89\end{array}$ & $\begin{array}{c}9565 \\
10 / 16 / 89\end{array}$ & $\begin{array}{c}9513 \\
11 / 22 / 91\end{array}$ & $\begin{array}{c}9156 \\
11 / 27 / 92\end{array}$ & $\begin{array}{c}9364 \\
5 / 27 / 93\end{array}$ \\
\hline $\begin{array}{l}\text { ALKALI EXTRACTION } \\
\% \text { transmission } \\
\text { analysis date }\end{array}$ & & $\begin{array}{c}0.2 \\
06 / 13 / 88\end{array}$ & $\begin{array}{l}\text { discon- } \\
\text { timued }\end{array}$ & & & & \\
\hline
\end{tabular}

notes: a) Sample dry (custy), visible yellow \& whire sulfates iron stained, resealed in poly drum $11 / 16 / 88$.

b) Sulfate sulfur value is questionable in view of previous results.

c) Sample dry (dusty), minor brown stains on a few particles. 


\begin{tabular}{|c|c|c|c|c|c|c|}
\hline $\begin{array}{l}\text { PSOC-1442 } \\
\text { collection date: }\end{array}$ & $\begin{array}{l}\text { DARCO } \\
33 / 28 / 85\end{array}$ & IigA, & 2o $\max =0$ & & & \\
\hline $\begin{array}{l}\text { Split ID } \\
\text { size } \\
\text { \% oxygen } \\
\text { note } \\
\text { dete sealed } \\
\text { date oxygen/subspl }\end{array}$ & $\begin{array}{l}\text { analysis } \\
\text { sample } 1 \\
-20 \text { mesh }\end{array}$ & $\begin{array}{l}30 \text { gai. } \\
3 \text { of } 3 \\
-1 / 4 " \\
3.16 \\
\text { a } \\
8 / 17 / 88\end{array}$ & $\begin{array}{l}30 \text { gal. } \\
3 \text { of } 3 \\
-1 / 4 " \\
10.6 \\
9 / 18 / 89\end{array}$ & $\begin{array}{l}30 \mathrm{gal} \\
3 \text { of } 3 \\
-1 / 4 " \\
18.8 \\
11 / 16 / 90\end{array}$ & $\begin{array}{l}30 \text { gel. } \\
3 \text { of } 3 \\
-1 / 4 " \\
11 / 19 / 91\end{array}$ & $\begin{array}{l}30 \mathrm{gal} \\
3 \text { of } 3 \\
-1 / 4 " \\
\text { b } \\
4 / 19 / 93\end{array}$ \\
\hline $\begin{array}{l}\text { PROXIMATE } \\
\% \text { mois ture } \\
\% \text { ash (dry) } \\
\text { analysis date }\end{array}$ & $\begin{array}{c}32.6 \\
11.3 \\
06 / 11 / 85\end{array}$ & $\begin{array}{c}30.5 \\
9.4 \\
09 / 14 / 88\end{array}$ & $\begin{array}{c}34.1 \\
10.9 \\
10 / 16 / 89\end{array}$ & $\begin{array}{c}35.8 \\
10.6 \\
1 / 23 / 91\end{array}$ & $\begin{array}{c}34.7 \\
9.5 \\
2 / 20 / 92\end{array}$ & $\begin{array}{c}33.7 \\
10.4 \\
5 / 27 / 93\end{array}$ \\
\hline $\begin{array}{l}\text { SULFUR FORUL (dry \%) } \\
\text { total sulfur } \\
\text { sulfate } \\
\text { pyritic } \\
\text { organic } \\
\text { analysis date }\end{array}$ & $\begin{array}{r}0.78 \\
0.02 \\
0.11 \\
0.65 \\
06 / 11 / 85\end{array}$ & $\begin{array}{c}1.05 \\
0.11 \\
0.10 \\
0.84 \\
09 / 22 / 88\end{array}$ & $\begin{array}{r}0.82 \\
0.08 \\
0.08 \\
0.66 \\
10 / 16 / 89\end{array}$ & $\begin{array}{r}0.83 \\
0.13 \\
0.11 \\
0.60 \\
1 / 23 / 91\end{array}$ & $\begin{array}{r}0.81 \\
0.07 \\
0.05 \\
0.70 \\
2 / 20 / 92\end{array}$ & $\begin{array}{r}0.81 \\
0.11 \\
0.08 \\
0.63 \\
5 / 27 / 93\end{array}$ \\
\hline $\begin{array}{l}\text { CALORTFIC VALUE (dry) } \\
\text { BTU/lb } \\
\text { analysis date }\end{array}$ & $\begin{array}{c}11375 \\
06 / 11 / 85\end{array}$ & & $\begin{array}{c}11108 \\
10 / 16 / 89\end{array}$ & $\begin{array}{c}11005 \\
1 / 25 / 91\end{array}$ & $\begin{array}{c}10958 \\
2 / 20 / 92\end{array}$ & $\begin{array}{c}10903 \\
5 / 27 / 93\end{array}$ \\
\hline
\end{tabular}

notes: a) Sample moist, good condition, container resealed 08/17/88. PSU MCL ash, moisture, sulfur forms.

b) Condensation on drum walls; no visible deterioration of coal. 


\begin{tabular}{|c|c|c|c|c|c|c|}
\hline $\begin{array}{c}\text { PSOC-1443 UNAArED I } \\
\text { collection date: }\end{array}$ & $\begin{array}{l}\text { Lower Hilc } \\
3 / 29 / 85\end{array}$ & Dx) IigA, & $0 \max =0$ & & & \\
\hline $\begin{array}{l}\text { Split I0 } \\
\text { size } \\
\text { \% head oxygen } \\
\text { note } \\
\text { date sealed } \\
\text { date oxygen/subspl }\end{array}$ & $\begin{array}{l}\text { analysis } \\
\text { sample } 1 \\
-20 \text { mesh }\end{array}$ & $\begin{array}{c}30 \text { gal. } \\
3 \text { of } 4 \\
-1^{4 *} \\
3.84 \\
a \\
8 / 22 / 88\end{array}$ & $\begin{array}{l}30 \text { gal } \\
3 \text { of } 4 \\
-1 / 4 " \\
6.8 \\
9 / 18 / 89\end{array}$ & $\begin{array}{l}30 \text { gel. } \\
3 \text { of } 4 \\
-i / 4 " \\
16.7 \\
11 / 16 / 90\end{array}$ & $\begin{array}{l}30 \text { gal. } \\
3 \text { of } 4 \\
-1 / 4^{n}\end{array}$ & $\begin{array}{c}30 \text { gal. } \\
3 \text { of } 4 \\
-1 / 4 " \\
\text { b } \\
4 / 19 / 93\end{array}$ \\
\hline $\begin{array}{l}\text { PROXIMATE } \\
\% \text { moisture } \\
\% \text { ash (dry) } \\
\text { analysis date }\end{array}$ & $\begin{array}{c}28.5 \\
21.4 \\
06 / 11 / 85\end{array}$ & $\begin{array}{c}29.2 \\
19.5 \\
09 / 14 / 88\end{array}$ & $\begin{array}{c}33.35 \\
20.97 \\
10 / 16 / 89\end{array}$ & $\begin{array}{l}35.6 \\
20.8 \\
1 / 23 / 91\end{array}$ & $\begin{array}{c}34.2 \\
16.7 \\
2 / 20 / 92\end{array}$ & $\begin{array}{c}33.9 \\
21.2 \\
5 / 27 / 93\end{array}$ \\
\hline $\begin{array}{l}\text { SULFUR FORis (dry \%) } \\
\text { total sulfur } \\
\text { sulfate } \\
\text { pyritic } \\
\text { organic } \\
\text { analysis date }\end{array}$ & $\begin{array}{c}0.75 \\
0.01 \\
0.09 \\
0.64 \\
06 / 11 / 85\end{array}$ & $\begin{array}{c}0.99 \\
0.03 \\
0.02 \\
0.94 \\
09 / 22 / 88\end{array}$ & $\begin{array}{c}6.83 \\
0.05 \\
0.02 \\
0.76 \\
10 / 16 / 89\end{array}$ & $\begin{array}{c}0.83 \\
0.05 \\
0.02 \\
0.76 \\
1 / 23 / 91\end{array}$ & $\begin{array}{c}0.90 \\
0.02 \\
0.02 \\
0.86 \\
2 / 20 / 92\end{array}$ & $\begin{array}{c}0.82 \\
0.04 \\
0.03 \\
0.75 \\
5 / 27 / 93\end{array}$ \\
\hline $\begin{array}{l}\text { CALORIFIC VALUE (dry) } \\
\text { BTU/lb } \\
\text { analysis date }\end{array}$ & $\begin{array}{c}9882 \\
06 / 11 / 85\end{array}$ & & $\begin{array}{c}9673 \\
10 / 16 / 89\end{array}$ & $\begin{array}{c}9681 \\
1 / 25 / 91\end{array}$ & $\begin{array}{c}c \\
10175 \\
2 / 20 / 92\end{array}$ & $\begin{array}{c}9591 \\
5 / 27 / 93\end{array}$ \\
\hline
\end{tabular}

notes: a) Good condition, moist, sample crushed to $-1 / 4^{\prime \prime}$ and resealed on $8 / 22 / 88$. PSU MCL ash, moisture, sulfur forms.

b) Sample moist, no visible deterioration.

c) High calorific value caused in part by low ash in this split. 


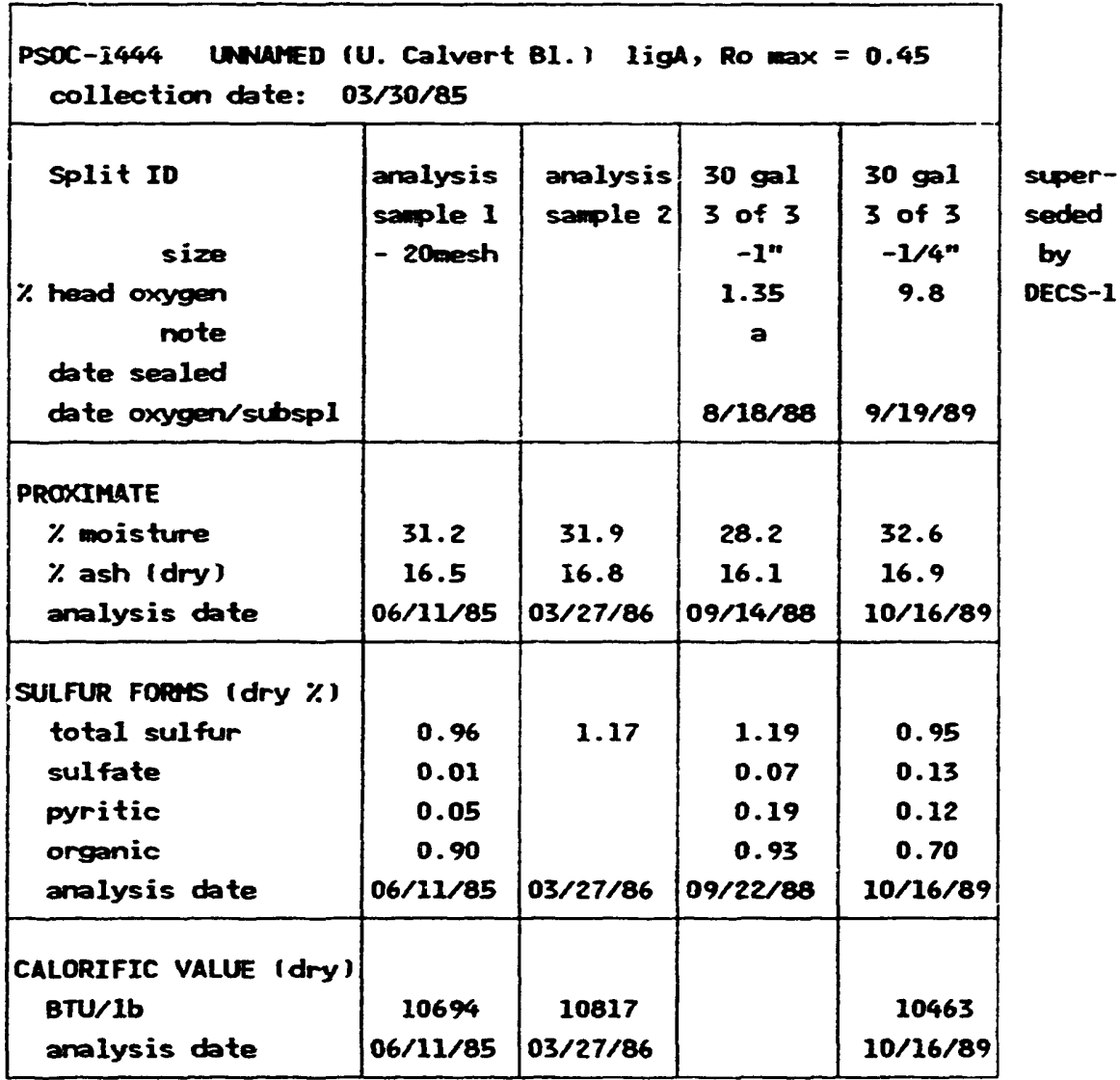

notes: a) Trace of visible sulfates, moist, sample was crushed to $-1 / 4 "$ and resealed on $8 / 18 / 88$.

PSU MCL ash, moisture, sulfur forms. 


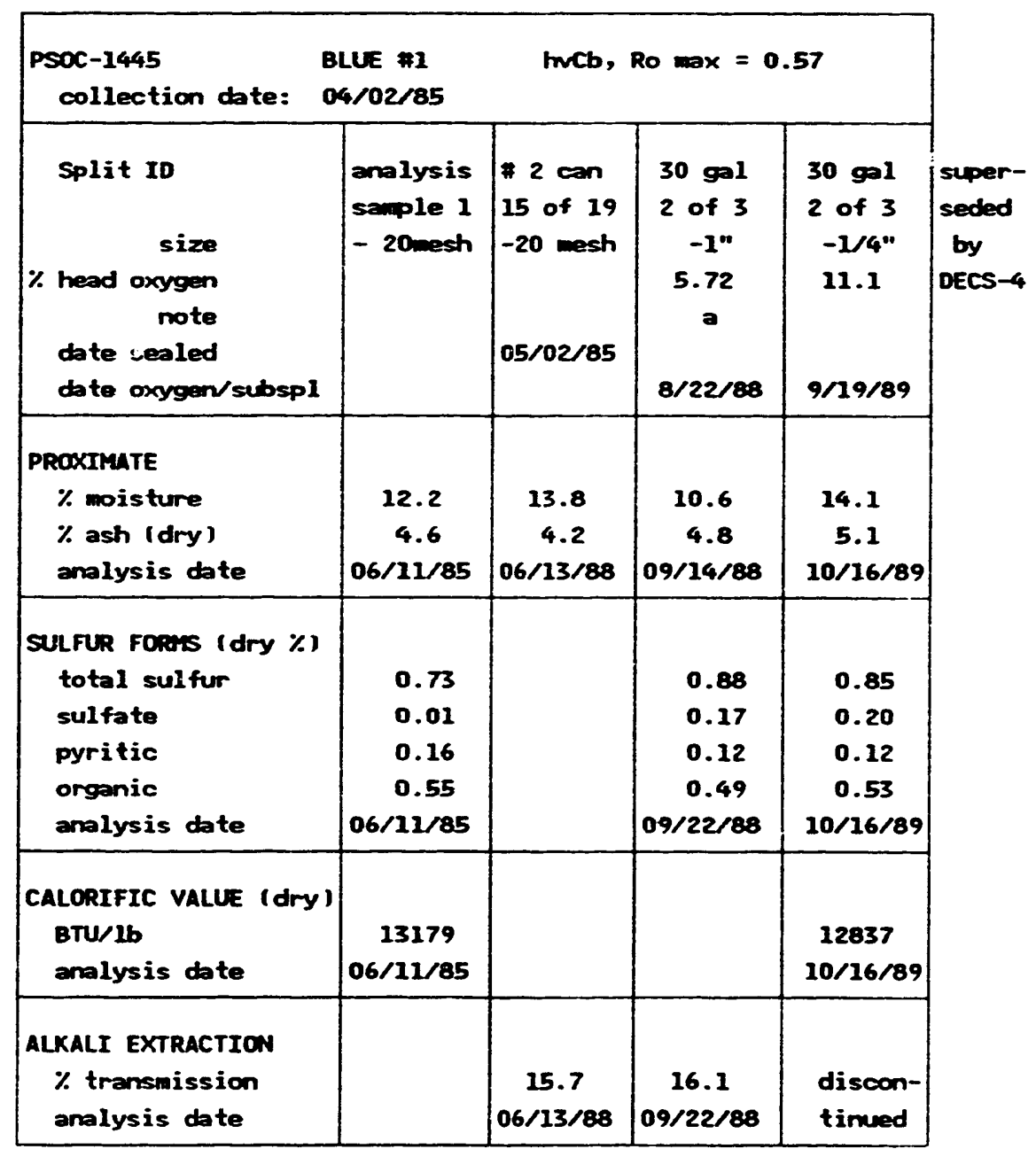

notes: a) Good condition, moist, sample crushed to $-1 / 4^{\prime \prime}$ and resealed on 08/22/88. PSU MCL ash, moisture, sulfur forms. 


\begin{tabular}{|c|c|c|c|c|c|c|c|c|}
\hline $\begin{array}{l}\text { PSOC-1446 } \\
\text { collection date: }\end{array}$ & $\begin{array}{l}\text { EN REXICO } \\
4 / 03 / 85\end{array}$ & 18 Incb, & Ro $\max =0$ & .59 & & & & \\
\hline $\begin{array}{l}\text { Split ID } \\
\text { size } \\
\text { \% head oxygen } \\
\text { note } \\
\text { date sealed } \\
\text { date oxygen/subspl }\end{array}$ & $\begin{array}{l}\text { analysis } \\
\text { sample } 1 \\
-20 \text { mesh }\end{array}$ & $\begin{array}{l}2 \text { can } \\
15 \text { of } 22 \\
-20 \text { mesh } \\
05 / 03 / 85\end{array}$ & $\begin{array}{c}30 \text { gal. } \\
3 \text { of } 3 \\
-1 / 4^{*} \\
12.3 \\
\text { a }\end{array}$ & $\begin{array}{l}5 \text { gal } \\
3 \text { of } 3 \\
-1 / 4^{\prime \prime} \\
20.6 \\
b \\
8 / 23 / 88\end{array}$ & $\begin{array}{l}30 \text { gal. } \\
3 \text { of } 3 \\
-1 / 4 " \\
16.5 \\
9 / 19 / 89\end{array}$ & $\begin{array}{l}30 \text { gal. } \\
3 \text { of } 3 \\
-1 / 4 " \\
19.6 \\
11 / 16 / 90\end{array}$ & $\begin{array}{l}30 \text { gal. } \\
3 \text { of } 3 \\
-1 / 4^{\circ} \\
11 / 19 / 91\end{array}$ & $\begin{array}{l}30 \text { gal. } \\
3 \text { of } 3 \\
-1 / 4 " \\
\text { C } \\
4 / 19 / 93\end{array}$ \\
\hline $\begin{array}{l}\text { PROXIMATE } \\
\% \text { moisture } \\
\% \text { ash (dry) } \\
\text { analysis date }\end{array}$ & $\begin{array}{c}11.2 \\
20.8 \\
06 / 10 / 85\end{array}$ & $\begin{array}{c}12.9 \\
19.9 \\
06 / 13 / 88\end{array}$ & $\begin{array}{c}9.7 \\
20.9 \\
09 / 14 / 88\end{array}$ & $\begin{array}{c}9.2 \\
21.2 \\
09 / 14 / 88\end{array}$ & $\begin{array}{c}12.2 \\
20.5 \\
10 / 16 / 89\end{array}$ & $\begin{array}{l}13.6 \\
20.5 \\
1 / 23 / 91\end{array}$ & $\begin{array}{c}12.2 \\
20.1 \\
2 / 20 / 92\end{array}$ & $\begin{array}{c}12.6 \\
21.0 \\
5 / 27 / 93\end{array}$ \\
\hline $\begin{array}{l}\text { SULFUR FORits (dry \%) } \\
\text { total sulfur } \\
\text { sulfate } \\
\text { Pyritic } \\
\text { organic } \\
\text { analysis date }\end{array}$ & $\begin{array}{c}0.85 \\
0.01 \\
0.45 \\
0.39 \\
06 / 10 / 85\end{array}$ & & $\begin{array}{c}1.03 \\
0.18 \\
0.16 \\
0.69 \\
09 / 22 / 88\end{array}$ & $\begin{array}{c}1.00 \\
0.17 \\
0.17 \\
0.66 \\
09 / 22 / 88\end{array}$ & $\begin{array}{c}0.93 \\
0.21 \\
0.13 \\
0.60 \\
10 / 16 / 89\end{array}$ & $\begin{array}{c}0.90 \\
0.25 \\
0.12 \\
0.54 \\
1 / 23 / 91\end{array}$ & $\begin{array}{c}0.85 \\
0.18 \\
0.06 \\
0.61 \\
2 / 20 / 92\end{array}$ & $\begin{array}{c}0.89 \\
0.25 \\
0.10 \\
0.54 \\
5 / 27 / 93\end{array}$ \\
\hline $\begin{array}{l}\text { CALORIFIC VALUE (dry) } \\
\text { BTU/lb } \\
\text { analysis date }\end{array}$ & $\begin{array}{c}10940 \\
06 / 10 / 85\end{array}$ & & & & $\begin{array}{c}10696 \\
10 / 16 / 89\end{array}$ & $\begin{array}{c}10619 \\
1 / 23 / 91\end{array}$ & $\begin{array}{c}10707 \\
2 / 20 / 92\end{array}$ & $\begin{array}{c}10537 \\
5 / 27 / 93\end{array}$ \\
\hline $\begin{array}{l}\text { ALKALI EXTRACTION } \\
\text { \% transmission } \\
\text { aralysis date }\end{array}$ & & $\begin{array}{c}38.3 \\
06 / 14 / 88\end{array}$ & $\begin{array}{c}37.1 \\
09 / 22 / 88\end{array}$ & & $\begin{array}{l}\text { discon- } \\
\text { tinued }\end{array}$ & & & \\
\hline
\end{tabular}

notes: a) Good condition, resealed 08/23/88 (split 1 ). PSU MCL ash, moisture, sulfur forms.

b) White sulfate crystals visible on top surface. Resealed on 08/23/88 (split 2). PSU MCL ash, moisture, sulfur forms.

c) Sample dusty; some particles completely coated with yellow deposits. 


\begin{tabular}{|c|c|c|c|c|c|c|c|}
\hline $\begin{array}{l}\text { PSOC-1447 } \\
\text { collection date: }\end{array}$ & $\begin{array}{l}\text { ED } \\
4 / 04 / 85\end{array}$ & mucb, & Ro $\max =0$ & & & & \\
\hline $\begin{array}{l}\text { Split ID } \\
\text { size } \\
\text { \% head oxygen } \\
\text { note } \\
\text { date sealed } \\
\text { date oxygen/subspl }\end{array}$ & $\begin{array}{l}\text { analysis } \\
\text { sample } 1 \\
\text { - 60mesh }\end{array}$ & $\begin{array}{l}\# 2 \text { can } \\
15 \text { of } 19 \\
-20 \text { mesh } \\
05 / 03 / 85\end{array}$ & $\begin{array}{c}30 \text { gal } \\
3 \text { of } 3 \\
-1 / 4 " \\
13.0 \\
a \\
9 / 01 / 88\end{array}$ & $\begin{array}{l}30 \text { gal } \\
3 \text { of } 3 \\
-1 / 4^{\circ} \\
9 / 19 / 89\end{array}$ & $\begin{array}{l}30901 \\
3 \text { of } 3 \\
-1 / 4^{\circ} \\
19.9 \\
11 / 16 / 90\end{array}$ & $\begin{array}{l}30 \text { gal. } \\
3 \text { of } 3 \\
-1 / 4 \cdot \\
11 / 19 / 91\end{array}$ & $\begin{array}{l}30 \text { gel. } \\
3 \text { of } 3 \\
-1 / 4 " \\
\text { b } \\
4 / 19 / 93\end{array}$ \\
\hline $\begin{array}{l}\text { PROXImATE } \\
\% \text { moisture } \\
\% \text { ash (dry) } \\
\text { amalysis date }\end{array}$ & $\begin{array}{c}10.1 \\
5.7 \\
06 / 10 / 85\end{array}$ & $\begin{array}{c}12.3 \\
5.1 \\
06 / 13 / 88\end{array}$ & $\begin{array}{c}10.1 \\
5.8 \\
10 / 12 / 88\end{array}$ & $\begin{array}{c}12.2 \\
6.1 \\
10 / 16 / 89\end{array}$ & $\begin{array}{c}12.8 \\
5.9 \\
1 / 23 / 91\end{array}$ & $\begin{array}{c}11.8 \\
6.1 \\
2 / 20 / 92\end{array}$ & $\begin{array}{c}12.4 \\
5.7 \\
5 / 27 / 93\end{array}$ \\
\hline $\begin{array}{l}\text { SULFUR Forits (dry \%) } \\
\text { total sulfur } \\
\text { sulfate } \\
\text { pyritic } \\
\text { organic } \\
\text { analysis date }\end{array}$ & $\begin{array}{c}0.41 \\
0.01 \\
0.23 \\
0.17 \\
06 / 10 / 85\end{array}$ & & $\begin{array}{c}0.39 \\
0.01 \\
0.04 \\
0.34 \\
01 / 17 / 89\end{array}$ & $\begin{array}{c}0.42 \\
0.02 \\
0.02 \\
0.38 \\
10 / 16 / 89\end{array}$ & $\begin{array}{c}0.40 \\
0.03 \\
0.02 \\
0.35 \\
1 / 23 / 91\end{array}$ & $\begin{array}{c}0.42 \\
0.01 \\
0.03 \\
0.38 \\
2 / 20 / 92\end{array}$ & $\begin{array}{c}0.41 \\
0.02 \\
0.02 \\
0.37 \\
5 / 27 / 93\end{array}$ \\
\hline $\begin{array}{l}\text { CALORIFIC VALUE (dry) } \\
\text { BTU/1b } \\
\text { analysis date }\end{array}$ & $\begin{array}{c}12983 \\
06 / 10 / 85\end{array}$ & & & $\begin{array}{c}12557 \\
10 / 16 / 89\end{array}$ & $\begin{array}{c}12595 \\
1 / 23 / 91\end{array}$ & $\begin{array}{c}12539 \\
2 / 20 / 92\end{array}$ & $\begin{array}{c}12537 \\
5 / 27 / 93\end{array}$ \\
\hline $\begin{array}{l}\text { ALKALI EXTRACTION } \\
\text { \% transmission } \\
\text { analysis date }\end{array}$ & & $\begin{array}{c}5.6 \\
06 / 14 / 88\end{array}$ & $\begin{array}{c}4.5 \\
10 / 05 / 88\end{array}$ & $\begin{array}{l}\text { discon- } \\
\text { timeed }\end{array}$ & & & \\
\hline
\end{tabular}

notes: a I cood condition, sample dry and dusty, lidis loose on secondary buckets, sample resealed on 09/01/88.

PSU MCL ash, moisture. PSU MCL sulfur forms (revised analysis reportl.

b) Sample dusty, no visible deterioration. 


\begin{tabular}{|c|c|c|c|c|c|c|c|}
\hline $\begin{array}{l}\text { PSOC-1448 } \\
\text { collection date: }\end{array}$ & $\begin{array}{l}\text { ORK CANYON } \\
\text { Y/08/85 }\end{array}$ & mab, & Ro $\max =0$ & & & & \\
\hline $\begin{array}{l}\text { Split ID } \\
\text { size } \\
\text { head oxygen } \\
\text { note } \\
\text { date sealed } \\
\text { date oxygen/subspl }\end{array}$ & $\begin{array}{l}\text { analysis } \\
\text { sample } 1 \\
-20 \text { mesh }\end{array}$ & $\begin{array}{l}\# 2 \text { can } \\
8 \text { of } 29 \\
-2 c \text { mesh } \\
05 / 07 / 85\end{array}$ & $\begin{array}{l}30 \text { gal } \\
3 \text { of } 3 \\
-1 / 4^{\prime \prime} \\
18.7 \\
a \\
8 / 29 / 88\end{array}$ & $\begin{array}{l}30 \text { gal } \\
3 \text { of } 3 \\
-1 / 4 " \\
19.5 \\
9 / 19 / 89\end{array}$ & $\begin{array}{l}30 \text { gal. } \\
3 \text { of } 3 \\
-1 / 4^{n} \\
20.7 \\
11 / 16 / 90\end{array}$ & $\begin{array}{l}30 \text { gal } \\
3 \text { of } 3 \\
-1 / 4 " \\
11 / 19 / 91\end{array}$ & $\begin{array}{l}30 \text { gal. } \\
3 \text { of } 3 \\
-1 / 4 \cdot \\
\text { b } \\
4 / 19 / 93\end{array}$ \\
\hline $\begin{array}{l}\text { PROXIMATE } \\
\% \text { moisture } \\
\% \text { ash (dry) } \\
\text { analysis date }\end{array}$ & $\begin{array}{c}1.6 \\
11.4 \\
06 / 10 / 85\end{array}$ & $\begin{array}{c}2.2 \\
11.1 \\
04 / 04 / 88\end{array}$ & $\begin{array}{c}1.1 \\
11.3 \\
09 / 19 / 88\end{array}$ & $\begin{array}{c}1.9 \\
11.1 \\
10 / 16 / 89\end{array}$ & $\begin{array}{c}2.2 \\
10.8 \\
10 / 03 / 91\end{array}$ & $\begin{array}{c}2.0 \\
11.5 \\
2 / 20 / 92\end{array}$ & $\begin{array}{c}2.0 \\
10.7 \\
5 / 27 / 93\end{array}$ \\
\hline $\begin{array}{l}\text { GIESELER FLUIDITY } \\
\text { max. ddpm } \\
\text { fluid range } \\
\text { analysis date }\end{array}$ & $\begin{array}{r}5632 \\
72 \\
06 / 03 / 85\end{array}$ & $\begin{array}{r}2661 \\
89 \\
04 / 04 / 88\end{array}$ & $\begin{array}{r}659 \\
74 \\
09 / 08 / 88\end{array}$ & $\begin{array}{r}230 \\
74 \\
02 / 21 / 90\end{array}$ & $\begin{array}{c}1 \\
19 \\
09 / 04 / 91\end{array}$ & $\begin{array}{l}\text { discon- } \\
\text { timued }\end{array}$ & \\
\hline $\begin{array}{l}\text { SULFUR FORMS (dry \%) } \\
\text { total sulfur } \\
\text { sulfate } \\
\text { pyritic } \\
\text { organic } \\
\text { analysis date }\end{array}$ & $\begin{array}{c}0.46 \\
0.01 \\
0.04 \\
0.41 \\
06 / 10 / 85\end{array}$ & & $\begin{array}{c}0.49 \\
0.02 \\
0.05 \\
0.42 \\
01 / 17 / 89\end{array}$ & $\begin{array}{c}0.46 \\
0.03 \\
0.03 \\
0.40 \\
10 / 16 / 89\end{array}$ & $\begin{array}{c}0.48 \\
0.02 \\
0.07 \\
0.39 \\
10 / 03 / 91\end{array}$ & $\begin{array}{r}0.44 \\
0.02 \\
0.04 \\
0.38 \\
2 / 20 / 92\end{array}$ & $\begin{array}{c}0.94 \\
0.04 \\
0.04 \\
0.36 \\
5 / 27 / 93\end{array}$ \\
\hline $\begin{array}{l}\text { CALORIFIC VALUE (dry) } \\
\text { BTU/lb } \\
\text { analysis date }\end{array}$ & $\begin{array}{c}13467 \\
06 / 10 / 85\end{array}$ & & & $\begin{array}{c}13198 \\
10 / 16 / 89\end{array}$ & $\begin{array}{c}13353 \\
10 / 03 / 91\end{array}$ & $\begin{array}{c}13229 \\
2 / 20 / 92\end{array}$ & $\begin{array}{c}13234 \\
5 / 27 / 93\end{array}$ \\
\hline $\begin{array}{l}\text { ALKALI EXTRACTION } \\
\% \text { transmission } \\
\text { analysis date }\end{array}$ & & $\begin{array}{c}100.1 \\
06 / 01 / 88\end{array}$ & $\begin{array}{c}97.7 \\
09 / 23 / 88\end{array}$ & & $\begin{array}{l}90.8 \\
9 / 04 / 91\end{array}$ & $\begin{array}{c}85.3 \\
9 / 02 / 92\end{array}$ & $\begin{array}{c}92.8 \\
7 / 01 / 93\end{array}$ \\
\hline
\end{tabular}

notes: a) PSU MCL ash, moisture. PSU MCL sulfur forms (revised analys is report).

b) No visible deterioration. 


\begin{tabular}{|c|c|c|c|c|c|c|}
\hline $\begin{array}{l}\text { PSOC-1451 } \\
\text { collection date: }\end{array}$ & $\begin{array}{l}\text { ITTSBURGH } \\
\text { 5/01/85 }\end{array}$ & InvAb, & Ro $\max =0$ & .77 & & \\
\hline $\begin{array}{l}\text { Split ID } \\
\text { size } \\
\text { \% head oxygen } \\
\text { note } \\
\text { date sealed } \\
\text { date oxygen/subspl }\end{array}$ & $\begin{array}{l}\text { analysis } \\
\text { sample } 1 \\
-20 \text { mesh }\end{array}$ & $\begin{array}{l}\text { analysis } \\
\text { sample } 2 \\
-20 \text { wesh }\end{array}$ & $\begin{array}{l}\text { analysis } \\
\text { sample } 3 \\
-20 \text { mesh }\end{array}$ & $\begin{array}{c}\# 2 \text { can } \\
1 \text { of ? } \\
-20 \text { wesh } \\
11 / 03 / 87\end{array}$ & $\begin{array}{l}5991 . \\
-1 / 4 " \\
a\end{array}$ & $\begin{array}{l}30 \text { gal } \\
1 \text { of } 3 \\
-1 / 4^{\circ} \\
14.6 \\
9 / 20 / 89\end{array}$ \\
\hline $\begin{array}{l}\text { PROXIMATE } \\
\% \text { moisture } \\
\% \text { ash (dry) } \\
\text { analysis date }\end{array}$ & $\begin{array}{c}2.6 \\
14.2 \\
06 / 11 / 85\end{array}$ & $\begin{array}{c}2.5 \\
13.7 \\
02 / 14 / 86\end{array}$ & $\begin{array}{c}2.5 \\
13.9 \\
02 / 18 / 86\end{array}$ & $\begin{array}{c}3.6 \\
13.9 \\
04 / 06 / 88\end{array}$ & $\begin{array}{c}1.8 \\
13.4 \\
09 / 14 / 88\end{array}$ & $\begin{array}{l}\text { discon- } \\
\text { tinued }\end{array}$ \\
\hline $\begin{array}{l}\text { GIESELER FLUIDITY } \\
\text { max. ddpm } \\
\text { fluid range } \\
\text { analysis date }\end{array}$ & $\begin{array}{r}8525 \\
80 \\
05 / 29 / 85\end{array}$ & & & $\begin{array}{r}59 \\
62 \\
04 / 06 / 88\end{array}$ & $\begin{array}{c}225 \\
63 \\
08 / 17 / 83\end{array}$ & $\begin{array}{l}\text { to be } \\
\text { resamp- } \\
\text { led }\end{array}$ \\
\hline $\begin{array}{l}\text { SULfUR forats (dry \%) } \\
\text { total sulfur } \\
\text { sulfate } \\
\text { pyritic } \\
\text { organic } \\
\text { analysis date }\end{array}$ & $\begin{array}{c}1.36 \\
0.01 \\
0.82 \\
0.53 \\
06 / 10 / 85\end{array}$ & $\begin{array}{r}1.37 \\
02 / 12 / 86\end{array}$ & $\begin{array}{r}1.38 \\
02 / 18 / 86\end{array}$ & & $\begin{array}{c}1.39 \\
0.15 \\
0.40 \\
0.84 \\
09 / 22 / 88\end{array}$ & \\
\hline $\begin{array}{l}\text { CALORIfIC VALUE (dry) } \\
\text { BTU/Ib } \\
\text { ralysis date }\end{array}$ & $\begin{array}{c}12863 \\
06 / 10 / 85\end{array}$ & $\begin{array}{c}12855 \\
02 / 12 / 86\end{array}$ & $\begin{array}{c}12893 \\
02 / 18 / 86\end{array}$ & & & \\
\hline $\begin{array}{l}\text { ALKALI EXTRACTION } \\
\% \text { transmission } \\
\text { analysis date }\end{array}$ & & & & $\begin{array}{c}99.5 \\
06 / 02 / 88\end{array}$ & & \\
\hline
\end{tabular}

notes: a) Sample used for restocking. entire sample gr and to -20 mesh. PSU MCL ash, moisture, sulfur forms.

b) Superseded by DECS -12 


\begin{tabular}{|c|c|c|c|c|}
\hline $\begin{array}{l}\text { PSOC-1461 } \\
\text { collection date: }\end{array}$ & $\begin{array}{l}\text { Aнmoth } \\
5 / 24 / 85\end{array}$ & an, & $0 \max =5$ & \\
\hline $\begin{array}{l}\text { Split ID } \\
\text { size } \\
\text { \% head oxygen } \\
\text { note } \\
\text { ote sealed } \\
\text { date oxygen/subspl }\end{array}$ & $\begin{array}{l}\text { analys is } \\
\text { sample } 1 \\
\text { - 60mesh }\end{array}$ & $\begin{array}{c}30 \text { gal } \\
3 \text { of } 3 \\
-1 / 4= \\
19.7 \\
a \\
08 / 07 / 85 \\
9 / 23 / 88\end{array}$ & $\begin{array}{l}30 \text { gal } \\
3 \text { of } 3 \\
-1 / 4^{\circ 1} \\
19.9 \\
9 / 19 / 89\end{array}$ & $\begin{array}{l}30 \% 1 \\
3 \text { of } 3 \\
-1 / 9 . \cdots \\
20.6 \\
11 / 16 / 90\end{array}$ \\
\hline $\begin{array}{l}\text { PROXImate } \\
\% \text { moisture } \\
\% \text { ash (dry) } \\
\text { analysis date }\end{array}$ & $\begin{array}{c}3.1 \\
24.2 \\
10 / 08 / 85\end{array}$ & $\begin{array}{c}3.0 \\
21.6 \\
10 / 12 / 88\end{array}$ & $\begin{array}{c}3.5 \\
23.3 \\
10 / 16 / 89\end{array}$ & $\begin{array}{l}3.8 \\
24.8 \\
1 / 23 / 91\end{array}$ \\
\hline $\begin{array}{l}\text { SULFUR FORIS (dry \%) } \\
\text { total sulfur } \\
\text { sulfate } \\
\text { pyritic } \\
\text { organic } \\
\text { amalysis date }\end{array}$ & $\begin{array}{c}0.74 \\
0.02 \\
0.36 \\
0.37 \\
10 / 08 / 85\end{array}$ & $\begin{array}{c}0.64 \\
0.04 \\
0.26 \\
0.34 \\
01 / 17 / 89\end{array}$ & $\begin{array}{c}0.65 \\
0.02 \\
0.21 \\
0.42 \\
10 / 16 / 89\end{array}$ & $\begin{array}{c}0.6 \bar{c} \\
0.06 \\
0.28 \\
0.29 \\
1 / 23 / 91\end{array}$ \\
\hline $\begin{array}{l}\text { CALORIFIC VALUE Idry } \\
\text { BTU/lb } \\
\text { analysis date }\end{array}$ & $\begin{array}{c}10860 \\
10 / 08 / 85\end{array}$ & & $\begin{array}{r}10975 \\
10 / 16 / 89\end{array}$ & $\begin{array}{c}10716 \\
1 / 25 / 91\end{array}$ \\
\hline
\end{tabular}

notes: a) Good condition, sample dry. PSU MCL ash, moisture. PSU MCL sulfur forms (revised analysis report). 


\begin{tabular}{|c|c|c|c|c|c|c|}
\hline $\begin{array}{l}\text { PSUC-1468 } \\
\text { collection date: }\end{array}$ & $\begin{array}{l}\text { XCK MOUNTA } \\
/ 03 / 85\end{array}$ & [N an, & $0 \max =5$. & & & \\
\hline $\begin{array}{l}\text { Split ID } \\
\text { size } \\
\text { \% head oxygen } \\
\text { note } \\
\text { date sealed } \\
\text { date oxygen/subsipl }\end{array}$ & $\begin{array}{l}\text { analys is } \\
\text { sample } 1 \\
-60 \text { mesh }\end{array}$ & $\begin{array}{l}30 \text { gal. } \\
1 \text { of } 3 \\
-1 / 4 " \\
17.8 \\
a \\
8 / 07 / 85 \\
9 / 25 / 88\end{array}$ & $\begin{array}{l}30 \text { gal. } \\
1 \text { of } 3 \\
-1 / 4 " \\
20.3 \\
9 / 28 / 89\end{array}$ & $\begin{array}{l}30 \text { ga } 1 \\
1 \text { of } 3 \\
-1 / 4 " \\
16.5 \\
11 / 16 / 90\end{array}$ & $\begin{array}{l}30 \text { gal. } \\
3 \text { of } 3 \\
-1 / 4 "\end{array}$ & $\begin{array}{l}30 \text { gal. } \\
3 \text { of } 3 \\
-1 / 4 " * \\
b \\
4 / 19 / 93\end{array}$ \\
\hline $\begin{array}{l}\text { PROXIMATE } \\
\% \text { moir ture } \\
\% \text { ash (dry) } \\
\text { analysis date }\end{array}$ & $\begin{array}{c}4.5 \\
6.8 \\
10 / 08 / 85\end{array}$ & $\begin{array}{c}3.5 \\
6.6 \\
10 / 12 / 88\end{array}$ & $\begin{array}{c}4.6 \\
6.6 \\
12 / 14 / 89\end{array}$ & $\begin{array}{l}5.3 \\
6.6 \\
1 / 23 / 91\end{array}$ & \begin{tabular}{c|}
5.2 \\
7.0 \\
$11 / 27 / 92$
\end{tabular} & $\begin{array}{l}5.2 \\
7.0 \\
5 / 27 / 33\end{array}$ \\
\hline $\begin{array}{l}\text { SULFUR FORAs (dry \%) } \\
\text { total sulfur } \\
\text { sulfate } \\
\text { Pyritic } \\
\text { organic } \\
\text { analysis date }\end{array}$ & $\begin{array}{c}0.49 \\
0.01 \\
0.11 \\
0.37 \\
10 / 08 / 85\end{array}$ & $\begin{array}{c}0.43 \\
0.01 \\
0.07 \\
0.35 \\
01 / 17 / 89\end{array}$ & $\begin{array}{c}0.39 \\
0.03 \\
0.06 \\
0.29 \\
12 / 14 / 89\end{array}$ & $\begin{array}{c}0.41 \\
0.01 \\
0.05 \\
0.35 \\
1 / 23 / 91\end{array}$ & $\begin{array}{c}0.41 \\
0.01 \\
0.07 \\
0.33 \\
11 / 27 / 92\end{array}$ & $\begin{array}{c}0.38 \\
0.02 \\
0.05 \\
032 \\
5 / 27 / 93\end{array}$ \\
\hline $\begin{array}{l}\text { CALORIFIC VALUE (dry) } \\
\text { BTU/lb } \\
\text { analys is date }\end{array}$ & $\begin{array}{c}13748 \\
10 / 08 / 85\end{array}$ & & $\begin{array}{c}13671 \\
12 / 14 / 89\end{array}$ & $\begin{array}{c}13784 \\
1 / 25 / 91\end{array}$ & $\begin{array}{c}13596 \\
11 / 27 / 92\end{array}$ & $\begin{array}{c}13753 \\
5 / 27 / 93\end{array}$ \\
\hline
\end{tabular}

notes: a) Sample dry, good condition. PSU MCL ash, moisture. PSU MCL sulfur forms (revised analysis report).

b) Sample dusty, no visible deterioration. 


\begin{tabular}{|c|c|c|c|c|c|c|c|}
\hline $\begin{array}{l}\text { PSOC-1469 } \\
\text { collection date: }\end{array}$ & $\begin{array}{l}\text { IARY LEE } \\
16 / 10 / 85\end{array}$ & huBb, & Ro $\max =$ & & & & \\
\hline $\begin{array}{l}\text { Split ID } \\
\text { size } \\
\text { \% head oxygen } \\
\text { note } \\
\text { date sealed } \\
\text { date oxygen/subspl }\end{array}$ & $\begin{array}{l}\text { analys is } \\
\text { sample } 1 \\
-60 \text { mesh }\end{array}$ & $\begin{array}{l}\# 2 \text { can } \\
26 \text { of } 31 \\
-20 \text { mesh } \\
10 / 01 / 85\end{array}$ & $\begin{array}{c}30 \text { gal. } \\
3 \text { of } 3 \\
-1 / 4^{\circ 1} \\
15.0 \\
a \\
8 / 29 / 88\end{array}$ & $\begin{array}{l}30 \mathrm{gal} \\
3 \text { of } 3 \\
-1 / 4 " \\
18.1 \\
9 / 28 / 89\end{array}$ & $\begin{array}{l}30 \text { gal. } \\
3 \text { of } 3 \\
-1 / 4 " 1 \\
19.9 \\
11 / 16 / 90\end{array}$ & $\begin{array}{l}30 \text { gal. } \\
3 \text { of } 3 \\
-1 / 4 " \\
11 / 20 / 91\end{array}$ & $\begin{array}{l}30 \text { gal. } \\
3 \text { of } 3 \\
-1 / 4 " \\
b \\
5 / 06 / 93\end{array}$ \\
\hline $\begin{array}{l}\text { PROXIMATE } \\
\% \text { moisture } \\
\% \text { ash (dry) } \\
\text { analysis date }\end{array}$ & $\begin{array}{c}1.9 \\
21.0 \\
10 / 08 / 85\end{array}$ & $\begin{array}{c}1.9 \\
21.1 \\
04 / 08 / 88\end{array}$ & $\begin{array}{c}1.3 \\
20.7 \\
09 / 19 / 88\end{array}$ & $\begin{array}{c}2.3 \\
20.8 \\
02 / 06 / 90\end{array}$ & $\begin{array}{c}2.3 \\
20.8 \\
10 / 03 / 91\end{array}$ & $\begin{array}{c}2.2 \\
25.5 \\
2 / 20 / 92\end{array}$ & $\begin{array}{c}2.5 \\
20.8 \\
5 / 27 / 93\end{array}$ \\
\hline $\begin{array}{l}\text { GIESELER FLUIDITY } \\
\text { max. ddpm } \\
\text { fluid range } \\
\text { analysis date }\end{array}$ & $\begin{array}{r}659 \\
73 \\
09 / 21 / 85\end{array}$ & $\begin{array}{r}189 \\
71 \\
04 / 07 / 88\end{array}$ & $\begin{array}{c}21 \\
64 \\
09 / 09 / 88\end{array}$ & $\begin{array}{c}8 \\
54 \\
01 / 03 / 90\end{array}$ & $\begin{array}{l}0 \\
0 \\
9 / 03 / 91\end{array}$ & $\begin{array}{l}\text { discon- } \\
\text { timued }\end{array}$ & \\
\hline $\begin{array}{l}\text { SULFUR FoRts (dry \%) } \\
\text { total sulfur } \\
\text { sulfate } \\
\text { Pyritic } \\
\text { organic } \\
\text { analysis date }\end{array}$ & $\begin{array}{c}0.80 \\
0.02 \\
0.26 \\
0.52 \\
10 / 08 / 85\end{array}$ & & $\begin{array}{c}0.84 \\
0.06 \\
0.14 \\
0.64 \\
01 / 17 / 89\end{array}$ & $\begin{array}{c}0.78 \\
0.07 \\
0.13 \\
0.58 \\
02 / 06 / 90\end{array}$ & $\begin{array}{c}0.77 \\
0.02 \\
0.07 \\
0.68 \\
10 / 03 / 91\end{array}$ & $\begin{array}{c}0.70 \\
0.06 \\
0.12 \\
0.52 \\
2 / 20 / 92\end{array}$ & $\begin{array}{c}0.77 \\
0.10 \\
0.13 \\
0.54 \\
5 / 27 / 93\end{array}$ \\
\hline $\begin{array}{l}\text { CALORIFIC VALUE (dry) } \\
\text { BTU/Ib } \\
\text { analysis date }\end{array}$ & $\begin{array}{c}11838 \\
10 / 08 / 85\end{array}$ & & & $\begin{array}{c}11742 \\
02 / 06 / 90\end{array}$ & $\begin{array}{c}11697 \\
10 / 03 / 91\end{array}$ & $\begin{array}{c}c \\
10949 \\
2 / 20 / 92\end{array}$ & $\begin{array}{c}11635 \\
5 / 27 / 93\end{array}$ \\
\hline $\begin{array}{l}\text { ALKALI EXTRACTION } \\
\% \text { transmission } \\
\text { analysis date }\end{array}$ & & $\begin{array}{c}99.8 \\
06 / 02 / 88\end{array}$ & $\begin{array}{c}95.5 \\
09 / 23 / 88\end{array}$ & $\begin{array}{c}94.4 \\
01 / 03 / 90\end{array}$ & $\begin{array}{c}94.6 \\
9 / 03 / 91\end{array}$ & $\begin{array}{c}86.2 \\
9 / 02 / 92\end{array}$ & $\begin{array}{c}95.1 \\
7 / 01 / 93\end{array}$ \\
\hline
\end{tabular}

notes: a) Good condition, sample dry. PSU MCL ash, moisture.

PSU MCL sulfur forms (revised analysis report).

b) No visible deterioration.

c) Low calorific value caused in part by high ash in this split. 


\begin{tabular}{|c|c|c|c|c|c|c|c|c|}
\hline $\begin{array}{l}\text { PSOC-1470 } \\
\text { collection date: }\end{array}$ & $\begin{array}{l}\text { 2ATT } \\
6 / 12 / 85\end{array}$ & trubb, & Ro $\max =0$ & .89 & & & & \\
\hline $\begin{array}{l}\text { Split ID } \\
\text { size } \\
\text { \% head oxygen } \\
\text { note } \\
\text { date sealed } \\
\text { date oxygen/subspl }\end{array}$ & $\begin{array}{l}\text { analysis } \\
\text { sample } 1 \\
-60 \text { mesh }\end{array}$ & $\begin{array}{l}\# 2 \text { can } \\
19 \text { of } 33 \\
-20 \text { mesh } \\
10 / 29 / 85\end{array}$ & $\begin{array}{c}30 \text { gal. } \\
2 \text { of } 2 \\
-1 " \\
8.6 \\
a\end{array}$ & $\begin{array}{l}5 \text { gal. } \\
2 \text { of } 4 \\
-1 / 4 " \\
17.9 \\
b \\
8 / 29 / 88\end{array}$ & $\begin{array}{c}30 \text { gal. } \\
2 \text { of } 2 \\
-1 / 4 " \\
7.3 \\
9 / 28 / 89\end{array}$ & $\begin{array}{l}30 \text { gal. } \\
2 \text { of } 2 \\
-1 / 4^{\prime \prime} \\
15.4 \\
11 / 16 / 90\end{array}$ & $\begin{array}{l}30 \text { gal. } \\
2 \text { of } 2 \\
-1 / 4^{\prime \prime} \\
11 / 20 / 91\end{array}$ & $\begin{array}{c}30 \text { gal. } \\
2 \text { of } 2 \\
-1 / 4 " \\
\text { c } \\
5 / 06 / 93\end{array}$ \\
\hline $\begin{array}{l}\text { PROXIMATE } \\
\% \text { moisture } \\
\% \text { ash (dry) } \\
\text { analysis date }\end{array}$ & $\begin{array}{c}2.9 \\
20.6 \\
10 / 08 / 85\end{array}$ & $\begin{array}{c}3.0 \\
21.0 \\
04 / 14 / 88\end{array}$ & $\begin{array}{c}2.5 \\
20.5 \\
09 / 19 / 88\end{array}$ & $\begin{array}{c}3.1 \\
20.3 \\
09 / 19 / 88\end{array}$ & $\begin{array}{c}3.6 \\
20.6 \\
2 / 28 / 90\end{array}$ & $\begin{array}{c}3.5 \\
21.0 \\
10 / 03 / 91\end{array}$ & $\begin{array}{c}3.7 \\
21.6 \\
2 / 20 / 92\end{array}$ & $\begin{array}{c}4.3 \\
21.0 \\
5 / 27 / 93\end{array}$ \\
\hline $\begin{array}{l}\text { GIESELER FLUIDITY } \\
\text { max. ddpm } \\
\text { fluid range } \\
\text { analysis date }\end{array}$ & $\begin{array}{r}487 \\
66 \\
10 / 11 / 85\end{array}$ & $\begin{array}{r}3 \\
33 \\
04 / 14 / 88\end{array}$ & $\begin{array}{c}55 \\
56 \\
09 / 12 / 88\end{array}$ & & $\begin{array}{c}4 \\
38 \\
2 / 28 / 90\end{array}$ & $\begin{array}{c}0 \\
0 \\
9 / 06 / 91\end{array}$ & $\begin{array}{l}\text { discon- } \\
\text { timued }\end{array}$ & \\
\hline $\begin{array}{l}\text { SULFUR FORHS (dry \%) } \\
\text { total sulfur } \\
\text { sulfate } \\
\text { pyritic } \\
\text { organic } \\
\text { analysis date }\end{array}$ & $\begin{array}{c}2.50 \\
0.04 \\
1.88 \\
0.58 \\
10 / 08 / 85\end{array}$ & & $\begin{array}{c}2.56 \\
0.37 \\
1.16 \\
1.03 \\
01 / 17 / 89\end{array}$ & $\begin{array}{c}2.58 \\
0.62 \\
0.96 \\
1.00 \\
01 / 17 / 89\end{array}$ & $\begin{array}{c}2.48 \\
0.47 \\
1.11 \\
0.90 \\
2 / 28 / 90\end{array}$ & $\begin{array}{c}2.36 \\
0.45 \\
1.00 \\
0.90 \\
10 / 03 / 91\end{array}$ & $\begin{array}{c}2.40 \\
0.61 \\
0.50 \\
1.29 \\
2 / 20 / 92\end{array}$ & $\begin{array}{c}2.41 \\
0.79 \\
0.74 \\
0.88 \\
5 / 27 / 93\end{array}$ \\
\hline $\begin{array}{l}\text { CALORIFIC VALUE (dry) } \\
\text { BTU/Ib } \\
\text { analysis date }\end{array}$ & $\begin{array}{c}11599 \\
10 / 08 / 85\end{array}$ & & & & $\begin{array}{c}11458 \\
2 / 28 / 90\end{array}$ & $\begin{array}{c}11385 \\
10 / 03 / 91\end{array}$ & $\begin{array}{c}11185 \\
2 / 20 / 92\end{array}$ & $\begin{array}{c}11105 \\
5 / 27 / 93\end{array}$ \\
\hline $\begin{array}{l}\text { ALKALI EXTRACTION } \\
\% \text { transmission } \\
\text { analysis date }\end{array}$ & & $\begin{array}{c}98.8 \\
06 / 03 / 88\end{array}$ & $\begin{array}{c}95.8 \\
09 / 32 / 88\end{array}$ & & $\begin{array}{c}92.9 \\
2 / 22 / 90\end{array}$ & $\begin{array}{c}83.0 \\
9 / 06 / 91\end{array}$ & $\begin{array}{c}94.8 \\
9 / 03 / 92\end{array}$ & $\begin{array}{c}92.1 \\
7 / 02 / 93\end{array}$ \\
\hline
\end{tabular}

notes: a) Sample moist, trace of yellow sulfate on top surface sample was crushed to -1 " and then split, plastometer data fluid range estimated. PSU MCL ash, moisture. PSU MCL sulfur forms (revised analysis report).

b) white fibrous sulfate visible on surface. PSU MCL ash, moisture. PSU MCL sulfur forms ( revised analysis report).

c) Occasional masses of white fibrous sulfates visible on surface. 


\begin{tabular}{|c|c|c|c|c|c|c|c|}
\hline $\begin{array}{l}\text { PSOC-1471 } \\
\text { collection date: }\end{array}$ & $\begin{array}{l}\text { EE WEE } \\
6 / 13 / 85\end{array}$ & huAb, & Ro $\max =$ & & & & \\
\hline $\begin{array}{l}\text { Split ID } \\
\text { size } \\
\text { \% head oxygen } \\
\text { note } \\
\text { date sealed } \\
\text { date oxygen/subspl }\end{array}$ & $\begin{array}{l}\text { analys is } \\
\text { sample } 1 \\
-60 \text { mesh }\end{array}$ & $\begin{array}{l}\# 2 \text { can } \\
20 \text { of } 37 \\
-20 \text { mesh } \\
10 / 29 / 85\end{array}$ & $\begin{array}{c}30 \text { gal. } \\
1 \text { of } 3 \\
-1 / 4 " \\
15.7 \\
a \\
08 / 21 / 85 \\
8.30 / 88\end{array}$ & $\begin{array}{l}30 \text { gal. } \\
1 \text { of } 3 \\
-1 / 4^{\circ} \\
20.3 \\
9 / 28 / 89\end{array}$ & $\begin{array}{l}30 \text { gal. } \\
1 \text { of } 3 \\
-1 / 4^{\prime \prime} \\
20.4 \\
12 / 20 / 90\end{array}$ & $\begin{array}{l}30 \mathrm{gal} \\
1 \text { of } 3 \\
-1 / 4 " \\
11 / 20 / 91\end{array}$ & $\begin{array}{l}30 \text { gal. } \\
\text { 1 of } 3 \\
-1 / 4^{\prime \prime} \\
\text { b } \\
5 / 06 / 93\end{array}$ \\
\hline $\begin{array}{l}\text { PROXIMATE } \\
\% \text { moisture } \\
\% \text { ash (dry) } \\
\text { analysis date }\end{array}$ & $\begin{array}{c}2.5 \\
8.0 \\
10 / 08 / 85\end{array}$ & $\begin{array}{c}2.7 \\
7.7 \\
04 / 15 / 88\end{array}$ & $\begin{array}{c}2.2 \\
7.9 \\
10 / 12 / 88\end{array}$ & $\begin{array}{c}3.0 \\
8.0 \\
4 / 17 / 90\end{array}$ & $\begin{array}{c}2.1 \\
8.9 \\
10 / 03 / 91\end{array}$ & $\begin{array}{c}2.9 \\
6.5 \\
2 / 20 / 92\end{array}$ & $\begin{array}{l}3.1 \\
7.9 \\
5 / 27 / 93\end{array}$ \\
\hline $\begin{array}{l}\text { GIESELER FLUIDITY } \\
\text { max. ddpm } \\
\text { fluid range } \\
\text { analysis date }\end{array}$ & $\begin{array}{r}90 \\
60 \\
10 / 16 / 85\end{array}$ & $\begin{array}{r}116 \\
66 \\
04 / 15 / 88\end{array}$ & $\begin{array}{c}3 \\
39 \\
10 / 14 / 88\end{array}$ & $\begin{array}{c}2 \\
22 \\
2 / 23 / 90\end{array}$ & $\begin{array}{l}0 \\
0 \\
9 / 10 / 91\end{array}$ & $\begin{array}{l}\text { discon- } \\
\text { timued }\end{array}$ & \\
\hline $\begin{array}{l}\text { SULFUR FORMS (dry \%) } \\
\text { total sulfur } \\
\text { sulfate } \\
\text { pyritic } \\
\text { organic } \\
\text { analysis date }\end{array}$ & $\begin{array}{c}1.04 \\
0.03 \\
0.22 \\
0.79 \\
10 / 08 / 85\end{array}$ & & $\begin{array}{c}1.01 \\
0.10 \\
0.13 \\
0.78 \\
01 / 17 / 89\end{array}$ & $\begin{array}{c}1.06 \\
0.11 \\
0.11 \\
0.84 \\
4 / 17 / 90\end{array}$ & $\begin{array}{c}1.03 \\
0.14 \\
0.13 \\
0.76 \\
10 / 03 / 91\end{array}$ & $\begin{array}{l}1.00 \\
0.05 \\
0.09 \\
0.85 \\
2 / 20 / 92\end{array}$ & $\begin{array}{c}1.02 \\
0.14 \\
0.07 \\
0.81 \\
5 / 27 / 93\end{array}$ \\
\hline $\begin{array}{l}\text { CALORIFIC VALUE (dry) } \\
\text { BTU/Ib } \\
\text { analysis date }\end{array}$ & $\begin{array}{c}13715 \\
10 / 08 / 85\end{array}$ & & & $\begin{array}{c}13539 \\
4 / 17 / 90\end{array}$ & $\begin{array}{c}13370 \\
10 / 03 / 91\end{array}$ & $\begin{array}{c}13787 \\
2 / 20 / 92\end{array}$ & $\begin{array}{c}13516 \\
5 / 27 / 93\end{array}$ \\
\hline $\begin{array}{l}\text { ALKALI EXTRACTION } \\
\% \text { transmission } \\
\text { analysis date }\end{array}$ & & $\begin{array}{c}94.7 \\
06 / 03 / 88\end{array}$ & $\begin{array}{c}97.2 \\
10 / 05 / 88\end{array}$ & $\begin{array}{c}87.1 \\
2 / 23 / 90\end{array}$ & $\begin{array}{l}88.2 \\
9 / 10 / 91\end{array}$ & $\begin{array}{c}90.5 \\
9 / 03 / 92\end{array}$ & $\begin{array}{c}96.6 \\
7 / 06 / 93\end{array}$ \\
\hline
\end{tabular}

notes: a) Dry, good condition. PSU MCL ash, moisture.

PSU MCL sulfur forms (revised analysis report).

b) Minor amounts of yellow coatings on some particles. 


\begin{tabular}{|c|c|c|c|c|c|c|c|}
\hline $\begin{array}{l}\text { PSOC-1474 } \\
\text { collection date: }\end{array}$ & $\begin{array}{l}\text { PLASHDAM } \\
16 /=1 / 85\end{array}$ & mb, & Ro $\max =1$ & & & & \\
\hline $\begin{array}{l}\text { Split ID } \\
\text { size } \\
\text { \% head oxygen } \\
\text { note } \\
\text { date sealed } \\
\text { date oxygen/subspl }\end{array}$ & $\begin{array}{l}\text { analysis } \\
\text { sample } 1 \\
-60 \text { mesh }\end{array}$ & $\begin{array}{l}\# 2 \text { can } \\
26 \text { of } 33 \\
-20 \text { mesh } \\
10 / 29 / 85\end{array}$ & $\begin{array}{c}30 \text { gal } \\
3 \text { of } 3 \\
-1 / 4 " \\
16.3 \\
a \\
08 / 27 / 85 \\
8 / 30 / 88\end{array}$ & $\begin{array}{c}30 \text { gal. } \\
3 \text { of } 3 \\
-1 / 4 " 1 \\
16.3 \\
9 / 28 / 89\end{array}$ & $\begin{array}{l}30 \text { ga } 1 \\
3 \text { of } 3 \\
-1 / 4 " \\
12 / 20 / 90\end{array}$ & $\begin{array}{l}30 \text { gal. } \\
3 \text { of } 3 \\
-1 / 4 " \\
11 / 20 / 91\end{array}$ & $\begin{array}{l}30 \text { gal. } \\
3 \text { of } 3 \\
-1 / 4 " \\
\text { b } \\
5 / 06 / 93\end{array}$ \\
\hline $\begin{array}{l}\text { PROXIMATE } \\
\% \text { moisture } \\
\% \text { ash (dry) } \\
\text { analysis date }\end{array}$ & $\begin{array}{c}1.6 \\
12.4 \\
10 / 22 / 85\end{array}$ & $\begin{array}{c}1.1 \\
13.4 \\
04 / 18 / 88\end{array}$ & $\begin{array}{c}1.2 \\
12.3 \\
10 / 12 / 88\end{array}$ & $\begin{array}{c}1.9 \\
12.4 \\
2 / 28 / 90\end{array}$ & $\begin{array}{c}1.8 \\
13.0 \\
10 / 03 / 91\end{array}$ & $\begin{array}{c}1.8 \\
17.4 \\
2 / 21 / 92\end{array}$ & $\begin{array}{c}2.1 \\
12.4 \\
5 / 26 / 93\end{array}$ \\
\hline $\begin{array}{l}\text { GIESELER FLUIDITY } \\
\text { max. ddpm } \\
\text { fluid range } \\
\text { analysis date }\end{array}$ & $\begin{array}{r}14490 \\
99 \\
09 / 17 / 85\end{array}$ & $\begin{array}{r}743 \\
83 \\
04 / 18 / 88\end{array}$ & $\begin{array}{c}556 \\
84 \\
10 / 18 / 88\end{array}$ & $\begin{array}{c}310 \\
78 \\
2 / 28 / 90\end{array}$ & $\begin{array}{l}0 \\
0 \\
9 / 11 / 91\end{array}$ & $\begin{array}{l}\text { discon- } \\
\text { tirued }\end{array}$ & \\
\hline $\begin{array}{l}\text { SULFUR FORIS (dry \%) } \\
\text { total sulfur } \\
\text { sulfate } \\
\text { Pyritic } \\
\text { organic } \\
\text { analysis date }\end{array}$ & $\begin{array}{c}0.73 \\
0.01 \\
0.16 \\
0.56 \\
10 / 22 / 85\end{array}$ & & $\begin{array}{c}0.70 \\
0.05 \\
0.11 \\
0.54 \\
01 / 17 / 89\end{array}$ & $\begin{array}{c}0.68 \\
0.07 \\
0.07 \\
0.54 \\
4 / 17 / 90\end{array}$ & $\begin{array}{c}0.68 \\
0.07 \\
0.10 \\
0.51 \\
10 / 03 / 91\end{array}$ & $\begin{array}{c}0.61 \\
0.03 \\
0.04 \\
0.54 \\
2 / 21 / 92\end{array}$ & $\begin{array}{r}0.66 \\
0.09 \\
0.06 \\
0.51 \\
5 / 27 / 93\end{array}$ \\
\hline $\begin{array}{l}\text { CALORIFIC VALUE (dry) } \\
\text { BTU/lb } \\
\text { analysis date }\end{array}$ & $\begin{array}{c}13588 \\
10 / 22 / 85\end{array}$ & & & $\begin{array}{c}13428 \\
4 / 17 / 90\end{array}$ & $\begin{array}{c}13370 \\
10 / 03 / 91\end{array}$ & $\begin{array}{c}C \\
12642 \\
2 / 21 / 92\end{array}$ & $\begin{array}{r}13478 \\
5 / 27 / 93\end{array}$ \\
\hline $\begin{array}{l}\text { ALKALI EXTRACTION } \\
\text { \% transmission } \\
\text { analysis date }\end{array}$ & & $\begin{array}{c}98.5 \\
06 / 03 / 88\end{array}$ & $\begin{array}{c}98.9 \\
10 / 05 / 88\end{array}$ & $\begin{array}{c}92.6 \\
2 / 28 / 90\end{array}$ & $\begin{array}{r}94.8 \\
9 / 11 / 91\end{array}$ & $\begin{array}{c}93.5 \\
9 / 03 / 92\end{array}$ & $\begin{array}{c}97.9 \\
7 / 07 / 93\end{array}$ \\
\hline
\end{tabular}

notes: a) Dry, good condition. PSU MCL ash, moisture.

PSU MCL sulfur forms (revised analysis report).

b) No visible deterioration.

c) Low calorific value caused in part by high ash in this split. 


\begin{tabular}{|c|c|c|c|c|c|}
\hline $\begin{array}{l}\text { PSOC-1475 } \\
\text { collection date: }\end{array}$ & $\begin{array}{l}\text { 1khorn \#3 } \\
6 / 18 / 85\end{array}$ & Invab, & Ro $\max =0$ & 97 & \\
\hline $\begin{array}{l}\text { Split I0 } \\
\text { size } \\
\text { \% head oxygen } \\
\text { note } \\
\text { date sealed } \\
\text { date oxygen/subspl }\end{array}$ & $\begin{array}{l}\text { analysis } \\
\text { sample } 1 \\
-60 \text { mesh }\end{array}$ & $\begin{array}{l}\# 2 \text { can } \\
27 \text { of } 33 \\
-20 \text { mesh } \\
10 / 29 / 85\end{array}$ & $\begin{array}{c}30 \text { gal. } \\
4 \text { of } 4 \\
-1 / 4 " \\
9.5 \\
a \\
08 / 28 / 88 \\
9 / 01 / 88\end{array}$ & $\begin{array}{l}30 \text { gal. } \\
4 \text { of } 4 \\
-1 / 4 " \\
13.5 \\
9 / 28 / 89\end{array}$ & $\begin{array}{l}30 \mathrm{gal} \\
4 \text { of } 4 \\
-1 / 4 "\end{array}$ \\
\hline $\begin{array}{l}\text { PROXIMATE } \\
\% \text { moisture } \\
\% \text { ash (dry) } \\
\text { analysis date }\end{array}$ & $\begin{array}{c}3.4 \\
3.3 \\
10 / 23 / 85\end{array}$ & $\begin{array}{c}4.3 \\
1.5 \\
04 / 19 / 88\end{array}$ & $\begin{array}{c}2.9 \\
2.9 \\
10 / 12 / 88\end{array}$ & $\begin{array}{c}3.5 \\
3.2 \\
4 / 17 / 90\end{array}$ & $\begin{array}{c}3.7 \\
3.4 \\
10 / 03 / 91\end{array}$ \\
\hline $\begin{array}{l}\text { OIESELER FLUIDITY } \\
\text { max. ddpm } \\
\text { fluid range } \\
\text { analysis date }\end{array}$ & $\begin{array}{r}177 \\
59 \\
09 / 18 / 85\end{array}$ & $\begin{array}{r}15 \\
59 \\
04 / 19 / 88\end{array}$ & $\begin{array}{c}3 \\
33 \\
10 / 20 / 88\end{array}$ & $\begin{array}{c}2 \\
30 \\
3 / 02 / 90\end{array}$ & $\begin{array}{c}1 \\
2 \\
9 / 12 / 91\end{array}$ \\
\hline $\begin{array}{l}\text { SULFUR FORAs (dry \%) } \\
\text { total sulfur } \\
\text { sulfate } \\
\text { pyritic } \\
\text { organic } \\
\text { analysis date }\end{array}$ & $\begin{array}{c}0.98 \\
0.02 \\
0.32 \\
0.64 \\
10 / 23 / 85\end{array}$ & & $\begin{array}{c}0.93 \\
0.06 \\
0.22 \\
0.65 \\
01 / 17 / 89\end{array}$ & $\begin{array}{c}0.87 \\
0.08 \\
0.20 \\
0.59 \\
4 / 17 / 90\end{array}$ & $\begin{array}{c}0.92 \\
0.10 \\
0.22 \\
0.61 \\
10 / 03 / 91\end{array}$ \\
\hline $\begin{array}{l}\text { CALORIFIC VALUE (dry) } \\
\text { BTU/lb } \\
\text { analysis date }\end{array}$ & $\begin{array}{c}14540 \\
10 / 23 / 85\end{array}$ & & & $\begin{array}{c}14392 \\
4 / 17 / 90\end{array}$ & $\begin{array}{c}14348 \\
10 / 03 / 91\end{array}$ \\
\hline $\begin{array}{l}\text { ALKALI EXTRACTION } \\
\text { \% transmission } \\
\text { analysis date }\end{array}$ & & $\begin{array}{c}95.9 \\
06 / 03 / 88\end{array}$ & $\begin{array}{c}97.4 \\
10 / 05 / 88\end{array}$ & $\begin{array}{c}92.1 \\
3 / 02 / 90\end{array}$ & $\begin{array}{c}92.5 \\
9 / 12 / 91\end{array}$ \\
\hline
\end{tabular}

note: a) Sample discrepancy with ash analysis. PSU MCL ash, moisture. PSU MCL sulfur forms ( re' sed analysis report). 


\begin{tabular}{|c|c|c|c|c|c|c|c|}
\hline $\begin{array}{l}\text { PSOC-1482 } \\
\text { collection date: }\end{array}$ & $\begin{array}{l}\text { AGEL } \\
7 / 01 / 85\end{array}$ & ligh, & Ro $\max =0$ & & & & \\
\hline $\begin{array}{l}\text { Spiit ID } \\
\text { size } \\
\text { \% head oxygen } \\
\text { note } \\
\text { date sealed } \\
\text { date oxygen/subspl }\end{array}$ & $\begin{array}{l}\text { arralysis } \\
\text { sampie } 1 \\
\text { - 60mesh }\end{array}$ & $\begin{array}{l}\text { analys is } \\
\text { sample } 2 \\
-20 \text { mesh }\end{array}$ & $\begin{array}{c}30 \text { gal } \\
1 \text { of } 3 \\
-1 / 4^{\prime \prime} \\
1.6 \\
a \\
08 / 29 / 85 \\
9 / 23 / 88\end{array}$ & $\begin{array}{c}30 \mathrm{gal} \\
1 \text { of } 3 \\
-1 / 4^{n} \\
6.6 \\
9 / 28 / 89\end{array}$ & $\begin{array}{l}30 \mathrm{sel} \\
1 \text { of } 3 \\
-1 / 4^{\circ} \\
12 / 20 / 90\end{array}$ & $\begin{array}{l}30 \text { gal. } \\
1 \text { of } 3 \\
-1 / 4 " \\
11 / 20 / 91\end{array}$ & $\begin{array}{l}30 \text { gal. } \\
1 \text { of } 3 \\
-1 / 4 " \\
\text { b } \\
5 / 06 / 93\end{array}$ \\
\hline $\begin{array}{l}\text { PROXImATE } \\
\% \text { mois ture } \\
\% \text { ash (dry) } \\
\text { analysis date }\end{array}$ & $\begin{array}{c}34.7 \\
9.0 \\
10 / 24 / 85\end{array}$ & $\begin{array}{c}34.5 \\
8.5 \\
12 / 11 / 86\end{array}$ & $\begin{array}{c}32.9 \\
7.4 \\
10 / 12 / 88\end{array}$ & $\begin{array}{c}34.4 \\
9.2 \\
2 / 06 / 90\end{array}$ & $\begin{array}{c}36.4 \\
9.0 \\
1 / 23 / 91\end{array}$ & $\begin{array}{c}34.4 \\
6.9 \\
2 / 21 / 92\end{array}$ & $\begin{array}{c}35.6 \\
9.1 \\
5 / 27,93\end{array}$ \\
\hline $\begin{array}{l}\text { SULFUR FORis (dry \%) } \\
\text { total sulfur } \\
\text { sulfate } \\
\text { pyritic } \\
\text { organic } \\
\text { analysis date }\end{array}$ & $\begin{array}{c}0.86 \\
0.02 \\
0.09 \\
0.75 \\
10 / 24 / 85\end{array}$ & $12 / 11 / 86$ & $\begin{array}{c}0.82 \\
0.14 \\
0.22 \\
0.46 \\
01 / 17 / 89\end{array}$ & $\begin{array}{c}0.88 \\
0.17 \\
0.20 \\
0.51 \\
2 / 06 / 90\end{array}$ & $\begin{array}{r}0.82 \\
0.18 \\
0.14 \\
0.51 \\
1 / 23 / 91\end{array}$ & $\begin{array}{c}0.79 \\
0.11 \\
0.07 \\
0.61 \\
2 / 21 / 92\end{array}$ & $\begin{array}{c}0.85 \\
0.25 \\
0.08 \\
0.53 \\
5 / 27 / 93\end{array}$ \\
\hline $\begin{array}{l}\text { CALORIFIC VALUE (dry) } \\
\text { BTU/Ib } \\
\text { analysis cate }\end{array}$ & $\begin{array}{c}11118 \\
10 / 24 / 85\end{array}$ & & & $\begin{array}{c}10695 \\
2 / 06 / 90\end{array}$ & $\begin{array}{c}10783 \\
1 / 25 / 91\end{array}$ & $\begin{array}{c}10872 \\
2 / 21 / 92\end{array}$ & $\begin{array}{c}10583 \\
5 / 27 / 93\end{array}$ \\
\hline
\end{tabular}

notes: a) Sample moist, trace yellow \& white sulfates. PSU MCL ash, moisture. PSU MCL sulfur forms (revised analysis report).

b) Minor amounts of yellow coatings and white acicular crystals. 


\begin{tabular}{|c|c|c|c|c|c|}
\hline $\begin{array}{l}\text { PSOC-1485 } \\
\text { collection date: }\end{array}$ & $\begin{array}{l}\text { CSEBUD } \\
7 / 05 / 86\end{array}$ & subB, & Ro $\max =0$ & & \multirow[b]{2}{*}{$\begin{array}{l}\text { super- } \\
\text { seded } \\
\text { by } \\
\text { DECS-10 }\end{array}$} \\
\hline $\begin{array}{l}\text { Split Io } \\
\text { size } \\
\text { \% head oxygen } \\
\text { note } \\
\text { date sealed } \\
\text { date oxygen/subspl }\end{array}$ & $\begin{array}{l}\text { analysis } \\
\text { sample } 1 \\
-60 \text { mesh }\end{array}$ & $\begin{array}{l}2 \text { can } \\
15 \text { of } 31 \\
-20 \text { mesh } \\
04 / 01 / 86\end{array}$ & $\begin{array}{c}30 \text { gal } \\
3 \text { of } 3 \\
-1 / 4^{\prime \prime} \\
8.1 \\
a \\
03 / 25 / 86 \\
9 / 26 / 88\end{array}$ & $\begin{array}{l}30 \text { gal. } \\
3 \text { of } 3 \\
-1 / 4^{\prime \prime} \\
10.4 \\
9 / 2>/ 89\end{array}$ & \\
\hline $\begin{array}{l}\text { Proximate } \\
\% \text { mois ture } \\
\% \text { ash (dry) } \\
\text { analysis date }\end{array}$ & $\begin{array}{c}25.4 \\
11.4 \\
04 / 24 / 8\end{array}$ & $\begin{array}{c}25.0 \\
10.7 \\
06 / 13 / 88\end{array}$ & $\begin{array}{c}21.9 \\
10.4 \\
10 / 12 / 88\end{array}$ & $\begin{array}{c}25.1 \\
11.4 \\
12 / 14 / 89\end{array}$ & \\
\hline $\begin{array}{l}\text { SULFUR FORAs (dry \%) } \\
\text { total sulfur } \\
\text { sulfate } \\
\text { pyritic } \\
\text { organic } \\
\text { analysis date }\end{array}$ & \begin{tabular}{|c}
0.88 \\
0.00 \\
0.02 \\
0.86 \\
$04 / 24 / 86$
\end{tabular} & & $\begin{array}{c}0.86 \\
0.08 \\
0.23 \\
0.55 \\
01 / 17 / 89\end{array}$ & $\begin{array}{c}0.77 \\
0.13 \\
0.24 \\
0.40 \\
12 / 14 / 89\end{array}$ & \\
\hline $\begin{array}{l}\text { CALORIFIC VALUE (dry) } \\
\text { BTU/lb } \\
\text { analysis date }\end{array}$ & $\begin{array}{c}11620 \\
04 / 24 / 86\end{array}$ & & & $\begin{array}{c}11449 \\
12 / 14 / 89\end{array}$ & \\
\hline $\begin{array}{l}\text { ALKALI EXTRACTION } \\
\% \text { transmission } \\
\text { analysis date }\end{array}$ & & $\begin{array}{c}0.2 \\
06 / 15 / 88\end{array}$ & $\begin{array}{c}0.4 \\
10 / 06 / 88\end{array}$ & $\begin{array}{l}\text { discon- } \\
\text { tinued }\end{array}$ & \\
\hline
\end{tabular}

notes: a) Sample moist, good condition. PSU MCL ash, moisture. PSU MCL sulfur forms (revised analysis report). 


\begin{tabular}{|c|c|c|c|c|c|c|c|}
\hline $\begin{array}{l}\text { PSOC-1486 } \\
\text { collection date: }\end{array}$ & $\begin{array}{l}\text { IE DIRTY } \\
7 / 09 / 85\end{array}$ & subs, & Ro $\max =c$ & & & & \\
\hline $\begin{array}{l}\text { Split ID } \\
\text { size } \\
\text { \% head oxygen } \\
\text { note } \\
\text { date sealed } \\
\text { date oxygen/subspl }\end{array}$ & $\begin{array}{l}\text { analysis } \\
\text { sample } 1 \\
-60 \text { mesh }\end{array}$ & $\begin{array}{l}2 \text { can } \\
17 \text { of } 31 \\
-20 \text { mesh } \\
04 / 02 / 86\end{array}$ & $\begin{array}{c}30 \text { sal } \\
3 \text { of } 3 \\
-1 / 4 " 1 \\
4.3 \\
a \\
03 / 26 / 86 \\
9 / 26 / 88\end{array}$ & $\begin{array}{c}30 \text { gal. } \\
3 \text { of } 3 \\
-1 / 4 " \\
7.0 \\
9 / 29 / 89\end{array}$ & $\begin{array}{l}30 \text { gol. } \\
3 \text { of } 3 \\
-1 / 4^{\circ} \\
12 / 20 / 90\end{array}$ & $\begin{array}{l}30 \text { of } \\
3 \text { of } 3 \\
-1 / 0+1 \\
11 / 20 / 91\end{array}$ & $\begin{array}{c}30 \text { gal. } \\
3 \text { of } 3 \\
-1 / 4^{\circ} \\
\text { c } \\
5 / 06 / 93\end{array}$ \\
\hline $\begin{array}{l}\text { ProxImate } \\
\% \text { mois ture } \\
\% \text { ash (dry) } \\
\text { analysis date }\end{array}$ & $\begin{array}{c}20.1 \\
19.4 \\
04 / 24 / 86\end{array}$ & $\begin{array}{c}18.8 \\
18.7 \\
06 / 14 / 88\end{array}$ & $\begin{array}{c}17.3 \\
17.2 \\
10 / 12 / 88\end{array}$ & $\begin{array}{c}19.5 \\
19.3 \\
12 / 14 / 89\end{array}$ & $\begin{array}{l}20.6 \\
20.1 \\
1 / 23 / 91\end{array}$ & $\begin{array}{c}18.7 \\
14.1 \\
2 / 21 / 92\end{array}$ & $\begin{array}{c}19.4 \\
19.1 \\
5 / 27 / 93\end{array}$ \\
\hline $\begin{array}{l}\text { SUlfuR Forins (dry \%) } \\
\text { total sulfur } \\
\text { sulfate } \\
\text { pyritic } \\
\text { organic } \\
\text { analysis date }\end{array}$ & $\begin{array}{c}0.94 \\
0.01 \\
0.25 \\
0.68 \\
04 / 24 / 86\end{array}$ & & $\begin{array}{c}0.91 \\
0.17 \\
0.16 \\
0.58 \\
01 / 17 / 89\end{array}$ & $\begin{array}{r}0.77 \\
0.27 \\
0.12 \\
0.38 \\
12 / 14 / 89\end{array}$ & $\begin{array}{r}0.90 \\
0.28 \\
0.12 \\
0.50 \\
1 / 23 / 91\end{array}$ & $\begin{array}{c}\text { (b) } \\
0.68 \\
0.03 \\
0.03 \\
0.61 \\
2 / 21 / 92\end{array}$ & $\begin{array}{c}0.86 \\
0.27 \\
0.05 \\
0.54 \\
5 / 27 / 93\end{array}$ \\
\hline $\begin{array}{l}\text { CALORIFIC VALUE (dry) } \\
\text { BTU/1b } \\
\text { analysis date }\end{array}$ & $\begin{array}{c}10447 \\
04 / 24 / 86\end{array}$ & & & $\begin{array}{c}10267 \\
12 / 14 / 89\end{array}$ & $\begin{array}{r}10116 \\
1 / 23 / 91\end{array}$ & $\begin{array}{c}\text { d } \\
10919 \\
2 / 21 / 92\end{array}$ & $\begin{array}{c}10016 \\
5 / 27 / 93\end{array}$ \\
\hline $\begin{array}{l}\text { ALKALI EXTRACTION } \\
\text { \% transmission } \\
\text { analysis date }\end{array}$ & & $\begin{array}{c}0.0 \\
06 / 15 / 88\end{array}$ & $\begin{array}{c}0.0 \\
10 / 06 / 88\end{array}$ & $\begin{array}{l}\text { discon- } \\
\text { timued }\end{array}$ & & & \\
\hline
\end{tabular}

notes: a) Sample dry, trace yellow sulfate. PSU MCL ash, moisture. PSU MCL sulfur forms (revised analysis report).

b) Sulfate analysis questionable in view of results from other years.

c) Traces of brown and yellow deposits on particles, particularly deeper in barrel. White streaks on barrel.

d) High calorific value caused in part by low ash in this split. 


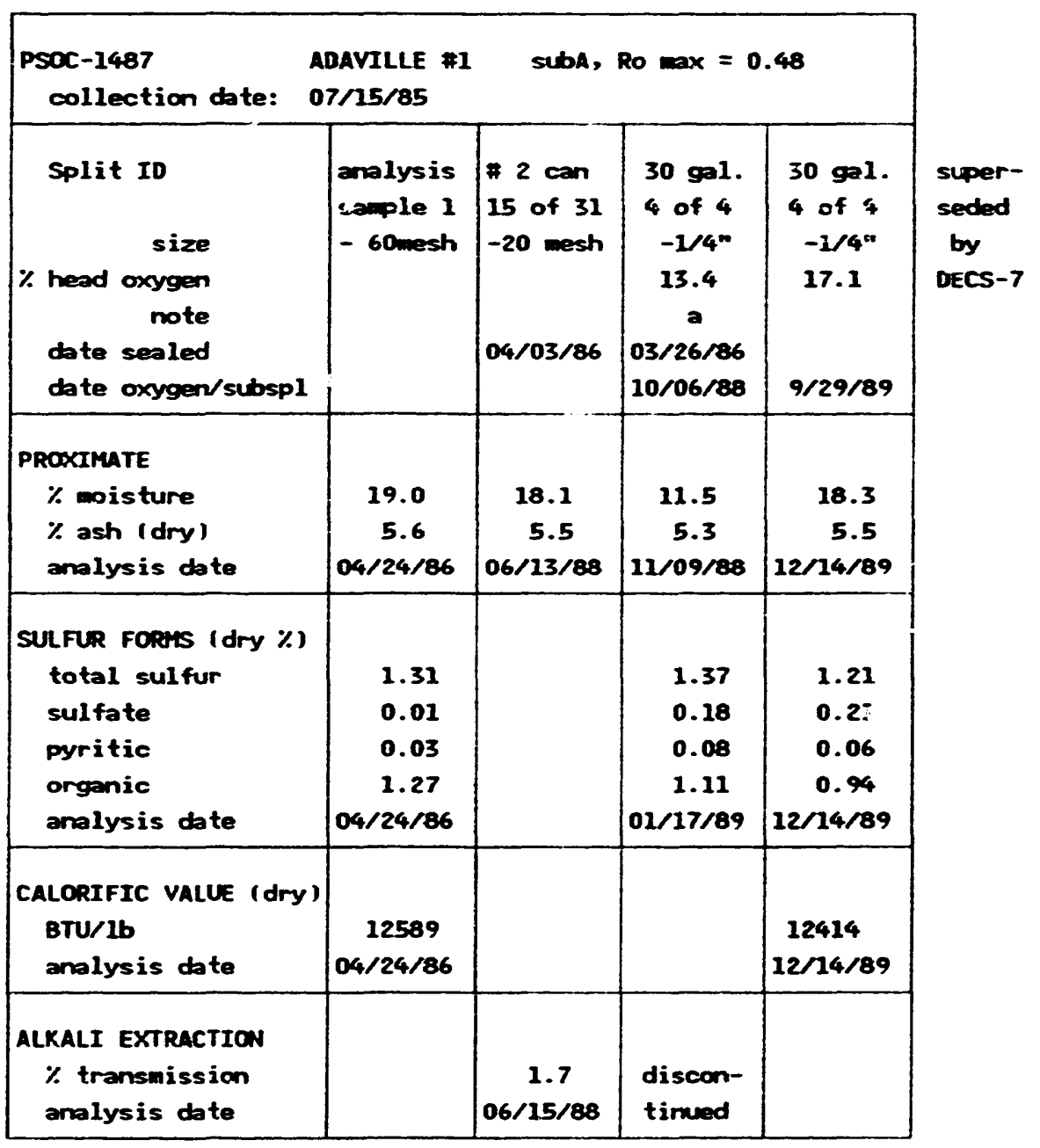

notes: Sample dry, trace yellow sulfate. PSU MCL sulfur forms (revised analysis report). 


\begin{tabular}{|c|c|c|c|c|}
\hline $\begin{array}{l}\text { PSOC-1488 } \\
\text { collection date: }\end{array}$ & $\begin{array}{l}\text { OIETZ } \\
37 / 17 / 85\end{array}$ & subs, & Ro $\max =0$. & 47 \\
\hline $\begin{array}{l}\text { Split ID } \\
\text { size } \\
\text { \% head oxygen } \\
\text { note } \\
\text { dote sealed } \\
\text { dote oxygen/subspl }\end{array}$ & $\begin{array}{l}\text { amaiysis } \\
\text { sample } 1 \\
-60 \text { mesh }\end{array}$ & $\begin{array}{c}30 \text { gal } \\
3 \text { of } 4 \\
-1 / 40 \\
3.4 \\
a \\
3 / 27 / 86 \\
10 / 03 / 88\end{array}$ & $\begin{array}{c}30 \mathrm{gal} \\
3 \text { of } 4 \\
-1 / 4^{4} \\
9.9 \\
10 / 03 / 89\end{array}$ & $\begin{array}{l}\text { super- } \\
\text { saded } \\
\text { by } \\
\text { DECS-9 }\end{array}$ \\
\hline $\begin{array}{l}\text { PROXImaTe } \\
\text { \% moisture } \\
\% \text { ash (dry) } \\
\text { analysis date }\end{array}$ & $\begin{array}{c}23.7 \\
5.4 \\
04 / 25 / 86\end{array}$ & $\begin{array}{c}14.6 \\
4.6 \\
11 / 09 / 88\end{array}$ & $\begin{array}{c}23.1 \\
5.2 \\
12 / 14 / 89\end{array}$ & \\
\hline $\begin{array}{l}\text { SULFU FOpis (dry \%) } \\
\text { total sulfur } \\
\text { sulfate } \\
\text { pyritic } \\
\text { organic } \\
\text { aralysis date }\end{array}$ & $\begin{array}{c}0.50 \\
0.00 \\
0.02 \\
0.48 \\
04 / 25 / 86\end{array}$ & $\begin{array}{c}0.61 \\
0.06 \\
0.08 \\
0.47 \\
01 / 17 / 89\end{array}$ & $\begin{array}{c}0.38 \\
0.08 \\
0.04 \\
0.26 \\
12 / 14 / 89\end{array}$ & \\
\hline $\begin{array}{l}\text { CALORIFIC VALUE (dry) } \\
\text { BTU/Ib } \\
\text { anolysis date }\end{array}$ & $\begin{array}{c}12519 \\
04 / 25 / 86\end{array}$ & & $\begin{array}{c}12387 \\
12 / 14 / 89\end{array}$ & \\
\hline
\end{tabular}

notes: a) Sample moist, good condition. PSU MCL sulfur forms (revised analysis report). 


\begin{tabular}{|c|c|c|c|c|c|c|}
\hline $\begin{array}{l}\text { PSOC-1489 } \\
\text { collection date: }\end{array}$ & $\begin{array}{l}\text { UST } \\
7 / 19 / 85\end{array}$ & ligA, & Ro $\max =$ & & & \\
\hline $\begin{array}{l}\text { Split ID } \\
\text { size } \\
\text { \% head oxygen } \\
\text { note } \\
\text { date sealed } \\
\text { date oxygen/subspl }\end{array}$ & $\begin{array}{l}\text { analysis } \\
\text { sample } 1 \\
-6 \text { mesh }\end{array}$ & $\begin{array}{c}30 \text { gal. } \\
4 \text { of } 4 \\
-1 / 4 " \\
10.6 \\
a \\
10 / 06 / 88\end{array}$ & $\begin{array}{l}30 \text { gal. } \\
4 \text { of } 4 \\
-1 / 4^{\circ 1} \\
14.3 \\
9 / 29 / 89\end{array}$ & $\begin{array}{l}30 \mathrm{gel} \\
4 \text { of } 4 \\
-1 / 4 \cdot\end{array}$ & $\begin{array}{l}30 \text { pl. } \\
4 \text { of } 4 \\
-1 / 4 " \\
11 / 20 / 91\end{array}$ & $\begin{array}{l}30 \text { gal. } \\
4 \text { of } 4 \\
-1 / 4= \\
\text { c } \\
5 / 06 / 93\end{array}$ \\
\hline $\begin{array}{l}\text { PROXIMATE } \\
\% \text { moisture } \\
\% \text { ash (dry) } \\
\text { analysis date }\end{array}$ & $\begin{array}{c}37.2 \\
11.0 \\
04 / 25 / 86\end{array}$ & $\begin{array}{c}20.1 \\
10.5 \\
11 / 09 / 88\end{array}$ & $\begin{array}{c}34.8 \\
10.9 \\
12 / 14 / 89\end{array}$ & $\begin{array}{c}37.4 \\
10.1 \\
1 / 23 / 91\end{array}$ & $\begin{array}{c}34.9 \\
6.5 \\
2 / 21 / 92\end{array}$ & $\begin{array}{c}34.2 \\
9.8 \\
5 / 24 / 93\end{array}$ \\
\hline $\begin{array}{l}\text { SULFUR FORis (dry \%) } \\
\text { total sulfur } \\
\text { sulfate } \\
\text { pyritic } \\
\text { organic } \\
\text { analysis date }\end{array}$ & $\begin{array}{c}0.77 \\
0.00 \\
0.02 \\
0.75 \\
04 / 25 / 86\end{array}$ & $\begin{array}{c}0.77 \\
0.26 \\
0.25 \\
0.26 \\
01 / 17 / 89\end{array}$ & $\begin{array}{c}0.52 \\
0.32 \\
0.15 \\
0.04 \\
12 / 14 / 89\end{array}$ & $\begin{array}{r}0.69 \\
0.26 \\
0.10 \\
0.33 \\
1 / 23 / 91\end{array}$ & $\begin{array}{c}\text { (b) } \\
0.38 \\
0.08 \\
0.03 \\
0.27 \\
2 / 21 / 92\end{array}$ & $\begin{array}{c}0.65 \\
0.31 \\
0.05 \\
0.29 \\
5 / 27 / 93\end{array}$ \\
\hline $\begin{array}{l}\text { CALORIFIC VALUE (dry) } \\
\text { BTU/Ib } \\
\text { analysis date }\end{array}$ & $\begin{array}{c}10668 \\
04 / 25 / 86\end{array}$ & & $\begin{array}{c}10515 \\
12 / 14 / 89\end{array}$ & $\begin{array}{r}10483 \\
1 / 31 / 91\end{array}$ & $\begin{array}{c}d \\
10556 \\
2 / 21 / 92\end{array}$ & $\begin{array}{c}10328 \\
5 / 27 / 93\end{array}$ \\
\hline
\end{tabular}

notes: a) Sample moist, trace yellow and white sulfate.

PSU MCL sulfur forms (revised analysis report).

b: Sulfate analysis questionable on view of results from other years.

c) Moderate amount of white coatings on particles.

d) High calorific value caused in part by low ash in this split. 


\begin{tabular}{|c|c|c|c|c|c|c|c|c|}
\hline $\begin{array}{l}\text { PSOC-1490 } \\
\text { collection date: }\end{array}$ & $\begin{array}{l}\text { RAZTL BLOC } \\
12985\end{array}$ & hucb, & Ro $\max =$ & .45 & & & & \\
\hline $\begin{array}{l}\text { Split ID } \\
\text { size } \\
\text { \% head oxygen } \\
\text { note } \\
\text { date sealed } \\
\text { date oxygen/subspl }\end{array}$ & $\begin{array}{l}\text { analysis } \\
\text { saple } 1 \\
-60 \text { mesh }\end{array}$ & $\begin{array}{l}\text { analysis } \\
\text { sample } 2 \\
-60 \text { mesh }\end{array}$ & $\begin{array}{l}2 \text { can } \\
15 \text { of } 24 \\
-20 \text { mesh } \\
10 / 30 / 85\end{array}$ & $\begin{array}{l}30 \text { gal } \\
3 \text { of } 3 \\
-1 / 4^{\circ} \\
16.4 \\
a \\
10 / 22 / 85 \\
10 / 06 / 8: 3\end{array}$ & $\begin{array}{c}30 \mathrm{gal} \\
3 \text { of } 3 \\
-1 / 4^{\circ "} \\
16.8 \\
9 / 29 / 89\end{array}$ & $\begin{array}{l}30 \text { gal. } \\
3 \text { of } 3 \\
-1 / 4^{\prime \prime} \\
12 / 20 / 90\end{array}$ & $\begin{array}{l}30 \text { gal. } \\
3 \text { of } 3 \\
-1 / 4^{\circ} \\
11 / 20 / 91\end{array}$ & $\begin{array}{l}30 \text { gal. } \\
3 \text { of } 3 \\
-1 / 4^{\circ} \\
b \\
5 / 06 / 93\end{array}$ \\
\hline $\begin{array}{l}\text { PROXIMATE } \\
\% \text { mois ture } \\
\% \text { ash (dry) } \\
\text { analysis date }\end{array}$ & $\begin{array}{c}15.1 \\
5.4 \\
12 / 23 / 85\end{array}$ & $\begin{array}{c}15.2 \\
5.3 \\
05 / 30 / 86\end{array}$ & $\begin{array}{c}15.5 \\
5.2 \\
06 / 14 / 88\end{array}$ & $\begin{array}{c}9.1 \\
5.2 \\
11 / 09 / 88\end{array}$ & $\begin{array}{r}16.5 \\
5.3 \\
12 / 14 / 89\end{array}$ & $\begin{array}{l}18.7 \\
5.1 \\
2 / 23 / 91\end{array}$ & $\begin{array}{c}15.7 \\
3.9 \\
2 / 21 / 91\end{array}$ & $\begin{array}{c}17.2 \\
5.2 \\
5 / 27 / 93\end{array}$ \\
\hline $\begin{array}{l}\text { SULFUR FORrs } \\
\text { total sulfur } \\
\text { sulfate } \\
\text { pyritic } \\
\text { organic } \\
\text { analysis date }\end{array}$ & $\begin{array}{c}0.71 \\
0.01 \\
0.09 \\
0.61 \\
12 / 23 / 85\end{array}$ & 0.73 & & $\begin{array}{c}0.76 \\
0.03 \\
0.11 \\
0.62 \\
01 / 17 / 89\end{array}$ & $\begin{array}{c}0.67 \\
0.10 \\
0.06 \\
0.51 \\
12 / 14 / 89\end{array}$ & $\begin{array}{c}0.76 \\
0.06 \\
0.07 \\
0.62 \\
1 / 23 / 91\end{array}$ & $\begin{array}{c}0.75 \\
0.06 \\
0.02 \\
0.66 \\
2 / 21 / 92\end{array}$ & $\begin{array}{c}0.70 \\
0.07 \\
0.04 \\
0.59 \\
5 / 27 / 93\end{array}$ \\
\hline $\begin{array}{l}\text { CALORIFIC VALUE (dry) } \\
\text { BTU/Ib } \\
\text { analysis date }\end{array}$ & $\begin{array}{c}13960 \\
12 / 23 / 85\end{array}$ & $\begin{array}{c}13966 \\
05 / 30 / 86\end{array}$ & & & $\begin{array}{c}13692 \\
12 / 14 / 89\end{array}$ & $\begin{array}{c}13715 \\
1 / 31 / 91\end{array}$ & $\begin{array}{c}13847 \\
2 / 21 / 92\end{array}$ & $\begin{array}{c}13602 \\
5 / 27 / 93\end{array}$ \\
\hline $\begin{array}{l}\text { ALKALI EXTRACTION } \\
\% \text { transmission } \\
\text { analysis date }\end{array}$ & & & $\begin{array}{c}95.9 \\
06 / 15 / 88\end{array}$ & & $\begin{array}{c}77.8 \\
1 / 10 / 90\end{array}$ & $\begin{array}{c}71.8 \\
6 / 03 / 91\end{array}$ & $\begin{array}{c}72.8 \\
9 / 09 / 92\end{array}$ & $\begin{array}{c}86.3 \\
7 / 07 / 93\end{array}$ \\
\hline
\end{tabular}

notes: a) Sample moist, good condition. PSU MCL sulfur forms (revised analysis report).

b) No visible deterioration. 


\begin{tabular}{|c|c|c|c|c|c|c|c|c|}
\hline $\begin{array}{l}\text { PSOC-1491 } \\
\text { collection date: }\end{array}$ & $\begin{array}{l}\text { DNOTANA \#6 } \\
\text { 37/29/85 }\end{array}$ & twcb, $R$ & 0 $\max =0$. & & & & & \\
\hline $\begin{array}{l}\text { Split ID } \\
\text { size } \\
\text { \% head oxygen } \\
\text { note } \\
\text { date sealed } \\
\text { date oxygen/subspl }\end{array}$ & $\begin{array}{l}\text { aralysis } \\
\text { sample } 1 \\
-60 \text { mesh }\end{array}$ & $\begin{array}{l}\text { analysis } \\
\text { sample } 2\end{array}$ & $\begin{array}{l}\text { \# } 2 \text { can } \\
10 \text { of } 29 \\
-20 \text { mosh } \\
10 / 30 / 85\end{array}$ & $\begin{array}{c}30 \text { sal. } \\
1 \text { of } 3 \\
-1 / 4^{\circ} \\
15.0 \\
3 \\
10 / 14 / 88\end{array}$ & $\begin{array}{c}30 \text { gol. } \\
1 \text { of } 3 \\
-1 / 4^{\circ \prime} \\
16.4 \\
10 / 03 / 89\end{array}$ & $\begin{array}{l}30 \text { gal. } \\
1 \text { of } 3 \\
-1 / 4^{n \prime} \\
12 / 20 / 90\end{array}$ & $\begin{array}{l}30 \text { gal. } \\
1 \text { of } 3 \\
-1 / 4^{\circ} \\
11 / 20 / 91\end{array}$ & $\begin{array}{c}30 \text { gal. } \\
1 \text { of } 3 \\
-1 / 40 \\
b \\
5 / 06 / 93\end{array}$ \\
\hline $\begin{array}{l}\text { PROXImATE } \\
\% \text { moisture (a.d) } \\
\% \text { ash (dry) } \\
\text { amalysis date }\end{array}$ & $\begin{array}{c}11.5 \\
15.8 \\
05 / 28 / 86\end{array}$ & $\begin{array}{c}11.5 \\
15.2 \\
05 / 30 / 86\end{array}$ & $\begin{array}{c}9.9 \\
14.2 \\
3 / 10 / 88\end{array}$ & $\begin{array}{c}9.6 \\
14.9 \\
02 / 01 / 89\end{array}$ & $\begin{array}{r}11.3 \\
15.2 \\
12 / 14 / 89\end{array}$ & $\begin{array}{c}12.8 \\
15.1 \\
1 / 23 / 9 x\end{array}$ & $\begin{array}{c}11.1 \\
10.7 \\
2 / 21 / 92\end{array}$ & $\begin{array}{l}11.7 \\
15.2 \\
5 / 28.93\end{array}$ \\
\hline $\begin{array}{l}\text { GIESELER FLUIDITY } \\
\text { max. ddom } \\
\text { fluid range } \\
\text { analysis ite }\end{array}$ & $\begin{array}{r}28 \\
61 \\
11 / 27 / 85\end{array}$ & & $\begin{array}{c}0 \\
0 \\
3 / 21 / 88\end{array}$ & $\begin{array}{c}0 \\
0 \\
11 / 21 / 88\end{array}$ & $\begin{array}{l}\text { discon- } \\
\text { timued }\end{array}$ & & & \\
\hline $\begin{array}{l}\text { SUlfuR FOrets (dry) } \\
\text { total sulfur } \\
\text { sulfate } \\
\text { pyritic } \\
\text { organic } \\
\text { amalysis dote }\end{array}$ & $\begin{array}{c}2.82 \\
0.02 \\
2.22 \\
0.57 \\
12 / 23 / 85\end{array}$ & $05 / 30 / 86$ & & & \begin{tabular}{r|}
2.56 \\
1.30 \\
0.60 \\
0.66 \\
$12 / 14 / 89$
\end{tabular} & \begin{tabular}{r|}
2.78 \\
1.37 \\
0.53 \\
0.88 \\
$1 / 23 / 91$
\end{tabular} & $\begin{array}{c}2.56 \\
1.03 \\
0.26 \\
1.27 \\
2 / 21 / 92\end{array}$ & $\begin{array}{r}2.80 \\
1.52 \\
0.42 \\
0.85 \\
5 / 28.93\end{array}$ \\
\hline $\begin{array}{l}\text { CALORIFIC VALUE (dry) } \\
\text { BTU/1b } \\
\text { analysis date }\end{array}$ & $\begin{array}{c}12134 \\
12 / 23 / 85\end{array}$ & $\begin{array}{c}12292 \\
05 / 30 / 86\end{array}$ & & & $\begin{array}{c}11613 \\
12 / 14 / 89\end{array}$ & $\begin{array}{c}11505 \\
1 / 23 / 91\end{array}$ & $\begin{array}{c}c \\
12229 \\
2 / 21 / 92\end{array}$ & $\begin{array}{c}11320 \\
5 / 28 / 93\end{array}$ \\
\hline $\begin{array}{l}\text { ALKALI EXTRACTION } \\
\% \text { transmission } \\
\text { analysis date }\end{array}$ & & & $\begin{array}{c}95.1 \\
06 / 07 / 88\end{array}$ & $\begin{array}{c}93.6 \\
12 / 09 / 88\end{array}$ & $\begin{array}{c}97.3 \\
1 / 08 / 90\end{array}$ & $\begin{array}{c}88.5 \\
6 / 03 / 91\end{array}$ & $\begin{array}{c}86.7 \\
9 / 16 / 92\end{array}$ & $\begin{array}{c}90.3 \\
7 / 07 / 93\end{array}$ \\
\hline
\end{tabular}

notes: a) Sample moist, good condition. PSU MCL ash, moisture;

$s$ forms (revised)

b) Trace of white acicular crystals.

c) High calorific value caused in aprt by low ash in this split. 


\begin{tabular}{|c|c|c|c|c|c|c|c|c|}
\hline $\begin{array}{l}\text { PSOC-1492 } \\
\text { collection date: }\end{array}$ & $\begin{array}{l}\text { LLINOIS } \$ 5 \\
8 / 01 / 85\end{array}$ & mucb, & $\max =0$ & & & & & \\
\hline $\begin{array}{l}\text { Split ID } \\
\text { size } \\
\text { \% head oxygen } \\
\text { note } \\
\text { cate sealed } \\
\text { date oxygen/subspl }\end{array}$ & $\begin{array}{l}\text { amalysis } \\
\text { sample } 1 \\
-60 \text { mesh }\end{array}$ & $\begin{array}{l}\text { analys is } \\
\text { sample } 2\end{array}$ & $\begin{array}{l}\# 2 \text { can } \\
31 \text { of } 31 \\
-20 \text { mesh } \\
11 / 14 / 85\end{array}$ & $\begin{array}{l}30 \text { gal } \\
1 \text { of } 3 \\
-1 / 4 " \\
16.1 \\
a \\
10 / 14 / 88\end{array}$ & $\begin{array}{l}30 \mathrm{gal} \\
1 \text { of } 3 \\
-1 / 4 " \\
18.3 \\
10 / 0 \geq / 89\end{array}$ & $\begin{array}{l}30 \text { gal. } \\
1 \text { of } 3 \\
-1 / 4 " \\
12 / 21 / 90\end{array}$ & $\begin{array}{l}30 \text { gal. } \\
1 \text { of } 3 \\
-1 / 4 \cdot \\
11 / 20 / 91\end{array}$ & $\begin{array}{l}30 \text { gal. } \\
1 \text { of } 3 \\
-1 / 4= \\
b \\
5 / 06 / 93\end{array}$ \\
\hline $\begin{array}{l}\text { PROXIMATE } \\
\% \text { moisture (a.d) } \\
\% \text { ash (dry) } \\
\text { analysis date }\end{array}$ & $\begin{array}{c}9.6 \\
10.6 \\
12 / 23 / 85\end{array}$ & $\begin{array}{c}9.5 \\
10.5 \\
05 / 29 / 86\end{array}$ & $\begin{array}{c}9.2 \\
9.9 \\
03 / 14 / 88\end{array}$ & $\begin{array}{c}8.0 \\
10.2 \\
02 / 01 / 89\end{array}$ & $\begin{array}{c}9.8 \\
10.5 \\
02 / 06 / 90\end{array}$ & $\begin{array}{l}10.9 \\
10.6 \\
1 / 23 / 91\end{array}$ & $\begin{array}{c}9.6 \\
9.0 \\
2 / 21 / 92\end{array}$ & $\begin{array}{l}9.9 \\
10.6 \\
5 / 28 / 93\end{array}$ \\
\hline $\begin{array}{l}\text { GIESELER FLUIDITY } \\
\text { max. ddpm } \\
\text { fluid range } \\
\text { analysis date }\end{array}$ & $\begin{array}{r}6 \\
56 \\
11 / 29 / 85\end{array}$ & & $\begin{array}{r}5 \\
58 \\
03 / 14 / 88\end{array}$ & $\begin{array}{c}0 \\
0 \\
11 / 21 / 88\end{array}$ & $\begin{array}{l}\text { discon- } \\
\text { timued }\end{array}$ & & & \\
\hline $\begin{array}{l}\text { SULFUR FORIS (dry) } \\
\text { total sulfur }(3.90) \\
\text { sulfate }(0.03) \\
\text { pyritic }(1.82) \\
\text { organic }(2.05) \\
\text { analysis date }\end{array}$ & $\begin{array}{r}3.90 \\
0.03 \\
1.82 \\
2.05 \\
12 / 23 / 85\end{array}$ & 4.35 & & $\begin{array}{c}4.10 \\
0.91 \\
0.76 \\
2.43 \\
2 / 12 / 90\end{array}$ & \begin{tabular}{r|}
3.90 \\
0.94 \\
0.62 \\
2.34 \\
$02 / 06 / 90$
\end{tabular} & \begin{tabular}{c|}
3.77 \\
0.98 \\
0.59 \\
2.20 \\
$1 / 23 / 91$
\end{tabular} & \begin{tabular}{c|}
3.35 \\
0.65 \\
0.23 \\
2.47 \\
$2 / 21 / 92$
\end{tabular} & $\begin{array}{l}3.74 \\
1.16 \\
0.54 \\
2.04 \\
5 / 28 / 93\end{array}$ \\
\hline $\begin{array}{l}\text { CALORIFIC VALUE (dry) } \\
\text { BTU/lb } \\
\text { analysis date }\end{array}$ & $\begin{array}{c}12758 \\
12 / 23 / 85\end{array}$ & $\begin{array}{c}12722 \\
05 / 29 / 86\end{array}$ & & & $\begin{array}{c}12237 \\
02 / 06 / 90\end{array}$ & $\begin{array}{c}12246 \\
1 / 23 / 91\end{array}$ & $\begin{array}{c}12462 \\
2 / 21 / 92\end{array}$ & $\begin{array}{c}12026 \\
5 / 28 / 95\end{array}$ \\
\hline $\begin{array}{l}\text { ALKALI EXTRACTION } \\
\% \text { transmission } \\
\text { amalysis date }\end{array}$ & & & $\begin{array}{c}86.9 \\
06 / 07 / 88\end{array}$ & $\begin{array}{c}93.4 \\
12 / 09 / 88\end{array}$ & $\begin{array}{c}86.2 \\
01 / 11 / 90\end{array}$ & & $\begin{array}{c}79.4 \\
9 / 21 / 92\end{array}$ & $\begin{array}{c}83.3 \\
7 / 07 / 93\end{array}$ \\
\hline
\end{tabular}

notes: a s Sample dry, good condition. PSU MCL ash, moisture; s forms (revised).

b) Trace of brown powdery coatings. 


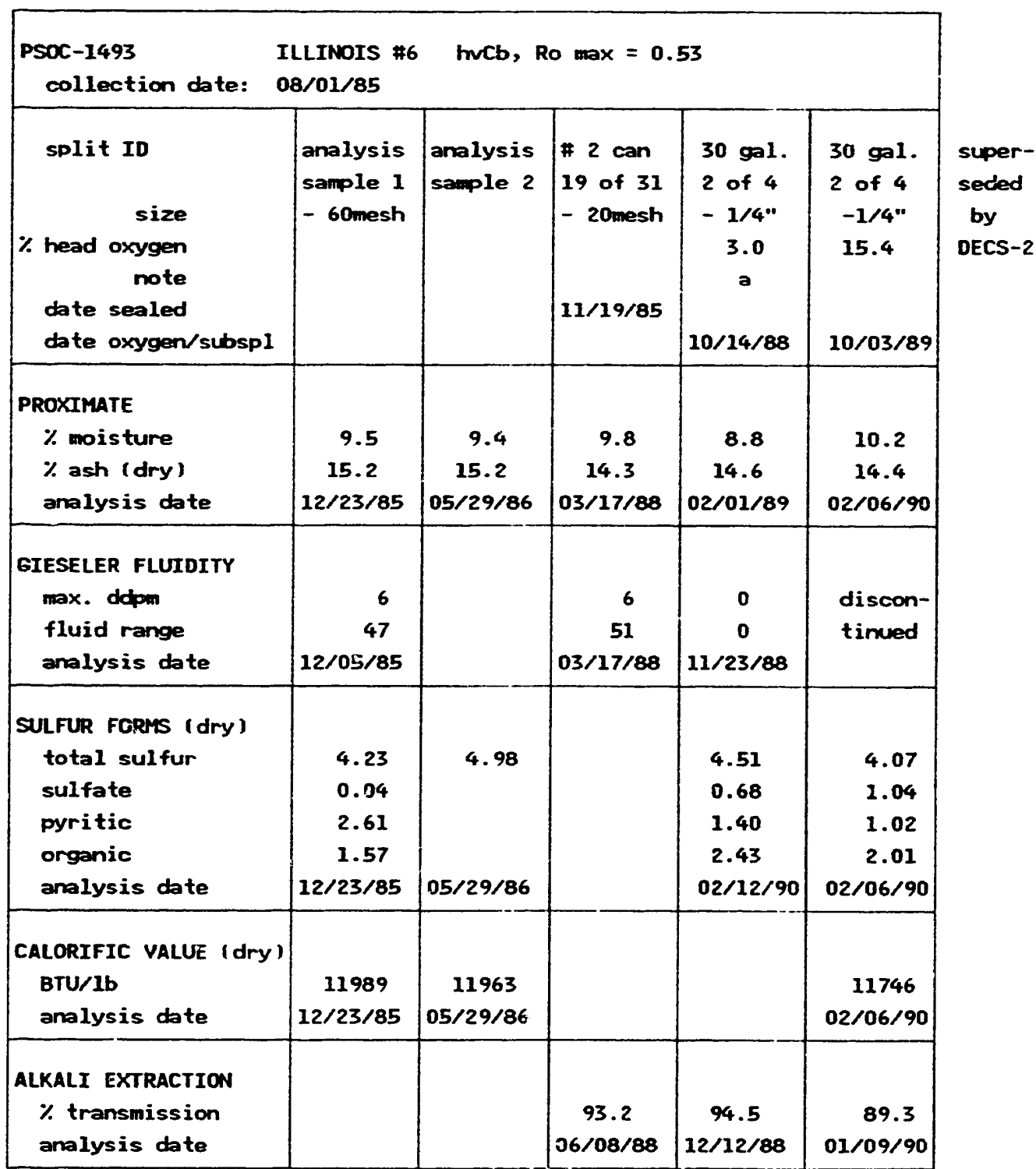

notes: a) Sample moist, trace yellow sulfate. PSU MCL ash, moistures $S$ forms (revised). 


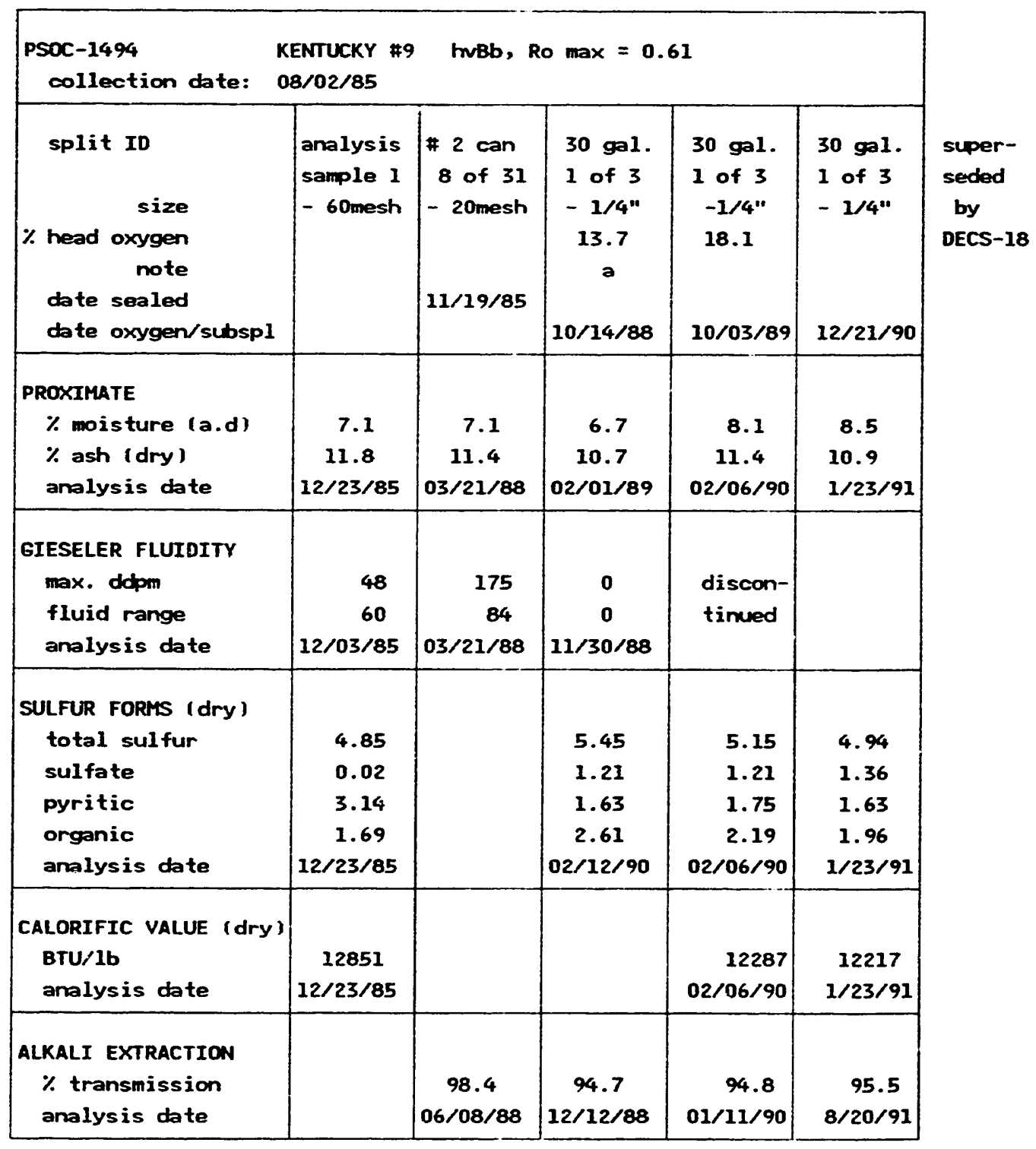

notes: a) Sample dry, iron stained. PSU MCL ash, moisture; S forms (revised). 


\begin{tabular}{|c|c|c|c|c|c|c|c|c|}
\hline $\begin{array}{l}\text { PSOC-1495 } \\
\text { collection date: }\end{array}$ & $\begin{array}{l}\text { EVIER-WHE } \\
\text { 8/05/85 }\end{array}$ & LER mucb, & Ro $\max =0$ & .62 & & & & \\
\hline $\begin{array}{l}\text { spijt ID } \\
\text { size } \\
\text { \% head oxygen } \\
\text { note } \\
\text { date sealed } \\
\text { date oxygen/subspl }\end{array}$ & $\begin{array}{l}\text { analysis } \\
\text { sample } 1 \\
\text { - 60mesh }\end{array}$ & $\begin{array}{l}\text { analys is } \\
\text { sample } 2 \\
-60 \text { mesh }\end{array}$ & $\begin{array}{l}\# 2 \text { can } \\
23 \text { of } 31 \\
-20 \text { mesh } \\
11 / 21 / 85\end{array}$ & $\begin{array}{c}30 \text { gal } \\
3 \text { of } 3 \\
-1 " \\
15.8 \\
\text { a } \\
10 / 14 / 88\end{array}$ & $\begin{array}{l}30 \text { gal. } \\
1 \text { of } 3 \\
-1 / 4^{\prime \prime} \\
17.9 \\
10 / 11 / 89\end{array}$ & $\begin{array}{l}30 \text { gal. } \\
1 \text { of } 3 \\
-1 / 4 " \\
12 / 21 / 90\end{array}$ & $\begin{array}{l}30 \text { gal. } \\
1 \text { of } 3 \\
-1 / 4 " \\
11 / 20 / 91\end{array}$ & $\begin{array}{c}30 \text { gal. } \\
1 \text { of } 3 \\
-1 / 4 " \\
\text { b } \\
5 / 06 / 93\end{array}$ \\
\hline $\begin{array}{l}\text { PROXIMATE } \\
\% \text { moisture (a.d) } \\
\% \text { ash (dry) } \\
\text { analysis date }\end{array}$ & $\begin{array}{c}13.4 \\
16.1 \\
12 / 30 / 85\end{array}$ & $\begin{array}{c}13.3 \\
16.1 \\
05 / 30 / 86\end{array}$ & $\begin{array}{c}10.2 \\
14.9 \\
03 / 22 / 88\end{array}$ & $\begin{array}{c}11.0 \\
15.1 \\
02 / 01 / 89\end{array}$ & $\begin{array}{c}13.0 \\
15.8 \\
02 / 06 / 90\end{array}$ & $\begin{array}{c}14.4 \\
15.3 \\
1 / 23 / 91\end{array}$ & $\begin{array}{c}13.4 \\
11.2 \\
2 / 21 / 92\end{array}$ & $\begin{array}{c}13.3 \\
15.9 \\
5 / 28 / 93\end{array}$ \\
\hline $\begin{array}{l}\text { GIESELER FLUIDITY } \\
\text { max. ddpw } \\
\text { fluid range } \\
\text { analysis date }\end{array}$ & $\begin{array}{r}2 \\
45 \\
12 / 04 / 85\end{array}$ & & $\begin{array}{r}0 \\
0 \\
03 / 22 / 88\end{array}$ & $\begin{array}{c}0 \\
0 \\
12 / 02 / 88\end{array}$ & $\begin{array}{l}\text { discon- } \\
\text { timued }\end{array}$ & & & \\
\hline $\begin{array}{l}\text { SULFUR FORAS (dry) } \\
\text { total sulfur } \\
\text { sulfate } \\
\text { pyritic } \\
\text { organic } \\
\text { analysis date }\end{array}$ & $\begin{array}{c}6.04 \\
0.03 \\
3.77 \\
2.24 \\
12 / 30 / 85\end{array}$ & $\begin{array}{c}7.02 \\
0.22 \\
3.43 \\
3.37 \\
06 / 18 / 86\end{array}$ & & $\begin{array}{c}6.47 \\
1.89 \\
1.49 \\
3.09 \\
02 / 12 / 90\end{array}$ & $\begin{array}{c}6.05 \\
1.97 \\
1.59 \\
2.49 \\
02 / 06 / 90\end{array}$ & $\begin{array}{r}5.92 \\
2.15 \\
0.08 \\
3.68 \\
1 / 23 / 91\end{array}$ & $\begin{array}{c}6.21 \\
1.15 \\
1.47 \\
3.59 \\
2 / 21 / 92\end{array}$ & $\begin{array}{r}5.96 \\
2.35 \\
0.61 \\
3.00 \\
5 / 28 / 93\end{array}$ \\
\hline $\begin{array}{l}\text { CALORIFIC VALUE (dry) } \\
\text { BTU/lb } \\
\text { analysis date }\end{array}$ & $\begin{array}{c}11879 \\
12 / 20 / 85\end{array}$ & & & & $\begin{array}{c}11085 \\
02 / 06 / 90\end{array}$ & $\begin{array}{c}11097 \\
1 / 31 / 91\end{array}$ & $\begin{array}{c}c \\
12172 \\
2 / 21 / 92\end{array}$ & $\begin{array}{c}10841 \\
5 / 28 / 93\end{array}$ \\
\hline $\begin{array}{l}\text { ALKALI EXTRACTION } \\
\% \text { transmission } \\
\text { analysis date }\end{array}$ & & & $\begin{array}{c}87.4 \\
06 / 07 / 88\end{array}$ & $\begin{array}{c}93.0 \\
12 / 12 / 88\end{array}$ & $\begin{array}{c}94.6 \\
01 / 12 / 90\end{array}$ & $\begin{array}{c}85.0 \\
2 / 20 / 91\end{array}$ & $\begin{array}{c}97.1 \\
9 / 21 / 92\end{array}$ & $\begin{array}{r}90.6 \\
7 / 08 / 93\end{array}$ \\
\hline
\end{tabular}

notes: a) PSU MCL ash, moisture; S forms (revised).

b) Abundant white acicular crystals and some yellow/brown coatings.

c) High calorific value caused in part by low ash in this split. 


\begin{tabular}{|c|c|c|c|c|c|c|c|}
\hline $\begin{array}{l}\text { PSOC-1496 } \\
\text { collection date: }\end{array}$ & BO & tucb, & Ro $\max =0$ & & & & \\
\hline $\begin{array}{l}\text { Split ID } \\
\text { size } \\
\text { \% head oxygen } \\
\text { note } \\
\text { date sealed } \\
\text { date oxygen/subspl }\end{array}$ & $\begin{array}{l}\text { analysis } \\
\text { sample } 1 \\
-60 \text { mesh }\end{array}$ & $\begin{array}{l}\text { 2 Can } \\
13 \text { of } 30 \\
-20 \text { mesh } \\
11 / 27 / 85\end{array}$ & $\begin{array}{l}30 \text { gal. } \\
3 \text { of } 3 \\
-1 / 4 " 1 \\
12.8 \\
\text { a } \\
10 / 14 / 88\end{array}$ & $\begin{array}{l}30 \text { gal. } \\
3 \text { of } 3 \\
-1 / 4 " \\
19.0 \\
10 / 11 / 89\end{array}$ & $\begin{array}{l}30 \text { gal. } \\
3 \text { of } 3 \\
-1 / 4 " \\
12 / 21 / 90\end{array}$ & $\begin{array}{l}30 \text { gal. } \\
3 \text { of } 3 \\
-1 / 4 " \\
11 / 21 / 91\end{array}$ & $\begin{array}{l}30 \text { gal. } \\
3 \text { of } 3 \\
-1 / 41 \\
\text { b } \\
5 / 06 / 93\end{array}$ \\
\hline $\begin{array}{l}\text { Proximate } \\
\% \text { moisture (a.d) } \\
\% \text { ash (dry) } \\
\text { analysis date }\end{array}$ & $\begin{array}{c}7.9 \\
29.4 \\
12 / 23 / 85\end{array}$ & $\begin{array}{c}7.3 \\
29.5 \\
03 / 28 / 88\end{array}$ & $\begin{array}{c}6.3 \\
27.4 \\
02 / 01 / 89\end{array}$ & $\begin{array}{c}8.3 \\
28.5 \\
02 / 06 / 90\end{array}$ & $\begin{array}{c}8.8 \\
28.0 \\
1 / 23 / 91\end{array}$ & $\begin{array}{c}6.1 \\
27.3 \\
2 / 21 / 92\end{array}$ & $\begin{array}{c}8.5 \\
28.8 \\
6 / 14 / 93\end{array}$ \\
\hline $\begin{array}{l}\text { GIESELER FLUIDITY } \\
\text { max. didpm } \\
\text { fluid range } \\
\text { analysis date }\end{array}$ & $\begin{array}{r}6 \\
44 \\
12 / 04 / 85\end{array}$ & $\begin{array}{r}6 \\
39 \\
03 / 28 / 88\end{array}$ & $\begin{array}{c}0 \\
0 \\
12 / 02 / 88\end{array}$ & $\begin{array}{l}\text { discon- } \\
\text { timued }\end{array}$ & & & \\
\hline $\begin{array}{l}\text { SULFUR FORMS (dry \%) } \\
\text { total sulfur } \\
\text { sulfate } \\
\text { pyritic } \\
\text { organic } \\
\text { analysis date }\end{array}$ & $\begin{array}{r}6.04 \\
0.03 \\
3.31 \\
2.69 \\
12 / 23 / 85\end{array}$ & & $\begin{array}{c}6.23 \\
2.09 \\
1.75 \\
2.39 \\
02 / 12 / 90\end{array}$ & $\begin{array}{r}5.74 \\
2.02 \\
1.93 \\
1.79 \\
02 / 06 / 90\end{array}$ & $\begin{array}{c}5.78 \\
2.22 \\
2.14 \\
1.42 \\
1 / 23 / 91\end{array}$ & $\begin{array}{c}5.73 \\
1.26 \\
1.19 \\
3.28 \\
2 / 21 / 92\end{array}$ & $\begin{array}{c}6.09 \\
2.68 \\
1.35 \\
2.06 \\
6 / 14 / 93\end{array}$ \\
\hline $\begin{array}{l}\text { CALORIFIC VALUE (dry) } \\
\text { BTU/lb } \\
\text { analysis date }\end{array}$ & $\begin{array}{c}9881 \\
12 / 23 / 85\end{array}$ & & & $\begin{array}{c}9619 \\
02 / 06 / 90\end{array}$ & $\begin{array}{c}9498 \\
1 / 23 / 91\end{array}$ & $\begin{array}{c}9912 \\
2 / 21 / 92\end{array}$ & $\begin{array}{c}9199 \\
6 / 14 / 93\end{array}$ \\
\hline $\begin{array}{l}\text { ALKALI EXTRACTION } \\
\% \text { transmission } \\
\text { analysis date }\end{array}$ & & $\begin{array}{c}94.4 \\
06 / 30 / 88\end{array}$ & $\begin{array}{c}94.7 \\
12 / 12 / 88\end{array}$ & $\begin{array}{c}80.9 \\
01 / 12 / 90\end{array}$ & $\begin{array}{c}95.6 \\
8 / 22 / 91\end{array}$ & $\begin{array}{c}97.2 \\
9 / 21 / 92\end{array}$ & $\begin{array}{r}97.5 \\
7 / 08 / 93\end{array}$ \\
\hline
\end{tabular}

notes: a) Sample dry, trace white sulfate. PSU MCL ash, moisture (low ash may be due to lab not sample split); S forms (revised).

b) Abundant white acicular crystals and some yellow/brown coatings. 


\begin{tabular}{|c|c|c|c|c|c|c|c|c|}
\hline $\begin{array}{l}\text { PSOC-1497 } \\
\text { collection date: }\end{array}$ & $\begin{array}{l}\text { ILLINOIS \#2 } \\
28 / 08 / 85\end{array}$ & twucb, & Ro $\max =0$ & .40 & & & & \\
\hline $\begin{array}{l}\text { Split ID } \\
\text { size } \\
\begin{array}{l}\text { \% head oxygen } \\
\text { note }\end{array} \\
\text { date sealed } \\
\text { date oxygen/subspl }\end{array}$ & $\begin{array}{l}\text { analys is } \\
\text { sample } 1 \\
-60 \text { mesh }\end{array}$ & $\begin{array}{l}\text { analysis } \\
\text { sample } 2\end{array}$ & $\begin{array}{l}\# 2 \text { can } \\
13 \text { of } 30 \\
-20 \text { mesh } \\
12 / 03 / 85\end{array}$ & $\begin{array}{c}30 \text { gal } \\
1 \text { of } 3 \\
-1 / 4 " \\
11.9 \\
a \\
10 / 14 / 88\end{array}$ & $\begin{array}{l}30 \text { gel. } \\
1 \text { of } 3 \\
-1 / 4^{\prime \prime} \\
16.0 \\
10 / 04 / 89\end{array}$ & $\begin{array}{l}30 \text { gal. } \\
1 \text { of } 3 \\
-1 / 4^{\prime \prime} \\
12 / 21 / 90\end{array}$ & $\begin{array}{l}30 \text { gal. } \\
1 \text { of } 3 \\
-1 / 4 " \\
11 / 21 / 91\end{array}$ & $\begin{array}{c}30 \text { gal. } \\
1 \text { of } 3 \\
-1 / 4 " 1 \\
\text { b } \\
5 / 06 / 93\end{array}$ \\
\hline $\begin{array}{l}\text { PROXIMATE } \\
\% \text { moisture (a.d) } \\
\% \text { ash (dry) } \\
\text { analysis date }\end{array}$ & $\begin{array}{c}13.5 \\
12.0 \\
12 / 23 / 85\end{array}$ & $\begin{array}{c}13.4 \\
11.7 \\
05 / 30 / 86\end{array}$ & $\begin{array}{c}14.6 \\
12.3 \\
03 / 29 / 88\end{array}$ & $\begin{array}{c}12.1 \\
10.8 \\
02 / 01 / 88\end{array}$ & $\begin{array}{c}13.2 \\
11.5 \\
02 / 06 / 90\end{array}$ & $\begin{array}{c}13.9 \\
11.8 \\
1 / 23 / 91\end{array}$ & $\begin{array}{c}12.3 \\
6.8 \\
2 / 21 / 92\end{array}$ & $\begin{array}{c}13.4 \\
11.5 \\
6 / 14 / 93\end{array}$ \\
\hline $\begin{array}{l}\text { GIESELER FLUIDITY } \\
\text { max. ddpm } \\
\text { fluid range } \\
\text { analysis date }\end{array}$ & $\begin{array}{r}83 \\
65 \\
12 / 06 / 85\end{array}$ & & $\begin{array}{c}146 \\
87 \\
03 / 29 / 88\end{array}$ & $\begin{array}{c}0 \\
0 \\
12 / 05 / 88\end{array}$ & $\begin{array}{l}\text { discon- } \\
\text { timued }\end{array}$ & & & \\
\hline $\begin{array}{l}\text { SULFUR FORAS (dry \%) } \\
\text { total sulfur } \\
\text { suifate } \\
\text { Pyritic } \\
\text { organic } \\
\text { analysis date }\end{array}$ & $\begin{array}{c}5.04 \\
0.02 \\
4.00 \\
1.02 \\
12 / 23 / 85\end{array}$ & $\begin{array}{c}5.93 \\
0.35 \\
3.51 \\
2.06 \\
06 / 18 / 86\end{array}$ & & $\begin{array}{c}5.33 \\
2.20 \\
1.21 \\
1.92 \\
02 / 12 / 90\end{array}$ & $\begin{array}{r}4.96 \\
2.16 \\
1.43 \\
1.37 \\
02 / 06 / 90\end{array}$ & $\begin{array}{r}5.13 \\
2.41 \\
1.17 \\
1.55 \\
1 / 23 / 91\end{array}$ & $\begin{array}{r}4.24 \\
1.92 \\
0.54 \\
1.79 \\
2 / 21 / 92\end{array}$ & $\begin{array}{r}5.02 \\
2.51 \\
0.96 \\
1.56 \\
6 / 14 / 93\end{array}$ \\
\hline $\begin{array}{l}\text { CALORIFIC VALUE (dry) } \\
\text { BTU/1b } \\
\text { analysis date }\end{array}$ & $\begin{array}{c}12823 \\
12 / 23 / 85\end{array}$ & $\begin{array}{c}12778 \\
05 / 30 / 86\end{array}$ & & & $\begin{array}{c}11748 \\
02 / 06 / 90\end{array}$ & $\begin{array}{r}11633 \\
1 / 23 / 91\end{array}$ & $\begin{array}{c}12628 \\
2 / 21 / 92\end{array}$ & $\begin{array}{r}11421 \\
6 / 14 / 93\end{array}$ \\
\hline $\begin{array}{l}\text { ALKALI EXTRACTION } \\
\% \text { transmission } \\
\text { analysis date }\end{array}$ & & & $\begin{array}{c}96.3 \\
06 / 08 / 88\end{array}$ & $\begin{array}{c}93.5 \\
12 / 14 / 88\end{array}$ & $\begin{array}{r}88.9 \\
01 / 23 / 90\end{array}$ & $\begin{array}{c}89.1 \\
8 / 23 / 91\end{array}$ & $\begin{array}{c}95.4 \\
9 / 22 / 92\end{array}$ & $\begin{array}{r}89.9 \\
7 / 08 / 93\end{array}$ \\
\hline
\end{tabular}

notes: a) Sample dry, visible white sulfate. PSU MCL ash, moisture;

$s$ forms (revised).

b) Trace of brown and white coatings. 


\begin{tabular}{|c|c|c|c|c|c|c|c|}
\hline $\begin{array}{l}\text { PSOC-1498 } \\
\text { collection date: }\end{array}$ & $\begin{array}{l}\text { ADEE } \\
9 / 24 / 35\end{array}$ & trucb, & Ro $\max =0$ & & & & \\
\hline $\begin{array}{l}\text { Split ID } \\
\text { size } \\
\text { \% head oxygen } \\
\text { note } \\
\text { date sealed } \\
\text { date oxygen/subspl }\end{array}$ & $\begin{array}{l}\text { analysis } \\
\text { sample } 1 \\
-60 \text { mesh }\end{array}$ & $\begin{array}{l}\# 2 \text { Can } \\
15 \text { of } 31 \\
-20 \text { mesh } \\
12 / 13 / 85\end{array}$ & $\begin{array}{c}30 \text { gal. } \\
3 \text { of } 3 \\
-1 / 4 " \\
15.6 \\
a \\
10 / 14 / 88\end{array}$ & $\begin{array}{l}30 \text { gal. } \\
3 \text { of } 3 \\
-1 / 4^{\circ "} \\
17.1 \\
10 / 04 / 89\end{array}$ & $\begin{array}{l}30 \text { gal. } \\
3 \text { of } 3 \\
-1 / 4 " \\
12 / 21 / 90\end{array}$ & $\begin{array}{l}30 \text { gal. } \\
3 \text { of } 3 \\
-1 / 4^{\prime \prime} \\
11 / 21 / 91\end{array}$ & $\begin{array}{l}30 \text { gal. } \\
3 \text { of } 3 \\
-1 / 4 " 1 \\
\text { b } \\
5 / 06 / 93\end{array}$ \\
\hline $\begin{array}{l}\text { PROXIMATE } \\
\% \text { moisture } \\
\% \text { ash (dry) } \\
\text { analysis date }\end{array}$ & $\begin{array}{c}9.6 \\
7.1 \\
03 / 13 / 86\end{array}$ & $\begin{array}{c}9.6 \\
7.3 \\
06 / 14 / 88\end{array}$ & $\begin{array}{c}9.8 \\
6.6 \\
02 / 01 / 89\end{array}$ & $\begin{array}{c}11.1 \\
7.1 \\
02 / 06 / 90\end{array}$ & $\begin{array}{c}11.9 \\
7.2 \\
1 / 23 / 91\end{array}$ & $\begin{array}{c}10.8 \\
7.6 \\
2 / 21 / 92\end{array}$ & $\begin{array}{c}11.5 \\
7.3 \\
6 / 14 / 93\end{array}$ \\
\hline $\begin{array}{l}\text { SULFUR FORAs (dry \%) } \\
\text { total sulfur } \\
\text { sulfate } \\
\text { pyritic } \\
\text { organic } \\
\text { analysis date }\end{array}$ & $\begin{array}{c}0.52 \\
0.00 \\
0.03 \\
0.48 \\
03 / 13 / 86\end{array}$ & & $\begin{array}{c}0.55 \\
0.02 \\
0.04 \\
0.49 \\
02 / 12 / 90\end{array}$ & $\begin{array}{c}0.46 \\
0.01 \\
0.02 \\
0.43 \\
02 / 06 / 90\end{array}$ & $\begin{array}{c}0.51 \\
0.01 \\
0.03 \\
0.47 \\
1 / 23 / 91\end{array}$ & $\begin{array}{r}0.53 \\
0.02 \\
0.03 \\
0.48 \\
2 / 21 / 92\end{array}$ & $\begin{array}{c}0.47 \\
0.01 \\
0.02 \\
0.44 \\
6 / 14 / 93\end{array}$ \\
\hline $\begin{array}{l}\text { CALORIFIC VALUE (dry) } \\
\text { BTU/lb } \\
\text { analysis date }\end{array}$ & $\begin{array}{c}12762 \\
03 / 13 / 86\end{array}$ & & & $\begin{array}{c}12520 \\
02 / 06 / 90\end{array}$ & $\begin{array}{r}12438 \\
1 / 31 / 91\end{array}$ & $\begin{array}{c}12429 \\
2 / 21 / 92\end{array}$ & $\begin{array}{c}12368 \\
6 / 14 / 93\end{array}$ \\
\hline $\begin{array}{l}\text { ALKALI EXTRACTION } \\
\% \text { transmission } \\
\text { analysis date }\end{array}$ & & $\begin{array}{c}23.5 \\
06 / 16 / 88\end{array}$ & $\begin{array}{c}38.8 \\
12 / 14 / 88\end{array}$ & $\begin{array}{l}\text { discon- } \\
\text { tinued }\end{array}$ & & & \\
\hline
\end{tabular}

notes: a) Sample dry (dusty), good condition. PSU MCL ash, mois: ture; s forms (revised).

b) No visible deterioration. 


\begin{tabular}{|c|c|c|c|c|c|c|c|}
\hline $\begin{array}{l}\text { PSOC-1499 } \\
\text { collection date: }\end{array}$ & $\begin{array}{l}\text { OLORADO B } \\
9 / 26 / 85\end{array}$ & mubb, & Ro $\max =0$ & & & & \\
\hline $\begin{array}{l}\text { Split ID } \\
\text { size } \\
\text { \% head oxygen } \\
\text { note } \\
\text { date sealed } \\
\text { date oxygen/subspl }\end{array}$ & $\begin{array}{l}\text { analysis } \\
\text { sample } 1 \\
-60 \text { mesh }\end{array}$ & $\begin{array}{l}\# 2 \text { can } \\
15 \text { of } 31 \\
-20 \text { mesh } \\
12 / 17 / 85\end{array}$ & $\begin{array}{c}30 \text { gal } \\
3 \text { of } 4 \\
-1 / 4 " \\
17.1 \\
\text { a } \\
10 / 14 / 88\end{array}$ & $\begin{array}{l}30 \text { gal. } \\
3 \text { of } 4 \\
-1 / 4 " \\
18.3 \\
10 / 11 / 89\end{array}$ & $\begin{array}{l}30 \mathrm{gal} \\
3 \text { of } 4 \\
-1 / 4^{\prime \prime} \\
12 / 21 / 90\end{array}$ & $\begin{array}{l}30 \text { gal. } \\
3 \text { of } 4 \\
-1 / 4 " \\
11 / 21 / 91\end{array}$ & $\begin{array}{l}30 \text { gal } \\
3 \text { of } 4 \\
-1 / 4 " \\
b \\
6 / 05 / 93\end{array}$ \\
\hline $\begin{array}{l}\text { PROXImate } \\
\% \text { moisture } \\
\% \text { ash (dry) } \\
\text { analysis date }\end{array}$ & $\begin{array}{c}3.4 \\
5.9 \\
03 / 13 / 86\end{array}$ & $\begin{array}{c}4.2 \\
4.4 \\
04 / 22 / 88\end{array}$ & $\begin{array}{c}3.7 \\
5.3 \\
02 / 03 / 89\end{array}$ & $\begin{array}{c}4.4 \\
5.7 \\
02 / 28 / 90\end{array}$ & $\begin{array}{c}4.8 \\
6.0 \\
10 / 03 / 91\end{array}$ & $\begin{array}{c}4.1 \\
7.2 \\
10 / 14 / 92\end{array}$ & $\begin{array}{c}4.7 \\
5.9 \\
6 / 14 / 93\end{array}$ \\
\hline $\begin{array}{l}\text { GIESELER FLUIDITY } \\
\text { max. ddpm } \\
\text { fluid range } \\
\text { analys is date }\end{array}$ & $\begin{array}{r}4 \\
43 \\
12 / 17 / 85\end{array}$ & $\begin{array}{r}4 \\
44 \\
04 / 22 / 88\end{array}$ & $\begin{array}{c}2 \\
36 \\
12 / 05 / 88\end{array}$ & $\begin{array}{c}1 \\
18 \\
02 / 01 / 90\end{array}$ & $\begin{array}{c}1 \\
34 \\
8 / 21 / 91\end{array}$ & $\begin{array}{c}0 \\
0 \\
9 / 24 / 92\end{array}$ & $\begin{array}{l}\text { discon- } \\
\text { timued }\end{array}$ \\
\hline $\begin{array}{l}\text { SULFUR FORTS (dry \%) } \\
\text { total sulfur } \\
\text { sulfate } \\
\text { pyritic } \\
\text { organic } \\
\text { analysis date }\end{array}$ & $\begin{array}{c}0.67 \\
0.00 \\
0.02 \\
0.65 \\
03 / 13 / 86\end{array}$ & & $\begin{array}{c}0.74 \\
0.04 \\
0.04 \\
0.66 \\
02 / 12 / 90\end{array}$ & $\begin{array}{r}0.64 \\
0.01 \\
0.02 \\
0.61 \\
02 / 28 / 90\end{array}$ & $\begin{array}{r}0.65 \\
0.01 \\
0.05 \\
0.59 \\
10 / 03 / 91\end{array}$ & $\begin{array}{c}0.64 \\
0.02 \\
0.04 \\
0.58 \\
10 / 14 / 92\end{array}$ & $\begin{array}{c}0.66 \\
0.01 \\
0.03 \\
0.62 \\
6 / 14 / 93\end{array}$ \\
\hline $\begin{array}{l}\text { CALORIFIC VALUE (dry) } \\
\text { BTU/1b } \\
\text { analysis date }\end{array}$ & $\begin{array}{c}13780 \\
03 / 13 / 86\end{array}$ & & & $\begin{array}{c}13597 \\
02 / 28 / 90\end{array}$ & $\begin{array}{c}13519 \\
10 / 03 / 91\end{array}$ & $\begin{array}{c}13320 \\
10 / 14 / 92\end{array}$ & $\begin{array}{l}13415 \\
6 / 14 / 93\end{array}$ \\
\hline $\begin{array}{l}\text { ALKALI EXTRACTION } \\
\text { \% transmission } \\
\text { analysis date }\end{array}$ & & $\begin{array}{c}98.7 \\
06 / 06 / 88\end{array}$ & & $\begin{array}{c}91.8 \\
02 / 01 / 90\end{array}$ & $\begin{array}{c}93.9 \\
8 / 21 / 91\end{array}$ & $\begin{array}{c}89.8 \\
9 / 24 / 92\end{array}$ & $\begin{array}{c}96.6 \\
7 / 08 / 93\end{array}$ \\
\hline
\end{tabular}

notes: a) Sample dry (dusty), good condition. PSU MCL ash, moisture? S forms (revised).

b) No visible deterioration. 


\begin{tabular}{|c|c|c|c|c|c|}
\hline $\begin{array}{l}\text { PSOC-1500 } \\
\text { collection date: }\end{array}$ & $\begin{array}{l}\text { ASIN B } \\
9 / 26 / 85\end{array}$ & mont, & Ro $\max =1$ & 45 & \multirow[b]{2}{*}{$\begin{array}{l}\text { super- } \\
\text { seded } \\
\text { by } \\
\text { DECS-3 }\end{array}$} \\
\hline $\begin{array}{l}\text { Split ID } \\
\text { size } \\
\text { \% head oxygen } \\
\text { note } \\
\text { date sealed } \\
\text { date oxygen/subspl }\end{array}$ & $\begin{array}{l}\text { analysis } \\
\text { sample } 1 \\
\text { - 60mesh }\end{array}$ & $\begin{array}{l}\text { \# } 2 \text { can } \\
15 \text { of } 31 \\
-20 \text { mesh } \\
02 / 11 / 86\end{array}$ & $\begin{array}{c}36 \text { gel } \\
2 \text { of } 4 \\
-1 / 4= \\
19.7 \\
a \\
10 / 19 / 88\end{array}$ & $\begin{array}{c}30 \text { gal. } \\
2 \text { of } 4 \\
-1 / 4 " \\
19.4 \\
10 / 11 / 89\end{array}$ & \\
\hline $\begin{array}{l}\text { PROXIMATE } \\
\% \text { moisture } \\
\% \text { ash (dry) } \\
\text { analysis date }\end{array}$ & $\begin{array}{c}4.4 \\
23.4 \\
03 / 13 / 86\end{array}$ & $\begin{array}{c}2.2 \\
21.8 \\
04 / 25 / 88\end{array}$ & $\begin{array}{c}1.6 \\
24.2 \\
02 / 03 / 89\end{array}$ & $\begin{array}{c}2.8 \\
22.8 \\
03 / 01 / 90\end{array}$ & \\
\hline $\begin{array}{l}\text { GIESELER FLUIDITY } \\
\text { max. ddpm } \\
\text { fluid range } \\
\text { analysis date }\end{array}$ & $\begin{array}{r}267 \\
83 \\
03 / 13 / 86\end{array}$ & $\begin{array}{r}284 \\
84 \\
04 / 25 / 88\end{array}$ & $\begin{array}{c}30 \\
70 \\
01 / 17 / 89\end{array}$ & $\begin{array}{c}23 \\
66 \\
02 / 02 / 90\end{array}$ & \\
\hline $\begin{array}{l}\text { SULFUR FORAs (dry \%) } \\
\text { total sulfur } \\
\text { sulfate } \\
\text { pyritic } \\
\text { organic } \\
\text { analysis date }\end{array}$ & $\begin{array}{c}0.76 \\
0.00 \\
0.32 \\
0.44 \\
03 / 13 / 86\end{array}$ & & $\begin{array}{c}0.84 \\
0.13 \\
0.17 \\
0.54 \\
02 / 12 / 89\end{array}$ & $\begin{array}{c}0.74 \\
0.08 \\
0.19 \\
0.47 \\
03 / 01 / 90\end{array}$ & \\
\hline $\begin{array}{l}\text { CALORIFIC VALUE (dry) } \\
\text { BTU/lb } \\
\text { analysis date }\end{array}$ & $\begin{array}{c}11986 \\
03 / 13 / 86\end{array}$ & & & $\begin{array}{c}12005 \\
03 / 01 / 90\end{array}$ & \\
\hline $\begin{array}{l}\text { ALKALI EXTRACTION } \\
\text { \% transmission } \\
\text { analysis date }\end{array}$ & & $\begin{array}{c}99.0 \\
06 / 06 / 88\end{array}$ & & $\begin{array}{c}99.1 \\
02 / 02 / 90\end{array}$ & \\
\hline
\end{tabular}

notes: a) Sample moist, good condition. PSU MCL ash, moisture, $s$ forms (revised). 


\begin{tabular}{|c|c|c|c|c|c|c|c|}
\hline $\begin{array}{l}\text { PSOC-1501 } \\
\text { collection date: }\end{array}$ & $\begin{array}{l}\text { UANTTA C } \\
9 / 27 / 85\end{array}$ & meb, & Ro $\operatorname{tax}=0$ & & & & \\
\hline $\begin{array}{l}\text { Split ID } \\
\text { size } \\
\text { \% head oxygen } \\
\text { note } \\
\text { date sealed } \\
\text { date oxygen/subspl }\end{array}$ & $\begin{array}{l}\text { analys is } \\
\text { sample } 1 \\
-60 \text { mesh }\end{array}$ & $\begin{array}{l}\text { \# } 2 \text { can } \\
16 \text { of } 29 \\
-20 \text { mesh } \\
03 / 03 / 86\end{array}$ & $\begin{array}{c}30 \text { gal. } \\
3 \text { of } 3 \\
-1 / 4^{\prime \prime} \\
16.4 \\
10 / 19 / 88\end{array}$ & $\begin{array}{c}30 \text { gal. } \\
3 \text { of } 3 \\
-1 / 4^{\circ 1} \\
17.7 \\
10 / 12 / 89\end{array}$ & $\begin{array}{l}30 \text { gal } \\
3 \text { of } 3 \\
-1 / 4 " \\
2 / 05 / 91\end{array}$ & $\begin{array}{l}30 \text { gal. } \\
3 \text { of } 3 \\
-1 / 4^{\prime \prime} \\
11 / 21 / 91\end{array}$ & $\begin{array}{c}30 \text { gol. } \\
3 \text { of } 3 \\
-1 / 4^{n} \\
\text { a } \\
5 / 06 / 93\end{array}$ \\
\hline $\begin{array}{l}\text { PROXIMATE } \\
\% \text { moisture } \\
\because \text { ash (dry) } \\
\text { amalysis date }\end{array}$ & $\begin{array}{c}5.8 \\
5.6 \\
03 / 13 / 86\end{array}$ & $\begin{array}{c}6.8 \\
5.4 \\
04 / 26 / 88\end{array}$ & $\begin{array}{c}6.0 \\
5.5 \\
02 / 10 / 89\end{array}$ & $\begin{array}{c}6.5 \\
5.6 \\
02 / 06 / 90\end{array}$ & $\begin{array}{c}7.6 \\
5.7 \\
2 / 08 / 91\end{array}$ & $\begin{array}{c}6.5 \\
5.8 \\
2 / 21 / 92\end{array}$ & $\begin{array}{c}6.4 \\
5.5 \\
6 / 14 / 93\end{array}$ \\
\hline $\begin{array}{l}\text { GIESELER FLUIDITY } \\
\text { max. datpm } \\
\text { fluid range } \\
\text { analysis date }\end{array}$ & $\begin{array}{r}1 \\
24 \\
03 / 20 / 86\end{array}$ & $\begin{array}{r}1 \\
25 \\
04 / 26 / 88\end{array}$ & $\begin{array}{c}0 \\
0 \\
02 / 02 / 89\end{array}$ & $\begin{array}{l}\text { discon- } \\
\text { timued }\end{array}$ & & & \\
\hline $\begin{array}{l}\text { SULfUR Forats (dry \%) } \\
\text { total sulfur } \\
\text { sulfate } \\
\text { pyritic } \\
\text { organic } \\
\text { analysis date }\end{array}$ & $\begin{array}{c}0.64 \\
0.00 \\
0.02 \\
0.62 \\
03 / 13 / 86\end{array}$ & & $\begin{array}{c}0.57 \\
0.02 \\
0.03 \\
0.53 \\
02 / 10 / 89\end{array}$ & $\begin{array}{c}0.61 \\
0.01 \\
0.03 \\
0.57 \\
02 / 06 / 90\end{array}$ & $\begin{array}{r}0.63 \\
0.01 \\
0.03 \\
0.59 \\
2 / 08 / 91\end{array}$ & $\begin{array}{c}0.63 \\
0.01 \\
0.02 \\
0.60 \\
2 / 21 / 92\end{array}$ & $\begin{array}{c}0.62 \\
0.01 \\
0.02 \\
0.60 \\
6 / 14 / 93\end{array}$ \\
\hline $\begin{array}{l}\text { CALORIFIC VALUE (dry) } \\
\text { BTU/Ib } \\
\text { analys is date }\end{array}$ & $\begin{array}{c}13579 \\
03 / 13 / 86\end{array}$ & & $\begin{array}{c}13368 \\
02 / 10 / 89\end{array}$ & $\begin{array}{c}13342 \\
02 / 06 / 90\end{array}$ & $\begin{array}{c}13317 \\
2 / 15 / 91\end{array}$ & $\begin{array}{c}13257 \\
2 / 21 / 92\end{array}$ & $\begin{array}{r}13286 \\
6 / 14 / 93\end{array}$ \\
\hline $\begin{array}{l}\text { ALKALI EXTRACTION } \\
\text { \% transmission } \\
\text { analysis date }\end{array}$ & & $\begin{array}{c}94.3 \\
06 / 06 / 88\end{array}$ & $\begin{array}{c}90.9 \\
02 / 10 / 89\end{array}$ & $\begin{array}{c}92.0 \\
01 / 23 / 90\end{array}$ & $\begin{array}{c}89.8 \\
8 / 26 / 91\end{array}$ & $\begin{array}{c}90.5 \\
9 / 22 / 92\end{array}$ & $\begin{array}{r}92.7 \\
7 / 09 / 93\end{array}$ \\
\hline
\end{tabular}

notes: a) No visible deterioration. 


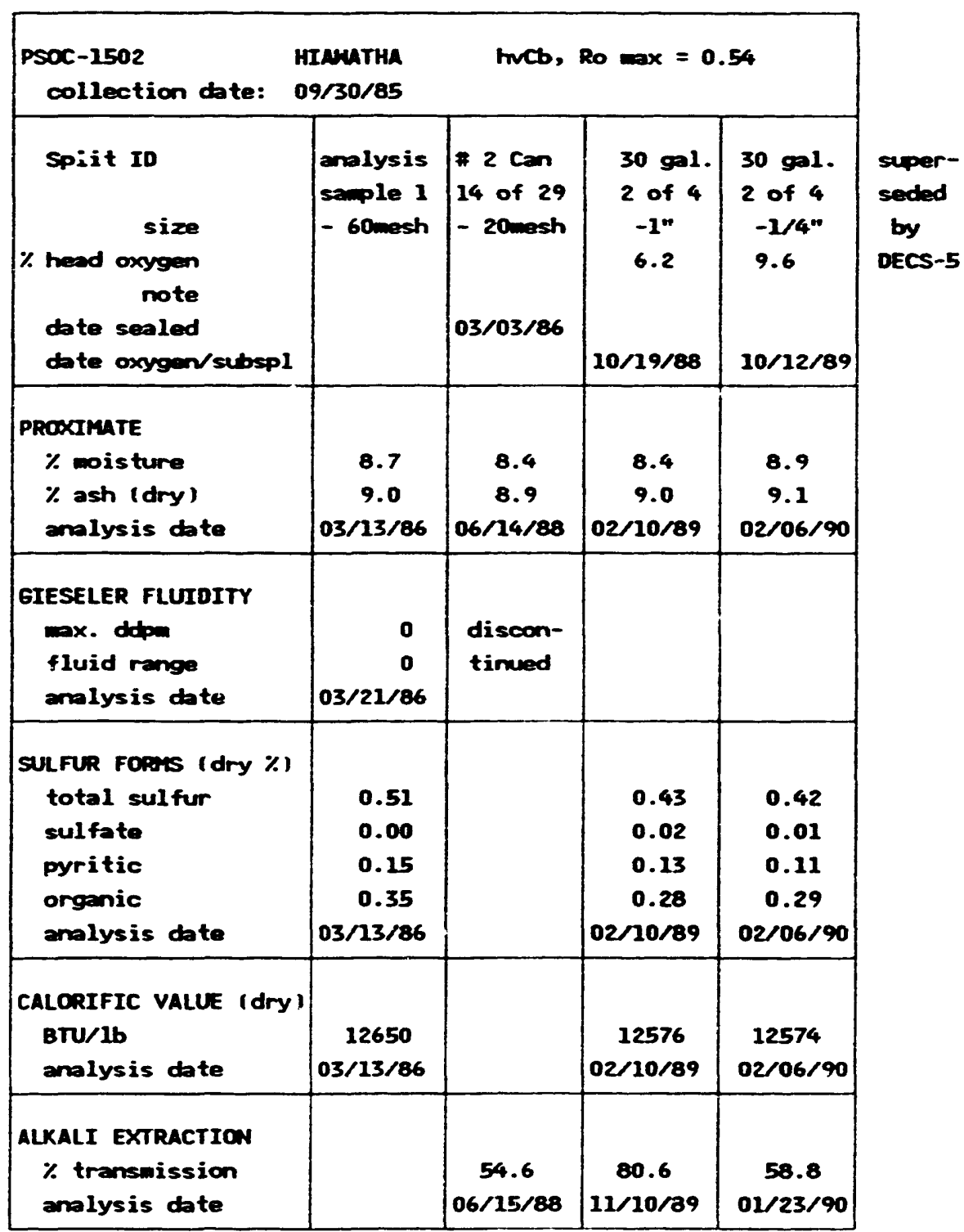




\begin{tabular}{|c|c|c|c|c|c|}
\hline $\begin{array}{l}\text { PSOC-1503 } \\
\text { collection dete? }\end{array}$ & $\begin{array}{l}\text { LIND CANYO } \\
\text { 0/01/85 }\end{array}$ & Incb, & Ro $\max =0$ & & \multirow{5}{*}{$\begin{array}{l}\text { super- } \\
\text { seded } \\
\text { by } \\
\text { DECS-6, } \\
\text { DECS-16, } \\
\text { DECS-17 }\end{array}$} \\
\hline $\begin{array}{l}\text { Split I0 } \\
\text { size } \\
\text { \% heed oxygen } \\
\text { note } \\
\text { date sealed } \\
\text { dote oxygen/subspl }\end{array}$ & $\begin{array}{l}\text { analysis } \\
\text { sample } 1 \\
\text { - 60mesh }\end{array}$ & $\begin{array}{l}2 \text { can } \\
15 \text { of } 31 \\
-20 \text { mesh } \\
03 / 20 / 86\end{array}$ & $\begin{array}{c}30 \text { gal } \\
3 \text { of } 4 \\
-1 \% \\
16.9 \\
10 / 19 / 88\end{array}$ & $\begin{array}{c}30 \text { gal. } \\
3 \text { of } 4 \\
-1 / 4^{m} \\
16.6 \\
10 / 12 / 89\end{array}$ & \\
\hline $\begin{array}{l}\text { PRoxImuTE } \\
\% \text { misture } \\
\% \text { ash (dry) } \\
\text { analysis date }\end{array}$ & $\begin{array}{c}10.4 \\
3.9 \\
04 / 25 / 86\end{array}$ & $\begin{array}{c}5.1 \\
4.0 \\
04 / 26 / 88\end{array}$ & $\begin{array}{c}5.2 \\
4.0 \\
02 / 10 / 89\end{array}$ & $\begin{array}{c}5.6 \\
4.1 \\
02 / 06 / 90\end{array}$ & \\
\hline $\begin{array}{l}\text { GIESELER FLITOITY } \\
\text { max. ddpm } \\
\text { fluid range } \\
\text { analysis date }\end{array}$ & $\begin{array}{r}2 \\
39 \\
03 / 24 / 86\end{array}$ & $\begin{array}{r}0 \\
0 \\
04 / 26 / 88\end{array}$ & $\begin{array}{l}\text { discon- } \\
\text { timued }\end{array}$ & & \\
\hline $\begin{array}{l}\text { SULFUR FORits (dry \%) } \\
\text { total sulfur } \\
\text { sulfate } \\
\text { pyritic } \\
\text { organic } \\
\text { analysis date }\end{array}$ & $\begin{array}{c}0.54 \\
0.00 \\
0.02 \\
0.52 \\
04 / 25 / 86\end{array}$ & & $\begin{array}{c}0.47 \\
0.01 \\
0.17 \\
0.29 \\
02 / 10 / 89\end{array}$ & $\begin{array}{c}0.48 \\
0.02 \\
0.02 \\
0.44 \\
02 / 06 / 90\end{array}$ & \\
\hline $\begin{array}{l}\text { CALORIFIC VALUE (dry) } \\
\text { BTU/lb } \\
\text { analysis date }\end{array}$ & $\begin{array}{c}14069 \\
04 / 25 / 86\end{array}$ & & $\begin{array}{c}13984 \\
02 / 10 / 89\end{array}$ & $\begin{array}{c}13832 \\
02 / 06 / 90\end{array}$ & \\
\hline $\begin{array}{l}\text { ALKALI EXTRACTION } \\
\text { \% transmission } \\
\text { analysis date }\end{array}$ & & $\begin{array}{c}94.5 \\
06 / 06 / 88\end{array}$ & $\begin{array}{c}86.1 \\
02 / 10 / 89\end{array}$ & $\begin{array}{c}84.6 \\
01 / 24 / 90\end{array}$ & \\
\hline
\end{tabular}




\begin{tabular}{|c|c|c|c|c|c|}
\hline $\begin{array}{l}\text { PSOC-1504 } \\
\text { collection date: }\end{array}$ & $\begin{array}{l}\text { PPER SUNAY } \\
\text { 0/02/85 }\end{array}$ & SIDE met & , Ro max & 0.80 & \\
\hline $\begin{array}{l}\text { Split ID } \\
\text { size } \\
\text { \% head oxygen } \\
\text { note } \\
\text { date sealed } \\
\text { date oxygen/subspl }\end{array}$ & $\begin{array}{l}\text { analysis } \\
\text { sample } 1 \\
-60 \text { mesh }\end{array}$ & $\begin{array}{l}2 \text { can } \\
15 \text { of } 31 \\
-20 \text { mesh } \\
03 / 21 / 86\end{array}$ & $\begin{array}{c}30 \text { gal. } \\
4 \text { of } 4 \\
-1 / 40 \\
18.7 \\
a \\
03 / 18 / 86 \\
10 / 28 / 88\end{array}$ & $\begin{array}{c}30 \mathrm{gal} \\
4 \text { of } 4 \\
-1 / 4^{\circ} \\
19.2 \\
10 / 12 / 89\end{array}$ & $\begin{array}{l}30 \text { gal. } \\
4 \text { of } 4 \\
-1 / 4 " \\
2 / 05 / 91\end{array}$ \\
\hline $\begin{array}{l}\text { PrOXIMATE } \\
\% \text { moisture } \\
\% \text { ash (dry I } \\
\text { analysis dote }\end{array}$ & $\begin{array}{c}3.4 \\
7.5 \\
04 / 25 / 86\end{array}$ & $\begin{array}{c}2.8 \\
7.9 \\
05 / 04 / 88\end{array}$ & $\begin{array}{c}2.6 \\
7.3 \\
02 / 10 / 89\end{array}$ & $\begin{array}{c}3.0 \\
7.7 \\
02 / 06 / 90\end{array}$ & $\begin{array}{c}3.9 \\
7.6 \\
2 / 08 / 91\end{array}$ \\
\hline $\begin{array}{l}\text { GIESELER FLUIOITY } \\
\text { max. ddpm } \\
\text { fluid range } \\
\text { analysis bte }\end{array}$ & $\begin{array}{r}17 \\
51 \\
03 / 24 / 86\end{array}$ & $\begin{array}{r}13 \\
58 \\
05 / 04 / 88\end{array}$ & $\begin{array}{c}0 \\
0 \\
02 / 02 / 89\end{array}$ & $\begin{array}{l}\text { discon- } \\
\text { tinued }\end{array}$ & \\
\hline $\begin{array}{l}\text { SULFUR FOrus (dry \%) } \\
\text { total sulfur } \\
\text { sulfate } \\
\text { pyritic } \\
\text { organic } \\
\text { analysis date }\end{array}$ & $\begin{array}{c}0.77 \\
0.02 \\
0.04 \\
0.71 \\
04 / 24 / 86\end{array}$ & & $\begin{array}{c}0.82 \\
0.02 \\
0.03 \\
0.77 \\
02 / 12 / 90\end{array}$ & \begin{tabular}{r|}
0.73 \\
0.02 \\
0.02 \\
0.70 \\
$02 / 06 / 90$
\end{tabular} & $\begin{array}{r}0.75 \\
0.02 \\
0.03 \\
0.71 \\
2 / 08 / 91\end{array}$ \\
\hline $\begin{array}{l}\text { CALORIFIC VALUE Idry } \\
\text { BTU/lb } \\
\text { analys is date }\end{array}$ & $\begin{array}{c}13640 \\
04 / 24 / 86\end{array}$ & & $\begin{array}{c}13512 \\
02 / 10 / 89\end{array}$ & $\begin{array}{r}13218 \\
02 / 06 / 90\end{array}$ & $\begin{array}{c}13519 \\
2 / 15 / 91\end{array}$ \\
\hline $\begin{array}{l}\text { ALKALI EXTRACTION } \\
\text { \% transmission } \\
\text { analysis date }\end{array}$ & & $\begin{array}{c}97.2 \\
06 / 07 / 88\end{array}$ & $\begin{array}{c}91.8 \\
02 / 10 / 89\end{array}$ & $\begin{array}{r}87.4 \\
01 / 24 / 90\end{array}$ & $\begin{array}{c}94.4 \\
8 / 26 / 91\end{array}$ \\
\hline
\end{tabular}

superseded by DeCS -15

notes: a I Sample dry, good condition. PSU S forms (revised). 


\begin{tabular}{|c|c|c|c|c|c|c|c|}
\hline $\begin{array}{l}\text { P5OC-1506 } \\
\text { collection date: }\end{array}$ & $\begin{array}{l}\text { WNER } \\
\text { W/14/85 }\end{array}$ & INAB, & Ro $\max =0$ & .96 & & & \\
\hline $\begin{array}{l}\text { Split I0 } \\
\text { size } \\
\text { \% head oxygen } \\
\text { note } \\
\text { date sooled } \\
\text { date oxygen/subspl }\end{array}$ & $\begin{array}{l}\text { analysis } \\
\text { sample } 1 \\
-60 \text { mesh }\end{array}$ & $\begin{array}{l}2 \text { can } \\
15 \text { of } 31 \\
-20 \text { mesh } \\
04 / 24 / 86\end{array}$ & $\begin{array}{c}30 \text { gil } \\
3 \text { of } 3 \\
-1 / 4 n \\
19.4 \\
a \\
04 / 22 / 86 \\
10 / 28 / 88\end{array}$ & $\begin{array}{c}30 \text { gal } \\
3 \text { of } 3 \\
-1 / 4^{\circ} \\
19.8 \\
10 / 13 / 89\end{array}$ & $\begin{array}{l}30 \text { gal } \\
3 \text { of } 3 \\
-1 / 4 \% \\
2 / 05 / 91\end{array}$ & $\begin{array}{l}30 \text { gal. } \\
3 \text { of } 3 \\
-1 / 4 \times \\
11 / 21 / 91\end{array}$ & $\begin{array}{l}30 \mathrm{gel} \\
3 \text { of } 3 \\
-1 / 4^{\circ} \\
b \\
4 / 13 / 93\end{array}$ \\
\hline $\begin{array}{l}\text { PROXIMATE } \\
\% \text { moisture } \\
\% \text { ash (dry) } \\
\text { analysis date }\end{array}$ & $\begin{array}{c}1.3 \\
13.6 \\
10 / 04 / 85\end{array}$ & $\begin{array}{c}1.8 \\
14.0 \\
05 / 05 / 88\end{array}$ & $\begin{array}{c}1.1 \\
14.7 \\
02 / 10 / 89\end{array}$ & $\begin{array}{c}1.5 \\
14.5 \\
03 / 01 / 90\end{array}$ & $\begin{array}{c}1.6 \\
15.0 \\
10 / 03 / 91\end{array}$ & $\begin{array}{c}1.6 \\
14.5 \\
10 / 21 / 92\end{array}$ & $\begin{array}{l}1.5 \\
14.3 \\
6 / 14 / 93\end{array}$ \\
\hline $\begin{array}{l}\text { GIESELER FLUIDITY } \\
\text { max. dom } \\
\text { fluid range } \\
\text { analysis date }\end{array}$ & $\begin{array}{r}29881 \\
90 \\
04 / 25 / 86\end{array}$ & $\begin{array}{r}6645 \\
89 \\
05 / 05 / 88\end{array}$ & $\begin{array}{c}4889 \\
79 \\
02 / 02 / 89\end{array}$ & $\begin{array}{c}2870 \\
81 \\
02 / 09 / 90\end{array}$ & $\begin{array}{c}782 \\
78 \\
6 / 26 / 91\end{array}$ & $\begin{array}{c}1426 \\
76 \\
2 / 19 / 93\end{array}$ & $\begin{array}{c}1646 \\
75 \\
7 / 01 / 93\end{array}$ \\
\hline $\begin{array}{l}\text { SUlfuR foies (dry \%) } \\
\text { total sulfur } \\
\text { sulfate } \\
\text { pyritic } \\
\text { organic } \\
\text { analysis date }\end{array}$ & $\begin{array}{r}0.70 \\
0.02 \\
0.14 \\
0.54 \\
10 / 05 / 85\end{array}$ & & $\begin{array}{r}0.72 \\
0.05 \\
0.07 \\
0.60 \\
02 / 12 / 90\end{array}$ & $\begin{array}{c}0.65 \\
0.06 \\
0.08 \\
0.51 \\
03 / 01 / 90\end{array}$ & $\begin{array}{r}0.66 \\
0.09 \\
0.09 \\
0.48 \\
10 / 03 / 91\end{array}$ & \begin{tabular}{c|}
0.63 \\
0.09 \\
0.05 \\
0.49 \\
$10 / 21 / 92$
\end{tabular} & $\begin{array}{c}0.65 \\
0.08 \\
0.04 \\
0.54 \\
6 / 14 / 93\end{array}$ \\
\hline $\begin{array}{l}\text { CALORIFIC VALUE } \\
\text { BTU/lb dry } \\
\text { analysis dete }\end{array}$ & $\begin{array}{c}13325 \\
10 / 05 / 85\end{array}$ & & $\begin{array}{c}13024 \\
02 / 10 / 89\end{array}$ & $\begin{array}{c}13095 \\
03 / 01 / 90\end{array}$ & $\begin{array}{c}12991 \\
10 / 03 / 91\end{array}$ & $\begin{array}{c}13033 \\
10 / 21 / 92\end{array}$ & $\begin{array}{c}13048 \\
6 / 14 / 93\end{array}$ \\
\hline $\begin{array}{l}\text { ALKALI EXTRACTION } \\
\% \text { transmission } \\
\text { analysis date }\end{array}$ & & $\begin{array}{c}98.8 \\
06 / 08 / 88\end{array}$ & $\begin{array}{c}95.4 \\
02 / 13 / 89\end{array}$ & $\begin{array}{c}98.9 \\
02 / 09 / 90\end{array}$ & $\begin{array}{c}99.2 \\
6 / 26 / 91\end{array}$ & $\begin{array}{c}99.1 \\
9 / 25 / 92\end{array}$ & $\begin{array}{c}97.3 \\
7 / 09 / 93\end{array}$ \\
\hline
\end{tabular}

notes: a ) Sample dry, good condition; PSU S forms (revisedI.

b) Sample dry, no visible deterioration. 


\begin{tabular}{|c|c|c|c|c|}
\hline \begin{tabular}{|c|}
$\begin{array}{r}\text { PSOC-1507 } \\
\text { collection date: }\end{array}$ \\
Split ID
\end{tabular} & $\begin{array}{l}\text { BEULAH } \\
07 / 02 / 85\end{array}$ & \multicolumn{3}{|c|}{ ligA, Ro $\max =0.37$} \\
\hline $\begin{array}{l}\text { Split ID } \\
\text { size } \\
\text { \% head oxygen } \\
\text { note } \\
\text { date sealed } \\
\text { date oxygen/subspl }\end{array}$ & $\begin{array}{l}\text { analysis } \\
\text { sample } 1 \\
-60 \text { mesh }\end{array}$ & $\begin{array}{c}30 \text { gal. } \\
1 \text { of } 3 \\
-1 \% \\
6.7 \\
\text { a } \\
04 / 10 / 86 \\
10 / 28 / 88\end{array}$ & $\begin{array}{c}30 \text { gal. } \\
1 \text { of } 3 \\
-1 / 4^{\circ 1} \\
7.1 \\
10 / 13 / 89\end{array}$ & $\begin{array}{l}\text { super- } \\
\text { seded } \\
\text { by } \\
\text { DECS-11 }\end{array}$ \\
\hline $\begin{array}{l}\text { PROXImATE } \\
\% \text { moisture } \\
\% \text { ash (dry) } \\
\text { analysis cate }\end{array}$ & $\begin{array}{c}35.6 \\
12.5 \\
04 / 24 / 86\end{array}$ & $\begin{array}{c}32.8 \\
12.5 \\
02 / 10 / 89\end{array}$ & $\begin{array}{c}31.9 \\
13.3 \\
02 / 06 / 90\end{array}$ & \\
\hline $\begin{array}{l}\text { SULfuR FORrs (dry \%) } \\
\text { total sulfur } \\
\text { sulfate } \\
\text { pyritic } \\
\text { organic } \\
\text { analysis date }\end{array}$ & $\begin{array}{c}1.43 \\
0.01 \\
0.02 \\
1.40 \\
04 / 24 / 86\end{array}$ & $\begin{array}{c}1.41 \\
0.19 \\
0.48 \\
0.74 \\
02 / 12 / 90\end{array}$ & $\begin{array}{c}1.23 \\
0.16 \\
0.51 \\
0.56 \\
02 / 06 / 90\end{array}$ & \\
\hline $\begin{array}{l}\text { CALORIFIC VALUE (dry) } \\
\text { BTU/lb } \\
\text { analysis date }\end{array}$ & $\begin{array}{c}10627 \\
04 / 24 / 86\end{array}$ & $\begin{array}{c}10585 \\
02 / 10 / 89\end{array}$ & $\begin{array}{c}10420 \\
02 / 06 / 90\end{array}$ & \\
\hline
\end{tabular}

notes: a) Sample moist, trace white and yellow sulfate.

PSU $s$ forms (revised). 


\begin{tabular}{|c|c|c|c|c|c|}
\hline $\begin{array}{l}\text { PSOC-1508 } \\
\text { collection date: }\end{array}$ & $\begin{array}{l}\text { OCAHONTAS } \\
1 / 05 / 85\end{array}$ & \#3 lvb, & Ro $\max =$ & 85 & \\
\hline $\begin{array}{l}\text { Split ID } \\
\text { size } \\
\text { \% head oxygen } \\
\text { : rote } \\
\text { date sealed } \\
\text { date oxygen/subspl }\end{array}$ & $\begin{array}{l}\text { analysis } \\
\text { sample } 1 \\
-60 \text { mesh }\end{array}$ & $\begin{array}{l}\# 2 \text { can } \\
15 \text { of } 31 \\
-20 \text { mesh } \\
12 / 04 / 85\end{array}$ & $\begin{array}{c}30 \mathrm{gal} \\
6 \text { of } 6 \\
-1 / 4 " \\
20.7 \\
a \\
11 / 14 / 85 \\
10 / 28 / 88\end{array}$ & $\begin{array}{c}30 \text { gal. } \\
6 \text { of } 6 \\
-1 / 4 " \\
20.9 \\
10 / 13 / 89\end{array}$ & $\begin{array}{l}30 \text { gal. } \\
6 \text { of } 6 \\
-1 / 4 " \\
2 / 05 / 91\end{array}$ \\
\hline $\begin{array}{l}\text { PROXIMATE } \\
\% \text { moisture } \\
\% \text { ash (dry) } \\
\text { analysis date }\end{array}$ & $\begin{array}{c}1.1 \\
7.4 \\
12 / 23 / 85\end{array}$ & $\begin{array}{c}1.9 \\
7.1 \\
05 / 06 / 88\end{array}$ & $\begin{array}{c}1.4 \\
7.4 \\
02 / 10 / 89\end{array}$ & $\begin{array}{c}1.3 \\
7.5 \\
02 / 06 / 90\end{array}$ & $\begin{array}{c}2.4 \\
7.2 \\
2 / 15 / 91\end{array}$ \\
\hline $\begin{array}{l}\text { GIESELER FLUIDITY } \\
\text { max. ddpm } \\
\text { fluid range } \\
\text { analysis date }\end{array}$ & $\begin{array}{r}10 \\
44 \\
12 / 07 / 85\end{array}$ & $\begin{array}{r}3 \\
41 \\
05 / 06 / 88\end{array}$ & $\begin{array}{c}0 \\
0 \\
02 / 02 / 89\end{array}$ & $\begin{array}{l}\text { discon- } \\
\text { tinued }\end{array}$ & \\
\hline $\begin{array}{l}\text { SULFUR FORMS (dry \%) } \\
\text { total sulfur } \\
\text { sulfate } \\
\text { pyritic } \\
\text { orgenic } \\
\text { analysis date }\end{array}$ & $\begin{array}{c}0.64 \\
0.00 \\
0.14 \\
0.50 \\
12 / 23 / 85\end{array}$ & & $\begin{array}{c}0.57 \\
0.02 \\
0.24 \\
0.31 \\
02 / 10 / 89\end{array}$ & \begin{tabular}{|c|}
0.61 \\
0.03 \\
0.07 \\
0.51 \\
$02 / 06 / 90$
\end{tabular} & $\begin{array}{c}0.60 \\
0.02 \\
0.07 \\
0.51 \\
2 / 15 / 91\end{array}$ \\
\hline $\begin{array}{l}\text { CALORIFIC VALUE (dry) } \\
\text { BTU/1b } \\
\text { analysis date }\end{array}$ & $\begin{array}{c}14542 \\
12 / 23 / 85\end{array}$ & & $\begin{array}{c}14489 \\
02 / 10 / 89\end{array}$ & $\begin{array}{r}14345 \\
02 / 06 / 90\end{array}$ & $\begin{array}{c}14410 \\
2 / 15 / 91\end{array}$ \\
\hline $\begin{array}{l}\text { ALKALI EXTRACTION } \\
\text { \% transmission } \\
\text { analysis date }\end{array}$ & & $\begin{array}{c}100.2 \\
06 / 09 / 88\end{array}$ & $\begin{array}{c}96.9 \\
02 / 13 / 89\end{array}$ & $\begin{array}{c}97.7 \\
01 / 25 / 90\end{array}$ & $\begin{array}{c}95.8 \\
8 / 27 / 91\end{array}$ \\
\hline
\end{tabular}

notes: a) Sample dry, good condition. 


\begin{tabular}{|c|c|c|c|c|c|c|}
\hline $\begin{array}{l}\text { PSOC-1515 } \\
\text { collection date: }\end{array}$ & $\begin{array}{l}\text { ENMSYLVAN } \\
5 / 08 / 86\end{array}$ & A C & a, Ro $\max$ & $=2.80$ & & \\
\hline $\begin{array}{l}\text { Split ID } \\
\text { size } \\
\text { \% head oxygen } \\
\text { note } \\
\text { date sealed } \\
\text { date oxygen/subspl }\end{array}$ & $\begin{array}{l}\text { analys is } \\
\text { sample } 1 \\
-60 \text { mesh }\end{array}$ & $\begin{array}{c}30 \mathrm{gal} \\
4 \text { of } 4 \\
-1 / 4 " \\
19.8 \\
a \\
08 / 08 / 86 \\
10 / 28 / 88\end{array}$ & $\begin{array}{l}30 \text { gal. } \\
4 \text { of } 4 \\
-1 / 4 " \\
20.3 \\
10 / 13 / 89\end{array}$ & $\begin{array}{l}30 \text { gal. } \\
4 \text { of } 4 \\
-1 / 4^{\prime \prime}\end{array}$ & $\begin{array}{l}30 \text { gal. } \\
4 \text { of } 4 \\
-1 / 4 " \\
11 / 21 / 91\end{array}$ & $\begin{array}{l}30 \text { gal. } \\
4 \text { of } 4 \\
-1 / 4 " \\
b \\
5 / 06 / 93\end{array}$ \\
\hline $\begin{array}{l}\text { PROXIMATE } \\
\% \text { moisture } \\
\% \text { ash (dry) } \\
\text { analysis date }\end{array}$ & $\begin{array}{c}2.4 \\
29.2 \\
09 / 25 / 86\end{array}$ & $\begin{array}{c}2.2 \\
28.3 \\
02 / 10 / 89\end{array}$ & $\begin{array}{c}2.1 \\
28.3 \\
02 / 06 / 90\end{array}$ & $\begin{array}{c}3.5 \\
30.0 \\
2 / 08 / 91\end{array}$ & $\begin{array}{c}2.1 \\
33.1 \\
2 / 21 / 92\end{array}$ & $\begin{array}{c}2.4 \\
29.7 \\
6 / 14 / 93\end{array}$ \\
\hline $\begin{array}{l}\text { SULFUR FORIS (dry \%) } \\
\text { total sul fur } \\
\text { sulfate } \\
\text { pyritic } \\
\text { organic } \\
\text { analysis date }\end{array}$ & $\begin{array}{c}0.58 \\
0.00 \\
0.10 \\
0.49 \\
09 / 25 / 86\end{array}$ & $\begin{array}{c}0.63 \\
0.13 \\
0.04 \\
0.46 \\
02 / 12 / 90\end{array}$ & $\begin{array}{r}0.59 \\
0.10 \\
0.06 \\
0.43 \\
02 / 06 / 90\end{array}$ & $\begin{array}{r}0.60 \\
0.10 \\
0.06 \\
0.44 \\
2 / 08 / 91\end{array}$ & $\begin{array}{c}0.53 \\
0.06 \\
0.03 \\
0.44 \\
2 / 21 / 92\end{array}$ & $\begin{array}{c}0.60 \\
0.10 \\
0.04 \\
0.46 \\
6 / 14 / 93\end{array}$ \\
\hline $\begin{array}{l}\text { CALORIFIC VALUE (dry) } \\
\text { BTU/lb } \\
\text { analys is date }\end{array}$ & $\begin{array}{c}10642 \\
09 / 16 / 86\end{array}$ & $\begin{array}{c}10677 \\
02 / 10 / 89\end{array}$ & $\begin{array}{c}10657 \\
02 / 06 / 90\end{array}$ & $\begin{array}{c}10360 \\
2 / 15 / 91\end{array}$ & $\begin{array}{c}9812 \\
2 / 21 / 92\end{array}$ & $\begin{array}{c}10386 \\
6 / 14 / 93\end{array}$ \\
\hline
\end{tabular}

notes: a) Sample moist, good condition. PSU S forms (revised).

b) No visible deterioration. 


\begin{tabular}{|c|c|c|c|c|c|c|c|}
\hline $\begin{array}{l}\text { PSOC-1516 LOME } \\
\text { collection date: }\end{array}$ & $\begin{array}{l}\text { R KITTANNI } \\
5 / 29 / 86\end{array}$ & NG Ivb, & Ro $\max =1$ & & & & \\
\hline $\begin{array}{l}\text { Split ID } \\
\text { size } \\
\text { \% head oxygen } \\
\text { note } \\
\text { date sealed } \\
\text { date oxygen/subspl }\end{array}$ & $\begin{array}{l}\text { analys is } \\
\text { sample } 1 \\
-60 \text { mesh }\end{array}$ & $\begin{array}{l}\# 2 \text { can } \\
15 \text { of } 31 \\
-20 \text { mesh } \\
08 / 20 / 86\end{array}$ & $\begin{array}{c}30 \mathrm{gal} \\
4 \text { of } 4 \\
-1 / 4 " \\
20.5 \\
a \\
08 / 08 / 86 \\
10 / 28 / 88\end{array}$ & $\begin{array}{l}30 \mathrm{gal} \\
4 \text { of } 4 \\
-1 / 4 " \\
20.6 \\
10 / 13 / 89\end{array}$ & b & $\begin{array}{l}30 \text { gal. } \\
4 \text { of } 4 \\
-1 / 4 " \\
11 / 21 / 91\end{array}$ & $\begin{array}{c}30 \text { gal. } \\
4 \text { of } 4 \\
-1 / 4 " \\
\text { c } \\
5 / 06 / 93\end{array}$ \\
\hline $\begin{array}{l}\text { PROXIMATE } \\
\% \text { moisture } \\
\% \text { ash (dry) } \\
\text { analysis date }\end{array}$ & $\begin{array}{c}2.0 \\
10.3 \\
09 / 25 / 86\end{array}$ & $\begin{array}{c}2.1 \\
9.4 \\
05 / 09 / 88\end{array}$ & $\begin{array}{c}2.1 \\
10.3 \\
02 / 10 / 89\end{array}$ & $\begin{array}{c}1.9 \\
10.5 \\
2 / 28 / 90\end{array}$ & & $\begin{array}{c}1.9 \\
15.4 \\
2 / 21 / 92\end{array}$ & $\begin{array}{c}2.5 \\
10.5 \\
6 / 15 / 93\end{array}$ \\
\hline $\begin{array}{l}\text { GIESELER FLUIDITY } \\
\text { max. ddpm } \\
\text { fluid range } \\
\text { analysis date }\end{array}$ & $\begin{array}{r}53 \\
69 \\
08 / 20 / 86\end{array}$ & $\begin{array}{r}2 \\
25 \\
05 / 09 / 88\end{array}$ & $\begin{array}{c}2 \\
\text { nd } \\
02 / 06 / 89\end{array}$ & $\begin{array}{l}0 \\
0\end{array}$ & & $\begin{array}{l}\text { discon- } \\
\text { timued }\end{array}$ & \\
\hline $\begin{array}{l}\text { SULFUR FORis I dry \% ) } \\
\text { total sulfur } \\
\text { sulfate } \\
\text { pyritic } \\
\text { organic } \\
\text { analysis date }\end{array}$ & $\begin{array}{r}1.40 \\
0.01 \\
0.61 \\
0.78 \\
09 / 25 / 86\end{array}$ & & $\begin{array}{r}1.33 \\
0.01 \\
0.81 \\
0.50 \\
02 / 10 / 89\end{array}$ & $\begin{array}{c}1.44 \\
0.29 \\
0.36 \\
0.79 \\
02 / 28 / 90\end{array}$ & & $\begin{array}{c}1.56 \\
0.22 \\
0.38 \\
0.96 \\
2 / 21 / 92\end{array}$ & $\begin{array}{c}1.39 \\
0.37 \\
0.29 \\
0.74 \\
6 / 15 / 93\end{array}$ \\
\hline $\begin{array}{l}\text { CALORIFIC VALUE (dry) } \\
\text { BTU/lb } \\
\text { analysis date }\end{array}$ & $\begin{array}{c}14025 \\
09 / 25 / 86\end{array}$ & & $\begin{array}{c}13822 \\
02 / 10 / 89\end{array}$ & $\begin{array}{c}13752 \\
02 / 28 / 90\end{array}$ & & $\begin{array}{c}12915 \\
2 / 21 / 92\end{array}$ & $\begin{array}{r}13708 \\
6 / 15 / 93\end{array}$ \\
\hline $\begin{array}{l}\text { ALKALI EXTRACTION } \\
\% \text { transmission } \\
\text { analysis date }\end{array}$ & & $\begin{array}{c}99.9 \\
06 / 08 / 88\end{array}$ & $\begin{array}{c}95.9 \\
02 / 15 / 89\end{array}$ & $\begin{array}{c}98.8 \\
02 / 12 / 90\end{array}$ & & $\begin{array}{c}96.9 \\
9 / 22 / 92\end{array}$ & $\begin{array}{r}99.7 \\
7 / 09 / 93\end{array}$ \\
\hline
\end{tabular}

notes: a I Sample dry, good condition.

b) quality monitoring not performed - recollection was planned at the time quality monitoring splitting was performed.

c) Trace of yellow coatings. 


\begin{tabular}{|c|c|c|c|c|c|c|c|}
\hline $\begin{array}{l}\text { PSOC-1517 } \\
\text { collection date: }\end{array}$ & $\begin{array}{l}\text { HIO \#5 } \\
6 / 09 / 86\end{array}$ & mBb, & Ro $\max =$ & & & & \\
\hline $\begin{array}{l}\text { Split ID } \\
\text { size } \\
\text { \% head oxygen } \\
\text { note } \\
\text { date sealed } \\
\text { date oxygen/subspl }\end{array}$ & $\begin{array}{l}\text { analysis } \\
\text { sample } 1 \\
-60 m e s h\end{array}$ & $\begin{array}{l}\# 2 \text { can } \\
16 \text { of } 31 \\
-20 \text { mesh } \\
08 / 22 / 86\end{array}$ & $\begin{array}{c}30 \text { gal } \\
2 \text { of } 3 \\
-1 " \\
16.4 \\
a \\
08 / 07 / 86 \\
10 / 28 / 88\end{array}$ & $\begin{array}{l}30 \mathrm{gal} \\
2 \text { of } 3 \\
-1 / 4^{\prime \prime} \\
18.7 \\
10 / 13 / 89\end{array}$ & $\begin{array}{l}30 \text { gal. } \\
2 \text { of } 3 \\
-1 / 4 " \\
2 / 05 / 91\end{array}$ & $\begin{array}{l}30 \text { gal. } \\
2 \text { of } 3 \\
-1 / 4 " \\
11 / 25 / 91\end{array}$ & $\begin{array}{l}30 \text { gal. } \\
2 \text { of } 3 \\
-1 / 4^{\prime \prime} \\
\text { c } \\
5 / 06 / 93\end{array}$ \\
\hline $\begin{array}{l}\text { PROXIMATE } \\
\% \text { moisture } \\
\% \text { ash (dry) } \\
\text { analysis date }\end{array}$ & $\begin{array}{c}4.2 \\
4.8 \\
09 / 25 / 86\end{array}$ & $\begin{array}{c}4.0 \\
4.7 \\
05 / 10 / 88\end{array}$ & $\begin{array}{c}4.3 \\
5.8 \\
02 / 10 / 89\end{array}$ & $\begin{array}{c}4.5 \\
5.7 \\
02 / 28 / 90\end{array}$ & $\begin{array}{c}5.3 \\
5.6 \\
10 / 03 / 91\end{array}$ & $\begin{array}{c}4.1 \\
3.2 \\
2 / 21 / 92\end{array}$ & $\begin{array}{c}5.4 \\
5.9 \\
6 / 14 / 93\end{array}$ \\
\hline $\begin{array}{l}\text { GIESELER FLUIDITY } \\
\text { max. ddpm } \\
\text { fluid range } \\
\text { analys is date }\end{array}$ & $\begin{array}{r}1509 \\
75 \\
09 / 03 / 86\end{array}$ & $\begin{array}{r}13 \\
50 \\
05 / 10 / 88\end{array}$ & $\begin{array}{c}77 \\
60 \\
02 / 06 / 89\end{array}$ & $\begin{array}{c}18 \\
52 \\
02 / 13 / 90\end{array}$ & $\begin{array}{c}1(b) \\
2 \\
8 / 26 / 91\end{array}$ & $\begin{array}{l}\text { discon- } \\
\text { timued }\end{array}$ & \\
\hline $\begin{array}{l}\text { SULFUR FORMS (dry \%) } \\
\text { total sulfur } \\
\text { sulfate } \\
\text { Pyritic } \\
\text { organic } \\
\text { analysis date }\end{array}$ & $\begin{array}{c}1.92 \\
0.01 \\
0.70 \\
1.21 \\
09 / 25 / 86\end{array}$ & & $\begin{array}{c}2.09 \\
0.01 \\
0.98 \\
1.10 \\
02 / 10 / 89\end{array}$ & $\begin{array}{r}2.19 \\
0.32 \\
1.17 \\
0.70 \\
02 / 28 / 90\end{array}$ & $\begin{array}{r}2.08 \\
0.43 \\
0.97 \\
0.68 \\
10 / 23 / 91\end{array}$ & $\begin{array}{l}1.40 \\
0.13 \\
0.39 \\
0.88 \\
2 / 21 / 92\end{array}$ & $\begin{array}{r}2.30 \\
0.51 \\
0.97 \\
0.82 \\
6 / 14 / 93\end{array}$ \\
\hline $\begin{array}{l}\text { CALORIFIC VALUE (dry) } \\
\text { BTU/lb } \\
\text { analysis date }\end{array}$ & $\begin{array}{c}14258 \\
09 / 25 / 86\end{array}$ & & $\begin{array}{c}13933 \\
02 / 10 / 89\end{array}$ & $\begin{array}{c}13841 \\
02 / 28 / 90\end{array}$ & $\begin{array}{c}13814 \\
10 / 03 / 91\end{array}$ & $\begin{array}{c}14320 \\
2 / 21 / 92\end{array}$ & $\begin{array}{r}13663 \\
6 / 14 / 93\end{array}$ \\
\hline $\begin{array}{l}\text { ALKALI EXTRACTION } \\
\% \text { transmission } \\
\text { analysis date }\end{array}$ & & $\begin{array}{c}98.0 \\
06 / 09 / 88\end{array}$ & $\begin{array}{c}98.1 \\
02 / 15 / 89\end{array}$ & $\begin{array}{c}87.0 \\
02 / 13 / 90\end{array}$ & $\begin{array}{c}96.4 \\
8 / 26 / 91\end{array}$ & $\begin{array}{c}97.1 \\
9 / 23 / 92\end{array}$ & $\begin{array}{r}98.2 \\
7 / 12 / 93\end{array}$ \\
\hline
\end{tabular}

notes: a I Sample dry, good condition.

b) No consecutive readings with fluidity.

c) No visible deterioration. 


\begin{tabular}{|c|c|c|c|c|c|c|c|}
\hline \multicolumn{8}{|c|}{$\begin{array}{l}\text { PSOC-1518 M. KITTANIING (OHIO \#6) mBb, Ro max }=0.69 \\
\text { collection date: 06/10/86 }\end{array}$} \\
\hline $\begin{array}{l}\text { Split ID } \\
\text { size } \\
\text { \% head oxygen } \\
\text { note } \\
\text { date sealed } \\
\text { date oxygen/subspl }\end{array}$ & $\begin{array}{l}\text { analysis } \\
\text { sample } 1 \\
-60 \text { mesh }\end{array}$ & $\begin{array}{l}\# 2 \text { can } \\
15 \text { of } 31 \\
-20 \text { mesh } \\
08 / 26 / 86\end{array}$ & $\begin{array}{c}30 \text { gal } \\
3 \text { of } 3 \\
-1 / 4 " \\
18.5 \\
a \\
08 / 07 / 86 \\
10 / 28 / 88\end{array}$ & $\begin{array}{c}30 \text { gal } \\
3 \text { of } 3 \\
-1 / 4^{\prime \prime} \\
19.1 \\
\\
10 / 16 / 89\end{array}$ & $\begin{array}{l}30 \text { gal. } \\
3 \text { of } 3 \\
-1 / 4 " \\
2 / 05 / 91\end{array}$ & $\begin{array}{l}30 \text { gal. } \\
3 \text { of } 3 \\
-1 / 4 " \\
11 / 25 / 91\end{array}$ & $\begin{array}{l}30 \text { gal. } \\
3 \text { of } 3 \\
-1 / 4 \cdot \\
\text { b } \\
5 / 10 / 93\end{array}$ \\
\hline $\begin{array}{l}\text { PROXIMATE } \\
\% \text { moisture } \\
\% \text { ash (dry) } \\
\text { analysis date }\end{array}$ & $\begin{array}{c}5.3 \\
9.9 \\
09 / 25 / 86\end{array}$ & $\begin{array}{c}5.0 \\
9.3 \\
05 / 11 / 88\end{array}$ & $\begin{array}{c}5.8 \\
9.6 \\
03 / 07 / 89\end{array}$ & $\begin{array}{c}5.9 \\
9.5 \\
02 / 06 / 90\end{array}$ & $\begin{array}{c}6.8 \\
9.8 \\
2 / 08 / 91\end{array}$ & $\begin{array}{l}4.3 \\
8.4 \\
2 / 21 / 92\end{array}$ & $\begin{array}{c}6.2 \\
9.6 \\
6 / 14 / 93\end{array}$ \\
\hline $\begin{array}{l}\text { GIESELER FLUIDITY } \\
\text { max. ddpa } \\
\text { fluid range } \\
\text { analysis date }\end{array}$ & $\begin{array}{r}113 \\
62 \\
09 / 04 / 86\end{array}$ & $\begin{array}{r}118 \\
66 \\
05 / 11 / 88\end{array}$ & $\begin{array}{c}1 \\
3 \\
3 / 03 / 89\end{array}$ & $\begin{array}{l}\text { discon- } \\
\text { timued }\end{array}$ & & & \\
\hline $\begin{array}{l}\text { SULFUR FORits (dry \%) } \\
\text { total sulfur } \\
\text { sulfate } \\
\text { pyritic } \\
\text { organic } \\
\text { analysis date }\end{array}$ & $\begin{array}{r}3.86 \\
0.12 \\
2.26 \\
1.48 \\
09 / 25 / 86\end{array}$ & & $\begin{array}{c}3.44 \\
0.91 \\
1.05 \\
1.48 \\
03 / 07 / 89\end{array}$ & $\begin{array}{r}3.77 \\
1.06 \\
0.88 \\
1.83 \\
02 / 06 / 90\end{array}$ & $\begin{array}{r}3.62 \\
1.16 \\
0.55 \\
1.91 \\
1 / 23 / 91\end{array}$ & $\begin{array}{l}2.98 \\
0.46 \\
0.35 \\
2.17 \\
2 / 21 / 92\end{array}$ & $\begin{array}{l}3.67 \\
1.30 \\
0.49 \\
1.88 \\
6 / 14 / 93\end{array}$ \\
\hline $\begin{array}{l}\text { CALORIFIC VALUE (dry) } \\
\text { BTU/1b } \\
\text { analysis date }\end{array}$ & $\begin{array}{c}13074 \\
09 / 25 / 86\end{array}$ & & $\begin{array}{r}12258 \\
03 / 07 / 89\end{array}$ & $\begin{array}{c}12538 \\
02 / 06 / 90\end{array}$ & $\begin{array}{c}12434 \\
2 / 15 / 91\end{array}$ & $\begin{array}{c}12902 \\
2 / 21 / 92\end{array}$ & $\begin{array}{l}12352 \\
6 / 14 / 93\end{array}$ \\
\hline $\begin{array}{l}\text { ALKALI EXTRACTION } \\
\text { \% transmission } \\
\text { analysis date }\end{array}$ & & $\begin{array}{c}97.4 \\
06 / 09 / 88\end{array}$ & $\begin{array}{c}94.8 \\
03 / 06 / 89\end{array}$ & $\begin{array}{c}92.4 \\
01 / 25 / 90\end{array}$ & $\begin{array}{c}91.2 \\
8 / 17 / 91\end{array}$ & $\begin{array}{c}88.5 \\
9 / 23 / 92\end{array}$ & $\begin{array}{l}94.9 \\
7 / 12 / 93\end{array}$ \\
\hline
\end{tabular}

notes: a) Sample dry, large visible white sulfate crystals.

b) White encrustations and brown coatings on some particles. 


\begin{tabular}{|c|c|c|c|c|c|c|c|}
\hline $\begin{array}{l}\text { PSOC-1519 } \\
\text { collection date: }\end{array}$ & $\begin{array}{l}\text { ITTSBURGH } \\
6 / 19 / 86\end{array}$ & truBb, & Ro $\max =$ & & & & \\
\hline $\begin{array}{l}\text { Split ID } \\
\text { size } \\
\text { \% head oxygen } \\
\text { note } \\
\text { date sealed } \\
\text { date oxygen/subspl }\end{array}$ & $\begin{array}{l}\text { analysis } \\
\text { sample } 1 \\
-60 \text { mesh }\end{array}$ & $\begin{array}{l}\# 2 \text { can } \\
15 \text { of } 31 \\
-20 \text { mesh } \\
08 / 29 / 86\end{array}$ & $\begin{array}{l}30 \text { gal. } \\
1 \text { of } 3 \\
-1 / 4^{\prime \prime} \\
18.3 \\
a \\
8 / 06 / 86 \\
9 / 24 / 88\end{array}$ & $\begin{array}{l}35 \text { gal. } \\
1 \text { of } 3 \\
-1 / 4 " 1 \\
18.4 \\
10 / 16 / 89\end{array}$ & b & $\begin{array}{l}30 \text { \%al. } \\
1 \text { of } 3 \\
-1 / 4 " \\
11 / 25 / 91\end{array}$ & $\begin{array}{l}30 \text { gal. } \\
1 \text { of } 3 \\
-1 / 4^{\circ} \\
\text { c } \\
4 / 13 / 93\end{array}$ \\
\hline $\begin{array}{l}\text { PROXIMATE } \\
\% \text { mois ture } \\
\% \text { ash (dry) } \\
\text { analysis date }\end{array}$ & $\begin{array}{c}3.1 \\
10.5 \\
09 / 25 / 86\end{array}$ & $\begin{array}{c}3.5 \\
9.8 \\
05 / 12 / 88\end{array}$ & $\begin{array}{c}1.5 \\
10.2 \\
10 / 12 / 88\end{array}$ & $\begin{array}{c}3.3 \\
11.7 \\
02 / 28 / 90\end{array}$ & & $\begin{array}{c}5.6 \\
13.7 \\
10 / 15 / 92\end{array}$ & $\begin{array}{l}4.5 \\
11.8 \\
6 / 14 / 93\end{array}$ \\
\hline $\begin{array}{l}\text { GIESELER FLUIDITY } \\
\text { max. ddpm } \\
\text { fluid range } \\
\text { analysis date }\end{array}$ & $\begin{array}{r}3548 \\
77 \\
12 / 15 / 86\end{array}$ & $\begin{array}{r}1409 \\
80 \\
05 / 12 / 88\end{array}$ & $\begin{array}{c}800 \\
67 \\
10 / 21 / 88\end{array}$ & $\begin{array}{c}17 \\
39 \\
02 / 14 / 90\end{array}$ & & $\begin{array}{c}3 \\
27 \\
9 / 18 / 92\end{array}$ & $\begin{array}{l}81 \\
53 \\
7 / 02 / 93\end{array}$ \\
\hline $\begin{array}{l}\text { SULFUR FORMS (dry \%) } \\
\text { total sulfur } \\
\text { sulfate } \\
\text { pyritic } \\
\text { organic } \\
\text { analysis date }\end{array}$ & $\begin{array}{c}1.94 \\
0.10 \\
0.02 \\
1.82 \\
09 / 25 / 86\end{array}$ & & $\begin{array}{c}1.93 \\
0.47 \\
0.60 \\
0.86 \\
09 / 17 / 89\end{array}$ & $\begin{array}{c}1.97 \\
0.55 \\
0.42 \\
1.00 \\
02 / 28 / 90\end{array}$ & & $\begin{array}{r}2.62 \\
1.16 \\
0.51 \\
0.95 \\
10 / 15 / 92\end{array}$ & $\begin{array}{l}2.02 \\
0.73 \\
0.29 \\
1.00 \\
6 / 14 / 93\end{array}$ \\
\hline $\begin{array}{l}\text { CALORIFIC VALUE (dry) } \\
\text { BTU/Ib } \\
\text { analysis date }\end{array}$ & $\begin{array}{c}13382 \\
09 / 25 / 86\end{array}$ & & & $\begin{array}{c}12959 \\
02 / 28 / 90\end{array}$ & & $\begin{array}{c}12221 \\
10 / 15 / 92\end{array}$ & $\begin{array}{c}12825 \\
6 / 14 / 93\end{array}$ \\
\hline $\begin{array}{l}\text { ALKALI EXTRACTION } \\
\text { \% transmission } \\
\text { analysis date }\end{array}$ & & $\begin{array}{c}81.4 \\
06 / 09 / 88\end{array}$ & $\begin{array}{c}85.0 \\
10 / 06 / 88\end{array}$ & $\begin{array}{c}70.7 \\
02 / 28 / 90\end{array}$ & & $\begin{array}{c}70.6 \\
9 / 18 / 92\end{array}$ & $\begin{array}{r}77.2 \\
7 / 02 / 93\end{array}$ \\
\hline
\end{tabular}

notes: a) PSU MCL ash, moisture.

b) Quality monitoring not performed - recollection was planned at the time quality monitoring splitting was performed.

c) No visible deterioration. 


\begin{tabular}{|c|c|c|c|c|}
\hline \multirow{2}{*}{$\begin{array}{l}\text { PSOC-1520 } \\
\text { collection date: } \\
\text { Split ID } \\
\text { size } \\
\% \text { head oxygen } \\
\text { note } \\
\text { date sealed } \\
\text { date oxygen/subspl }\end{array}$} & $\begin{array}{l}\text { SHITH ROLAA } \\
08 / 18 / 86\end{array}$ & \multicolumn{3}{|c|}{ subC, Ro $\max =0.43$} \\
\hline & $\begin{array}{l}\text { analysis } \\
\text { sample } 1 \\
-60 \text { mesh }\end{array}$ & $\begin{array}{l}\text { analysis } \\
\text { sample } 2 \\
-60 \text { mesh }\end{array}$ & $\begin{array}{c}5 \mathrm{gl} . \\
-1 / 4^{-\prime} \\
a\end{array}$ & $\begin{array}{l}30 \mathrm{gal} \\
4 \text { of } 5 \\
-10 \\
12.1\end{array}$ \\
\hline $\begin{array}{l}\text { PROXIMATE } \\
\% \text { moisture } \\
\% \text { ash (dry) } \\
\text { analysis date }\end{array}$ & $\begin{array}{c}29.7 \\
12.4 \\
09 / 16 / 86\end{array}$ & $\begin{array}{c}26.7 \\
12.6 \\
10 / 05 / 87\end{array}$ & $\begin{array}{c}24.8 \\
10.5 \\
09 / 14 / 88\end{array}$ & $\begin{array}{r}27.3 \\
12.7 \\
02 / 06 / 90\end{array}$ \\
\hline $\begin{array}{l}\text { SULFUR FORis (dry \%) } \\
\text { total sulfur } \\
\text { sulfate } \\
\text { pyritic } \\
\text { organic } \\
\text { analysis date }\end{array}$ & $\begin{array}{c}1.21 \\
0.01 \\
0.05 \\
1.15 \\
09 / 16 / 86\end{array}$ & $10 / 05 / 87$ & $\begin{array}{c}1.24 \\
0.34 \\
0.14 \\
0.76 \\
09 / 22 / 88\end{array}$ & $\begin{array}{c}1.18 \\
0.24 \\
0.23 \\
0.71 \\
02 / 06 / 90\end{array}$ \\
\hline $\begin{array}{l}\text { CALORIFIC VALUE (dry) } \\
\text { BTU/1b } \\
\text { analysis date }\end{array}$ & $\begin{array}{c}11291 \\
09 / 16 / 86\end{array}$ & & & $\begin{array}{c}11006 \\
02 / 06 / 90\end{array}$ \\
\hline
\end{tabular}

notes: a) Entire sample used for restocking \#2 cans. PSU MCL ash, moisture, sulfur forms. 


\begin{tabular}{|c|c|c|c|c|c|c|c|}
\hline $\begin{array}{l}\text { PSOC-1521 } \\
\text { collection date: }\end{array}$ & $\begin{array}{l}\text {. HARTSHOF } \\
\text { 8/22/86 }\end{array}$ & Ivb, & Ro $\max =1$ & & & & \\
\hline $\begin{array}{l}\text { Split ID } \\
\text { size } \\
\text { \% head oxygen } \\
\text { note } \\
\text { date sealed } \\
\text { date oxygen/subspl }\end{array}$ & $\begin{array}{l}\text { analysis } \\
\text { sample } 1 \\
-60 \text { mesh }\end{array}$ & $\begin{array}{l}\text { \# } 2 \text { can } \\
15 \text { of } 31 \\
-20 \text { mesh } \\
08 / 29 / 86\end{array}$ & $\begin{array}{c}30 \text { gal } \\
3 \text { of } 3 \\
-1 / 4^{\prime \prime} \\
18.8 \\
a \\
08 / 07 / 86 \\
10 / 28 / 88\end{array}$ & $\begin{array}{l}30 \text { gal } \\
3 \text { of } 3 \\
-1 / 4 \times \\
19.7 \\
10 / 23 / 89\end{array}$ & $\begin{array}{l}30 \text { gal. } \\
3 \text { of } 3 \\
-1 / 4^{\mathrm{m}}\end{array}$ & $\begin{array}{l}30 \mathrm{gal} \\
3 \text { of } 3 \\
-1 / 4^{n}\end{array}$ & $\begin{array}{l}30 \text { gal. } \\
3 \text { of } 3 \\
-1 / 4^{n} \\
b \\
5 / 10 / 93\end{array}$ \\
\hline $\begin{array}{l}\text { PROXIMATE } \\
\% \text { mois ture } \\
\% \text { ash (dry) } \\
\text { analysis date }\end{array}$ & $\begin{array}{c}2.7 \\
11.2 \\
09 / 26 / 86\end{array}$ & $\begin{array}{c}2.1 \\
10.0 \\
05 / 13 / 88\end{array}$ & $\begin{array}{c}2.5 \\
11.4 \\
03 / 07 / 89\end{array}$ & $\begin{array}{c}2.6 \\
10.0 \\
02 / 06 / 90\end{array}$ & $\begin{array}{c}4.6 \\
11.5 \\
2 / 08 / 91\end{array}$ & $\begin{array}{c}2.3 \\
18.5 \\
2 / 21 / 92\end{array}$ & $\begin{array}{l}3.4 \\
11.5 \\
6 / 15 / 93\end{array}$ \\
\hline $\begin{array}{l}\text { GIESELER FLUIDITY } \\
\text { max. ddpm } \\
\text { fluid range } \\
\text { analys is date }\end{array}$ & $\begin{array}{r}4 \\
38 \\
09 / 15 / 86\end{array}$ & $\begin{array}{r}0 \\
0 \\
05 / 13 / 88\end{array}$ & $\begin{array}{l}\text { discon- } \\
\text { timued }\end{array}$ & & & & \\
\hline $\begin{array}{l}\text { SULFUR FOPAls (dry \%) } \\
\text { total sulfur } \\
\text { sulfate } \\
\text { Pyritic } \\
\text { organic } \\
\text { analysis date }\end{array}$ & $\begin{array}{c}0.80 \\
0.00 \\
0.02 \\
0.77 \\
09 / 26 / 86\end{array}$ & & $\begin{array}{c}0.79 \\
0.19 \\
0.16 \\
0.43 \\
03 / 07 / 89\end{array}$ & $\begin{array}{r}0.85 \\
0.18 \\
0.15 \\
0.52 \\
02 / 06 / 90\end{array}$ & $\begin{array}{r}0.84 \\
0.22 \\
0.19 \\
0.43 \\
2 / 08 / 91\end{array}$ & $\begin{array}{r}0.67 \\
0.10 \\
0.14 \\
0.42 \\
2 / 21 / 92\end{array}$ & $\begin{array}{c}0.81 \\
0.21 \\
0.09 \\
0.50 \\
6 / 15 / 93\end{array}$ \\
\hline $\begin{array}{l}\text { CALORIFIC VALUE (dry) } \\
\text { BTU/lb } \\
\text { analysis date }\end{array}$ & $\begin{array}{c}13612 \\
09 ; 26 / 86\end{array}$ & & $\begin{array}{c}13538 \\
03 / 07 / 89\end{array}$ & $\begin{array}{r}13825 \\
02 / 06 / 90\end{array}$ & $\begin{array}{c}13493 \\
2 / 15 / 91\end{array}$ & $\begin{array}{c}c \\
11953 \\
2 / 21 / 92\end{array}$ & $\begin{array}{c}13512 \\
6 / 15 / 93\end{array}$ \\
\hline $\begin{array}{l}\text { ALKALI EXTRACTION } \\
\% \text { transmission } \\
\text { analysis date }\end{array}$ & & $\begin{array}{c}99.5 \\
06 / 10 / 88\end{array}$ & $\begin{array}{c}96.1 \\
03 / 06 / 89\end{array}$ & $\begin{array}{r}96.8 \\
01 / 25 / 90\end{array}$ & $\begin{array}{c}99.0 \\
8 / 27 / 91\end{array}$ & $\begin{array}{c}98.0 \\
9 / 23 / 92\end{array}$ & $\begin{array}{c}99.6 \\
7 / 12 / 93\end{array}$ \\
\hline
\end{tabular}

notes: a) : ample moist, good condition.

b) No visible deterioration.

c) Low calorific value caused in part by high ash in this split. 


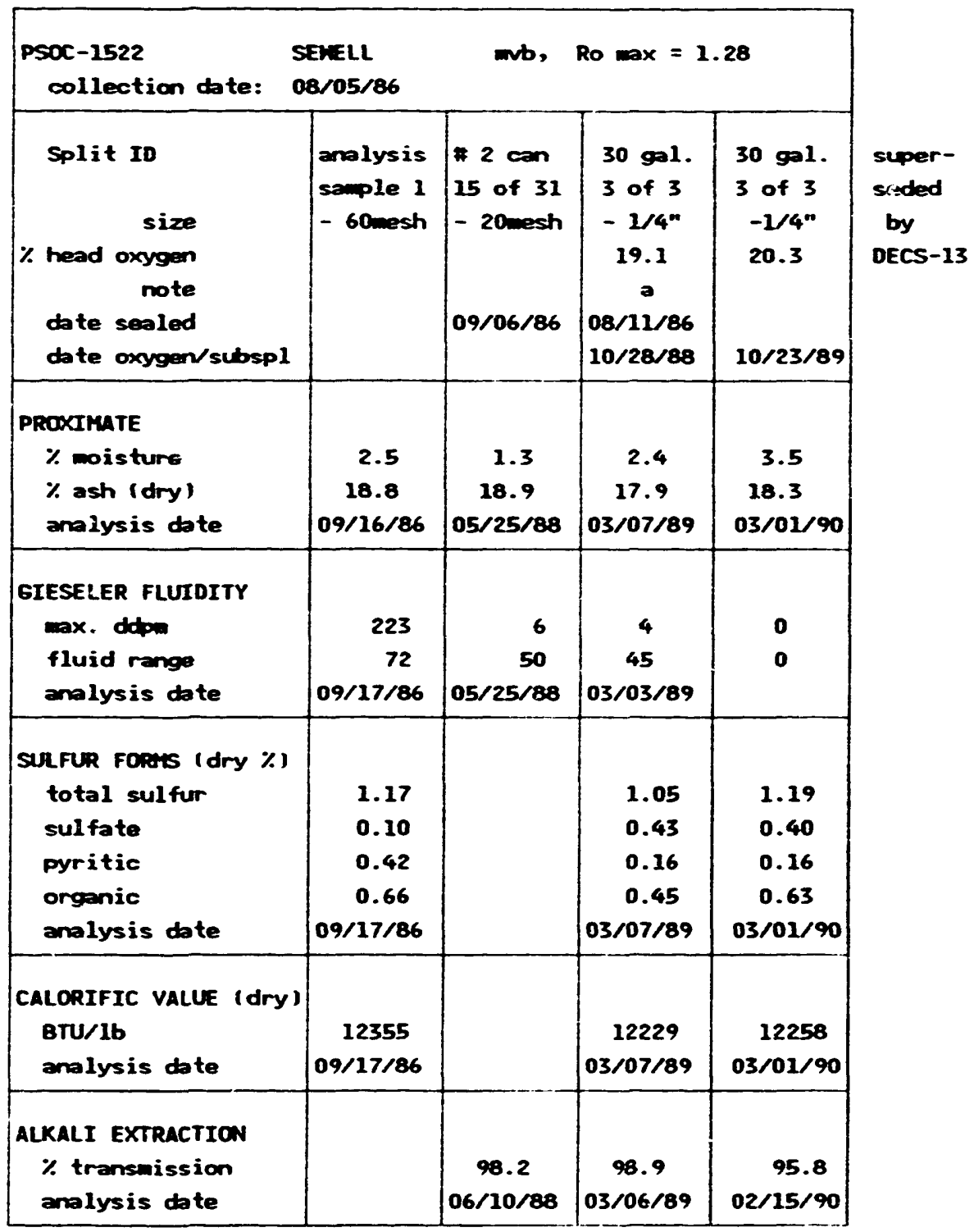

notes: a ) Sample dry, good condition. 


\begin{tabular}{|c|c|c|c|c|c|c|c|}
\hline $\begin{array}{l}\text { PSOC-1523 } \\
\text { collection date: }\end{array}$ & $\begin{array}{l}5 \text { BLOCK } \\
8 / 06 / 86\end{array}$ & InBb, & Ro $\max =$ & & & & \\
\hline $\begin{array}{l}\text { Split ID } \\
\text { size } \\
\text { \% head oxygen } \\
\text { note } \\
\text { date sealed } \\
\text { cate oxygen/subspl }\end{array}$ & $\begin{array}{l}\text { analysis } \\
\text { sample } 1 \\
-60 \text { mesh }\end{array}$ & $\begin{array}{l}22 \text { can } \\
15 \text { of } 31 \\
-20 \text { mesh } \\
09 / 08 / 86\end{array}$ & $\begin{array}{c}30 \text { gal } \\
3 \text { of } 3 \\
-1 / 4 " \\
20.1 \\
a \\
11 / 16 / 88\end{array}$ & $\begin{array}{l}30 \text { gal. } \\
3 \text { of } 3 \\
-1 / 4^{\prime \prime} \\
20.4 \\
10 / 23 / 89\end{array}$ & $\begin{array}{l}30 \text { gal. } \\
3 \text { of } 3 \\
-1 / 4 " 1 \\
2 / 05 / 91\end{array}$ & $\begin{array}{l}30 \text { gal. } \\
3 \text { of } 3 \\
-1 / 4 " \\
9 / 15 / 92\end{array}$ & $\begin{array}{c}30 \text { gal. } \\
3 \text { of } 3 \\
-1 / 4^{\circ} \\
\text { b } \\
5 / 10 / 93\end{array}$ \\
\hline $\begin{array}{l}\text { PROXIMATE } \\
\% \text { mois ture } \\
\% \text { ash (dry) } \\
\text { analysis date }\end{array}$ & $\begin{array}{c}2.6 \\
16.1 \\
09 / 25 / 86\end{array}$ & $\begin{array}{c}2.7 \\
14.5 \\
05 / 26 / 88\end{array}$ & $\begin{array}{c}2.5 \\
25.6 \\
03 / 07 / 89\end{array}$ & $\begin{array}{c}2.9 \\
15.9 \\
02 / 28 / 90\end{array}$ & $\begin{array}{c}3.2 \\
15.9 \\
10 / 03 / 91\end{array}$ & $\begin{array}{c}3.2 \\
16.1 \\
10 / 14 / 92\end{array}$ & $\begin{array}{c}3.2 \\
15.8 \\
6 / 14 / 93\end{array}$ \\
\hline $\begin{array}{l}\text { GIESELER FLUIDITY } \\
\text { max. ddpo } \\
\text { fluid range } \\
\text { analysis date }\end{array}$ & $\begin{array}{r}415 \\
58 \\
09 / 18 / 86\end{array}$ & $\begin{array}{r}10 \\
49 \\
05 / 26 / 88\end{array}$ & $\begin{array}{c}5 \\
33 \\
03 / 01 / 89\end{array}$ & $\begin{array}{c}3 \\
30 \\
02 / 16 / 90\end{array}$ & $\begin{array}{c}2 \\
6 \\
8 / 19 / 91\end{array}$ & $\begin{array}{c}0 \\
0 \\
9 / 26 / 92\end{array}$ & $\begin{array}{c}2 \\
36 \\
8 / 02 / 93\end{array}$ \\
\hline $\begin{array}{l}\text { SULFUR FOins (dry \%) } \\
\text { total sul fur } \\
\text { sulfate } \\
\text { pyritic } \\
\text { organic } \\
\text { analysis date }\end{array}$ & \begin{tabular}{|c}
0.71 \\
0.00 \\
0.02 \\
0.69 \\
$09 / 25 / 86$
\end{tabular} & & $\begin{array}{c}0.68 \\
0.06 \\
0.04 \\
0.57 \\
03 / 07 / 89\end{array}$ & $\begin{array}{c}0.72 \\
0.06 \\
0.03 \\
0.63 \\
02 / 28 / 90\end{array}$ & $\begin{array}{r}0.71 \\
0.05 \\
0.05 \\
0.62 \\
10 / 03 / 91\end{array}$ & $\begin{array}{r}0.70 \\
0.09 \\
0.05 \\
0.57 \\
10 / 14 / 92\end{array}$ & $\begin{array}{c}0.71 \\
0.06 \\
0.02 \\
0.63 \\
6 / 14 / 93\end{array}$ \\
\hline $\begin{array}{l}\text { CALORIFIC VALUE (dry) } \\
\text { BTU/lb } \\
\text { analysis date }\end{array}$ & $\begin{array}{c}12425 \\
09 / 25 / 86\end{array}$ & & $\begin{array}{c}12391 \\
03 / 07 / 89\end{array}$ & $\begin{array}{c}12340 \\
02 / 28 / 90\end{array}$ & $\begin{array}{c}12345 \\
10 / 03 / 91\end{array}$ & $\begin{array}{c}12248 \\
10 / 14 / 92\end{array}$ & $\begin{array}{c}12299 \\
6 / 14 / 93\end{array}$ \\
\hline $\begin{array}{l}\text { ALKALI EXTRACTION } \\
\text { \% transmission } \\
\text { amalysis date }\end{array}$ & & $\begin{array}{c}96.3 \\
06 / 10 / 88\end{array}$ & $\begin{array}{c}95.5 \\
03 / 06 / 89\end{array}$ & $\begin{array}{c}90.0 \\
02 / 16 / 90\end{array}$ & $\begin{array}{c}94.6 \\
8 / 19 / 91\end{array}$ & $\begin{array}{c}76.8 \\
9 / 16 / 92\end{array}$ & $\begin{array}{c}93.9 \\
8 / 27 / 93\end{array}$ \\
\hline
\end{tabular}

notes: a I Sample dry, good condition.

b) No visible deterioration. 


\begin{tabular}{|c|c|c|c|c|c|}
\hline \multicolumn{5}{|c|}{$\begin{array}{l}\text { PSOC-1524 UPPER RITTANATIN mab, Ro max = } 1.06 \\
\text { collection dote: 10/23/86 }\end{array}$} & \multirow{5}{*}{$\begin{array}{l}\text { super- } \\
\text { seded } \\
\text { by } \\
\text { DECS-14 }\end{array}$} \\
\hline $\begin{array}{l}\text { Split ID } \\
\text { size } \\
\text { \% head oxygen } \\
\text { note } \\
\text { date sealed } \\
\text { date oxygen/subspl }\end{array}$ & $\begin{array}{l}\text { amalysis } \\
\text { sample } 1 \\
-60 \text { mesh }\end{array}$ & $\begin{array}{l}2 \text { Can } \\
15 \text { of } 30 \\
-20 \text { mesh } \\
11 / 04 / 86\end{array}$ & $\begin{array}{c}30 \text { sal } \\
3 \text { of } 3 \\
-1 / 4 \% \\
20.3 \\
9 \\
11 / 16 / 88\end{array}$ & $\begin{array}{l}30 \mathrm{gal} \\
3 \text { of } 3 \\
-1 / 4^{\circ} \\
20.4\end{array}$ & \\
\hline $\begin{array}{l}\text { PROXImaTE } \\
\% \text { misture } \\
\% \text { ash (dry ) } \\
\text { analysis date }\end{array}$ & $\begin{array}{c}1.6 \\
11.0 \\
12 / 25 / 86\end{array}$ & $\begin{array}{c}1.5 \\
11.5 \\
05 / 31 / 88\end{array}$ & $\begin{array}{c}1.8 \\
10.9 \\
03 / 07 / 89\end{array}$ & $\begin{array}{c}2.0 \\
11.5 \\
02 / 28 / 90\end{array}$ & \\
\hline $\begin{array}{l}\text { GIESELER FLUIDITY } \\
\text { mx. ddpin } \\
\text { fluid range } \\
\text { analysis date }\end{array}$ & $\begin{array}{r}30000 \\
110 \\
03 / 17 / 87\end{array}$ & $\begin{array}{r}4589 \\
91 \\
05 / 31 / 88\end{array}$ & $\begin{array}{c}3907 \\
88 \\
03 / 03 / 89\end{array}$ & $\begin{array}{c}752 \\
82 \\
02 / 19 / 90\end{array}$ & \\
\hline $\begin{array}{l}\text { SUlfuR FOpets (dry \%) } \\
\text { total sulfur } \\
\text { sulfate } \\
\text { pyritic } \\
\text { organic } \\
\text { analysis date }\end{array}$ & $\begin{array}{r}1.85 \\
0.02 \\
1.39 \\
0.44 \\
11 / 25 / 86\end{array}$ & & $\begin{array}{c}1.76 \\
0.18 \\
1.08 \\
0.51 \\
03 / 07 / 89\end{array}$ & $\begin{array}{r}2.01 \\
0.25 \\
0.93 \\
0.83 \\
02 / 28 / 90\end{array}$ & \\
\hline $\begin{array}{l}\text { CALORIFIC VALUE (dry) } \\
\text { BTU/lb } \\
\text { analysis date }\end{array}$ & $\begin{array}{c}13656 \\
11 / 17 / 86\end{array}$ & & $\begin{array}{r}13469 \\
03 / 07 / 89\end{array}$ & $\begin{array}{c}13378 \\
02 / 23 / 90\end{array}$ & \\
\hline $\begin{array}{l}\text { ALKALI EXTRACTION } \\
\% \text { transmission } \\
\text { analysis date }\end{array}$ & & $\begin{array}{c}98.3 \\
06 / 10 / 83\end{array}$ & $\begin{array}{c}98.2 \\
03 / 08 / 89\end{array}$ & $\begin{array}{c}93.5 \\
02 / 19 / 90\end{array}$ & \\
\hline
\end{tabular}

notes: a) Sample dry, good condition. 


\begin{tabular}{|c|c|c|c|c|c|c|}
\hline $\begin{array}{l}\text { PSOC-1532 } \\
\text { collection date: }\end{array}$ & $\begin{array}{l}\text { (Usibel1i } \\
9 / 11 / 87\end{array}$ & subc, & to $\max =0$ & & & \\
\hline $\begin{array}{l}\text { Split ID } \\
\text { size } \\
\text { \% head oxygen } \\
\text { note } \\
\text { date sealed } \\
\text { date oxygen/subspl }\end{array}$ & $\begin{array}{l}\text { analysis } \\
\text { sample } 1 \\
-60 \text { mesh }\end{array}$ & $\begin{array}{c}30 \mathrm{gal} \\
2 \text { of } 2 \\
-1 / 4^{\circ} \\
15.7 \\
a \\
11 / 16 / 88\end{array}$ & $\begin{array}{l}30 \text { gal. } \\
2 \text { of } 2 \\
-1 / 4^{\circ} \\
17.6 \\
10 / 24 / 89\end{array}$ & $\begin{array}{l}30 \text { gel. } \\
2 \text { of } 2 \\
-1 / 4^{\circ}\end{array}$ & $\begin{array}{l}30 \text { gel. } \\
2 \text { of } 2 \\
-1 / 4^{\prime \prime}\end{array}$ & $\begin{array}{l}30 \text { gal } \\
2 \text { of } 2 \\
-1 / 4^{\circ} \\
\text { b } \\
5 / 10 / 93\end{array}$ \\
\hline $\begin{array}{l}\text { PROXIMATE } \\
\% \text { moisture } \\
\% \text { ash (dry) } \\
\text { analysis date }\end{array}$ & $\begin{array}{c}23.7 \\
9.5 \\
11 / 12 / 87\end{array}$ & $\begin{array}{c}24.3 \\
9.2 \\
03 / 08 / 89\end{array}$ & $\begin{array}{c}25.8 \\
10.7 \\
02 / 06 / 90\end{array}$ & $\begin{array}{c}26.9 \\
9.6 \\
2 / 08 / 91\end{array}$ & $\begin{array}{c}25.3 \\
7.9 \\
2 / 21 / 92\end{array}$ & $\begin{array}{c}26.2 \\
10.1 \\
6 / 14 / 93\end{array}$ \\
\hline $\begin{array}{l}\text { SulfuR Foins (dry \%) } \\
\text { total sulfur } \\
\text { sulfate } \\
\text { pyritic } \\
\text { organic } \\
\text { analysis cate }\end{array}$ & $\begin{array}{c}0.22 \\
0.02 \\
0.45 \\
0.16 \\
11 / 12 / 87\end{array}$ & $\begin{array}{c}0.17 \\
0.01 \\
0.03 \\
0.13 \\
03 / 08 / 89\end{array}$ & $\begin{array}{c}0.18 \\
0.01 \\
0.04 \\
0.13 \\
02 / 06 / 90\end{array}$ & $\begin{array}{c}0.23 \\
0.01 \\
0.02 \\
0.20 \\
2 / 08 / 91\end{array}$ & $\begin{array}{r}0.20 \\
0.00 \\
0.01 \\
0.18 \\
2 / 21 / 92\end{array}$ & $\begin{array}{r}(c) \\
0.20 \\
0.02 \\
0.03 \\
0.15 \\
6 / 14 / 93\end{array}$ \\
\hline $\begin{array}{l}\text { CALORIfIC VALUE (dry) } \\
\text { BTulb } \\
\text { analysis date }\end{array}$ & $\begin{array}{c}10867 \\
11 / 12 / 87\end{array}$ & $\begin{array}{c}10705 \\
03 / 08 / 89\end{array}$ & $\begin{array}{c}10589 \\
02 / 06 / 90\end{array}$ & $\begin{array}{c}10530 \\
2 / 15 / 91\end{array}$ & $\begin{array}{c}10599 \\
2 / 21 / 92\end{array}$ & $\begin{array}{c}10444 \\
6 / 14 / 93\end{array}$ \\
\hline
\end{tabular}

notes: a) Sample moist, good condition

b) Sample moist at bottom of barrel, trace white coatings.

c) Changed from reported total $S$ value of $1.96 \%$ which was 10 times too high. 


\begin{tabular}{|c|c|c|c|c|c|c|}
\hline $\begin{array}{l}\text { PSOC-1533 } \\
\text { collection date: }\end{array}$ & $\begin{array}{l}(\text { Usibelli) } \\
9 / 11 / 87\end{array}$ & ligh, & $0 \max =0$ & & & \\
\hline $\begin{array}{l}\text { Split I0 } \\
\text { size } \\
\text { head oxygen } \\
\text { note } \\
\text { date sealed } \\
\text { date oxygen/subspl }\end{array}$ & $\begin{array}{l}\text { analysis } \\
\text { sample } 1 \\
-60 \text { mesh }\end{array}$ & $\begin{array}{l}30 \text { gal } \\
2 \text { of } 2 \\
-1 / 4 \times \\
17.2 \\
a \\
11 / 16 / 88\end{array}$ & $\begin{array}{l}30 \text { gei. } \\
2 \text { of } 2 \\
-1 / 4 \times 1 \\
18.6 \\
10 / 24 / 89\end{array}$ & $\begin{array}{l}30 \mathrm{gal} \\
2 \text { of } 2 \\
-1 / 4^{-1}\end{array}$ & $\begin{array}{l}30 \text { gal. } \\
2 \text { of } 2 \\
-1 / 4^{\circ}\end{array}$ & $\begin{array}{l}30 \text { gal. } \\
2 \text { of } 2 \\
-1 / 4 \times \\
\text { b } \\
5 / 10 / 93\end{array}$ \\
\hline $\begin{array}{l}\text { PROXIMATE } \\
\% \text { moisture } \\
\% \text { ash (dry) } \\
\text { analysis date }\end{array}$ & $\begin{array}{c}26.4 \\
8.3 \\
12 / 11 / 87\end{array}$ & $\begin{array}{c}26.4 \\
8.3 \\
03 / 07 / 89\end{array}$ & \begin{tabular}{c|}
28.3 \\
9.1 \\
$02 / 06 / 90$
\end{tabular} & $\begin{array}{c}29.8 \\
8.2 \\
2 / 08 / 91\end{array}$ & $\begin{array}{c}27.9 \\
7.5 \\
2 / 21 / 92\end{array}$ & $\begin{array}{c}28.8 \\
8.6 \\
6 / 14 / 93\end{array}$ \\
\hline $\begin{array}{l}\text { SULFUR Forits (dry \%) } \\
\text { total sulfur } \\
\text { sulfate } \\
\text { pyritic } \\
\text { organic } \\
\text { analysis date }\end{array}$ & $\begin{array}{c}0.16 \\
0.02 \\
0.05 \\
0.09 \\
12 / 11 / 87\end{array}$ & $\begin{array}{c}0.10 \\
0.01 \\
0.03 \\
0.05 \\
03 / 07 / 89\end{array}$ & $\begin{array}{r}0.10 \\
0.02 \\
0.04 \\
0.04 \\
02 / 06 / 90\end{array}$ & $\begin{array}{c}0.16 \\
0.01 \\
0.02 \\
0.12 \\
2 / 08 / 91\end{array}$ & $\begin{array}{c}0.11 \\
0.01 \\
0.02 \\
0.08 \\
2 / 21 / 92\end{array}$ & $\begin{array}{c}(c) \\
0.14 \\
0.02 \\
0.04 \\
0.08 \\
6 / 14 / 93\end{array}$ \\
\hline $\begin{array}{l}\text { CALORIFIC VALUE (dry) } \\
\text { BTU/1b } \\
\text { analysis date }\end{array}$ & $\begin{array}{c}10620 \\
12 / 11 / 87\end{array}$ & $\begin{array}{c}10523 \\
03 / 07 / 89\end{array}$ & $\begin{array}{c}10466 \\
02 / 06 / 90\end{array}$ & $\begin{array}{c}10434 \\
2 / 25 / 91\end{array}$ & $\begin{array}{r}10426 \\
2 / 21 / 92\end{array}$ & $\begin{array}{c}10388 \\
6 / 14 / 93\end{array}$ \\
\hline
\end{tabular}

notes: a I Sample moist, good condition.

b) Sample dry at top of barr ", moist belows trace brown coatings

c) Changed from reported tocal s value of $1.36 \%$ which was 10 times too high. 


\begin{tabular}{|c|c|c|c|c|c|c|}
\hline $\begin{array}{l}\text { PSOC-1534 } \\
\text { collection date: }\end{array}$ & $\begin{array}{l}\text { ATERFALL } \\
9 / 14 / 87\end{array}$ & subc, & $0 \max =0$ & & & \\
\hline $\begin{array}{l}\text { Split I0 } \\
\text { size } \\
\text { \% head oxygen } \\
\text { note } \\
\text { date sealed } \\
\text { date oxygen/subspl }\end{array}$ & $\begin{array}{l}\text { analys is } \\
\text { sample } 1 \\
-60 \text { mesh }\end{array}$ & $\begin{array}{l}30 \text { gal. } \\
2 \text { of } 2 \\
-1 / 4 \cdot \\
13.6 \\
\text { a } \\
11 / 16 / 88\end{array}$ & $\begin{array}{l}30 \text { gal. } \\
2 \text { of } 2 \\
-1 / 4 " 1 \\
15.2 \\
10 / 24 / 89\end{array}$ & $\begin{array}{l}30 \mathrm{gal} \\
2 \text { of } 2 \\
-1 / 4^{\prime \prime} \\
2 / 05 / 91\end{array}$ & $\begin{array}{l}30 \text { gal. } \\
2 \text { of } 2 \\
-1 / 4 " \\
11 / 25 / 91\end{array}$ & $\begin{array}{l}30 \text { gal. } \\
2 \text { of } 2 \\
-1 / 4 " \\
b \\
5 / 10 / 93\end{array}$ \\
\hline $\begin{array}{l}\text { PRCXIMATE } \\
\% \text { moisture } \\
\% \text { ash (dry) } \\
\text { amalysis date }\end{array}$ & $\begin{array}{c}21.8 \\
11.1 \\
11 / 12 / 87\end{array}$ & $\begin{array}{c}23.1 \\
10.8 \\
03 / 07 / 89\end{array}$ & $\begin{array}{r}23.8 \\
11.4 \\
02 / 06 / 90\end{array}$ & $\begin{array}{c}24.9 \\
11.5 \\
2 / 08 / 91\end{array}$ & $\begin{array}{c}23.7 \\
8.3 \\
2 / 21 / 92\end{array}$ & $\begin{array}{c}23.8 \\
11.5 \\
5 / 10 / 93\end{array}$ \\
\hline $\begin{array}{l}\text { SULFUR Forits (dry \%) } \\
\text { total sulfur } \\
\text { sulfate } \\
\text { pyritic } \\
\text { organic } \\
\text { analysis date }\end{array}$ & $\begin{array}{c}0.15 \\
0.01 \\
0.05 \\
0.09 \\
11 / 12 / 87\end{array}$ & $\begin{array}{c}0.13 \\
0.02 \\
0.01 \\
0.10 \\
03 / 07 / 89\end{array}$ & $\begin{array}{c}0.12 \\
0.01 \\
0.01 \\
0.09 \\
02 / 06 / 90\end{array}$ & $\begin{array}{c}0.17 \\
0.01 \\
0.02 \\
0.14 \\
2 / 08 / 91\end{array}$ & $\begin{array}{c}0.16 \\
0.01 \\
0.01 \\
0.14 \\
2 / 21 / 92\end{array}$ & $\begin{array}{r}0.14 \\
0.01 \\
0.01 \\
0.13 \\
5 / 10 / 93\end{array}$ \\
\hline $\begin{array}{l}\text { CALORIFIC VALUE (dry) } \\
\text { BTU/lb } \\
\text { analysis date }\end{array}$ & $\begin{array}{c}11082 \\
11 / 12 / 87\end{array}$ & $\begin{array}{c}10965 \\
03 / 07 / 89\end{array}$ & $\begin{array}{c}10832 \\
02 / 06 / 90\end{array}$ & $\begin{array}{c}10753 \\
2 / 15 / 91\end{array}$ & $\begin{array}{c}10979 \\
2 / 21 / 92\end{array}$ & $\begin{array}{c}10657 \\
6 / 14 / 93\end{array}$ \\
\hline
\end{tabular}

notes: a) Sample moist, good condition.

b) Sample moist at bottom of barrel; trace white crystals and brown coatings. 


\begin{tabular}{|c|c|c|c|c|c|c|c|}
\hline $\begin{array}{c}\text { PSOC-1535 DEADFA } \\
\text { collection date: } 1\end{array}$ & $\begin{array}{l}\text { LL SYMCLIN } \\
0 / 08 / 87\end{array}$ & E \#4 sub/ & , Ro $\max$ & 0.73 & & & \\
\hline $\begin{array}{l}\text { Split ID } \\
\text { size } \\
\text { \% head ixygen } \\
\text { note } \\
\text { date seeled } \\
\text { date oxygen/subspl }\end{array}$ & $\begin{array}{l}\text { analysis } \\
\text { sample } 1 \\
-60 \text { mesh }\end{array}$ & $\begin{array}{l}\text { \#2 Can } \\
-20 \text { mesh } \\
12 / 14 / 87\end{array}$ & $\begin{array}{c}30 \text { gal } \\
2 \text { of } 2 \\
-1 / 4 " \\
20.8 \\
a \\
11 / 16 / 88\end{array}$ & $\begin{array}{l}30 \text { gal. } \\
2 \text { of } 2 \\
-1 / 4^{\prime \prime} \\
19.2 \\
10 / 25 / 89\end{array}$ & $\begin{array}{l}30 \text { gal. } \\
2 \text { of } 2 \\
-1 / 4 " \\
2 / 05 / 91\end{array}$ & $\begin{array}{l}30 \text { gal. } \\
2 \text { of } 2 \\
-1 / 4^{\prime \prime} \\
11 / 25 / 91\end{array}$ & $\begin{array}{l}30 \text { gal. } \\
2 \text { of } 2 \\
-1 / 4 " \\
c \\
5 / 10 / 93\end{array}$ \\
\hline $\begin{array}{l}\text { PROXIMATE } \\
\% \text { moisture } \\
\% \text { ash (dry) } \\
\text { amalysis date }\end{array}$ & $\begin{array}{c}10.1 \\
3.3 \\
12 / 23 / 87\end{array}$ & $\begin{array}{c}10.2 \\
3.4 \\
06 / 14 / 88\end{array}$ & $\begin{array}{c}10.1 \\
3.4 \\
03 / 07 / 89\end{array}$ & $\begin{array}{r}10.6 \\
3.7 \\
02 / 06 / 90\end{array}$ & $\begin{array}{c}11.5 \\
3.5 \\
2 / 08 / 91\end{array}$ & $\begin{array}{c}10.4 \\
3.4 \\
2 / 21 / 92\end{array}$ & $\begin{array}{c}10.1 \\
3.4 \\
5 / 10 / 93\end{array}$ \\
\hline $\begin{array}{l}\text { GIESELER FLUIDITY } \\
\text { max. ddpm } \\
\text { fluid range } \\
\text { amalysis date }\end{array}$ & $\begin{array}{l}\mathbf{0} \\
\mathbf{0}\end{array}$ & $\begin{array}{l}\text { discon- } \\
\text { tinued }\end{array}$ & & & & & \\
\hline $\begin{array}{l}\text { SULFUR FORMS (dry \%) } \\
\text { total sulfur } \\
\text { sulfate } \\
\text { pyritic } \\
\text { organic } \\
\text { analysis date }\end{array}$ & $\begin{array}{r}\text { (b) } \\
0.15 \\
0.02 \\
0.01 \\
0.18 \\
12 / 23 / 87\end{array}$ & & $\begin{array}{c}0.16 \\
0.01 \\
0.01 \\
0.14 \\
03 / 07 / 89\end{array}$ & $\begin{array}{r}0.13 \\
0.01 \\
0.01 \\
0.12 \\
02 / 06 / 90\end{array}$ & $\begin{array}{c}0.16 \\
0.01 \\
0.04 \\
0.11 \\
2 / 08 / 91\end{array}$ & $\begin{array}{c}0.23 \\
0.01 \\
0.02 \\
0.21 \\
2 / 21 / 92\end{array}$ & $\begin{array}{c}0.17 \\
0.00 \\
0.01 \\
0.16 \\
5 / 10 / 93\end{array}$ \\
\hline $\begin{array}{l}\text { CALORIFIC VALUE (dry) } \\
\text { BTU/lb } \\
\text { analysis date }\end{array}$ & $\begin{array}{c}12107 \\
12 / 23 / 87\end{array}$ & & $\begin{array}{c}12085 \\
03 / 07 / 89\end{array}$ & $\begin{array}{c}12074 \\
02 / 06 / 90\end{array}$ & $\begin{array}{c}12050 \\
2 / 15 / 91\end{array}$ & $\begin{array}{c}12069 \\
2 / 21 / 92\end{array}$ & $\begin{array}{r}11978 \\
5 / 10 / 93\end{array}$ \\
\hline $\begin{array}{l}\text { ALKALI EXTRACTION } \\
\% \text { transmission } \\
\text { analysis date }\end{array}$ & & $\begin{array}{c}0.1 \\
06 / 16 / 88\end{array}$ & $\begin{array}{l}\text { discon- } \\
\text { tinued }\end{array}$ & & & & \\
\hline
\end{tabular}

notes: a) Sample dry, good condition.

b) Changed from reported total S value of 1.46 which was 10 times too high.

c) Trace white acicular crystals. 


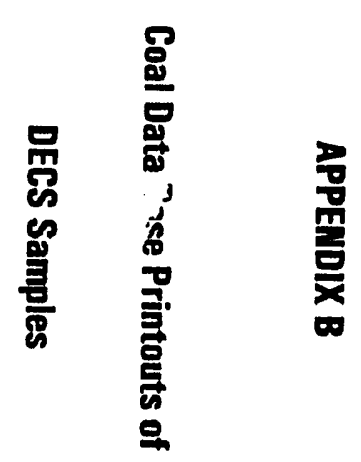


SAMPLE IDENTIFICATION: -

$\begin{array}{ll}\text { PSU NUMBER } & \rightarrow \text { DECS }-1 \\ \text { SEAM NAME } & \rightarrow \text { BOTTOM } \\ \text { ALTERNATE SEAM NAME } & \rightarrow> \\ \text { APPARENT RANK } & \rightarrow \text { SUBBIT C }\end{array}$

APPARENT RANK $\rightarrow$ SUBBIT C

SAMPLE HISTORY:

SAMPLING DATE \& AGENCY

$->12 / 11 / 89 ; P S U$

SAMPLE TYPE

$\rightarrow$ CHANNEL-WHOLE SEAM

ADDITIONAL INFORMATION

$\rightarrow$

MINE INFORMATION:

RESERVES

$\rightarrow$

ANNUAL PRODUCTIION

$\rightarrow 5500$ THOUSAND TONS
SAMPLE LOCATION:

COUNTRY $\rightarrow$ USA

STATE $\quad \rightarrow$ TEXAS

COUNTY $\rightarrow$ FREESTONE

TOWNSHIP $\quad \rightarrow$

CITY $\quad \rightarrow$ FAIRFIELD

COAL PROVINCE $\rightarrow$ GULF

REGION $\rightarrow$

MAP REFERENCE: LATITUDE $\rightarrow 31 \mathrm{D} 51 \mathrm{M} 10 \mathrm{~S}$

LONGITUDE $\rightarrow \quad 96 \mathrm{D} 3 \mathrm{M} 59 \mathrm{~S}$

QUADRANGLE $\rightarrow$ YOUNG $\left(7.5^{\prime}\right)$

LIFE EXPECTANCY $\rightarrow 25$ YEARS

MINING METHOD $\quad \rightarrow$ STRIP
* penn state coal data base *\#

\#\#

** DECS -1

** $11 / 16 / 93$ \#*

** PAGE

$\stackrel{+\infty}{\infty}$

\section{SEAM INFORMATION:}

AGE OF SEAM

FORMATION

SEAM THICKNESS $\rightarrow$ EOCENE

$\rightarrow$ Upper Wilcox

-> Upper Calvert Bluff

$$
\rightarrow \quad 9 \mathrm{FT} . \quad 6 \mathrm{IN} \text {. }
$$

** SEAM PROFILE *\#

\section{$\rightarrow g r$ siltstone}

$\rightarrow$ duil, brown, fibrous

$\rightarrow$ moderately bright, black

-> dull, brown, thlckness varles

$\rightarrow$ moderately bright, black

$\rightarrow$ dull, brown, fibrous

$\rightarrow$ brown shale w/coaly stringers 


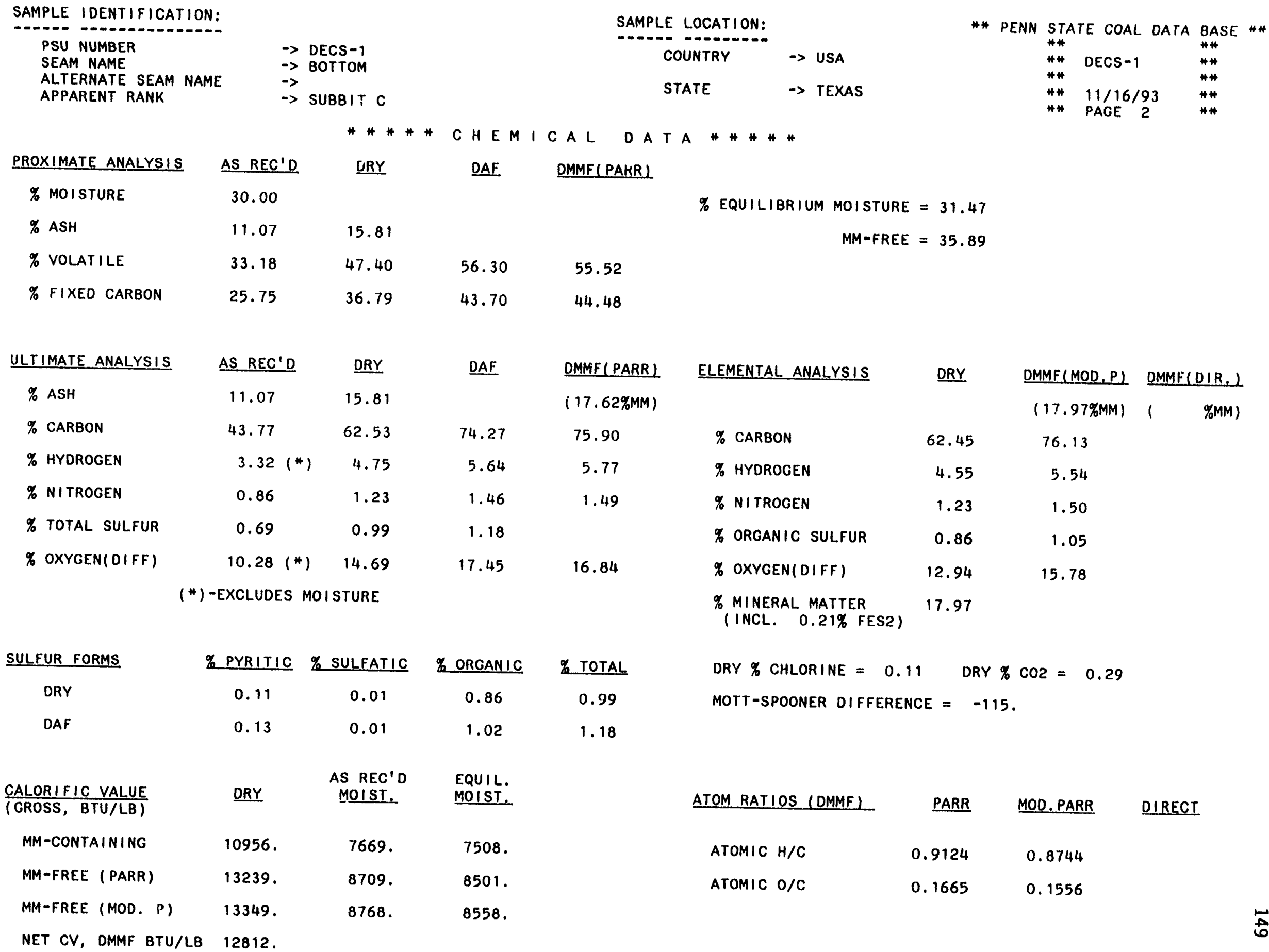


SAMPLE IDENTIFICATION:

PSU MUMBER

PSU NUMBER

SEAM NAME

ALTERNATE SEAM NAME

APPARENT RANK

$$
\begin{aligned}
& \rightarrow>\text { DECS }-1 \\
& \rightarrow>\text { BOTTOM } \\
& -> \\
& \rightarrow>\text { SUBBIT C }
\end{aligned}
$$

$\begin{array}{ll}\text { SAMPLE LOCATION: } & \\ \text { COUNTRY } & \rightarrow \text { USA } \\ \text { STATE } & \rightarrow \text { TEXAS }\end{array}$

$$
\begin{aligned}
& \text { * * * * R A N K } \\
& \text {-> SUBBIT C } \\
& \rightarrow \text { SUBBIT C } \\
& \rightarrow
\end{aligned}
$$

APPARENT RANK (AS REC'D MOIST.)

ASTM RANK (EQUIL. MOIST.)

REFLECTANCE RANK CATEGORY
\#\# PENN STATE COAL DATA BASE \#\#

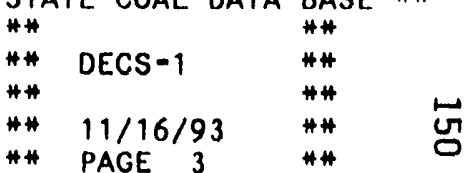

\footnotetext{
***** PETROG GAPHIC D A T A *\#\#**
}

MACERAL COMPOSITION (WHITE LIGHT AND FLUORESCENCE ANALYSIS )

MACERAL COMPOSIT

DMM

TEXT- ULM- HUMO- GEL- CORPO- SPOR- CUT- RES- ALG- FUS- SEMI- MAC- SCLERO-

OMMF VOL \% HUMINITE $=78.4$

ULMINITE REFLECTANCE DATA: HIGH: $0.51 \quad$ LOW: $0.20 \quad$ MEAN-MAX: 0.35

\section{* * \# CAKING ANDMECHAN I CAL PROPERTIES}

GIESELER COAL PLASTOMETER:

INIT. SOFTENING TEMP

TEMP. MAX. FLUIDITY

SOLIDIFICATION TEMP.

FLUIO TEMP. RANGE

MAXIMUM FLUIDITY

WASHABILITY DATA AVAILABLE?
GRAY-KING COKE TYPE

HARDGROVE GRINDABILITY 68.2

FREE SWELLING INDEX

1.0 


\begin{tabular}{ll} 
SAMPLE IDENTIFICATION: & \\
\hline PSU NUMBER & $\rightarrow$ DECS -1 \\
SEAM NAME & $\rightarrow$ BOTTOM \\
ALTERNATE SEAM NAME & $\rightarrow>$ \\
APPARENT RANK & $\rightarrow$ SUBBIT C
\end{tabular}

MAJOR ELEMENT ANALYSIS MAJOR ELEMENT ANALYSIS

OXIDE $\%$ OF HTA
ELEMENT \% OF
TOTAL DRY COAL
RACE ELEMENT ANALYSIS

$\begin{array}{ccccc}\text { S102 } & \text { AL2O3 } & \text { TIO2 } & \text { FE203 } & \text { MGO } \\ 45.6 & 17.0 & 1.26 & 4.18 & 2.68 \\ \text { SI } & \text { AL } & \text { TI } & \text { FE } & \text { MG } \\ 3.7 & 1.5 & 0.13 & 0.50 & 0.28\end{array}$

SAMPLE LOCATION:

COUNTRY

STATE

-> USA

$\%$ HTA $=17.14$

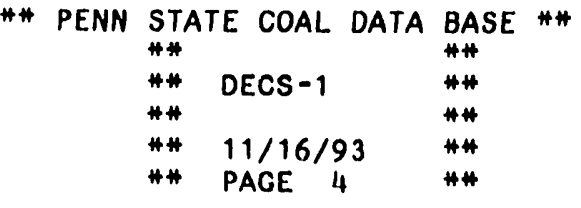

$\begin{array}{lll}* * & \\ * * & \text { DECS }-1 & * * \\ * * & & * * \\ * * & 11 / 16 / 93 & * * \\ * * & \text { PAGE } 4 & * *\end{array}$

\begin{tabular}{|c|c|c|c|c|c|c|c|c|c|c|}
\hline & $\begin{array}{l}\text { PPM } \\
\text { HTA }\end{array}$ & $\begin{array}{c}\text { PPM } \\
\text { TOTAL COAL }\end{array}$ & & $\begin{array}{l}\text { PPM } \\
\text { HTA }\end{array}$ & $\begin{array}{c}\text { PPM } \\
\text { TOTAL COAL }\end{array}$ & & $\begin{array}{l}\text { PPM } \\
\text { HTA. }\end{array}$ & $\begin{array}{c}\text { PPM } \\
\text { TOTAL COAL }\end{array}$ & VOLAT I LES & $\begin{array}{c}\text { PPM } \\
\text { TOTAL COAL }\end{array}$ \\
\hline$A G$ & & & $\mathrm{GE}$ & & & SN & & & AS & \\
\hline B & & & LA & & & SR & 2960. & 507. & $B R$ & \\
\hline$B A$ & 1344. & 230 & L.I & & & $T H$ & & & $C D$ & \\
\hline$B E$ & 5.0 & 0.9 & MN & 1239. & 212 . & $U$ & & & $\mathrm{CL}$ & 1100. \\
\hline BI & & & MO & & & $v$ & 35. & 6. & $F$ & \\
\hline$C E$ & & & NB & & & $Y$ & & & HC & \\
\hline CO & & & NI & $<25$. & $<4$. & YB & & & SB & \\
\hline$C R$ & 95. & 16. & PB & & & $Z N$ & 50. & 9. & SE & \\
\hline $\mathrm{CU}$ & 130 & 22. & $\mathrm{RB}$ & 30. & 5. & $Z R$ & 270 & 46. & & \\
\hline GA & & & SC & & & & & & & \\
\hline
\end{tabular}


SAMPLE IDENTIFICATION: SAMPLE IOENTIFICATION: PSU NUMBER

SEAM NAME

ALTERNATE SEAM NAME APPARENT RANK
SAMPLE LOCATION:

SAMPLE LOCATION:

COUNTRY

-> USA

STATE

$\rightarrow$ TEXAS
* penn state coal data base \#\# $\begin{array}{llll}* * & & \\ * * & \text { DECS }-1 & * * & \\ * * & & * * & \\ * * & 11 / 16 / 93 & * \# & 5 \\ * & \text { PAGE } 5 & * * & N\end{array}$

ASH FUSION TEMPERATURES (ALL TEMPERATURES IN DEGREES F)

REDUCING OXIDIZING

INITIAL DEFORMATION

SOFTENING

HEMISPHER ICAL

FLUID
1840.

2000.

2060.

2100.
2120.

2220.

2280.

2330 . 
SAMPLE IDENTIFICATION:

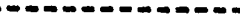

PSU NUMBER

SEAM NAME

ALTERNATE SEAM NAME

APPARENT RANK

$\rightarrow$ DECS -2

$\rightarrow$ ILLINOIS \#6

$\rightarrow$ HERRIN

$\rightarrow$ HVC

SAMPLE HISTORY:

SAMPLE HISTORY:

SAMPLING DATE \& AGENCY

$->12 / 13 / 89 ; P S U$

SAMPLE TYPE

ADDITIONAL INFORMATION

MINE INFORMATION:

MINE INFORMATION

RESERVES

ANNUAL PRODUCTION

$\rightarrow$

$\rightarrow$

$\rightarrow$ CHANNEL-WHOLE SEAM

LONGITUDE $\rightarrow 89 \mathrm{D} 43 \mathrm{M} 19 \mathrm{~S}$

QUADRANGLE $\rightarrow$ TIIden $\left(7.5^{\prime}\right)$

COUNTY

$\rightarrow$ RANDOLPH

TOWNSHIP $\quad \rightarrow$

CITY $\rightarrow$ SPARTA

COAL PROVINCE $\rightarrow$ INTERIOR

$$
\text { REGION } \rightarrow \text { EASTERN }
$$

LIFE EXPECTANCY $\rightarrow$

MINING METHOD $\quad \rightarrow$ STRIP
* PENN STATE COAL DATA BASE **

\#\#

*\# DECS -2

** $11 / 16 / 93$

** PAGE 1

\#\#

SEAM INFORMATION:

AGE OF SEAM

GROUP

FORMATION

SEAM THICKNESS
-> PENNSYL.

$\rightarrow$ Kewanee

-> Carbondale

$\rightarrow \quad 6 \mathrm{FT} . \quad 3 \mathrm{IN}$. 
SAMPLE IDENTIFICATION: -....- -.............. PSU NUMBER SEAM NAME

ALTERNATE SEAM NAME APPARENT RANK

$$
\begin{aligned}
& \rightarrow \text { DECS }-2 \\
& \Rightarrow \text { ILLINOIS \#6 } \\
& \rightarrow \text { HERRIN } \\
& \rightarrow \text { HVC }
\end{aligned}
$$

SAMPLE LOCATION:

COUNTRY $\rightarrow$ USA

STITE $\quad \rightarrow$ ILLINOIS
* penn state coal data base **

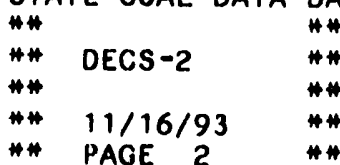

\section{PROXIMATE ANALYSIS}

\% MOISTURE

$\%$ ASH

$\%$ Volatille

\% FIXED CARBON

\section{ULTIMATE ANALYSIS}

$\%$ ASH

\% CARBON

$\%$ HYOROGEN

\% NITROGEN

\% TOTAL SULFUR

\% OXYGEN(DIFF)

\section{AS REC'D}

10.43

14.47

\section{AS REC'D}

(*)-EXCLUDES MOISTURE

SULFUR FORMS

DRY

DAF

CALORIFIC VALUE

(GROSS, BTU/LB)

\section{MM-CONTAININO}

MM-FREE (PARR)

MM-FREE (MOD. P)

NET CV, DMMF BTU/LB
DAF

DMMF (PARR)

\% EQUILIBRIUM MOISTURE $=11.51$

$$
\text { MM-FREE }=13.89
$$

34.16

40.93

14.47

58.66

$4.08(*)$

0.99

4.05

$7.31(*)$
\% PYRITIC \% SULFATIC

2.27

2.71

0.01

0.01

QRY

AS REC'D

MOIST.

11880 .

10641.

14556.

12708 .

14585.

12744 .
38.14

45.49

43.77

56.23

DAF DMMFIPARR

(19.94\%MM)

78.11

81.80

5.70

1.39

1.32

5.39

9.73

11.12

14061.

$\begin{array}{cc}\text { \% ORGANIC \% TOTAL } \\ 2.24 & 4.52 \\ 2.67 & 5.39\end{array}$

EQUIL. MOIST.

12522.

12559.

\section{ELEMENTAL ANALYSIS}

\% CARBON

$\%$ HYDROGEN

\% NITROGEN

\% oRGanic SULFUR

\% OXYGEN(DIFF)

\% MINERAL MATTER (INCL. 4.25\% FES2)

QRY DMMFIMODE) DMME(DIR.) (19.39\%MM) qMM)

DRY \% CHLORINE $=0.12$ DRY \% $602=0.66$

MOTT-SPOONER DIFFERENCE $=24$.

ATOM RATIOS (DMMF)_ PARR MOD.PARR OIRECT.

$\begin{array}{lll}\text { ATOMIC H/C } & 0.8363 & 0.8083 \\ \text { ATOMIC O/C } & 0.1020 & 0.0869\end{array}$


SAMPLE IDENTIFICATION: SAMPLE IDENTIFICATION:

PSU NUMBER

SEAM NAME

ALTERNATE SEAM NAME

APPARENT RANK
SAMPLE LOCATION:

COUNTRY $\rightarrow$ USA

STATE.
* penn state coal data base *

$\begin{array}{ll}* * & \\ \# * & \text { DECS }-2 \\ \# * & 11 / 16 / 93\end{array}$

**** rank calculat lons*****

APPARENT RANK (AS REC'D MOIST.)

$\rightarrow \quad H V C$

$\rightarrow \quad$ HVC

INTERNATIONAL RANK :

AS REC'D MOIST. $\rightarrow$

$\rightarrow \quad$ HVC

REFLECTANCE RANK CATEGORY

$\rightarrow \quad H V C$

**** PETROGRAPHIC DATA\#\#*\#*

MACERAL COMPOSITION ( WHITE LIGHT AND FLUORESCENCE ANALYSIS )

MACERAL COMPOSITION

VITRINITE VITRINITE FUSINITE FUSINITE MACRINITE MICRINITE TINITE INITE

\begin{tabular}{|c|c|c|c|c|c|c|c|c|c|c|c|c|}
\hline DMMF VOL. \% & 86.7 & 0.0 & 1.1 & 2.2 & 1.7 & 0.2 & 0.0 & 6.9 & 0.2 & 0.1 & 0.8 & \\
\hline DRY VOL. \% & 77.4 & 0.0 & 1.0 & 2.0 & 1.5 & 0.2 & 0.0 & 6.2 & 0.2 & 0.1 & 0.7 & $10.7 \% \mathrm{MM}$ \\
\hline DRY WT. \% & 70.0 & 0.0 & 1.0 & 1.9 & 1.5 & 0.2 & 0.0 & 4.7 & 0.1 & 0.1 & 0.6 & $19.9 \% \mathrm{MM}$ \\
\hline
\end{tabular}

REFLECTANCE DATA

VITRINITE :

HIGH:

0.65 LOW:

0.42

MEAN-MAX:

0.52

GRAY-KING COKE TYPE

HARDGROVE GRINDABILITY $\quad 57.8$

FREE SWELLING INDEX

3.5

VICKERS HARDNESS NUMBER 
SAMPLE IDENTIFICATION:

PSI MUMBER

SEAM MAME

ALTERNATE SEAM NAME

ALPARENT RANK

$\rightarrow$ DECS-2

$\rightarrow$ ILLINOIS \#6

$\rightarrow$ HERRIN

$\rightarrow \quad H V C$

MAJOR ELEMENT ANALYSIS

$\begin{array}{cccc} & \text { S102 } & \text { AL203 } & \text { T102 } \\ \text { OXIDE \% OF HTA } & 49.2 & 19.1 & 0.89 \\ & \text { SI } & \text { AL } & \text { TI }\end{array}$

ELEMENT \% OF

TOTAL DRY COAL
SAMPLE LOCATION:

COUNTRY $\rightarrow$ USA

STATE

$\rightarrow$ ILLINOIS

\% HTA $=15.97$

$\begin{array}{cccc}\text { FE203 } & \text { MGO } & \text { CAO } & \text { NA2O } \\ 20.4 & 0.82 & 5.18 & 0.71 \\ \text { FE } & \text { MG } & \text { CA } & \text { MA }\end{array}$

K20

1.87

k

2. 3

0.08

0.08

0.25

0.01

4.52

TRACE ELEMENT ANALYSIS

PPM PPM

HTA TOTAL COAL

GE

AG

B

LA

BA 269.

43. LI

BE

8.0

1.3

MN

387.

62. U

BI

MO

CE

NB

co

NI

CR

130.

21. PB

CU

10. RB

GA

60.

85.

14. YB

20.

\% HTA $=15.97$

PPM
HTA

PPM

TOTAL COAL

VOLATILES PPM

TOTAL COAL

AS

Bk

CD

CL

1200.

F

HG

SB

sE

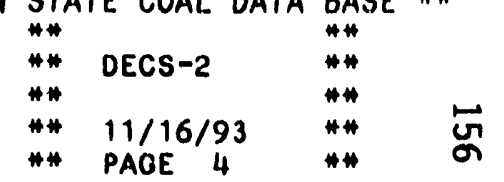

$14 . \quad \mathrm{ZR}$

610.

97.

26. 
SAMPLE IOENTIFICATION:

$\begin{array}{ll}\text { PSU NUMBER } & \rightarrow \text { DECS }-2 \\ \text { SEAM NAME } & \rightarrow \text { ILLINOIS } \# 6 \\ \text { ALTERNATE SEAM NAME } & \rightarrow \text { HERRIN } \\ \text { APPARENT RANK } & \rightarrow \text { HVC }\end{array}$

APPARENT RANK
SAMPLE LOCATION:

SAMPLE LOCATION:

COUNTRY

$\rightarrow$ USA

STATE

$\rightarrow$ ILLINOIS

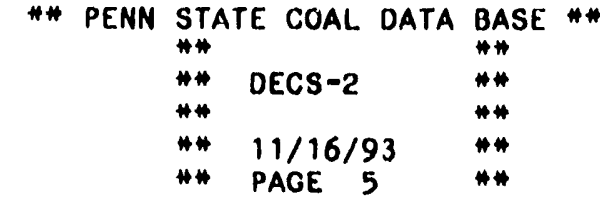

**
$* *$ DECS -2
$* *$
$* *$
$*$ PAGE 5

ASH FUSION TEMPERATURES (ALL TEMPERATURES IN DEGREES F)

ASH FUSION TEMPERATURES

INITIAL DEFORMATION

SOFTENING

HEMISPHER ICAL

FLUID

\section{REDUCING OXIDIZING}

1900.

2240 .

2035.2340

2100.2400

$2180 . \quad 2450$.

FLUID 
SAMPLE IDENTIFICATION:

$\begin{array}{ll}\text { PSU NUMBER } & \rightarrow \text { DECS }-3 \\ \text { SEAM NAME } & \rightarrow \text { COAL BASIN M } \\ \text { ALTERNATE SEAM NAME } & \rightarrow>\text { DUTCH CREEK } \\ \text { APPARENT RANK } & \rightarrow \text { MED VOL }\end{array}$

SAMPLE HISTORY:

SAMPLING DATE \& AGENCY

$\rightarrow \quad 5 / 31 / 90$.

SAMPLE TYPE

$\rightarrow$ CHANNEL-WHOLE SEAM

ADDITIONAL INFORMATION

MINE INFORMATION:

MINE INFORMATION:

\section{RESERVES}

ANNUAL PRODUCTION

$\rightarrow$ U. CRET.

$\rightarrow$ MESAVERDE

$\rightarrow$ WILLIAMS FORK

$\rightarrow \quad 9 \mathrm{FT} . \quad 0 \mathrm{IN}$.
SEAM INFORMATION:

AGE OF SEAM

GROUP

FORMATION

SEAM THICKNESS
SAMPLE LOCATION:

COUNTRY

$\rightarrow$ USA

STATE

$\rightarrow$ COLORADO

COUNTY

$\rightarrow$ GUNNISON

$\begin{array}{ll}\text { TOWNSHIP } & \rightarrow \\ \text { CITY } & \rightarrow \\ \end{array}$

COAL PROVINCE $\rightarrow$ ROCKY MOUNTAIN REGION $\rightarrow$ UINTA

MAP REFERENCE: LATITUDE $\rightarrow \begin{array}{rllllll} & 39 & D & 11 & \mathrm{M} & 13 & \mathrm{~S}\end{array}$ LONGITUDE $\rightarrow 107 \mathrm{D} 22 \mathrm{M} 7 \mathrm{~S}$

QUADRANGLE $\rightarrow$ PLACITA $\left(7.5^{\prime}\right)$

LIFE EXPECTANCY ->

MINING METHOD $\quad \rightarrow$ UNDERGROUND
** penn state coal data base **

\#*

* DECS -3

\#* $11 / 16 / 93$

* PAGE 1 


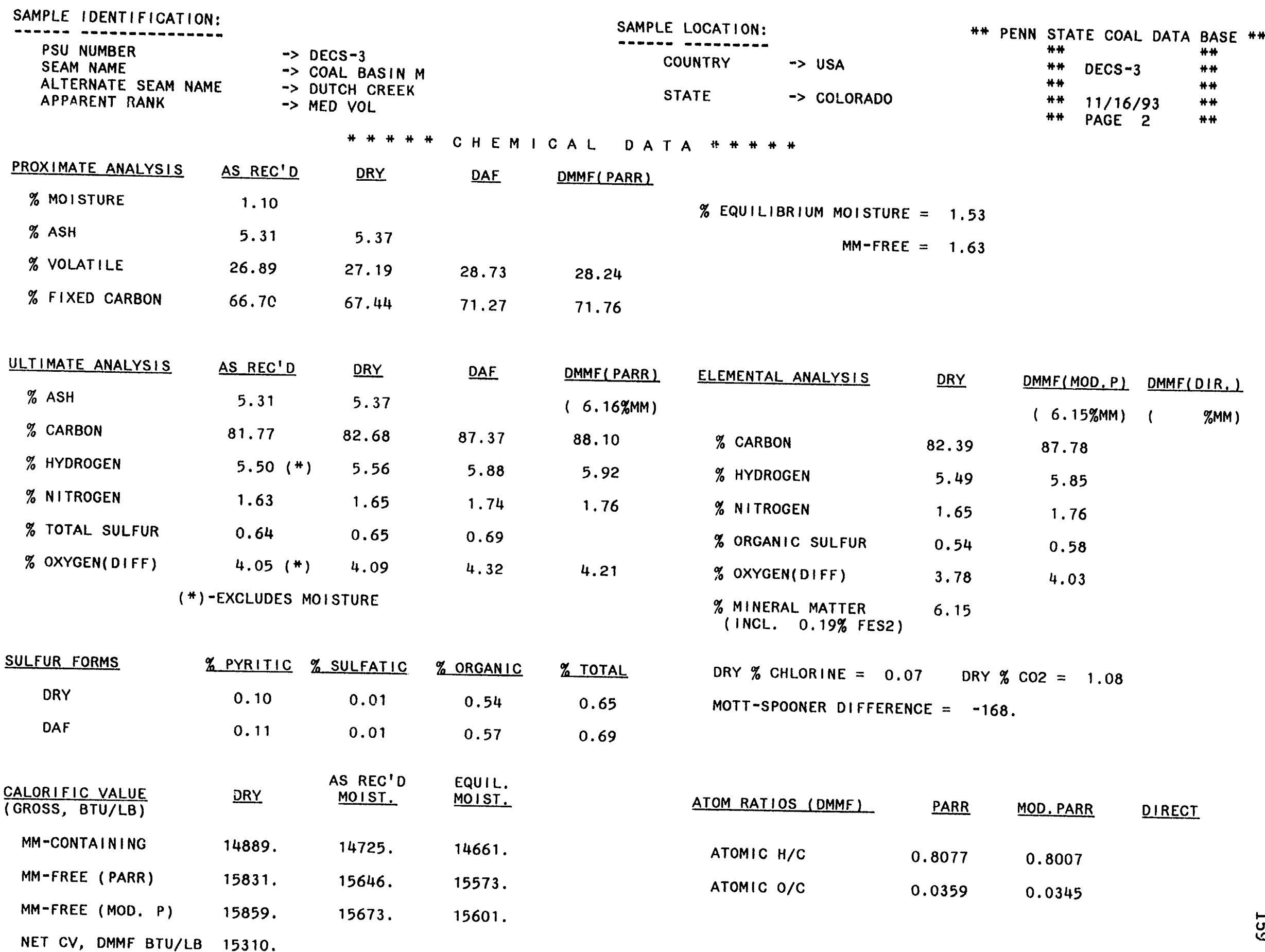


SAMPLE IDENTIFICATION:

SAMPLE IDENTIFICATION:

PSU NUMBER

SEAM NAME

ALTERNATE SEAM NAME

APPARENT RANK $\rightarrow$ DECS -3

$\rightarrow$ COAL BASIN M

$\rightarrow$ DUTCH CREEK

\begin{tabular}{ll} 
SAMPLE LOCATION: & \\
\hline COUNTRY & $\rightarrow$ USA \\
STATE & $\rightarrow$ COLORADO
\end{tabular}

** pENN STATE cOAL dATA base *\#

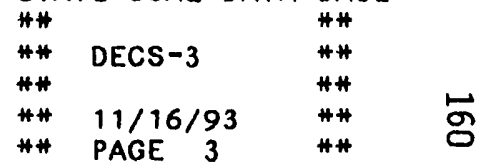

APPARENT RANK (AS REC'D MOIST, )

ASTM RANK (EQUIL. MOIST.)

REFLECTANCE PA CA CATEGORY

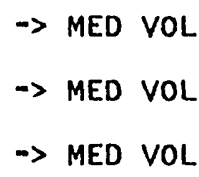

$\rightarrow$ MED VOL

$\rightarrow$ MED VOL

$\rightarrow$ MED VOL

C A L C ULATIONS***\#*

INTERNATIONAL RANK :

AS REC'D MOIST. $\rightarrow$

EQUIL. MOIST.

REPORTED RANK :
MACERAL COMPOSITION ( WHITE LIGHT ANALYSIS )

MACERAL COMPOSITION

PSEUDO- SEMI

VITRINITE VITRINITE FUSINITE FUSINITE MACRINITE MICRINITE TINITE INITE

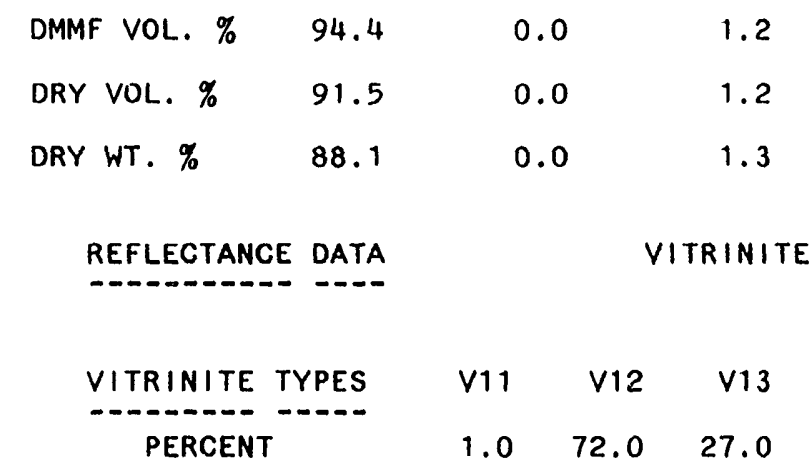

$\begin{array}{lllll}2.0 & 2.2 & 0.0 & 0.0 & 0.0 \\ 1.9 & 2.1 & 0.0 & 0.0 & 0.0\end{array}$

0.2

0.0

0.0

0.2

0.0

0.0

$3.1 \% \mathrm{MM}$

$2.0 \quad 2.2$

0.2

0.0

0.0

$6.2 \% \mathrm{BM}$
**\#* C A K ING A N D MECHAN I C A L

GIESELER COAL PLASTOMETER:

INIT. SOFTENING TEMP

399

TEMP. MAX. FLUIDITY

461

SOLIDIFICATION TEMP.

499

FLUID TEMP. RANGE

100

MAXIMUM FLUIDITY

WASHAB ILITY DATA AVAILABLE?
HIGH: 1.38 LOW: 1.19 MEAN-MAX: 1.28

GRAY-KING COKE TYPE

HARDGROVE GRINDABILITY

103.0

FREE SWELLING INDEX

9.0

VICKERS HARONESS NUMBER 
SAMPLE IDENTIFICATION: SAMPLE IDENTIFICATION: PSU NUMBER

SEAM NAME

ALTERNATE SEAM NAME

APPARENT RANK

$\rightarrow$ DECS -3

$\rightarrow$ COAL BASIN M

$\rightarrow$ DUTCH CREEK

$\rightarrow$ MED VOL

MAJOR ELEMENT ANALYSIS

MAJOR ELEMENT ANALYSIS

OXIDE \% OF HTA

S102 AL203 T102

$\begin{array}{lll}24.8 & 22.7 & 0.82\end{array}$

10.8

3.52

SI AL TI

ELEMENT \% OF

TOTAL DRY COAL

$\begin{array}{lllll}0.6 & 0.6 & 0.03 & 0.4 & 0.11\end{array}$

SAMPLE LOCATION:

$\begin{array}{ll}\text { COUNTRY } & \rightarrow \text { USA } \\ \text { STATE } & \rightarrow \text { COLORADO }\end{array}$

** penn state coal data base \#\# \#*

** DECS -3

\#*

** PAGE 4

TRACE ELEMENT ANALYSIS

TRACE ELEMENT ANALYSIS

\% HTA $=5.26$

$\begin{array}{lc}\text { PPM } & \text { PPM } \\ \text { HTÁ } & \text { TOTAL COAL }\end{array}$

PPM

HTA

PPM

TOTAL COAL

$\begin{array}{lc}\text { PPM } & \text { PPM } \\ \text { HTA } & \text { TOTAL COAL }\end{array}$

VOLATILES PPM

AG

GE

SN

B

LA

SR

3805 .

200.

AS

BR

CD

CL

700.

BE

4.0

0.2 MN

310.

16. U

110.

6.

$$
\text { F }
$$

HG

SB

$<25$.

$<1$.

YB

65.

3.

SE

20.

1. $\mathrm{ZR}$

150.

8. 
SAMPLE IDENTIFICATION:

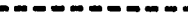

PSU NUMBER

SEAM NA.ME

ALTERNATE SEAM NAME

$\rightarrow$ DECS -3

$\rightarrow$ COAL BASIN M

$\rightarrow$ DUTCH CREEK

$\rightarrow \rightarrow$ MED VOL
SAMPLE LOCATION:

COUNTRY

$\rightarrow$ USA

STATE

-> COLORADO
* penn state coal data base *\# \#\# \#\# \#\# DECS-3 \#*

*\# DCCS-3 \#*

\#\# 11/16/93 \#\#

$\begin{array}{lll}* * & 11 / 16 / 93 \\ * * & \text { PAGE } 5\end{array}$

ASH FUSION TEMPERATURES (ALL TEMPERATURES IN DEGREES F)

REDUCING OXIDIZING

INITIAL DEFORMATION

2020.

2270.

SOFTENING

2130 .

2365.

HEMISPHERICAL

2200 .

2420 .

FLUID

2250 . 2460 .


SAMPLE IDENTIFICATION:

$\begin{array}{ll}\text { PSU NUMBER } & \rightarrow \text { DECS }-4 \\ \text { SEAM NAME } & \rightarrow \text { BLUE } \\ \text { ALTERNATE SEAM NAME } & \rightarrow \# 1 \\ \text { APPARENT RANK } & \rightarrow \text { HVC }\end{array}$

\section{SAMPLE HISTORY:}

SAMPLING DATE \& AGENCY

$\Rightarrow 6 / 4 / 90 ; P S U$

SAMPLE TYPE

$\rightarrow$ CHANNEL-WHOLE SEAM

ADDITIONAL INFORMATION

MINE INFORMATION:

\section{RESERVES}

ANNUAL PRODUCTION
SAMPLE LOCATION:

COUNTRY $\rightarrow$ USA

STATE $\quad \rightarrow$ NEW MEXICO

COUNTY $\quad \rightarrow$ MCKINLEY

TOWNSHIP $\quad \rightarrow$

CITY $\quad \rightarrow$ WINDOW ROCK, AZ

COAL PROVINCE $\rightarrow$ ROCKY MOUNTAIN

REGION $\rightarrow$ SAN JUAN RIVER

MAP REFERENCE: LATITUDE $\rightarrow \quad 35042 \mathrm{M} 53 \mathrm{~s}$

LONGITUDE $\rightarrow 108 \mathrm{D} \quad 55 \mathrm{M} 22$

QUADRANGLE-> TSE BONITA SCHOOL $\left(7.5^{\prime}\right)$

LIFE EXPECTANCY $\quad \rightarrow$

MINING METHOD $\quad \rightarrow$ STRIP

SEAM INFORMATION:

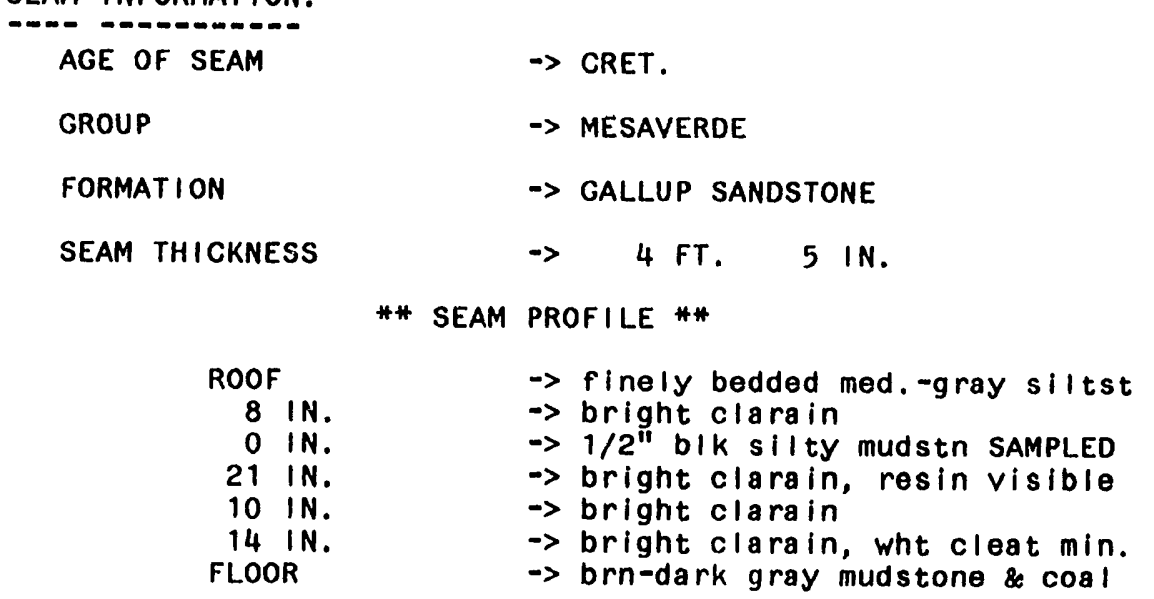




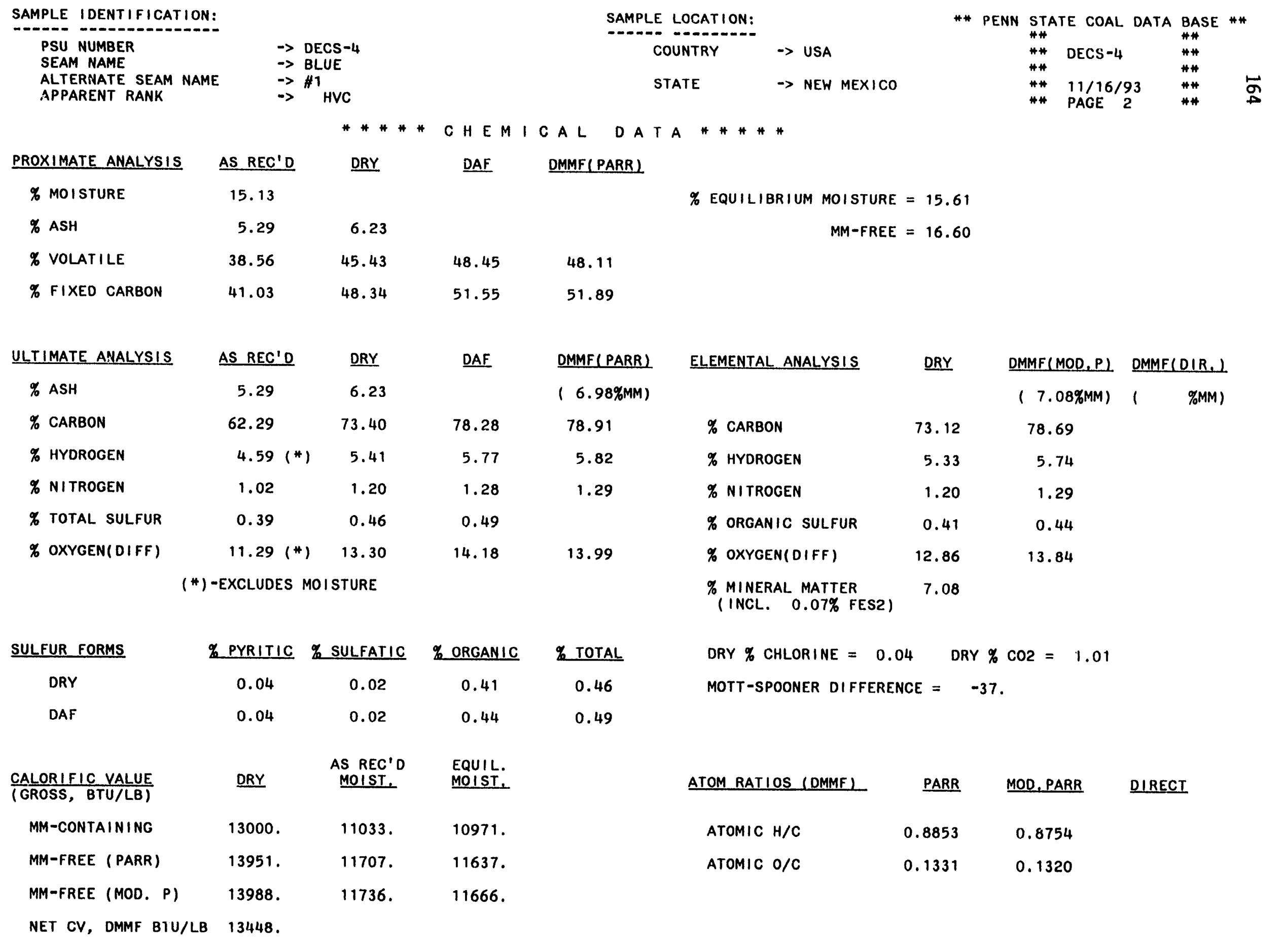


SAMPLE IDENTIFICATION: PSU NUMBER

SEAM NAME

ALTERNATE SEAM NAME

APPARENT RANK

$\Rightarrow$ DECS -4
$\Rightarrow$ BLUE
$\Rightarrow \# 1$
$\Rightarrow$ HVC

\begin{tabular}{ll} 
SAMPLE LOCATION: & \\
\hline COUNTRY & $\rightarrow$ USA \\
STATE & $\rightarrow$ NEW MEXICO
\end{tabular}

* penn state coal data base *\# $\begin{array}{llll}* * & & * * \\ * * & \text { DECS }-4 & * * \\ * * & & * * \\ * * & 11 / 16 / 93 & * * \\ * * & \text { PAGE } & \# *\end{array}$

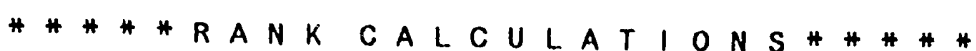

APPARENT RANK (AS REC'D MOIST.)

ASTM RANK (EQUIL. MOIST.)

$\rightarrow \quad H V C$

$\rightarrow \quad H V C$

$\rightarrow \quad$ HVC

INTERNATIONAL RANK :

AS RE.C'D MOIST. $\rightarrow$
EQUIL. MOIST. $\rightarrow$

REPORTED RANK :

$\rightarrow \quad H V C$

***** PETROGRA P H I C D A T A *\#\#\#\#

MACERAL COMPOSITION ( WHITE LIGHT AND FLUORESCENCE ANALYSIS

MACERAL COMPOSITION

VITRINITE VITRINITE FUSINITE FUSINITE MACRINITE MICRINITE TINITE INITE

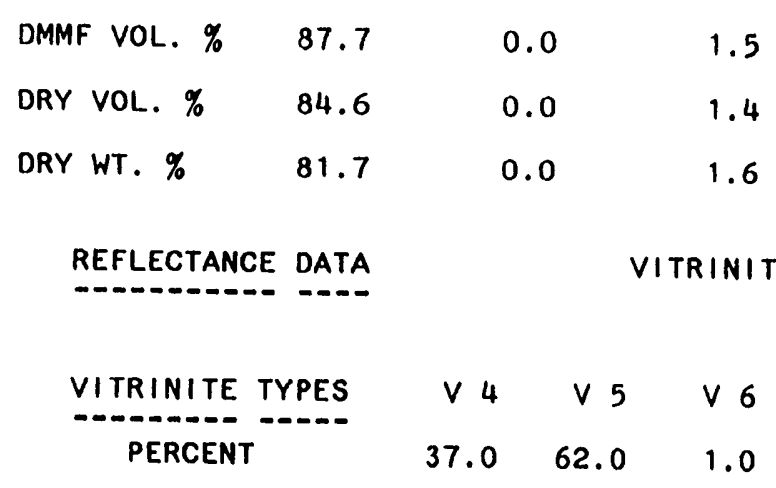

**** C A K I N G

$\begin{array}{lll}3.5 & 2.5 & 0.0 \\ 3.4 & 2.4 & 0.0 \\ 3.5 & 2.5 & 0.0\end{array}$

0.0

4.5

RESINITE ALGINITE CUTINITE

.4

3.5

0.0

$0.0 \quad 4.3$

$0.0 \quad 3.6$

\begin{abstract}
0.3
\end{abstract}
0.0

0.0

0.3

0.0

0.0

$3.5 \% \mathrm{MM}$

0.2

0.0

0.0

$7.0 \% \mathrm{MM}$

GIESELER COAL PLASTOMETER:

INIT. SOFTENING TEMP

TEMP. MAX. FLUIDITY

SOLIDIFICATION TEMP.

FLUID TEMP. RANGE

MAXIMUM FLUIDITY

WASHABILITY DATA AVAILABLE? NO
HIGH: 0.62 LOW: 0.43 MEAN-MAX: 0.51 
SAMPLE IDENTIFICATION:

$\begin{array}{ll}\text { PSU NUMBER } & ->\text { DECS }-4 \\ \text { SEAM NAME } & ->\text { BLUE } \\ \text { ALTERNATE SEAM NAME } & ->\# 1 \\ \text { APPARENT RANK } & ->\text { HVC }\end{array}$

\section{MAJOR ELEMENT ANALYSIS}

MANOR ELEMENT ANALYSIS

OXIDE \% OF HTA
ELEMENT $\%$ OF
TOTAL DRY COAL
TACE ELEMENT ANALYSIS

TRACE ELEMENT ANALYSIS

$\begin{array}{lccc} & \begin{array}{c}\text { PPM } \\ \text { HTA }\end{array} & \text { TOTAL COAL } & \\ \text { AG } & & & \text { GE } \\ \text { B } & & & \text { LA } \\ \text { BA } & 1344 . & 81 . & \text { LI } \\ \text { BE } & 17 . & 1 . & \text { MN } \\ \text { BI } & & & \text { MO } \\ \text { CE } & & & \text { NB } \\ \text { CO } & & & \text { NI } \\ \text { CR } & 110 . & & \text { PB } \\ \text { CU } & 90 . & 5 . & \text { RB } \\ \text { GA } & & 5 C\end{array}$

GE

MO

NB

SC
SAMPLE LOCATION:

$$
\begin{array}{ll}
\text { COUNTRY } & \rightarrow \text { USA } \\
\text { STATE } & \rightarrow \text { NEW MEXICO }
\end{array}
$$

$\%$ HTA $=6.04$

$\begin{array}{cccccccccr}\text { S102 } & \text { AL2O3 } & \text { TIO2 } & \text { FE203 } & \text { MGO } & \text { CAO } & \text { NA20 } & \text { K20 } & \text { P205 } & \text { S03 } \\ 57.4 & 21.7 & 1.29 & 6.08 & 1.88 & 7.14 & 0.79 & 0.79 & 0.14 & 2.10 \\ \text { SI } & \text { AL } & \text { TI } & \text { FE } & \text { MG } & \text { CA } & \text { NA } & \text { K } & \text { P } & \text { S } \\ 1.6 & 0.7 & 0.05 & 0.26 & 0.07 & 0.31 & 0.04 & 0.04 & 0.004 & 0.46\end{array}$

$\%$ HTA $=6.04$

$\begin{array}{lc}\text { PPM } & \text { PPM } \\ \text { HTA } & \text { TOTAL COAL }\end{array}$

PPM

HTA

PPM

TOTAL COAL

SN

1776.

107.

VOLATILES

PPM

SR

19. U

310.

$<25$.
* penn state coal data base \#\#

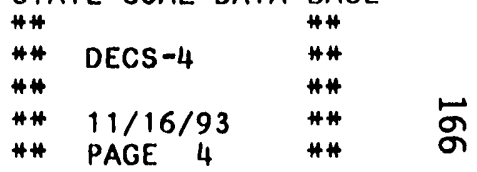

ڤே 
SAMPLE IDENTIFICATION: SAMPLE IDENTIFICATIOH:

PSU NUMBER

SEAM NAME

ALTERNATE SEAM NAME

APPARENT RANK

$\rightarrow$ DECS -4

$\rightarrow$ BLUE

$\rightarrow \# 1$

$\rightarrow$ HVC
SAMPLE LOCATION:

SAMPLE LOCATION:

COUNTRY

$\rightarrow$ USA

STATE

$\rightarrow$ NEW MEXICO
** penn state coal data base ** \#*

\#\# DECS -4 \#*

\#\# $11 / 16 / 93 \quad$ \#*

** PAGE 5 **

ASH FUSION TEMPERATURES (ALL TEMPERATURES IN DEGREES F)

AEDUCIMG OXIDIZIMG

$\begin{array}{lll}\text { INITIAL DEFORMATION } & 2100 . & 2250 . \\ \text { SOFTENING } & 2210 . & 2300 . \\ \text { HEMISPHERICAL } & 2265 . & 2345 . \\ \text { FLUID } & 2300 . & 2390 .\end{array}$


SAMPLE IDENTIFICATION:

DAMPLE TOE

PSU NUMBER $\quad \rightarrow$ DECS -

SEAM NAME $\rightarrow$ HIAWATHA

ALTERNATE SEAM NAME

$\rightarrow \quad$ HVC

SAMPLE HISTORY

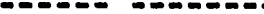

SAMPLING DATE \& AGENCY

$\rightarrow 6 / 6 / 90 ; P S U$

SAMPLE TYPE

$\rightarrow$ CHANNEL-WORKING SECTION

ADDITIONAL INFORMATION

MINE INFORMATION:

RESERVES

ANNUAL PRODUCTION

$\rightarrow$

$\rightarrow$

$\rightarrow$ U. CRET.

$\rightarrow$ MESAVERDE

$\rightarrow$ BLACKHAWK

$\rightarrow \quad 9 \mathrm{FT} . \quad 2 \mathrm{IN}$.

SEAM INFORMATION:

AGE OF SEAM

GROUP

FORMATION

SEAM THICKNES
SAMPLE LOCATION:

COUNTRY

$\rightarrow$ USA

STATE $\quad \rightarrow$ UTAH

COUNTY $\quad \rightarrow$ SEVIER

TOWNSHIP $\quad \rightarrow$

CITY $\quad \rightarrow$ EMERY

COAL PROVINCE $\rightarrow$ ROCKY MOUNTAIN

REGION $\rightarrow$ UINTA

$\begin{array}{llllllll}\text { MAP REFERENCE: LATITUDE } & \rightarrow & 38 & 0 & 57 & M & 55 & S \\ \text { LONGITUDE } & \rightarrow & 111 & D & 23 & M & 15 & \text { S }\end{array}$

LONGITUDE $\rightarrow 1110023 \mathrm{M} 15 \mathrm{~S}$

\#\# SEAM PROFILE \#\#

ROOF

22 IN.

16 IN.

4 IN.

68 IN

FLOOR

$\rightarrow$ not accessible

$\rightarrow$ c.0Bl in mine roof NOT SAMPLED

$\rightarrow$ coal

$\rightarrow$ zone of discont. $5 \mathrm{~mm}$ partings

$\rightarrow \cos 1$

$\rightarrow$ gray silty mudstone

*\# PENN STATE COAL DATA bASE **

\#*

\#\# DECS -5

\#\# $11 / 16 / 93$ \#\#

* PAGE 1

ळ
LIFE EXPECTANCY $\rightarrow$

MINING METHOD $\quad \rightarrow$ UNDERGROUND 
SAMPLE IDENTIFICATION: SAMPLE IDENTIFICATION: PSU NUMBER SEAM NAME

ALTERNATE SEAM NAME APPARENT RANK

$$
\begin{aligned}
& \rightarrow> \\
& \Rightarrow E C S-5 \\
& \Rightarrow \text { HIAWATHA } \\
& \rightarrow> \\
& \rightarrow \text { HVC }
\end{aligned}
$$

SAMPLE LOCATION:

$$
\begin{array}{ll}
\text { COUNTRY } & \rightarrow \text { USA } \\
\text { STATE } & \rightarrow \text { UTAH }
\end{array}
$$

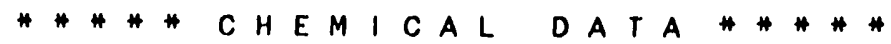

\section{PROXIMATE ANALYSIS}

AS REC'D DRY

\% moI STURe

\% ASH

7.54

\% VOLATILE

\% FIXED CARBon

$$
7.90
$$

37.29

\begin{tabular}{|c|c|c|c|c|}
\hline ULTIMATE ANALYSIS & AS REC'D & DRY & DAF & DMMFIPAR \\
\hline$\%$ ASH & 7.90 & 8.54 & & $(9.53 \% \mathrm{M}$ \\
\hline \% CARBON & 67.22 & 72.70 & 79.49 & 80.36 \\
\hline$\%$ HYDROGEN & $4.58(*)$ & 4.95 & 5.41 & 5.47 \\
\hline \% NITROGEN & 1.05 & 1.14 & 1.25 & 1.26 \\
\hline$\%$ TOTAL SULFUR & 0.52 & 0.56 & 0.61 & \\
\hline$\%$ OXYGEN(DIFF) & $11.20(*)$ & 12.11 & 13.24 & 12.91 \\
\hline \multicolumn{5}{|c|}{ (*)-EXCLUDES MOISTURE } \\
\hline SULFUR FORMS & \% PYRITIC & \% SULFATIC & \% ORGANIC & Y TOTAL \\
\hline DRY & 0.27 & 0.01 & 0.28 & 0.56 \\
\hline DAF & 0.30 & 0.01 & 0.31 & 0.61 \\
\hline$\frac{\text { CALORIFIC VALUE }}{\text { (GROSS, BTU/LB) }}$ & $\underline{\text { ORY }}$ & $\begin{array}{l}\text { AS REC'D } \\
\text { MOIST. }\end{array}$ & $\begin{array}{l}\text { EQUIL. } \\
\text { MOIST. }\end{array}$ & \\
\hline MM-CONTAINING & 12760 & 11798. & 11755 & \\
\hline MM-FREE (PARR) & 14073 & 12910 & 12858 & \\
\hline MM-FREE (MOD. P) & 14131. & 12959. & 12906 & \\
\hline
\end{tabular}

47.27

51.13

8.54

DAF

DMMF (PARR)

$\begin{array}{lll} & \text { DAF } & \text { DMMFIPARR } \\ 54 & & \\ 33 & 44.10 & 43.58 \\ 13 & 55.90 & 56.42\end{array}$

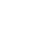

\% EQUILIBRIUM MOISTURE $=7.88$

$$
\begin{aligned}
\text { MM-FREE } & =8.66
\end{aligned}
$$

penn state coal data base ** ** DECS -5 \#* DECS -5 ** $11 / 16 / 93$ \#\# PAGE 2 
SAMPLE IDENTIFICATION:

PSU NUMBER

SEAM NAME

ALTERNATE SEAM NAME

APPARENT RANK $\rightarrow$ DECS -5

$\rightarrow$ HIAWATHA

$\rightarrow$ HVC
COUNTRY $\rightarrow$ USA

STATE $\quad \rightarrow$ UTAH
SAMPLE LOCATION:
* penn state coal data base **

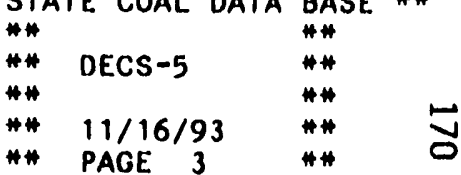

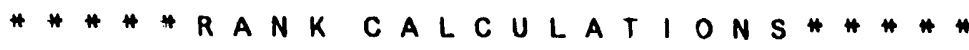

APPARENT RANK (AS REC'D MOIST.)

ASTM RANK (EQU!L. MOIST.)

REFLECTANCE RANK CATEGORY

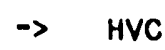

$\rightarrow \quad H V C$

$\rightarrow \quad H V B$
INTERNAT IONAL RANK :

AS REC'D MOIST. $\rightarrow$

EQUIL. MOIST.

REPORTED RANK : $\quad \rightarrow$ HVC
***** PETROGRAPHIC DATA \#\#***

MACERAL COMPOSITION ( WHITE LIGHT AND FLUORESCENCE ANALYSIS )

MACERAL COMPOSITION

SCLERO- SPORVITRINITE VITRINITE FUSINITE FUSINITE MACRINITE MICRINITE TINITE INITE
RESINITE ALGINITE CUTINITE

$\begin{array}{lrrrr}\text { DMMF VOL. \% } & 65.5 & 0.0 & 3.7 \\ \text { DRY VOL. \% } & 62.3 & 0.0 & 3.5 \\ \text { DRY WT. \% } & 59.6 & 0.0 & 3.7 \\ & & & & \\ \text { REFLECTANCE DATA } & & & \text { VITRINIT } \\ \text { PITRINITE TYPES } & V 4 & V 5 & V 6 \\ \text { PERCENT } & & 2.0 & 56.0 & 42.0\end{array}$

$\begin{array}{lll}0.8 & 0.0 & 11.8\end{array}$

$\begin{array}{lll}0.8 & 0.0 \quad 11.2\end{array}$

0.8

$0.0 \quad 9.1$
2.2

0.3

0.5

$9.2 \quad 5.2$

5.4

HIGH:

0.68

LOW:

0.48

MEAN-MAX
$0.5 \quad 4.8 \% \mathrm{MM}$

$0.4 \quad 9.5 \% \mathrm{MM}$
GIESELER COAL PLASTOMETER:

INIT. SOFTENING TEMP

TEMP. MAX. FLUIDITY

SOLIDIFICATION TEMP.

FLUID TEMP. RANGE

MAXIMUM FLUIDITY

WASHABILITY DATA AYAILABLE? MO
GRAY-KING COKE TYPE

HARDGROVE GRINDABILITY

43.1

FREE SWELLING INDEX

1.0

VICKERS HARDNESS NUMBER

no 
SAMPLE IOENTIFICATION:

\section{SAMPLE IOENTIFICATION:}

SEAM NAME

ALTERNATE SEAM NAME

APPARENT RANK

$\rightarrow$ DECS -5

$\rightarrow$ HIAWATHA

$\Rightarrow \quad H V C$

MAJOR ELEMENT ANALYSIS

MAJOR ELEMENT ANALYSIS

OXIDE \% OF HTA

S102 AL203 T102

44.1

11.0

0.84

SI $\quad A L$

TOEMENT \% OF

$\begin{array}{ll}1.8 & 0.5\end{array}$

TI

0.04

FE203

8. 10

FE

0.49

TRACE ELEMENT ANALYSIS

TRACE ELEMENT ANALYSIS
SAMPLE LOCATION:

COUNTRY $\rightarrow$ USA

STATE

-> UTAH

\% HTA $=8.61$

NAOO

$8.40 \quad 4.29$

1.73

CA

0.52

0.09

NA

0.27

$\%$ HTA $=8.61$

$\begin{array}{lc}\text { PPM } & \text { PPM } \\ \text { HTA } & \text { TOTAL COAL }\end{array}$

PPM $P P M$
HTA

AG

GE

B

$B A$

806.

69.

BE

$<3.0$

$<0.3$

MN

BI

CE

co

CR

cu

85.

150.

GA

$\begin{array}{rrr} & M O \\ & & \text { NB } \\ & & \text { NI } \\ 85 . & 7 . & \text { PB } \\ 150 . & 13 . & \text { RB } \\ & & \text { SC }\end{array}$

$\begin{array}{ll} & S N \\ & S R \\ & T H \\ & U \\ & \\ & Y \\ & Y \\ \text { 1. } & Y B \\ & Z N \\ & Z R\end{array}$

TOTAL COAL

SN

465.

HTA TOTAL COAL

1691.

146.

9.

110.

35.

15.
* PENN STATE coal data base ** ** ** DECS -5
** D DCS
* PAGE 4
\#*
\#*


SAMPLE IDENTIFICATION:

$\begin{array}{ll}\text { PSU NUMBER } & \rightarrow \text { DECS }-5 \\ \text { SEAM NAME } & \rightarrow \text { HIAWATHA } \\ \text { ALTERNATE SEAM NAME } & \rightarrow \text { HVC } \\ \text { APPARENT RANK } & \rightarrow \text { HV }\end{array}$

ALPAREMT RANK

$\rightarrow \quad H V C$ ASH FUSION TEMPERATURES (ALL TEMPERATURES IN DEGREES F)

SOFTENING

HEMISPHER:CAL

FLUID
REDUCING

1920.

2045.

2100 .

2170 .
OXIDIZING

2150 .

2205.

2245.

2300 .
SAMPLE LOCATION:

COVIYTRY

-> USA

STATE

$\rightarrow$ UTAH i\#* PENN STATE COAL DATA BASE \#* H* \#\# DECS -5

\#* 11/16/93

H* PAGE 5 
SAMPLE IDENTIFICATION:

$\begin{array}{ll}\text { PSU NUMBER } & \rightarrow \\ \text { SEAM NAME } & \rightarrow \text { DECS }-6 \\ \text { ALTERNATE SEAM NAME } & \rightarrow \text { BIInd Canyon } \\ \text { APPARENT RANK } & \rightarrow \text { HVA }\end{array}$

\section{SAMPLE HISTORY:}

SAMPLING DATE \& AGENCY

$\rightarrow 6 / 7 / 90 ; P S U$

SAMPLE TYPE

$\rightarrow$ CHANNEL-WORKING SECTION

ADDITIONAL INFORMATION $\rightarrow$ TOP $8^{\prime}$ OF $8^{\prime} 6^{\prime \prime}$ SEAM

MINE INFORMATION:

MINE INFORMATION:

RESERVES

ANNUAL PRODUCTION
SAMPLE LOCATION:

country

$\rightarrow$ USA

STATE $\quad \rightarrow$ UTAH

COUNTY $\rightarrow$ Emery

TOWNSHIP $\quad \rightarrow$

CITY $\quad \rightarrow$ Huntington

COAL PROVINCE $\rightarrow$ ROCKY MOUNTAIN REGION $\rightarrow$ UINTA

MAP REFERENCE: LATITUDE $\rightarrow \quad 39 \mathrm{D} \quad 21 \mathrm{M} 44 \mathrm{~S}$

LONGITUDE $\rightarrow 111 \mathrm{D} 10 \mathrm{M} 4 \mathrm{~s}$

QUADRANGLE-> Mahogany Polnt (7.5')

LIFE EXPECTANCY $\rightarrow>$

MINING METHOD $\quad \rightarrow$ UNDERGROUND

SEAM INFORMATION:

\section{SEAM INFORMATION:}

AGE OF SEAM

GROUP

FORMATION

SEAM THICKNESS $\rightarrow$ U. CRET.

-> Mesaverde

$\rightarrow$ Blackhawk

$\rightarrow \quad 8 \mathrm{FT} . \quad 6 \mathrm{IN}$.

** SEAM PROFILE **

$\vec{\rightarrow}$ coal

ROOF
5 IN.
2 IN.
89
IN.
6 IN.
FLOOR

ROOF

5 in.

6 iN.

FLOOR
$\Rightarrow$ high ash $(30-40 \%)$ li thotype

$\rightarrow$ coal with abundant resin

$\rightarrow$ below mine floor NOT SAMPLED

$\rightarrow$ not accessible
** PENN STATE coAl dATA BASE \#\# ** \#* DECS -6

*\# 11/16/93

** PAGE 1 
SAMPLE IDENTIFICATION:

PSU NUMBER
SEAM NAME
ALTERNATE SEAM NAME
APPARENT RANK

$\Rightarrow$ DECS -6
$\Rightarrow$ BIind Canyon
$\rightarrow>$ HVA

SAMPLE LOCATION:

COUNTRY $\rightarrow$ USA

$\rightarrow$
* penn state coal data base \#\# \#\# \#\# \#\# ** DECS -6

\#\# 11/16/93

* PAGE 2

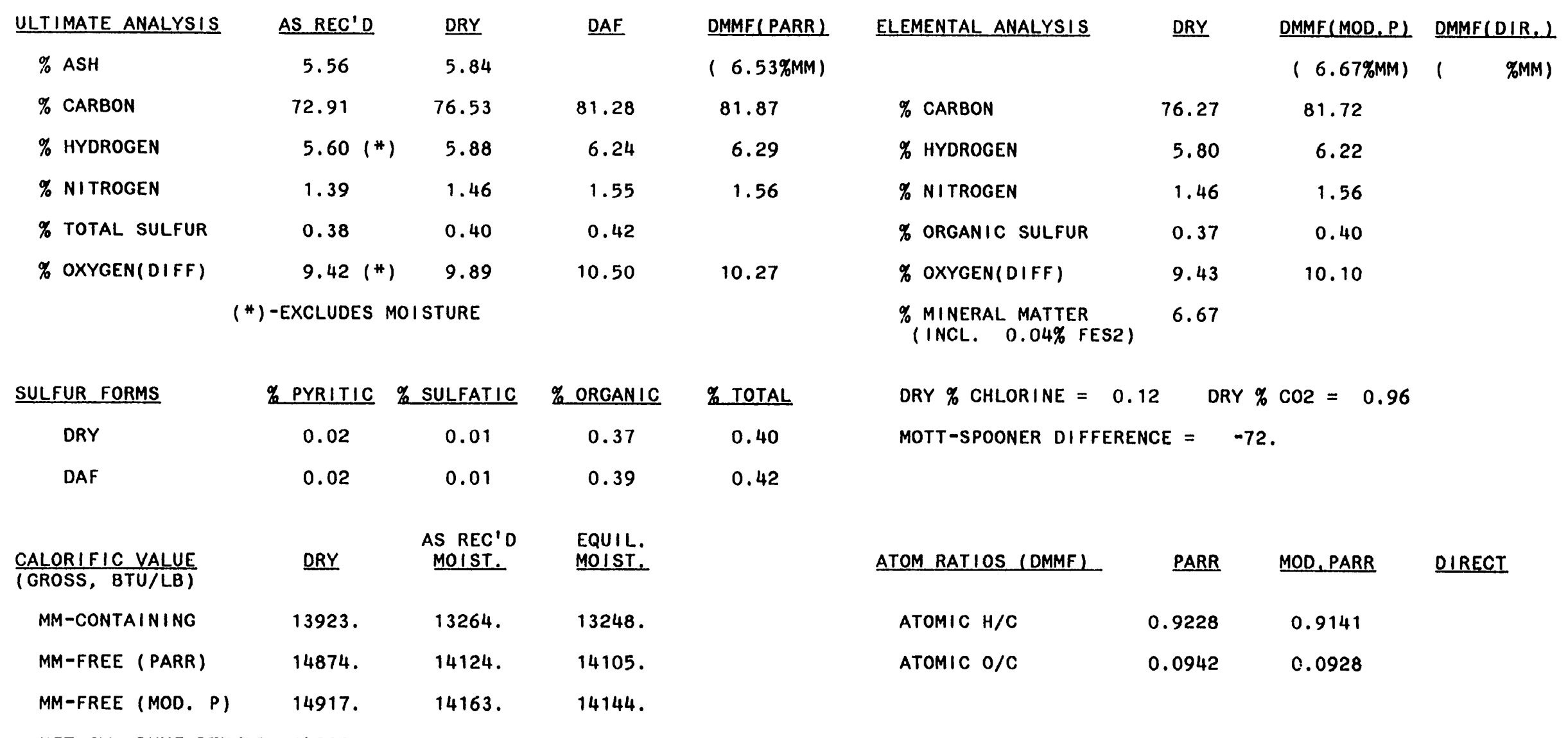

\begin{tabular}{|c|c|c|c|c|c|c|c|}
\hline PROXIMATE ANALYSIS & AS REC'D & $\underline{\text { DRY }}$ & $\underline{\text { DAF }}$ & DMMF(PARR) & & & \\
\hline$\%$ MOISTURE & 4.73 & & & & \% EQUILIBRIUM & MOISTURE = & 4.85 \\
\hline$\%$ ASH & 5.56 & 5.84 & & & & MM-FREE $=$ & 5.18 \\
\hline$\%$ VOLATILE & 42.40 & 44.50 & 47.26 & 46.94 & & & \\
\hline$\%$ FIXED CARBON & 47.31 & 49.66 & 52.74 & 53.06 & & & \\
\hline
\end{tabular}

NET CV, DMMF BTU/LB 14333. 
S,MMPLE IDENTIFICATION: SAMPLE IDENTIFICATION: 'SU NUMBER SEAM NAME

ALTERNATE SEAM NAME

APPARENT RANK

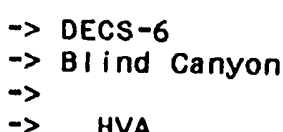

$\rightarrow$ DECS -6

$\rightarrow$

SAMPLE LOCATION:

COUNTRY $\rightarrow$ USA

STATE

$\rightarrow$ UTAH
*\# PENN STATE COAL DATA BASE \#\#

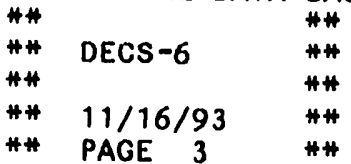

APPARENT RANK (AS REC'D MOIST.)

ASTM RANK (EQUIL. MOIST.)

REFLECTANCE RANK CATEGORY

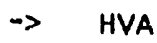

$\rightarrow \quad$ HVA

$\rightarrow \quad H V B$

C ALCULATIONSH*\#\#*

INTERNATIONAL RANK :

AS REC'D MOIST. $->$

EQUIL. MOIST.

REPORTED RANK :

***\#* PETROGRAPHIC DATA \#*\#**

MACERAL COMPOSITION ( WHITE LIGHT ANO FLUORESCENCE ANALYSIS )

MACERAL COMPOSITION PSEUDO- SEMI-

SEMI -

SCLERO- SPOR-

VITRINITE FUSINITE FUSINITE MACRINITE MICRINITE TINITE INITE

\begin{tabular}{|c|c|c|c|c|c|c|c|c|c|c|c|c|}
\hline DMMF VOL. \% & 69.1 & 0.0 & 1.8 & 5.6 & 5.3 & 0.9 & 0.0 & 11.1 & 5.0 & 0.4 & 0.8 & \\
\hline DRY VOL. \% & 66.8 & 0.0 & 1.7 & 5.4 & 5.1 & 0.9 & 0.0 & 10.7 & 4.8 & 0.4 & 0.8 & $3.3 \% \mathrm{MM}$ \\
\hline DRY WT. \% & 64.5 & 0.0 & 1.9 & 5.6 & 5.3 & 0.9 & 0.0 & 9.7 & 4.4 & 0.4 & 0.7 & $6.5 \% \mathrm{MM}$ \\
\hline
\end{tabular}

REFLECTANCE DATA

VITRINITE :

HIGH:

0.82

LOW:

0.58

MEAN-MAX:

0.66

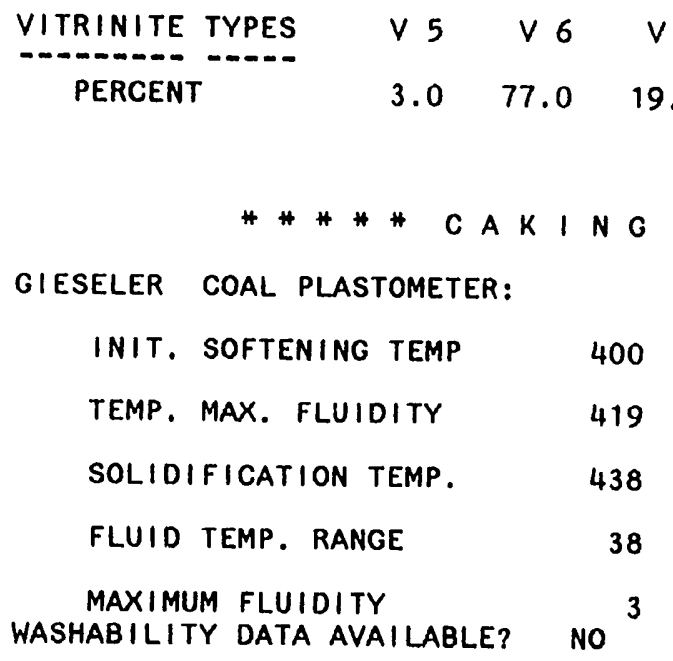

GRAY-KING COKE TYPE

HARDGROVE GRINDABILITY

44.5

FREE SWELLING INDEX

VICKERS HARDNESS NUMBER

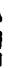


SAMPLE IDENTIFICATION: SAMPLE IDENTIFICATION: PSU NUMBER

SEAM NAME

ALTERNATE SEAM NAME.

APPARENT RANK

$\rightarrow$ DECS -6

$\rightarrow$ Blind Canyon

$\rightarrow$ HVA

MAJOR ELEMENT ANALYSIS

MAJOR ELEMENT ANALYSIS

OXIDE \% OF HTA

S102 AL203

50.3

15.3

T102

0.96

FE203

6.88

TI

FE

ELEMENT \% OF

SI $A L$

$1.5 \quad 0.5$

0.04

0.30

SAMPLE LOCATION:

$\begin{array}{ll}\text { COUNTRY } & \rightarrow \text { USA } \\ \text { STATE } & \rightarrow \text { UTAH }\end{array}$

* penn state coal data base **

\#* ${ }^{* *}$ DECS-6

on

TRACE ELEMENT ANALYSIS

TRACE ELEMENT ANALYSIS

$\begin{array}{lc}\text { PPM } & \text { PPM } \\ \text { HTA } & \text { TOTAL COAL }\end{array}$

PPM PPM

HTA TOTAL COAL

\% HTA $=6.26$

\begin{tabular}{|c|c|c|c|c|c|c|c|c|c|c|}
\hline & $\begin{array}{l}\text { PPM } \\
\text { HTA }\end{array}$ & $\begin{array}{c}\text { PPM } \\
\text { TOTAL COAL }\end{array}$ & & $\begin{array}{l}\text { PPM } \\
\text { HTA }\end{array}$ & $\begin{array}{c}\text { PPM } \\
\text { TOTAL COAL }\end{array}$ & & $\begin{array}{l}\text { PPM } \\
\text { HTA }\end{array}$ & $\begin{array}{c}\text { PPM } \\
\text { TOTAL COAL }\end{array}$ & VOLATILES & $\begin{array}{c}\text { PPM } \\
\text { TOTAL COAL }\end{array}$ \\
\hline$A G$ & & & GE & & & SN & & & AS & \\
\hline B & & & LA & & & SR & 1353. & 85. & BR & \\
\hline BA & 1433 & 90. & LI & & & $\mathrm{TH}$ & & & $C D$ & \\
\hline BE & 4.0 & 0.3 & MN & 155. & 10. & $u$ & & & $\mathrm{CL}$ & 1200 \\
\hline 81 & & & MO & & & $v$ & 140 & 9. & $F$ & \\
\hline$C E$ & & & NB & & & $Y$ & & & HG & \\
\hline $\mathrm{CO}$ & & & NI & $<25$. & $<2$. & YB & & & SB & \\
\hline CR & 95. & 6. & PB & & & $Z N$ & 70. & 4. & SE & \\
\hline $\mathrm{CU}$ & 150. & 9. & $R B$ & 25. & 2. & $Z R$ & 470 & 29. & & \\
\hline GA & & & SC & & & & & & & \\
\hline
\end{tabular}


SAMPLE IDENTIFICATION:

PSU NUMBER

SEAM NAME

ALTERNATE SEAM NAME

APPARENT RANK

$\rightarrow$ DECS -6

$\rightarrow$ BIInd Canyon

HVA
SAMPLE LOCATION:

SAMPLE LOCATION:

COUNTRY

$\rightarrow$ USA

STATE

$\rightarrow$ UTAH
* PENN state coAl dATA base \#* ** D* \#\#

\#* $11 / 16 / 93$

* PAGE 5

ASH FUSION TEMPERATURES (ALL TEMPERATURES IN DEGREES F)

INITIAL DEFORMATION

SOFTENING

HEMISPHERICAL

FLUID
REDUCING

1900.

2020 .

2090.

2240.
OXIDIZING

2250.

2345.

2400.

2440. 
SAMPLE IDENTIFICATION:

SAMPLE IOENTIFICATION

PSU NUMBER

SEAM NAME

ALTERNATE SEAM NAME

APPARENT RANK

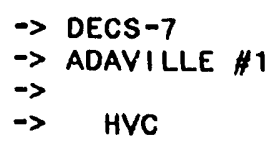

SAMPLE HISTORY:

SAMPLING DATE \& AGENCY

$\rightarrow 6 / 8 / 90 ; P S U$

SAMPLE TYPE

$\rightarrow$ CHANNEL-WHOLE SEAM

ADDITIONAL INFORMATION

MINE INFORMATION:

MINE INFORMATION:

\section{RESERVES}

ANNUAL PRODUCTION

$\rightarrow$ U. CRET.

$\rightarrow$

$\rightarrow$ ADAVILLE

$\rightarrow 120 \mathrm{FT} . \quad 0 \mathrm{IN}$

SEAM INFORMATION:

AGE OF SEAM

GROUP

FORMATION

SEAM THICKNESS

\section{SAMPLE LOCATION:}

COUNTRY $\rightarrow$ USA

STATE $\quad \rightarrow$ WYOMING

COUNTY

$\rightarrow$ LINCOLN

TOWNSHIP

CITY

$\rightarrow$

$\rightarrow$ KEMMERER

COAL PROVINCE $\rightarrow$ POCKY MOUNTAIN

REGION $\rightarrow$ HAMS FORK

MAP REFERENCE: LATITUDE $\rightarrow 41 \mathrm{D} \quad 47 \mathrm{M} 23 \mathrm{~S}$

LONGITUDE $\rightarrow 110 \mathrm{D} 36 \mathrm{M} 32 \mathrm{~S}$

QUADRANGLE-> KEMMERER $\left(7.5^{\prime}\right)$ $\rightarrow$

$\rightarrow$ channel sample taken as $257^{\prime}$ trench on pit floor

LIFE EXPECTANCY $\rightarrow$

MINING METHOD

$\rightarrow$ STRIP 
SAMPLE IDENTIFICATION: SAMPLE IDENTIFICATION: PSU NUMBER

SEAM NAME

ALTERNATE SEAM NAME

APPARENT RANK

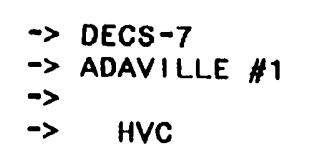

SAMPLE LOCATION:

COUNTRY $\rightarrow$ USA

STATE $\quad \rightarrow$ WYOMING

***** CHEMICAL DATA \#\#\#\#*

PROXIMATE ANALYSIS

$\%$ MOISTURE

AS REC'D DRY

$\%$ ASH

17.34

\% VOLATILE

\% FIXED CARBON

\begin{abstract}
3.45
\end{abstract}
4.17

38.11

46.10

41.11

49.73

DAF

OMMF(PARR)

\% EQUILIBRIUM MOISTURE $=18.40$

MM-FREE $=19.15$
ULTIMATE ANALYSIS

\section{AS REC'D DRY}

$\%$ ASH

\% CARBON

$\%$ HYDROGEN

\% Nitrogen

\% TOtal sulfur

\% OXYGEN(DIFF)

$4.38(*)$

0.82

0.81

12.12 (*)
$3.45 \quad 4.17$

$61.09 \quad 73.90$

5.30

0.99

0.98

14.66

(*)-EXCLUDES MOISTURE

\section{SULFUR FORMS}

DRY

DAF

MM-CONTAINING

MM-FREE (PARR)

MM-FREE (MOD. P)

NET CV, OMMF BTU/LB

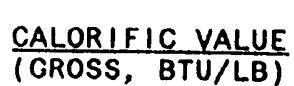

$\begin{array}{ccccc}\text { \% PYRITIC } & \text { \% SULFATIC } & \text { \% ORGANIC } & \text { \% TOTAL } \\ 0.10 & 0.01 & 0.87 & 0.98 \\ 0.10 & 0.01 & 0.91 & 1.02\end{array}$

DRY AS REC'D EQUIL.

$13068 . \quad 10802 . \quad 10663$.

$13710 . \quad 11230 . \quad 11079$.

$13720.11243 . \quad 11093$.
** peNn state coAl data base \#\#

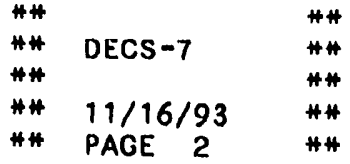


SAMPLE IDENTIFICATION: SAMPLE IDENTIFICATION: PSU NUMBER

SEAM NAME

ALTERNATE SEAM NAME

APPARENT RANK

$\begin{array}{ll}\text { SAMPLE LOCATION: } & \\ \text { COUNTRY } & \rightarrow \text { USA } \\ \text { STATE } & \rightarrow \text { WYOMING }\end{array}$

* penn state coal data base *\# $\# \#$ **

\#\# $11 / 16 / 93$ \#\# PAGE 3

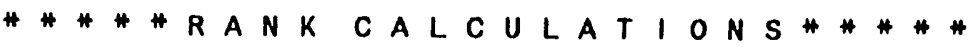

APPARENT RANK (AS REC'D MOIST.)

$\rightarrow \quad H V C$

$\rightarrow \quad H V C$

$\rightarrow$
INTERNATIONAL RANK :

AS REC'D MOIST. $\rightarrow$

EQUIL. MOIST. $\rightarrow$

REPORTED RANK : $\quad \rightarrow \quad$ HVC

REFLECTANICE RANK CATEGORY

**** PETROGRAPHIC DATA

MACERAL COMPOSITION ( WHITE LIGHT AND FLUORESCENCE ANALYSIS )

MACERAL COMPOSITION

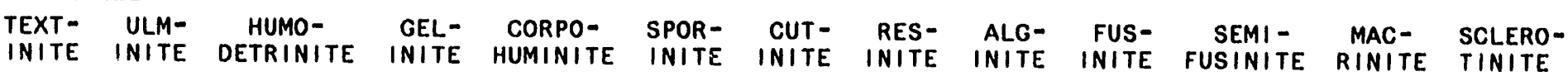

DMMF

$52.9 \quad 1.3 \quad 7.9$

3.10 .0

$0.1 \quad 0.0$

0.3

\begin{abstract}
0.5
\end{abstract}
0.9

0.5

DRY WT. \% $0.0 \quad 30.9 \quad 50.2$

$1.2 \quad 7.5$

2.90 .0

$\begin{array}{llll}0.1 & 0.0 & 0.3 & 0.5\end{array}$

$0.9 \quad 0.5$

$5.0 \% \mathrm{MM}$

DMMF VOL \% HUMINITE $=94.6$

ULMINITE REFLECTANCE DATA:

HIGH: $\quad 0.56$

LOW: $\quad 0.27$

MEAN-MAX:

0.45

**\#* C A K I N G

GIESELER COAL PLASTOMETER:

INIT. SOFTENING TEMP

TEMP. MAX, FLUIDITY

SOLIDIFICATION TEMP.

FLUID TEMP. RANGE

MAXIMUM FLUIDITY

WASHABILITY DATA AVAILABLE? NO
GRAY-KING COKE TYPE

HARDGROVE GRINDABILITY

FREE SWELLING INDEX

VICKERS HARONESS NUMBER 
SAMPLE IDENTIFICATION:

DENTIFICATION:

$\rightarrow$ DECS -7

SEAM NAME

$\rightarrow$ ADAVILLE \#1

ALIERNATE SEAM

$\rightarrow$. HVC

MAJOR ELEMENT ANALYSIS

MAUOR ELEMENT ANALYSIS

$\begin{array}{lccccr} & \text { SIO2 } & \text { AL2O3 } & \text { TIO2 } & \text { FE2O3 } & \text { MGO } \\ \text { OXIDE \% OF HTA } & 50.2 & 18.7 & 0.68 & 4.49 & 3.41 \\ \text { ELEMENT \% OF } & \text { SI } & \text { AL } & \text { TI } & \text { FE } & \text { MG } \\ \text { TOTAL DRY COAL } & 1.0 & 0.4 & 0.02 & 0.14 & 0.09\end{array}$

TRACE ELEMENT ANALYSIS

TRACE ELEMENT ANALYSIS

PPM PPM

HTA TOTAL COAL

PPM PPM

HTA TOTAL COAL

GE

$A G$

B

\section{BA}

BE

$<3.0$

91.

LA

B

CE

co

CR

85.

4. $\mathrm{PB}$

CU

GA

$<25$.

1. YB

20.

1. $\quad Z R$

SAMPLE LOCATION:

COUNTRY

$\rightarrow$ USA

STATE

-> WYOMING

** peni state coAl data base *

\begin{tabular}{|c|c|}
\hline \#\# & DECS -7 \\
\hline & $\begin{array}{l}11 / 16 / 93 \\
\text { PAGE }\end{array}$ \\
\hline
\end{tabular}

\% HTA $=4.43$

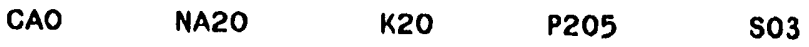

$\begin{array}{lllll}8.76 & 0.38 & 0.41 & 0.18 & 10.5\end{array}$

CA NA

K

$\mathbf{s}$

0.28

0.01

0.02

0.003

1.0
$\%$ HTA $=4.43$

PPM PPM
HTA TOTAL COAL

TAL COAL

SN

$S R$

1945.

86.

VOLATILES PPM

AS

BR

CD

$\mathrm{CL}$

600.

$<10$

$<0.4$

$\mathbf{F}$

HG

SB

SE

95.

4.

380.

17. 
SAMPLE IDENTIFICATION:

SEAM NAME

ALTERNATE SEAM NAME

APPARENT RANK

$\rightarrow$ DECS -7

$\Rightarrow$ ADAVILLE

HVC
SAMPLE LOCATION:

COUNTRY

$\rightarrow$ USA

STATE

$\rightarrow$ WYOMING
* PENN STATE COAL DATA bASE :*

HH

*\# DECS -7

\#\# $11 / 16 / 93$

* PAGE 5

ASH FUSION TEMPERATURES (ALL TEMPERATURES IN DEGREES F

INITIAL DEFORMATION

SOFTENING

HEMISPHER ICAL

FLUID
REDUCING OXIDIZING

2120

2200.

2200.2300

2260 . 2345

2330 . 2390 . 
SAMPLE IDENTIFICATION: -

PSU NUMBER

ALTERNATE SEAM NAME

APPARENT RANK

$\rightarrow$ DECS -8

$\rightarrow$ SMITH-ROLAND

$\rightarrow$ WYODAK

$\rightarrow$ SUBBIT C

SAMPLE HISTORY:

SAMPLING DATE AGENCY

$\Rightarrow 6 / 11 / 90 ; P S U$

SAMPLE TYPE

ADDITIONAL INFORMATION

MINE INFORMATION:

\section{RESERVES}

ANNUAL PRODUCTION

$\rightarrow$

$\Rightarrow$ RUN OF MINE

$\rightarrow$ COLLECTED FROM AUTOMATIC SAMPLER

SAMPLE LOCATION:

$\begin{array}{ll}\text { COUNTRY } & \rightarrow \text { USA } \\ \text { STATE } & \rightarrow \text { WYOMING } \\ \text { COUNTY } & \rightarrow \text { CAMPBELL }\end{array}$

** penN state coAl dATA base \#* ** OHA COAL DATA BASE

** DECS -0

* $11 / 16 / 93$

*\# PAGE 1 \#*

COUNTY

$\rightarrow$

TOWNSHIP

CITY

$\rightarrow$ GILLETTE

COAL PROVINCE $\rightarrow$ NORTHERN GREAT PLAINS REGION $\rightarrow$ FORT UNION

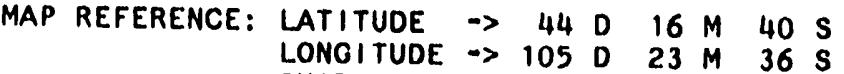
QUADRANGLE $\rightarrow$ GILLETTE EAST $\left(7.5^{\prime}\right)$

LIFE EXPECTANCY $\rightarrow$

MINING METHOO $\rightarrow$ STRIP

\section{SEAM INFORMATION:}

AGE OF SEAM

GROUP

FORMATION

SEAM THICKNESS
-> PALEO.

$\rightarrow$

$\rightarrow$ FORT UNION

$\rightarrow \quad 90 \mathrm{FT} . \quad 8 \mathrm{IN}$

** SEAM PROFILE **

ROOF

$480 \mathrm{IN}$.

240 IN.

0 IN

$120 \mathrm{IN}$

$8 \mathrm{IN}$.

$240 \mathrm{IN}$.

FLOOR $\rightarrow 40^{\prime}$ coal (Roland seam)

$\rightarrow 20^{\prime}$ coal (Roland seam)

$\rightarrow$ parting, thickness unspecifled

$\rightarrow 10$ coal (Smith seam)

$\rightarrow$ parting

$\rightarrow 20^{\prime} \mathrm{COal}(\mathrm{Sm} / \mathrm{th}$ seam) 


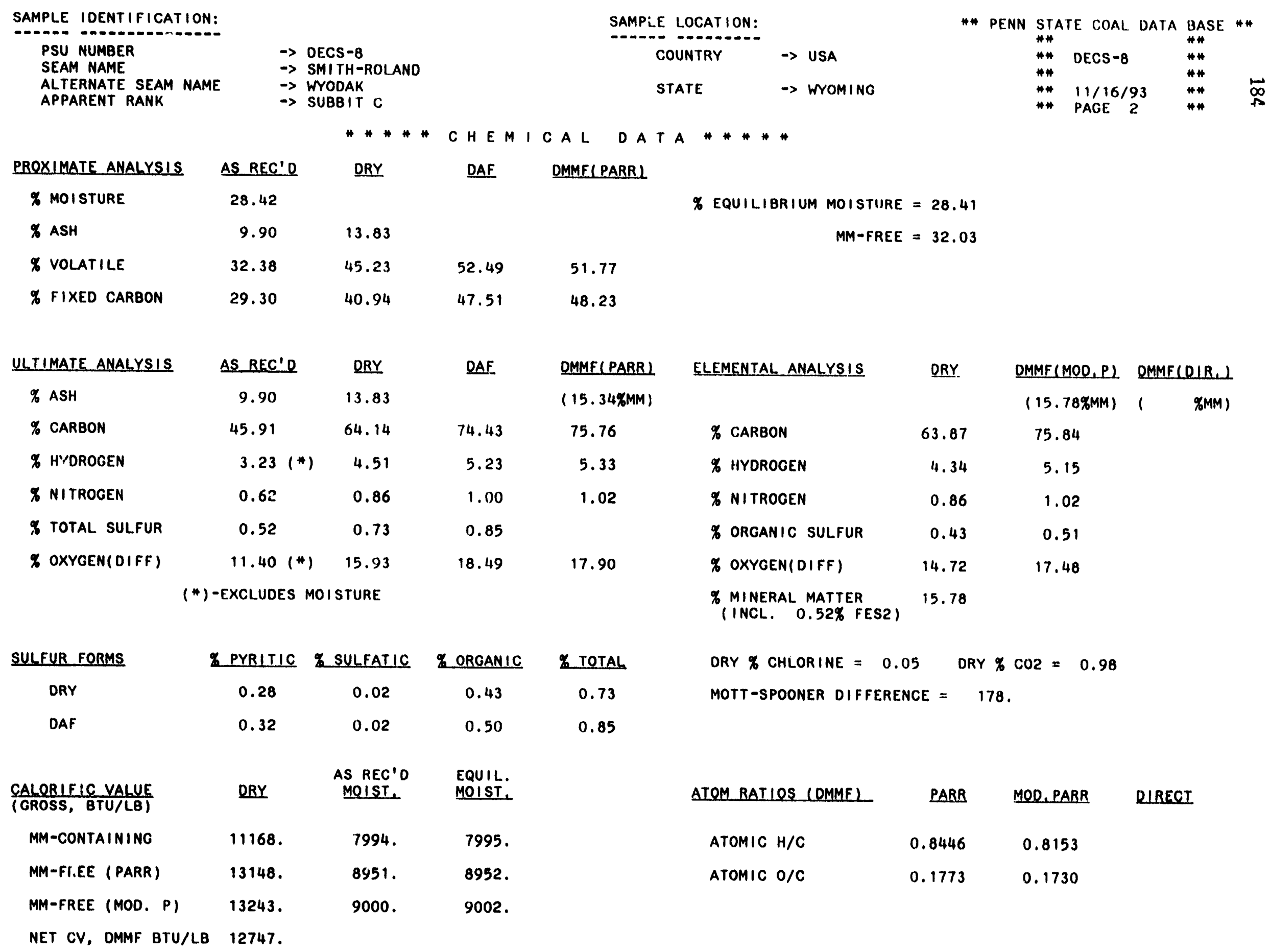


SAMPLE IDENTIFICATION: SAMPLE IOENTIFICATION: PSU NUMBER

SEAM NAME

ALTERNATE SEAM NAME

APPARENT RANK

$\rightarrow$ DECS -8

$\rightarrow$ SMITH-ROLAND

$\rightarrow$ WYODAK

$\rightarrow$ SUBBIT $C$
SAMPLE LOCATION:

COUNTRY

$\rightarrow$ USA

STATE

$\rightarrow$ WYOMING
** penn state coal data base ** H* *H DECS-8

* $11 / 16 / 93$ *H PAGE 3
APPARENT RANK (AS REC'D MOIST.)

ASTM RANK (EQUIL. MOIST.)

REFLECTANCE RANK CATEGORY
* * R A N K

$\rightarrow$ SUBBIT C

$\rightarrow$ SUBBIT C

$\rightarrow$

CALCULATIONS*****

INTERNATIONAL RANK :

AS REC'D MOIST. $\rightarrow$

EQUIL. MOIST.

REPORTED RANK : $\quad \rightarrow$ SUBBIT C

***** PETROGRAPHIC DATA *****

MACERAL COMPOSITION ( WHITE LIGHT AND FLUORESCENCE ANALYSIS )

MACERAL COMPOSITION

TEXT- ULM- HUMO- GEL- CORPO- SPOR- CUT- RES- ALG- FUS- SEMI-
INITE INITE DETRINITE INITE HUMINITE INITE INITE INITE INITE INITE FUSINITE RINITE TINITE

DMMF

VOL. \% $\quad 0 . C \quad 32.6$

DETRINITE

B. 0

4. 0

re

(n)

0.3

0.1

INITE

SINIT

RINITE

DRY WT. \% $0.0 \quad 27.6$

29.0

$6.8 \quad 3.4$

9.6

$0.1 \quad 0.3$

0.12 .2

3.0

2.5

0.1

OMMF VOL \& HLMINITE $=\quad 78.9$

ULMINITE REFLECTANCE DATA:

HIGII: $\quad 0.45$

LOW: $\quad 0.26$

MEAN-MAX:

0.37

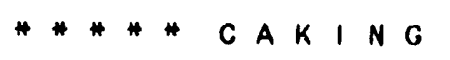

A N D

MECHA I CAL

GIESELER COAL PLASTOMETER:

INIT. SOFTENING TEMP

GRAY-KING COKE TYPE

TEMP. MAX. FLUIOITY

HARDGROVE GRINDABILITY

SOLIOIFICATION TEMP.

FREE SWELLING INDEX

FLUID TEMP. RANGE

VICKERS HARDNESS NUMBER

MAXIMUM FLUIDITY

WASHABILITY DATA AVAILABLE? NO 
SAMPLE IDENTIFICATION: SAMPLE IDENTIFICATION:

PSU NUMBER

SEAM NAME

ALTERNATE SEAM NAME

APPARENT RANK $\rightarrow$ DECS -8

$\rightarrow$ SMITH-ROLAND

$\rightarrow$ WYODAK

$\rightarrow$ SUBBIT C
SAMPLE LOCATION:

COUNTRY $\rightarrow$ USA

STATE $\quad \rightarrow$ WYOMING

$\%$ HTA $=13.19$

MAJOR ELEMENT ANALYSIS

$\begin{array}{lccc} & \text { S102 } & \text { AL203 } & \text { TIO2 } \\ \text { OXIDE \% OF HTA } & 40.7 & 21.8 & 1.05 \\ & \text { SI } & \text { AL } & \text { TI } \\ \text { ELEMENT \% OF } & & 1.5 & 0.08\end{array}$

$\begin{array}{cc}\text { FE2.03 } & \text { MGO } \\ 6.23 & 3.56 \\ \text { FE } & \text { MG }\end{array}$

$\begin{array}{rr}\text { CAO } & \text { NA2O } \\ 13.3 & 0.86 \\ \text { CA } & \text { NA }\end{array}$

k20

0.77

K

1.3

0.08

0.03

$\%$ HTA $=13.19$

TRACE ELEMENT ANALYSIS

\section{PPM \\ HTA \\ PPM}

$A G$

$\mathrm{AC}$

B

BA

$B E$$$
3851 .
$$

4.0

508.

0.5

CE

c.0

CR

$\mathrm{CU}$

GA

120.

150

16.

20.

$\begin{array}{lc}\text { PPM } & \text { PPM } \\ \text { HTA } & \text { TOTAL COAL }\end{array}$

GE

LA

MO

NB

NI

55.

45.
SC
\#\# PENIN STATE COAL DATA BASE \#\#

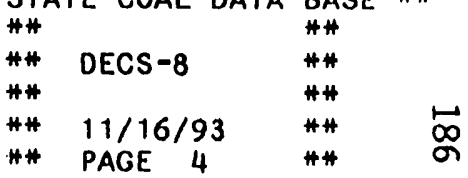

* PAGE 4 
SAMPLE IDENTIFICATION:

- -O-. - - -
PSU NUMBER
ALTERNATE SEAM NAME
$\rightarrow$ DECS -8
$\rightarrow$ SMITH-ROLANO
$\rightarrow$ WYOOAK
$\rightarrow$ SUBBIT C
APPARENT RANK

(ALL TEMPERATURES IN DEGREES F)

ASH FUSION TEMPERATURES

REDUCING OXIDIZING

INITIAL DEFORMATION

SOFTENING

2000 .

2200

HEMI SPHER ICAL

FLUID
2145.2300

2210.2345
2100.2260 .
SAMPLE LOCATION:

COUNTRY

$\rightarrow$ USA

STATE

$\rightarrow$ WYOMING
\#* PENN STATE COAL dATA BASE \#\#<smiles>[11CH3]</smiles>

\#\#

** DECS -8

\#\# 11/16/93

* PAGE 5

\#\#

\#*

$\# \#$ 
SAMPLE IDENTIFICATION:

SAMPLE IDEI

PSU NUMBER

SEAM NAME

ALTERNATE SEAM NAME

APPARENT RANK

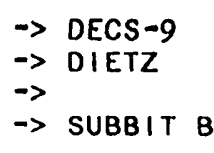

SAMPLE HISTORY:

SAMPLING DATE \& AGENCY

SAMPLE TYPE

ADDITIONAL INFORMATION

MINE INFORMATION:

MINE INFORMATION:

RESERVES

ANNUAL PRODUCTION

$\rightarrow$

$\rightarrow$

$\rightarrow$ AIR ROTARY DRILL CUTTINGS, WHOLE SEAM
SAMPLE LOCATION:

COUNTRY $\rightarrow$ USA

STATE $\quad \Rightarrow$ MONTANA

COUNTY

$\rightarrow$ BIGHORN

TOWNSHIP $\rightarrow$

CITY $\rightarrow$ DECKER

COAL PROVINCE $\rightarrow$ NORTHERN GREAT PLAINS REGION $\rightarrow$ FORT UNION

MAP REFERENCE: LATITUDE $\rightarrow \quad 45 \mathrm{D} \quad 6 \mathrm{M} 41 \mathrm{~S}$

LONGITUDE $\rightarrow 106 \mathrm{D} 53 \mathrm{M} 53$

QUADRANGLE-> PEARL SCHOOL (7.5')
MINING METHOD $\rightarrow$ STRIP

SEAM INFORMATION:

SEAM INFORMATION:

AGE OF SEAM

$\rightarrow$ PALEO.

GROUP

FORMATION

->

$\rightarrow$ FORT UNION

SEAM THICKNESS

$\Rightarrow 83 \mathrm{FT} . \quad 8 \mathrm{iN}$.

** pENN STATE COAL DATA bASE **

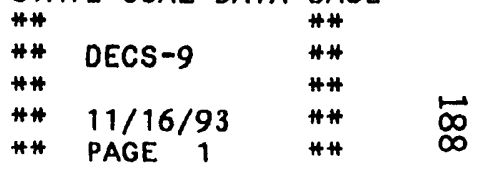

$$
\Rightarrow \quad 83 \mathrm{FT} . \quad 8 \mathrm{IN} .
$$


SAMPLE IDENTIFIGATION: PSU NUMBER

SEAM NAME

ALTERNATE SEAM NAME

APPARENT RANK

$\rightarrow$ DECS -9

$\rightarrow$ DIETZ

$\rightarrow$ SUBBIT B
SAMPLE LOCATION:

COUNTRY $\rightarrow$ USA

STATE $\quad \rightarrow$ MONTANA
* penn state coal data base *\# \#\# \#\# \#\# DECS -9

\#*

H+ $11 / 16 / 93$

* *\#* CHEM I C A L D A T A

\begin{tabular}{|c|c|c|c|c|}
\hline PROXIMATE ANALYSIS & AS REC D & DRY & DAF & DMMF (PARF \\
\hline$\%$ MOISTURE & 24.68 & & & \\
\hline$\%$ ASH & 4.80 & 6.37 & & \\
\hline$\%$ VOLATILE & 33.46 & 44.43 & 47.45 & 47.10 \\
\hline$\%$ FIXED CARBON & 37.06 & 49.20 & 52.55 & 52.90 \\
\hline
\end{tabular}

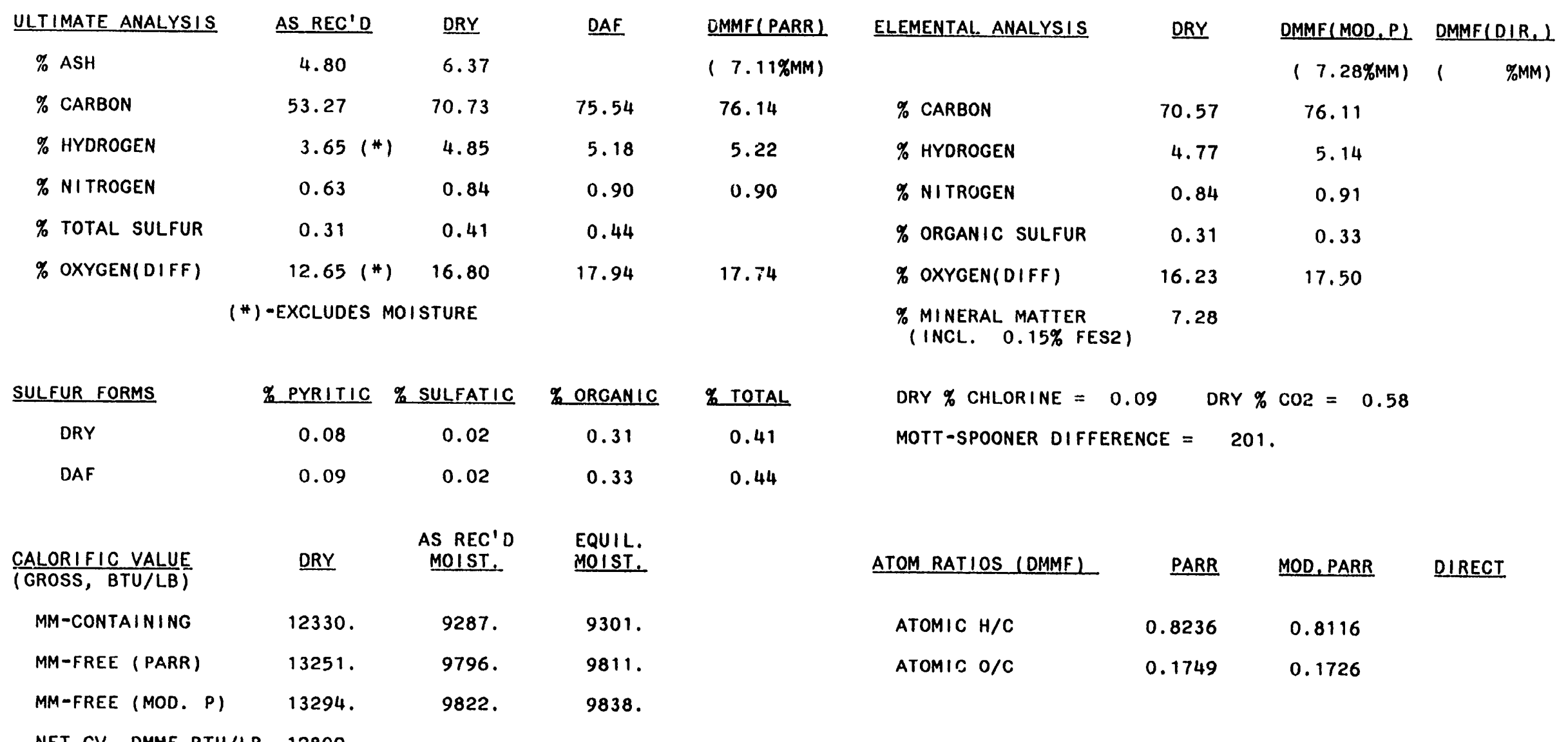

MM- RREE $=26.00$

NET CV, DMMF BTU/LB 12809. 
SAMPLE IOENTIFICATION:

CAMPLE IOENTIFICATION:

PSU NUMBER

SEAM NAME

ALTERNATE SEAM NAME

APPARENT RANK

$$
\begin{aligned}
& \Rightarrow \text { DECS }-9 \\
& \Rightarrow \text { DIETZ } \\
& \Rightarrow \\
& \Rightarrow \text { SUBBIT B }
\end{aligned}
$$

\begin{tabular}{ll} 
SAMPLE LOCATION: & \\
\hline COUNTRY & $\rightarrow$ USA \\
STATE & $\rightarrow$ MONTANA
\end{tabular}

\#* PEN

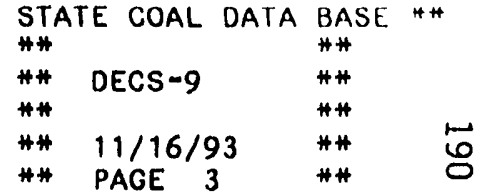

APPARENT RANK (AS REC'D MOIST.)

ASTM RANK (EQUIL. MOIST.)

REFLECTANCE RANK CATEGORY
\#\#\#\#RANK

$\rightarrow$ SUBBIT B

$\rightarrow$ SUBBIT B

$\rightarrow$
A L C U L A T I O N S \#\#\#\#\#

INTERNATIONAL RANK :

AS REC'D MOIST. $\rightarrow$

\begin{tabular}{|c|c|c|c|c|c|c|c|c|c|c|c|c|c|c|}
\hline & $\begin{array}{l}\text { TEXT- } \\
\text { INITE }\end{array}$ & $\begin{array}{l}\text { ULM- } \\
\text { INITE }\end{array}$ & $\begin{array}{c}\text { HUMO- } \\
\text { DETRINITE }\end{array}$ & $\begin{array}{l}\text { GEL- } \\
\text { INITE }\end{array}$ & $\begin{array}{l}\text { CORPO- } \\
\text { HUMINITE }\end{array}$ & $\begin{array}{l}\text { SPOR- } \\
\text { INITE }\end{array}$ & $\begin{array}{l}\text { CUT- } \\
\text { INITE }\end{array}$ & $\begin{array}{l}\text { RES- } \\
\text { INITE }\end{array}$ & $\begin{array}{l}\text { ALG- } \\
\text { INITE }\end{array}$ & $\begin{array}{l}\text { FUS- } \\
\text { INITE }\end{array}$ & $\begin{array}{l}\text { SEMI- } \\
\text { FUSINITE }\end{array}$ & $\begin{array}{l}\text { MAC- } \\
\text { RINITE }\end{array}$ & $\begin{array}{l}\text { SCLERO- } \\
\text { TINITE }\end{array}$ & \\
\hline $\begin{array}{l}\text { DMMF } \\
\text { VOL. \% }\end{array}$ & 0.0 & 43.4 & 42.0 & 1.5 & 0.8 & 5.1 & 0.2 & 0.0 & 0.0 & 2.3 & 2.3 & 1.7 & 0.7 & \\
\hline DRY WT. \% & 0.0 & 40.3 & 39.0 & 1.4 & 0.7 & 4.7 & 0.2 & 0.0 & 0.0 & 2.1 & 2.1 & 1.6 & 0.7 & $7.1 \%$ MM. \\
\hline DMMF VOL \% & HUMIN & ITE $=$ & 87.7 & & & & & & & & & & & \\
\hline ULMINITE & EFLECT & VCE DA & & HIGH: & 0.47 & LOW & 0.2 & & MEAN & -MAX: & 0.38 & & & \\
\hline
\end{tabular}

EQUIL. MOIST.

REPORTED RANK : $\quad \rightarrow$ SUBBIT B

MACERAL COMPOSITION (WHITE LIGHT AND FLUORESCENCE ANALYSIS )

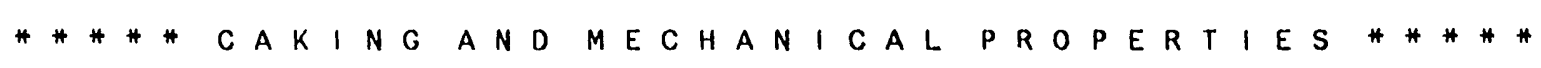

GIESELER COAL PLASTOMETER:

INIT. SOFTENING TEMP

TEMP. MAX. FLUIDITY

SOLIDIFICATION TEMP.

FLUID TEMP. RANGE

MAXIMUM FLUIDITY

WASHABILITY DATA AVAILABLE? NO
GRAY-KING COKE TYPE

HARDGROVE GRINDABILITY $\quad 40.8$

FREE SWELLING INDEX

VICKERS HARDNESS N'MMRER 
SAMPLE IDENTIFICATION: -10
0

PSU NUMBER $\rightarrow$ DECS -9

SEAM NAME

ALTERNATE SEAM NAME

$\rightarrow$ DIET

$\rightarrow$

APPARENT RANK
SAMPLE LOCATION:

COUNTRY

$\rightarrow$ USA

STATE

$\rightarrow$ MONTANA
\#\# pENN state coal daTA base \#

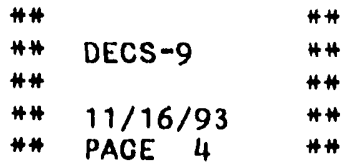

MAJOR ELEMENT ANALYSIS MANOR ELEMENT ANALYSIS

$\begin{array}{lccccc} & \text { SIO2 } & \text { AL203 } & \text { TIO2 } & \text { FE203 } & \text { MGO } \\ \text { OXIOE \% OF HTA } & 36.2 & 18.1 & 1.20 & 4.90 & 4.52 \\ \text { ELEMENT \% OF } & \text { SI } & \text { AL } & \text { TI } & \text { FE } & \text { MG } \\ \text { TOTAL ORY COAL } & 1.0 & 0.6 & 0.04 & 0.20 & 0.16\end{array}$

TRACE ELEMENT ANALYSIS

TRACE ELEMENT ANALYSIS

\section{PPM}

HTA

PPM

TOTAL COAL

BA

11644.

4.0

BI

CE

co

CR

85.

210

GA
GE

LA

694.

0.2

TOTAL COAL

$0.2 \quad M M$

MO

NB

NI

5. $P B$

13.

$\mathrm{SC}$

40.
\% HTA $=5.96$

$\begin{array}{ccccc}\text { CAO } & \text { NA2O } & \text { K20 } & \text { P205 } & \text { S03 } \\ 13.5 & 5.55 & 1.01 & 0.36 & 13.4 \\ \text { CA } & \text { NA } & K & P & \mathrm{~S} \\ 0.6 & 0.25 & 0.05 & 0.01 & 0.4\end{array}$

$\%$ HTA $=5.96$

$\begin{array}{lcl}\text { PPM } & \text { PPM } & \text { VOLATILES } \\ \text { HTA } & \text { TOTAL COAL }\end{array}$

AS

BR

CD

CL 900 .

250.

15.

F

HG

SB

$<25 . \quad$ 1. $\quad$ YB

2. $\quad Z R$

130.

8.

SE 
SAMPLE IDENTIFICATION:

SAMPLE IOENTIFICATION:
PSU NUMBER
$\rightarrow$ DECS -9
SEAM NAME
ALTEPNATE SEAM NAME
$\rightarrow$ DIETZ
$\rightarrow$ SUBBIT B

SAMPLE LOCATION:

$\begin{array}{ll}\text { COUNTRY } & \rightarrow \text { USA } \\ \text { STATE } & \rightarrow \text { MONTANA }\end{array}$

** penn state coal data base ** *\# * DECS -9

\#\# $11 / 16 / 93$

* PAGE 5

ASH FUSION TEMPERATURES (ALL TEMPERATURES IN DEGREES F) -.- -....-

REDUCING

OXIDIZING

INITIAL DEFORMATION

1910.

2050.

\section{SOFTENING}

1990.

2145.

HEMISPHER ICAL

2040

2210.

FLUID

2090 . 2240 .


SAMPLE IDENTIFICATION: SAMPLE IDENTIFICATION: PSU NUMBER

SEAM NAME

ALTERNATE SEAM NAME

APPARENT RANK

$\rightarrow$ DECS -10

$\rightarrow$ ROSEBUD

$\rightarrow$ SUBBIT B

SAMPLE HISTCRY:

SAMPLING DATE \& AGENCY

$->6 / 13 / 90 ; P S U$

SAMPLE TYPE

ADDITIONAL INFORMATION

MINE INFORMATION:

RESERVES

ANNUAL PRODUCTION

$\rightarrow$ CHANNEL-WORKING SECTION

$\rightarrow>6 "$ high pyrite coal below pit floor not sampled

$\rightarrow$ USA

COUNTRY

STATE $\quad \rightarrow$ MONTANA

COUNTY $\rightarrow$ ROSEBUD

TOWNSHIP $\quad \rightarrow$

CITY $\rightarrow$ COLSTRIP

COAL PROVINCE $\rightarrow$ NORTHERN GREAT PLAINS

REGION $\rightarrow$ FORT UNION

MAP REFERENCE: LATITUDE $\rightarrow 45 \mathrm{D} 48 \mathrm{M} 30 \mathrm{~s}$

LONGITUDE $\Rightarrow 106 \quad \mathrm{D} 39 \mathrm{M} 30 \mathrm{~s}$

QUADRANGLE $\rightarrow>$ COLSTRIP SW $\left(7.5^{\prime}\right)$
LIFE EXPECTANCY $\rightarrow$

MINING METHOD $\quad \rightarrow$ STRIP

\section{SEAM INFORMATION:}

-..- -.........-

AGE OF SEAM

$\rightarrow$ PALEO.

GROUP

FORMATION

SEAM THICKNESS

$\rightarrow$

$\rightarrow$ FORT UNION

$\rightarrow \quad 22$ FT. 2 IN. 
SAMPLE IDENTIFICATION:

PSU NUMBER
SEAM NAME
ALTERNATE SEAM NAME
APPARENT RANK

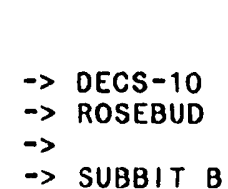

SAMPLE LOCATION:

SAMPLE LOCATION:

$\begin{array}{ll}\text { COUNTRY } & \rightarrow \text { USA } \\ \text { STATE } & \rightarrow \text { MONTANA }\end{array}$

* penn staite coal data base *

** DECS 10 \#*

H* DECS -10

** $11 / 16 / 93$

\#\# PAGE 2

\begin{tabular}{|c|c|c|c|c|}
\hline PROXIMATE ANALYSIS & AS REC'D & DRY & DAF & DMMF (PAI) \\
\hline \% MOISTURE & 21.58 & & & \\
\hline$\%$ ASH & 9.85 & 12.56 & & \\
\hline$\approx$ VOLATILE & 32.68 & 41.67 & 47.66 & 46.86 \\
\hline$\%$ FIXED CARBON & 35.89 & 45.77 & 52.34 & 53.14 \\
\hline
\end{tabular}

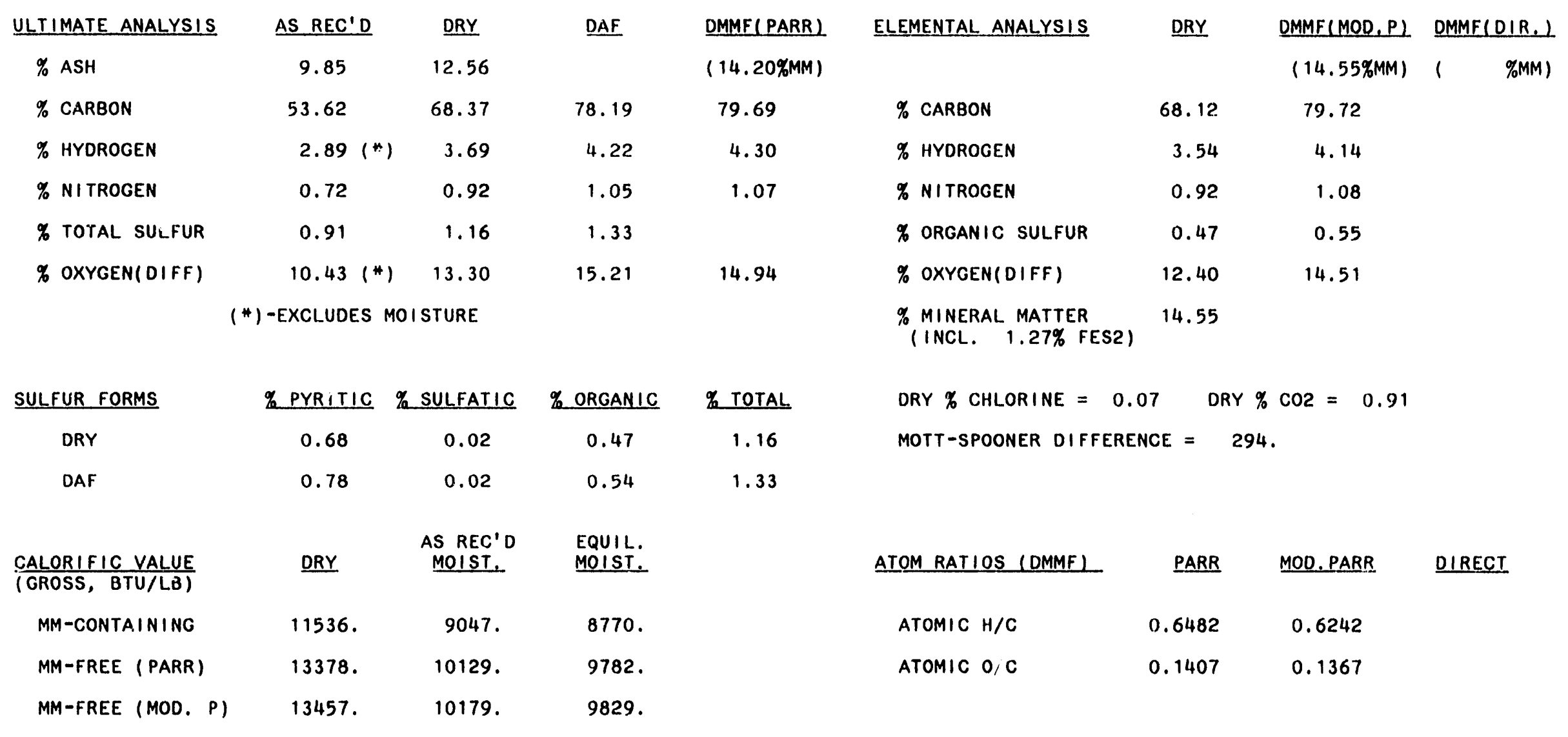

$\%$ EQUILIBRIUM MOISTURE $=23.98$

MM-FREE $=26.96$

NET CV, DMMF BTU/LB 13057. 
SAMPLE IDENTIFICATION: PSU NUMBER PSU NUMBER

SEAM NAME

ALTERNATE SEAM NAME APPARENT RANK

$\rightarrow$ DECS -10
$\Rightarrow$ ROSEBUD
$\Rightarrow$
$\rightarrow$ SUBSIT

SAMPLE LOCATION:

COUNTFY $\rightarrow$ USA

STATE
** penN state coal data base *\#

$\begin{array}{lll}* * & & \\ * * & \text { DECS }-10 & * \\ * * & * \\ * * & 11 / 16 / 93 & * * \\ * * & \text { PAGE 3 } & * *\end{array}$

APPARENT RANK (AS REC'D MOIST.)

ASTM RANK (EQUIL. MOIST.)

REFLECTANCE RANK CATEGORY
\# * \# * R A N K C A L C U L A T I O N S \#\#\#\#*

$\rightarrow$ SUBBIT B

$\rightarrow$ SUBBIT B

$->$
INTERNATIONAL RANK :

AS REC'D MOIST. $\rightarrow$

EQUIL. MOIST.

REPORTED RANK :

$\rightarrow$ SUBBIT B

**\#*\# PETROGRAPH I C DATA \#\#\#\#*

MACERAL COMPOSITION ( WHITE LIGHT AND FLUORESCENCE ANALYSIS)

\begin{tabular}{|c|c|c|c|c|c|c|c|c|c|c|c|c|c|c|}
\hline DMMF & $\begin{array}{l}\text { TEXT - } \\
\text { INITE }\end{array}$ & $\begin{array}{l}\text { ULM- } \\
\text { INITE }\end{array}$ & $\begin{array}{l}\text { HUMO- } \\
\text { DETRINITE }\end{array}$ & $\begin{array}{l}\text { GEL- } \\
\text { INITE }\end{array}$ & $\begin{array}{l}\text { CORPO- } \\
\text { HUMINITE }\end{array}$ & $\begin{array}{l}\text { SPOR- } \\
\text { INITE }\end{array}$ & $\begin{array}{l}\text { CUT- } \\
\text { INITE }\end{array}$ & $\begin{array}{l}\text { RES- } \\
\text { INITE }\end{array}$ & $\begin{array}{l}\text { ALG- } \\
\text { INITE }\end{array}$ & $\begin{array}{l}\text { FUS- } \\
\text { INITE }\end{array}$ & $\begin{array}{l}\text { SEMI- } \\
\text { FUSINITE }\end{array}$ & $\begin{array}{l}\text { MAC- } \\
\text { RINITE }\end{array}$ & $\begin{array}{l}\text { SCLERO- } \\
\text { TINITE }\end{array}$ & \\
\hline VOL. \% & 0.0 & 34.8 & 37.4 & 0.6 & 0.7 & 9.7 & 0.0 & 0.4 & 0.0 & 4.6 & 6.6 & 5.1 & 0.1 & \\
\hline $\begin{array}{l}\text { DRY WT. \% } \\
\text { DMMF VOL \% }\end{array}$ & $\begin{array}{l}0.0 \\
\text { HUMIN }\end{array}$ & $T E=$ & $\begin{array}{l}32.1 \\
73.5\end{array}$ & 0.5 & 0.6 & 8.3 & 0.0 & 0.3 & 0.0 & 3.9 & 5.7 & 4.4 & 0.1 & $14.2 \% \mathrm{MM}$. \\
\hline ULMINITE R & EFLECT & CE DA & & HIGH: & 0.49 & LOW: & 0.3 & & MEAN & MAX: & 0.42 & & & \\
\hline
\end{tabular}

***** CAKING ANDMECHAN I C A L

PROPERTIES ${ }^{\circ}$ O * *

GIESELER COAL PLASTOMETER:

GRAY-KING COKE TYPE

INIT. SOFTENING TEMP

TEMP. MAX. FLUIDITY

HARDGROVE GRINDABILITY

SOLIDIFICATION TEMP.

FREE SWELLING INDEX

FLUID TEMP. RANGE

VICKERS HARDNESS NUMBER

MAXIMUM FLUIDITY

WASHAB: LITY DATA AVAILABLE? 
SAMPLE IDENTIFICATION:

$\begin{array}{ll}\text { PSU NUMBER } & \rightarrow \text { DECS }-10 \\ \text { SEAM NAME } & \rightarrow \text { ROSEBUD } \\ \text { ALTERNATE SEAM NAME } & \rightarrow>\text { SUBBIT } B \\ \text { APPATENT RANK } & \rightarrow\end{array}$

MAJOR ELEMENT ANALYSIS

MAJOR ELEMENT ANALYSIS

$\begin{array}{lccccc} & \text { S102 } & \text { AL203 } & \text { TI02 } & \text { FE203 } & \text { MGO } \\ \text { OXIDE \% OF HTA } & 37.0 & 17.9 & 0.76 & 9.14 & 3.31 \\ \begin{array}{l}\text { ELEMENT \% OF } \\ \text { TOTAL DRY COAL }\end{array} & \text { SI } & \text { AL } & \text { TI } & \text { FE } & \text { MG } \\ & 2.3 & 1.3 & 0.06 & 0.85 & 0.26\end{array}$

TRACE ELEMENT ANALYSIS

$\begin{array}{lc}\text { PPM } & \text { PPM } \\ \text { HTA } & \text { TOTAL COAL }\end{array}$

$A G$

GE

B

BA

6628.

4.0

879.

0.5

BI

CE

co

CR

cU

50.

70.

GA

PPM PPM

TOTAL COAL

$<25$.

< 3. YB

25.

3. $\quad Z R$

SAMPLE LOCATION:

-...-. -.......

COUNTRY $\quad \rightarrow$ USA

STATE

$\rightarrow$ MONTANA

\% HTA $=13.26$

$\begin{array}{ccccc}\text { CAO } & \text { NA2O } & \text { K20 } & \text { P205 } & \text { S03 } \\ 13.2 & 1.17 & 0.48 & 0.31 & 16.2 \\ \text { CA } & \text { NA } & \text { K } & \text { P } & \text { S } \\ 1.3 & 0.12 & 0.05 & 0.02 & 1.2\end{array}$

\% HTA $=13.26$

PPM

PPM

TOTAL COAL

VOLATILES PPM

.......... TOTAL COAL

AS

4059.

538.

$B R$

CD

CL $\quad 700$.

15.

2.

F

HG

SB

SE

35.

5.

38.

290. 
SAMPLE IDENTIFICATION:

SAMPLE IDENTIFICATION:

PSU NUMBER

SEAM NAME

ALTERNATE SEAM NAME

APPARENT RANK

$\rightarrow$ DECS -10

$\rightarrow$ ROSEBUD

$\rightarrow>$

$\rightarrow$ SUBBIT B
SAMPLE LOCATION:

SAMPLE LOCATION.

COUNTRY

-> USA

STATE

$\rightarrow$ MONTANA
* penN state coal data base *\#

*\# DECS -10

*\# $11 / 16 / 93$

* PAGE 5

ASH FUSION TEMPERATURES (ALL TEMPERATURES IN DEGREES F)

- - - - - . -

REDUCING OXIDIZING

INITIAL DEFORMATION

2080 .

2200.

SOFTENINC

HEMISPHERICAL

2155.

2260 .

FLUID

2200 .

2300 .

2250. 
SAMPLE IDENTIFICATION:

$\begin{array}{ll}\text { PSU NUMBER } & \rightarrow \text { DECS }-11 \\ \text { SEAM NAME } & \rightarrow \text { BEULAH } \\ \text { ALTERNATE SEAM NAME } & \rightarrow \\ \text { APPARENT RANK } & \rightarrow \text { LIGNITE }\end{array}$

SAMPLE HISTORY:

SAMPLING DATE \& AGENCY

$\rightarrow 6 / 15 / 90 ;$ PSU

SAMPLE TYPE

ADDITIONAL INFORMATION

MINE INFORMATION:

RESERVES

ANNUAL PRODUCTION

-> 9999 THOUSAND TONS
SAMPLE LOCATION:

COUNTRY $\quad \rightarrow$ USA

STATE $\rightarrow$ NORTH DAKOTA

COUNTY $\rightarrow$ MERCER

TOWNSHIP $\quad \rightarrow$

CITY $\quad \rightarrow$ BEULAH

COAL PROVINCE $\rightarrow$ NORTHERN GREAT PLAINS REGION $\rightarrow$ FORT UNION

MAP REFERENCE: LATITUDE $\rightarrow 477 \mathrm{D} 23 \mathrm{M} 29 \mathrm{~S}$ LONGITUDE $\rightarrow 101 \mathrm{D} \quad 47 \mathrm{M} 59 \mathrm{~S}$

$\rightarrow$ Face loosened by blasting; no description. OUADRANGLE $\rightarrow$ BEULAH NE $\left(7.5^{\prime}\right)$

\begin{tabular}{|c|c|c|}
\hline & 5 , UAL U & \\
\hline *\# & DECS - 11 & $\# \#$ \\
\hline & $11 / 16 / 93$ & $\begin{array}{l}\text { H+ } \\
\text { H* }\end{array}$ \\
\hline
\end{tabular}

LIFE EXPECTANCY $\rightarrow 50$ YEARS

MINING METHOD $\rightarrow$ STRIP

SEAM INFORMATION:

AGE OF SEAM

GROUP

FORMATION

SEAM THICKNESS
-> PALEO.

$\rightarrow$

$\rightarrow$ FORT UNION

$\rightarrow \quad 17 \mathrm{FT} . \quad 0 \mathrm{IN}$. 
SAMPLE IDENTIFICATION: OAMPE IOENTIFICATION: PSU NUMBER

SEAM NAME

ALTERNATE SEAM NAME

APPARENT RANK

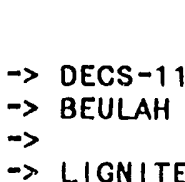

SAMPLE LOCATION:

COUNTRY $\rightarrow$ USA

STATE $\quad \rightarrow$ NORTH DAKOTA
* penn state coal data base \#*

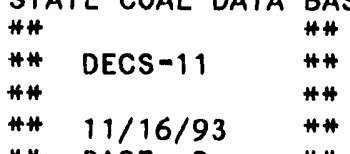

\#\# *\# CHEMICAL DATA\#\#\#\#

\section{PROXIMATE ANALYSIS}

AS REC'D DRY

\% MOISTURE

33.38

DAF

DMMF(PARR)

$\% \mathrm{ASH}$

6.37

9.56

\% VOLATILE

\% FIXED CARBON

37.36

56.08

22.89

34.36

62.01

37.99

ULTIMATE ANALYSIS

of ASH

AS REC'D $\quad$ DRY

$6.37 \quad 9.56$

\% CARBON

$\%$ HYDROGEN

\% NITROGEN

\% TOTAL SULFUR

\% OXYGEN(DIFF)

$44.07 \quad 66.15$

$2.68\left(^{*}\right) \quad 4.03$

0.60

0.90

0.49

0.74

$12.40\left(^{*}\right)$

18.62

(*)-EXCLUDES MOISTURE

SULFUR FORMS

DRY

DAF

CALORIFIC VALUE

(GROSS, BTU/LB)

MM-CONTAINING

MM-FREE (PARR)

MM-FREE (MOD. P)

\% PYRITIC \% SULFATIC

$\begin{array}{ll}0.31 & 0.03 \\ 0.34 & 0.03\end{array}$

\% ORGANIC

0.39

\% TOTAL

0.43

0.74

0.82
AS REC'D EQUIL.

DRY MOIST. MOIST.

$11062 . \quad 7370 . \quad 7166$.

12350. 7910. 7676.

12409. 7940.7703.
\% EQUILIBRIUM MOISTURE $=35.22$

MM-FREE $=37.92$

NET CV, DMMF BTU/LB 11989

DMMF (PARR)

ELEMENTAL ANALYSIS

DRY

DMMF(MOD,P) DMMF(DIR,)

(10.73\%MM)

y. CARBON

\% HYDROGEN

\% NITROGEN

\% ORGANIC SULFUR

\% OXYGEN(DIFF)

\% mineral matter

(INCL. $0.58 \%$ FES2)

\subsection{5}

(10.99\%MM)

\%MM)

DRY \% CHLORINE $=0.08$ DRY $\%$ CO2 $=0.38$

MOTT-SPOONER DIFFERENCE $=179$.

PARR

MOD. PARR

DIRECT

$\begin{array}{lll}\text { ATOMIC H/C } & 0.7317 & 0.7114 \\ \text { ATOMIC O/C } & 0.2064 & 0.2019\end{array}$


SAMPLE IDENTIFICATION:

$\begin{array}{ll}\text { PSU NUMBER } & \rightarrow \text { DECS }-11 \\ \text { SEAM NAME } & ->\text { BEULAH } \\ \text { ALTERNATE SEAM NAME } & \rightarrow> \\ \text { APPARENT RANK } & \rightarrow \text { LIGNITE }\end{array}$

SAMPLE LCCATION:

- DAME LOCATION:

COUNTRY $\rightarrow$ USA

STATE $\rightarrow$ NORTH DAKOTA
* penn state coal data base *\#

$\begin{array}{lll}* * & \\ * * & \text { DECS }-11 & * * \\ * * & * * \\ * * & 11 / 16 / 93 & * * \\ * * & \text { PAGE } 3\end{array}$

APPARENT RANK (AS REC'D MOIST.)

ASTM RANK (EQUIL. MOIST.)

REFLECTANCE RANK CATEGORY

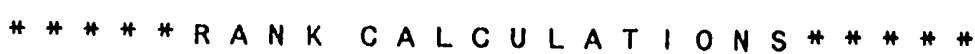

$\rightarrow$ LIGNITE

$\rightarrow$ LIGNITE

$\rightarrow$
INTERN GTIONAL RANK :

AS REC'D MOIST. $\rightarrow$

EQUIL. MOIST. $\rightarrow$

REPORTED RANK : $\rightarrow$ LIGNITE

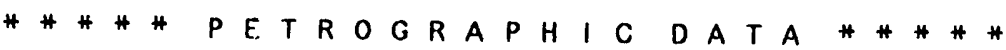

MACERAL COMPOSITION ( WHITE LIGHT AND FLUORESCENCE ANALYSIS )

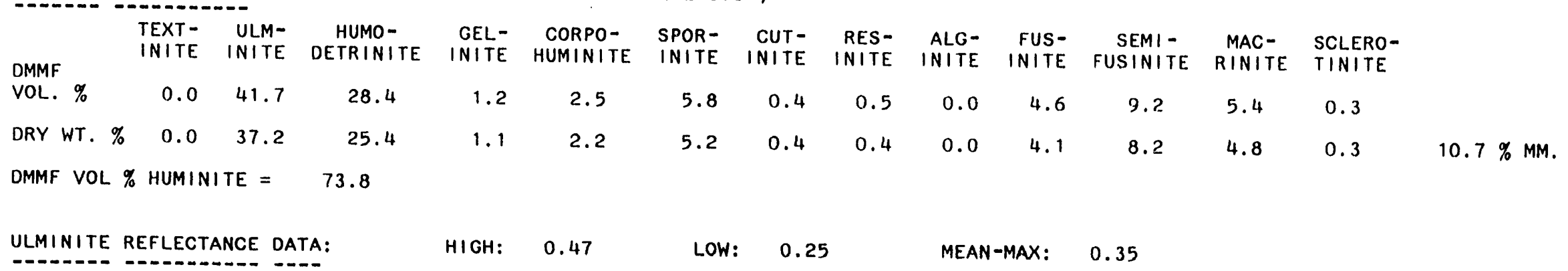

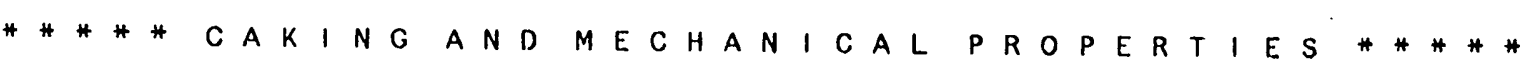

GIESELER COAL PLASTOMETER:

GRAY-KING COKE TYPE

INIT. SOFTENING TEMP

HARDGROVE GRINDABILITY

TEMP. MAX. FLUIDITY

FREE SWELLING INDEX

SOLIDIFICATION TEMP.

VICKERS HARDNESS NUMBER

FLUID TEMP. RANGE

MAXIMUM FLUIDITY

WASHABILITY DATA AVAILABLË? 
SAMPLE IDENTIFICATION:

PSU NUMBER $\quad \rightarrow$ DECS -11

SEAM NAME

ALTERNATE SEAM NAME

$\rightarrow$ BEULAH

APPARENT RANK

- LIGNITE

MAJOR ELEMENT ANALYSIS

$\begin{array}{lccccc} & \text { S102 } & \text { AL203 } & \text { T102 } & \text { FE203 } & \text { MGO } \\ \text { OXIDE \% OF HTA } & 11.5 & 11.1 & 0.57 & 9.29 & 9.94 \\ \text { ELEMENT \% OF } & \text { SI } & \text { AL } & \text { TI } & \text { FE } & \text { MG } \\ \text { TOTAL DRY COAL } & 0.5 & 0.6 & 0.03 & 0.61 & 0.56\end{array}$

TRACE ELEMENT ANALYSIS

TRACE ELEMENT ANALYSIS

PPM
HTA

PPM PPM

HTA TOTAL COAL

GE

$A G$

B

BA

9225.

7.0

868.

0.7

LA

BE

B I

CE

co

CR

$\mathrm{CU}$

55.

95.

GA

SN

620

58. U

$<25$.

$<2$. YB

2. $\quad \mathrm{ZR}$
SAMPLE LOCATION:

COUNTRY

-> USA

STATE

$\rightarrow$ NORTH DAKOTA
** pENN STATE coal dATA base \#\# ** ***

\#* DECS -1

\#* 11/16/93

\#\# PAGE 4

$\%$ HTA $=9.41$

$\begin{array}{ccccc}\text { CAO } & \text { NA2O } & \text { K2O } & \text { P2C5 } & \text { S03 } \\ 27.4 & 9.39 & 0.59 & 0.49 & 18.2 \\ \text { CA } & \text { NA } & K & P & S\end{array}$

1.8

0.66

0.05

0.02

0.7

$\%$ HTA $=9.41$

PPM PPM VOLATILES PPM

HTA TOTAL COAL

8202.

772.

AS

BR

CD

CL $\quad 800$

145.

14.

F

HO

$\mathrm{SB}$

120.

11.

SE

160.

15. 
SAMPLE IDENTIFICATION: -

PSU NUMBER

ALTERNATE SEAM NAME

$\rightarrow$ DECS -11

$\rightarrow$ BEULAH

$\rightarrow \rightarrow$

$\rightarrow$ LIGNITE
SAMPLE LOCATION:

SAMPLE LOCATION:

COUNTRY $\quad \rightarrow$ USA

STATE

$\rightarrow$ NORTH DAKOTA
*\# PENn STATE coAl dATA base \#\#

** DECS -11

\#* $11 / 16 / 93$

** PAGE 5

$\# *$

ASH FUSION TEMPERATURES (ALL TEMPERATURES IN DEGREES F) ASH FUSION TEMPERATURES

REDUCING OXIDIZING

INITIAL DEFORMATION

2000 .

2200 .

SOFTENING

2070.

2270 .

HEMISPHERICAL

2100 . 2330

FLUID

2140 . 2380


SAMPLE IDENTIFICATION:

SAMPLE IDENTIFIOATION:

PSU NUMBER

SEAM NAME

ALTERNATE SEAM NAME

APPARENT RANK

$\rightarrow$ DECS-12

$\rightarrow$ PITTSBURGH

$\rightarrow \# 8$

$\rightarrow$ HVA

SAMPLE HISTORY:

SAMPLING DATE. \& AGENCY

- 7/25/90; PSU

SAMPLE TYPE

$\rightarrow$ CHANNEL-WHOLE SEAM

ADDITIONAL INFORMATION

MINE INFORMATION:

\section{RESERVES}

ANNUAL PROOUCTION
$->$

$\rightarrow$ PENNSYL.

-> MONONGAHELA

$\rightarrow$

$\Rightarrow \quad 6 \mathrm{FT} .6 \mathrm{iN}$.

* SeAM profille \#\#
SAMPLE LOCATION:

SAMPLE LOCATION:

COUNTRY

-> USA

STATE

$\rightarrow$ PENNSYLVANIA

COUNTY

$\rightarrow$ GKEENE

TOWNSHIP

$\rightarrow$
$\rightarrow$ GRAYSVILLE

CITY

COAL PROVINCE $\rightarrow$ EASTERN REGION $\rightarrow$ APPALACHIAN

MAP REFERENCE: LATITUDE $\rightarrow \quad 39 \mathrm{D} \quad 55 \mathrm{M} \quad 58 \mathrm{~S}$

LONGITUDE $\rightarrow 80$ D $33 \mathrm{M}$

QUADRANGLE $\rightarrow>$ WINO RIDGE $\left(7.5^{\prime}\right)$

LIFE EXPECTANCY $\rightarrow$

MINING METHOD $\rightarrow$ UNDERGROUND
* penn state coal data base **

$\begin{array}{lll}* * & & \\ * * & \text { DECS }-12 & * * \\ * * & & * * \\ * * & 11 / 16 / 93 & * * \\ * * & \text { PAGE } 1 & * *\end{array}$

\section{SEAM INFORMATION:}

AGE OF SEAM

GROUP

FORMATION

SEAM THICKNESS $\rightarrow$ hard gray mudstone

$\rightarrow$ rider: bright clarain \& shale

$\rightarrow$ bright clarain w/ occ. vitrain

$\rightarrow$ shale parting \& vitrain

$\rightarrow$ bright clarain $w /$ occ. vitrain

-> hard gray mudstone, clay 
SAMPLE IDENTIFICATION: SAU NUMBER PSU NUMBER SEAM NAME ALTERNATE SEAM NAME APPARENT RANK $\rightarrow$ PITTSBURGH

$\rightarrow \# 8$ $\rightarrow$ DECS -12
SAMPLE LOCATION:

SAMPLE LOCATION:

COUNTRY $\quad \rightarrow$ USA

STATE $\rightarrow$ PENNSYLVANIA
* penn state coal data base **

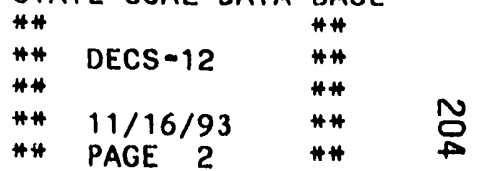

***** CHEM I C A L D A T A \#\#*\#*
PROXIMATE ANALYSIS

\% MOISTURE

AS REC'D $\quad \underline{\text { DRY }}$

$\% \mathrm{ASH}$

\% volatille

\% FIXED CARBON

10.25

36.02

53.73

\subsection{0}

10.00

35.16

52.44
40.13

59.87

$\underline{\text { DAF }}$

$10.00 \quad 10.25$

$\%$ ASH

\% CARBON

$\%$ NITROGEN

\% total sulfur

\% OXYGEN(DIFF)

$\begin{array}{ll}72.99 & 74.78\end{array}$

$4.99\left(^{*}\right) \quad 5.11$

$1.09 \quad 1.12$

$7.33(*) \quad 7.51$
$\%$ HYDROGEN
$1.20 \quad 1.23$

(*) -EXCludes moISTURE

\section{SULFUR FORMS}

DRY

DAF

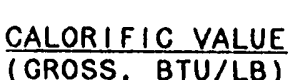

MM-CONTAINING

MM-FREE (PARR)

MM-FREE (MOD. P)

NET CV, DMMF BTU/LB

14795.

$\begin{array}{ccccc}\text { \% PYRITIC } & \text { \% SULFATIC } & \text { \% ORGANIC } & \text { \% TOTAL } \\ 0.38 & 0.01 & 0.73 & 1.12 \\ 0.42 & 0.01 & 0.81 & 1.25\end{array}$

DRY AS REC'D EQUIL.

$13532 . \quad 13207 . \quad 13142$.

15259.14846 .14763$.

15332. $14916 . \quad 14833$.
\% equilibrium moIsture $=2.88$

MM-FREE $=3.26$
83.32

5.69

1.37

1.25

8.37

8.15

(11.69\%MM)

84.68

5.79

1.39

1.25

\section{ELEMENTAL ANALYSIS}

$\%$ CARBON

\% HYDROGEN

\% Nitrogen

\% ORGANIC SULFUR

\% OXYGEN(DIFF)

\% MINERAL matTER

(INCL. 0.71\% FES2)
74.69

4.98

1.23

0.73

6.49

11.88
MOTT-SPOONER DIFFERENCE $=61$.

\section{ATOM RATIOS (DMMF) PARR MOD, PARR}

$\begin{array}{lll}\text { ATOMIC H/C } & 0.8208 & 0.8016 \\ \text { ATOMIC O/C } & 0.0722 & 0.0653\end{array}$

$(11.88 \% M M) \quad 1 \quad \% M M)$

84.75

5.66

1.40

0.83

7.37

0.0653 
SAMPLE IDENTIFICATION:

$\begin{array}{ll}\text { PSU NUMBER } & ->\text { DECS }-12 \\ \text { SEAM NAME } & ->\text { PITTSBURGH } \\ \text { ALTERNATE SEAM NAME } & ->\# 8 \\ \text { APPARENT RANK } & ->\text { HVA }\end{array}$

SAMPLE LOCATION:

COUNTRY $\quad \rightarrow$ USA

STATE $\rightarrow$ PENNSYLVANIA
** penn state coal data base \#\#

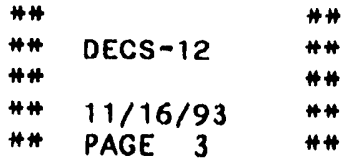

*

***** r a nk calgulat l ons n ****

APPARENT RANK (AS REC'D MOIST.)

ASTM RANK (EQUIL. MOIST.)

REFLECTANCE RANK CATEGORY

$$
\begin{array}{ll}
\rightarrow & H V A \\
\rightarrow & H V A \\
\rightarrow & H V A
\end{array}
$$

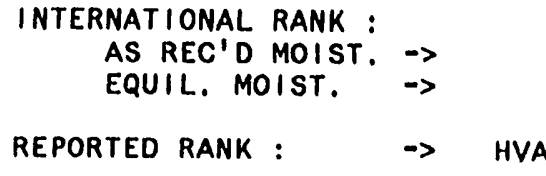

MACERAL COMPOSITION ( WHITE LIGHT ANALYSIS ) PSEUDO-
VITRINITE VITRINITE FUSINITE FUSINITE MACRINITE MICRINITE TINITE INITE

RESINITE ALGINITE CUTINITE

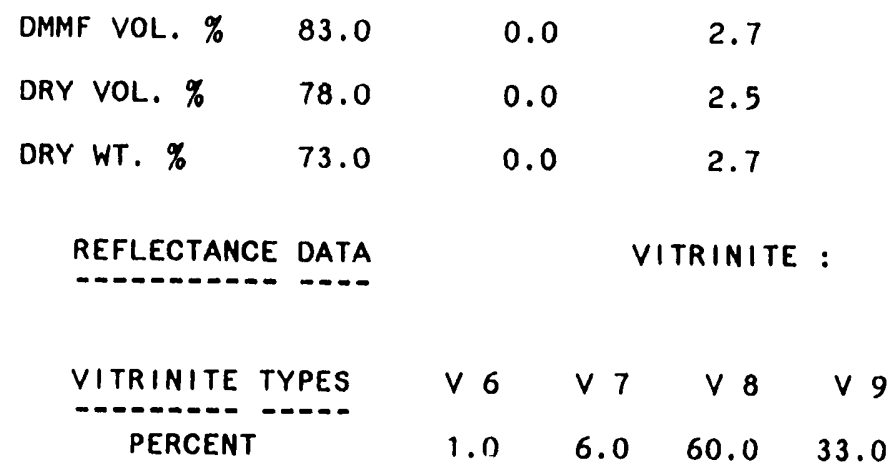

$4.0 \quad 1.4$

$3.8 \quad 1.3$

$3.8 \quad 1.3$

HIGH:

$\begin{array}{lll}0.8 & 0.0 & 7.1 \\ 0.8 & 0.0 & 6.7\end{array}$

0.8
1.0

0.9

0.8

0.99

LOW:

0.68
0.0

0.0

0.0
**** CAKING

GIESELER COAL PLASTOMETER:

INIT. SOFTENING TEMP

TEMP. MAX. FLUIDITY

SOLIDIFICATION TEMP.

FLUID TEMP. RANGE

MAXIMUM FLUIDITY

WASHABILITY DATA AVAILABLE? NO
387

440

477

90

20002 
SAMPLE IDENTIFICATION:

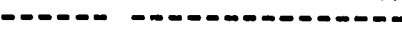
PSU NUMBER SEAM NAME

ALTERNATE SEAM NAME

APPARENT RANK $\rightarrow$ DECS -12

$\rightarrow$ PITTSBURGH

$\rightarrow \# 8$
SAMPLE LOCATION:

$\begin{array}{ll}\text { COUNTRY } & \rightarrow \text { USA } \\ \text { STATE } & \rightarrow \text { PENNSYLVANIA }\end{array}$

* penn state coal data base \#\#

$\begin{array}{llll}* * & & \\ * * & \text { DECS }-12 & * * & \\ * * & & * * & \\ * * & 11 / 16 / 93 & * * & \tilde{O} \\ * * & \text { PAGE } & * * & \sigma\end{array}$

MAJOR ELEMENT ANALYSIS

-.-.-

OXIDE \% OF HTA

$5102 \quad$ AL203 T102

FE203

$\begin{array}{lll}55.8 & 25.8 & 1.21\end{array}$

$$
6.37
$$

MGO

CAO

NA2O

K20

0.49

2.19

P205

$\mathrm{SO} 3$

SI AL

FE

MG

.20

NA

$3.1 \quad 1.6$

0.09

0.53

0.07

0.27

0.04

0.22

0.56

2.10

TOTAL DRY COAL

TRACE ELEMENT ANALYSIS

TRACE ELEMENT ANALYSIS

$\begin{array}{lc}\text { PPM } & \text { PPM } \\ \text { HTA } & \text { TOTAL COAL }\end{array}$

PPM

HTA

PPM

TOTAL COAL

\% HTA $=11.89$

PPM PTA TOTAL

TOTAL COAL

AG

GE

B

BA

717.

85. LI

BE

$1.0 \quad M N$

155.

BI

$C E$

co

CR

180.

21. PB

cu

9. $\mathrm{RB}$

GA

$\begin{array}{ccc}8.0 & 1.0 & \text { MN } \\ & & \text { MO } \\ & & \text { NB } \\ & & \text { NI } \\ 180 . & 21 . & \text { PB } \\ 75 . & 9 . & \text { RB } \\ & & \text { SC }\end{array}$

SN
SR
TH.
UH
V
Y

$930 . \quad 111$.

VOLATILES PPM

. TOTAL COAL

AS

BR

CD

CL 2300 .

60.

7.

95.

11. YB

130.

15.

12. $Z R$

200.

24. 
SAMPLE IDENTIFICATION: SAMPLE IOENTIFICATION:

PSU NUMBER

SEAM NAME

ALTERNATE SEAM NAME

$\rightarrow$ DECS -12

$\rightarrow$ PITTSBURCH

$\rightarrow \# 8$

$\rightarrow H V A$

ASH FU'SION TEMPERATURES (ALL TEMPERATURES IN DEGREES F)

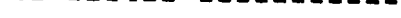
REOUCING
OXIDIZING

INITIAL DEFORMATION

2225.

2420

SOFTENING

HEMI SPHERICAL

FLUID

2330 . 2510

$2390 . \quad 2565$

2445.2600
SAMPLE LOCATION:

SAMPLE LOCATION:

COUNTRY $\rightarrow$ USA

STATE

$\rightarrow$ PENNSYLVANIA
* penN state coAl data base **

\#* DECS-12

** 11/16/93

* PAGE 5 
SAMPLE IDENTIFICATION:

$\begin{array}{ll}\text { PSU NUMBER } & ->\text { DECS }-13 \\ \text { SEAM NAME } & ->\text { SEWELL } \\ \text { ALTERNATE SEAM NAME } & -> \\ \text { APPARENT RANK } & ->\text { MED VOL }\end{array}$

\section{SAMPLE HISTORY:}

SAMPLING DATE \& AGENCY

$->12 / 13 / 90 ; P S U$

SAMPLE TYPE

$\rightarrow$ CHANNEL-WHOLE SEAM

ADDITIONAL INFORMATION

MINE INFORMATION:

MINE INFORMATION:

RESERVES

ANNUAL PRODUCTION

\section{SAMPLE LOCATION:}

COUNTRY

-> USA

STATE

$\rightarrow$ WEST VIRGINIA

COUNTY

-> GREENBRIER

TOWNSHIP

CITY

$\rightarrow$ NEW HAVEN

$\rightarrow>$ BELLBURN

COAL PROVINCE $\rightarrow$ EASTERN REGION $\rightarrow$ APPALACHIAN

MAP REFERENCE: LATITUDE $\rightarrow \quad 38$ D 1 M $29 \mathrm{~S}$

LONGITUDE $\rightarrow 80 \mathrm{D} 46 \mathrm{mi} 30 \mathrm{~S}$

QUADRANGLE $\rightarrow$ CORLISS $\left(7.5^{\prime}\right)$

SEAM INFORMATION:

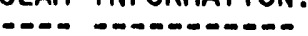

AGE OF SEAM

$\rightarrow$ PENNSYL.

GROUP

-> POTTSVILLE

FORMATION

$\rightarrow$ NEW RIVER

SEAM THICKNESS

$\rightarrow \quad 1 \mathrm{FT} . \quad 8 \mathrm{IN}$.

* SEAM PROFIlle **

LIFE EXPECTANCY $\rightarrow$

MINING METHOD $\rightarrow$
ROOF
FLOOR
$\rightarrow$ hard gray mudstone
$\rightarrow$ bright clarain, shale lenses
$\rightarrow$ gray shale ecoal (gradational) 


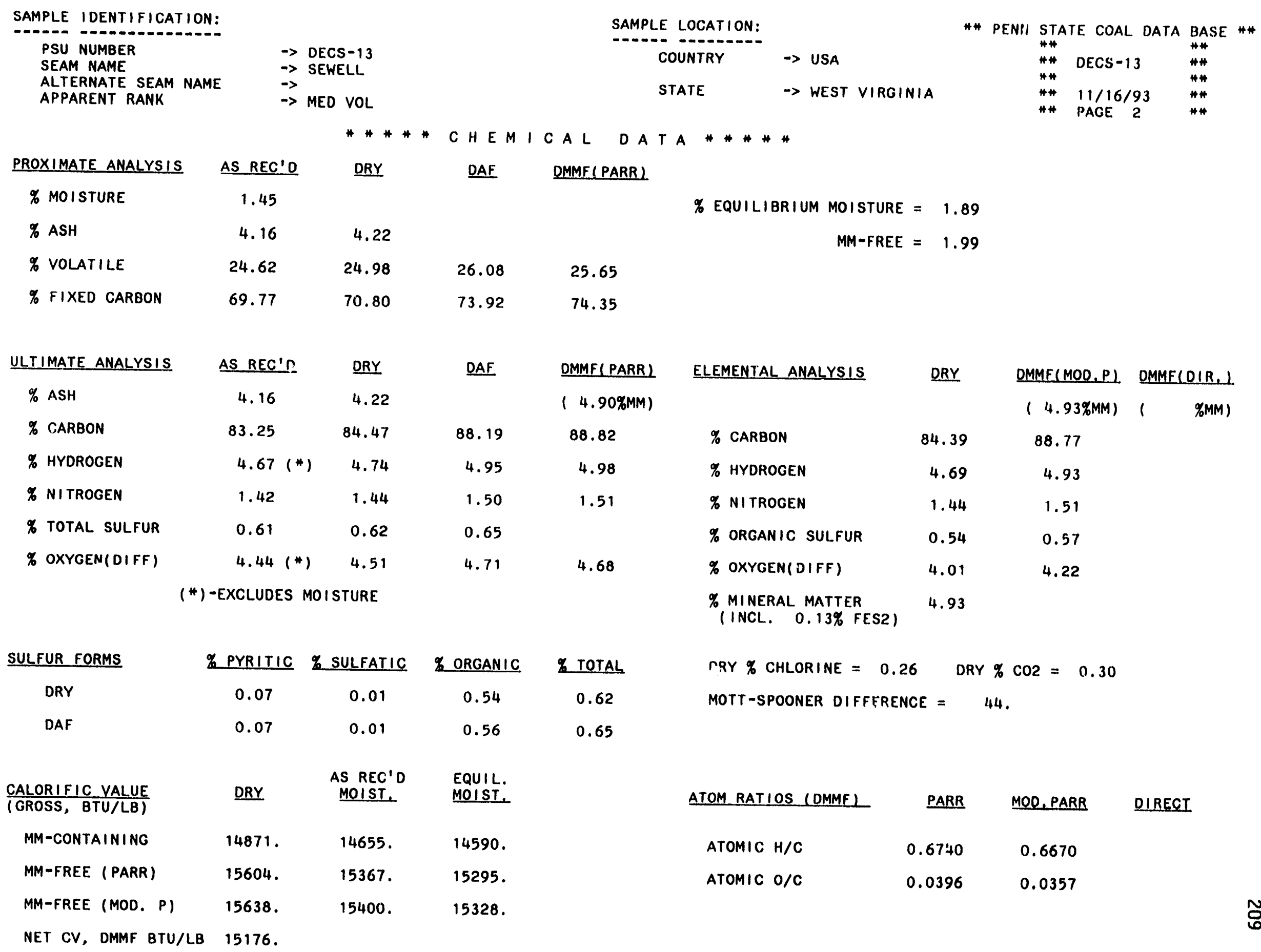


SAMPLE IDENTIFICATION: SAMPLE IDENTIFICATION: PSU NUMBER

SEAM NAME

ALTERNATE SEAM NAME

APPARENT RANK

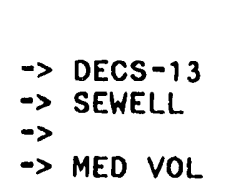

SAMPLE LOCATION: COUNTRY

$\rightarrow$ USA

STATE
** PF.Nn state coal data base \#\#

$\begin{array}{llll}* * & \\ * * & \text { DECS }-13 & * * \\ * * & & * * \\ * * & 11 / 16 / C) & * * & \\ * * & \text { PAGE 3 } & * * & 0\end{array}$

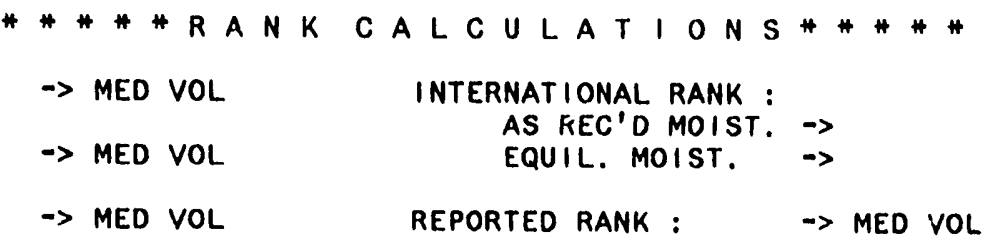

$\rightarrow$ MED VOL

$\rightarrow$ MED VOL

INTERNATIONAL RANK :

AS REC'D MOIST. $\rightarrow$
EQUIL. MOIST.

$\rightarrow$ MED VOL

REPORTED RANK : $\quad \rightarrow$ MED VOL

APPARENT RANK (AS REC'D MOIST.)

ASTM RANK (EQUIL. MOIST.)

***** PETROGRAPH I C D A T A

MACERA:- COMPOSITION ( WHITE LIGHT ANALYSIS)

MACERA. COMPOSITION

$\begin{array}{lcc}\text { PSEUDO- } & \text { SEMI- } & \text { SCLERO- SPOR- } \\ \text { VITRINITE } & \text { FUSINITE FUSINITE MACRINITE MICRINITE } & \text { TINITE }\end{array}$

OMMF VOL. \% 77.

DRY VOL. \% 75.3

DRY WT. \%

72.3

0.0

5.2

7.4

4.5

4.4

4.5

5.6

7.5

HIGH: $\quad 1.48$

0.9

0.9

0.03 .1

RESINITE

1.4

ALGINITE CUTINITE

REFLECTANCE DATA

VITRINITE :

0.9

0.0

3.0

1.4

1.3

0.0

0.3

0.0

2.8

LOW: $\quad 1.22$

0.0

$0.32 .4 \% \mathrm{MM}$

REFLECTANCE DATA

\begin{tabular}{lrrr} 
VITRINITE TYPES & $V 12$ & $V 13$ & V14 \\
\hline PERCENT & 14.0 & 75.0 & 11.0
\end{tabular}

**\#\# C A K ING

GIESELER COAL PLASTOMETER:

INIT. SOFTENING TEMP 404

TEMP. MAX. FLUIDITY 456

SOLIDIFICATION TEMP. 493

FLUID TEMP. RANGE 89

MAXIMUM FLUIDITY 909

WASHABILITY DATA AVAILABLE? NO
GRAY-KING COKE TYPE

HARDGROVE GRINDABILITY 82.1

FREE SWELLING INDEX

8.0

VICKERS HARDNESS NUMBER
1

1.35 
SAMPLE IDENTIFICATION: SAMPLE IOENTI HICATION:

PSU NUMBER

SEAM NAME

ALTERNATE SEAM NAME

$\Rightarrow$ DECS -13

$\rightarrow$ SEWELL

$\rightarrow$ MED VOL
SAMPLE LOCATION:

COUNTRY $\rightarrow$ USA

STATE

$\rightarrow$ WEST VIRGINIA
* penn state coal data base *\#

$\begin{array}{lll}* * & \\ * * & \text { DECS }-13 & * \\ * * & * \\ * * & 11 / 16 / 93 & * * \\ * * & \text { PAGE } 4 & * *\end{array}$

$* *$

MAJOR ELEMENT ANALYSIS

$\begin{array}{lcccccrrrrr} & \text { S1O2 } & \text { AL2O3 } & \text { TIO2 } & \text { FE203 } & \text { MGO } & \text { CAO } & \text { NA20 } & \text { K20 } & \text { P205 } & \text { S03 } \\ \text { OXIOE \% OF HTA } & 52.8 & 33.3 & 1.02 & 4.59 & 0.97 & 1.78 & 0.70 & 2.29 & 1.16 & 0.20 \\ \text { ELEMENT \% OF } & \text { SI } & \text { AL } & \text { TI } & \text { FE } & \text { MG } & \text { CA } & \text { NA } & \text { K } & \text { P } & \text { S } \\ \text { TOTAL DRY COAL } & 1.0 & 0.7 & 0.03 & 0.14 & 0.02 & 0.05 & 0.02 & 0.08 & 0.02 & 0.62\end{array}$

TRACE ELEMENT ANALYSIS

TRACE ELEMENT AMALYSIS

PPM PPM

\% HTA $=4.23$

AG

B

BA 2597.

BE 24 .

BI

CE

co

CR

CU

260.

260.

GA
$1 . \quad M N$

PPM
HTA

SN

AS

2452.

104.

BR

C.O

CL 2600

270.

F

HG

270.

11. YB

95.

4. $2 R$

150.

6.

SB

SE 
SAMPLE IDENTIFICATION: PSU NUMBER

PSU NUMBER

SE.AM NAME

$\rightarrow$ DECS -13

ALTERNATE SEAM NAME

$\rightarrow$ SEWELL

$\rightarrow$ MED VOL
SAMPLE LOCATION:

SAMPLE LOCATION:

COUNTRY

$\rightarrow$ USA

STATE

$\rightarrow$ WEST VIRIGINIA
* penn state coal data base *\#

$\# \#$

Cs -13

H\# $11 / 16 / 93$

* PAGE 5
\#*

**

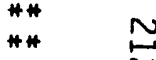

ASH FUSION TEMPERATURES (ALL TEMPERATURES IN DEGREES F)

REDUCING OXIDIZING

INITIAL DEFORMATION

SOFTENING

$2750 .+$

$2800 .+$

HEMISPHERICAL

$2800 .+2800 .+$

FLUID

$2800 .+2800 .+$

$2800 .+2800$. 
SAMPLE IDENTIFICATION:

PSU NUMBER

SEAM NAME

ALTERNATE SEAM NAME

APPARENT RANK

$\rightarrow$ DECS -14

$\rightarrow$ UPPER KITTANNING

$\rightarrow \quad$ HVA

SAMPLE HISTORY:

SAMPLING DATE \& AGENCY

$\rightarrow 12 / 14 / 90 ; P S U$

SAMPLE TYPE

$\rightarrow$ CHANNEL-WORKING SECTION

ADDITIONAL INFORMATION

MINE INFORMATION:

RESERVES

ANNUAL PRODUCTION

$\rightarrow$

$\rightarrow$ PENNSYL.

$\rightarrow$ ALLEGHENY

$\rightarrow$

SEAM THICKNESS
MINE INFORMATION:

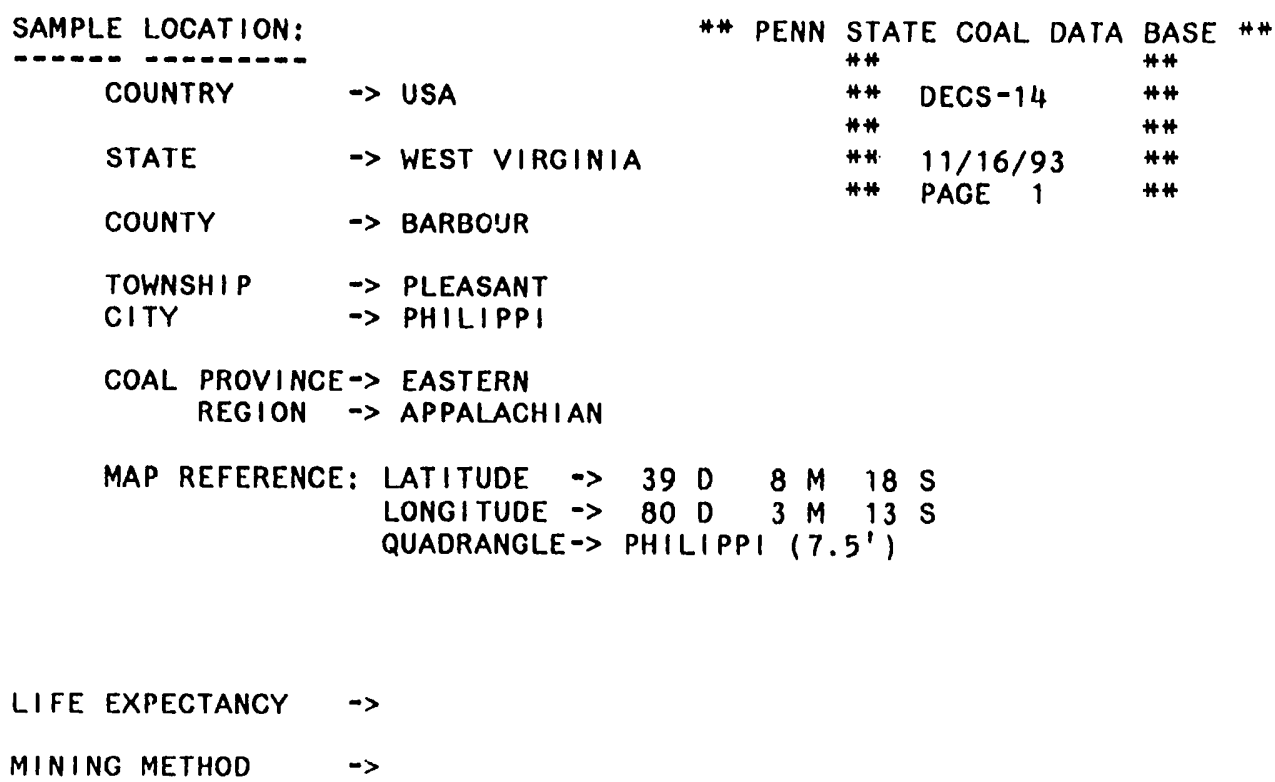

* penn state coal data base \#\# \#* \#\# DECS -14 \#\#

\#\#

** $11 / 16 / 93 \quad$ \#\#

* PAGE 1 \#\#

LIFE EXPECTANCY $\quad \rightarrow$

MINING METHOD $\quad \rightarrow$ 
SAMPLE IDENTIFICATION: PSU NUMBER

SEAM NAME

ALTERNATE SEAM NAME APPARENT RANK

$\rightarrow>$
$\Rightarrow$ DECS -14
$\rightarrow$ UPPER KITTANNING
$\rightarrow$ HVA

SAMPLE LOCATION:

SAMPLE LOCATION:

$\begin{array}{ll}\text { COUNTRY } & \rightarrow \text { USA } \\ \text { STATE } & \rightarrow \text { WEST VIRGINIA }\end{array}$

** PENN STATE COAL DATA bASE \#* \#\# DECS -14 \#\#

$\#+$

* $11 / 16 / 93$

\#\#

PAGE

\section{PROXIMATE ANALYSIS}

AS REC'D

1.46

\% moisture

$\%$ ASH

$\%$ VOLATILE

\% FIXED CARBON

10.37

10.52

32.11

56.06

32.59

56.89

36.42

DAF

DMMF (PARR)

ULTIMATE ANALYSIS

$\%$ ASH

\% CARBON

$\%$ HYDROGEN

\% NITROgen

\% total sulfur

\% OXYGEN(DIFF)

(*) -EXCLUDES MOISTURE

\section{SULFUR FORMS}

DRY

DAF

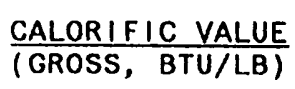

MM-CONTAINING

MM-FREE (PARR)

MM-FREE (MOD. P)

\% PYRITIC \& SULFATIC

0.94

0.01

1.05

0.01

DRY AS REC'D

MOIST,

13678.

\begin{abstract}
13478.
\end{abstract}
13424.

15503.

15245.

15175.

15577.

15317.

15246.
\% EQUILIBRIUM MOISTURE $=1.86$

MM-FREE $=2.12$

35.40

64.60

DAF DMMF(PARR

ELEMENTAL ANALYSIS

$\underline{\text { DRY }}$

DMMF (MOD.P) DMMFELDIR.L

(12.35\%MM)

$85.20 \quad 86.98$

5.46

5.58

1.48

\% CARBON

$\%$ HYDROGEN

76.19

(12.51\%MM)

\%MM)

1.45

$\%$ NITROGEN

4.77

5.45

\% ORGANIC SULFUR

1.30

1.49

.07

5.95

$\%$ OXYGEN(DIFF)

0.85

0.97

\% MINERAL MATter

(INCL. $1.76 \%$ FES2)

4.38

5.00

12.51

ORY \% CHLORINE $=0.37$ DRY \% CO2 $=0.20$

MOTT-SPOONER DIFFERENCE $=-62$.

1.80

2.01

NET CV, DMMF BTU/LB 15058.

ATOM RATIOS DMME

ATOMIC H/C

PARR MOD. PARR

DIRECT

ATOMIC O/C

0.7704

0.7523

0.0514

0.0431 
SAMPLE IDENTIFICATION: PAMU NUMBER OOL PSU NUMBER

ALTERNATE SEAM NAME APPARENT RANK $\rightarrow$ DECS -14 $\rightarrow$ UPPER KITTANNING

$\rightarrow$ HVA

\begin{tabular}{ll} 
SAMPLE LOCATION: & \\
\hline COUNTRY & $\rightarrow$ USA \\
STATE & $\rightarrow$ WEST VIRGINIA
\end{tabular}

*\# penn state coal data base \#\#

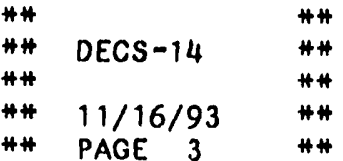

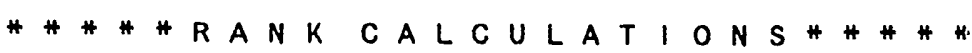

APPARENT RANK (AS REC'D MOIST.) $\rightarrow \quad$ HVA

$\rightarrow \quad$ HVA

$\rightarrow \quad$ HVA
INTERNATI ONAL RANK :

EQUIL. MOIST.

REPORTED RANK :
AS REC'D MOIST. $\rightarrow$

REFLECTANCE RANK CATEGORY

***\#* PETROGRAPHIC D A T A \#\#\#\#\#

MACERAL COMPOSITION ( WHITE LIGHT ANALYSIS)

MACERAL COMPOSITION PSEUDO-

PSEUDO- SEMI- SCLERO- SPORVITRINITE VITRINITE FUSINITE FUSINITE MACRINITE MICRINITE TINITE INITE

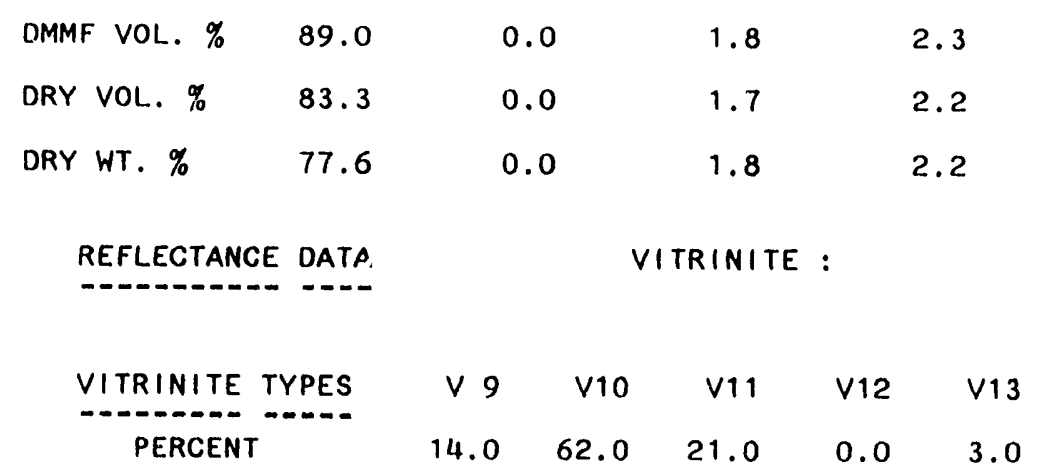

**\#** CAKING ANDMECHAN I CAL.

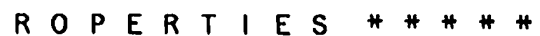

GIESELER COAL PLASTOMETER:

GRAY-KING COKE TYPE

INIT. SOFTENING TEMP $\quad 376$

HARDGROVE GRINDABILITY

68.9

TEMP. MAX. FLUIDITY 446

FREE SWELLING INDEX

8.0

SOLIDIFICATION TEMP. $\quad 485$

VICKERS HARDNESS NUMBER

FLUID TEMP. RANGE

109

MAXIMUM FIUIDITY 29930 
SAMPLE IDENTIFICATION:

PSU NUMBER
SEAM NAME

ALTERNATE SEAM NAME

APPARENT RANK

$\rightarrow$ DECS -14

$\rightarrow$ UPPER KITTANNING

$\rightarrow$

$\rightarrow \quad H V A$

MAJOR ELEMENT ANALYSIS

OXIDE \% OF HTA

S102 AL2O3 T102

46.1

ELEMENT \% OF

TOTAL DRY COAL

$$
\text { SI }
$$

2.3

29.5

AL

1.6

TRACE ELEMENT ANALYSIS
MAJOR ELEMENT ANALYSIS
SAMPLE LOCATION:

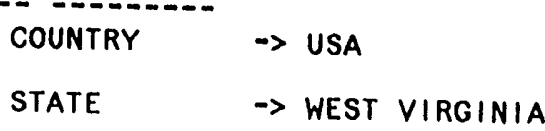

* penn state coal data base ** $\begin{array}{ll}* \# & \\ \# * & \text { DECS }-14 \\ * * & \end{array}$

\begin{tabular}{|c|c|c|c|c|c|c|c|c|c|c|}
\hline & $\begin{array}{l}\text { PPM } \\
\text { HTA }\end{array}$ & $\begin{array}{c}\text { PPM } \\
\text { TOTAL COAL }\end{array}$ & & $\begin{array}{l}\text { PPM } \\
\text { HTA }\end{array}$ & $\begin{array}{c}\text { PPM } \\
\text { TOTAL COAL }\end{array}$ & & $\begin{array}{l}\text { PPM } \\
\text { HTA }\end{array}$ & $\begin{array}{c}\text { PPM } \\
\text { TOTAL COAL }\end{array}$ & VOLATILES & $\begin{array}{c}\text { PPM } \\
\text { TOTAL COAL }\end{array}$ \\
\hline AG & & & $G E$ & & & SN & & & AS & \\
\hline B & & & LA & & & SR & 592. & 62. & $B R$ & \\
\hline BA & 448. & 47. & LI & & & TH & & & $C D$ & \\
\hline BE & 10 & 1. & MN & 77. & 8. & $U$ & & & $\mathrm{CL}$ & 3700. \\
\hline$C E$ & & & $\begin{array}{l}\text { MO } \\
\text { NB }\end{array}$ & & & $\begin{array}{l}V \\
Y\end{array}$ & 70 & 7. & $F$ & \\
\hline $\mathrm{CO}$ & & & NI & 55. & 6. & YB & & & SB & \\
\hline CR & 170 & 18. & PB & & & $Z N$ & 190. & 20 & $S E$ & \\
\hline $\begin{array}{l}\mathrm{CU} \\
\mathrm{GA}\end{array}$ & 95. & 10. & $\begin{array}{l}\text { RB } \\
\text { SC }\end{array}$ & 100 & 11. & ZR & 220 & 23 & & \\
\hline
\end{tabular}


SAMPLE IDENTIFICATION: PSU NUMBER

SEAM NAME

ALTERNATE SEAM NAME

APPARENT RANK

$\rightarrow$ DECS -14

$\rightarrow$ UPPER KITTANNING

$\rightarrow$

$\rightarrow \quad H V A$
SAMPLE LOCATION: SAMPLE LOCATION: COUNTRY $\rightarrow$ USA

STATE

$\rightarrow$ WEST VIRGINIA
* penn state coAl dATA base \#\# \#\# \#\# \#\# DECS -14 \#\# \#* \#* $11 / 16 / 93 \quad$ \#* * PAGE 5

ASH FUSION TEMPERATURES (ALL TEMPERATURES IN DEGREES F)

\section{ASH FUSION TEMPERATURES}

INITIAL DEFORMATION

SOFTENING

HEMISPHER I CAL

FLUID

\section{REDUCING \\ OXIDIZING}

2310.

2520.

2400.

2600 .

2460 .

2640.

2510.

2685. 
SAMPLE IDENTIFICATION: PSU NUMBER

SEAM NAME

ALTERNATE SEAM NAME

APPARENT RANK
SAMPLE LOCATION:

COUNTRY $\rightarrow$ USA

STATE $\quad \rightarrow$ UTAH

COUNTY

$\rightarrow$ Carbon

TOWNSHIP

CITY

$\rightarrow$

COAL PROVINCE $\rightarrow$ ROCKY MOUNTAIN REGION $\rightarrow$ UINTA

MAP REFERENCE: LATITUDE

$39 \mathrm{D} 35 \mathrm{M} 30 \mathrm{~s}$ LUATINE $\rightarrow 110 \mathrm{D} 21 \mathrm{M} 26 \mathrm{~S}$ QUADRANGLE $\rightarrow$ Patmos Head $\left(7.5^{\prime}\right)$

ADDITIONAL INFORMATION

-> upper split of Lower Sunnyside seam

MINE INFORMATION:

\section{RESERVES}

ANNUAL PRODUCTION
$->$

$\rightarrow$
LIFE EXPECTANCY $\rightarrow$

MINING METHOD $\quad \rightarrow$ UNDERGROUND

\section{SEAM INFORMATION:}

\section{AGE OF SEAM}

GROUP

FORMATION

SEAM THICKNESS $\rightarrow$ U. CRET.

$\rightarrow$ Mesaverde

$\rightarrow$ Blackhawk

$\rightarrow \quad 4 \mathrm{FT} . \quad 7 \mathrm{IN}$.

*\# SEAM PROFILE **

$\rightarrow$ gray mudstone

$\rightarrow$ clarain, with some vitrain

$\rightarrow$ clarain, abundant resin

$\rightarrow$ discont. gray shale parting

$\rightarrow$ clarain

$\rightarrow$ gray/black laminated mudstone
* penn state coal oata base *\#

\#\#

\#\# DECS-15 \#\#

\#*

\#* 11/16/93 \#\# N

34 IN.

2 IN

FLOOR 
SAMPLE IDENTIFICATION: PSU MUMBER PSU NUMBER

ALTERNATE SEAM NAME APPARENT RANK

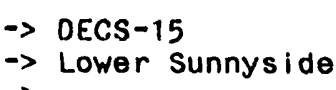

PROXIMATE ANALYSIS

\% moIstuRE

AS REC'D $\quad \underline{\text { DRY }}$

DAF

DMMF (PARR)

$\% \mathrm{ASH}$

\% VOLATILE

\% FIXED CARBON

2.71

9.86

10.13

36.86

37.89

50.57

51.98

42.16

57.84

41.31

58.69

\section{ANALYSIS}

AS REC'D

$9.86 \quad 10.13$

\% CARU.

72.28

74.29

\% HYDROGEN

$\%$ NITROGEN

\% total sulfur

\% OXYGEN(DIFF)

4.99

1.59

$1.62 \quad 1.67$

$7.13(*) \quad 7.33$

(*)-EXCLUDES MOISTURE

SULFUR FORMS

DRY

DAF

\% PYRITIC \% SULFATIC

0.83

0.01

0.92

0.01

DRY

AS REC'D

CALORIFIC VALUE

MM-CONTAIN INC

MM-FREE (PARR)

MM-FREE (MOD. P)

14967.

MOIST.

ORGANIC

0.83

0.92

1.67

1.86
EQUIL.

MOIST.

12858.

14435 .

14486.
DMMF (PARR

(11.86\%MM)

84.29

5.66

1.80

8.25
\% EQUILIBRIUM MOISTURE $=3.15$

MM-FREE $=3.56$
** penn state coal data base \#*

$\begin{array}{lll}* * & & * * \\ * * & \text { DECS }-15 & * * \\ * * & & * * \\ * * & 11 / 16 / 93 & * * \\ * * & \text { PACE 2 } & * *\end{array}$

NET CV, DMMT BTU/LB 14496

ELEMENTAL ANALYSIS

\% CARBON

\% HYDROGEN

\% NITROGEN

\% organic sulfur

\% OXYGEN(DIFF)

\% MINERAL MATTER

(INCL. $1.55 \%$ FES2)
QRY

74.17

4.87

1.59

0.83

6.62

11.92

ORY \% CHLORINE $=0.16$ DRY \% CO2 $=0.43$

MOTT-SPOONER DIFFERENCE $=-93$.

\section{ATOM RATIOS (DMMF) PARR MOD,PARR DIRECT}

$\begin{array}{lll}\text { ATOMIC H/C } & 0.8068 & 0.7894 \\ \text { ATOMIC O/C } & 0.0735 & 0.0670\end{array}$


SAMPLE IDENTIFICATION: CAMPLE IDEN PSU NUMBER

SEAM NAME

ALTERNATE SEAM NAME APPARENT RANK
SAMPLE LOCATION:

COUNTRY $\rightarrow$ USA

STATE
* penn state coAl data base **

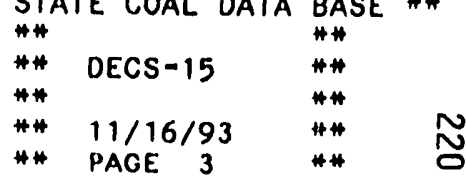

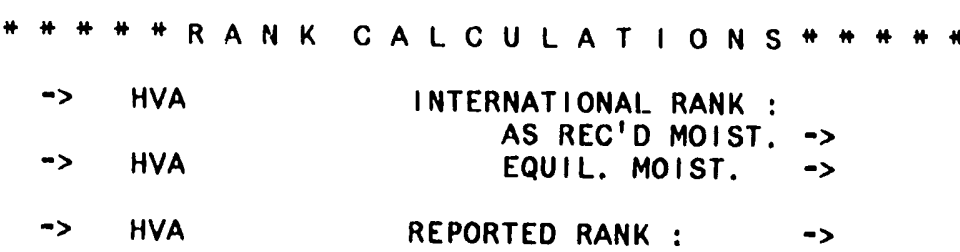

$\rightarrow \quad$ HVA

$\rightarrow \quad H V A$

$\Rightarrow \quad$ HVA

INTERNATIONAI- RANK :

AS REC'D MOIST. $\rightarrow$
EQUIL. MOIST. $\rightarrow$

REPORTED RANK : $\quad \rightarrow$

REFLECTANCE RANK CATEGORY

*\#** PETROGRAPHIC D A T A

MACERAL COMPOSITION ( WHITE LIGHT AND FLUORESCENCE ANALYSIS )

MACERAL COMPOSITION

PSEUDO-
VITRINITE VITRINITE FUSINITE FUSINITE MACRINITE MICRINITE SCLERO- SPCR-

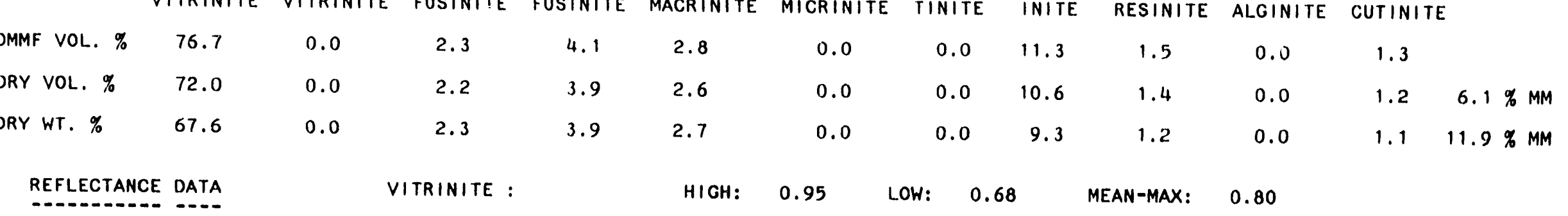

\begin{tabular}{lrrrr} 
VITRINITE TYPES & $\vee 6$ & $V 7$ & $\vee 8$ & $\vee 9$ \\
\hline PERCENT & 1.0 & 56.0 & 40.0 & 3.0
\end{tabular}

*\#\#\# CAKING ANDMECHAN I CAL PROPERTIES

GIESELER COAL PLASTOMETER:

INIT. SOFTENING TEMP 402

TEMP. MAX. FLUIDITY 442

SOLIDIFICATION TEMP. $\quad 478$

FLUID TEMP. RANGE 76

MAXIMUM FLUIDITY 959

WASHABILITY DATA AVAILABLE? NO

GRAY-KING COKE TYPE

HARDGROVE GRINDABILITY 54.2

FREE SWELLING INDEX

4.5

VICKERS HARDNESS NUMBER

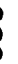


SAMPLE IDENTIFICATION:

SAMPLE IDENTIFICATION:

SEAM NAME

ALTERNATE SEAM NAME

APPARENT RANK

$\rightarrow$ DECS -15

$\rightarrow$ Lower Sunnyside

$\rightarrow$ HVA
PSU NUMBER

SAMPLE LOCATION:

-

COUNTRY $\rightarrow$ USA

STATE

$\rightarrow$ UTAH

$\%$ HTA $=10.08$

MAJOR ELEMENT ANALYSIS

MANOR ELEMENT ANALYSIS

OXIDE \% OF HTA

AL203

TIO2

\begin{abstract}
FE203
\end{abstract}
11.6

MGO

1.03

FE

$\begin{array}{lll}\text { SI } & A L & T I\end{array}$

ELEMENT \% OF

TOTAL DRY COAL
0.06

0.8
* PENN state coAl data base **

$\begin{array}{lll}* * & & * * \\ * * & \text { DECS }-15 & * * \\ * * & & * * \\ * * & 11 / 16 / 93 & \# * \\ * * & \text { PAGE 4 } & * *\end{array}$

TRACE ELEMENT ANALYSIS

TRACE ELEMENT ANALYSIS

$\begin{array}{lc}\text { PPM } & \text { PPM } \\ \text { HTA } & \text { TOTAL COAL }\end{array}$

$\begin{array}{lc}\text { PPM } & \text { PPM } \\ \text { HTA } & \text { TOTAL COAL }\end{array}$

HTA TOTAL COAL

GE

LA

LI

BA

BE

7.0

$0.7 \quad M N$

232.

MO

NB

CE

co

NI

CR

90.

9. PB

CU

65.

7. RB

GA 
SAMPLE IOENTIFICATION:

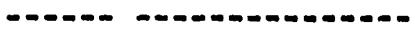
PSU NUMBER
SEAM NAME
ALTERNATE SEAM NAME
$\rightarrow$ DECS -15
APPARENT RANK
$\rightarrow$ Lower Sunnyside
$\rightarrow$ HVA

SAMPLE LOCATION:

SAMPLE LOCATION:

COUNTRY

$\rightarrow$ USA

STATE

$\rightarrow$ UTAH
* penn state coal oATa base ** ** 4* DECS - 15

\#* 11/16/93

* PAGE 5

ASH FUSION TEMPERATURES (ALL TEMPERATURES IN DEGREES F)

ASH FUSION TEMPERATURES

INITIAL DEFORMATION

\section{SOFTENING}

HEMISPHERICAL

FLUID
REDUCING

2415.

2480 .

2560 .

2635.
OXIDIZINO

2655.

2695.

$2700 .+$

$2700 .+$ 
SAMPLE IDENTIFICATION:

PSU NUMBER

PSU NUMBER

SEAM NAME

ALTERNATE SEAM NAME

APPARENT RANK

$\rightarrow$ DECS -16

$\rightarrow$ Bl ind Canyon

$\rightarrow$

$\rightarrow \quad H V B$

SAMPLE HISTORY:

SAMPLING DATE \& AGENCY $\rightarrow 5 / 7 / 91 ;$ PSU

SAMPLE TYPE

$\rightarrow$ CHANNEL-WORKING SECTION

ADDITIONAL INFORMATION

$\rightarrow$

MINE INFORMATION:

-.-. -.-.-..-.--

RESERVES

ANNUAL PRODUCTION

$->$

$\rightarrow$
SAMPLE LOCATION:

COUNTRY $\rightarrow$ USA

STATE $\quad \rightarrow$ UTAH

COUNTY $\rightarrow$ EmerY

TOWNSHIP

CITY

$\rightarrow$

$\rightarrow$ Huntington

COAL PROVINCE $\rightarrow$ ROCKY MOUNTAIN REGION $\rightarrow$ UINTA

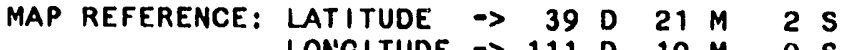
GUADRANGLE $\rightarrow>$ Mahogany Point (7.5')

LIFE EXPECTANCY $\rightarrow$

MINING METHOD $\rightarrow$ UNDERGROUND
** pENN STATE coal dATA base \#*

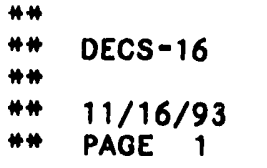

$\rightarrow$ U. CRET

$\rightarrow$ Mesaverde

$\rightarrow$ Blackhawk

FORMATION

SEAM THICKNESS

$\rightarrow \quad 7 \mathrm{FT}$.

6 IN.

** SEAM PROFILE **

NFORMATION:

AGE OF SEAM

Group

$\rightarrow$ gray to black mudstone

$\rightarrow$ mudstone w/ vitrain stringers

$\rightarrow$ hard bright clarain, resin

$\rightarrow$ bright clarain, abund. resin

$\rightarrow$ coal below floor NOT SAMPLED

$\rightarrow$ not exposed 


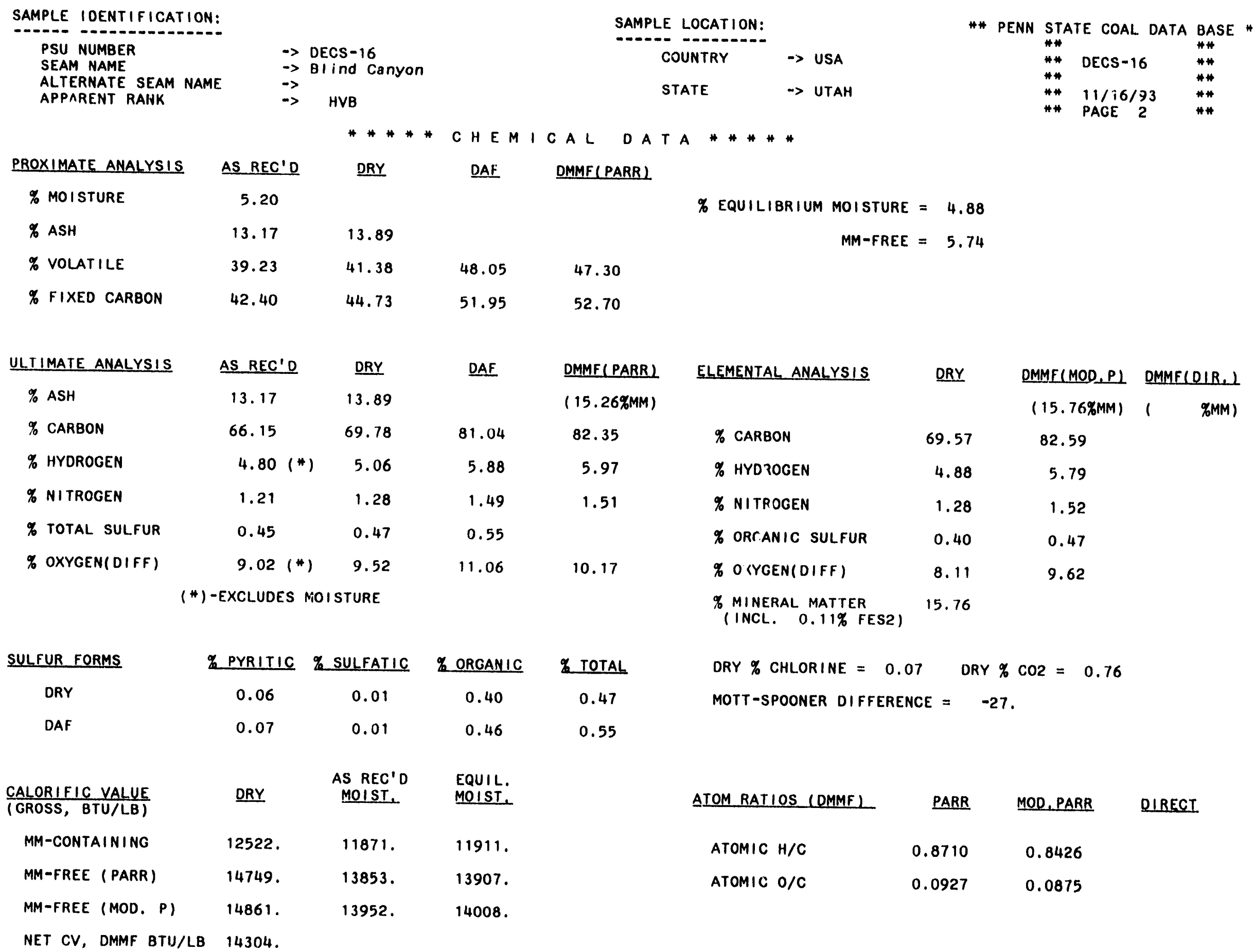


SAMPLE IDENTIFICATION:

SAMPLE IOENTIEICATION:

PSU NUMBER

SEAM NAME

ALTERNATE SEAM NAME

APPARENT RANK
SAMPLE LOCATION:

COUNTRY $\rightarrow$ USA

STATE $\quad \rightarrow$ UTAH
* penn state coal data base * * ** \#* DECS -16

* $11 / 16 / 93$

** PAGE 3
**

\#*

$* *$ $\rightarrow$ Blind Canyon

$\rightarrow \quad H V B$

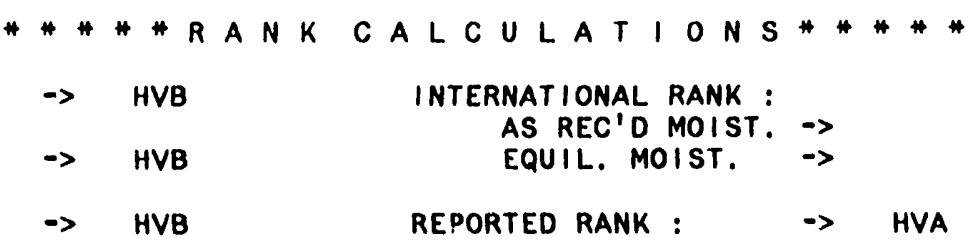

$\rightarrow \quad H V B$

$\rightarrow \quad H V B$

$\rightarrow \quad H V B$

INTERNATIONAL RANK :

AS REC'D MOIST. $\rightarrow$

EQUIL. MOIST.

REPORTED RANK : $\quad \rightarrow \quad$ HVA

REFLECTANCE RANK CATEGORY

***** PETROgRAPHI C DATA

MACERAL COMPOSITION ( WHITE LIGHT AND FLUORESCENCE ANALYSIS )

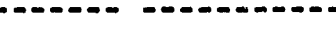

PSEUDO-
VITRINITE VITRINITE FUSINITE FUSINITE MACRINITE MICRINITE TINITE INITE

RESINITE ALGINITE CUTINITE

\begin{tabular}{|c|c|c|c|c|c|c|c|c|c|c|c|c|}
\hline DMMF VOL. $\%$ & 76.7 & 0.0 & 1.1 & 4.7 & 3.0 & 0.6 & 0.0 & 8.2 & 5.1 & 0.0 & 0.8 & \\
\hline DRY VOL. \% & 70.6 & 0.0 & 1.0 & 4.3 & 2.8 & 0.6 & 0.0 & 7.5 & 4.7 & 0.0 & $0 . i$ & $8.0 \% \mathrm{MM}$ \\
\hline DRY WT. $\%$ & 65.0 & 0.0 & 1.1 & 4.3 & 2.7 & 0.5 & 0.0 & 6.4 & 4.0 & 0.0 & 0.6 & $15.3 \% \mathrm{MM}$ \\
\hline
\end{tabular}

REFLECTANCE DATA

VITRINITE :

HIGH:

0.73

LOW:

0.52

MEAN-MAX:

0.64

\begin{tabular}{lrrr} 
VITRINITE TYPES & $V 5$ & $V 6$ & $V 7$ \\
\hline PERCENT & 26.0 & 61.0 & 13.0
\end{tabular}

$* * *$ c a K

GIESELER COAL PLASTOMETER:

INIT. SOFTENING TEMP

396

TEMP. MAX. FLUIDITY

420

SOLIDIFICATION TEMP.

438

FLUID TEMP. RANGE

MAXIMUM FLUIDITY

WASHABILITY DATA AVAILABLE?
42

No ${ }^{6}$
GRAY-KING COKE TYPE

HARDGROVE GRINDABILITY $\quad 46.6$

FREE SWELLING INDEX

$? .0$ 
SAMPLE IDENTIFICATION: SAMPLE IDENTIFICATION: PSU NUMBER

SEAM NAME

ALTERNATE SEAM NAME

APPARENT RANK

$\rightarrow$ DECS -16

$\rightarrow$ Blind Canyon

$\rightarrow$ HVB

MAJOR ELEMENT ANALYSIS

MAJOR ELEMENT ANALYSIS

OXIDE \% OF HTA

$5102 \quad$ AL203 TlO2

62.1

SI

$21.5 \quad 1.01$

F.LEMENT \% OF

TOTAL DRY COAL
SAMPLE LOCATION:

$\begin{array}{ll}\text { COUNTRY } & \rightarrow \text { USA } \\ \text { STATE } & \rightarrow \text { UTAH }\end{array}$

* penn state coal data base ** ** ** DECS -16

* $11 / 16 / 93$

TRACE ELEMENT ANALYSIS

PPM PPM

GE

$A G$

B

BA

BE

BI

CE

co

CR

CU

130.

70.

$\begin{array}{rrr}4.0 & 0.5 & \text { MN } \\ & & \text { MO } \\ & & \text { NB } \\ & & \text { NI } \\ 130 . & 17 . & \text { PB } \\ 70 . & 9 . & \text { RB } \\ & & \text { SC }\end{array}$

65.

9. $Z R$

$\%$ HTA $=13.45$

$\begin{array}{ccccccc}\text { FE203 } & \text { MGO } & \text { CAO } & \text { NA2O } & \text { K2O } & \text { P205 } & \text { SO3 } \\ 3.83 & 1.11 & 4.21 & 2.35 & 1.51 & 0.17 & 2.30 \\ \text { FE } & \text { MG } & \text { CA } & \text { NA } & K & P & \text { S } \\ 0.36 & 0.09 & 0.40 & 0.23 & 0.17 & 0.01 & 0.47\end{array}$

$\%$ HTA $=13.45$

PPM PPM

HTA TOTAL COAL

$\begin{array}{lc}\text { PPM } & \text { PPM } \\ \text { HTA } & \text { TOTAL COAL. }\end{array}$

45.

12.

90.

80.

11. $\mathrm{YB}$
VLATILES PPM TOTAL COAL

AS

BR

CD

CL $\quad 700$.

$F$

HG

SB

SE

105.

14.

50 
SAMPLE IDENTIFICATION:

$\begin{array}{ll}\text { PSU NUMBER } & \rightarrow \text { DECS }-16 \\ \text { SEAM NAME } & \rightarrow \text { BI irid Canyon } \\ \text { ALTERNATE SEAM NAME } & \rightarrow \\ \text { APPARENT RANK } & \rightarrow\end{array}$

APPARENT RANK
SAMPLE LOCATION:

SOUNTRY

$\rightarrow$ USA

STATE

$\rightarrow$ UTAH
** PENN STATE COAL DATA BASE \#\# *\# \#\# DECS -16 \#\# $11 / 16 / 93$ \#\# \#\# PAGE 5 \#\#

ASH FUSION TEMPERATURES (ALL TEMPERATURES IN DEGREES F)

\section{ASH FUSION TEMPERATURES}

INITIAL DEFORMATION

SOFTENING

HEMISPHERICAL

FLUID
REDUCING

OXIDIZING

2280.

2315.

2360.

2420.
2385.

2425.

2485.

2660. 
SAMPLE IDENTIFICATION:

$\begin{array}{ll}\text { PSU NUMBER } & \rightarrow \text { DECS }-17 \\ \text { SEAM NAME } & \rightarrow \text { BIInd Canyon } \\ \text { ALTERNATE SEAM NAME } & \rightarrow\end{array}$

APPARENT RANK

$\rightarrow \quad H V A$

\section{SAMPLE HISTORY:}

SAMPLING DATE \& AGENCY

$\rightarrow$ 8/ 5/91; PSU

SAMPLE TYPE

$\rightarrow$ CHANNEL-SUBSECTION

ADDITIONAL INFORMATION

MINE INFORMATION:

MINE INFORMATION:

RESERVES

ANNUAL PRODUCTION

$\rightarrow$

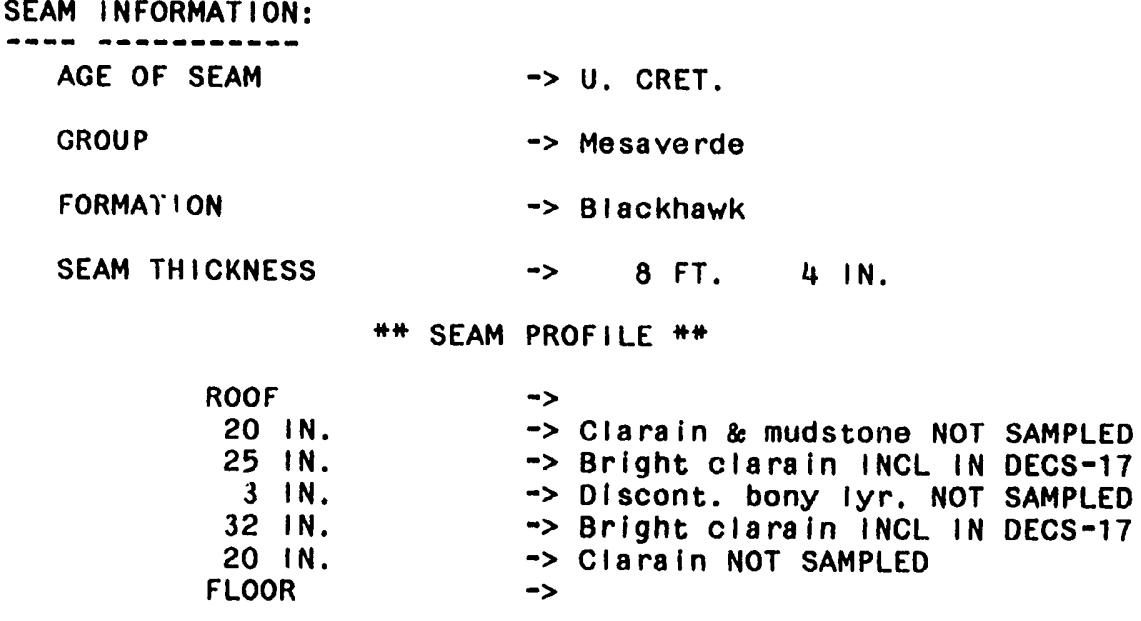

$\rightarrow$ U. CRET.

$\rightarrow$ Mesaverde

-> Blackhawk

$\Rightarrow \quad 8 \mathrm{FT} . \quad 4 \mathrm{IN}$.

** SEAM PROFILE **

ROOF
20 IN.
25 IN.
3 IN.
32 IN.
20 IN.
FLOOR

$\rightarrow$

$\rightarrow$ Clarain \& mudstone NOT SAMPLED

$\rightarrow$ Bright clarain INCL IN DECS-17

$\rightarrow$ Discont , bony IYr. NOT SAMPLED

$\rightarrow$ Bright clara in INCL IN DECS -17

$\rightarrow$ Claraín NOT SAMPLED

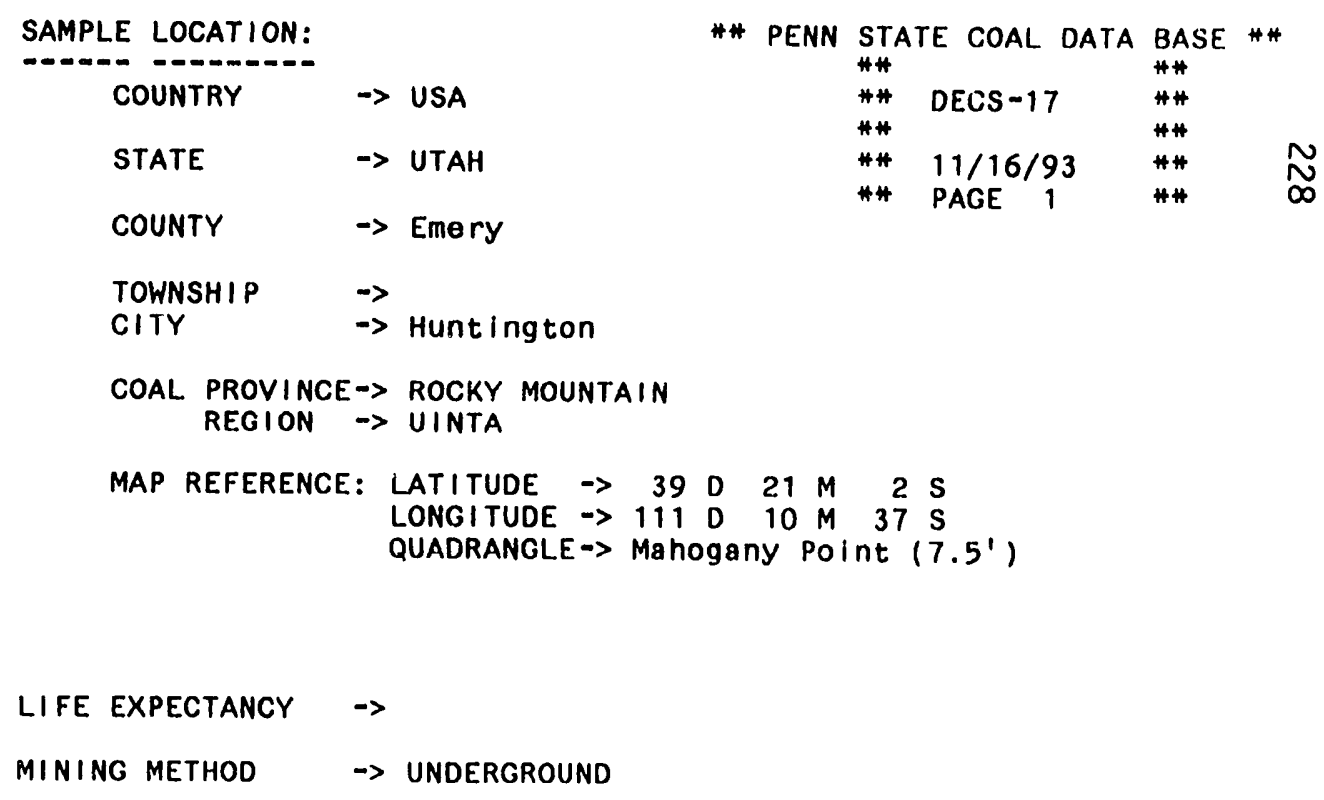




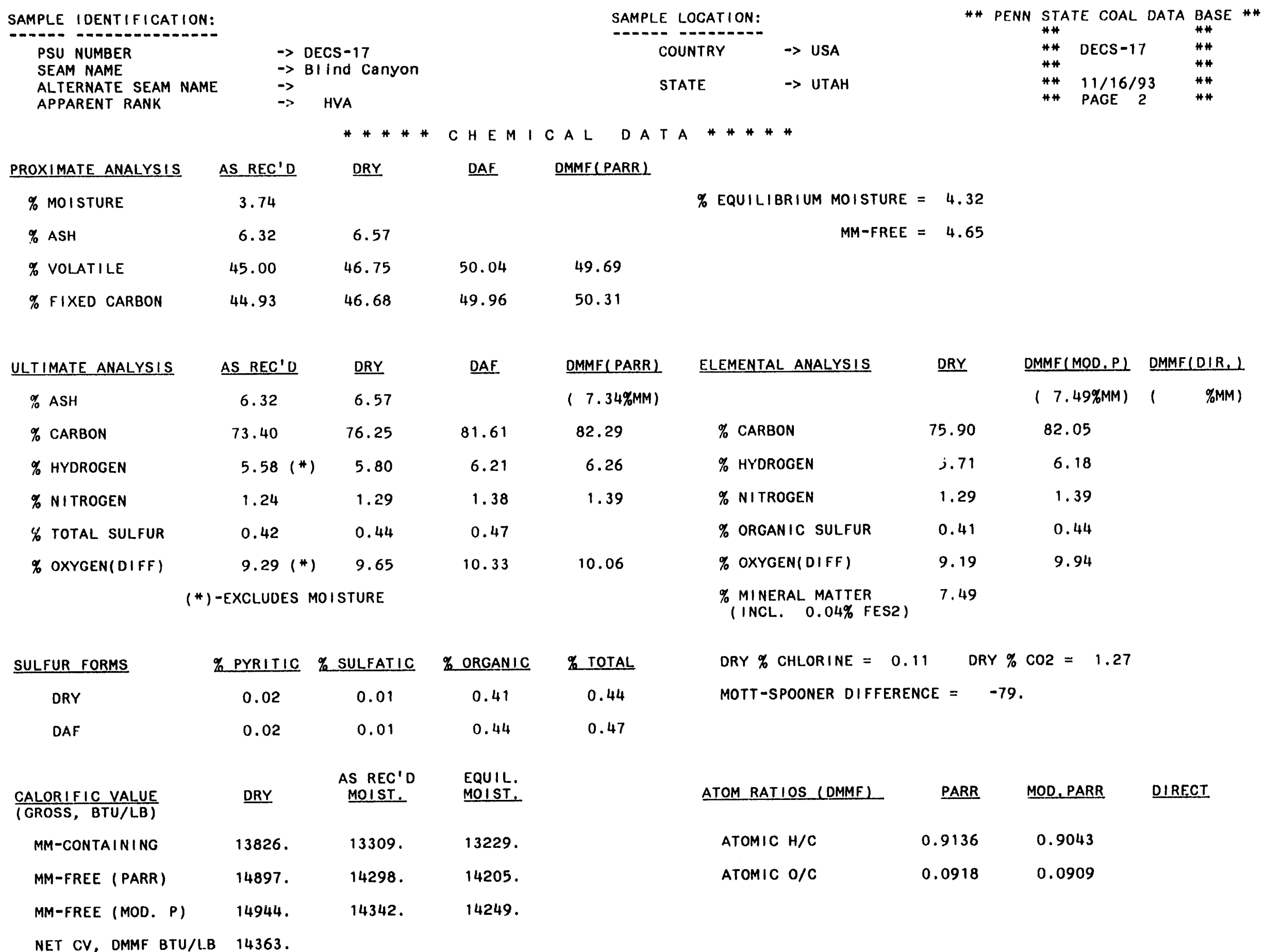




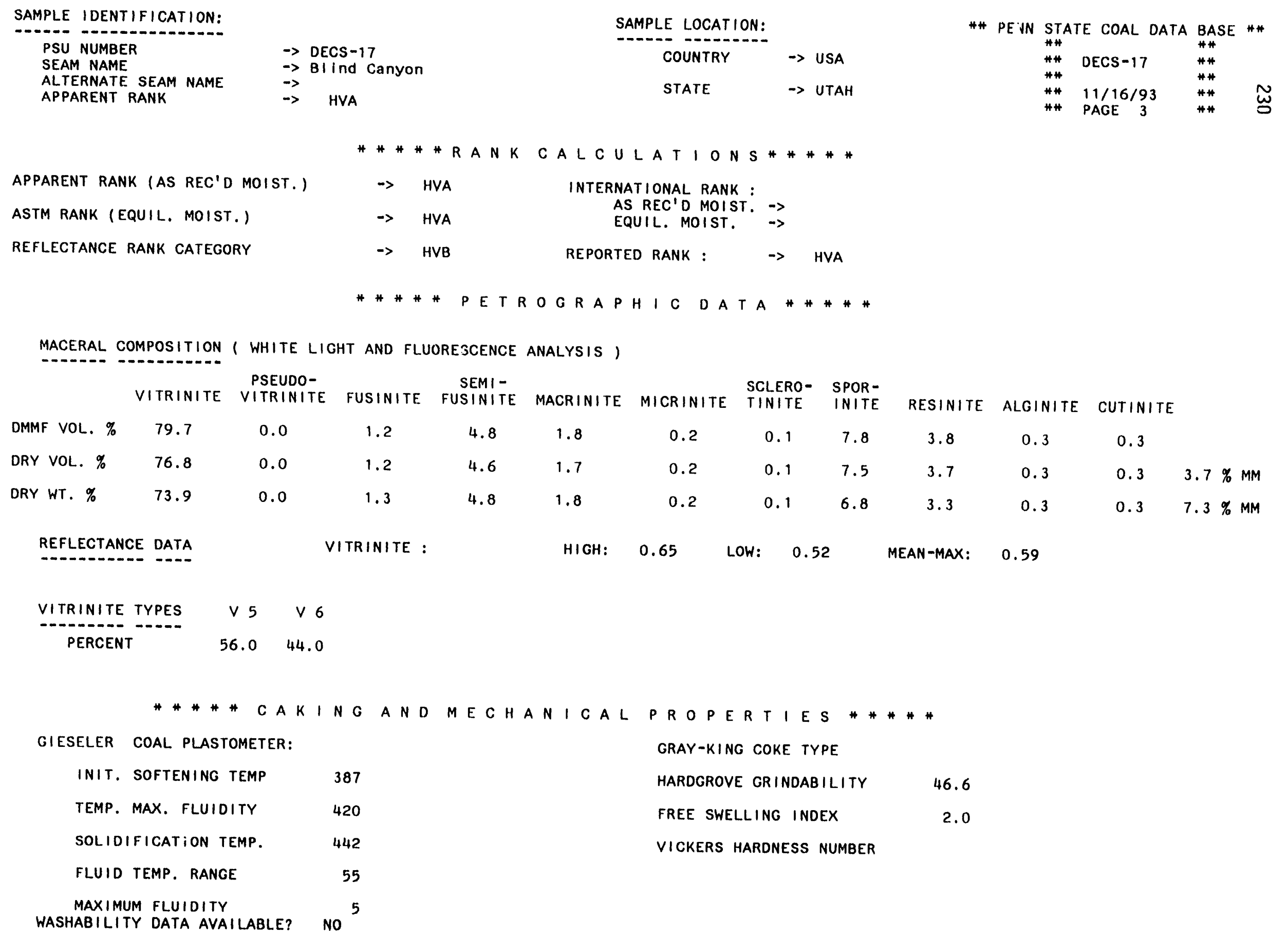


SAMPLE IDENTIFICATION:

-..-.- -............. SEAM NAME

ALTERNATE SEAM NAME

APPARENT RANK

$\rightarrow$ DECS -17
$\rightarrow$ Blind Canyon
$\rightarrow>$ HVA

MAJOR ELEMENT ANALYSIS

MAJOR ELEMENT ANALYSIS

OXIDE \% OF HTA

$5102 \quad A L 203$

47.6

9.23

SI AL

$1.4 \quad 0.31$

TOTAM T\% OF

TRACE ELEMENT ANALYSIS

TRACE ELEMENT ANALYSIS
SAMPLE LOCATION:

COUNTRY $\rightarrow$ USA

STATE $\quad->$ UTAH
* penn state coal daTA base \#*

H*

** DECS -17

\#\# $11 / 16 / 93$

** PAGE 4
$\# *$

\#\#

\begin{tabular}{|c|c|c|c|c|c|c|c|c|c|c|}
\hline & $\begin{array}{l}\text { PPM } \\
\text { HTA }\end{array}$ & $\begin{array}{c}\text { PPM } \\
\text { TOTAL COAL }\end{array}$ & & $\begin{array}{l}\text { PPM } \\
\text { HTA }\end{array}$ & $\begin{array}{c}\text { PPM } \\
\text { TOTAL COAL. }\end{array}$ & & $\begin{array}{l}\text { PPM } \\
\text { HTA }\end{array}$ & $\begin{array}{c}\text { PPM } \\
\text { TOTAL COAL }\end{array}$ & VOLAT ILES & $\begin{array}{c}\text { PPM } \\
\text { TOTAL COAL }\end{array}$ \\
\hline AG & & & $\mathrm{GE}$ & & & SN & & & AS & \\
\hline B & & & LA & & & SR & & & BR & \\
\hline BA & & & LI & & & TH & & & $C D$ & \\
\hline BE & $<3.0$ & $<0.2$ & $M N$ & 155. & 10. & $\mathbf{U}$ & & & $\mathrm{CL}$ & 1100. \\
\hline BI & & & MO & & & V & 140 & 9. & $F$ & \\
\hline CE & & & NB & & & $Y$ & & & HG & \\
\hline CO & & & NI & $<25$. & 2 & YB & & & SB & \\
\hline CR & 95. & 6. & PB & & & $Z N$ & 50. & 3. & SE & \\
\hline $\mathrm{CU}$ & 135. & 9. & RB & 20. & 1. & $Z R$ & 440. & 28. & & \\
\hline GA & & & SC & & & & & & & \\
\hline
\end{tabular}


SAMPLE IDENTIFICATION:

SAMPLE IDENTIFICATION:

SEAM NAME

ALTERNATE SEAM NAME

APPARENT RANK

$\Rightarrow$ DECS -17

$\rightarrow$ Bilind Canyon

$\rightarrow$

HVA
SAMPLE LOCATION:

COUNTRY

$\rightarrow$ USA

STATE

$\Rightarrow$ UTAH
\#\# penN state coal data base \#\#

\#* OECS 17

\#\# DECS -17

\#\# 11/16/93

* PAGE 5

\#*

\#*

$\stackrel{N}{\omega}$

ASH FUSION TEMPERATURES (ALL TEMPERATURES IN DEGREES F)

REDUCING OXIDIZING

INITIAL DEFORMATION 2100.

\section{SOFTENING}

1900 . 2200

HEMISPHERICAL

1965.

2245 .

FLUID

2020 .

2290. 
SAMPLE IOENTIF ICATION: SAMPLE IOENTIFICATION:

$\begin{array}{ll}\text { PSU NUMBER } & \rightarrow \text { DECS }-18 \\ \text { SEAM NAME } & \rightarrow \text { Kentucky } \# 9 \\ \text { ALTERNATE SEAM NAME } & \rightarrow \\ \text { APPARENT RANK } & \rightarrow\end{array}$

SAMPLE HISTORY:

$\rightarrow 10 / 10 / 91 ; P S U$

SAMPLE TYPE

-> CHANNEL-WHOLE SEAM

ADDITIONAL INFORMATION
SAMPLING DATE \& AGENCY

\section{SAMPLE LOCATION: \\ COMPLE LOCATION:}

COUNTRY

$\rightarrow$ USA

STATE

$\rightarrow$ KENTUCKY

COUNTY

$\rightarrow$ Union

TOWNSHIP

CITY

$\rightarrow$ Waverly

COAL PROVINCE $\rightarrow$ INTERIOR REGION $\rightarrow$ EASTERN

$\begin{array}{rllllllll}\text { MAP REFERENCE: } & \text { LATITUDE } & \rightarrow & 37 & \mathrm{D} & 38 & \mathrm{M} & 48 & \mathrm{~S} \\ \text { LONGITUDE } & \rightarrow & 87 & \mathrm{D} & 48 & \mathrm{M} & 37 & \mathrm{~S}\end{array}$ LONGITUDE $\rightarrow \quad 87$ D $48 \mathrm{M}, 37$
QUADRANGLE $\rightarrow$ Waverly $\left(7.5^{\prime}\right)$

MINE INFORMATION:

MINE INFORMATION:

\section{RESERVES}

ANNUAL PRODUCTION
LIFE EXPECTANCY $\rightarrow$

MINING METHOD $\rightarrow$ UNDERGROUND $\rightarrow$

$\rightarrow$

-> PENNSYL.

$\rightarrow$

$\rightarrow$ Carbondale

$\rightarrow \quad 5 \mathrm{FT} . \quad 2 \mathrm{IN}$.

\#* SEAM PROFILE \#\#

* penn state coal data base \#*

* DECS -18

*\# 11/16/93

* PAGE 1

SEAM INFORMATION:

AGE OF SEAM

GROUP

FORMATION

SEAM THICKNESS

$\begin{array}{cl}\text { ROOF } & \rightarrow \text { gray mudstone, coal stringers } \\ 6 \text { IN. } & \rightarrow \text { brt clarain, } 1-3 \mathrm{~mm} \text { pyrite btm }\end{array}$

$\begin{array}{cl}\text { RoOF } & \rightarrow \text { gray mudstone, coal stringers } \\ 6 \text { IN. } & \rightarrow \text { brt clarain, } 1-3 \mathrm{~mm} \text { pyrite btm } \\ 27 \text { IN. } & \rightarrow \text { brt clarain, calcite on cleat }\end{array}$

27 IN.

$\rightarrow 1 \mathrm{~cm}$ ptg; brt clarain, vitraín

$\rightarrow$ pyrite zone NOT SAMPLED

8 IN. $\quad \Rightarrow$ brt clarain, vitrain, parting

FLOOR

dk gray mudstone, slickensides 


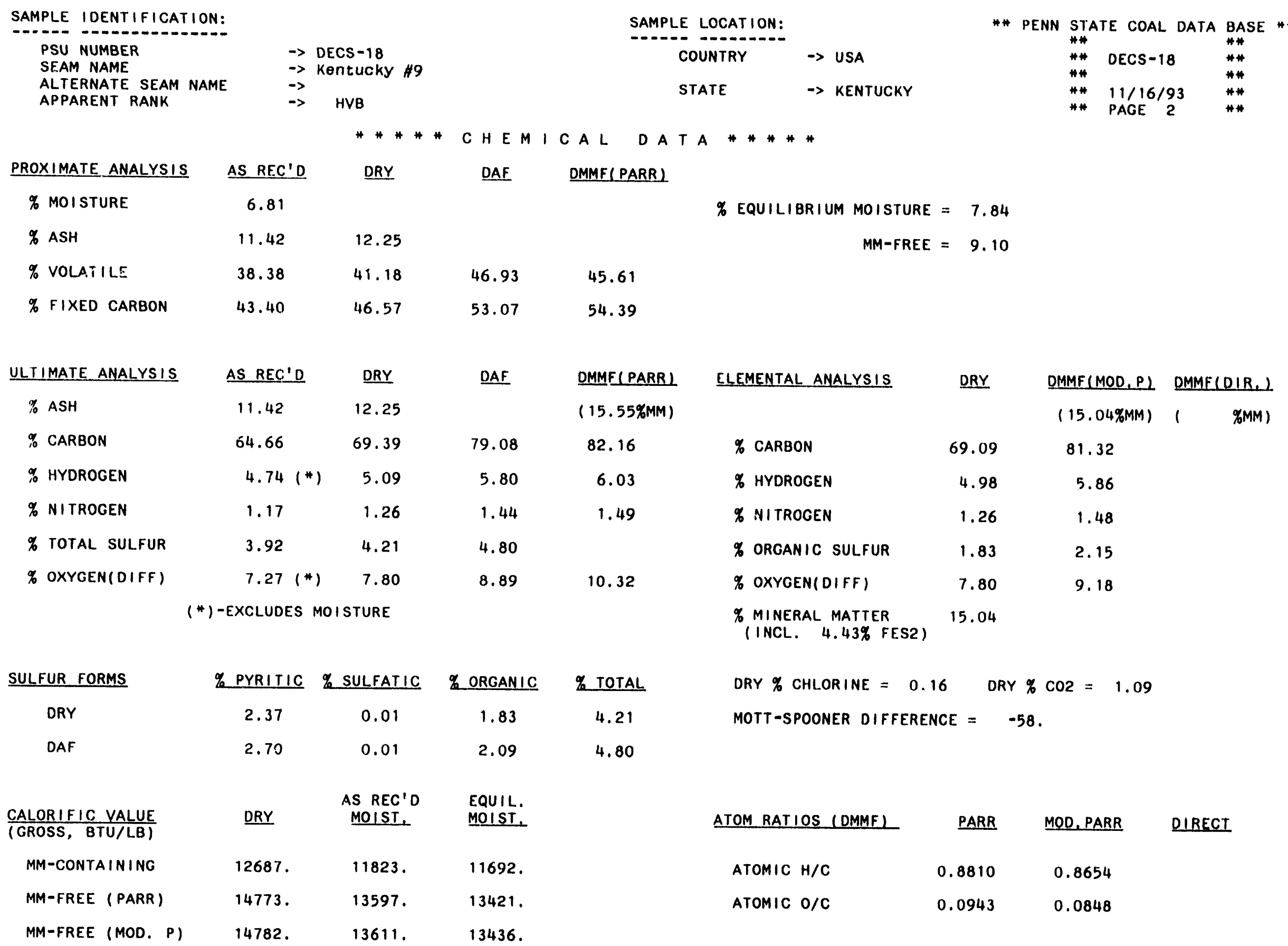


SAMPLE IDENTIFICATION: SAMPLE IDENTIFICATION:

PSU NUMBER

SEAM NAME

ALTERNATE SEAM NAME

APPARENT RANK
SAMPLE LOCATION:

COUNTRY $\rightarrow$ USA

STATE

$\rightarrow$ Kentucky \#9

HVB
** PENN STATE COAL DATA base **

** DECS -18

(1)

* $11 / 16 / 93$

* PAGE 3

APPARENT RANK (AS REC'D MOIST, )

**** R A N K

$\rightarrow \quad$ HVB

$\rightarrow \quad H V B$

$\rightarrow \quad$ HVC
INTERNATIONAL RANK :

AS REC'D MOIST. $\rightarrow$

EQUIL. MOIST.

REPORTED RANK : $\rightarrow$

REFLECTANCE RANK CATEGORY

***** PETROG RA P H I C D A T A *\#***

MACERAL COMPOSITION ( WHITE LIGHT AND FLUORESCENCE ANALYSIS )

OSEUDO-

VITRINITE VITRINITE FUSINITE FUSINITE MACRINITE MICRINITE TINITE INITE
RESINITE ALGINITE CUTINITE

\begin{tabular}{lrrrr} 
DMMF VOL. \% & 85.7 & 0.0 & 1.9 \\
DRY VOL. \% & 78.7 & 0.0 & 1.7 \\
ORY WT. \% & 72.3 & 0.0 & 1.9 \\
& & & & \\
REFLECTANCE DATA & & & VITRINIT \\
\hline & & & & \\
VITRINITE TYPES & $V 4$ & $V 5$ & $V 6$ \\
\hline PERCENT & 10.0 & 67.0 & 23.0
\end{tabular}

$\begin{array}{lll}0.0 & 0.5 & 1.2\end{array}$

2.2

\begin{abstract}
0.8
\end{abstract}

\begin{abstract}
0.0
\end{abstract}
6.0

$2.0 \quad 1.6$

0.7

0.0

5.5

$2.0 \quad 1.5$

0.7

0.0

4.7

0.0

0.5

1.1

8.2. $\%$ MM

0.0

0.4

$0.9 \quad 15.5 \%$ MM
HIGH:

0.67

LOW:

0.44

MEAN-MAX:

0.56

**** CAK I NG

ANDMECHANICAL

GIESELER COAL PLASTOMETER:

GRAY-KING COKE TYPE

INIT. SOFTENING TEMP 376

TEMP. MAX. FLUIDITY 421

SOLIDIFICATION TEMP. $\quad 456$

HARDGROVE GRINDABILITY

56.3

FREE SWELLING INDEX

2.0

FLUID TEMP. RANGE

VICKERS HARONESS NUMBER

MAXIMUM FLUIDITY

WASHABILITY DATA AVAILABLE? NO 
SAMPLE IDENTIFICATION:

CAMPLE IOENTIFICATION:

\begin{tabular}{|c|c|}
\hline $\begin{array}{l}\text { PSU NUMBER } \\
\text { SEAM NAME } \\
\text { ALTERNATE SEAM NAME } \\
\text { APPARENT RANK }\end{array}$ & $\begin{array}{l}\Rightarrow \text { DECS }-18 \\
\Rightarrow \text { Kentucky H9 } \\
\Rightarrow \text { HVB } \\
\Rightarrow \quad \text { H }\end{array}$ \\
\hline
\end{tabular}

MAJOR ELEMENT ANALYSIS

MAJOR ELEMENT ANALYSIS

$\begin{array}{lccccc}\text { OXIDE \% OF HTA } & \text { S102 } & \text { AL203 } & \text { TIO2 } & \text { FE203 } & \text { MGO } \\ \begin{array}{l}\text { ELEMENT \% OF } \\ \text { TOTAL DRY COAL }\end{array} & 41.2 & 15.6 & 0.75 & 22.0 & 0.70 \\ & \text { SI } & \text { AL } & \text { TI } & \text { FE } & \text { MG } \\ & 2.4 & 1.0 & 0.06 & 1.9 & 0.05\end{array}$

TRACE ELEMENT ANALYSIS

\section{SAMPLE LOCATION: \\ COUNTRY $\rightarrow$ USA \\ STATE \\ $\rightarrow$ KENTUCKY}

* penn state coal data base **

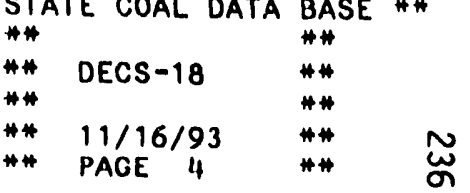

$\%$ HTA $=12.55$

$\begin{array}{ccccc}\text { CAO } & \text { NA2O } & \text { K2O } & \text { P205 } & \text { S03 } \\ 8.08 & 0.66 & 1.89 & 0.04 & 9.40 \\ \text { CA } & \text { NA } & K & P & S \\ 0.73 & 0.06 & 0.20 & 0.002 & 4.21\end{array}$

\% HTA $=12.55$

PPM PPM

HTA TOTAL COAL

VOLATILES PPM

HTA TOTAL COAL

SN

GE

169.

21.

AS

BR

CD

CL 1600.

150.

19.

F

HG

SB

SE

150.

19. 
SAMPLE IDENTIFICATION: PSU NUMBER

ALTERMATE SEAM NAME

APPARENT RANK

$\rightarrow$ DECS -18

$\rightarrow$ Kentucky

$\rightarrow \quad$ HVB
SAMPLE LOCATION:

COUNTRY

$\rightarrow$ USA

STATE

$\rightarrow$ KENTUCKY
** penn state coal data base \#\#

**

** DECS -18

** $11 / 16 / 93$

* PAGE 5

ASH FUSION TEMPERATURES (ALL TEMPERATURES IN DEGREES F) ASH FUSION TEMPERATURES
REDUCINC
OXIDIZING

INITIAL DEFORMATION

1755.

2100.

SOFTENING

1900.

2200.

HEMI SPHERICAL

2000.

2260.

FLUID

2040.

2310. 
SAMPLE IDENTIFICATION:

PSU NUMBER

SEAM NAME

ALTERNATE SEAM NAME

APPARENT RANK

$$
\begin{aligned}
& \Rightarrow \text { DECS }-19 \\
& \Rightarrow \text { PoCahontas \#3 } \\
& \Rightarrow \text { LOW VOL }
\end{aligned}
$$

SAMPLE HISTORY

SAMPLING DATE \& AGENCY

$->12 / 17 / 91$; PSU

\section{SAMPLE TYPE}

$\rightarrow$ CHANNEL-WHOLE SEAM

ADDITIONAL INFORMATION

$\rightarrow$

MINE INFORMATION:

MINE INFORMATION:

\section{RESERVES}

ANNUAL PRODUCTION

$\rightarrow 1855$ THOUSAND TONS

\section{SAMPLE LOCATION:} SAMPLE LOCATION:

$\begin{array}{ll}\text { COUNTRY } & \rightarrow \text { USA } \\ \text { STATE } & \rightarrow \text { VIRGINIA } \\ \text { COUNTY } & \rightarrow \text { BUChENAN }\end{array}$

** DECS 19

\#* DECS -19

\#* 11/16/93

**

COUNTY $\rightarrow$ Buchanan

* PAUE 1

TOWNSHIP $\quad \rightarrow$

CITY $\quad \rightarrow$ Oakwood

COAL PROVINCE $\rightarrow$ EASTERN

REGION $\rightarrow$ APPALACHIAN

MAP REFERENCE: LATITUDE $\rightarrow \quad 37 \mathrm{D} 12 \mathrm{M} 45 \mathrm{~s}$

LONGITUDE $\rightarrow 82 \mathrm{D} 2 \mathrm{M} 30 \mathrm{~S}$

QUADRANGLE $\rightarrow$ Vansont $\left(7.5^{\prime}\right)$

LIFE EXPECTANCY $\rightarrow 1$ YEARS

MINING METHOD $\rightarrow$ UNDERGROUND

\section{SEAM INFORMATION:} AGE OF SEAM

GROUP

\section{FORMATION}

SEAM THICKNESS $\rightarrow$ PENNSYL.

$\rightarrow$ Pottsville

-> Pocahontas

$\rightarrow \quad 4 \mathrm{FT} . \quad 10 \mathrm{IN}$.

** SEAM PROFILE **

$\begin{aligned} & \text { ROOF } \\ & 4 \text { IN. } \\ & 0 \text { IN. } \\ & 14 \text { IN. } \\ & 1 \text { IN. } \\ & 25 \text { IN. } \\ & 14 \text { IN. } \\ & \text { FLOOR }\end{aligned}$

$\rightarrow$ gray mudstone

$\rightarrow$ bright clarain with vitrain

$\rightarrow$ gray mudstone < $1 / 2^{\prime \prime}$

$\rightarrow$ clarain

$\rightarrow$ gray mudstone NOT SAMPLED

$\rightarrow$ bright clarain, friable

$\rightarrow$ clarain w/ ptgs, less friable

$\rightarrow$ gray mudstone 
SAMPLE IDENTIFICATION: PSU NUMBER SEAM NAME

ALTERNATE SEAM NAME

APPARENT RANK

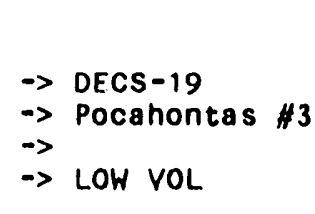

SAMPLE LOCATION: COUNTRY $\quad \rightarrow$ USA

Pocahontas \#3

$\rightarrow$ LOW VOL
** penn state coal data base * \#* *

\#\# $11 / 16 / 93$

*t+

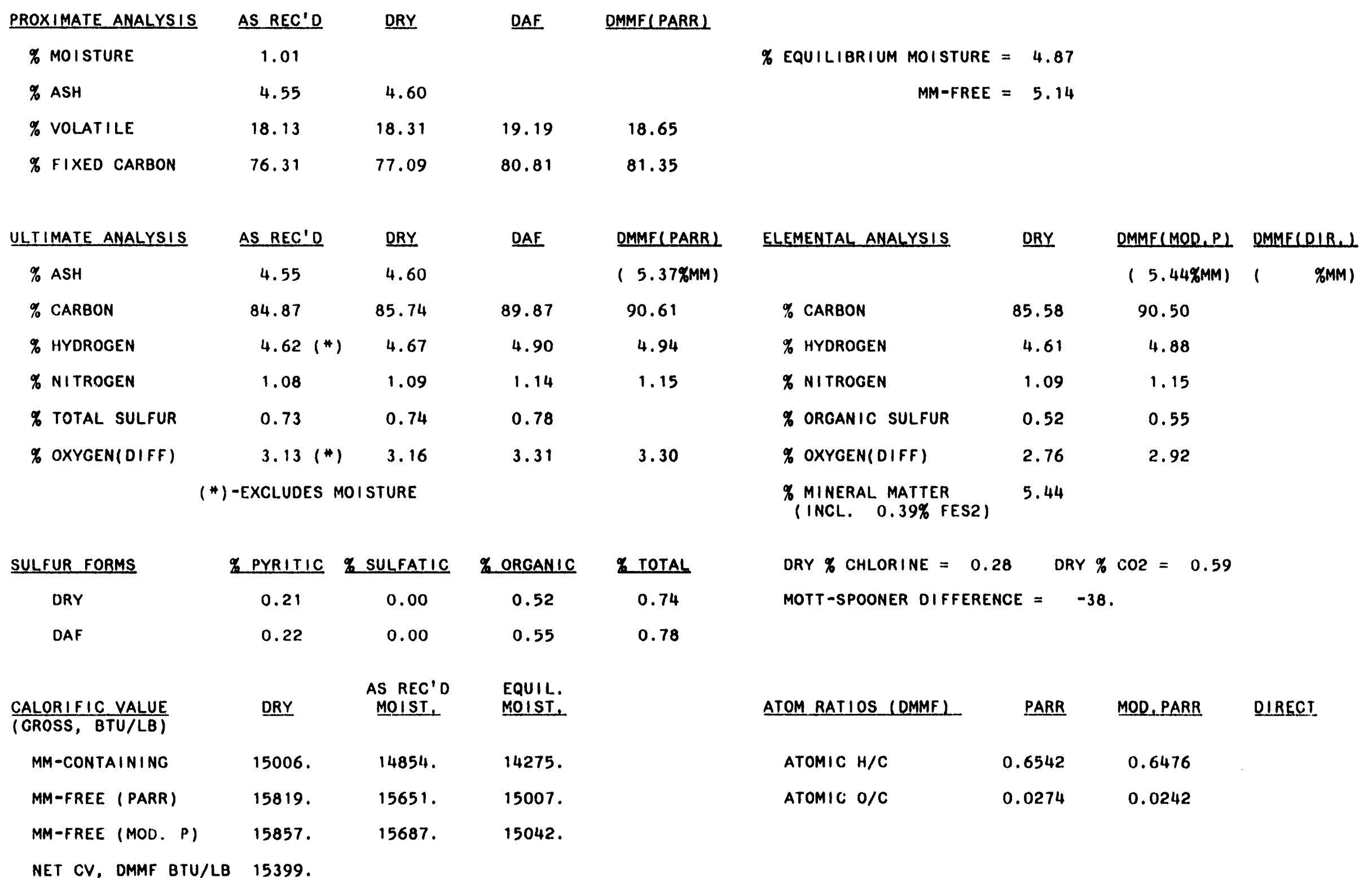


SAMPLE IDENTIFICATION: PSU NUMBER

\section{SEAM NAME}

ALTERNATE SEAM NAME

APPARENT RAN

\section{SAMPLE LOCATION: \\ COUNTRY $\rightarrow$ USA \\ STATE. \\ $\rightarrow$ VIRGINIA}

$\rightarrow$ LOW YOL
\#\# PENN STi : COAL DATA BASE \#\#

$\begin{array}{llll}* * & & * * & \\ * * & \text { DECS }-19 & * \# & \\ * * & & * * & \\ * * & 11 / 16 / 93 & * * & N \\ * * & \text { PACE 3 } & * * & \text { \& }\end{array}$

APPARENT RANK (AS REC'D MOIST.) ASTM RANK (EQUIL. MOIST.) REFLECTANCE RANK CATEGORY

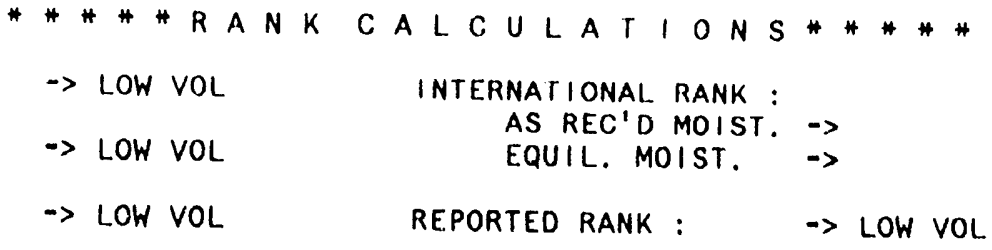

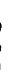

MACERAL COMPOSITION ( WHITE LIGHT ANALYSIS )

PSEUDO-
VITRINITE VITRINITE FUSINITE FUSINITE MACRINITE MICRINITE TINITE INITE

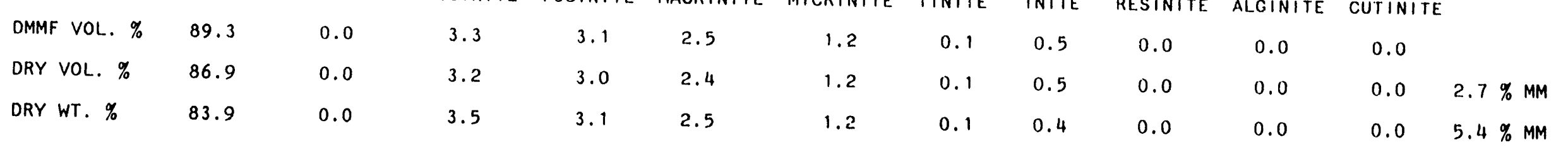

REFLECTANCE DATA

VITRINITE :

HIGH:

1.83 LOW:

1.57

MEAN-MAX:

1.71

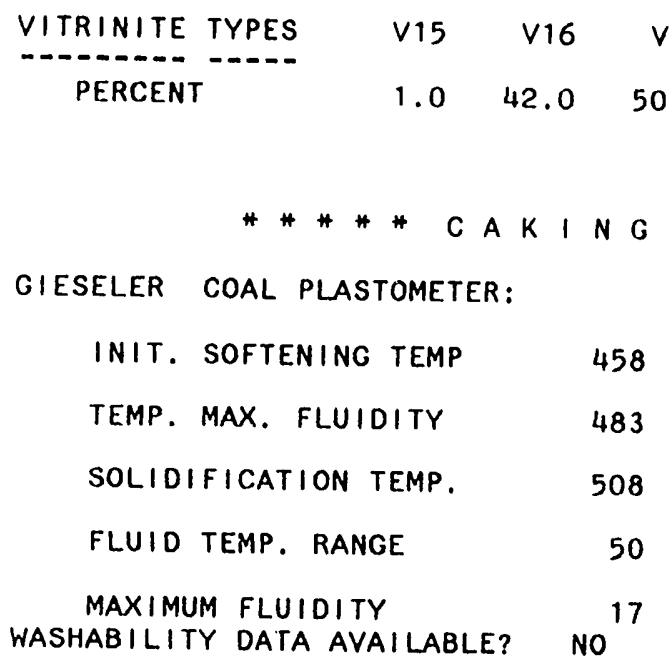

GRAY-KING COKE TYPE

HARDGROVE GRINDABILITY $\quad 101.6$

FREE SWELLING INDEX

6.0

VICKERS HARDNESS NUMBER 
SAMPLE IDENTIFICATIUN:

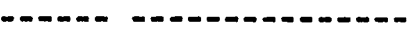

PSU NUMBER

SEAM NAME

ALTERNATE SEAM NAME

APPARENT RANK

$\rightarrow$ DECS -19

$\rightarrow$ Pocahontas \#3

$\rightarrow$ LOW VOL

MAJOR ELEMENT ANALYSIS

MAJOR ELEMENT ANALYSIS

OXIDE \% OF HTA

S102 AL203

28.6

19.6

TI 02

0.95

FE203

17.0

ELEMENT \% OF

TOTAL DRY COAL

$\begin{array}{lll}0.7 & 0.6 & 0.03\end{array}$

0.7
SAMPLE LOCATION:

SAMPLE LOCATION:

COUNTRY

$\rightarrow$ USA

STATE

$\rightarrow$ VIRGINIA
** PENN STATE COAL DATA bASE *\#

$\begin{array}{ll}* * & \\ \# * & \text { DECS }-19 \\ \# * & \\ * * & 11 / 16 / 93 \\ * & \text { PAGE } 4\end{array}$

TRACE ELEMENT ANALYSIS

$\begin{array}{lcccc} & \begin{array}{c}\text { PPM } \\ \text { HTA }\end{array} & \text { TOTAL COAL } & & \text { PPM } \\ \text { AG } & & & \text { GE } & \\ \text { B } & & & \text { LA } & \\ \text { BA } & 3493 . & 193 . & \text { LI } & \\ \text { BE } & 25 . & 1 . & \text { MN } & 387 . \\ \text { BI } & & & \text { MO } & \\ \text { CE } & & & \text { NB } & \\ \text { CO } & & & \text { NI } & 130 . \\ \text { CR } & 180 . & 10 . & \text { PB } & \\ \text { CU } & 260 . & 14 . & \text { RB } & 35 . \\ \text { GA } & & & \text { SC } & \end{array}$

PPM PPM
HTA

$\%$ HTA $=5.52$

FE MG

MGO

CAO NA2O

K20

P205

S03

2.49

$15.2 \quad 1.14$

0.83

0.04

13.0

0.08

0.6

NA

k

$\mathrm{S}$

0.05

0.04

0.001

0.7

$\%$ HTA $=5.52$

PPM
HTA

PPM

VOLATILES PPM

SN

OTAL COAL

AS

2452.

135.

BR

CD

CL 2800 .

F

HG

SB

7. YB

110.

6.

SE 
SAMPLE IDENTIFICATION:

SAMPLE IDENTIFICATION:

PSU NUMBER

SEAM NAME

ALTERNATE SEAM NAME

APPARENT RANK

$\rightarrow$ DECS -19

$\rightarrow$ Pocahontas \#3

$\rightarrow$

$\rightarrow$ LOW VOL
SAMPLE LOCATION:

SAMPLE LOCATION:

COUNTRY

$\rightarrow$ USA

STATE

$\rightarrow$ VIRGINIA
** pENN STATE COAL dATA base **

\begin{tabular}{|c|c|}
\hline DECS -19 & $\begin{array}{l}* \# \\
\# * \\
* \#\end{array}$ \\
\hline $\begin{array}{l}11 / 16 / 93 \\
\text { PAGE } 5\end{array}$ & $\begin{array}{l}* * \\
* *\end{array}$ \\
\hline
\end{tabular}

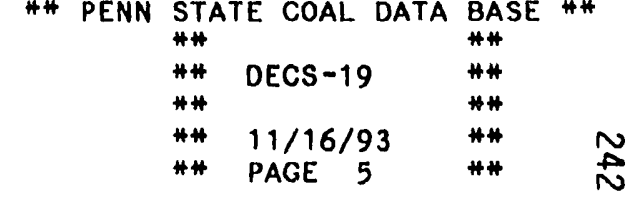

ASH FUSION TEMPERATURES (ALL TEMPERATURES IN DEGREES F)

ASH FUSION TEMPERATURES

\section{INITIAL DEFORMATION}

SOFTENINC

HEMISPHER ICAL

FLUID

\section{REDUCING OXIDIZING}

1910

2300 .

2030.2380

2090.2440
2140 . 2475
2140 .
2475. 
SAMPLE IDENTIFICATION:

$\begin{array}{ll}\text { PSU NUMBER } & \rightarrow \text { DECS }-20 \\ \text { SEAM NAME } & \rightarrow \text { EIkhorn \#3 } \\ \text { ALTERNATE SEAM NAME } & \rightarrow> \\ \text { APPARENT RANK } & \rightarrow\end{array}$

SAMPLE HISTORY:

SAMPLING DATE \& AGENCY $\rightarrow 12 / 18 / 91$; PSU

SAMPLE TYPE

$\rightarrow$ CHANNEL -WHOLE SEAM

ADOITIONAL INFORMATION

MINE INFORMATION:

MINE INFORMATION:

RESERVES

ANNUAL PROOUCTION

$\rightarrow$

$\rightarrow$

$\rightarrow$ PENNSYL.

$\rightarrow$

$\rightarrow$ Breathitt

$\Rightarrow \quad 5 \mathrm{FT} . \quad 4 \mathrm{IN}$

* SEAM PROFILE \#\#

SEAM INFORMATION:

AGE OF SEAM

GROUP

FORMATION

SEAM THICKNESS

\section{SAMPLE LOCATION:}

COUNTRY

$\rightarrow$ USA

STATE $\quad \rightarrow$ KENTUCKY

COUNTY $\rightarrow$ Floyd

TOWNSHIP $\quad \rightarrow$

CITY $\quad \rightarrow$ Teaberry

COAL PROVINCE $\rightarrow$ EASTERN

REGION $\rightarrow$ APPALACHIAN

MAP REFERENCE: LATITUDE $\rightarrow>37 \mathrm{D} 25 \mathrm{M} 33 \mathrm{~S}$

LONGITUDE $\rightarrow \quad 82 \mathrm{D} 36 \mathrm{M} 43 \mathrm{~S}$

QUADRANGLE $\rightarrow$ PikeVille (7.5')

LIFE EXPECTANCY $\rightarrow 2$ YEARS

MINING METHOD $\rightarrow$ UNDERGROUND
* penn state coal data base *\#

* DECS-20

** $11 / 16 / 93$

$\begin{array}{ll}* * & 11 / 16 / 93 \\ * & \text { PAGE } 1\end{array}$

H\#

\#\#

$\# *$

\#*

$\rightarrow$ gray laminated mudstone

$\begin{aligned} \text { ROOF } & \rightarrow \text { gray laminated mudstone } \\ 30 \text { IN. } & \rightarrow \text { clarain, occ. pyrite on cleat } \\ 9 \text { IN. } & \rightarrow \text { gray mudstone ptg NOT SAMPLED } \\ 1 \text { IN. } & \rightarrow \text { mudstone ce clarain NOT SAMPLED } \\ 24 \text { IN. } & \rightarrow \text { clarain, occ. } 5 \text { mm partings } \\ \text { FLOOR } & \rightarrow \text { gray mudstone }\end{aligned}$




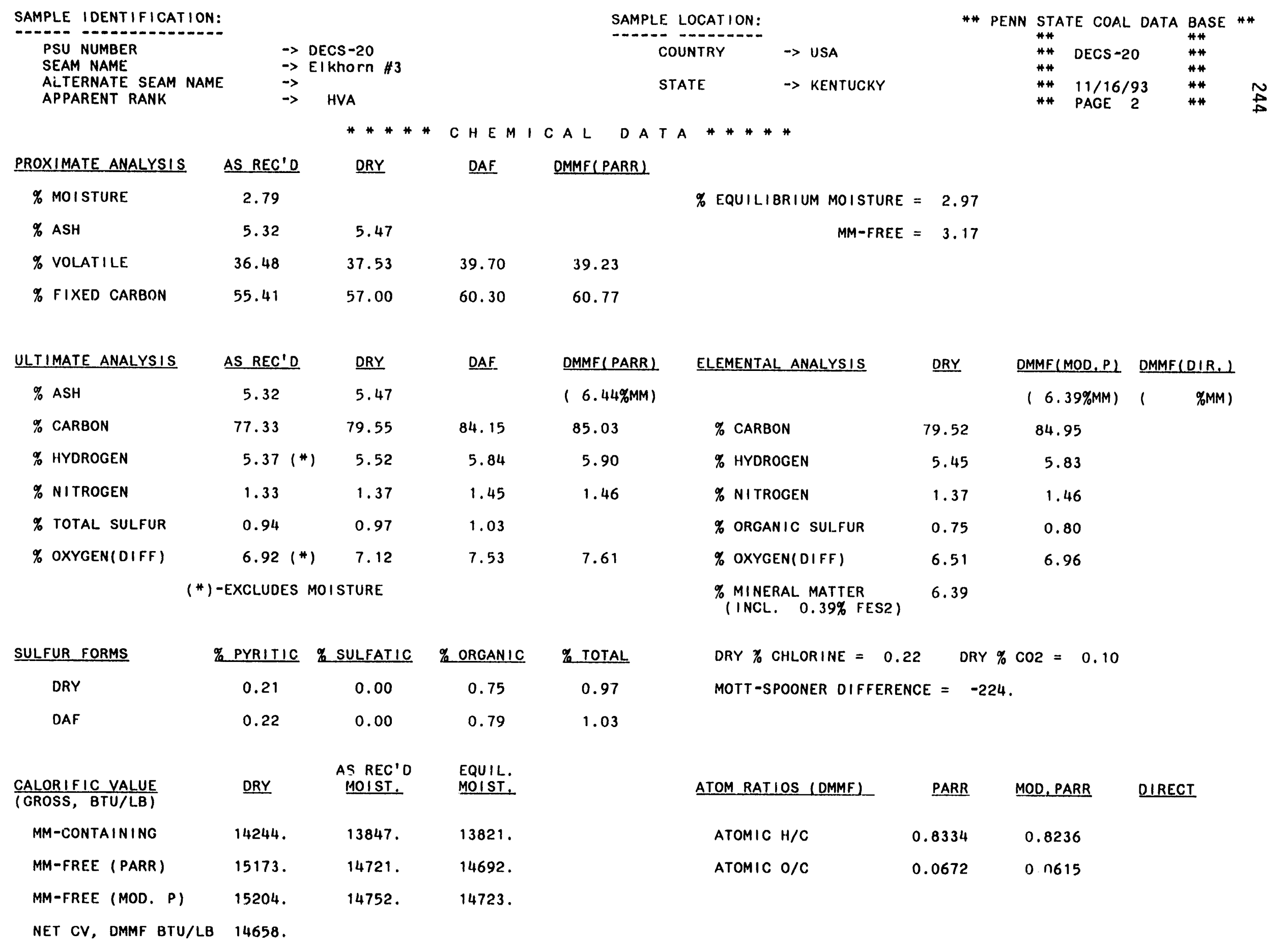


SAMPLE IDENTIFICATION: SAMPLE IDENTIFICATION:

PSU NUMBER $\quad \rightarrow$ DECS -20

SEAM NAME

ALTERNATE SEAM NAME

APPARENT RANK

$\rightarrow$ Elkhorn \#3

$\rightarrow$ HVA

\begin{tabular}{ll} 
SAMPLE LOCATION: & \\
\hline COUNTRY & $\rightarrow$ USA \\
STATE & $\rightarrow$ KENTUCKY
\end{tabular}

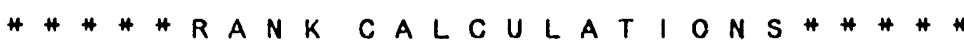

APPARENT RANK (AS REC'D MOIST.)

$\rightarrow$

$\rightarrow \quad$ HVA

$\rightarrow \quad$ HVA
INTERNAT IONAL RANK :

AS REC'D MOIST, $\rightarrow$

EQUIL. MOIST

REPORTED RANK :

$\rightarrow \quad$ HVA
REFLECTANCE RANK CATEGORY

****\# PETROGRAPH C C DATA *****

MACERAL COMPOSITION ( WHITE LIGHT AND FLUORESCENCE ANALYSIS )

MACERAL COMPOSITION

$\begin{array}{lcc}\text { PSEUDO- } & \text { SEMI- } & \\ \text { VITRINITE FUSINITE FUSINITE MACRINITE MICRINITE TINITE } & \text { SPOR- } \\ \text { INITE }\end{array}$

RESINITE ALGINITE CUTINITE

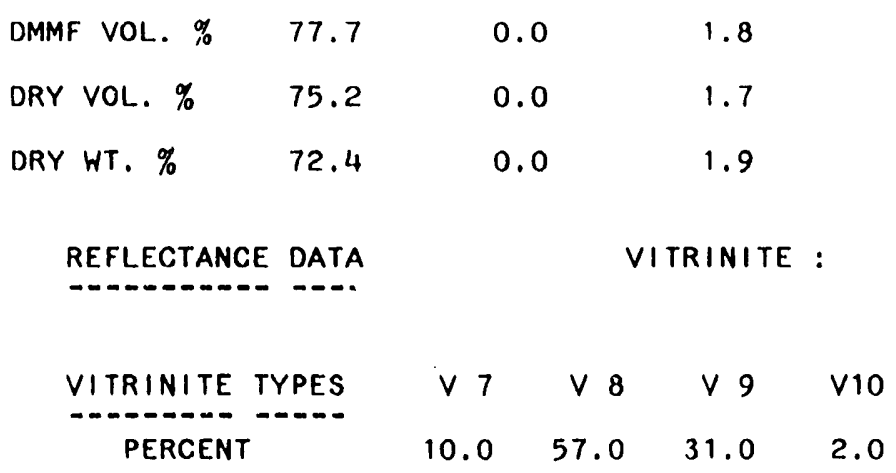

**\#* CAKING A N D MECHAN I C A L

GIESELER COAL PLASTOMETER:

GRAY-KING COKE TYPE

INIT. SOFTENING TEMP 403

HARDGROVE GRINDABILITY

52.1

TEMP. MAX. FLUIDITY

439

FREE SWELLING INOEX

4.0

SOLIDIFICATION TEMP. $\quad 469$

VICKERS HARDNESS NUMBER

FLUID TEMP. RANGE

66

MAXIMUM FLUIDITY

1612

WASHABILITY DATA AVAILABLE? NO 
SAMPLE IDENTIFICATION:

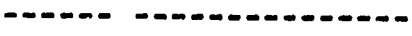
PSU NUMBER SEAM NAME

ALTERNATE SEAM NAME

APPARENT RANK

$$
\begin{aligned}
& \rightarrow \text { DECS-20 } \\
& \rightarrow \text { EIkhorn } \# 3 \\
& \rightarrow> \\
& \rightarrow \text { HVA }
\end{aligned}
$$

MAJOR ELEMENT ANALYSIS

MAUOR ELEMENT ANALYSIS

$\begin{array}{lccc} & \text { S102 } & \text { AL203 } & \text { T102 } \\ \text { OXIDE \% OF HTA } & 47.8 & 31.3 & 1.15 \\ \text { ELEMENT \% OF } & \text { SI } & \text { AL } & \text { TI } \\ \text { TOTAL DRY COAL } & 1.3 & 0.9 & 0.04\end{array}$

TRACE ELEMENT ANALYSIS
SAMPLE LOCATION:

COUNTRY $\quad \rightarrow$ USA

STATE

-> KENTUCKY

$\%$ HTA $=5.68$

$\begin{array}{ccccccc}\text { FE203 } & \text { MGO } & \text { CAO } & \text { NA2O } & \text { K20 } & \text { P205 } & \text { S03 } \\ 9.20 & 1.24 & 3.26 & 1.16 & 2.72 & 0.014 & 1.80 \\ \text { FE } & \text { MG } & \text { CA } & \text { NA } & \text { K } & P & S \\ 0.37 & 0.04 & 0.13 & 0.05 & 0.13 & 0.001 & 0.97\end{array}$

\begin{tabular}{|c|c|c|c|c|c|c|c|c|c|c|}
\hline & $\begin{array}{l}\text { PPM } \\
\text { HTA }\end{array}$ & $\begin{array}{c}\text { PPM } \\
\text { TOTAL COAL }\end{array}$ & & $\begin{array}{l}\text { PPM } \\
\text { HTA }\end{array}$ & $\begin{array}{c}\text { PPM } \\
\text { TOTAL COAL }\end{array}$ & & $\begin{array}{l}\text { PPM } \\
\text { HTA }\end{array}$ & $\begin{array}{c}\text { PPM } \\
\text { TOTAL COAL }\end{array}$ & VOLATILES & $\begin{array}{c}\text { PPM } \\
\text { TOTAL COAL }\end{array}$ \\
\hline$A G$ & & & $\mathrm{GE}$ & & & SN & & & AS & \\
\hline$B$ & & & LA & & & SR & 2368. & 134. & BR & \\
\hline$B A$ & 2239. & 127 & LI & & & $\mathrm{TH}$ & & & $C D$ & \\
\hline$B E$ & 55. & 3. & $M N$ & 232 & 13. & $U$ & & & $\mathrm{CL}$ & 2200. \\
\hline BI & & & MO & & & V & 290. & 16. & $\mathrm{~F}$ & \\
\hline$C E$ & & & NB & & & $Y$ & & & $H G$ & \\
\hline $\mathrm{CO}$ & & & NI & 220 & 12. & YB & & & SB & \\
\hline CR & 170 & 10. & PB & & & $Z N$ & 240 & 14. & SE & \\
\hline $\mathrm{CU}$ & 300 & 17. & $R B$ & 95. & 5. & $Z R$ & 170 & 10. & & \\
\hline GA & & & SC & & & & & & & \\
\hline
\end{tabular}

* penn state coal data base **

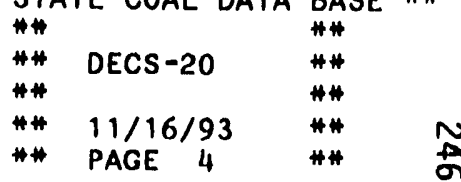


SAMPLE IDENTIFICATION:

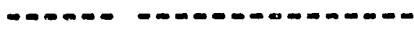
PSU NUMBER

SEAM NAME

ALTERNATE SEAM NAME

APPARENT RANK $\rightarrow$ DECS -20

$\rightarrow$ Elkhorn \#3

$\overrightarrow{->}$

$\rightarrow \quad$ HVA
SAMPLE LOCATION:

SAMPLE LOCATION:

COUNTRY $\quad \rightarrow$ USA

STATE

-> KENTUCKY
* penn state coal data base \#\# $\# *$

\#\# $11 / 16 / 93$

** PAGE 5
ASH FUSION TEMPERATURES (ALL TEMPERATURES IN DEGREES F)

REDUCING OXIDIZING SOFTEN ING

HEMISPHERICAL

FLUID
INITIAL DEFORMATION
2180 .

2370 .

2300 .

2355.

2420.
2460 .

2505.

2550. 
SAMPLE IDENTIFICATION:

PSU NUMBER
SEAM NAME
ALTERNATE SEAM NAME

$\rightarrow$ DECS -21

$\rightarrow$ Lykens Valley $\# 2$

APPARENT RANK

$\rightarrow$ Lykens \#2

AN

SAMPLE HISTORY:

SAMPLING DATE \& AGENCY

$->10 / 7 / 92$; PSU

SAMPLE TYPE

$\rightarrow$ CHANNEL-WHOLE SEAM

ADDITIONAL INFORMATION

$\rightarrow$ Coal fractured by blasting

MINE INFORMATION:

MINE INFORMATION:

RESERVES

$->$

ANNUAL PRODUCTION
SAMPLE LOCATION:

SAMPLE L

COUNTRY $\quad \rightarrow$ USA

STATE $\quad \rightarrow$ PENNSYLVANIA

COUNTY

-> Columbia

TOWNSHIP

CITY

$\rightarrow$ Conygham

COAL PROVINCE-> EASTERN REGION $\rightarrow$ ANTHRACITE

MAP REFERENCE: LATITUDE $\Rightarrow 40046 \mathrm{M} 34 \mathrm{~S}$ LONGITUDE $\rightarrow 76 \mathrm{D} 22 \mathrm{M} 43 \mathrm{~S}$ QUADRANGLE-> Mount Carmel (7.5')

LIFE EXPECTANCY $\rightarrow$

MINING METHOD $\rightarrow$ UNDERGROUND
SEAM INFORMATION: AGE OF SEAM

GROUP

$\rightarrow$ PENNSYL.

$\rightarrow$ Pottsville

FORMATION

SEAM THICKNESS
$->$
$\rightarrow$
$6 \mathrm{FT}$.
6 IN.

$\begin{aligned} \text { ROOF } & \rightarrow \text { Coarse-grained sandstone } \\ 18 \text { IN. } & \rightarrow \text { Blucky bright clarain } \\ 45 \text { IN. } & \rightarrow \text { Fractured bright clarain } \\ 4 \text { IN. } & \rightarrow \text { Dul I coal "middilngs" } \\ 11 \text { IN. } & \rightarrow \text { Bright clarain, less fractured } \\ \text { FLOOR } & \rightarrow \text { Laminated dark gray shale }\end{aligned}$


SAMPLE IDENTIFICATION:

CAMPE IDENTIFICATION:

PSU NUMBER

SEAM NAME

ALTERNATE SEAM NAME

APPARENT RANK

$\rightarrow$ OECS -21
$\rightarrow$ Lykens Valley $\# 2$
$\rightarrow$ Lykens $\# 2$
$\rightarrow$ AN

SAMPLE LOCATION:

SAMPLE LOCATION:

COUNTRY $\quad \rightarrow$ USA

$\rightarrow$ AN
* penn state coal data base "*

** DECS -21 **

*

\#\# 11/16/93 *\#

* PAOE

$* *$

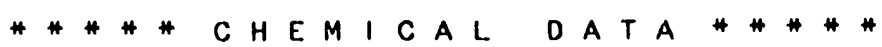

$\rightarrow$ PENNSYLVANIA

PROXIMATE ANALYSIS

$\%$ MOISTURE

$\%$ ASH

\% VOLATILE

\% FIXED CARBON

ULTIMATE ANALYSIS

$\%$ ASH

\% CARBON

\% HYDROGEN

$\%$ NITROGEN

\% total sulfur

\% OXYGEN(DIFF)

\section{AS REC'D ORY}

3.99

10.71

4.33

80.97

AS REC'D ORY

$10.71 \quad 11.15$

77.06

$3.42(*)$

0.68

0.48

$3.67(*)$

11.15

4.51

84.34

80.26

3.56

0.71

0.50

3.82

(*)-EXCLUDES MOISTURE

SULFUR FORMS

DRY

DAF

CALORIFIC VALUE

(GROSS, BTU/LB)

MM-CONTAININC

MM-FREE (PARR)

MM-FREE (MOD. P)

\% PYRITIC \% sulfatic

0.06

0.00

\% ORGANIC

0.43

0.48

AS REC'D EQUIL.

DRY MOIST. MOIST.

13284. $12754 . \quad 13005$.

15122.

14437.

14760.

15204.

14513.

14839.
\% EQUILIBRIUM MOISTURE $=2.10$

MM-FREE $=2.40$

NET CV, DMMF BTU/LB 14826 . 

SAMPLE IOENTIFICATION: PSU NUMBER SEAM NAME

ALTERNATE SEAM NAME

APPARENT RANK $\rightarrow$ DECS -21

$\rightarrow$ Lykens Valley \#2

$\rightarrow$ Lykens $\# 2$

$\rightarrow \quad$ AN
SAMPLE LOCATION:

COUNTRY $\rightarrow$ USA

STATE

\begin{tabular}{|c|c|}
\hline \#\# & DECS -21 \\
\hline \#\# & $\begin{array}{l}11 / 16 / 93 \\
\text { PAGE } 3\end{array}$ \\
\hline
\end{tabular}

* penn state coal data base **
*

\#* $\quad \tilde{O}$
APPARENT RANK (AS REC'D MOIST.)

ASTM RANK (EQUIL. MOIST.)

REFLECTANCE RANK CATEGORY

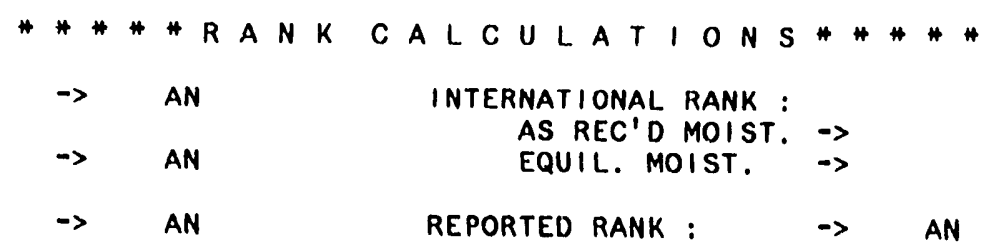

INTERNAT IONAL RANK :

AS REC'D MOIST. $\rightarrow$ EQUIL. MOIST.

REPORTED RANK :

$\rightarrow \quad A N$

***** PETROGRAPHIC DATA *****

MACERAL COMPOSITION ( WHITE LIGHT ANALYSIS )

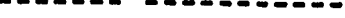

PSEUDO-

VITRINITE VITRINITE FUSINITE FUSINITE MACRINITE MICRINITE TINITE INITE

RESINITE ALGINITE CUTINITE

\begin{tabular}{|c|c|c|c|c|c|c|c|c|c|c|c|c|}
\hline DMMF VOL. \% & 87.1 & 0.0 & 2.5 & 8.3 & 2.0 & 0.1 & 0.0 & 0.0 & 0.0 & 0.0 & 0.0 & \\
\hline DRY VOL. \% & 81.6 & 0.0 & 2.3 & 7.8 & 1.9 & 0.1 & 0.0 & 0.0 & 0.0 & 0.0 & 0.0 & $6.3 \% \mathrm{MM}$ \\
\hline DRY WT. \% & 75.9 & 0.0 & 2.4 & 7.5 & 1.8 & 0.1 & 0.0 & 0.0 & 0.0 & 0.0 & 0.0 & $12.3 \% \mathrm{MM}$ \\
\hline
\end{tabular}

REFLECTANCE DATA

VITRINITE :

LOW: $\quad 4.26$

MEAN-MAX

5.19

REFLECTANCE OATA

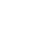

NUMBER OF V-TYPES IS GREATER THAN 10; CONSULT ORIGINAL DATA FOR INFORMATION \#\#\#\#*

***** CAKING AND MECHAN I CA L

GIESELER COAL PLASTOMETER:

INIT. SOFTENING TEMP

TEMP. MAX. FLUIDITY

SOLIDIFICATION TEMP.

FLUID TEMP. RANGF.

MAXIMUM FLUIDITY

WASHABILITY DATA AVAILABLE?
P R O PERTIES* * * * *

GRAY-KING COKE TYPE

HARDGROVE GRINDAB ILITY

31.6

FREE SWELLING INDEX

0.0

VICKERS HARDNESS NUMBER 
SAMPLE IDENTIFICATION:

$\begin{array}{ll}\text { PSU NUMBER } & \rightarrow \text { DECS }-21 \\ \text { SEAM NAME } & \rightarrow \text { Lykens Valley } \# 2 \\ \text { ALTERNATE SEAM NAME } & \rightarrow \text { Lykens } \# 2\end{array}$

$\rightarrow$ Lykens

$\rightarrow \quad$ AN

MAJOR ELEMENT ANALYSIS

MAUOR ELEMENT ANALYSIS

$\begin{array}{lccc} & \text { S102 } & \text { AL203 } & \text { TIO2 } \\ \text { OXIDE \% OF HTA } & 55.1 & 30.7 & 2.27 \\ \text { ELEMENT \% OF } & \text { SI } & \text { AL } & \text { TI } \\ \text { TOTAL DRY COAL } & 2.9 & 1.8 & 0.15\end{array}$

FE203
4.24
FE
0.33
SAMPLE LOCATION:
-..... - - - -

COUNTRY $\rightarrow$ USA

STATE

$\rightarrow$ PENNSYLVANIA

* penn state coal data base *

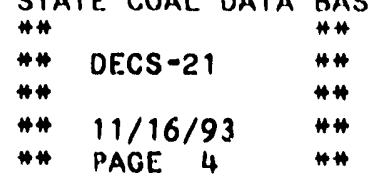

\author{
$\%$ HTA $=11.14$
}

$\begin{array}{cccccc}\text { MCO } & \text { CAO } & \text { NA2O } & K 20 & \text { P205 } & S 03 \\ 0.88 & 0.56 & 0.22 & 4.93 & 0.04 & 0.20 \\ \text { MG } & \text { CA } & \text { NA } & K & P & S \\ 0.06 & 0.04 & 0.02 & 0.46 & 0.002 & 0.50\end{array}$

TRACE ELEMENT ANALYSIS

TRACE ELEMENT ANALYSIS

$\begin{array}{lc}\text { PPM } & \text { PPM } \\ \text { HTA } & \text { TOTAL COAL }\end{array}$

\section{PPM PPM}

HTA

TOTAL COAL

AG

B

BA

BE

1164.

45.

130.

BI

CE

co

CR

210.

220 .

GA
5.

GE

LA

LI

MN

MO

NB

NI

23. $P B$

25. RB

(1)

$\%$ HTA $=11.14$

$\begin{array}{lll}\text { PPM } & \text { PPM } & \text { VOLATILES PPM } \\ \text { HTA } & \text { TOTAL COAL } & \end{array}$

AS

BR

CD

$\mathrm{CL} \quad 400$.

190. 21.

F

HG

SB

17. YB

130.

14.

SE

22. $Z R \quad 500.56$. 
SAMPLE IDENTIFICATION:

SEAM NAME

ALTERNATE SEAM NAME

APPARENT RANK

$\rightarrow$ DECS -21

$\rightarrow$ Lykens Valley \#2

$\rightarrow$ Lykens \#2

$\rightarrow \quad$ AN

ASH FUSION TEMPERIATURES (ALL TEMPERATURES IN DEGREES F) ASH FUSION TEMPERATURES

SOFTENING

HEMI SPHERICAL

FLUID
REDUCING OXIDIZING

$2800 .+2800 .+$

$2800+2800+$

$2800 .+2800 .+$
$2730 .+2800+$
SAMPLE LOCATION: COUNTRY

-> USA

STATE

$\rightarrow$ PENNSYLVANIA
* penn state coal data base **

OA

H* $11 / 16 / 93$

* PAGE 5

\#*

**

**

岕 
SAMPLE IDENTIFICATION:

PSU NUMBER

ALTERNATE SEAM NAME

APPARENT RANK

$\rightarrow$ DECS-22

$\rightarrow$ Upper Kittanning

$\rightarrow \quad$ HVA

SAMPLE HISTORY:

SAMPLING DATE \& AGENCY

$\rightarrow \quad 6 / 14 / 93 ; P S U$

SAMPLE TYPE

ADOITIONAL INFORMATION

$\rightarrow$ CHANNEL-LITHOTYPE

$\rightarrow$ Cannel coal pod sheped deposit; see PSOC-1560 Ro

$\rightarrow$ USA

COUNTRY

STATE $\quad \rightarrow$ PENNSYLVANIA

COUNTY

$\rightarrow$ Armstrong

TOWNSHIP $\quad \rightarrow$ Mahoning

CITY $\quad \rightarrow$ DIstant

COAL PROVINCE $\rightarrow$ EASTERN

REGION $\rightarrow$ APPALACHIAN

MAP REFERENCE: LATITUDE $\rightarrow \quad 400058 \mathrm{M} 21 \mathrm{~S}$

ONGITUDE $\rightarrow 79 \mathrm{D} \quad 18 \mathrm{M} 23$

QUADRANGLE $\rightarrow$ Distant $\left(7.5^{\prime}\right)$

MINE INFORMATION:

MINE INFORMATION:

RESERVES

ANNUAL PRODUCTION
$->$

$\rightarrow$
LIFE EXPECTANCY $\rightarrow$

MINING METHOD $\rightarrow$ UHDERGROUND
SEAM INFORMATION: AGE OF SEAM

GROUP

FORMATION

SEAM THICKNESS
-> PENNSYL.

$\rightarrow$ Al legheny

$\rightarrow$

$\Rightarrow \quad 9 \mathrm{FT} . \quad 2 \mathrm{IN}$.

** SEAM PROFILE **

$\begin{aligned} & \text { ROOF } \\ & 16 \text { IN. } \\ & 29 \text { IN. } \\ & 27 \text { IN. } \\ & 8 \text { IN. } \\ & 30 \text { IN. } \\ & \text { FLOOR }\end{aligned}$

$\rightarrow$ Irregular contact

$\rightarrow$ Blocky fow band

DECS -22

$\rightarrow$ Very blocky DECS-22

$\rightarrow$ Blocky, some partings DECS-22

$\rightarrow$ Blocky, not fractured DECS-22

$\rightarrow$ Clarain, well banded PSOC-1560

FLOOR 
SAMPLE IDENTIFICATION: PSU NUMBER PSU NUMBER

ALTERNATE SEAM NAMF

APPARENT RANK

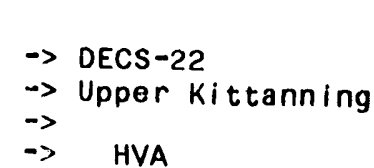

SAMPLE LOCATION:

SAMPLE LOCATION:

$\begin{array}{ll}\text { COUNTRY } & \text {-> USA } \\ \text { STATE } & \rightarrow \text { PENNSYLVANIA }\end{array}$

* penn state coal data base *\#

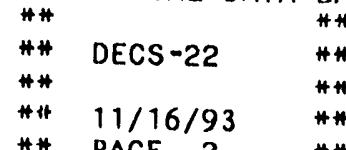

STATE $\quad \rightarrow$ PENNSYLVANIA $\rightarrow$ HVA *\#*** CHEMICA L DATA *\#***

\section{PROXIMATE ANALYSIS}

\% MOISTURE

AS REC'D DRY

0.91

$\% \mathrm{ASH}$

\% VOLATILE

\% FIXED CARBON

ULTIMATE ANALYSIS

$\%$ ASH

\% CARBON

$\%$ HYDROGEN

\% NITROGEN

\% total sulfur

\% OXYGEN (DIFF)

(*) -EXCLUDES MOISTURE

\section{SULFUR FORMS}

DRY

DAF

$\frac{\text { CALORIFIC VALUE }}{\text { (GROSS, BTU/LB) }}$

MR:-CONTAINING

MM-FREE (PARR)

MM-FREE (MOD. P)

NET CV, DMMF BTU/LB

$\begin{array}{cccc}\text { \% PYRITIC } & \% \text { SULFATIC } & \text { \% ORGANIC } \\ 1.15 & 0.03 & 0.53 \\ 1.50 & 0.04 & 0.69 \\ 11786 . & 11679 . & 11630 . \\ 15827 . & 15633 . & 15546 . \\ 16039 . & 15840 . & 15751 .\end{array}$

\% EQUILIBRIUM MOISTURE $=1.32$

MM-FREE $=1.80$
$30.17 \quad 30.45$

39.68

37.75

$\begin{array}{llll}45.86 & 46.28 & 60.32 & 62.25\end{array}$

\section{AS REC'D \\ $23.06 \quad 23.27$}

64.30

64.89

$4.44\left(^{*}\right) \quad 4.48$

$1.35 \quad 1.36$

1.69

1.71

4.29

$\underline{D A F}$

DMMF (PARR)

ELEMENTAL ANALYSIS

(26.07\%MM)

$84.57 \quad 87.77$

5.814

1.77

2.23

5.59

6.06

1.84

4.33

15471. 
SAMPLE IDENTIFICATION:

PSU NUMBER

SEAM NAME

ALTERNATE SEAM NAME

APPARENT RANK

$\rightarrow$ DECS -22

$\rightarrow$ Upper Kittanning

$\rightarrow$ HVA
SAMPLE LOCATION:

COUNTRY $\rightarrow$ USA

STATE $\rightarrow$ PENNSYLVANIA
** penn state coal data base ** \#\# \#\# \#\# DECS -22

\#\# $11 / 16 / 93$

* PAGE 3

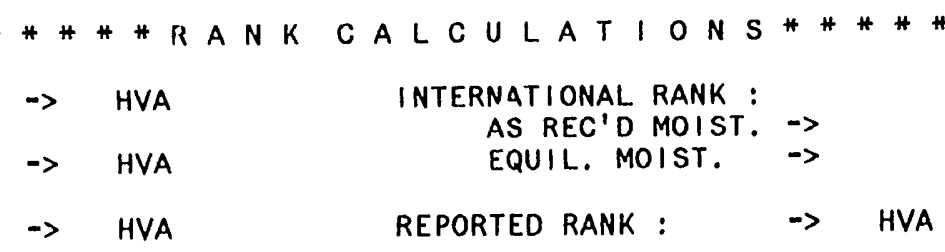

$\rightarrow \quad H V A$

$\rightarrow \quad$ HVA

$\rightarrow \quad$ HVA

INTERNATIONAL RANK :

AS REC'D MOIST, $\rightarrow$

EQUIL. MOIST.

REPORTED RANK : $\quad \rightarrow \quad$ HVA

REFLECTANCE RANK CATEGORY

***** PETROGRAPHIC D A T A * * * *

MACERAL COMPOSITION ( WHITE LIGHT AND FLUORESCENCE ANALYSIS )

MACERAL COMPOSITION PSEUDO$\begin{array}{llll} & \text { SEMI- } & \text { SCLERU- SPOR- } \\ \text { FUSINITE FUSINITE MACRINITE MICRINITE TINITE INITE }\end{array}$ VITRINITE VITRINITE RESINITE ALGINITE CUTINITE

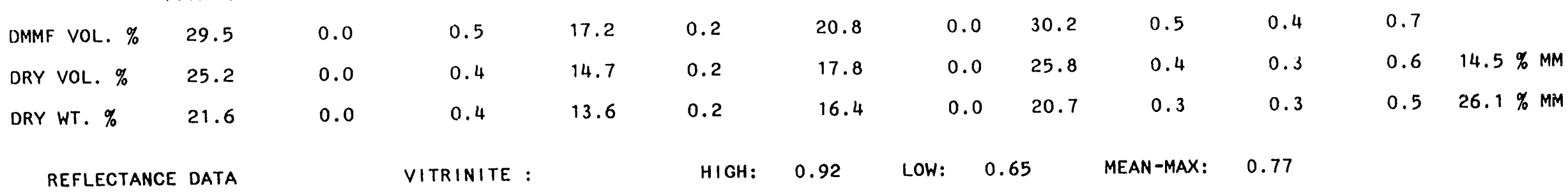

REFLECTANCE DATA

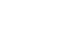

\begin{tabular}{lrrrr} 
VITRINITE TYPES & $\vee 6$ & $V 7$ & $\vee 8$ & $V 9$ \\
\hline PERCENT & 8.0 & 68.0 & 20.0 & 4.0
\end{tabular}

***** CA K I N G A N D MECHAN I C A L

GIESELER COAL PLASTOMETER:

INIT. SOFTENING TEMP 416

TEMP. MAX. FLUIDITY 444

SOLIDIFICATION TEMP. 482

FLUID TEMP. RANGE 66

MAXIMUM FLUIDITY 11719

WASHABILITY DATA AVAILABLE? NO
PROPERT I E S

GRAY-KING COKE TYPE

HARDGROVE GRINDAB ILITY 31.0

FREE SWELLING INDEX

1.0

VICKERS HARDNESS NUMBER 
SAMPLE IDENTIFICATION: SAMPLE IDENTIFICATION: PSU NUMBER

SEAM NAME

ALTERNATE SEAM NAME

APPARENT RANK

$\rightarrow$ DECS -22

$\rightarrow$ Upper Kittanning

$\rightarrow$ HVA

MAJOR ELEMENT ANALYSIS

MAJOR ELEMENT ANALYSIS

$\begin{array}{cc} & \$ 102 \\ \text { OXIDE \% OF HTA } & 62.0 \\ \text { ELEMENT \% OF } & S 1 \\ \text { TOTAL DRY COAL } & 6.6 \\ & \\ \text { PPM } & \\ \text { HTA } & \text { TOTAL COAL }\end{array}$

AL203 T102

$23.2 \quad 1.49$

$A L$

TI

$2.8 \quad 0.20$

AG

B

\section{BA}

BE

358.

BI

CE

co

CR

cu

GA

\begin{tabular}{ll} 
SAMPLE LOCATION: & \\
\hline COUNTRY & $\rightarrow$ USA \\
STATE & $\rightarrow$ PENNSYLVANIA
\end{tabular}

** penn state coal data base \#\#

$\begin{array}{ll}* \# & \\ * * & \text { DECS }-22 \\ * * & 11 / 16 / 93 \\ * * & \text { PAGE } 4\end{array}$

\#\#

\#\#+\# \#\#
$\%$ HTA $=22.81$

FE203 MGO

CAO NA2O

K20

P205

SO3

$8.71 \quad 1.01$

0.53

0.28

2.74

0.04

0.10

FE

MG

CA

NA

K

$$
\mathrm{P}
$$

$\mathrm{s}$

$1.39 \quad 0.14$

0.09

0.05

0.52

0.004

1.71
TOTAL COAL

GE

10.

LA

PPM PPM

HTA TOTAL COAL

PPM
HTA

$\%$ HTA $=22.81$

TOTAL COAL

SN

SR

169.

39.

18.

77.

MO

NB

NI

180.

41. PB

150.

34. RB

SC

sc


SAMPLE IDENTIFICATION: CAMPLE IDENTIFICATION:

PSU NUMBER

SEAM NAME

ALTERNATE SEAM NAME

APPARENT RANK

$\rightarrow$ DECS -22

$\rightarrow$ Upper Kittanning

$\rightarrow$

$\rightarrow \quad$ HVA
SAMPLE LOCATION:

COUNTRY

$\rightarrow$ USA

STATE

$\rightarrow$ PENNSYLVANIA
*\# PENN STATE COAL DATA bASE *\# \#\# DECS -22

\#* $11 / 16 / 93$

ASH FUSION TEMPERATURES (ALL TEMPERATURES IN DEGREES F) ASH FUSION TEMPERATURES

REDUCING OXIDIZING

INITIAL DEFORMATION

2600.

$2725 .+$

SOFTENING

2645.

2765 .

HEMISPHERICAL

2680 .

$2780 .+$

FLUID

$2740 .+2800 .+$ 


\author{
APPENDIX C \\ List of Agencies Supplied with \\ Coal Samples andlor Data \\ 4/08/88 through 9/07/93
}

Numbers in parentheses are multiple requests which were separately processed. 
Abilene Christian University (4)

Advanced Fuel Research (8)

AGL

Air Products Corporation

Akita University (Japan)

Akita University. Chemical Eng. for Resources Dept. (Japan)

Alcoa Research Lab

Alcon Coal and Land Co.

Allied Coal Testing

Amax Coal Company Research and Development Center (3)

American Lignite Co. (2)

Amoco 0 il Company. Research \& Development Division (15)

Anthracite Industries Co.

ARCO

ARCO Coal Company

ARCO 0 il and Gas Co.

Argonne National Laboratory

Arizona State University. Chemistry and Biochemistry Dept.

Arizona State University. Geology Department

Arizona State University. Mechanical \& Aerospace Engineering Department

Arkansas Tech University. Arkansas Mining Institute

Asea Brown Boveri Ltd. (Switzerland)

Atlas Powder Co.

Auburn University. Chemical Engineering Department (5)

Babcock \& Wilcox Co. (2)

BCR National Laboratories (6)

Boarshead Resource Development Co.

Borden Chemical Co.

Brigham Young University (5)

Brigham Young University. College of Engineering Technology

Brookhaven National Laboratory (2)

Brown University

Brown University, Engineering Div.

Bruce MacDonald, Consultant

Buckeye Industrial Mining Co. (2)

Cal Tech

Calgon Carbon Corporation (2)

California Institute of Technology

CANMET Energy Mines \& Resources (Canada) (3)

Carnegie-Mellon University. Metallurgical Eng. \& Materials Science Dept.

Central Research Institute, Electric Power Industry (Japan) (2)

Centre Lime \& Stone

Chalmers University of Technology (Sweden)

Chemrol1 Enterprises

Chevron Oilfield Research Co. (6)

City College of New York. Chemical Enaineering Department (2)

Clark Atlanta University. Chemistry Department (6)

Clarke Rajchel Engineering (2)

Coal Cleaning Test Facility. Homer City, PA 
Coal Technology Corp. (2)

Colorado School of Mines. Chemical Engineering Department (3)

CONOCO Research Center

Consolidation Coal Company

Consortium for Fossil Fuel Liquefaction Science (3)

Davis Coal Co.

Decision Focus Co.

Dow Chemical/DESTEC

Duke University Medical Center

EFH Coal Co.

EG \& G Idaho

Electrochem, Inc.

Emory University. Chemistry Department

Encyclopedia Britannica

Energy Ventures Analysis Inc.

Engineering Resources

ENSR Consultants and Engineers

ERM Inc.

Exxon Production Research Co. (3)

Exxon Research \& Development Co. (5)

Fossil Energy Research Company

Foster-Wheeler Development Corp.

Frankl in \& Marshall College. Geology Department

Gannon University

Gas Research Institute

Genesis Research Corp. (2)

General Electric Co.

Geological Survey of Denmark

Geological Survey of Wyoming

Gould Energy, Warner Labs Division

Grambling State University. Chemistry Department (7)

Hacettepe University, Chemistry Department (Turkey) (2)

Hampton University. Engineering Department

Hess and Fisher Engineers

Howard University. Chemistry Department (2)

Idea. Inc.

Idemitsu Kosan Co., Ltd. (Japan)

Illinois State Geological Survey

Indianapolis Power \& Light Co. (4)

Industrial Mining Co. (2)

Industrial Quimica del Nalon (Spain)

Institute of Gas Technology (4)

Instituto Nacional del Carbon (Spain)

Interpower of Pennsylvania

J.K. Research (4)

Jet Propulsion Laboratory

Juniata College

Kansai University. Chemical Engineering Dept.

Dr. J. Kelly, Mining Engineer 
Kentucky Center for Applied Energy Research (2)

Korea Advanced Institute of Science and Technology

Lehigh University. Chemistry Department (2)

Louisiana State University. Chemical Engineering Department

Louisiana Tech

Martin Marietta Energy Systems

Michigan Biotechnology Institute

Michigan State University

Missouri Geological Survey

Monash University. Chemistry Deptartment (Australia) (2)

MPR Associates

National Refractories and Minerals Corporation

National University of Colombia

New Energy and Development Org.

New York State Electric \& Gas

New York University. Chemistry Department

New York University. Medical Center

NIOSH (2)

NIR Systems (2)

NKK America, Inc.

Northeastern University (3)

Northern Indiana Public Service Co.

Notre Dame University. Geology Department

Oak Ridge National Laboratory (7)

Ohio Coal Development Office

Ohio Coal Research Board

Ohio State University. Chemistry Department

Ohio University, Geological Sciences Department

Ohio University. Chemical Engineering Department (5)

Pacific Northwest Laboratory

Peabody Development Co.

PEN Coal Corp.

Pendygraft \& Plews. Attorneys

Pennsylvania Department of Environmental Resources

Pennsylvania Geological Survey

Pennsylvania State University. Architectural Engineering Department

Pennsylvania State University. Chemical Engineering Dept.

Pennsyivania State University. Dust Control Center (3)

Pennsyivania State University, Energy and Fuels Research Center (4)

Pennsyivania State University. Fuel Science Department (125)

Pennsylvania State University. Geography Department

Pennsyivania State University. Geosciences Department (26)

Pennsylvania State University, Materials Research Lab (2)

Pennsyivania State University. Mineral Processing Section (44)

Pennsylvania State University. Mining Engineering Department (4)

Pennsylvanio "tate University. Nuclear Engineering Department

Pennsylvania State University, Polymer Science Department (17)

Perfect View, Inc. (5)

Physical Science Inc. 
Potomac Electric Power Company

Praxis Engineers Inc.

PSI Technology Co. (3)

Ralph Gray Services

RDI, Inc.

Research Triangle Institute

RLN Co.

Rochester and Pittsburgh Coal Co. (2)

Russian Academy of Sciences. Kemerovo Scientific Centre

Sandia National Laboratory (3)

Science Applications International Co. (2)

Science Ventures. Inc. (3)

Scott Paper Co.

Sekei University. Industrial Chemistry Department (Japan) (2)

Seoul National University. Chemical Engineering Dept. (Korea)

She11 Development Co. (5)

Solar Energy Research Institute

Solar Sources, Inc.

Southern Illinois University. Coal Research Center

Southern Illinois University, Geology Department ( 7 )

Southern Illinois University. Mechanical Engineering Department (5)

Southern Illinois University. Dept. of Mechanical Eng. \& Energy Processes

Southern Research Institute

Spackman, W. . consultant

Standard Laboratories

Stanford University (4)

Stanford University. Mechanical Engineering Dept. (2)

State University of New York at Binghamton. Chemistry Department (2)

State University of New York at Buffalo (2)

Stock Equipment Company (2)

Stone and Webster Engineering (2)

Superior Adsorbents. Inc.

TA Instruments

Technical Research Centre of Finland

Technical University of Denmark

Technology and Management Servvices, Inc.

Tennessee Valley Authority

Texaco Research Center (2)

Texas A \& M University (2)

Texas A \& M University. Mechanical Engineering Dept. (2)

Textron Defense Systems

Tohoku University. Institute for Chemical Reaction Science (2) (Japan)

TRW Corp.

Tuskegee University. Chemical Engineering Dept. (2)

University of Alabama. Geology Department (2)

University of Alaska. Mineral Industries Research Lab

University of Arkansas. Engineering Research Center (2)

Iniversity of British Columbia, Geological Sciences Department (Canada)

University of California at Berkeley. Materials Science and Mineral Eng.Dept. 
University of California at Davis, Chemical Engineering Department (4)

University of Canterbury (New Zealand) (2)

University of Chicago. Chemistry Department

University of Chicago. Geophysical Sciences Department

University of Cincinnati. Chemical Engineering Department

University of Delaware

University of Delaware. Chemical Engineering Department

University of Hartford. Biology Dept.

University of Illinios

University of Illinois. Mechanical \& Industrial Engineering Department (7)

University of Kentucky (4)

University of Kentucky. Center for Applied Energy Research

University of Kentucky. Consortium for Fossil Fuel Liquefaction Science (6)

University of Kentucky. Geology Department (2)

University of Kentucky. Mechanical Engineering Depar sent

University of Kentucky. School of Biological Sciences

University of London, Chemistry Department (England)

University of Lund. Analytical Chemistry Dept. (Sweden)

University of New Hampshire

University of New Hampshire. Dept. of Chemical Engineering

University of New South Wales. Applied Geology Department (Australia)

University of North Dakota

University of North Dakota. Energy and Envirormental Research Center (10)

University of Pierre and Marie Curie (France)

University of Pittsburgh

University of Pittsburgh. Chemical Engineering Department (3)

University of South Carolina (2)

University of Southern Mississippi. Chemistry \& Biochemistry Dept. (2)

University of Texas at Arlington. Chemistry Department (2)

University of Toledo. Geology Dept.

University of Toronto (2)

University of Utah (5)

University of Utah. Center for Microanalysis (3)

University of Utah. Chemistry Dept. (3)

University of Utah. Fuels Engineering Department (11)

University of Utah. Metallurgy \& Metallurgical Engineering Department (4)

University of Wisconsin, Anthropology Dept.

Unocal Science and Technology Division (8)

U.S. Bureau of Mines (4)

U.S. Department of Energy

U.S. Department of Energy. Morgantown (9)

U.S. Department of Energy. Pittsburgh (21)

U.S. Geological Survey (6)

Usibelli Mining Co.

USSR Academy of Sciences

Viking Systems International (5)

West Virginia University. Chemical Engineering Dept. (4)

West Virginia University. Chemistry Department

West Virginia University. Geology and Geography Department 

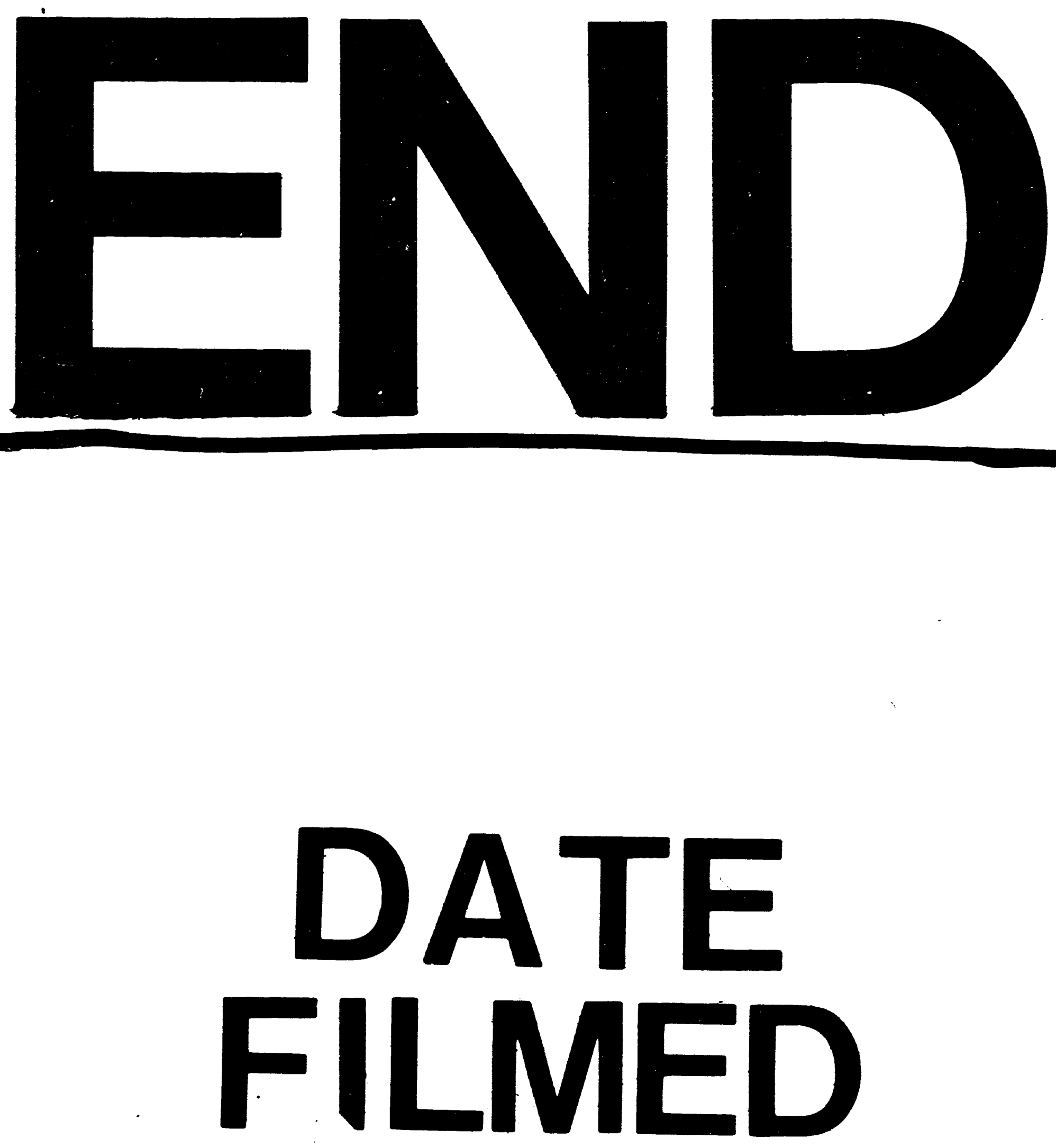

$3 / 30 / 94$ 